Ulrich Harteisen, Silke Neumeyer, Susanne Schlagbauer, Kilian Bizer, Stephan Hensel und Lukas Krüger

\title{
Grünes Band - Modellregion für Nachhaltigkeit
}

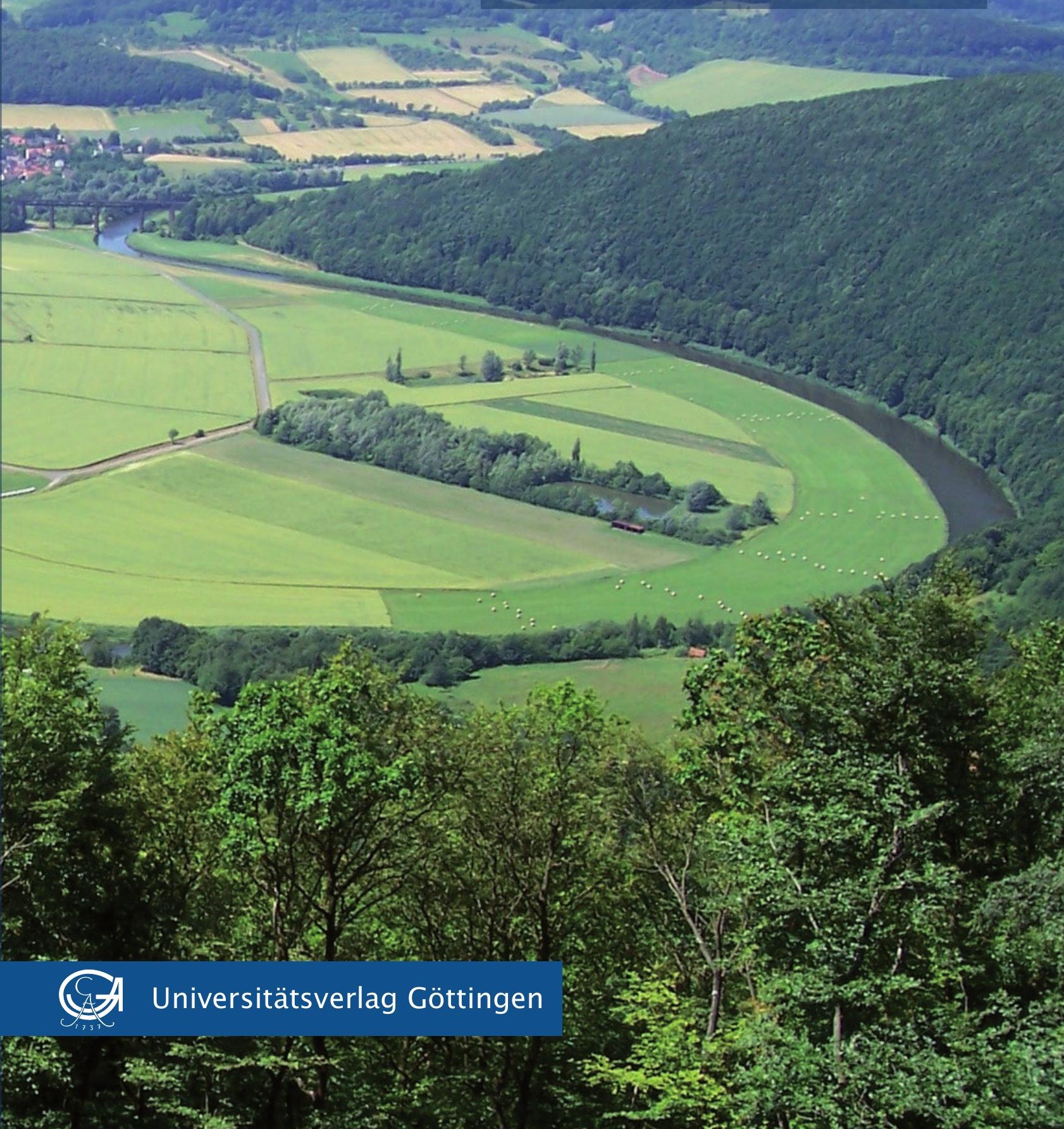



Ulrich Harteisen, Silke Neumeyer, Susanne Schlagbauer Kilian Bizer, Stephan Hensel, Lukas Krüger

Grünes Band - Modellregion für Nachhaltigkeit

This work is licensed under the Creative Commons License 3.0 "by-nd", allowing you to download, distribute and print the document in a few copies for private or educational use, given that the document stays unchanged and the creator is mentioned. You are not allowed to sell copies of the free version.

SORIERIGHISRESERVED 
erschienen im Universitätsverlag Göttingen 2010 
Ulrich Harteisen, Silke Neumeyer, Susanne Schlagbauer,

Kilian Bizer, Stephan Hensel, Lukas Krüger

Grünes Band Modellregion für Nachhaltigkeit
Abschlussbericht des
Forschungsvorhabens

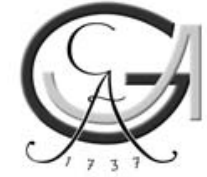

Universitätsverlag Göttingen 2010 
Bibliographische Information der Deutschen Nationalbibliothek

Die Deutsche Nationalbibliothek verzeichnet diese Publikation in der Deutschen Nationalbibliographie; detaillierte bibliographische Daten sind im Internet über $<$ http://dnb.ddb.de> abrufbar.

Der Gesamtabschlussbericht entstand im Rahmen des Verbundprojektes „Grünes Band - Modellregion für Nachhaltigkeit" und wurde mit Mitteln des Bundesministeriums für Bildung und Forschung (BMBF) unter dem Förderkennzeichen FKZ 0330838 gefördert und in Zusammenarbeit mit dem Bundesministerium für Umwelt, Naturschutz und Reaktorsicherheit umgesetzt.

Bundesministerium für Bildung und Forschung (BMBF)

Forschung für Nachhaltigkeit; Wissenschaft und Gesellschaft

Heinemannstr. 2

53175 Bonn - Bad Godesberg

Projektleitung:

HAWK Hochschule für angewandte Wissenschaft und Kunst Fakultät Ressourcenmanagement

Prof. Dr. Ulrich Harteisen

H/W

HAWK HOCHSCHULE FÖR ANGEWANDTE WISSENSCHAFT UND KUNST

Georg-August Universität Göttingen

Wirtschaftswissenschaftliche Fakultät

Prof. Dr. Kilian Bizer

GEORG-AUGUST-UNIVERSITÄT GÖTTINGEN

Dieses Buch ist auch als freie Onlineversion über die Homepage des Verlags sowie über den OPAC der Niedersächsischen Staats- und Universitätsbibliothek (http://www.sub.uni-goettingen.de) erreichbar und darf gelesen, heruntergeladen sowie als Privatkopie ausgedruckt werden. Es gelten die Lizenzbestimmungen der Onlineversion. Es ist nicht gestattet, Kopien oder gedruckte Fassungen der freien Onlineversion zu veräußern.

Satz und Layout: Susanne Schlagbauer, Silke Neumeyer

Umschlaggestaltung: Margo Bargheer

Titelabbildung: Heinz Sielmann Stiftung: Werraschleife bei Lindewerra

(C) 2010 Universitätsverlag Göttingen

http:/ / univerlag.uni-goettingen.de

ISBN: 978-3-941875-60-9 


\section{Projektpartner}

Bundesamt für Naturschutz

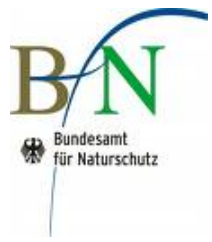

BUND BN Bayern

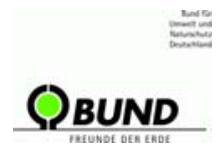

Alpenforschungsinstitut

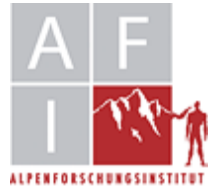

Die inhaltliche Verantwortung liegt bei den Autoren.

Die Autoren danken dem Fördermittelgeber für die freundliche Unterstützung.

Göttingen, Mai 2010 



\section{Inhaltsverzeichnis}

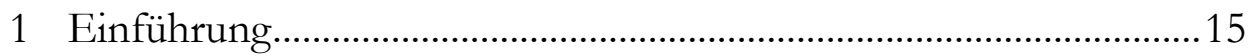

$1.1 \quad$ Problemstellung .......................................................................15

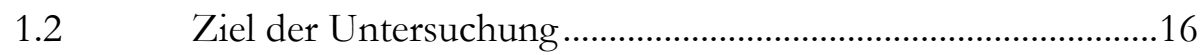

1.3 Aufbau der Untersuchung..........................................................19

$1.4 \quad$ Untersuchungsbeteiligte ............................................................20

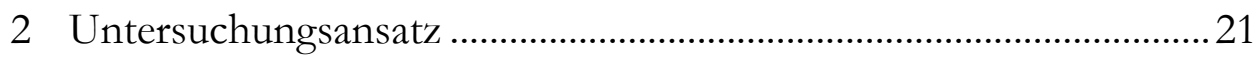

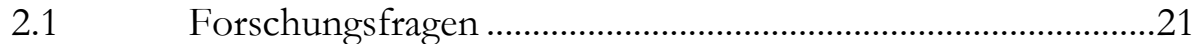

2.2 Methoden und Vorgehensweise der

Maßnahmenperspektive..............................................................23

2.2.1 (Regionale) Nachhaltigkeit als Managementprozess .............................23

2.2.2 Maßnahmenbasismatrix und Nachhaltigkeitsspinne .............................25

2.2.3 Nachhaltigkeitsspinne und regionale Verhaltensweisen .......................27

2.2.4 Regionale Nachhaltigkeitsinstitutionen ..................................................29

2.3 Methoden und Vorgehensweise der Netzwerkanalyse .............32

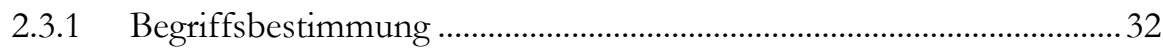

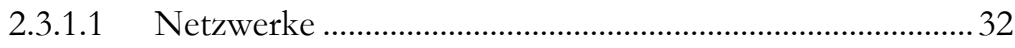

2.3.1.2 Netzwerkmerkmale und Erfolgsfaktoren............................33

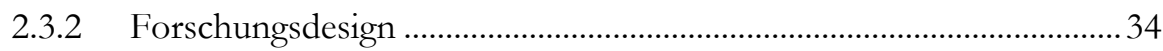

2.3.3 Empirische Datenerhebung und Analysemethoden ..............................35

2.3.3.1 Strukturelle Netzwerkanalyse (und Abgrenzung des Untersuchungstaumes) ..........................................................36

2.3.3.2 Qualitative Netzwerkanalyse .................................................38

2.3.4 Operationalisierung von (Projekt-) Netzwerken: Merkmale und

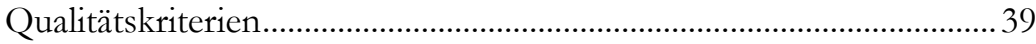

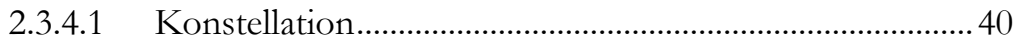

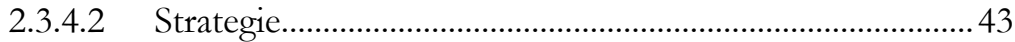

2.3.4.3 Instrumente ......................................................................... 47

2.4 Interdisziplinärer Brückenschlag zwischen Netzwerkanalyse und regionaler Nachhaltigkeit......................................................50 
2.4.1 Interdisziplinäre Verknüpfung...............................................................50

2.4.2 Regionale Managementregeln und Netzwerkmerkmale -

Dynamische Perspektive.

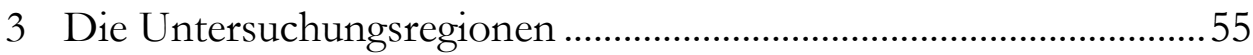

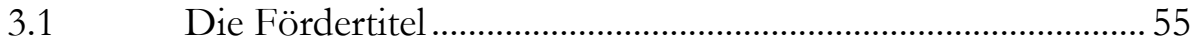

3.1.1 Erprobungs- und Entwicklungsvorhaben

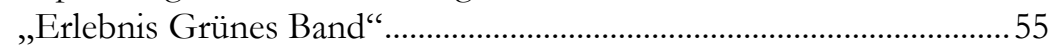

3.1.2 Naturschutzgroßprojekte.....................................................................58

3.2 E+E Region Elbe-Altmark-Wendland .................................... 61

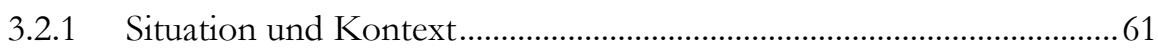

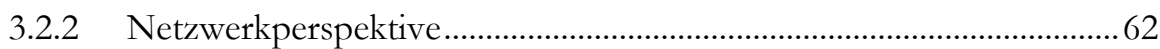

3.2.2.1 Strukturelle Analyse des Projektnetzwerks ........................62

3.2.2.2 Analyse der Netzwerkmerkmale und ihrer strategischen Qualitäten .....................................................70

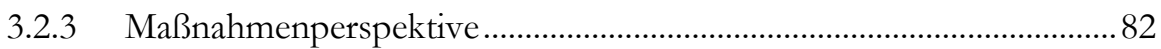

3.2.3.1 Maßnahmenbasismatrix ....................................................... 83

3.2.3.2 Nachhaltigkeitsspinne und regionale Verhaltensweisen .................................................................93

3.2.3.3 Nachhaltigkeitsinstitutionen.................................................96

3.2.4 Beispielhafte Synthese zwischen den Perspektiven ...............................98

3.3 E+E Region Thüringer Wald \& Schiefergebirge/Frankenwald ...................................................100

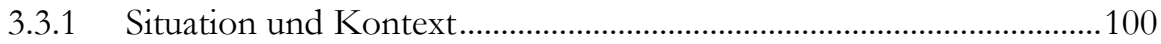

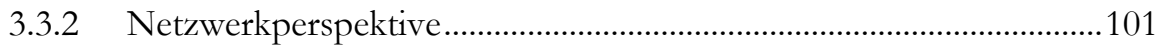

3.3.2.1 Strukturelle Analyse des Projektnetzwerks ......................101

3.3.2.2 Analyse der Netzwerkmerkmale ........................................109

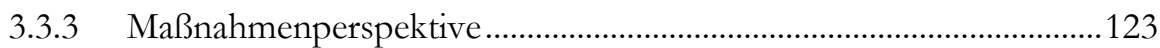

3.3.3.1 Maßnahmenbasismatrix ..................................................124

3.3.3.2 Nachhaltigkeitsspinne und regionale Verhaltensweisen.................................................................134

3.3.3.3 Nachhaltigkeitsinstitutionen ................................................137

3.3.4 Beispielhaft Synthese der Perspektiven ...............................................139

3.4 Naturschutzgroßprojekt Schaalsee-Landschaft ...................... 141

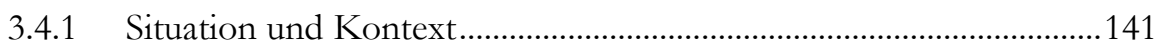

3.4.2 Netzwerkperspektive ............................................................................ 141

3.4.2.1 Strukturelle Analyse des Projektnetzwerks .....................142 
3.4.2.2 Analyse der Netzwerkmerkmale und ihrer strategischen Qualität(en) 152

3.4.2.3 Ergänzende Situationsanalyse ...........................................164

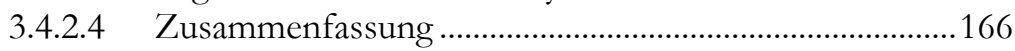

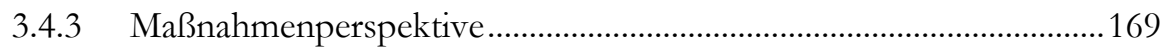

3.4.3.1 Maßnahmenbasismatrix .................................................. 169

3.4.3.2 Nachhaltigkeitsspinne und regionale Verhaltensweisen.......................................................... 183

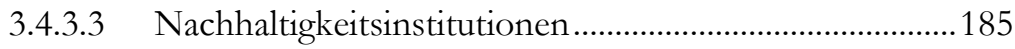

3.4.4 Beispielhafte Synthese zwischen den Perspektiven............................187

3.5 Naturschutzgroßprojekt Grünes Band Eichsfeld-Werratal. 190

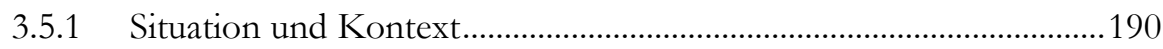

3.5.2 Inhaltliche und methodische Anpassung der disziplinären

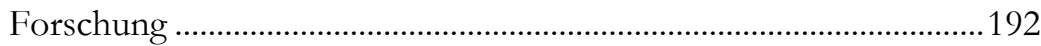

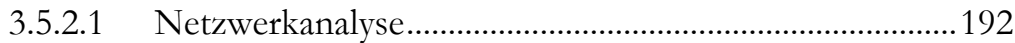

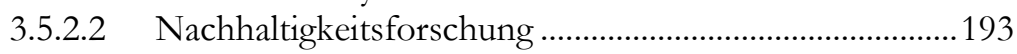

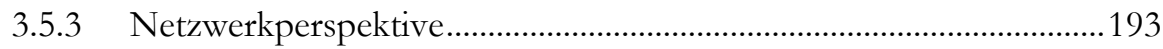

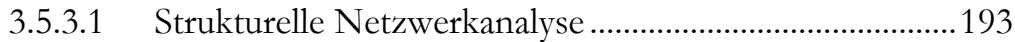

3.5.3.2 Qualitative Analyse ............................................................204

3.5.3.3 Ansatzpunkte für Empfehlungen..................................208

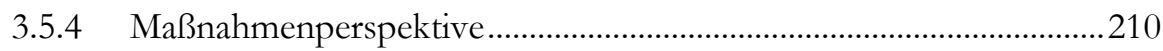

3.5.4.1 Maßnahmenbasismatrix ...................................................210

3.5.4.2 Nachhaltigkeitsspinne und regionale

3.5.4.3 Nachhaltigkeitsinstitutionen....................................... 214

4 Perspektiven und Empfehlungen.......................................................215

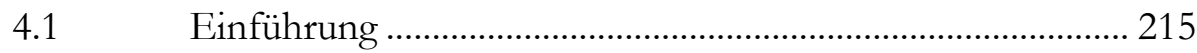

Empfehlungen zur Netzwerkarbeit ................................... 216

4.2.1 Konstellation: Breite Beteiligung........................................................216

4.2.2 Konstellation: Multiplikatoren........................................................218

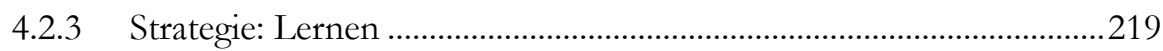

4.2.4 Strategie: Transparenz, Offenheit, Flexibilität....................................221

4.2.5 Strategie: Kooperation und win-win................................................223

4.2.6 Strategie: Arbeiten auf verschiedenen Ebenen ...................................223

4.2.7 Instrumente: Leitbild, Visionen und Ziele .........................................226

4.2.8 Instrumente: Prozessmanagement .....................................................22 


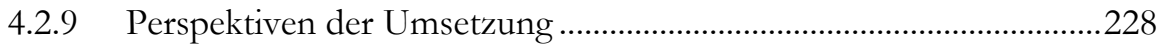

4.3 Regionale Nachhaltigkeit organisieren................................ 231

4.3.1 Perspektiven für den Fördermittelgeber ............................................235

4.3.2 Perspektiven für den Fördermittelempfänger...................................236

5 Fazit und Ausblick..................................................................239

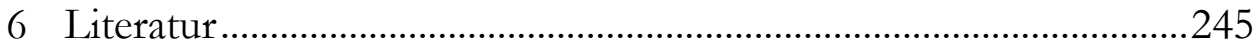

Anhang................................................................................... 254 


\section{Abbildungsverzeichnis}

Abbildung 1: Beispiel einer Nachhaltigkeitsspinne .................................................27

Abbildung 2: Forschungsdesign der Netzwerkanalyse ............................................. 35

Abbildung 3: Verknüpfung der Netzwerkanalyse mit der Maßnahmenanalyse .....51

Abbildung 4: Methodische Verknüpfung zwischen Nachhaltigkeit und

Netzwerken

Abbildung 5: Die Lage der Modellregionen des E+E-Vorhabens „Erlebnis

Grünes Band" am Grünen Band. Rot gekennzeichnet sind die beiden

Untersuchungsregionen des Forschungsvorhabens (Karte zur Verfügung gestellt

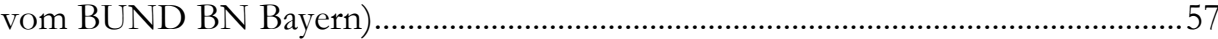

Abbildung 6: Verteilung der Fachbereiche, Institutionengruppen und Ost-WestVerteilung der Akteure der Grundgesamtheit und der Analyseeinheit des

Netzwerks Elbe-Altmark-Wendland .64

Abbildung 7: Netzwerkgraph des Netzwerks Elbe-Altmark-Wendland ..................66

Abbildung 8: Ost-West-Verteilung im Netzwerk Elbe-Altmark-Wendland...........68

Abbildung 9: Verteilung der Kontakthäufigkeiten im Netzwerk Elbe-Altmark-

Wendland

Abbildung 10: Zufriedenheit der Akteure mit der Netzwerkarbeit in der Untersuchungsregion im Netzwerk Elbe-Altmark-Wendland................................ 70

Abbildung 11: Maßnahmenbasismatrix für das E+E Vorhaben Elbe-Altmark-

Wendland

Abbildung 12: Nachhaltigkeitsspinne des E+E Projekts

Elbe-Altmark-Wendland

Abbildung 13: Verteilung der Fachbereiche, Institutionengruppen und Ost-WestVerteilung der Akteure der Grundgesamtheit und der Analyseeinheit im Netzwerk Thüringer Wald \& Schiefergebirge/ Frankenwald. 103

Abbildung 14: Graph des Netzwerks Thüringer Wald \& Schiefergebirge / Frankenwald 
Abbildung 15: Ost-West-Vernetzung des Netzwerks Thüringer Wald \&

Schiefergebirge / Frankenwald. .107

Abbildung 16: Verteilung der Kontakthäufigkeiten im Netzwerk Thüringer Wald $\&$ Schiefergebirge/Frankenwald 108

Abbildung 17: Zufriedenheit der Akteure im Netzwerk Thüringer Wald \& Schiefergebirge/Frankenwald.

Abbildung 18: Organigramm des Netzwerks Thüringer Wald \& Schiefergebirge /

Frankenwald (Quelle: Regionalverbund Thüringer Wald (2008, unveröff.), verändert)

Abbildung 19: Maßnahmenbasismatrix für das E+E Projekt Thüringer Wald \&

Schiefergebirge/Frankenwald

Abbildung 20: Nachhaltigkeitsspinne der Modellregion Thüringer Wald \&

Schiefergebirge/Frankenwald

Abbildung 21: NGP Schaalsee-Landschaft - Verteilung der Fachbereiche,

Institutionengruppen und räumliche Verteilung im Netzwerk

Abbildung 22: NGP Schaalsee-Landschaft - Graph des Projektnetzwerks

Abbildung 23: NGP Schaalsee-Landschaft - Länderübergreifende Vernetzung im

Projektnetzwerk .146

Abbildung 24: NGP Schaalsee-Landschaft - Alte (schwarz) und neue (blau)

Kontakte im Netzwerk 148

Abbildung 25: NGP Schaalsee-Landschaft - Verteilung der Kontakthäufigkeit..149

Abbildung 26: NGP Schaalsee-Landschaft - Häufigkeit der Kontaktarten

Abbildung 27: NGP Schaalsee-Landschaft -Zufriedenheit der Akteure mit

Einbindung in Projektnetzwerk

Abbildung 28: NGP Schaalsee-Landschaft - Maßnahmenbasismatrix

Abbildung 29: Milcherzeugung in MV im Jahr 2007 in den Landkreisen,

Statistisches Landesamt MV (2008)...

Abbildung 30: NGP Schaalsee-Landschaft - Absolute Eigentumsbildung des

ZSL 1994 - 2008; Quelle: ZSL (2008)

Abbildung 31: NGP Schaalsee-Landschaft - Nachhaltigkeitsspinne 
Abbildung 32: NGP Grünes Band Eichsfeld-Werratal : Großräumige Lage des Projektgebietes (Quelle: Heinz Sielmann Stiftung, 2007)

Abbildung 33: NGP Grünes Band Eichsfeld-Werratal - Verteilung der

Fachbereiche, Institutionengruppe und räumliche Verteilung im Netzwerk 195

Abbildung 34: NGP Grünes Band Eichsfeld-Werratal - Graph des

Projektnetzwerks

Abbildung 35: NGP Grünes Band Eichsfeld-Werratal: Länderübergreifende

Vernetzung im Projektnetzwerk.

Abbildung 36: NGP Grünes Band Eichsfeld-Werratal - Oben: Neue Kontakte (blau) im Gesamtnetzwerk;

Unten: Selektion aller neuen Kontakte im Netzwerk

Abbildung 37: NGP Grünes Band Eichsfeld-Werratal - Verteilung der

Kontakthäufigkeit

Abbildung 38: NGP Grünes Band Eichsfeld-Werratal - Häufigkeit der

Kontaktarten

Abbildung 39: Ebenen der Projektarbeit von Naturschutzgroßprojekten im

Kontext regionaler Nachhaltigkeit (Quelle: Eigene Darstellung) 210

Abbildung 40: NGP Schaalsee-Landschaft - Maßnahmenbasismatrix

Abbildung 41: Der „Nachhaltigkeitsbeirat“ und seine Einbindung in die unterschiedlichen Förderprojekte .225

Abbildung 42: Arbeitshilfe für die Maßnahmenebene

Abbildung 43: Beispiel für die Anwendung des Arbeitsblattes 235

\section{Tabellenverzeichnis}

Tabelle 1: Beispiel einer Maßnahmenbasismatrix

Tabelle 2: Überblick über die durchgeführten Interviews zu

Nachhaltigkeitsinstitutionen 30

Tabelle 3: Zusammenfassung der regionalen Nachhaltigkeitsinstitutionen

Tabelle 4: Merkmale regionaler Netzwerke und ihre Zusammenfassung 40 
Tabelle 5: Konstellation: Breite Beteiligung in der Region

Elbe-Altmark-Wendland

Tabelle 6: Konstellation: Multiplikatoren in der Region Elbe-Altmark-

Wendland

Tabelle 7: Konstellation: Personelle Kontinuität in der Region Elbe-Altmark-

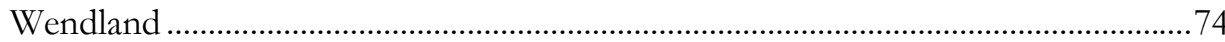

Tabelle 8: Strategie: Lernen in der Region Elbe-Altmark-Wendland.......................75

Tabelle 9: Strategie: Arbeiten auf verschiedenen Ebenen in der Region Elbe-

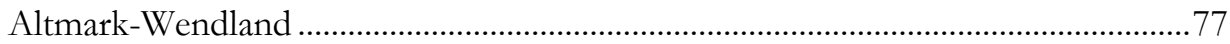

Tabelle 10: Strategie: Kooperation und win-win-Lösungen in der Region Elbe-

Altmark-Wendland

Tabelle 11: Strategie: Probleme und Lösungswille in der Region Elbe-Altmark-

Wendland .78

Tabelle 12: Strategie: Transparenz, Offenheit und Flexibilität in der Region Elbe-

Altmark-Wendland .79

Tabelle 13: Instrument: Leitbild in der Region Elbe-Altmark-Wendland. .80

Tabelle 14: Instrument: Prozessmanagement in der Region Elbe-Altmark-

Wendland

Tabelle 15: Synopse regionaler und nationaler Verhaltensweisen

Tabelle 16: Konstellation: Breite Beteiligung in der Region Thüringer Wald \&

Schiefergebirg/Frankenwald.

Tabelle 17: Konstellation: Multiplikatoren in der Region Thüringer Wald \&

Schiefergebirge/Frankenwald

Tabelle 18: Konstellation: Personelle Kontinuität in der Region Thüringer Wald \&

Schiefergebirge/Frankenwald.

Tabelle 19: Strategie: Lernen in der Region Thüringer Wald \&

Schiefergebirge/Frankenwald.

Tabelle 20: Strategie: Arbeiten auf verschiedenen Ebenen in der Region Thüringer

Wald \& Schiefergebirge/Frankenwald

Tabelle 21: Strategie: Kooperation und win-win in der Region Thüringer Wald \&

Schiefergebirge/Frankenwald 
Tabelle 22: Strategie: Probleme und Lösungswille in der Region Thüringer Wald \& Schiefergebirge/Frankenwald. 118

Tabelle 23: Strategie: Transparenz, Offenheit und Flexibilität in der Region

Thüringer Wald \& Schiefergebirge/Frankenwald

Tabelle 24: Instrumente: Leitbild in der Region Thüringer Wald \&

Schiefergebirge/Frankenwald.

Tabelle 25: Instrument: Prozessmanagement in der Region Thüringer Wald \&

Schiefergebirge/Frankenwald.

Tabelle 26: Synopse regionale und nationale Verhaltensweisen 136

Tabelle 27: NGP Schaalsee-Landschaft - Konstellation: Breite Beteiligung (D = diskursiv, $\mathrm{A}=$ akteursorientiert, $\mathrm{I}=$ integrativ;). .154

Tabelle 28: NGP Schaalsee-Landschaft - Konstellation: Multiplikatoren..... 155

Tabelle 29: NGP Schaalsee-Landschaft - Konstellation: Personelle Kontinuität 156

Tabelle 30: NGP Schaalsee-Landschaft - Strategie: Lernen ....

Tabelle 31: NGP Schaalsee-Landschaft - Strategie: Transparenz, Offenheit, Flexibilität

Tabelle 32: NGP Schaalsee-Landschaft - Strategie: Kooperation und win-win...160

Tabelle 33: NGP Schaalsee-Landschaft - Strategie: Arbeiten auf verschiedenen

Ebenen 161

Tabelle 34: NGP Schaalsee-Landschaft - Instrumente: Leitbilder, Visionen, Ziele 162

Tabelle 35: NGP Schaalsee-Landschaft - Instrumente: Prozessmanagement......164 Tabelle 36: NGP Schaalsee-Landschaft - Übersicht der regionalen Förderkulisse 166

Tabelle 37: NGP Schaalsee-Landschaft - Mögliche Nachhaltigkeitswirkungen der Sekundäreffekte der Projektmaßnahmen Teil I. 179

Tabelle 38: NGP Schaalsee-Landschaft: Mögliche Nachhaltigkeitswirkungen der Sekundäreffekte der Projektmaßnahmen Teil II 180

Tabelle 39: NGP Schaalsee-Landschaft - Regionale Verhaltensweisen für Nachhaltigkeit 
Tabelle 40: NGP Schaalsee-Landschaft: - Überblick über bestehende Nachhaltigkeitsinstitutionen in den Landkreisen .....................................................186

Tabelle 41: Fallbeispiele für die Wirkung des Netzwerks auf Maßnahmen ..........187

Tabelle 42: Korrespondierende regionale und nationale Managementregeln.......188

Tabelle 43: Region Eichsfeld-Werratal - Regionale Verhaltensweisen für

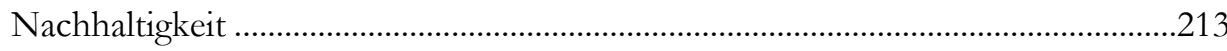

Tabelle 44: NGP Grünes Band Eichsfeld-Werratal - Beispiele von

Nachhaltigkeitsinstitutionen in den Landkreisen .................................................214

Tabelle 45: Werkzeuge für Netzwerkarbeit: Zuständigkeiten, bediente Merkmale und strategische Qualitäten ...............................................................................229 


\section{Abkürzungen}

$\begin{array}{ll}\text { BfN } & \begin{array}{l}\text { Bundesamt für Naturschutz } \\ \text { Bundesministerium für Umwelt, Naturschutz und Reaktor- } \\ \text { sicherheit } \\ \text { Erprobungs- und Entwicklungsvorhaben }\end{array} \\ \text { EZE } & \text { Entwicklungszielbereiche } \\ \text { HAWK } & \text { Hochschule für angewandte Wissenschaft und Kunst } \\ \text { ISEK } & \text { Integrierte Stadtentwicklungskonzept } \\ \text { NGP } & \text { Naturschutzgroßprojekt } \\ \text { PAG } & \text { Projektbegleitende Arbeitsgruppe } \\ \text { PEPL } & \text { Pflege- und Entwicklungsplan } \\ \text { R } & \text { Regel } \\ \text { RWK } & \text { Regionale Wachstumskerne } \\ \text { TF } & \text { Themenfeld } \\ \text { TG } & \text { Testgebiet } \\ \text { ZMP } & \text { Zentrale Markt- und Preisberichtstelle } \\ \text { ZSL } & \text { Zweckverband Schaalsee-Landschaft }\end{array}$





\section{Einführung}

\subsection{Problemstellung}

Der Eiserne Vorhang in Europa hat einen quer durch Europa verlaufenden Grenzstreifen hervorgebracht, in dem sich die Natur über Jahrzehnte ungestört entfalten und entwickeln konnte. Nach dem Fall des Eisernen Vorhangs Ende der 1980er Jahre erhielt dieser Streifen einen neuen Namen: Grünes Band. Dieses „Grüne Band“ hat in Deutschland eine Länge von 1393 km mit einer Flächenausdehnung von ca. 14.000 ha und ist damit der längste Wald- und OffenlandBiotopverbund Deutschlands. Bund und Länder verpflichten sich zur Bewahrung des ökologischen Potenzials des Grünen Bandes. Der Bund setzt sich daher im Rahmen von Erprobungs- und Entwicklungsvorhaben (E+E-Vorhaben) „Erlebnis Grünes Band" sowie von Naturschutzgroßprojekten (NGP) für den Schutz und die Entwicklung des Grünen Bandes ein. Naturschutzgroßprojekte als klassische Instrumente des Naturschutzes haben zum Ziel, national bedeutsame und repräsentative Naturräume mit gesamtstaatlicher Bedeutung zu schützen und langfristig zu sichern. Als besondere Aufgabe wird den E+E-Vorhaben „Erlebnis Grünes Band“" neben Maßnahmen zur Sicherung der biologischen Vielfalt auch die Zusammenführung von Schutz- und Nutzaspekten übertragen. Eine besondere Herausforderung der Naturschutzförderung im Bereich des Grünen Bandes ist die Notwendigkeit einer „grenzübergreifenden“ Zusammenarbeit. So müssen 
Akteure zum einen über Landkreisgrenzen hinaus das Grüne Band in den Blick nehmen. Zum anderen ist es notwendig, dass sie Naturschutzförderung über die Grenzen der Bundesländer hinweg gestalten.

\subsection{Ziel der Untersuchung}

Mit der Bewahrung und Förderung des Nationalen Naturerbes „Grünes Band“ verbinden viele Kommunen und Landkreise in strukturschwachen Regionen entlang des Grünen Bandes die Hoffnung einer positiven wirtschaftlichen Entwicklung. In anderen Regionen wird der Naturschutz eher als Hindernis einer bereits eingeleiteten positiven wirtschaftlichen Entwicklung gesehen. Um langfristig erfolgreich zu sein, müssen die Initiativen am „Grünen Band“ langfristig zeigen, dass sie neben dem Erhalt des nationalen Naturerbes, den dort lebenden Menschen eine wirtschaftlich tragfähige Zukunft bieten, d.h. regionale Nachhaltigkeit realisieren können. Um Synergieeffekte zu diesem Ziel im Kontext von Naturschutzförderprojekten erreichen zu können, muss man mehr über die Wirkung der Förderung wissen, um entsprechende Optimierungen anstoßen zu können. Das Forschungsvorhaben „Grünes Band - Modellregion für Nachhaltigkeit" ${ }^{\text {"1 }}$, aus dem der vorliegende Abschlußbericht hervorgegangen ist, hat zum Ziel, die Wirkungen der Förderung zu analysieren. Dazu werden vier Untersuchungsregionen entlang des Grünen Bandes ausgewählt: Die Naturschutzgroßprojekte „SchaalseeLandschaft" und „Grünes Band Eichsfeld-Werratal“ sowie die Regionen des E+E Vorhabens „Erlebnis Grünes Band“ „Elbe-Altmark-Wendland“ und „Thüringer Wald und Schiefergebirge/Frankenwald“. Auswahlkriterien sind die Bundesförderung mit verschiedenen Förderinstrumenten sowie die Abdeckung eines repräsentativen Ausschnitts des Grünen Bandes. Die übergeordnete Arbeitshypothese des Forschungsvorhabens lautet: „Im Bereich des Grünen Bandes besteht die einmalige Chance im Sinne des Prinzips der Nachbaltigkeit ein Beispiel für das Miteinander von erfolgreicher wirtschaftlicher und ökologischer Entwicklung zu realisieren. "Diese Hypothese hinterfragend, wird eine interdisziplinäre Herangehensweise gewählt, die Nachhaltigkeitsforschung und Netzwerkforschung miteinander verbindet. Der interdisziplinäre Brückenschlag in diesem Forschungsvorhaben gelingt durch einen methodischen Ansatz, der eine Verbindung zwischen statischer und dynamischer Betrachtungsweise im Hinblick auf regionale Nachhaltigkeit herstellt. Der eine Brückenkopf (Netzwerkforschung) wird dabei durch Netzwerkmerkmale (dynamische Perspektive) und der andere Brückenkopf (Nachhaltigkeitsforschung) durch regionale Nachhaltigkeitsthemenfelder / Nachhaltigkeitsindikatoren gebildet (statische Perspektive). Die Brücke zwischen diesen Brückenköpfen, um im Bild zu bleiben, besteht darin, dass erfolgreich arbeitende Netzwerke unter bestimmten Vorausset-

1 Im weiteren Bericht mit Forschungsvorhaben bezeichnet. 
zungen einen erheblichen Beitrag leisten können, Nachhaltigkeit von Förderungen herzustellen, indem unterschiedliche und zum Teil auch konkurrierende Interessen in gemeinsame Ziele und Maßnahmenverwirklichung eingebunden sind.

Der Begriff „Nachhaltigkeit“ büßt durch die inflationäre Verwendung stark an Kontur ein. Stammt das Konzept ursprünglich aus der deutschen Forstwirtschaft des 19. Jahrhunderts, so ist es insbesondere durch den Brundtland-Bericht zum Leitbild politischen Handelns geworden und beeinflusst seit der UN-Konferenz für Umwelt und Entwicklung in Rio de Janeiro 1992 sämtliche Politikbereiche. ${ }^{2}$ Die in Rio verabschiedete Agenda ist unter dem Schlagwort „Agenda 21“ bekannt und enthält Empfehlungen im Bezug auf nachhaltiges Handeln, sowohl für Staaten als auch für die einzelnen Regionen und Bürger eines Landes (lokale Agenda 21). In der Folge wurden auf verschiedenen Ebenen (international, national, regional) Nachhaltigkeitsindikatoren-Sets entwickelt, die eine Operationalisierung des Ziels und eine Positionsbestimmung auf dem Weg einer nachhaltigen Entwicklung ermöglichen sollen. Mittlerweile existiert eine schwierig überschaubare Vielzahl derartiger Indikatorensets, die Nachhaltigkeit in sehr unterschiedlicher Weise (unterschiedliche thematische Schwerpunkte und Anzahl von Indikatoren) operationalisieren. Grundsätzlich kennzeichnet die meisten Indikatoren-Sets als gemeinsames Merkmal eine integrative Sichtweise verschiedener NachhaltigkeitsDimensionen (zumeist Ökologie, Ökonomie und Soziales) ${ }^{3}$. Das gilt auch für die deutsche Nachhaltigkeitsstrategie, die Deutschland aus den Empfehlungen der Agenda 21 entwickelt und am 17. April 2002 als nationale Nachhaltigkeitsstrategie beschlossen hat. ${ }^{4}$ Die Agenda 21 fordert ebenfalls dazu auf, auf lokaler Ebene Prozesse zu initiieren, die eine nachhaltige regionale Entwicklung zum Ziel haben. Bei der Entwicklung von lokalen Agenda 21-Prozessen haben sich zwei Ansätze herausgebildet: Einerseits ein „bottom-up“-Verfahren, in welchem die Region unter Einbeziehung der in ihr wirkenden Akteure ein Leitbild einer nachhaltigen regionalen Entwicklung erstellt und dieses dann mit selbst gewählten Indikatoren operationalisiert. Andererseits ist auch ein ,top-down“-Verfahren beobachtbar, bei dem sich eine Kommune an einem, durch eine externe Institution entwickelten Indikatorensatz orientiert. Die Existenz der beiden Verfahren nebeneinander führt dazu, dass sich auf regionaler Ebene ganz unterschiedliche Nachhaltigkeitsverständnisse herausbilden, die eine vergleichende Betrachtung (horizontal) erschweren bzw. verhindern. 5

Die Vernetzung regionaler Akteure ist für den regionalen Nachhaltigkeitsprozess von großer Bedeutung. ${ }^{6}$ Auch die Bundesregierung geht in ihrer Nationalen

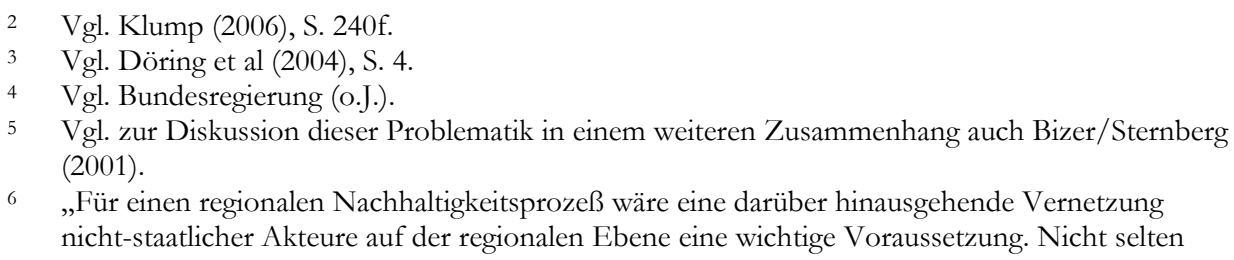


Nachhaltigkeitsstrategie davon aus, dass dem Idealzustand nur dann nahe gekommen werden kann, wenn die Bürger sich an der Verwirklichung dieser regulativen Idee beteiligen. ${ }^{7}$ Derzeit jedoch ist diese Kommunikation und Diskussion über die Verwirklichung noch nicht ausreichend ausgeprägt. ${ }^{8}$ Ein wesentlicher Bestandteil der nachhaltigen Entwicklung bildet seit der Rio-Konferenz 1992 die Partizipation. Immer wieder wird deutlich gemacht, dass nachhaltige Entwicklung eines partizipativen Vorgehens bedarf und die klassischen hierarchischen Instrumente dazu nicht geeignet sind. Da Nachhaltigkeit eine Querschnittsaufgabe 9 darstellt, ist die Einbindung verschiedener Akteure aus unterschiedlichen Bereichen und Ebenen notwendig. ${ }^{10}$ Den regionalen Akteuren müssen hierfür Instrumente zur Verfügung stehen, die ihnen sowohl genügend Möglichkeiten geben, ihre Interessen entsprechend zu vertreten, als auch sie zu entsprechender Eigeninitiative veranlassen. Netzwerke als eine Form von Governance ${ }^{11}$ fördern regionale Kooperationen ${ }^{12}$ und stellen damit ein „Partizipationsmodell " ${ }^{\text {" }}$ " dar, das die Operationalisierung des Leitbildes Nachhaltigkeit organisieren kann ${ }^{14}$. Sie gelten als ,„...] eine dem Nachhaltigkeitsleitbild gemäße Organisationsform, da sie systemisch agieren und ,gemeinschaftlichen Mehrwert' erzeugen können. “15 Netzwerke zeichnen sich dadurch aus, dass sie ,[...] offen sind für öffentliche wie private Akteure; zu einer selektiven Verdichtung des Informationsaustauschs beitragen, die Koordination erleichtern, weil Akteure in dauerhaften Beziehungen stehen und Vertrauen aufbauen; flexibel sind, wodurch sowohl die internen Beziehungen als auch die Beteiligung von Akteuren leicht an neue Erfordernisse angepasst werden können." "16

Wie sich der Beitrag von Netzwerken zur regionalen Nachhaltigkeit konkret gestaltet und vor allem, welche Voraussetzungen innerhalb der Netzwerke geschaffen sein müssen, damit ein Diskurs über Nachhaltigkeit gemäß deren An-

sind gesellschaftliche Interessen, die für die Umsetzung nachhaltiger Entwicklung von großer Bedeutung sind, auf regionaler Ebene kaum organisiert und dadurch nur schwer zu mobilisieren." Greif (2000), S. 112

7 Die Bundesregierung (16.04.2002)

8 „Weder entfaltet das Leitbild der nachhaltigen Entwicklung bislang visionäre Kraft noch besitzt es für die Menschen alltagspraktische Relevanz. Den Wenigsten ist bewusst, dass zuallererst ein jeder selbst aufgefordert ist, in seiner Bürgerrolle für eine bessere Zukunft einzutreten." Heinl (2005), S. 25.

$9 \quad$ Die Bundesregierung (16.04.2002.)

10 „Der Bedarf an neuen Organisationsmodellen um Nachhaltigkeit zu entwickeln, beschränkt sich nicht auf einzelne Handlungsbereiche in der Gesellschaft, sondern reicht von der Metaebene gesellschaftlicher Konsultationsprozesse bis zu der Mikroebene einzelwirtschaftlicher Aktivitäten.“ BfU Beratungsbüro für Umwelt- und Unternehmensentwicklung (2005), S. 215.

11 Wald, Jansen Dorothea (2007.)

12 Benz, Meincke (09.05.2007.)

13 BfU Beratungsbüro für Umwelt- und Unternehmensentwicklung (2005), S. 84.

14 BfU Beratungsbüro für Umwelt- und Unternehmensentwicklung (2005), S. 8

15 BfU Beratungsbüro für Umwelt- und Unternehmensentwicklung (2005), S. 216.

16 Benz, Meincke (09.05.2007), S. 17. 
forderungen stattfinden kann, ist derzeit nicht ausreichend untersucht und deshalb Gegenstand dieses Forschungsvorhabens.

Naturschutzfördertitel haben spezifisches Wirkungspotenzial im Naturschutz, das mit weiteren Folgewirkungen auch in anderen Bereichen einhergehen kann. Da seit der Rio-Konferenz 1992 auch regionale Akteure nachhaltige Entwicklungskonzepte entwickeln und ausführen, ist für diese Akteure ebenso wie für die Fördermittelgeber von Interesse wie das Erprobungs- und Entwicklungsvorhaben „Erlebnis Grünes Band“ und die Naturschutzgroßprojekte die regionale Nachhaltigkeit beeinflussen. Da die Naturschutzfördertitel unterschiedlich konzipiert sind und sich die regionale Nachhaltigkeit kontextbezogen gestaltet, werden keine allgemein gültigen Antworten gesucht. Ziel ist es zu sehen, welches Wirkungspotenzial hinsichtlich regionaler Nachhaltigkeit im konkreten Fall besteht. Das verdeutlicht, weshalb das Forschungsvorhaben auf einem interdisziplinären Ansatz aus Nachhaltigkeitsforschung und Netzwerkforschung beruht: Kein Prozess und kein Projekt ist durch sich allein nachhaltig - sie müssen entsprechend organisiert und geplant werden. Hierzu braucht es Akteure, die sich in bestimmten Strukturen, d.h. Netzwerken, organisieren. Wie diese Netzwerke gestaltet sein müssen, damit die Akteure regionale Nachhaltigkeit organisieren können, ist eine zentrale Frage des Vorhabens. Ebenso geht es darum, welche instrumentellen Hilfen sie brauchen, um sich auf dem Weg einer nachhaltigen Entwicklung zu orientieren? Im Rahmen dieses Forschungsvorhabens stehen diese beiden Fragen im Mittelpunkt.

\subsection{Aufbau der Untersuchung}

Kapitel 2 beschreibt die Vorgehensweise der Untersuchung, in dem es ausgehend von den Forschungsfragen (Abschnitt 2.1) die Methoden und Vorgehensweise der Maßnahmen- und Netzwerkperspektive entwickelt (Abschnitt 2.2 und Abschnitt 2.3). Regionale Nachhaltigkeit wird aus Sicht der Maßnahmenperspektive als ein Managementprozess verstanden, der mit geeigneten Methoden wie z.B. der Nachhaltigkeitsspinne auf potenzielle Beiträge zur Nachhaltigkeit hin zu überprüfen ist, um die empirisch ermittelten geeigneten regionalen Institutionen als Akteure in die Projektförderung einzubinden. In Abschnitt 2.3 wird geklärt, mit welchen Begriffbestimmungen das Forschungsdesign für Projektnetzwerke einhergeht, um eine umfassende empirische Erhebung zu ermöglichen. Aus diesen beiden Ansätzen folgt dann der interdisziplinäre Brückenschlag (Abschnitt 2.4), der den Zusammenhang zwischen Netzwerkanalyse und Nachhaltigkeit herstellt, indem er die regionalen Verhaltensweisen für Nachhaltigkeit mit strategischen Qualitätskriterien der Netzwerke (integrativ, akteursorientiert, diskursiv) zu einer dynamischen Perspektive verbindet.

Kapitel 3 nutzt diese Ergebnisse für die Untersuchung der Regionen. Dafür erfolgt zunächst eine kurze Rekapitulierung der Fördertitel (Abschnitt 3.1), um dann 
die zwei Regionen mit E+E-Vorhaben in der Untersuchungsregion „ElbeAltmark-Wendland“ (Abschnitt 3.2) und in der Untersuchungsregion „Thüringer Wald \& Schiefergebirge/Frankenwald“ (Abschnitt 3.3) sowie die zwei Regionen mit Naturschutzgroßprojekten, nämlich die Untersuchungsregion „Naturschutzgroßprojekt Schaalsee-Landschaft" (Abschnitt 3.3) und die Untersuchungsregion „Naturschutzgroßprojekt Grünes Band Eichsfeld-Werratal“ (Abschnitt 3.4), näher zu betrachten. Für alle vier Untersuchungsregionen werden die Nachhaltigkeitsund Netzwerksperspektive empirisch untersucht.

Aus diesen empirischen Ergebnissen entwickelt Kapitel 4 Perspektiven und Empfehlungen, die spezifisch zur Netzwerkarbeit verschiedene Konstellationen wie breite Beteiligung und Multiplikatoren sowie diverse Strategien wie Lernen oder Kooperation diskutieren (Abschnitt 4.2), um daraus für den Fördermittelgeber wie auch die Fördermittelempfänger Hinweise zu gewinnen, wie sie regionale Nachhaltigkeit im Kontext von NGP und E+E-Vorhaben besser organisieren können (Abschnitt 4.3).

Kapitel 5 bietet ein Fazit mit den zentralen Ergebnissen sowie einen Ausblick.

\subsection{Untersuchungsbeteiligte}

Das Forschungsvorhaben „Grünes Band - Modellregion für Nachhaltigkeit“ wurde im Zeitraum 01.10.2007 - 31.05.2010 durchgeführt. Das Forschungsvorhaben war ein Verbundprojekt der Fakultät Ressourcenmanagement der HAWK Hochschule für Angewandte Wissenschaft und Kunst und der Wirtschaftswissenschaftlichen Fakultät der Georg-August-Universität Göttingen. Fördermittelgeber war das Bundesministerium für Bildung und Forschung (BMBF) vertreten durch den Projektträger Jülich (PTJ). Das Bundesministerium für Umwelt, Naturschutz und Reaktorsicherheit (BMU) begleitete das Projekt zusammen mit dem Bundesamt für Naturschutz $(\mathrm{BfN})$ fachlich. Projektbeteiligt waren auch der BUND BN Bayern und das Alpenforschungsinstitut (AFI). Diesen allen danken die Autoren für ihre Bereitschaft sich fachlich und organisatorisch an diesem Vorhaben zu beteiligen.

Besonderen Dank aber schulden alle Projektbeteiligten den Akteuren in den vier Untersuchungsregionen, ohne deren Diskussions- und Auskunftsbereitschaft das Vorhaben nicht hätte gelingen können. Die vier Regionen sind das Naturschutzgroßprojekt Schaalsee-Landschaft und das Naturschutzgroßprojekt Grünes Band Eichsfeld-Werratal sowie das Erprobungs- und Entwicklungsvorhaben Erlebnis Grünes Band in der Modellregion Elbe-Altmark-Wendland sowie im Thüringer Wald \& Schiefergebirge/Frankenwald. 


\section{Untersuchungsansatz}

\subsection{Forschungsfragen}

Das Forschungsvorhaben geht von der Überlegung aus, dass die Bundesförderungen im Rahmen von Naturschutzgroßprojekten und des E+E Vorhabens „Erlebnis Grünes Band“, trotz sektoraler Ausrichtung auf den Naturschutz, eine Impulsfunktion für regionale Nachhaltigkeit erfüllen können. Wie die Förderungen auf die verschiedenen Handlungsfelder der regionalen Nachhaltigkeit tatsächlich direkt und bzw. oder indirekt - wirken, ist weder bekannt noch gibt es einen instrumentellen Rahmen, um diese Wirkungen der Förderung im Projektverlauf aufzudecken und für den Projekterfolg zu verwenden. Darüber hinaus wird angenommen, dass die regionalen Akteure - organisiert in Projektnetzwerken - diese Wirkung durch die Umsetzung der Förderung mit konkreten Maßnahmen wesentlich beeinflussen. In der Konzeption der Förderprogramme bzw. -richtlinien wird diesem Aspekt jedoch nur wenig Aufmerksamkeit zuteil. Die übergreifende Forschungsfrage lautet:

Wie wirken Naturschutzgroßprojekte und das E+E Vorbaben „Erlebnis Grünes Band“" auf regionale Nachbaltigkeit?

Diese Frage hat im Kontext der dargelegten Annahmen die Verknüpfung der Untersuchungen der Netzwerkanalysen mit Untersuchungen der Nachhaltigkeitsforschung nahe gelegt. Diese Frage wird von den beiden Forschungsperspektiven 
mit disziplinären Fragen (s. 2.2 und 2.3) unabhängig voneinander sowie beispielhaft unter einem interdisziplinär entwickelten Dach bearbeitet (s. 0).

Das Team der Nachhaltigkeitsforschung der Universität Göttingen geht der Frage nach, welche direkten und bzw. oder indirekten Wirkungen die geförderten Maßnahmen auf die regionale Nachhaltigkeit ausüben können. Diese Analyse wird in die Erforschung und Weiterentwicklung von Methoden und Instrumenten eines Nachhaltigkeitsmanagements im Kontext von Naturschutzförderprojekten eingebettet. Die Forschungshypothese lautet: Das Erprobungs- und Entwicklungsvorhaben (E+E-Vorhaben) „Erlebnis Grünes Band“ sowie Naturschutzgroßprojekte (NGP) wirken auf regionale Nachhaltigkeit.

Die Netzwerkanalyse der HAWK dient der Erhebung und Analyse der Projektnetzwerke, die sich im Kontext der betrachteten Förderprojekte gebildet haben. Für die Untersuchung leitend sind die Fragen, welche Netzwerkeigenschaften sie dazu befähigen bzw. sie darin behindern die Bundesförderung im Sinne eines Beitrags zur regionalen Nachhaltigkeit umzusetzen, wie die Konzeption der Fördertitel die Ausprägung dieser Eigenschaften beeinflusst und welche Netzwerkeigenschaften durch die Förderung gezielt im Sinne regionaler Nachhaltigkeit gefördert werden können. In der teamübergreifenden interdisziplinären Diskussion wurden folgende zwei Thesen formuliert:

\section{(1) Netzwerke sind Voraussetzung für regionale Nachbaltigkeit.}

Diese These gibt den Stand der wissenschaftlichen Erkenntnis, dass regionale Entwicklung und Maßnahmen erfolgreicher sind, wenn die Planungs- und Entscheidungsprozesse nicht Top-down gesteuert sind, sondern in einem ausgewogenen Verhandlungssystem von Macht-, Fachpromotoren und stakeholdern geführt werden, wieder. ${ }^{17}$

\section{(2) Es gibt Ausprägungen von Netzwerken, die einen optimalen Beitrag zur regionalen Nachbaltigkeit leisten.}

Stand der Forschung ist, dass bestimmte Netzwerkeigenschaften und Netzwerkstrukturen für die Leistungsfähigkeit der Netzwerke verantwortlich sind. In zahlreichen Studien (s. 2.3.4) wurden Netzwerkmerkmale identifiziert und konkretisiert. Den Studien ist jedoch gemeinsam, dass sie weniger auf Output-Faktoren abheben, als vielmehr die Qualität von Prozessen oder Ausprägungen von Netzwerken beachten. Diese Lücke greift das Forschungsteam auf und strebt an, grundsätzlich aufzudecken, wie sich welche Netzwerkausprägungen im Kontext der Förderprogramme E+E und NGP ausprägen. Die Annahme, dass Netzwerke die geeignete Organisationsform zur Umsetzung nachhaltiger Entwicklung sind,

17 Vgl. Kühn \& Moss (1998). 
soll mit diesem Forschungsbeitrag hinterfragt und ggf. unterstützt oder widerlegt werden.

Mit der Klärung der Forschungsfragen und -lücken können für den Fördermittelgeber und ebenso für regionale Akteure Empfehlungen zur Optimierung der Förderung bzw. der Projektnetzwerkarbeit abgeleitet werden.

\subsection{Methoden und Vorgehensweise der Maßnahmenper- spektive}

\subsection{1 (Regionale) Nachhaltigkeit als Managementprozess}

Vorbild für die Organisation von Nachhaltigkeitsprozessen auf regionaler Ebene ist die bundesnationale Nachhaltigkeitsstrategie und ihre Ausgestaltung als Managementkonzept. ${ }^{18}$ Das Konzept umfasst drei Komponenten. Die erste Komponente beinhaltet zehn Managementregeln, die allgemeine Grundsätze für im Sinne der nachhaltigen Entwicklung effektive Verhaltensweisen formulieren und im Folgenden im Wortlaut wiedergegeben sind:

„Grundregel“

1. Jede Generation muss ihre Aufgaben selbst lösen und darf sie nicht den kommenden Generationen aufbürden. Zugleich muss sie Vorsorge für absehbare zukünftige Belastungen treffen.

Regeln der Nachhaltigkeit für einzelne Handlungsbereiche

2. Erneuerbare Naturgüter (wie z. B. Wald oder Fischbestände) dürfen auf Dauer nur im Rahmen ihrer Fähigkeit zur Regeneration genutzt werden. Nicht erneuerbare Naturgüter (wie z. B. mineralische Rohstoffe oder fossile Energieträger) dürfen auf Dauer nur in dem Umfang genutzt werden, wie ihre Funktionen durch andere Materialien oder durch andere Energieträger ersetzt werden können.

3. Die Freisetzung von Stoffen darf auf Dauer nicht größer sein als die Anpassungsfähigkeit der natürlichen Systeme - z. B. des Klimas, der Wälder und der Ozeane.

18 Vgl. Die Bundesregierung (2008). 
4. Gefahren und unvertretbare Risiken für die menschliche Gesundheit sind zu vermeiden.

5. Der durch technische Entwicklungen und den internationalen Wettbewerb ausgelöste Strukturwandel soll wirtschaftlich erfolgreich sowie ökologisch und sozial verträglich gestaltet werden. Zu diesem Zweck sind die Politikfelder so zu integrieren, dass wirtschaftliches Wachstum, hohe Beschäftigung, sozialer Zusammenhalt und Umweltschutz Hand in Hand gehen.

6. Energie- und Ressourcenverbrauch sowie die Verkehrsleistung müssen vom Wirtschaftswachstum entkoppelt werden. Zugleich ist anzustreben, dass der wachstumsbedingte Anstieg der Nachfrage nach Energie, Ressourcen und Verkehrsleistungen durch Effizienzgewinne mehr als kompensiert wird. Dabei spielt die Schaffung von Wissen durch Forschung und Entwicklung sowie die Weitergabe des Wissens durch spezifische Bildungsmaßnahmen eine entscheidende Rolle.

7. Die öffentlichen Haushalte sind der Generationengerechtigkeit verpflichtet. Dies verlangt die Aufstellung ausgeglichener Haushalte durch Bund, Länder und Kommunen. In einem weiteren Schritt ist der Schuldenstand kontinuierlich abzubauen.

8. Eine nachhaltige Landwirtschaft muss nicht nur produktiv und wettbewerbsfähig, sondern gleichzeitig umweltverträglich sein sowie die Anforderungen an eine artgemäße Nutztierhaltung und den vorsorgenden, insbesondere gesundheitlichen Verbraucherschutz beachten.

9. Um den sozialen Zusammenhalt zu stärken, sollen

- Armut und sozialer Ausgrenzung soweit wie möglich vorgebeugt werden,

- allen Bevölkerungsschichten Chancen eröffnet werden, sich an der wirtschaftlichen Entwicklung zu beteiligen,

- notwendige Anpassungen an den demografischen Wandel frühzeitig in Politik, Wirtschaft und Gesellschaft erfolgen,

- alle am gesellschaftlichen und politischen Leben teilhaben.

10. Die internationalen Rahmenbedingungen sind gemeinsam so zu gestalten, dass die Menschen in allen Ländern ein menschenwürdiges Leben nach ihren eigenen Vorstellungen und im Einklang mit ihrer regionalen Umwelt führen und an den wirtschaftlichen Entwicklungen teilhaben können. Umwelt und Entwicklung bilden eine Einheit. Nachhaltiges globales 
Handeln orientiert sich an den Millenniums-Entwicklungszielen der Vereinten Nationen. In einem integrierten Ansatz ist die Bekämpfung von Armut und Hunger mit

- der Achtung der Menschenrechte,

- wirtschaftlicher Entwicklung,

- dem Schutz der Umwelt sowie

- verantwortungsvollem Regierungshandeln

zu verknüpfen.“ 19

Die zweite Komponente besteht aus einem Nachhaltigkeitsthementableau, das 21 Themenfelder beinhaltet, die für eine nachhaltige Entwicklung wichtig sind. Diese Themenfelder sind durch Nachhaltigkeitsindikatoren operationalisiert und mit Entwicklungszielvorgaben versehen. Jedem Themenfeld ist ein Nachhaltigkeitspostulat zugeordnet, das jeweils isoliert für ein Themenfeld Verhaltensempfehlungen formuliert, um eine günstige Entwicklung darin zu fördern.

Die dritte Komponente besteht darin, Fortschritte auf dem Weg einer nachhaltigen Entwicklung sichtbar zu machen und zu dokumentieren. Zu diesem Zweck erfolgt ein regelmäßiges Monitoring. Alle drei Komponenten zusammen verbinden eine statische Perspektive auf nachhaltige Entwicklung (Nachhaltigkeitsthementableau inklusive Indikatoren) mit einer dynamischen Perspektive (Nachhaltigkeitspostulate und Managementregeln).

Die Auswirkungen von regionalen Aktivitäten und Maßnahmen auf Nachhaltigkeit zu kennen, ist Voraussetzung dafür, Nachhaltigkeitsprozesse auf regionaler Ebene organisieren zu können. Vor dem Hintergrund eines solchen Managementkonzeptes lautet für die vier ausgesuchten Untersuchungsregionen die Forschungshypothese: „Maßnahmen, die im Rahmen von Erprobungs- und Entwicklungsvorhaben Erlebnis Grünes Band sowie Naturschutzgroßprojekten durchgeführt werden, wirken auf regionale Nachhaltigkeit“. Um dieser Hypothese nachgehen zu können, sind bestimmte Untersuchungsschritte, die in den nachstehenden Kapiteln 2.2.2 bis 2.2.4 dargestellt sind, ${ }^{20} \mathrm{zu}$ vollziehen.

\subsubsection{Maßnahmenbasismatrix und Nachhaltigkeitsspinne}

Das Leitbild „,regionaler Nachhaltigkeit“ ist vielschichtig. Der Begriff der Nachhaltigkeit wird in verschiedenen Gebrauchskategorien verwendet, die jeweils einen unterschiedlichen Grad an inhaltlicher Bestimmtheit aufweisen. Eine sehr verbreitete Gebrauchskategorie ist die des „Drei-Säulen-Modells“. Dieses versteht die drei Dimensionen Ökologie, Ökonomie und Soziales integrativ. Jede dieser drei

19 Die Bundesregierung (2008), S. 207 f.

20 Die Darstellung der Untersuchungsergebnisse in Kapitel 3 enthält für jede der vier Untersuchungsregionen dieselbe Struktur der Untersuchungsschritte. 
Dimensionen wird ferner durch Themenschwerpunkte inhaltlich ausdifferenziert. Diese Gebrauchskategorie des Nachhaltigkeitsbegriffes wird dieser Untersuchung zu Grunde gelegt. Um das Wirkungspotenzial des Erprobungs- und Entwicklungsvorhabens „Erlebnis Grünes Band“ und Naturschutzgroßprojekte im Hinblick auf die Beeinflussung von regionaler Nachhaltigkeit beurteilen zu können muss eine Beziehung zwischen den E+E-Vorhaben und Naturschutzgroßprojekten (NGP) und den Themenfeldern der regionalen Nachhaltigkeit hergestellt werden. Dies erfolgt durch die so genannte Maßnahmenbasismatrix. In Tabelle 1 sind in den Spalten der Matrix die einzelnen Maßnahmen des E+E-Vorhabens und des Naturschutzgroßprojekts Schaalsee-Landschaft ${ }^{21}$ abgetragen. In den Zeilen stehen die jeweiligen Themenfelder, die im Rahmen regionaler Nachhaltigkeit wichtig sind. Sie differenzieren die drei Dimensionen Ökologie, Ökonomie und Soziales inhaltlich aus.

Tabelle 1: Beispiel einer Maßnahmenbasismatrix

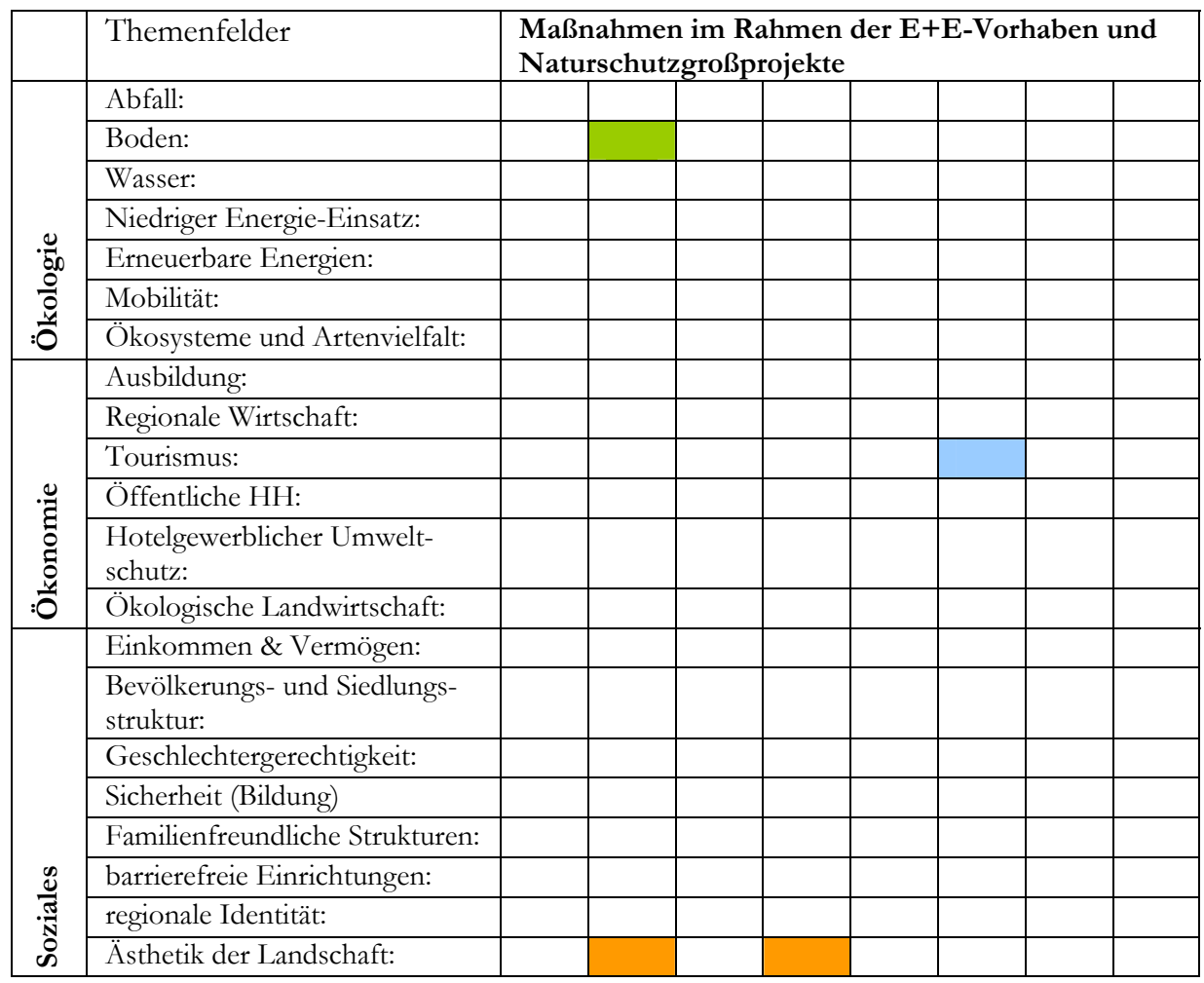

21 Bislang gibt es keine entsprechenden Maßnahmenkataloge aus dem NGP Grünes Band Eichsfeld-Werratal. 
Die Lesart erfolgt von den einzelnen Maßnahmen $\mathrm{M}_{\mathrm{i}}$ in der Spalte zu den 21 Themenfeldern in den Zeilen. Die Interpretation lautet folglich „Die Maßnahme $\mathrm{M}_{\mathrm{i}}$ wirkt direkt bzw. indirekt auf die Themenfelder 1 bis 21. Durch die Zuordnung des Maßnahmenwirkungspotenzials zu den Themenfeldern der regionalen Nachhaltigkeit entsteht in der Gesamtschau das Wirkungspotenzial in seiner Verteilungswirkung.

Eine Herausforderung im Rahmen dieses Forschungsvorhabens „Grünes Band - Modellregion für Nachhaltigkeit" besteht darin, dass viele Maßnahmen in den Untersuchungsregionen zum Zeitpunkt der Analyse noch nicht vollzogen sind beziehungsweise sich in der Umsetzungsphase befinden. Es wird daher die Annahme notwendig, dass alle Maßnahmen, wie sie in den Projektanträgen der E+E-Vorhaben und des Pflege- und Entwicklungsplans (PEPL) des Naturschutzgroßprojekts Schaalsee-Landschaft beschrieben waren auch so zur Umsetzung kommen. Die Wirkungsketten werden daher qualitativ entwickelt. Wo es möglich und sinnvoll ist, werden die zunächst auf Plausibilitätsüberlegungen basierenden möglichen Maßnahmenwirkungen durch Fallbeispiele aus der Literatur ergänzt. So entsteht eine statische Perspektive hinsichtlich der Maßnahmenwirkung auf regionale Nachhaltigkeit.

\subsubsection{Nachhaltigkeitsspinne und regionale Verhaltensweisen}

Die Maßnahmenbasismatrix kann auch als Kreisdiagramm dargestellt werden. Exemplarisch:

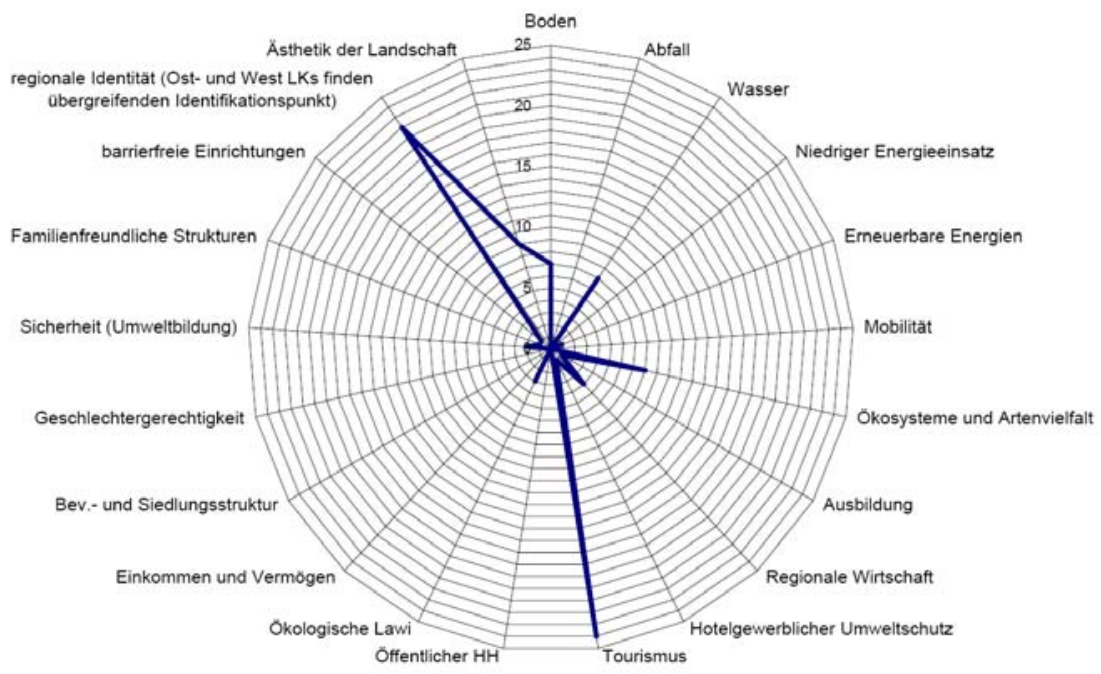


Hierbei sind die 21 Themenfelder der regionalen Nachhaltigkeit in einem äußeren Ring um das Kreisdiagramm gelegt. Vom Mittelpunkt des Kreisdiagramms ausgehend kann eine Darstellung der Maßnahmen, skaliert nach verschiedenen Kriterien, erfolgen. Dadurch entsteht eine netzartige Struktur, die an ein Spinnennetz erinnert und der „Nachhaltigkeitsspinne“ ihren Namen verleiht. Diese sind für das E+E-Vorhaben und die Naturschutzgroßprojekte unterschiedlich.

E+E-Vorbaben: Eine Möglichkeit stellt die Ermittlung der Maßnahmenkonzentration dar. Hierbei wird die Anzahl von Einzelmaßnahmen je Themenfeld gemessen. Themenfelder, die durch besonders viele Maßnahmen adressiert werden weisen entsprechend hohe „peaks“ auf. Ferner kann die Konzentration von finanzieller Zuwendung je Themenfeld dargestellt werden.

Naturschutrgroßprojekt „Schaalsee-Landschaft": Der Schwerpunkt der Maßnahmenperspektive lag auf den durch den ZSL erworbenen Flächen. Vor allem die fehlende Erfolgskontrolle erschwert den Nachweis von Nachhaltigkeitswirkungen einzelner Maßnahmen. Daher bietet die betrachtete Erwerbsentwicklung des Flächenankaufs eine Möglichkeit, die potenziellen Wirkungsgrößen abzuschätzen. Des Weiteren zielt die Nachhaltigkeitsdokumentation im Kontext von Flächen auf die Lösung eines weiteren Problems ab. Aufgrund der „Flickenteppichstruktur“ der Maßnahmenzielgebiete sowie sehr detaillierter Maßnahmenausgestaltung zielt das vorliegende Vorgehen auf eine Zusammenfassung von Einzelmaßnahmen $^{22} \mathrm{zu}$ „Entwicklungszielbereichen“ (EZB) ab. Stark verwandte Einzelmaßnahmen werden hierzu in Form von Flächenarten zu Bündeln zusammengefasst, die einen größeren Interpretationsspielraum hinsichtlich ihrer Nachhaltigkeitswirkungen zulassen

Bislang wird immer noch eine ausschließlich statische Perspektive beschrieben, schließlich ist die Nachhaltigkeitsspinne nichts anderes als die Maßnahmenbasismatrix in anderer Darstellungsform. Damit aus statischer Perspektive entsprechende Wirkungen in den Themenfeldern der regionalen Nachhaltigkeit festgestellt werden können (Beschreibung eines Zustandes), sind im Vorfeld entsprechende Prozesse zu initiieren. Gemäß dem Managementkonzept der bundesnationalen Nachhaltigkeitsstrategie können auch für die regionale Ebene Verhaltensweisen (Managementregeln und Nachhaltigkeitspostulate) ermittelt werden, die geeignet sind, um eine günstige Entwicklung in den Themenfeldern der regionalen Nachhaltigkeit herbeizuführen (dynamische Perspektive). Um diese dynamische

22 Vgl. Zweckverband Schaalsee-Landschaft (2006), S. 190 ff. 
Perspektive für die vier hier betrachteten Untersuchungsregionen zu erheben, wurden Interviews mit regionalen Akteuren unter diesem Aspekt ausgewertet.

\subsubsection{Regionale Nachhaltigkeitsinstitutionen}

Neben der Konzentration auf die reine Projektumsetzung besteht die Notwendigkeit einer langfristigen Verankerung der Projekte in den jeweiligen Untersuchungsregionen. E+E Projekte und Naturschutzgroßprojekte besitzen einen begrenzten Förderzeitraum, was unter Umständen dazu führen kann, dass nach dem Auslauf der Förderung die erreichten Ziele wieder auf die Ausgangssituation zurückfallen und auch die erarbeiteten Netzwerkstrukturen nicht erhalten werden können. Um dies zu verhindern, erscheint es sinnvoll, Anschlussmöglichkeiten für die jeweiligen Projektformen am Grünen Band zu finden. Um selbst tragende sowie nachhaltig-langfristige Strukturen für beide Projektformen zu etablieren, sind die bereits bestehenden Nachhaltigkeitsstrukturen jeder Region in die Projektplanung zu integrieren. In diesem Zusammenhang hat sich in den betrachteten Regionen gezeigt, dass oftmals bereits etablierte Nachhaltigkeitsinstitutionen existieren, die jedoch zumeist unter einem anderen Leitbild als der regionalen Nachhaltigkeit agieren. Dies hängt damit zusammen, dass in den Regionen Nachhaltigkeit schon lange eine wesentliche Rolle spielt, jedoch selten in einer so expliziten Form, wie es im Rahmen dieses Vorhabens untersucht wird.

Nachhaltigkeitsinstitutionen sorgen für eine Integration aller drei Säulen der Nachhaltigkeit, indem sie Abwägungsentscheidungen über einzelne fachpolitische Fragen hinaus vorbereiten, treffen oder vollziehen. Dabei ist die Gewichtung einzelner Nachhaltigkeitsaspekte innerhalb der jeweiligen Institution zunächst nebensächlich. So kann beispielsweise eine auf wirtschaftlichen Erfolg konzentrierte Institution als Nachhaltigkeitsinstitution identifiziert werden, wenn auch gleichzeitig ökologische und soziale Aspekte im Rahmen ihrer Entscheidungsfindung eine Rolle spielen.

Um dies zu erreichen, fand im Rahmen des Forschungsvorhabens eine Erhebung der regionalen Nachhaltigkeitsinstitutionen in jenen Landkreisen statt, die die Untersuchungsregionen berühren. Dabei lag der Schwerpunkt bewusst auf der Erhebung auch außerhalb der eigentlichen Untersuchungsregionen. Dies liegt in der Annahme begründet, dass innerhalb der Untersuchungsregion die wesentlichen Institutionen im Dialog mit dem Projektträger stehen bzw. sich gegebenenfalls äußern, wenn sie sich nicht genug in den Projektprozess integriert fühlen. Außerdem fand im Kontext der Netzwerkanalyse bereits die Analyse der wesentlichen und wichtigsten Akteure statt, so dass die damit repräsentierten Nachhaltigkeitsinstitutionen bereits erfasst sind.

Die Erhebung fand, wie bereits erwähnt, in jenen Landkreisen statt, die die Untersuchungsregion tangieren. Um dabei eine möglichst große Reichweite über die wesentlichen Nachhaltigkeitsinstitutionen in den Landkreisen zu erhalten, konzentrierte sich die Erhebung auf die einwohnerreichsten Städte. Dazu erfolgte 
ein leitfadengestütztes Interview mit den Bürgermeistern bzw. deren Stellvertretern (siehe Anhang 2). Auf Basis des Leitfadens konzentrierte sich die Erhebung auf bestehende Nachhaltigkeitsstrukturen und -institutionen des Landkreises und der Stadt. Dabei konnten die Bürgermeister die Position ihrer Stadt sowie ihre Erfahrung mit regionalen Nachhaltigkeitsinstitutionen der Region benennen.

Tabelle 2: Überblick über die durchgeführten Interviews zu Nachbaltigkeitsinstitutionen

\begin{tabular}{|l|l|}
\hline Untersuchungsregion & Stadt \\
\hline NGP Schaalsee-Landschaft & $\begin{array}{l}\text { Grevesmühlen } \\
\text { Ludwigslust } \\
\text { Ratzeburg }\end{array}$ \\
\hline NGP Grünes Band Eichsfeld-Werratal & Witzenhausen \\
& Heilbad Heiligenstadt \\
& Bleicherode \\
& Osterode am Harz \\
\hline E+E Elbe-Altmark-Wendland & Lüneburg \\
& Salzwedel \\
& Stendal \\
& Perleberg \\
\hline E+E Thüringer Wald/ & Bad Blankenburg \\
Thüringer Schiefergebirge/Frankenwald & Sonneberg \\
& Eisfeld \\
Schmalkalden \\
Gotha \\
\hline
\end{tabular}

Tabelle 2 gibt einen Überblick, in welchen Städten der Landkreise der Untersuchungsregionen die Befragung durchgeführt wurde.

Es ist dabei anzumerken, dass die durchgeführte Erhebung keinen Anspruch auf Vollständigkeit erheben kann. Vielmehr soll sie verdeutlichen, an welchen Institutionen sich eine Möglichkeit der Ankopplung realisieren lässt und wie die grundlegenden Strukturen der gängigen Nachhaltigkeitsinstitutionen aussehen, die sich identifizieren ließen.

Im Wesentlichen ergeben die Interviews vier gängige Institutionen, die mit Nachhaltigkeit direkt in Verbindung stehen: Integrierte Stadtentwicklungskonzepte (ISEK), Regionale Wachstumskerne (RWK), lokale Agenda 21 Initiativen sowie einzelne Personen (z.B. Bürgermeister).

Tabelle 3: Zusammenfassung der regionalen Nachbaltigkeitsinstitutionen

\begin{tabular}{|l|l|l|}
\multicolumn{1}{l}{ Stadt } & Institution & \multicolumn{1}{l|}{ Grundidee } \\
\hline Grevesmühlen & ISEK & $\begin{array}{l}\text { ISEK beschreibt Status quo, beinhaltet } \\
\text { Prognosen und Leitbilder. }\end{array}$ \\
\hline Ludwigslust & $\begin{array}{l}\text { ISEK, Lokale Agenda, Bürger- } \\
\text { werkstatt }\end{array}$ & $\begin{array}{l}\text { Ziele in der Lokalen Agenda beschrieben, } \\
\text { Bildung von elf Arbeitsgruppen }\end{array}$ \\
\hline Ratzeburg & $\begin{array}{l}\text { Arbeit im Rahmen des Pro- } \\
\text { gramms „Aktivregion Schleswig }\end{array}$ & $\begin{array}{l}\text { Arbeit im Programm „Ländliche Strukturen } \\
\text { und Entwicklungsanalysen“ (bis vor 2 Jah- }\end{array}$ \\
\hline
\end{tabular}




\begin{tabular}{|c|c|c|}
\hline & Holstein“ & $\begin{array}{l}\text { ren), Zusammenschluss auf regionaler Ebe- } \\
\text { ne mit anderen Gemeinden, Wirtschafts- } \\
\text { und Sozialpartnern um Maßnahmen und } \\
\text { Strategien zu erarbeiten. Nachhaltigkeits- } \\
\text { konzept: Integrierte Entwicklungsstrategie } \\
\text { für lokale Aktionsgruppe. }\end{array}$ \\
\hline Osterode & $\begin{array}{l}\text { Nachhaltigkeitsmanagement } \\
\text { obliegt dem Bürgermeister }\end{array}$ & $\begin{array}{l}\text { Nachhaltigkeit Grundlage des Handelns, } \\
\text { Leitbild für Stadt im Rahmen des Stadtmar- } \\
\text { ketings. }\end{array}$ \\
\hline Bleicherode & $\begin{array}{l}\text { Regionales Entwicklungskon- } \\
\text { zept „Westlicher Landkreis } \\
\text { Nordhausen“ }\end{array}$ & $\begin{array}{l}\text { Kommunale Arbeitsgemeinschaft } \\
\text { REK Westlicher Landkreis } \\
\text { Nordhausen als Auftraggeber für Entwick- } \\
\text { lungskonzept } \rightarrow \text { Maßnahmenkatalog und } \\
\text { Zielsetzung. }\end{array}$ \\
\hline Sonneberg & ISEK & $\begin{array}{l}\text { Entwicklungskonzepte für Stadtuntergebiete } \\
\text { (drei) mit Schwerpunkt demografischer } \\
\text { Wandel, innerstädtische Entwicklung (Flä- } \\
\text { chenverbrauch), Lösung nach Dringlichkeit. }\end{array}$ \\
\hline Schmalkalden & $\begin{array}{l}\text { Zusammenarbeit mit Nachhal- } \\
\text { tigkeitszentrum (Süd-West } \\
\text { Thüringen) in Nachbarstadt ( } 3 \\
\text { Mitarbeiter), ISEK }\end{array}$ & $\begin{array}{l}\text { Schwerpunkt Energiegewinnung und Ge- } \\
\text { bäudesanierung, Beachtung in Kommunal- } \\
\text { politik (Bsp.: Landesgartenschau, innerstäd- } \\
\text { tische Entwicklung), mindestens halbjährli- } \\
\text { ches Treffen mit Nachhaltigkeitszentrum. } \\
\text { Stadtentwicklungskonzept auf Nachhaltig- } \\
\text { keit ausgelegt }\end{array}$ \\
\hline Gotha & $\begin{array}{l}\text { Aktionsgruppe Lokale Agenda } \\
\text { 21, Nachhaltigkeitsbüro im } \\
\text { Stadtplanungsamt }\end{array}$ & Vier untergeordnete Arbeitsgruppen. \\
\hline $\begin{array}{l}\text { Bad Blanken- } \\
\text { burg }\end{array}$ & ISEK, „Rat der Bürgermeister“ & \\
\hline Eisfeld & & $\begin{array}{l}\text { Lokale Agenda } 21 \text { zwar unterzeichnet, aber } \\
\text { wenig bis keine Umsetzung. }\end{array}$ \\
\hline Witzenhausen & & Keine übergeordnete Institution. \\
\hline Heiligenstadt & & $\begin{array}{l}\text { Keine übergeordnete Institution; geplant ist } \\
\text { http://www.urlaub-am-mittelpunkt.de/ als } \\
\text { Plattform der Region für Touristen (nicht } \\
\text { fertig gestellt, Broschüre vorhanden). }\end{array}$ \\
\hline Stendal & $\begin{array}{l}\text { Regionalentwicklungskonzept, } \\
\text { ISEK, Städtedreieck, Städtenetz, } \\
\text { Lokale Agenda } 21\end{array}$ & \\
\hline Salzwedel & $\begin{array}{l}\text { RWK (für Region), für Stadt: } \\
\text { Stadtmarketing mit Wirtschafts- } \\
\text { förderungsamt, ISEK }\end{array}$ & \\
\hline Perleberg & ISEK, RWK & $\begin{array}{l}\text { Städtenetz, Kooperation mit Stadt Witten- } \\
\text { berge. }\end{array}$ \\
\hline Lüneburg & & $\begin{array}{l}\text { Keine übergeordnete Institution, Nachhal- } \\
\text { tigkeit wird über Foren oder Themenbezo- } \\
\text { gen bedacht. }\end{array}$ \\
\hline
\end{tabular}

Ausgehend von der Tabelle 3 lassen sich die gängigen Nachhaltigkeitsinstitutionen der Landkreise erkennen. Bei Planung der Förderprojekte sollten diese Insti- 
tutionen Berücksichtigung finden, um so die dauerhafte Etablierung der Projekte in der Region auch über die Grenzen der Untersuchungsregion sicherzustellen. Dazu erscheint es notwendig, mit Vertretern der jeweiligen Institution in Dialog zu treten und Perspektiven der Fortführung von Maßnahmen und Prozessen auszuloten. Diese Form des Dialogs lässt sich über Netzwerke organisieren.

\subsection{Methoden und Vorgehensweise der Netzwerkanalyse}

\subsubsection{Begriffsbestimmung}

\subsubsection{Netzwerke}

Im Allgemeinen wird ein Netzwerk als ,...eine durch Beziehungen bestimmten Typs verbundene Menge von sozialen Einheiten wie Personen, Positionen oder Organisationen“" 23 beschrieben. Netzwerke haben dabei keine eindeutigen Grenzen, sie sind durch Offenheit gekennzeichnet. In der Regionalentwicklung werden Netzwerken im Vergleich zu Kooperationen noch weitere Eigenschaften zugeordnet. Sie sind: 24

- Soziale Infrastrukturen: in eher losen und selbstorganisierten Verbünden können verschiedene Akteure an dem Netzwerk teilhaben, ohne dabei starr an die Erreichung eines bestimmten Zieles gebunden zu sein, wie dies bei Kooperationen der Fall wäre. Kooperationen können jedoch innerhalb des Netzwerkes entstehen. Mit ihrer Hilfe werden bestimmte, zeitlich befristete Ziele verwirklicht.

- Kooperationen höherer Ordnung: aufbauend auf bestehenden Kooperationen und potenziellen Kooperationen bilden sich Netzwerke.

- größer als Kooperationen: es sind mehr Akteure beteiligt, die ein Netzwerk bilden. Payer (2008) geht beispielsweise davon aus, dass ab 10 beteiligten Akteuren die Beziehungen nicht mehr als Kooperation zu bezeichnen sind, sondern sich Netzwerke ausbilden.

- längerfristig: Netzwerke bestehen bereits, wenn Kooperationen gebildet werden. Sie können folglich bestehende und zukünftige Kooperationen miteinander verknüpfen.

Die Gestalt der Netzwerke im Kontext von Förderungen korreliert mit einigen dieser Eigenschaften, weicht jedoch auch ab. Im Einklang mit der Definition von Payer (2008) stehen die betrachteten Netzwerke im Kontext der Förderung hinsichtlich der Größe, Zusammensetzung der Akteure und der Tatsache, dass sich

23 Pappi et al. (1987), S. 13.

24 nach Payer (2008). 
die Projektnetzwerke in einem System aus in den Regionen bestehenden, sich bildenden und potenziellen Kooperation und Netzwerken wieder finden. Abweichungen ergeben sich durch die steuernde Wirkung der Förderung. Die inhaltliche Orientierung des Netzwerks wird durch die Förderrichtlinien und Förderziele bestimmt und beeinflusst in Folge die Netzwerkbildung, d.h. Aktivierung von Akteuren. Die Zielorientierung wird zusätzlich von der aus der Förderung resultierenden Ausrichtung auf einen konkreten Zeitraum (Förderphase) geprägt, so dass sich erst nach der Förderphase zeigt, inwiefern sich die Netzwerke durch die Förderung die Fähigkeit der „Selbstorganisation“ angeeignet haben.

Aufgrund der regionalen Bedeutung der Projekte angesichts ihrer Gebietsgröße und der entsprechenden Mobilisierung von Akteuren in regionalen Handlungsräumen, wurde eine Anwendung des Forschungsstandes zu regionalen Netzwerken als sinnvoll und notwendig erachtet. Anders als bei der Betrachtung der Maßnahmen muss bei der Betrachtung der Netzwerke nicht nach den jeweiligen Fördertiteln unterschieden werden. Da es sich in beiden Fällen um Projektnetzwerke handelt, sind die theoretischen Überlegungen zu den Mechanismen in der Netzwerkarbeit übertragbar. Allerdings ist auf verschiedene Rahmenbedingungen wie die Laufzeit der Projekte Rücksicht zu nehmen, die in der Betrachtung und Auswertung der Fallstudien zu bedenken ist.

\subsubsection{Netzwerkmerkmale und Erfolgsfaktoren}

Das der Erfassung der Netzwerkmerkmale zugrunde liegende Verständnis ist, dass „Kooperation“ in der Region bzw. „kooperative Prozesse“ Teil regionaler Netzwerke sind bzw. durch sie entstehen. Die prozessorientierten Studien benennen zumeist „Erfolgsfaktoren“ für regionale Netzwerke und Prozesse. Von diesem Begriff wird im hier dargestellten Forschungsvorhaben Abstand genommen, da die wissenschaftliche Debatte über „Erfolg“ und deren Messbarkeit an dieser Stelle nicht geführt werden soll. Nichtsdestotrotz kann sich die durchgeführte Netzwerkanalyse nicht vollkommen von dem Erfolgsbegriff distanzieren. Zur Klarstellung sei an dieser Stelle unterstrichen, dass hier von erfolgreicher Netzwerkarbeit gesprochen wird, wenn die untersuchten Netzwerkkriterien in einer Weise ausgeprägt sind, die aus theoretisch hergeleiteter Sicht einen Beitrag der Netzwerkarbeit zur regionalen Nachhaltigkeit möglich erscheinen lassen.

Ein weiterer, viel diskutierter Aspekt im Kontext der Erforschung von Netzwerkmerkmalen, ist die Gewichtung dieser. Bei Adrian ${ }^{25}$ heißt es dazu:

„Die Gewichtung der genannten einzelnen Aspekte (Grundelemente von Regionalen Netzwerken synthetisiert aus den Ansätzen Milieukonzept, kreative Stadt-Konzept und Clusterbildung) und insbesondere die Frage, inwieweit sie bzw. deren Ausprägungen notwendige Bedingungen oder unterstützende ,Kann' - Faktoren darstellen, ist wohl durch empirische Forschung kaum zu eruieren. Resultat von Plausibilitätsüberlegungen muss es sein, dass

25 Vgl. Adrian (2003). 
in Abhängigkeit von der sperifischen Situation ein sperifisches Zusammenwirken vieler Faktoren erfolgsbestimmend oder-hemmend ist" ${ }^{\text {"26. }}$.

Da der hier vorliegende Forschungskontext klare Rahmenbedingungen liefert (Förderrichtlinien, Projektanträge, Regionen, Projektumsetzung) können die für die Projektnetzwerke der betrachteten Fördertitel förderlichen oder hemmenden Ausprägungen für den Beitrag zur regionalen Nachhaltigkeit erfasst werden.

\subsubsection{Forschungsdesign}

Der Forschungsprozess baut auf einer Literaturrecherche zur Erarbeitung eines Analyserahmens für die Netzwerke auf. Einschlägige wissenschaftliche Studien zu kooperativen Prozessen der Regionalentwicklung sowie der Netzwerkforschung im regionalen Kontext, die gerade im Zuge der Governance-Debatte an Impulsen gewann, werden herangezogen. Diese benennen erfolgversprechende Netzwerkeigenschaften für regionale Kooperationen und Netzwerke, denen insbesondere im Kontext regionaler Förderprogramme zunehmend Aufmerksamkeit geschenkt wird.

Der empirische Prozess durchläuft anschließend drei Ebenen der Projektnetzwerke, die Mikro-, die Meso- und Makroebene.

Auf der Mikroebene (Einzelakteur) wird das Netzwerk durch schriftliche Befragungen erhoben und quantitativ wie qualitativ analysiert. Auf dieser Ebene werden zudem leitfadengestützte Interviews mit regionalen Akteuren durchgeführt, die der Ergänzung der strukturellen Netzwerkdaten sowie der detaillierten Erfassung der Ausprägung der Netzwerkmerkmale sowie der Managementregeln im Netzwerk dienen.

Auf der Mesoebene (Interaktion) werden Regionale Workshops „,mit dem Projektnetzwerk" (Ausschnitt) durchgeführt. Sie dienen dazu, weitere Informationen zu den Forschungsfragen zu gewinnen bzw. die Ergebnisse zu reflektieren.

Auf Makroebene werden die gewonnenen Informationen und Daten zusammengeführt und durch Einbettung in die regionale Situation und regionalspezifischen Kontext, ausgewertet und analysiert. Eine netzwerk- bzw. projektübergreifende Analyse und Ableitung von Erkenntnissen mündet in die Erarbeitung der Perspektiven für Fördermittelgeber und Regionen.

26 Adrian (2003), S. 32. 


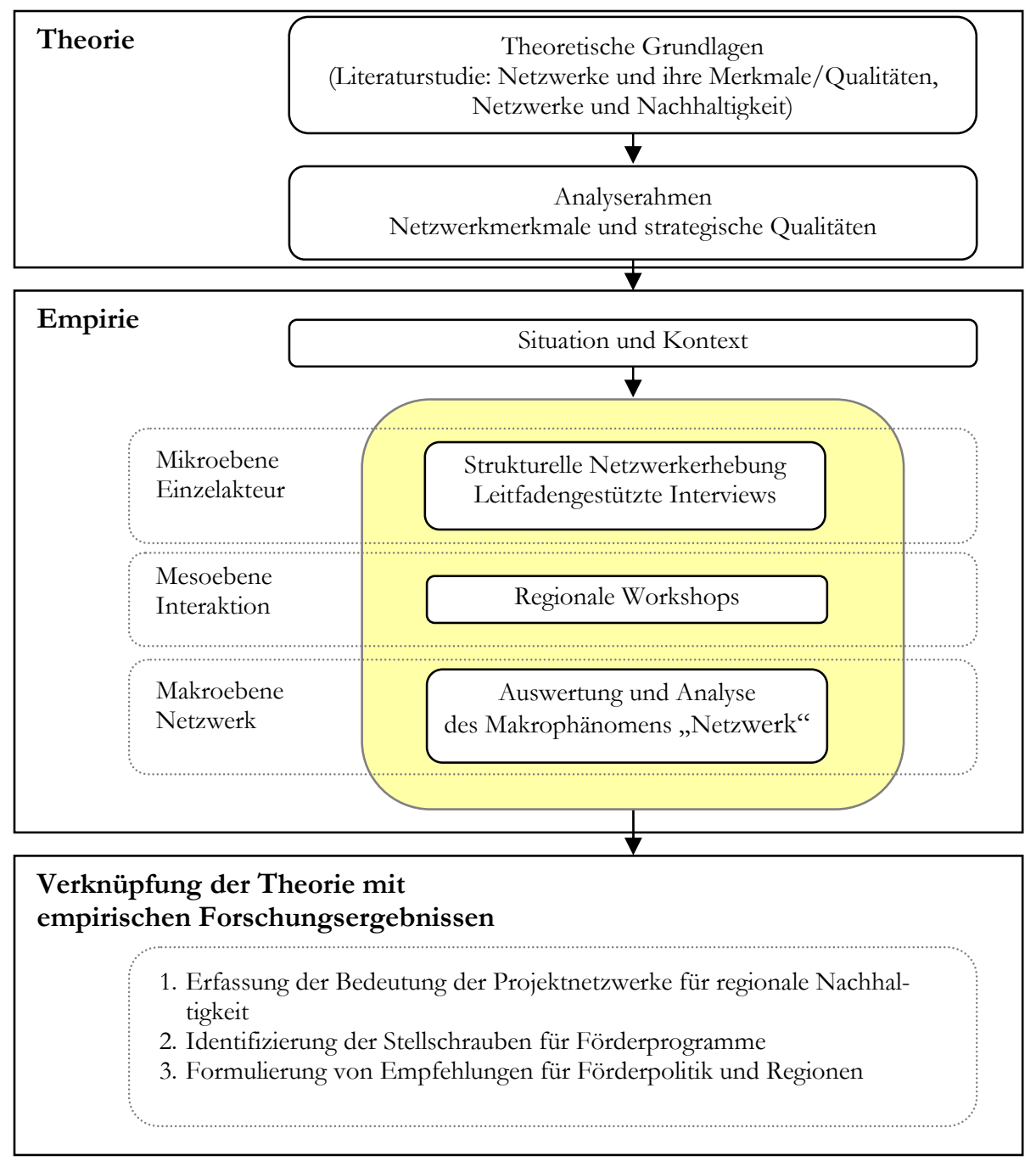

Abbildung 2: Forschungsdesign der Netzwerkanalyse

\subsubsection{Empirische Datenerhebung und Analysemethoden}

Untersuchungsleitend ist stets ein akteursorientiertes Vorgehen, welches durch den hohen Stellenwert des regionalen Erfahrungswissens im methodischen Ansatz gekennzeichnet ist. Die Netzwerkanalyse setzt mit Befragungen und Interviews, aber insbesondere auch mit regionalen Workshops unmittelbar bei dem für die Fragestellung relevanten Erfahrungswissen und den Perspektiven der regionalen Akteure an. Die gewählten Methoden beleuchten die interessierenden Aspekte der Beziehungen im Projektnetzwerk und setzen dicht an den Akteuren, deren indivi- 
dueller Wahrnehmung und Deutung der Kommunikations- und Netzwerkstrukturen an. Mit diesem Ansatz soll die Diskrepanz zwischen theoretischem Wissen und der Umsetzung von Förderprojekten geschlossen werden. Das „Qualitative“ der Untersuchung ist insbesondere auch in der Offenheit der Erhebung, des Sinnverstehens durch die Interviewer und der interpretativen Auswertung der Netzwerkdaten zu sehen.

\subsubsection{Strukturelle Netzwerkanalyse (und Abgrenzung des Untersuchungsraumes)}

Mittels schriftlicher Befragung (siehe Anhang) werden die Netzwerke, d.h. die Zusammensetzung der Akteure in den Projektnetzwerken sowie deren Beziehungen untereinander (Charakter und Intensität) und weitere netzwerkrelevante Aspekte erfragt. Diese liefern eine Datenbasis, die Beziehungsanalysen mittels visone ${ }^{27}$ ermöglicht, um bspw. Schlüsselakteure in den Projektnetzwerken zu identifizieren. Die Netzwerkanalyse als wissenschaftliche Methode, zeichnet sich auch hier dadurch aus, ,dass sie zur Untersuchung von Relationen und nicht von Attributen“ 28 , wie beispielsweise dem Alter oder Geschlecht, herangezogen wird. Nicht der einzelne Akteur steht im Vordergrund ,,sondern seine Einbettung in eine reale Struktur“"29. Die Attribute werden lediglich zur Beschreibung und Interpretation der Relationen herangezogen.

Die Befragung setzt jeweils an einer zentralen Organisationsstruktur, z.B. der PAG (NGP) oder Steuerungsgruppe $(\mathrm{E}+\mathrm{E})$, in den Netzwerken an (Ausgangsbasis). Die darin vertretenen Akteure werden nach den aus ihrer Sicht wichtigsten zehn Projektpartnern befragt. So wurde die Beantwortung des Fragebogens für die Befragten auf ein handhabbares $\mathrm{Maß}$ beschränkt sowie sichergestellt, dass die Befragten den Fokus auf das interessierende Projekt wahren. Die in der ersten Befragungsrunde erstmals genannten Akteure sind in einer zweiten Befragungsrunde für eine möglichst lückenlose Erfassung des Netzwerks erneut zu befragen. Damit wurde der realistische Ansatz mit Hilfe der Schneeballmethode zur Netzwerkerfassung gewählt, indem bei der Wahrnehmung der Akteure angesetzt wurde und diese die Netzwerkgrenzen bestimmt ${ }^{30}$. Im Kontext von Netzwerkanalysen ist zu beachten, dass die territoriale Begrenzung des Untersuchungsraumes generell nicht mit dem funktionalen Projektraum kongruent sein muss, ${ }^{31}$ da sich Netzwerke als soziale Konstrukte durch Akteure und eben nicht durch administrative oder geographische Grenzen bilden. Die untersuchten Projektnetzwerke sind aufgrund ihrer Einbettung in die Region ebenso wenig als räumlich begrenzt zu betrachten.

27 http://www.visone.info (letzter Zugriff: 27.04.2010).

28 Franke \& Wald, (2006), S. 154.

29 Wellmann in Zahl (2004), S. 9.

30 Der nominalistische Ansatz (d.h. der Forscher definiert das Netzwerk) wird aufgrund der Akteursvielfalt und deren ungewissen Streuung im Projektraum nicht als sinnvoll erachtet. Erst durch die Netzwerkanalyse können die tatsächlich relevanten Akteure identifiziert werden.

31 Vgl. Benz et al. (1999); Tröger-Weiss (1998); Maier et al. (2000). 
Das wichtigste Abgrenzungskriterium sind die „relevanten Akteure“ bzw. die „relevanten Beziehungen" für die Projekte. Als relevante Beziehungen sind alle projektbezogenen Beziehungen, die sich direkt (Umsetzung konkreter Projektmaßnahmen) oder indirekt (weitere Maßnahmen) durch dieses ergeben haben.

Es handelt sich außerdem um eine schließende Statistik, da ausgehend von einem erfassten Netzwerkausschnitt, Aussagen zum Gesamtnetzwerk erarbeitet werden. Insgesamt wurden 122 Fragebögen ausgefüllt zurückgesandt, auf die sich die Strukturanalysen beziehen.

Bei der Analyse der strukturellen Netzwerkdaten wird die Ausgangsbasis (s. o.) von der Grundgesamtheit - alle befragten und genannten Akteure - sowie die Analyseeinheit - alle, mindestens einmal genannten Akteure oder Akteure, die die Fragebogen ausgefüllt haben, unterschieden. Die gesamte Datenerhebung wird aus datenschutzrechtlichen Gründen anonymisiert ausgewertet.

Die strukturelle Netzwerkanalyse mit der Software visone ermöglicht es zudem, die Datensätze je Netzwerk hinsichtlich ihrer Beziehungsstrukturen und vor- sowie nachteiliger Positionen von Akteuren im Netzwerk zu analysieren. Auf Ebene des Gesamtnetzwerkes können auf Basis von Konzepten der Graphentheorie Maßzahlen ermittelt werden, die Informationen über das Beziehungssystem im Netzwerk liefern ${ }^{32}$. Durch verschiedene Zentralitätsanalysen werden zentrale Akteure herausgefiltert. Die Analyse erfolgt jeweils standardisiert, die Werte liegen daher immer zwischen 0 (niedrigster Wert) und 1 (höchster Wert). So zeigt die Gradzentralität (degree) auf Basis der ungerichteten Beziehungen an, welcher Akteur wie viele Beziehungen zu anderen pflegt und sich damit Kommunikationsvorteile verschafft (Kommunikationsaktivität). Die Nähezentralität (closeness) gibt die Nähe eines Akteurs zu allen anderen an und somit dessen Zugang zu effektiven, schnellen und unabhängigen Kontakten (Kommunikationseffektivität). Die Intermediationszentralität (betweenness) gibt an, welche Akteure aufgrund ihrer Position zwischen Paaren im Netzwerk eine besondere „Maklerposition“ innehaben (Kommunikationskontrolle). Um Auskunft über den Rang eines Akteurs im Netzwerk, d.h. dessen Prestige, zu gewinnen, ist die „indegree centrality“ des Netzwerks auf Basis gerichteter Beziehungen zu betrachten. Sie wird von der Zahl der empfangenen Werte eines Akteurs sowie der Stellung der ihn nominierenden Akteure bestimmt. ${ }^{33}$ Die Akteure mit dem meisten Prestige im Netzwerk werden mit drei Fragen ermittelt. Die Fragen 6 und 7 erfassen das verdeckte Prestige, in dem nach Abhängigkeiten (Rat) und Wichtigkeit bzw. Wertschätzung der Kontakte gefragt wird. Mit der Frage 8 wird direkt nach den Schlüsselpersonen im Projekt und bzw. oder in der Region gefragt und somit das offensichtliche Prestige erfasst. Zudem kann aufgrund der Kohäsion ein Netzwerkkern ermittelt werden, in dem die Beziehungen besonders dicht sind. Diese Akteure weisen damit eine besonders

32 Vgl. Schneider/ Janning (2007).

33 Vgl. Jansen (2006). 
intensive Beziehung zueinander auf und prägen durch deren intensive Interaktion die Netzwerkarbeit.

Die Akteure werden nach bestimmten Merkmalen definiert, die den Netzwerken ihren Charakter verleihen. So ist die Verteilung in sogenannte „Fachbereiche“ (siehe Netzwerkmerkmal Breite Beteiligung), Institutionen- oder Interessengruppen (Behördenvertreter, Vereine/Verbände, Privatunternehmen, private Akteure) sowie die Vernetzung der Akteure zwischen den alten und den neuen Bundesländern (,Ost-West-Verteilung“") interessant.

\subsubsection{Qualitative Netzwerkanalyse}

\section{Regionale Workshops}

In jeder Untersuchungsregion wurden zwei Regionale Workshops durchgeführt. Der erste im Anschluss an die strukturelle Erhebung des Netzwerks, um durch eine offene Diskussion zu ausgewählten Fragen (Projektnetzwerke, länderübergreifende Zusammenarbeit, regionale Nachhaltigkeit, Verknüpfung der Förderprojekte mit anderen Förderprogrammen in der Region, Fallbeispiele) ergänzende Daten- und Informationen zu gewinnen. Der Reflexionsworkshop am jeweiligen Ende der vier regionalen Untersuchungsphasen diente der Reflexion der Analyseergebnisse. Ergebnisprotokolle dienen dem Forschungsteam als Quelle und werden den Workshopteilnehmerinnen und -teilnehmern zur Verfügung gestellt.

\section{Leitfadengestütحte Experteninterviews}

Auf Basis der strukturellen Erhebungen sowie der jeweiligen regionalen Workshops wurde die Auswahl der zu interviewenden Schlüsselakteure getroffen. Diese müssen Zugriff auf umfassende regionale und projektbezogene Informationen haben sowie in irgendeiner Weise Verantwortung gegenüber dem Forschungsgegenstand tragen. ${ }^{34}$ Die relevanten Fachbereiche (siehe Merkmal Breite Beteiligung) werden dabei in jeder Region von einem regionalen Experten abgedeckt, so dass neun bis zwölf Interviews pro Region geführt wurden. Insgesamt liegen 42 Interviewtranskripte zur Analyse vor.

Die Interviewleitfäden (s. Anhang 4) umfassen zwei Themenkomplexe, die der Rückkopplung, Vertiefung und zusätzlichen Erfassung der Ausprägung der Netzwerkmerkmale dienen: dem Projektnetzwerk und dem Beitrag der Projektnetzwerke zur länderübergreifenden Kooperation. Die Interviewleitfäden sind für verschiedene Akteurstypen anzupassen, so für den Projektträger, die Schlüsselakteure im Projekt und eventuell fehlende Akteure.

34 Vgl. Mayer (2006). 
Die Interviews werden nach der Transkriptionsregel „so fein wie nötig“ zu Papier gebracht. ${ }^{35}$ Die Interviews werden mittels computergestützter (MaxQDA) Inhaltsanalyse ausgewertet. Der Analyserahmen aus Netzwerkmerkmalen und ihren Qualitätskriterien bildet zugleich das Codiersystem für die themenorientierte Inhaltsanalyse ${ }^{36}$ der Transkripte. Durch eine gegenseitige Kontrollcodierung am Beispiel jeweils eines Transkripts pro Untersuchungsregion wird die Gefahr der Über- oder Fehlinterpretation durch die Forscher eingeschränkt. Nach Abschluss der Codierung werden die identifizierten Netzwerkmerkmale mit Hilfe eines Stärken- und Schwächen-Rasters dargestellt. Dieses hat sich auch als geeignet für die Erfassung der strategischen Qualitäten erwiesen. Leere Zellen in den StärkenSchwächen-Tabellen stellen lediglich Datenlücken dar, so dass bezüglich des einen oder anderen Qualitätskriteriums keine Aussagen getroffen werden kann.

2.3.4 Operationalisierung von (Projekt-) Netzwerken: Merkmale und Qualitätskriterien

Die Schwierigkeit bei der Auswahl und Definition von relevanten Netzwerkmerkmalen liegt in dem Einfluss regionaler und überregionaler Rahmenbedingungen sowie der stets regionalspezifischen Ausgestaltung der Netzwerkmerkmale. Der Anspruch der Allgemeingültigkeit wird in der Netzwerkforschung bislang nicht angestrebt bzw. abgelehnt. ${ }^{37}$ Durch die Sichtung einschlägiger Studien wurden 17 Netzwerkmerkmale herausgearbeitet ${ }^{38}$, denen übergreifend eine besondere Bedeutung zugewiesen wurde. Diesen Netzwerkmerkmalen werden aufgrund ihrer Beschreibungen in der Literatur Qualitätskriterien zugeordnet, anhand derer die regionalspezifische Ausprägung der Netzwerkmerkmale erfasst werden können. Die Bedeutung der Merkmale für den Beitrag von Netzwerken zur regionalen Nachhaltigkeit kann nur dann beschrieben werden, wenn die Literaturstudie diesbezüglich Erkenntnisse liefert.

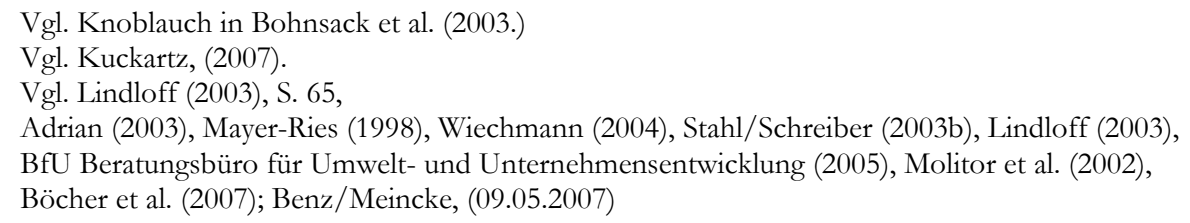


Tabelle 4: Merkmale regionaler Netzwerke und ibre Zusammenfassung

\begin{tabular}{|c|c|c|c|c|}
\hline & & $\begin{array}{l}\text { Merkmale } \\
\text { regionaler Netzwerke }\end{array}$ & & $\begin{array}{l}\text { Zusammenfassung der Merkmale } \\
\text { regionaler Netzwerke }\end{array}$ \\
\hline \multirow{7}{*}{ 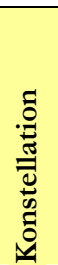 } & 1 & Breite Beteiligung & \multirow[t]{2}{*}{1} & \multirow[t]{2}{*}{ Breite Beteiligung } \\
\hline & 2 & Beteiligung der Wirtschaft & & \\
\hline & 3 & Starke Partner & \multirow[t]{4}{*}{2} & \multirow[t]{4}{*}{ Multiplikatoren } \\
\hline & \begin{tabular}{|l|l}
4 \\
\end{tabular} & Promotoren als Zugpferde & & \\
\hline & 5 & Politische Promotoren & & \\
\hline & 6 & Fachpromotoren/Regionale Expertengruppe & & \\
\hline & 7 & Personelle Kontinuität & 3 & Personelle Kontinuität \\
\hline \multirow{6}{*}{ 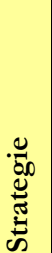 } & 8 & Lernen & \multirow[t]{2}{*}{4} & \multirow[t]{2}{*}{ Lernen } \\
\hline & 9 & Qualifizierung & & \\
\hline & 10 & Transparenz, Offenheit und Flexibilität & 5 & $\begin{array}{l}\text { Transparenz, Offenheit und Flexibili- } \\
\text { tät }\end{array}$ \\
\hline & 11 & Kooperation und win-win & 6 & Kooperation und win-win \\
\hline & 12 & Probleme und Lösungswille & 7 & Probleme und Lösungswille \\
\hline & 13 & Arbeiten auf verschiedenen Ebenen & 8 & Arbeiten auf verschiedenen Ebenen \\
\hline \multirow{4}{*}{ 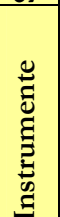 } & 14 & Leitbilder, Visionen, Ziele & 9 & Leitbilder, Visionen, Ziele \\
\hline & 15 & $\begin{array}{l}\text { Ausreichende Ressourcen für Projektmana- } \\
\text { gement }\end{array}$ & 10 & \multirow[t]{3}{*}{ Prozessmanagement } \\
\hline & 16 & (Externe) Moderation & \multirow[t]{2}{*}{11} & \\
\hline & 17 & $\begin{array}{l}\text { Kommunikation, Information und Öffent- } \\
\text { lichkeitsarbeit und Marketing }\end{array}$ & & \\
\hline
\end{tabular}

Aufgrund der aufgedeckten inhaltlichen Überlappungen und Zugehörigkeiten zwischen den Netzwerkmerkmalen konnte die Liste auf elf Merkmale reduziert werden (vgl. Tabelle 4). ${ }^{39}$

\subsubsection{Konstellation}

Netzwerke werden von ihren Akteuren geprägt, gestaltet und entwickelt. Die Konstellation der Akteure bestimmt folglich die Inhalte, Ausrichtung und Ar-

39 Die Reduzierung ergibt sich durch die Zusammenfassung von siebzehn Netzwerkmerkmalen auf insgesamt 11 Netzwerkmerkmale. Reduziert sind die Merkmale „breite Beteiligung“ und „Beteiligung der Wirtschaft“, da in unserem Verständnis das erste Merkmal das zweiten bedingt. Unter ,breiter Beteiligung“" wird folglich die Integration der relevanten Akteure aus den Bereichen Ökonomie, Ökologie und Soziales in gleichberechtigter und ausgewogener Weise verstanden. Des Weiteren wurden die drei Netzwerkmerkmale bezüglich der Promotoren sowie der Faktor „Starke Partner“ zu dem Merkmal „Multiplikatoren“ zusammengefasst. Da die Grenzen zwischen den „Starken Partnern“ und den „Promotoren“ fließend sind, bzw. Akteure auch beide Funktionen wahrnehmen können, erscheint eine Zusammenführung sinnvoll. Das Netzwerkmerkmal ist je nach regionalspezifischer Gegebenheit von einem Spektrum von politischen, fachlichen und regionalen Experten bzw. Entscheidungsträgern zu besetzen. Das Merkmal „Kommunikation und Öffentlichkeitsarbeit“" wurde aus Gründen der Vereinfachung und Entzerrung dem „kompetenten Prozessmanagement“ zugeordnet. Ebenso das Merkmal „Ausreichende Ressourcen für Projektmanagement", da auch für ein funktionierendes Prozessmanagement ausreichende personelle und finanzielle Mittel zur Verfügung gestellt werden müssen. 
beitsweise der Netzwerke. Die Konstellationsmerkmale beschreiben das „WER“ innerhalb der Netzwerke.

\section{Breite Beteiligung}

Voraussetzung für eine erfolgreiche Regionalentwicklung ist eine breite Beteiligung und Integration aller relevanten Akteure. ${ }^{40}$ Der Aspekt des „Integrativen“ muss sich in der Akteurskonstellation innerhalb regionaler Netzwerke - anhand einer „Durchmischung“"41 unterschiedlicher Akteure - widerspiegeln, denn nur dadurch können die regionalen humanen Potenziale und Ressourcen, die die Entwicklung gestalten müssen, ausgeschöpft werden. Besonders hervorgehoben wurde die Beteiligung der Wirtschaft. „Regionsbezogene Kooperationen brauchen Mitwirkung der Wirtschaft, um zu verhindern, dass im Kooperationsprozess unter der Dominanz kommunaler Interessen und politisch-administrativer Entscheidungskalküle der Zweck der Kooperation aus dem Blick verloren wird [...]“, aber auch „um wichtige Komplementärfunktionen wahrzunehmen, etwa bei [...] Maßnahmen zur Umweltverbesserung z.B. im Verkehrs- oder Energiebereich, bei der Wirtschaftsförderung oder der Umstrukturierung der Wirtschaft“ und „um finanzielle und technische Unterstützungsleistungen zu gewinnen [... $]^{\text {"42 }}$. Breite Beteiligung ist für regionale Nachhaltigkeit folglich bedeutend, da sie den Netzwerken durch Integration eine ,systemische Orientierung“43 gibt.

In diesem Zusammenhang sind folgende Qualitätskriterien zu beachten, die eine breite Beteiligung in den Projektnetzwerken sichern können:

\section{Qualitätskriterien}

- Projektträger und in Projektstrukturen (Gremien) integrierte Personen,

- Maßnahmenbezogene Partner des Projekts, Fach- oder Machtposition aus den Fachbereichen Naturschutz, Politik, Wirtschaftförderung, Privatwirtschaft, Landnutzung, Tourismus, Regionalmanagement/ entwicklung, Soziales (und Kultur und Bildung), Kommunalverwaltung

\section{Multiplikatoren}

Die Merkmale „Starke Partner“, „politische Promotoren“, „Fachpromotoren“ und „Zugpferde“ werden auf Basis der Literaturrecherche als „Multiplikatoren“ zusammengefasst, da sie, zwar mit unterschiedlicher Qualität, auf die gleichen Faktoren einwirken: Wahrnehmung, Akzeptanz, Unterstützung und Entwicklung

\footnotetext{
$40 \quad$ Vgl. Böcher et al. (2007), S. 21.

41 Selle (2000), S. 31.

42 Zeck (1998), S. 44.

43 BfU Beratungsbüro für Umwelt- und Unternehmensentwicklung (2005), S. 39.
} 
des Netzwerks bzw. der Projekte, die von Netzwerken bearbeitet werden. Im Kontext der regionalen Nachhaltigkeit sind Multiplikatoren insofern von zentraler Bedeutung, als sie für kreative Lösungsstrategien, die im Zuge des Such- und Gestaltungsprozesses zur Umsetzung des Leitbildes der Nachhaltigkeit erprobt werden müssen, gesellschaftliche Akzeptanz schaffen können.

Kaum eine der herangezogenen Studien verzichtet auf die herausragende Bedeutung der starken Partner für die Netzwerkarbeit hinzuweisen, da sie ,wesentlich die Dynamik der Prozesse beeinflussen können. Hierunter sind politische Entscheidungsträger wie Landräte oder Beigeordnete zu verstehen, aber auch anerkannte Persönlichkeiten aus der Wirtschaft oder der Zivilgesellschaft. " ${ }^{44}$ Das (persönliche) Engagement dieser Partner auf Basis informationeller, finanzieller, personeller oder (macht-)politischer Ressourcen ${ }^{45}$ oder deren ganz persönlichen „,soft skills“46, wirken unterstützend und motivierend.

Promotoren stehen den „starken Partnern“ sehr nahe und sind als „charismatische Persönlichkeiten“47, die sich für Prozesse und Netzwerke engagieren, als Motoren der Netzwerke und Initiativen zu verstehen. Sie können andere regionale Akteure für bestimmte Themen begeistern und motivieren. Von wesentlicher Bedeutung für diese Promotoren und ihren Beitrag zur Netzwerkarbeit ist ihr „Standing“48 innerhalb der Region. Sie lassen sich unterscheiden in politische Promotoren und Fachpromotoren.

Die Unterstützung und Mitwirkung der Politik in Netzwerken oder regionalen Prozessen hat sich als entscheidend für die Gewinnung öffentlicher Akzeptanz erwiesen sowie als Multiplikatorwirkung im Sinne des Anstoßes weiterer Akteure zur Mitwirkung oder eigenständiger Initiierung von Initiativen. Wie Selle (2000) feststellt, haben Vorhaben Aussicht auf Erfolg, ,wenn politische oder wirtschaftliche Macht im Spiel ist" ${ }^{\text {49. }}$. Während die politischen Promotoren Motivations- und Legitimationsfunktionen erfüllen, ${ }^{50}$ erfolgt „(...) die fachliche Bearbeitung der Kooperationsinhalte über eine (oder mehrere) Expertengruppen, deren Mitglieder aus den Institutionen der Region stammen (...)“51 und maßgeblich die inhaltliche Ausgestaltung der Projekte in den Netzwerken und die Überzeugung für diese prägen.

44 Lindloff (2003), S. 189.

45 Böcher et al. (2007), S. 20.

46 "[...] Voraussetzung ist, dass einflussreiche Repräsentanten der Region sich für die Kooperation engagieren, und zwar als Person, nicht nur als Institution." Adrian (2003), S. 43

47 Meister (2002), S. 58.

48 Böcher et al. (2007), S. 21.

49 Selle (2000), S. 151.

$50 \quad$ Vgl. Zeck (1998), S. 42.

51 Zeck (1998), S. 42. 


\section{Qualitätskriterium}

- Integration regionaler Meinungsführer und anerkannter Fachexperten relevanter Fachbereiche

\section{Personelle Kontinuität}

Netzwerkarbeit kann dann gelingen, wenn ein Kern aus aktiven Akteuren auch über die Initiierungsphase und Wachstumsphase hinaus besteht. Bei Netzwerkentwicklungen ist eine Beständigkeit eines Kreises an regionalen Akteuren, die die Aufgaben des Netzwerkmanagements übernehmen, von Bedeutung, da andernfalls „das Netzwerk zusammenbrechen“ kann und „schnell ein Gefühl von Überforderung und Intransparenz, das zum Abwandern von Mitgliedern und zu Frustration und Resignation bei den anderen führen kann" “. ${ }^{52}$ Personelle Kontinuität steht für kontinuierliche Netzwerkarbeit und somit für die Möglichkeit, Vertrauen und spezifisches Wissen im Netzwerk aufzubauen sowie eine stetige Weiterentwicklung zu gewährleisten. Insbesondere zentrale Akteure im Netzwerk können diese Qualität erfüllen. Dieses Merkmal ist somit sehr eng mit den zuvor beschrieben Multiplikatoren verbunden. Die positive Wirkung der Multiplikatoren ist in Abhängigkeit von deren kontinuierlichem Engagement zu sehen. Des Weiteren ist personelle Kontinuität im später dargestellten Prozessmanagement von Bedeutung. Kooperative Prozesse müssen aus „einem Guss“ kommen, um Abstimmungen, Verknüpfungen, Aufbauarbeit und Vernetzung im Netzwerk zu gestalten. Personelle Wechsel können zwar ggf. notwendig und hilfreich sein, sind jedoch zumeist mit mehr oder weniger gravierenden Brüchen im Prozess verbunden, so dass dies eine Gefahr für den Gesamtprozess darstellt.

\section{Qualitätskriterien}

- Kontinuierliches Engagement der Multiplikatoren im Netzwerk für das Projekt

- Kontinuierliche Betreuung des Prozessmanagement bzw. Netzwerkarbeit durch geeignete Person

\subsubsection{Strategie}

Unter Netzwerkstrategie werden jene Netzwerkmerkmale gefasst, die die Arbeitsund Funktionsweise des Netzwerks sowie seine Organisation näher beleuchten. Die Netzwerkstrategie legt im Wesentlichen offen, wie bestimmte Mechanismen im Zusammenspiel der einzelnen Akteure innerhalb des Netzwerks funktionieren.

52 BfU Beratungsbüro für Umwelt- und Unternehmensentwicklung (2005), S. 101-102. 


\section{Lernen}

Nachhaltigkeit ist weder eine klar definierbare Zielgröße, noch ein abschließend definiertes Leitbild aufgrund der Komplexität und Abstraktheit, das mit einfachen Strategien zu erreichen ist. Es bedarf eines Such-, Lern- und Gestaltungsprozesses. ${ }^{53}$ Dies kann nur durch den zuvor beschriebenen kooperativen und reflexiven Prozess gelingen, denn „Nachhaltigkeit erfordert ein Umdenken in der Regionalentwicklung, eine Veränderung von individuellen Einstellungen und Denkmustern. Dies kann nicht von oben herab verordnet werden, sondern muss in einem Lernprozess erarbeitet werden" $" 54$.

Diese Prozesse stellen einerseits Anforderungen an die regionalen Akteure, die „Sensibilität, Offenheit, Aufgeschlossenheit, Visionskraft und Kompetenz" aufweisen müssen. Andererseits werden Anforderungen an die Netzwerkstrukturen selbst benannt, die flexibel genug sein müssen, damit sich die „Lernerfahrung kontinuierlich auf sich selbst ,zurückspiegeln' und für die Gesamtentwicklung der Region nutzbar" ${ }^{46}$ gemacht werden kann. Durch eine angemessene Qualifizierung und ein Wissen über Beziehungen kann ein „Up-grading der Regionen“ 57 erreicht werden.

\section{Qualitätskriterien}

- direkter und regelmäßiger - sektorenübergreifender - Austausch zwischen den Akteuren des Netzwerks

- der Einsatz von reflexiven Instrumenten wie konkrete Evaluierungsmaßnahmen, Reflexionsworkshops, Monitoring etc.

- Erfahrungsaustausch mit Netzwerken ähnlicher bzw. gleicher inhaltlicher Ausrichtung

\section{Transparen₹, Offenheit und Flexibilität}

Transparenz steht in unmittelbarem Zusammenhang mit der Motivation der Akteure sich in Netzwerken zu engagieren, ${ }^{58}$ und ist durch eine ,gute Kommunikation [...] zwischen ,overhead' (Geschäftsstelle bzw. Lenkungsgruppe) und ,Basis' (Projekte, Beteiligte)“59 sowie eine „umfassende Information aller Interessierten“60 gekennzeichnet. Die Offenheit von Netzwerken im Sinne von intensiven Außen-

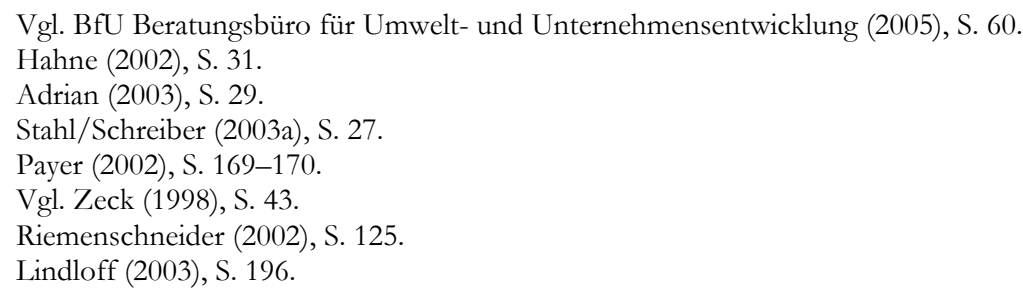


beziehungen fördert den dynamischen Charakter des Systems als Voraussetzung für dessen hohe kooperative Lernfähigkeit. ${ }^{61}$ Zur Offenheit der Netzwerke zählt dabei auch der Kontakt „(...) zu bislang noch nicht beteiligten Akteuren in der Region, zu benachbarten Landkreisen und den anderen Regionen (...) ${ }^{\text {‘62 }}$.

\section{Qualitätskriterien}

- Nachvollziehbarkeit von Abstimmungs- und Entscheidungsprozessen

- Fortwährende Zugangsmöglichkeit von neuen Akteuren ins Netzwerk

- Inhaltliche und organisatorische Flexibilität: Integration neuer Ideen, kreative Lösungen für regionalspezifische Herausforderungen

\section{Kooperation und win-win}

Wie die Belege zur „breiten Beteiligung“ bereits zeigen, sind Partizipation und Kooperation notwendige Bedingungen, um eine maximale Wirksamkeit der verfügbaren und immer auch begrenzten Ressourcen zu erreichen. Die Schaffung von win-win-Situationen steht in Wechselbeziehung mit der Motivation, denn nur wenn die Akteure einen Nutzen aus ihrem Engagement im Netzwerk ziehen, setzen sie sich und ihre Ressourcen ein. „Die Zielsetzung win-win, die in vielen Prozessen explizit verfolgt und gesucht wird, ist nach Selle die deutlichste Form, wie der Nutzen auch Erfolgsfaktor ist". ${ }^{63}$

\section{Qualitätskriterium}

- konkrete Projekt-/ Maßnahmenbeispiele, aus denen mindestens zwei Interessengruppen einen gemeinsamen Gewinn ziehen.

\section{Probleme und Lösungswille}

Motivation für Menschen zu handeln, kann ein gewisser Druck an subjektiv empfundenen Problemen sein. ${ }^{64}$ Spezifische Problemlagen einer Region müssen von den regionalen Akteuren als zentral und als subjektiv negativ auswirkend erfahren werden, so dass ein gemeinsamer regionaler Wille zur Lösung dieser Probleme entstehen kann. ${ }^{65}$ Allerdings führen regionale Probleme nicht zwangsweise zu einer Motivation regionaler Akteure.

\footnotetext{
61 Vgl. Adrian (2003), S. 28.

62 Böcher et al. (2007), S. 22.

63 Lindloff (2003), S. 202.

64 Vgl. Böcher et al. (2007), S. 16

65 Vgl. ebenda.
} 


\section{Qualitätskriterien}

- belegbare Bemühungen der Problembewältigung durch intensive Dialogphasen

- vereinbarte Kompromisse

\section{Arbeiten auf verschiedenen Ebenen}

Netzwerke als „soziale Infrastrukturen für die Verbindung zwischen Menschen und Organisationen mit ähnlichen Interessen und für die Transformation von potenziellen zu tatsächlichen Interaktionen zwischen ihnen "66 bedürfen besonderer Gelegenheiten zur Kommunikation. Netzwerke als komplexe soziale Konstrukte mit dem Anspruch einer breiten Beteiligung dürfen diese jedoch nicht auf Kosten von Effizienz oder Flexibilität tun, denn gerade in diesen Eigenschaften liegen die Vorteile von Netzwerken. ${ }^{67}$ Wie zuvor aufgezeigt, bedarf es eines intelligenten Umgangs mit den stets knappen regionalen Ressourcen - humanen, finanziellen oder der regionalen Motivation. So bietet sich eine funktionale Trennung der Entscheidungsebene und Entscheidungsvorbereitungsebene an. Dies macht die Kooperation außerdem tendenziell offener, weil die Experten bei der Entscheidungsvorbereitung weniger Rücksicht auf politische Restriktionen und damit auch fachfremde Aspekte nehmen müssen. Generell haben regionsbezogene Kooperationen nämlich häufig das Problem, dass die paritätische Abbildung der Interessen der Effizienz der Arbeit entgegenstehen kann. Es kommt zu einem Stillstand, weil sich praktisch gegen jede fachpolitisch gebotene Maßnahme jemand finden lässt, der sie nicht befürwortet. Häufig wird für die politische Repräsentanz und paritätische Interessenabbildung der regionalen Akteure daher neben der ,Lenkungsgruppe' eine weitere Organisationsebene geschaffen, z. B. Regionalversammlungen oder Regionalkonferenzen, die primär auf Informations-, Kontroll- und Mitsprachefunktion reduziert ist. ${ }^{68}$

Die Komplexität des Leitbilds der Nachhaltigkeit spiegelt sich im Aufbau und der Funktionsweise der dafür erforderlichen Netzwerke in den Regionen wider. Um dem Anspruch einer „breiten Beteiligung“ gerecht zu werden und zugleich zielorientiert sowie effizient zu arbeiten, muss die Netzwerkarbeit auf verschiedenen Ebenen mit eigenen Funktionen und Zielsetzungen agieren.

66 Payer (2008), S. 41.

67 „Organisationsflexibilität muss bei Netzwerken ausgeprägter sein als anderswo. Denn gerade in der Möglichkeit der Arbeitsteiligkeit und des Teilens auf vielen Ebenen (siehe das erste Basisprinzip) liegt einer der wichtigsten Vorteile von Netzwerken. Dies ist allerdings ohne eine hohe flexible Organisation als Ganzes - also des Netzwerks und auch der Organisation der beteiligten Partner - nicht möglich" BfU Beratungsbüro für Umwelt- und Unternehmensentwicklung (2005), S. 83-84.

68 Siehe beispielweise Zeck (1998), S. 42. 


\section{Qualitätskriterium}

- Vorhandensein projekt- bzw. regionsspezifischer Organisationsstrukturen im Netzwerk, mit denen eine Aufgabenteilung (Steuerung, Entscheidung, Umsetzung, Diskussion, Information) vorgenommen wird

\subsubsection{Instrumente}

Als Instrumente können jene Netzwerkmerkmale betrachtet werden, die den Akteuren innerhalb des Netzwerks ermöglichen, ihre Netzwerkarbeit zu gestalten. Sie sind in gewisser Weise das „Handwerkszeug“ der zentralen Netzwerkakteure um das Netzwerk aktiv zu steuern.

\section{Leitbilder, Visionen, Ziele}

Leitbilder spielen in der Raumentwicklung eine wichtige Rolle als Instrument, das „(...) die Vielfalt der divergierenden Interessen der von der räumlichen Planung Betroffenen zunächst in einer gemeinsam akzeptierten Idee, konkretisiert im räumlichen Leitbild, harmonisierend (...)“69 zusammenfasst. Leitbilder sorgen innerhalb von Netzwerken dafür, dass „(...) ein gemeinsamer Handlungsrahmen (...) für die Akteure entsteht ${ }^{“ 70}$. Denn „Verständigungen über allgemeine Werte und Orientierungen [...] sind notwendig ${ }^{671}$ um einen Orientierungs- sowie einen Aktionsrahmen für die Regionalentwicklung abzustecken. Innerhalb dieser Leitbilder werden dann Ziele für konkrete Probleme (s. o.) formuliert, die in Maßnahmen münden können.

Die herausragende Bedeutung von Leitbildern im Kontext der Nachhaltigkeit liegt darin, dass „,...)Stakeholder unterschiedlichster Herkunft und Kulturen aufeinander treffen, [so dass] ein gemeinsames Verständnis davon, wohin die Reise

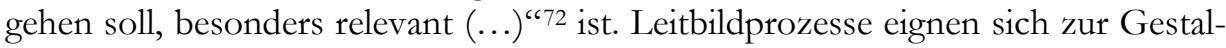
tung des zuvor beschriebenen Umdenkens bzgl. des Entwicklungsverständnisses (s. „Lernen“), wobei nicht nur das Leitbild, sondern auch der (Leitbild-)Prozess eine im Sinne der Nachhaltigkeit verantwortliche Kooperation hervorzubringen vermag. ${ }^{73}$

69 Spitzer (1995), S. 16.

70 Böcher et al. (2007), S. 22.

71 Selle (2000), S. 202.

72 BfU Beratungsbüro für Umwelt- und Unternehmensentwicklung (2005), S. 59.

73 Aus Sicht der nachhaltigen Regionalentwicklung steht bei diesen Verfahren der Prozessnutzen in Richtung Konkretisierung der nachhaltigen Regionalentwicklung höher als die Verabschiedung des Leitbildes selbst. Gelingt es, Prozess und Leitbild zu kommunizieren, das Akteursnetzwerk zu erweitern und Zielvereinbarungen zu treffen, so kann aus verständiger Kommunikation eine im Sinne der Nachhaltigkeit verantwortliche Kooperation erwachsen. Hahne (2002), S. 31. 
Nachhaltige Entwicklung zu gestalten ist eine Integrationsaufgabe. Um verschiedene gesellschaftliche Interessen und Bedürfnisse zu harmonisieren und der Entwicklung für jeden Einzelnen eine Orientierung zu geben, sind gemeinsam unterstützte Leitbilder, Visionen und Ziele unentbehrlich.

\section{Qualitätskriterien}

- das Vorhandensein eines Leitbildes

- die Qualität des Leitbildes hinsichtlich der Berücksichtigung der drei Dimensionen der Nachhaltigkeit

- die Bekanntheit und Verankerung des Leitbilds und damit seine Orientierungswirkung in den Köpfen der Netzwerkakteure

\section{Prozessmanagement}

Netzwerke sind komplexe Organisationsstrukturen aufgrund ihrer verzweigten Struktur und ihrer Offenheit. Um sie arbeitsfähig zu gestalten, bedarf es eines Prozessmanagements, das sich insbesondere um die Gestaltung von Moderation und Kommunikation im Netzwerk kümmert. Darunter fällt eine Fülle von Aufgaben, die von einer ausgewogenen Ressourcennutzung über Führung und Netzwerksteuerung hin zu Moderation und Coaching reichen. Diese Aufgaben erfordern eine hohe Kompetenz der verantwortlichen Akteure, da „dilettantisches Agieren im gesellschaftlichen Prozess [...] Erfolgschancen erheblich" "74 verringert.

Auch eine professionelle Öffentlichkeitsarbeit ist Teil des Prozessmanagements und für Netzwerke unverzichtbar. Frei nach dem Motto „Tue Gutes und rede darüber“" wird Öffentlichkeitsarbeit über Internet-Seiten, Newsletter oder Presseberichte betrieben. „Es ist kein Zufall, dass erfolgreiche NetzwerkkoordinatorInnen in den meisten Fällen über beträchtliche PR-Expertise entweder selbst verfügen oder zur Unterstützung heranziehen“. ${ }^{75}$ In Netzwerken für Nachhaltigkeit muss die „Gleichberechtigung der Beteiligten, ihrer Anliegen und ihrer Handlungsmöglichkeiten [...] durch eine geeignete Verfahrenssteuerung gesichert werden. [...] Moderation wird erforderlich, um die Gleichberechtigung der Positionen und Ge-

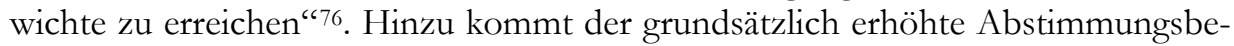
darf in Netzwerken für Nachhaltigkeit, da sie die Handlungsfelder Ökonomie, Ökologie und Soziales ebenso wie deren Akteure integrieren müssen. Zielkonflikte sind unvermeidbar und sind u.a. in den oben benannten Leitbildprozessen zu bearbeiten. Dazu bedarf es eines Prozessmanagements bzw. einer (externen) Moderation mit der nötigen Sensibilität für diese Komplexität, da stets die Balance

\footnotetext{
74 Böcher et al. (2007), S. 22.

75 Payer (2008), S. 42.

76 Adrian (2003), S. 24.
} 
zwischen Integration und Schwerpunktsetzung bzw. Ergebnisorientierung gewahrt sein muss. ${ }^{77}$ Das verdeutlicht auch das folgende Zitat:

„Regionale Diskurse und eine damit verbundene Schaffung von regionalen Kommunikations- und Kooperationsnetzwerken markieren somit einen wichtigen Schritt zur Etablierung eines regionalen Handlungsraumes, der als solcher von den relevanten Akteuren auch wahrgenommen und gestaltet werden kann. Ein funktionierender Nachbaltigkeitsprozess ist letztlich darauf angewiesen. "78

\section{Qualitätskriterien}

- Vorhandensein einer (externen) Moderation, die den Prozess steuernd begleitet.

- Aktive Teilhabe der Akteure verschiedener Sektoren

- Zufriedenheit der Akteure mit Prozess

- Präsenz des Netzwerks bzw. des Projekts in der Öffentlichkeit

- Wahrnehmung aller Aufgaben des Prozessmanagements durch Netzwerkmanager.

77 Vgl. BfU Beratungsbüro für Umwelt- und Unternehmensentwicklung (2005), S. 60.

78 Greif (2000), S. 113. 


\subsection{Interdisziplinärer Brückenschlag zwischen Netzwerk- analyse und regionaler Nachhaltigkeit}

Das Forschungsvorhaben verknüpft die Nachhaltigkeitsdiskussion mit der Frage nach der Rolle, die Netzwerke im Sinne der Nachhaltigkeit erfüllen sollten und der dazu nötigen Qualitätskriterien. Wenn man derartige Qualitätskriterien als Merkmale benennen kann, ergibt sich für den Bund die Möglichkeit, sie in seine Anforderungen aufzunehmen und damit die regionale Nachhaltigkeit zu beeinflussen. Dieser Abschnitt stellt die methodische Schnittstelle der beiden Forschungsstränge der Netzwerkanalyse und der Nachhaltigkeitsforschung auf der Basis der betrachteten Förderprojekte, dar.

Um auf die Frage eine Antwort zu finden, warum welche indikatorenbezogene Effekte festzustellen sind, ist es zusätzlich hilfreich, eine dynamische Perspektive einzunehmen, die den Prozess der Maßnahmenentwicklung und -umsetzung und damit die Rolle der Netzwerke mit in den Blick nimmt.

\subsubsection{Interdisziplinäre Verknüpfung}

Den Ausgangspunkt für die Verknüpfung bildet die Nationale Nachhaltigkeitsstrategie der Bundesrepublik Deutschland, die das Leitbild „Nachhaltigkeit" in ein Management-Konzept mit drei Komponenten übersetzt:

- Das Nachhaltigkeitsthementableau beinhaltet 21 Themenfelder, welche die vier Kategorien „Generationengerechtigkeit“, „Lebensqualität“, „sozialer Zusammenhalt“ und „Internationale Verantwortung“ inhaltlich ausdifferenzieren.

- Die korrespondierenden Verhaltensweisen beschreiben Verhaltensweisen, die eine nachhaltige Entwicklung in den Themenfeldern des Nachhaltigkeitsthementableaus begünstigen. Dies erfolgt zum einen durch zehn Managementregeln, die allgemeine Grundsätze für Verhaltensweisen im Sinne der nachhaltigen Entwicklung formulieren und integrativen, akteursorientierten und diskursiven Charakter haben. Zum anderen sind Handlungsempfehlungen (größtenteils) isoliert für jeden Themenbereich des Nachhaltigkeitsthementableaus in Form von Nachhaltigkeitspostulaten niedergeschrieben.

- Die begleitende Dokumentation erfasst in regelmäßigen Abständen den Stand der Nachhaltigkeitsindikatoren in den 21 Themenfeldern durch das Statistische Bundesamt sowie durch einen Fortschrittsbericht der Bundesregierung, der die Nachhaltigkeitsstrategie selbst reflektiert und weiterentwickelt. 
Die Nachhaltigkeitsstrategie bildet den Ausgangspunkt für die Untersuchung, weil der Bund als Fördermittelgeber auftritt und folglich bei der Ausrichtung seiner Förderpolitik eigene Zielkonzeptionen zugrunde legt. Das Managementkonzept des Bundes mit seinen drei Komponenten ist auf die regionale Ebene zu übersetzen, um erstens sichtbar zu machen, wie die Naturschutzfördertitel E+E und NGP auf Themenfelder der regionalen Nachhaltigkeit wirken (können), zweitens, wie durch die Fördertitel, regionale Nachhaltigkeit (mit)organisiert werden kann und um drittens aufzuzeigen, wie, d.h. mit welchen Instrumenten, die Entwicklung in den Themenfeldern dokumentiert werden kann.

Die Verknüpfung der Forschungsstränge geschieht innerhalb der zweiten Komponente, den Managementregeln der Nachhaltigkeit. Nachhaltigkeit ist sowohl auf Bundes- als auch auf Regionsebene als Leitbild zu verstehen, und erst die Diskussion über das Leitbild zwischen den relevanten Akteuren gibt diesem im regionalspezifischen Kontext eine handlungsweisende Bedeutung. Diese „Übersetzung“ ist folglich eine gesellschaftliche Aufgabe. ${ }^{79}$ Es müssen Strukturen vorhanden sein, die diese Aufgabe bewusst wahrnehmen, so auch in projektbezogenen Netzwerken. Die Erfassung und Analyse der Netzwerkmerkmale wird somit durch die interdisziplinäre Betrachtung der regionalen Managementregeln der Nachhaltigkeit und Nachhaltigkeitspostulate in den Untersuchungsregionen ergänzt. Die interdisziplinäre Forschungsfrage ist, welche Managementregeln und Nachhaltigkeitspostulate in den Förderprojekten Anwendung finden, ob und wie sie die geförderten Maßnahmen beeinflussen, und welche Merkmale ein in dem betrachteten Förderkontext erfolgreiches Netzwerk ausmachen. Als erfolgreich ist hier die Umsetzung im Sinne der Nachhaltigkeit zu verstehen, die mit Hilfe der strategischen Qualitäten, die die Nationale Nachhaltigkeitsstrategie vorgibt, zu definieren ist: akteursorientiert, integrativ, diskursiv. Die nachstehende Abbildung 3 verdeutlicht grafisch die zuvor beschriebene interdisziplinäre Verknüpfung von Netzwerk- und Nachhaltigkeitsforschung.

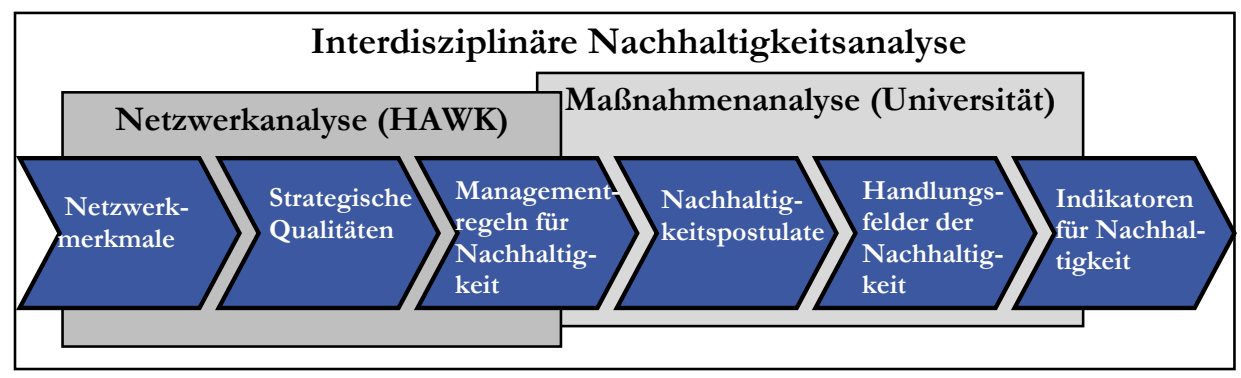

Abbildung 3: Verknüpfung der Netžwerkanalyse mit der Maßnabmenanalyse

79 Vgl. Die Bundesregierung (2008). 


\subsubsection{Regionale Managementregeln und Netzwerkmerkmale - Dynamische Perspektive}

Die dynamische Perspektive betrachtet den Prozess in den Regionen und ihren Netzwerken. Dieser Prozess wird durch die Managementregeln, mit denen Akteure ihr eigenes und das Verhalten anderer Akteure steuern, geprägt. Die Managementregeln für Nachhaltigkeit aus der Nationalen Nachhaltigkeitsstrategie geben für die Ausgestaltung der Netzwerke bestimmte Anforderungen im Sinne strategischer Qualitäten vor. Die Operationalisierung von Nachhaltigkeit stellt daher bestimmte Bedingungen an die Akteure und ihre Zusammenarbeit.

Aus den in der Nachhaltigkeitsstrategie formulierten Managementregeln lassen sich für das Nachhaltigkeitsmanagement strategische Qualitäten, die Netzwerke voraussichtlich erfüllen müssen, ableiten. Danach sind Netzwerke

- diskursivi ${ }^{80}$ auszugestalten. Nachhaltigkeit als Leitbild benötigt die Verhandlung unter den relevanten Akteuren. Da laut Regel 1 der Managementregeln alle Generationen dazu verpflichtet sind, ihre Aufgaben selbst zu lösen und zudem „Nachhaltige Entwicklung (...) auf Partizipation und Teilhabe" ${ }^{\text {"81 }}$ beruht, ist eine wesentliche Aufgabe in einer demokratisch ausgestalteten Gesellschaft die Beteiligung aller relevanten Akteure. Deshalb ist ein Diskurs bzw. eine Verhandlung dieser Akteure über die Art und Weise, wie diese Aufgaben zu lösen sind, zu führen. Darüber hinaus sollen laut Regel 9 ,alle am gesellschaftlichen und politischen Leben teilhaben“. Damit ist ein Auftrag an die Akteure formuliert, sich an der Realisierung des Leitbilds Nachhaltigkeit entsprechend zu beteiligen.

- akteursorientiert. Da Nachhaltigkeit, wie die Bundesregierung in der Nationalen Nachhaltigkeitsstrategie darlegt, nur gelingen kann, wenn ein ,.... Zusammenspiel aller relevanten Akteure (...)"82 stattfindet, stehen die Akteure im Mittelpunkt. Es handelt sich dabei um jene Akteure, die am jeweiligen Thema beteiligt oder davon betroffen sind. Die Anforderung „akteursorientiert" an die Netzwerke lässt sich ebenfalls aus den beiden Regeln 1 und 9 ableiten, da, wie bereits erläutert, ein Diskurs zwischen den relevanten Akteuren stattfinden muss.

80 Der Diskurs wird im Sinne der Theorie des kollektiven Handelns nach Habermas verstanden. Danach ist der Diskurs ein Prozess, in dem die Akteure ihre unterschiedlichen Ansprüche aushandeln: „Im Zentrum der Habermasschen Analyse stehen dabei der theoretische Diskurs zur Prüfung von Wahrheitsansprüchen und der praktische Diskurs, in den die Kommunikationsteilnehmer eintreten, wenn sie Ansprüche normativer Richtigkeit bzw. evaluativer Angemessenheit prüfen wollen" Schneider, (2009), S.199.

81 Die Bundesregierung (2008), S. 34.

82 Die Bundesregierung (2008), S. 206. 
- integrativ ${ }^{83}$ ausgerichtet. Da Nachhaltigkeit als „Querschnittsaufgabe“, viele Bereiche anspricht und keine Politikfelder unbeachtet lassen soll, ist eine integrative Ausrichtung Voraussetzung. In der Regel 5 der Managementregeln für Nachhaltigkeit ist die Aufgabe der Integration von Politikfeldern eindeutig benannt. Ein Netzwerk kann hierfür als Instrument gesehen werden.

Die Analyse der oben beschriebenen Netzwerkmerkmale wird um die Berücksichtigung ihrer strategischen Qualitäten ergänzt. Die in ein Stärken- und Schwächen-Raster eingefügten Merkmale werden bezüglich ihrer Wirkung auf eine oder mehrere strategische Qualitäten eingeordnet. Ziel ist es, Hinweise zu gewinnen, ob es Merkmale gibt, die sich in besonderer Weise auf die strategische Qualität der Netzwerke auswirken. Die Einordnung erfolgt fokussiert auf die offensichtliche strategische Qualität.

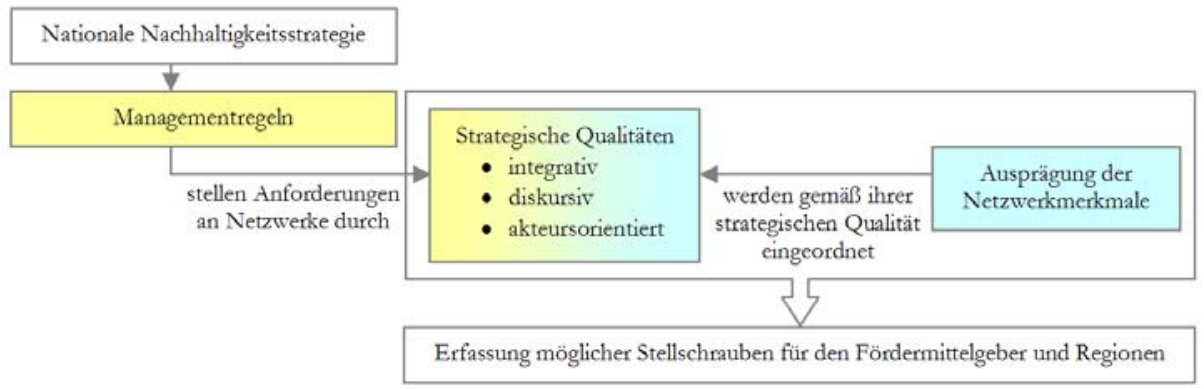

Abbildung 4: Methodische Verknüpfung zwischen Nachbaltigkeit und Netzwerken

Auf der Projektebene werden ergänzend für die betrachteten Projektnetzwerke jeweils Managementregeln der regionalen Nachhaltigkeit aus den durch die Netzwerkerhebungen gewonnenen Daten abgeleitet, die ebenfalls Einfluss auf die Ausprägung der Netzwerkmerkmale und deren strategische Qualität ausüben (siehe Abbildung 4). Die Betrachtung aller Merkmale, Ausprägungen, Qualitäten und Regeln, soll abschließend Auskunft darüber geben, welche Merkmale bei der Ausrichtung der Fördertitel zu berücksichtigen sind, wenn die regionale Nachhaltigkeit gefördert werden soll.

83 Es gibt unterschiedliche Verständnisse von Integration. Im Kontext des Forschungsvorhabens wird Integration daher verstanden als „,die Entstehung oder Herstellung einer Einheit oder Ganzheit aus einzelnen Elemente (!) oder die Fähigkeit einer Einheit oder Ganzheit, den Zusammenhalt der einzelnen Elemente auf der Basis gemeinsam geteilter Werte und Normen aufrechtzuerhalten."Nohlen (2008), S. 229. 



\section{Die Untersuchungsregionen}

\subsection{Die Fördertitel}

Die zur Untersuchung ausgewählten Naturschutzförderprojekte - Naturschutzgroßprojekte und das E+E Vorhaben „Erlebnis Grünes Band“ weisen Gemeinsamkeiten, jedoch auch erhebliche Unterschiede auf. Sie sind im Sinne der politischen Handlungsfähigkeit segmentiert, da sie primär Naturschutzziele verfolgen. Auch wenn im E+E-Vorhaben die Nachhaltigkeitsperspektive eher angelegt ist, da die Naturschutzförderung durch die Förderung touristischer Entwicklung flankiert wird, behält das Vorhaben seine sektorale Ausrichtung. Beide Fördertitel adressieren Regionen als Akteure, die jedoch im Unterschied zu vielen anderen Förderkulissen, nicht formal verfasst agieren. Die Förderung des BMU schafft neue regionale Handlungsräume auf Basis von naturschutz- bzw. landschaftsbezogener Zusammengehörigkeit.

\subsubsection{Erprobungs- und Entwicklungsvorhaben „Erlebnis Grünes Band“}

Der Fördertitel „Erprobungs- und Entwicklungsvorhaben im Bereich Naturschutz und Landschaftspflege" (E+E-Vorhaben) wurde 1987 vom Bundesumweltministerium eingerichtet. Mit diesem Fördertitel sollten Erfolg versprechende Naturschutzideen realisiert werden, wichtige Forschungsergebnisse in die Praxis umgesetzt werden und neue und verbesserte Anwendungen bereits erprobter 
Die Untersuchungsregionen

Methoden vertieft werden. Laut Richtlinie sollen die über diesen Titel geförderten Vorhaben „(...) dazu beitragen, Natur und Landschaft im besiedelten und unbesiedelten Bereich so zu schützen, zu pflegen oder zu entwickeln, dass

a) die Leistungsfähigkeit des Naturhaushalts,

b) die Nutzungsfähigkeit der Naturgüter

c) die Pflanzen- und Tierwelt sowie

d) die Vielfalt, Eigenart und Schönheit von Natur und Landschaft

als Lebensgrundlagen des Menschen und als Voraussetzung für seine Erholung in Natur und Landschaft nachhaltig gesichert werden." ${ }^{\text {"84 }}$

$\mathrm{E}+\mathrm{E}-$ Vorhaben gliedern sich in fünf Förderschwerpunkte ${ }^{85}$ :

1. „Artenvielfalt bewahren“. Unter diesem Schwerpunkt werden Vorhaben zum Schutz und zur Wiedereinbürgerung gefährdeter Pflanzen und Tiere umgesetzt.

2. „Naturschutzgerechte Regionalentwicklung vorantreiben“, dabei stehen vor allem die Umsetzung von Nutzungs- und Pflegekonzepten im Vordergrund.

3. „Wertvolle Lebensräume schützen“. Es sollen Verfahren und Methoden zur Renaturierung von Biotopen erprobt und weiterentwickelt werden.

4. „Gesellschaftliche Akzeptanz für den Naturschutz steigern“. Über verschiedene Ansätze zur Partizipation, Bildung und Information werden potentielle akzeptanzsteigernde Vorhaben durchgeführt werden.

5. „Naturschutz in die Stadtentwicklung integrieren“. Unter dem Stichwort „Ökologische Stadterneuerung“ wird beispielsweise naturschutzorientierte Entwicklung in suburbanen Räumen erprobt.

Das E+E-Vorhaben „Erlebnis Grünes Band“ wird dem Förderschwerpunkt „Gesellschaftliche Akzeptanz für den Naturschutz steigern“ zugeordnet. Dieses E+EVorhaben weist sich im Vergleich zu anderen Vorhaben durch eine Besonderheit aus. In E+E-Vorhaben ist ein Hauptvorhaben mit einer Modellregion üblich. In diesem Vorhaben sind es drei Hauptvorhaben und Modellregionen: ElbeAltmark-Wendland, Harz und Thüringer Wald \& Schiefergebirge/Frankenwald. In einer Voruntersuchung wurde ein naturschutzorientiertes touristisches Leitbild und Marketingkonzept entwickelt, das von März 2007 bis Dezember 2010 in diesen drei Hauptvorhaben erprobt und entwickelt wird. Gegenstand der Untersuchung des Forschungsvorhabens waren die Modellregionen Elbe-AltmarkWendland und Thüringer Wald \& Schiefergebirge/Frankenwald

84 Bundesministerium für Umwelt, Naturschutz und Reaktorsicherheit (1987), o. S.

85 BfN (2009). 


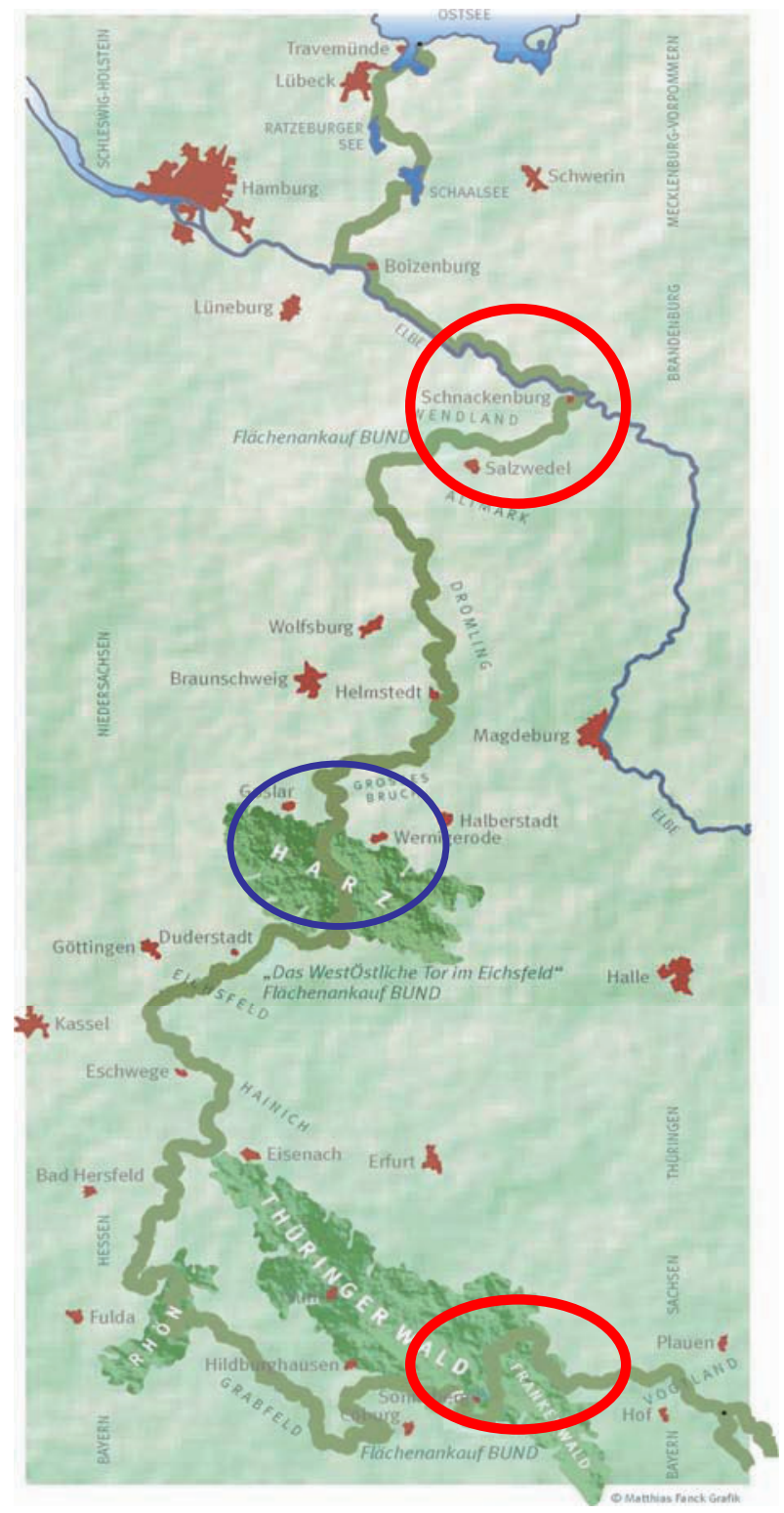

Abbildung 5: Die Lage der Modellregionen des E+E-Vorbabens „Erlebnis Grünes Band“ am Grünen Band. Rot gekennzeichnet sind die beiden Untersuchungsregionen des Forschungsvorbabens (Karte qur Verfügung gestellt vom BUND BN Bayern) 
Die Untersuchungsregionen

In der Vorstudie wurde als Oberziel für das Gesamtvorhaben die „Erhaltung des Grünen Bandes als einzigartiges Biotopverbundsystem nationaler Bedeutung“"86 formuliert. Darunter stehen zwei strategische Hauptziele: Erstens die „Akzeptanz des Grünen Bandes bei der einheimischen Bevölkerung “87, die von den Schlagworten „wirtschaftlicher Nutzen“ und „Teil der Identität“ untersetzt ist. Zweitens die „Unterstützung für das Grüne Band als erhaltenswertes Gut durch die Deutsche Bevölkerung“88, die durch „Bekanntheit bei der Bevölkerung“ und „Erlebbarkeit für Bevölkerung“"89 weiter konkretisiert wird.

Die Strukturen in den Hauptvorhaben sind komplex. Jedes Vorhaben ist länderübergreifend ausgerichtet und bindet aufgrund seiner Zielsetzung Akteure unterschiedlicher Fachbereiche ein. Die Koordination der drei Hauptvorhaben übernimmt die Wissenschaftliche Begleitung. Sie stellt auch den Austausch zwischen den einzelnen Modellregionen her.

Insgesamt verfügt das E+E-Vorhaben über ein Budget von 3.603.000 Euro und läuft von April 2005 (Start der Voruntersuchung) bis Dezember 2010 (Abschluss der wissenschaftlichen Begleitung).

\subsubsection{Naturschutzgroßprojekte}

Der Bund unterstützt die Länder seit 1979 im Rahmen von Naturschutzgroßprojekten bei der Erfüllung nationaler und supranationaler Naturschutzvereinbarungen. Ziele des Förderprogramms sind der „Schutz und die langfristige Sicherung national bedeutsamer und repräsentativer Naturräume mit gesamtstaatlicher Bedeutung“. Da der Bund lediglich über Rahmen gebende Gesetzeskompetenz im Politikbereich Naturschutz verfügt und die Länder für die Festlegung und Ausführung des Naturschutzrechtes zuständig sind, unterliegen die Naturschutzgroßprojekte besonderen Förderkriterien. Es handelt sich um Gebiete, „die im nationalen und internationalen Interesse für den Naturschutz außerordentlich wertvoll und für den betreffenden Lebensraumtyp in Deutschland besonders charakteristisch und repräsentativ sind“ und sich folglich durch „Repräsentanz“, „Großflächigkeit“, „Naturnähe“, „,Gefährdung“" und „,Beispielhaftigkeit“ auszeichnen.

Hauptinstrument des Programms ist die Schaffung von Eigentum zum Zweck des Natur- und Flächenschutzes. Alternativ können weitere Instrumente zur langfristigen Flächensicherung eingesetzt werden bspw. die langfristige Pacht oder Ausgleichszahlungen. Erklärtes Ziel des Förderprogramms ist, die Kerngebiete

86 BN-Bund Naturschutz in Bayern e.V./BUND-Bund für Umwelt und Naturschutz (2006), S. 109.

87 BN-Bund Naturschutz in Bayern e.V./BUND-Bund für Umwelt und Naturschutz (2006), S. 110 .

88 BN-Bund Naturschutz in Bayern e.V./BUND-Bund für Umwelt und Naturschutz (2006), S. 110 .

89 BN-Bund Naturschutz in Bayern e.V./BUND-Bund für Umwelt und Naturschutz (2006), S. 111. 
der Projektflächen bis zum Ende der jeweiligen Projektlaufzeit als Naturschutzgebiete auszuweisen und damit unter rechtlich verbindlichen Schutz zu stellen.

Die Förderung bzw. Finanzierung der Projekte übernehmen der Bund (75\%) und die Länder mit dem jeweiligen Projektträger (25\%) gemeinsam. Die Förderung kann sich über zwölf Jahre erstrecken und Projektträgerschaften übernehmen Landkreise, Städte, Gemeinden, Naturschutzorganisationen oder Zweckverbände.

Seit 1989 wurde das Programm der Naturschutzgroßprojekte um das Gewässerrandstreifenprogramm ergänzt, welches u.a. in der hier betrachteten Region Schaalsee-Landschaft Anwendung fand. Es strebt die Verbesserung der ökologischen Qualität der Fließgewässer durch den Erhalt und die Wiederherstellung ihrer Eigendynamik an.

Das BMU/BfN nimmt seit 2003 eine Optimierung der Förderrichtlinien von Naturschutzgroßprojekten vor. Die wesentlichen Optimierungspunkte ${ }^{90}$ sind:

- Einrichtung einer Moderationsphase über einen Zeitraum von bis zu drei Jahren. Diese ist jedoch in jedem Einzelfall gesondert zu prüfen und zu begründen. Eine projektbezogene Situations- und Interessenanalyse dient als Entscheidungsgrundlage.

- Durchführung einer Evaluation mittels einer Nutzwertanalyse: naturschutzfachliche Wirksamkeit der Maßnahmen, deren Wirtschaftlichkeit und die sozioökonomischen Auswirkungen im Projektgebiet.

- Gezielte sozioökonomische Erhebungen im Rahmen des PEPL, sofern diese in unmittelbarem Zusammenhang mit dem Naturschutzzweck stehen.

- Projektbegleitende Informationsmaßnahmen: Maßnahmen zur transparenten und nachhaltigen Vermittlung der Projektziele und Maßnahmenerfolge sowie zur öffentlichwirksamen Unterstützung der Planung und Durchführung von Naturschutzgroßprojekten.

- Verstärkte Förderung von Anträgen, die im Sinne eines „bottom up“Ansatzes erstellt wurden.

Naturschutzgroßprojekte werden somit seit 2005 zweiphasig mit einer Planungsbzw. Moderationsphase und einer Umsetzungsphase - nach einvernehmlichem Beschluss des Pflege- und Entwicklungsplans durch Projektträger, Land und BfN - durchgeführt.

In der ersten Phase wird ein Pflege- und Entwicklungsplan (PEPL) erarbeitet, der die zur Zielerreichung fachlich notwendigen Maßnahmen bestimmt sowie im Sinne einer Machbarkeitsstudie deren Umsetzungschancen abschätzt. Aufgrund des steigenden Bewusstseins für die Bedeutung der gesellschaftlichen Akzeptanz und den übersektoralen Einklang von regionalen Interessen im Kontext der Großprojekte finden parallel zur ökologischen Bestandsaufnahme auch sozioökonomische Untersuchungen statt, um die Interessen- und Akzeptanzsituation sowie

90 BfN (1993), BfN (2003), BfN (2007). 
ggf. das Konfliktpotenzial zu erfassen und Lösungswege aufzuzeigen. In der zweiten Phase erfolgt die Umsetzung der geplanten Maßnahmen, die begleitend zu evaluieren sind. Fördermittel werden für die Pflege- und Entwicklungsplanung und Moderation und zum Ankauf von Grundstücken sowie den Einsatz alternativer Instrumente, die Durchführung biotopeinrichtender und -lenkender Maßnahmen, projektbegleitende Informationsmaßnahmen und Evaluierungen sowie für Personal- und Sachkosten bereit gestellt. ${ }^{11}$

2008 erfolgte eine Umbenennung des Fördertitels in „,chance.natur - Bundesförderung Naturschutz“".

$91 \quad \operatorname{BfN}(2010)$ 


\subsection{E+E Region Elbe-Altmark-Wendland}

\subsubsection{Situation und Kontext}

Die Modellregion umfasst Gebiete der vier Bundesländer Niedersachsen, SachsenAnhalt, Brandenburg und Mecklenburg-Vorpommern. Daraus folgt ein insgesamt zersplittertes Geflecht von Akteuren und Zuständigkeiten. Zwischen dem ehemaligen Grenzfluss Elbe im Norden, dem Arendsee im Südosten und der Dummeniederung im Südwesten weist die Modellregion unterschiedliche Arten von Naturräumen auf. Bedeutsam sind die Elbe mit ihren Ufern sowie verschiedene Feuchtlebensräume (Reste von Auwäldern, Feuchtgrünland und verschiedene Arten von Kleingewässern). Trockenlebensräume (Binnendünen bzw. Trockenrasen) finden sich am Rande der Niederungsbereiche. Diese charakteristischen Naturräume sollen durch entsprechende Pflege- und Entwicklungsmaßnahmen erhalten bleiben. Die Modellregion ist zu einem großen Teil als Großschutzgebiet ausgewiesen und die Elbregion selbst ist als länderübergreifendes UNESCOBiosphärenreservat anerkannt.

Die landschaftlichen Potenziale in Wert zu setzen ist ein erklärtes Ziel in vielen Bereichen der Modellregion. Dies soll über den Aufbau touristischer Perspektiven gelingen. Eine Bestandsaufnahme und Ableitung von Perspektiven hinsichtlich der touristischen Inwertsetzung erfolgte im Rahmen einer Vorstudie ${ }^{92}$. Dabei wurden insbesondere die Tourismusbereiche Radwandern und Wassertourismus als Perspektiven identifiziert. Im Bereich von Kultur und Geschichte biete die Modellregion eine große Vielfalt von Denkmälern als Zeitzeugen der Geschichte, die touristisch in Wert gesetzt werden könnten.

Im Rahmen der Modellregion werden und wurden unterschiedliche Forschungsprojekte durchgeführt. Zum Beispiel verschiedene EU-Life-NaturProjekte mit der Zielsetzung, exemplarisch den Schutz charakteristischer Arten des Biosphärenreservates in nachhaltige Formen der Landnutzung zu integrieren, oder ein BMBF-Projekt zum Thema „Nachhaltiger Tourismus“.

92 Vgl. BN/BUND et al. (2006). 


\subsubsection{Netzwerkperspektive}

\subsubsection{Strukturelle Analyse des Projektnetzwerks}

\section{Datenbasis}

Ausgehend von den im Antrag benannten Mitgliedern der Steuerungsgruppe und geplanten Kooperationen wurden über zwei Runden insgesamt 64 Akteure befragt, wovon insgesamt 25 Akteure Rückmeldung gaben. Davon wiederum wurden 16 Fragebögen zurückgesendet, die restlichen 9 gaben an, entweder nicht involviert zu sein oder aus anderen Gründen den Fragebögen nicht ausfüllen zu können. Erfasst wurden so 79 Akteure (Grundgesamtheit), für 66 dieser Akteure konnten Beziehungen nachgewiesen werden (Analyseeinheit).

Die im Rücklauf erfassten 16 Akteure verteilen sich im Wesentlichen auf zwei Fachbereiche, den Naturschutz (5 Akteure) und den Tourismus (7 Akteure). Die Bereiche Regionalentwicklung, Kommunalverwaltung, Soziales/Kultur und Wirtschaftsförderung sind mit jeweils einem Akteur vertreten. Vertreter der Bereiche Politik, Wirtschaft und Landnutzung sind im Rücklauf nicht enthalten.

In der Verteilung der Institutionengruppen sind 44\% der Akteure Behördenvertreter, die Vereine und Verbände sind mit 38\% vertreten, und 18\% macht die Gruppe der (Wirtschafts-)Unternehmen aus. Zudem sind auf regionaler Ebene $50 \%$ der Akteure aus den neuen Bundesländern und 44\% der Akteure stammen aus den alten Bundesländern. 6\% der Akteure des Rücklaufs sind auf Landesebene (West) anzusiedeln.

Die aus dem Rücklauf ermittelten akteursbezogenen Daten liefern erste Indizien zu weiteren Merkmalen des Netzwerks. So ist die Verteilung des Alters der Akteure ein Hinweis auf die Integrationsfähigkeit des Netzwerks hinsichtlich der Generationengerechtigkeit. Ähnliches gilt für Aussagen zu den Ausbildungsabschlüssen. Die Dauer der beruflichen Tätigkeit hingegen liefert erste Indizien zur personellen Kontinuität im Projektnetzwerk und im regionalen Netzwerk.

Im Rücklauf ist eine deutliche Tendenz bezüglich Ausbildungsabschluss und Altersverteilung im Netzwerk zu erkennen. 69\% der Akteure besitzen einen Hochschulabschluss, während 25\% der Akteure einen Ausbildungsberuf erlernt haben. Des Weiteren befinden sich jeweils 44\% der Akteure in den Altersgruppen zwischen 40 bis 49 sowie 50 bis 59. Die aus dem Rücklauf ermittelten Daten lassen die Vermutung zu, dass das Projekt folglich vorwiegend Personen mittleren Alters und höheren Bildungsabschlusses anspricht.

Der Großteil der Akteure (44\%) ist seit mehr als zehn Jahren in seiner derzeitigen Position tätig. Mindestens seit einem Jahr sind 25\% tätig, seit mehreren Jahren sind 19\% in ihrer derzeitigen Position. Dies ist ein erster Hinweis dafür, dass der größte Teil des Netzwerks seit vielen Jahren in der Region verankert ist und 
damit eine Einbettung des Projekts in das regionale Netzwerk grundsätzlich gewährleistet werden kann.

Die in der Grundgesamtheit erfassten 79 Akteure verteilen sich im Wesentlichen auf zwei Fachbereiche, den Naturschutz (33 Akteure) und den Tourismus (25 Akteure). Alle anderen Akteursgruppen umfassen nie mehr als 10 Akteure (Politik: 8; Regionalentwicklung: 1, Kommunalverwaltung: 4, Soziales/Kultur: 5, Wirtschaftsförderung: 2, Wirtschaft: 1).

Die Verteilung der Institutionengruppen zeigt ein ausgewogenes Verhältnis von Verbandsvertretern und Vertretern aus Behörden. Allerdings ist insgesamt ein deutlicher Überhang dieser beiden Gruppen festzustellen. Während die Behördenvertreter $35 \%$ und die Verbands- bzw. Vereinsvertreter $38 \%$ der Grundgesamtheit ausmachen, sind Vertreter aus Privatwirtschaft mit $15 \%$ und politische Vertreter mit $11 \%$ vorhanden. Die Ost-West-Verteilung zeigt einen deutlichen Überhang auf Seiten der Vertreter aus den neuen Bundesländern (62\%) im Vergleich zu $37 \%$ an Vertretern aus den alten Bundesländern, 1\% der Akteure ist auf Landesebene (West) anzusiedeln. Der Überhang an Akteuren aus den neuen Bundesländern erklärt sich durch die Verteilung der Landkreise im Projektgebiet. Während auf östlicher Seite des Grünen Bandes vier Landkreise im Projektgebiet liegen, ist es auf westlicher Seite nur einer.

Aus dem Rücklauf und der Grundgesamtheit ergibt sich die Analyseeinheit mit 66 Akteuren (siehe Abbildung 6). Auch sie verteilen sich im Wesentlichen auf die zwei Fachbereiche Naturschutz (27 Akteure) und Tourismus (20 Akteure). Alle anderen Akteursgruppen umfassen nie mehr als 10 Akteure (Politik: 7; Regionalentwicklung: 1, Kommunalverwaltung: 3, Soziales/Kultur: 5, Wirtschaftsförderung: 2 , Wirtschaft: 1$)$. 

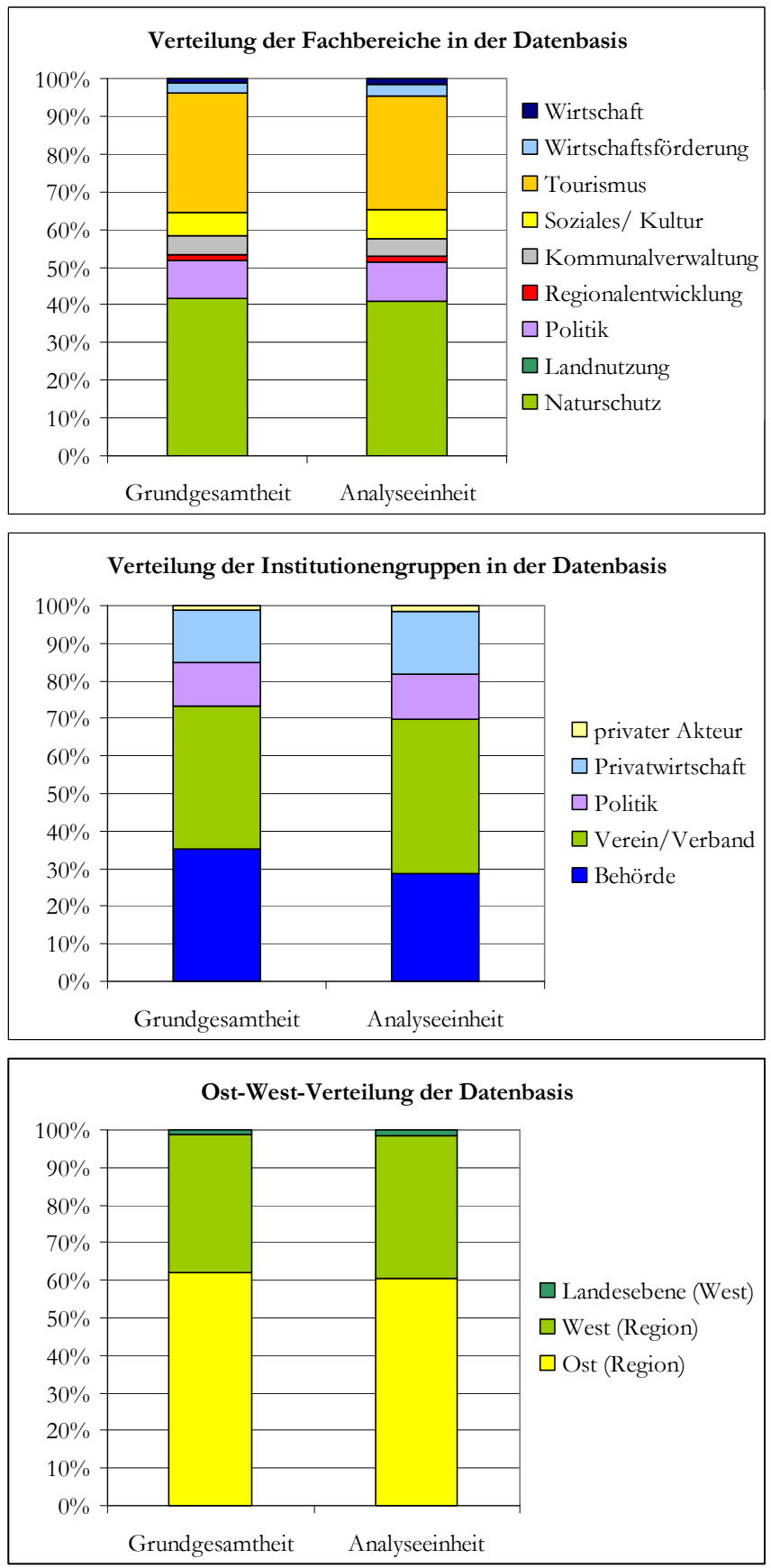

Abbildung 6: Verteilung der Fachbereiche, Institutionengruppen und Ost-West-Verteilung der Akteure der Grundgesamtheit und der Analyseeinheit des Netzwerks Elbe-Altmark-Wendland 
Die Akteure verteilen sich in den Institutionengruppen mit 29\% auf die Gruppe der Behörden, mit 41\% auf die Gruppe der Verbände und Vereine, 18\% sind aus dem unternehmerischen Bereich und 12\% sind politische Vertreter.

In der Ost-West-Verteilung zeigt sich, wie auch in der Grundgesamtheit mit 61\% ein deutlicher Überhang an Akteuren aus den neuen Bundesländern. Regionale Akteure aus den alten Bundesländern sind mit 38\% vertreten und 1\% der Akteure ist auf Landesebene (West) anzusiedeln.

Die Analyseeinheit stellt einen Ausschnitt des betrachteten Netzwerks dar. Alle weiteren Aussagen zum Netzwerk beziehen sich immer auf die Analyseeinheit. Betrachtet man Grundgesamtheit und Analyseeinheit im Vergleich (siehe Abbildung 8), so kann letztere repräsentativ für das gesamte Netzwerk eingeordnet werden.

Die Dominanz an Akteuren in den beiden Bereichen Naturschutz und Tourismus erklärt sich vorwiegend aus der Zielsetzung des Projekts, nämlich der „(...) Bewahrung wertvoller Lebensräume als entscheidendes Erlebnispotential (...)“93, der Erhaltung des Grünen Bandes als „(...) einzigartiger Erholungs- und Erlebnisraum $(\ldots)^{\text {‘94 }}$ sowie der Erzielung einer „,...) regionalen Wertschöpfung $(\ldots)^{\text {‘95 }}$ durch die Nutzung der Potentiale der Modellregion für einen naturnahen Tourismus. Das Vorhandensein unterschiedlicher Akteursgruppen deutet auf die fachbereichsübergreifende Ausrichtung des Projektnetzwerks hin. Lediglich die Gruppe der Landnutzer ist nicht vertreten.

\section{Konstellation}

Im Netzwerk der Untersuchungsregion Elbe-Altmark-Wendland zeigt sich ein relativ eng vernetzter Kern mit zahlreichen und vielfältigen Verbindungen zu anderen Akteuren außerhalb dieses Kerns (siehe Abbildung 7). Den Kern bilden vorwiegend Vertreter des Naturschutzes und Tourismus, aber auch Vertreter aus der Wirtschaftsförderung sind eingebunden. Es zeigt sich außerdem, dass zwar einige Akteure ihre Verbindungen vorwiegend sektoral pflegen, aber durchaus auch einige andere Akteure fachbereichsübergreifend im Netzwerk agieren. Eine Verbindung zwischen dem „Hauptnetzwerk“ und dem „Satelliten“ (siehe Abbildung 7) konnte nicht nachgewiesen werden. Da es sich bei den dargestellten Verbindungen um einen Ausschnitt des Netzwerks handelt, kann allerdings nicht der Rückschluss gezogen werden, dass diese Verbindung nicht existiert.

\footnotetext{
93 Trägerverbund Burg Lenzen e.V. (2006), S. 9.

94 Vgl. ebenda.

95 Vgl. ebenda.
} 


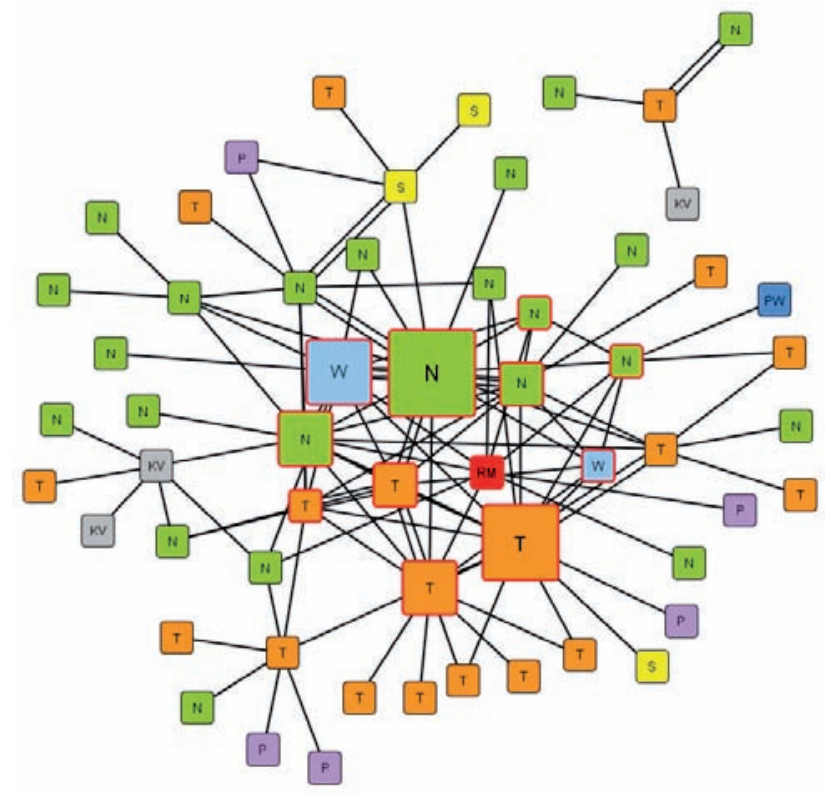

Abbildung 7: Netzwerkgraph des Netzwerks Elbe-Altmark-Wendland. $N=$ Naturschutz, $T=$ Tourismus, $P=$ Politik, $S=$ Soziales/Kultur, $K V=$ Kommunalverwaltung, $P W=$ Privatwirtschaft, $W=$ Wirtschaftsförderung, $\mathrm{RM}=$ Regionalentwicklung

Betrachtet man die Gradzentralität, so hat das höchste $\mathrm{Maß}$ an Zentralität der Projektträger $(0,304)$. Auch für die Nähezentralität und die Intermedationszentralität erweist sich der Projektträger als sehr zentral $(0,493$ bzw. 0,18). Damit zeigt sich, dass der Projektträger das höchste $\mathrm{Maß}$ an Kommunikationsaktivität, effizienz und -kontrolle im Netzwerk besitzt und damit auch am stärksten steuernd eingreifen kann.

Im Weiteren schließen sich an den Projektträger, die Gradzentralität betreffend, ein Vertreter aus dem Tourismus $(0,286)$ und ein Vertreter aus der Wirtschaftsförderung $(0,25)$ an. Die in der Abbildung 7 dargestellten weiteren beiden Positionen mit den Maßen 0,232 bzw. 0,214 werden jeweils von zwei Akteuren (jeweils ein Vertreter des Naturschutzes und ein Vertreter des Tourismus) eingenommen.

Die nähebasierte Zentralität ändert an dieser Reihenfolge nicht viel, lediglich zwischen den Vertreter des Tourismus und den Vertreter der Wirtschaftsförderung schiebt sich ein weiterer Akteur aus dem Tourismusbereich.

Bei der Intermediationszentralität verändert sich die Reihenfolge ab der dritten Position. Hier nimmt ein neuer Akteur aus dem Bereich Tourismus mit einem Wert von 0,131 die dritte Position ein. So besitzen nach dem Projektträger zwei 
Vertreter des Tourismus (mit den Werten 0,131 und 0,129) die größte Möglichkeit zur Kommunikationskontrolle, daran schließen sich ein Vertreter des Naturschutzes $(0,99)$ und ein Vertreter der Wirtschaftsförderung $(0,94)$ an.

Im Netzwerk der Untersuchungsregion waren nach dem Projektträger (indegree von 0,256) besonders zwei Akteure aus dem Bereich Naturschutz sowie ein Vertreter des Bereichs Tourismus am häufigsten um Rat gefragt (indegree von 0,103). Auffällig ist, dass einer der beiden Naturschutz-Akteure bei den Zentralitätsmaßen einen der hinteren Ränge einnahm.

Bei der Bedeutung des Kontakts setzt sich der Projektträger mit einem indegree von 0,255 deutlich gegenüber den anderen Akteuren ab. Die nächsten Akteure schließen mit einem indegree von 0,85 an. Ähnliches gilt für die Frage nach den Schlüsselakteuren. Auch hier setzt sich der Projektträger mit einem indegree von 0,273 deutlich von den anderen Akteuren ab. Den nächsten Akteuren, dem zentralen touristischen Akteur sowie einem Naturschutzvertreter wird ein indegree von 0,114 zugewiesen. An den Maßzahlen für das Prestige lässt sich daher deutlich die herausragende Bedeutung des Projektträgers für das Projektnetzwerk erkennen. Auch deswegen ist besonders er in der Position, das Netzwerk steuern zu können.

\section{Netzperkarbeit}

In der Netzwerkanalyse versteht man unter Strategie eines Netzwerks seine vorwiegende Arbeits- und Funktionsweise. Wie häufig, auf welche Weise und zu welchem Zweck innerhalb des Netzwerks der Kontakt aufgenommen wird, sind hierbei die wesentlichen strukturellen Merkmale. Aufgrund der Besonderheiten der Projektnetzwerke am Grünen Band wurde neben der Wirkung der Projekte auf die Vernetzung auch die länder- und sektorenübergreifende Vernetzung betrachtet.

33\% der Kontakte sind dabei durch das Projekt zustande gekommen, 67\% bestanden bereits vor dem Projekt. 15\% der neuen Kontakte sind länderübergreifend. Die Ost-West-Verteilung des Netzwerkkerns (siehe Abbildung 8) ist recht ausgewogen, so sind 42\% der Akteure aus dem Kern aus den alten Bundesländern, 58\% der Akteure aus den neuen Bundesländern. 


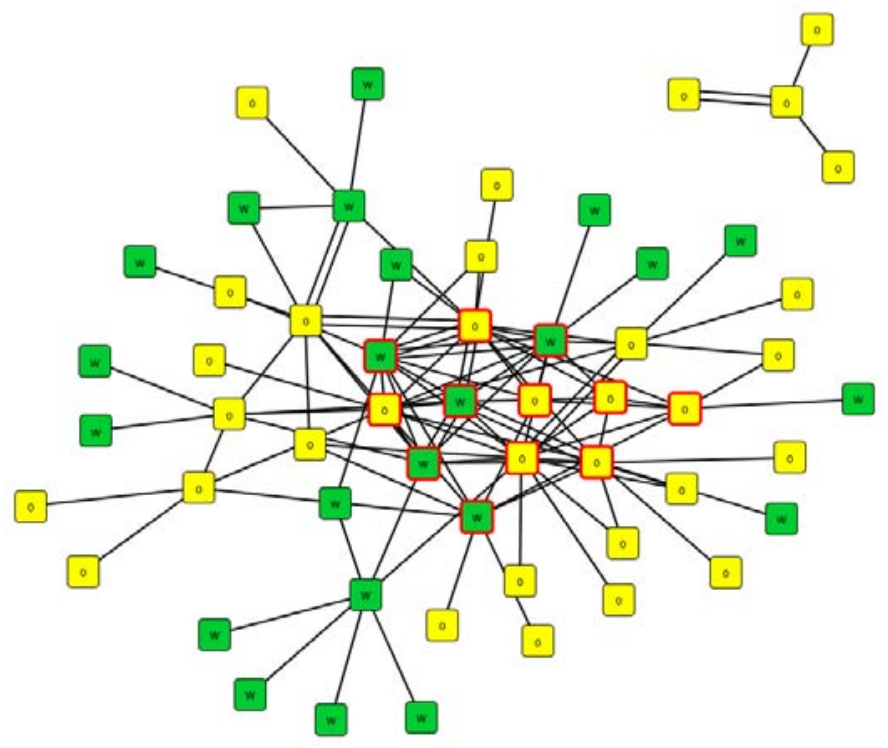

Abbildung 8: Ost-West-Verteilung im Netzwerk Elbe-Altmark-Wendland (grün = „West", gelb = „Ost", rot umrandet ist der Netzwerk.kern)

Zudem sind 30\% der neuen Kontakte sektorenübergreifend zwischen Tourismus und Naturschutz entstanden. Diese neu entstandenen Kontakte bestätigen eine vermutete positive Wirkung des Projekts auf die regionale länderübergreifende sowie die fachbereichsübergreifende Vernetzung.

Bei den Kontaktarten wurde unterschieden zwischen „Kontakt per Telefon oder Mail“, „Kontakt bei einer Arbeitsbesprechung“ im Rahmen des Projekts, zum Beispiel zur Planung und Gestaltung einer Maßnahme und „Kontakt während einer Projektveranstaltung“", sei es ein maßnahmenübergreifender Workshop oder ein Fest. Auffallend bei der Wahl der Kontaktarten ist vor allem die bedeutende Rolle der sogenannten „face-to-face“-Kommunikation in Form von Arbeitsbesprechungen (27\%) oder Projektveranstaltungen (39\%), die insgesamt 66\% ausmachen. Hieran wird die Bedeutung dieser Form der Kommunikation für die Gestaltung der Netzwerkarbeit deutlich.

Wesentlicher Zweck der Kontaktaufnahme war der Austausch von Informationen und die Gestaltung von Kooperationen. Der Austausch von Ressourcen spielt eine untergeordnete Rolle. In Förderprojekten wie dem E+E-Vorhaben ist der Austausch von Ressourcen, wie Geld oder Arbeitskraft nicht zu erwarten, da die finanzielle Abwicklung des Projekts hauptsächlich in der Verantwortung des Projektträgers liegt und die Fördermittel daher nicht an die weiteren Akteure wei- 
tergegeben werden. Der Zweck deutet außerdem darauf hin, dass die Akteure bei der Planung und Umsetzung der Maßnahmen maßgeblich eingebunden waren.

Zwischen der Mehrheit der Akteure bestehen lose Kontakte, so werden 46\% der Kontakte selten genutzt. 20\% der Akteure werden gelegentlich kontaktiert. Häufig werden von 35\% der Kontaktnehmer 27\% der Akteure kontaktiert.

Diese Verteilung verdeutlicht noch mal die Darstellung des Netzwerks (siehe Abbildung 9). Ein Teil der Akteure pflegt regelmäßigen Kontakt zueinander, während der Großteil der Akteure vergleichsweise lose in das Netzwerk eingebunden ist.

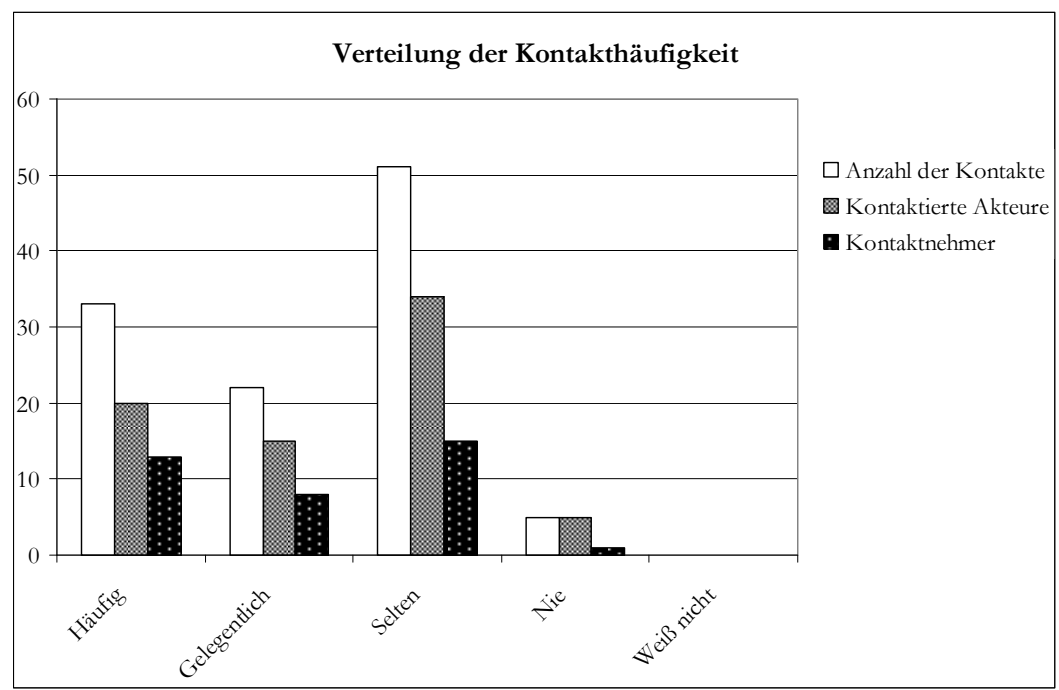

Abbildung 9: Verteilung der Kontakthäufigkeiten im Netzwerk. Elbe-Altmark-Wendland

12,5\% der Akteure mit positiver Rückmeldung fühlen sich sehr gut und 37,5\% fühlen sich gut in das Netzwerk eingebunden und sind mit ihrer jetzigen Position innerhalb des Netzwerks zufrieden. 31,25\% der Akteure sind mit ihrer Einbindung zufrieden. 12,5\% beurteilen die Einbindung als ausreichend und 6,25\% als mangelhaft. Besonders die Vertreter des Fachbereichs Naturschutz fühlen sich gut in das Netzwerk integriert. Für den Fachbereich Tourismus reicht die Spannweite der Zufriedenheit von „sehr gut“ bis „mangelhaft“. Dies mag sich aus der Vernetzung der einzelnen Akteure erklären. Es ist anzunehmen, dass sich besonders jene Akteure, die sich nicht im Kern des Netzwerks befinden, als nicht ausreichend integriert betrachten, was wiederum die Zufriedenheit mit dem Prozess schmälert. Besonders organisatorische Probleme, wie beispielsweise unzureichender Informationsfluss, bereiten den Akteuren Schwierigkeiten. 


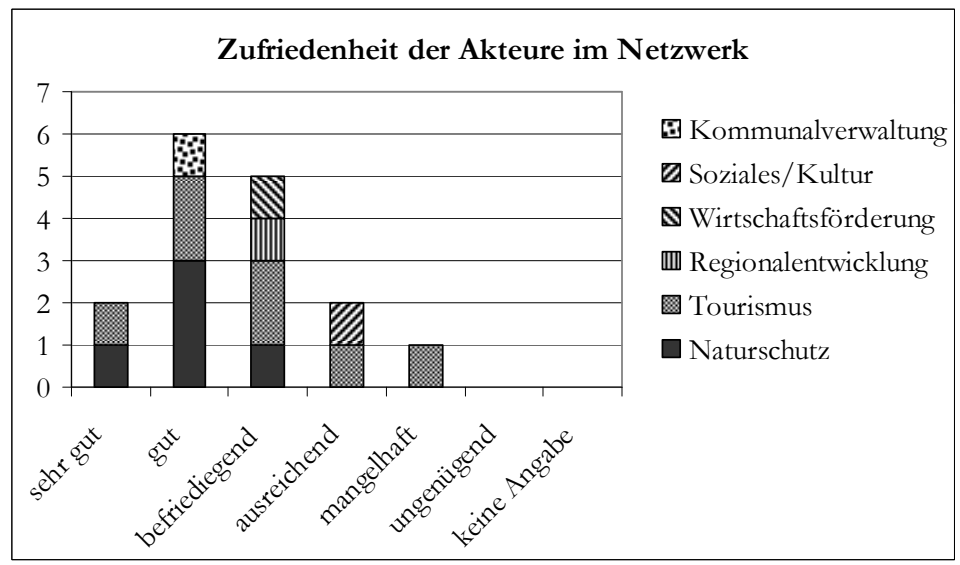

Abbildung 10: Zufriedenheit der Akteure mit der Netzwerkarbeit in der Untersuchungsregion im Netzwerk Elbe-Altmark-Wendland

\subsubsection{Analyse der Netzwerkmerkmale und ihrer strategischen Qualitäten}

Die Analyse der Netzwerkmerkmale und ihrer strategischen Qualitäten beruht auf der strukturellen Netzwerkanalyse, der Auswertung der geführten Interviews sowie der durchgeführten regionalen Workshops. Mit Hilfe eines StärkenSchwächenprofils werden die Netzwerkmerkmale und die Ausprägungen ihrer strategischen Qualitäten charakterisiert. Jedoch konnte nicht zwingend für jedes Kriterium ein Hinweis auf seine Ausprägung nachgewiesen werden, beispielsweise wenn das Kriterium nach Aussage der Akteure ausschließlich positiv ausgeprägt ist.

\section{Konstellation: „Breite Beteiligung“}

Hinsichtlich einer breiten Beteiligung im Rahmen des E+E-Vorhabens „Erlebnis Grünes Band“ muss einschränkend vorweg geschickt werden, dass das Projekt räumlich und vor allem thematisch begrenzt ist. Eine breite Beteiligung, wie es vergleichsweise in Leader-Prozessen möglich ist, ist daher gar nicht möglich. Gründe hierfür sind die inhaltliche Ausrichtung des Projekts auf das Grüne Band und der damit einhergehenden thematischen Begrenzung auf geschichtliche und naturschutzfachliche Themen.

Wie bereits in der strukturellen Erhebung festgestellt, hat sich im Projektnetzwerk ein verhältnismäßig stabiler Kern an Beteiligten manifestiert. Diese Akteure gehören überwiegend der fachbereichsübergreifenden Steuerungsgruppe des Projekts an. Die darüber hinaus beteiligten Akteure werden von dieser Steuerungsgruppe und dem Projektträger punktuell je nach Bedarf eingebunden, auch um die 
Belastung dieser Akteure möglichst gering zu halten. Die Aufgabe der breiten Beteiligung wird somit nicht ausschließlich von einem zentralen Akteur wahrgenommen, sondern verteilt sich auf verschiedene Positionen innerhalb des Netzwerks. Das kann sicherstellen, dass diese beteiligten Akteure auch interessiert und motiviert sind, am Projekt mitzuarbeiten. Neben dem Versuch über persönliche Kontakte Akteure zu gewinnen, wird versucht, durch Information und Pressearbeit weitere Akteure zu erreichen.

Das Interesse und die Motivation der bereits eingebundenen Akteure, was sich nicht nur in der Pressearbeit sondern auch in dem Versuch niederschlägt, weitere Akteure zu beteiligen, unterstützt maßgeblich die Akteursorientierung des Netzwerks.

Allerdings gelingt die Einbindung neuer Akteure nur zäh, was nicht zuletzt auf das fehlende Unternehmernetzwerk zurückzuführen ist. Ein solches Netzwerk wäre aufgrund der fachlichen und inhaltlichen Ausrichtung des Projekts wichtig, um die touristischen Produkte des E+E-Vorhabens zu etablieren. Jedoch scheint es nach Aussagen der Akteure bislang nicht zu gelingen, verschiedene touristische Leistungsträger einzubinden, um diese Lücke zu schließen. Der Grund hierfür mag zum einen in der zeitlichen Belastung des Leistungsträgers liegen. Viele der Hotels und Gaststätten werden als Familienbetrieb geführt, was grundsätzlich mit einer hohen zeitlichen Belastung der Akteure einhergeht. Projekte, wie das E+EVorhaben wecken daher meist nur dann Interesse, wenn ein direkter Nutzen erkennbar ist und der Aufwand der Beteiligung nicht zu hoch wird.

„Eine gewisse Eigenträgheit von vielen Leuten ist (...) da, (...) wenn ich jetz̨t an Unternehmen denke, die (...) von Servicekraft bis Koch bis Steuerberater bis Versicherungsvertreter alles in einem Haus [haben] und (...) kleine geführte Familienbetriebe sind. Alles sollen sie beherrschen irgendwo und jetzt kommt noch solche Entwicklungsprojektarbeit dazu. Da steht denen das alles über dem Kopf. Das ist denen einfach oft mal eine Nummer zu viel, die müssen eher intensiver betreut werden, an die Hand genommen werden, ihnen viel mehr erklärt werden. Persönliche individuelle Betreunng ist da erforderlich. Und das kann man natürlich in dem Maße teilweise gar nicht leisten." (Zitat aus einem Interview, Fachbereich Tourismus)

Darüber hinaus kann nach Bewilligung des Förderantrags eine breitere Beteiligung von weiteren Akteuren in der Regel nur kostenneutral realisiert werden. Die Möglichkeit neue Ideen und Maßnahmen in das Projekt einzubringen sind stark begrenzt. Dies ruft bei vielen Akteuren den Eindruck von „der Sack ist zu“ (Zitat aus einem Interview, Fachbereich Regionalentwicklung) hervor und verringert weiter die Motivation sich zu beteiligen. Diese Aspekte stehen ebenso wie das noch unzureichend ausgebaute Unternehmensnetzwerk sowie die fehlenden Vertreter der Landnutzer (Land- bzw. Forstwirtschaft), einer Akteursorientierung entgegen. Eine Verbindlichkeit der Beteiligung kann nach Einschätzung der interviewten Akteure nur durch weitere Geldmittel gelingen. 
Tabelle 5: Konstellation: Breite Beteiligung in der Region Elbe-Altmark-Wendland ( $D=$ diskursiv, $A$ = akteursorientiert, $I=$ integrativ;)

\begin{tabular}{|c|c|c|c|c|}
\hline Qualitätskriterien & Stärke & D & A & I \\
\hline \multirow[b]{2}{*}{$\begin{array}{l}\text { Projektträger und in Projektstruktu- } \\
\text { ren/ Gremien integrierte Personen. }\end{array}$} & $\begin{array}{l}\text { Die eingebundenen Akteure sind interes- } \\
\text { siert und motiviert }\end{array}$ & & $\mathrm{x}$ & \\
\hline & $\begin{array}{l}\text { Versuch, durch Information weitere Akteu- } \\
\text { re zu gewinnen. Ideen, um weitere Akteure } \\
\text { zu gewinnen vorhanden }\end{array}$ & & $\mathrm{x}$ & \\
\hline $\begin{array}{l}\text { Maßnahmenbezogene Partner des } \\
\text { Projekts repräsentieren relevante } \\
\text { Sektoren. }\end{array}$ & $\begin{array}{l}\text { Viele verschiedene Akteure versuchen } \\
\text { andere zu beteiligen }\end{array}$ & & $\mathrm{x}$ & \\
\hline Qualitätskriterien & Schwäche & $\mathbf{D}$ & $\mathbf{A}$ & I \\
\hline \multirow{3}{*}{$\begin{array}{l}\text { Projektträger und in Projektstruktu- } \\
\text { ren/ Gremien integrierte Personen. }\end{array}$} & $\begin{array}{l}\text { Die Motivation weiterer Akteure gelingt } \\
\text { nur sehr zäh }\end{array}$ & & $\mathrm{x}$ & \\
\hline & $\begin{array}{l}\text { „Der Sack ist zu“. Eine Einbindung weite- } \\
\text { rer Akteure kann bisher nur kostenneutral } \\
\text { geschehen }\end{array}$ & & $\mathrm{x}$ & \\
\hline & $\begin{array}{l}\text { Verbindlichkeit der Beteiligung nur durch } \\
\text { zusätzliche Geldmittel möglich }\end{array}$ & & $\mathrm{x}$ & \\
\hline \multirow{2}{*}{$\begin{array}{l}\text { Maßnahmenbezogene Partner des } \\
\text { Projekts repräsentieren relevante } \\
\text { Sektoren. }\end{array}$} & $\begin{array}{l}\text { Vertreter privater Unternehmen nicht } \\
\text { ausreichend eingebunden }\end{array}$ & & $\mathrm{x}$ & \\
\hline & Landnutzer fehlen. & & $\mathrm{x}$ & \\
\hline
\end{tabular}

Konstellation: „Multiplikeatoren“

Neben dem über die strukturelle Erhebung identifizierten zentralen Naturschutzvertreter gibt es eine Reihe weiterer Akteure von wesentlicher Bedeutung für das Netzwerk, die sich in der Steuerungsgruppe befinden. Dadurch wird sichergestellt, dass im zentralen Gremium des Netzwerks auch fachliche und regionale Experten vertreten sind, die im Projekt und der Region als Multiplikatoren fungieren. Dem Projektträger wird neben seiner eigenen Multiplikatorfunktion die wichtige Aufgabe eines „Dolmetschers“, also eines Vermittlers zwischen den vier beteiligten Bundesländern und den verschiedenen Fachbereichen zugewiesen. Die Akteure sehen hier die zentrale und bedeutende Aufgabe des Projektträgers, die sich auch in Moderationstätigkeiten desselben und den nachgewiesenen Zentralitätsmaßen widerspiegelt. Dies alles zusammengenommen macht ihn damit zu einem „Motor an der Spitze" des Netzwerks. Zudem kann diesem Kriterium keine Schwäche oder negative Ausprägung zugeordnet werden, was zusätzlich auf eine positive Wahrnehmung des Projektträgers und seiner Aufgaben durch die Akteure hindeutet. 
Die Besetzung der Steuerungsgruppe mit zentralen Akteuren, die als regionale Experten bezeichnet werden können erfüllt eine wichtige Funktion der Akteursorientierung. Die Multiplikatorenfunktion des Projektträgers ist als integrativ einzuordnen, da er als Vermittler unterschiedliche Interessen einbindet.

Ebenfalls relevant sind die politischen Promotoren als Multiplikatoren. Die Befragten bestätigen dem Projekt zwar eine grundsätzliche politische Verankerung, jedoch ist der politische Rückhalt des Projekts in jedem Bundesland unterschiedlich stark. Politische Partner wurden im Laufe des Projekts zwar gewonnen, jedoch wird die politische Bedeutung des Projekts in der Region eher als gering eingestuft. Mögliche Potentiale zur Integration unterschiedlicher Politikfelder können dadurch nicht ausgeschöpft werden.

Tabelle 6: Konstellation: Multiplikatoren in der Region Elbe-Altmark-Wendland $(D=\operatorname{diskursiv,~} A=$ akteursorientiert, $I=$ integrativ)

\begin{tabular}{|l|l|l|l|l|}
\hline Qualitätskriterien & Stärke & D & A & I \\
\hline \multirow{2}{*}{ Motor an der Spitze. } & Projektträger fungiert als „Dolmetscher“. & & $\mathrm{x}$ \\
\cline { 2 - 5 } & $\begin{array}{l}\text { Dem Projektträger kommt innerhalb des Netz- } \\
\text { werks das meiste Prestige zu. }\end{array}$ & & $\mathrm{x}$ \\
\hline \multirow{2}{*}{$\begin{array}{l}\text { Integration regionaler Meinungs- } \\
\text { führer und anerkannter Fachex- } \\
\text { perten aus den relevanten Sekto- } \\
\text { ren. }\end{array}$} & $\begin{array}{l}\text { Regionale Experten sind in der Steuerungsgrup- } \\
\text { pe vertreten. }\end{array}$ & $\begin{array}{l}\text { Politische Verankerung des Projekts ist grund- } \\
\text { sätzlich gegeben. }\end{array}$ & $\mathrm{x}$ & \\
\cline { 2 - 5 } & $\begin{array}{l}\text { Neben dem Projektträger erweisen sich Vertre- } \\
\text { ter vorwiegend aus dem Naturschutz, aber auch } \\
\text { des Tourismus als zentral. }\end{array}$ & $\mathrm{x}$ & \\
\hline Qualitätskriterien & Schwäche & D & A & I \\
\hline Motor an der Spitze. & - & & \\
\hline $\begin{array}{l}\text { Integration regionaler Meinungs- } \\
\text { führer und anerkannter Fachex- } \\
\text { perten aus den relevanten Sekto- } \\
\text { ren. }\end{array}$ & $\begin{array}{l}\text { Keine starke politische Bedeutung des Projekts. } \\
\text { Zürckhaltung der politischen Meinungsführer. }\end{array}$ & & $\mathrm{x}$ \\
\hline
\end{tabular}

\section{Konstellation: „Personelle Kontinuität“}

Dieses Netzwerkmerkmal wurde von keinem Akteur, weder in den Interviews, noch auf dem Workshop thematisiert. Dies könnte ein Indiz dafür sein, dass dieses Merkmal im Wesentlichen positiv ausgeprägt ist und aktuell kein Problemdruck zu bestehen scheint.

Der Projektträger ist seit vielen Jahren in der Region tätig und daher den Akteuren bereits seit langem bekannt. Diese Kontinuität schafft Vertrauen und damit 
die Grundlage für eine strukturierte Netzwerkarbeit, die sich positiv auf die Diskursivität des bestehenden Netzwerks auswirkt.

Die Struktur des Projekts bietet zudem durch Workshops touristischen Leistungsträgern die Möglichkeit zur Teilnahme am Projekt. Allerdings wechselt die Besetzung der Workshops stark, so dass durch viele, mehrfach geführte Diskussionen auch die Motivation der Akteure teilzunehmen abnimmt. Dies kann wiederum die strategische Qualität „diskursiv“ des Netzwerks behindern

Tabelle 7: Konstellation: Personelle Kontinuität in der Region Elbe-Altmark-Wendland $(D=$ diskursiv, $A=$ akteursorientiert, $I=$ integrativ)

\begin{tabular}{|l|l|l|l|l|}
\hline Qualitätskriterien & Stärke & D & A & I \\
\hline $\begin{array}{l}\text { Kontinuierliches Engagement der } \\
\text { Multiplikatoren im Netzwerk/ für } \\
\text { das Projekt }\end{array}$ & - & & & \\
\hline $\begin{array}{l}\text { Kontinuierliche Betreuung des } \\
\text { Prozessmanagement/ Netzwerkar- } \\
\text { beit durch geeignete Person. }\end{array}$ & $\begin{array}{l}\text { Projektträger fungiert als Koordinator, Mo- } \\
\text { derator und Organisator. }\end{array}$ & x & & \\
\hline Qualitätskriterien & Schwäche & D & A & I \\
\hline $\begin{array}{l}\text { Kontinuierliches Engagement der } \\
\text { Multiplikatoren im Netzwerk/ für } \\
\text { das Projekt }\end{array}$ & $\begin{array}{l}\text { Hohe Fluktuation im touristischen Bereich } \\
\text { verringert personelle Kontinuität (betrifft die } \\
\text { allgemeinen Leistungsträger, nicht die Multi- } \\
\text { plikatoren). }\end{array}$ & x & & \\
\hline $\begin{array}{l}\text { Kontinuierliche Betreuung des } \\
\text { Prozessmanagement/ Netzwerkar- } \\
\text { beit durch geeignete Person. }\end{array}$ & - & & & \\
\hline
\end{tabular}

\section{Strategie: Lernen}

Innerhalb des Netzwerks findet der Austausch hauptsächlich maßnahmenbezogen statt. Dies hat dazu geführt, dass der fachliche Austausch zwischen den Akteuren verstärkt wurde und so zu neuen Erkenntnissen über den jeweils anderen Fachbereich geführt hat. Dazu tragen die durchgeführten Produktworkshops bei, in denen die regionalen Akteure für die Entwicklung von touristischen Pauschalen fachlich unterstützt wurden. Dieser Unterstützung messen die Akteure eine hohe Bedeutung bei. Es fehlt allerdings ein Gesamtaustausch über alle einzelnen Projektbausteine hinweg, bzw. dieser ist nach Einschätzung einiger Akteure nicht ausreichend ausgeprägt. Es sind zwar Steuerungsgruppentreffen des Gesamtvorhabens vorgesehen und werden auch durchgeführt. Inwiefern die dabei ausgetauschten Informationen das Defizit beseitigen, konnte zum Zeitpunkt der Erhebung nicht geklärt werden.

Diese Workshops und Besprechungen dienen dem Austausch der Projektbeteiligten und damit den für die Nachhaltigkeit notwendigen Verhandlungen. Die bereits erwähnten $30 \%$ an häufigen Kontakten weisen auf einen regelmäßigen Austausch hin und sind einer Verhandlung oder Diskussion im Sinne der Nach- 
haltigkeit zuträglich. Die 46\% an seltenen Kontakten sind jedoch ein Indiz dafür, dass der Austausch zwischen den Akteuren jedoch nur in sehr großen, bzw. unregelmäßigen Abständen stattfindet. Dies kann zur Folge haben, dass notwendige Verhandlungen für ein gemeinsames Verständnis von Nachhaltigkeit nicht ausreichend intensiv geführt werden. Zudem wurde zum Zeitpunkt der Erhebung der Austausch zwischen den Modellregionen des E+E-Vorhabens durch die Befragten als zu gering bewertet. Beides ist der strategischen Qualität „diskursiv“ eher abträglich.

Ein weiteres zentrales Problem der grundsätzlich als positiv eingestuften Offenheit des Netzwerks ist die hohe Fluktuation der Teilnehmer in den einzelnen Projektworkshops. Dies verlangt, dass alle Akteure dort abgeholt werden, wo sie gerade in Sachen Informationen stehen. Dadurch muss Workshopzeit darauf verwandt werden, alle Akteure auf den jeweils gleichen Stand zu bringen. Dies führt bei Akteuren, die kontinuierlich diese Workshops besuchen dazu, dass die ,gefühlte“ Effizienz solcher Termine sich verringert, was zu Unzufriedenheiten auch innerhalb des Netzwerks führt. Dies wiederum verringert die diskursive Qualität des Netzwerks und die Motivation der sich regelmäßig beteiligenden Akteure, an den Workshops teilzunehmen.

Tabelle 8: Strategie: Lernen in der Region Elbe-Altmark-Wendland ( $D=$ diskursiv, $A=$ akteursorientiert, $I=$ integrativ)

\begin{tabular}{|c|c|c|c|c|}
\hline Qualitätskriterien & Stärke & D & $\mathbf{A}$ & $\mathbf{I}$ \\
\hline \multirow{3}{*}{$\begin{array}{l}\text { Direkter und regelmäßiger - sekto- } \\
\text { renübergreifender - Austausch zwi- } \\
\text { schen den Akteuren }\end{array}$} & $\begin{array}{l}\text { Maßnahmenbezogener Austausch im Pro- } \\
\text { jekt für Information und Kooperation. }\end{array}$ & $\mathrm{x}$ & & \\
\hline & $30 \%$ der erfassten Kontakte erfolgen häufig. & $\mathrm{x}$ & & \\
\hline & $\begin{array}{l}\text { Austausch vorwiegend in Projektveranstal- } \\
\text { tungen. }\end{array}$ & $\mathrm{x}$ & & \\
\hline $\begin{array}{l}\text { Einsatz reflexiver Instrumente (Eva- } \\
\text { luierungsmaßnahmen, Reflexions- } \\
\text { workshops, Monitoring etc.) }\end{array}$ & - & & & \\
\hline $\begin{array}{l}\text { Erfahrungsaustausch mit Netzwer- } \\
\text { ken ähnlicher inhaltlicher Ausrich- } \\
\text { tung }\end{array}$ & - & & & \\
\hline Qualitätskriterien & Stärke & D & $\mathbf{A}$ & I \\
\hline \multirow{2}{*}{$\begin{array}{l}\text { Direkter und regelmäßiger - sekto- } \\
\text { renübergreifender - Austausch zwi- } \\
\text { schen den Akteuren }\end{array}$} & $\begin{array}{l}46 \% \text { der erfassten Kontakte erfolgen nur } \\
\text { selten. }\end{array}$ & $\mathrm{x}$ & & $\mathrm{x}$ \\
\hline & $\begin{array}{l}\text { Hohe Fluktuation in den Workshops ver- } \\
\text { ringert ,gefühlte“ Effizienz. }\end{array}$ & $\mathrm{x}$ & & \\
\hline $\begin{array}{l}\text { Einsatz reflexiver Instrumente (Eva- } \\
\text { luierungsmaßnahmen, Reflexions- } \\
\text { workshops, Monitoring etc.) }\end{array}$ & $\begin{array}{l}\text { Kein Einsatz von Instrumenten zur Refle- } \\
\text { xion der Netzwerkarbeit. }\end{array}$ & $\mathrm{x}$ & & $\mathrm{x}$ \\
\hline
\end{tabular}


Die Untersuchungsregionen

\begin{tabular}{|l|l|l|l|l|}
\hline $\begin{array}{l}\text { Erfahrungsaustausch mit Netzwer- } \\
\text { ken ähnlicher inhaltlicher Ausrich- } \\
\text { tung }\end{array}$ & $\begin{array}{l}\text { Modellregionenübergreifend findet kein } \\
\text { ausreichender Austausch statt (zum Zeit- } \\
\text { punkt der Erhebung). }\end{array}$ & $\mathrm{x}$ & & \\
\hline
\end{tabular}

\section{Strategie: Arbeiten auf verschiedenen Ebenen}

Projektintern gliedert sich die Organisationsstruktur im Wesentlichen in drei Teile: der Projektträger, die fachübergreifend besetzte Steuerungsgruppe und die weiteren Akteure, die fallbezogen eingebunden werden. Der Steuerungsgruppe kommt als Bindeglied zwischen dem Projektträger und den weiteren Akteuren eine zentrale Bedeutung zu. Hier wird im Wesentlichen ein gemeinsames Verständnis für bestimmte Themen innerhalb des Netzwerks erarbeitet und in das weitere Projektnetzwerk transportiert. Sie dient somit auch der Diskussion und Entscheidungsfindung. Die Arbeit in der Steuerungsgruppe wird als vergleichsweise konfliktarm beschrieben. Aufgrund ihrer verbindenden Funktion und ihrer Funktion als Diskussionsplattform ist die Steuerungsgruppe als „diskursiv“ und ,akteursorientiert" einzuordnen.

Neben der Steuerungsgruppe kommt dem Projektträger ebenfalls eine wesentliche Bedeutung in der Organisationsstruktur zu. Hier wird die Bedeutung vor allem in der Koordination, Moderation und Organisation, also einer gewissen „Rahmenarbeit“ zwischen allen beteiligten Projektpartnern gesehen.

Um die Arbeitsweise des Projektnetzwerks möglichst effizient zu halten, werden weitere Akteure nur fallbezogen in das Projekt eingebunden. Außerdem soll dadurch vermieden werden, mögliche Belastungen dieser Akteure noch weiter zu erhöhen und die Projektstruktur zudem auch schlanker und damit leistungsfähiger zu halten:

„Und wir sind dann eben so, dass wir die [Akteure, die dann relevant sind für Landwirtschaft] dann eher fallberogen einbezieben wenn wir Naturschutzmaßnabmen machen, (...), dass man dann eben solche Abstimmungstermine macht, aber die eben nicht über 3 Jahre in irgendwelchen Gremien mitziebt." (Zitat aus einem Interview, Fachbereich Naturschutz)

Allerdings kann dies zu einer Überbelastung bereits stark eingebundener Akteure, zum Beispiel dem Projektträger, führen, wenn Möglichkeiten der Arbeitsteilung nicht ausreichend genutzt werden. 
Tabelle 9: Strategie: Arbeiten auf verschiedenen Ebenen in der Region Elbe-Altmark-Wendland ( $)$ diskursiv, $A=$ akteursorientiert, $I=$ integrativ)

\begin{tabular}{|c|c|c|c|c|}
\hline Qualitätskriterien & Stärke & $\mathbf{D}$ & $\mathbf{A}$ & $\mathbf{I}$ \\
\hline $\begin{array}{l}\text { Vorhandensein projekt- bzw. regionsspe- } \\
\text { zifischer Organisationsstrukturen im } \\
\text { Netzwerk, mit denen eine Aufgabentei- } \\
\text { lung (Steuerung, Entscheidung, Umset- } \\
\text { zung, Diskussion, Information) vorge- } \\
\text { nommen wird. }\end{array}$ & $\begin{array}{l}\text { Steuerungsgruppe agiert als Bindeglied } \\
\text { zwischen Projektträger und weiteren } \\
\text { Akteuren. }\end{array}$ & $\mathrm{x}$ & $\mathrm{x}$ & \\
\hline Qualitätskriterien & Schwäche & $\mathbf{D}$ & $\mathbf{A}$ & I \\
\hline $\begin{array}{l}\text { Vorhandensein projekt- bzw. regionsspe- } \\
\text { zifischer Organisationsstrukturen im } \\
\text { Netzwerk, mit denen eine Aufgabentei- } \\
\text { lung (Steuerung, Entscheidung, Umset- } \\
\text { zung, Diskussion, Information) vorge- } \\
\text { nommen wird. }\end{array}$ & 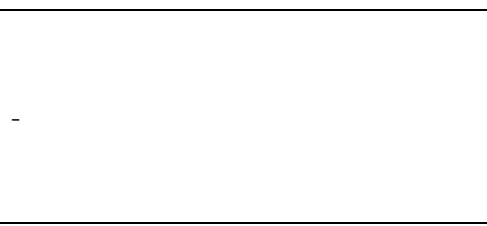 & & & \\
\hline
\end{tabular}

\section{Strategie: Kooperation und win-win}

Innerhalb des Projekts sticht die Maßnahme „Radrundkurs“ besonders hervor. Sie zeichnet sich dadurch aus, dass es hierbei dem Netzwerk gelungen ist, über Verhandlungen unterschiedliche Interessen einzubinden und in der Maßnahmenumsetzung entsprechend zu berücksichtigen, wodurch win-win-Situationen erzielt werden konnten. Die Ausprägung ist daher beispielhaft als „diskursiv“ einzuordnen, da das Netzwerk hier die länder- und sektorenübergreifenden notwendigen Verhandlungen geführt hat.

Tabelle 10: Strategie: Kooperation und win-win-Lösungen in der Region Elbe-Altmark-Wendland ( $D=$ diskursiv, $A=$ akteursorientiert, $I=$ integrativ)

\begin{tabular}{|l|l|c|c|c|}
\hline Qualitätskriterien & Stärke & D & A & I \\
\hline $\begin{array}{l}\text { Projekt-/ Maßnahmen, aus denen min- } \\
\text { destens zwei Interessengruppen einen } \\
\text { gemeinsamen Gewinn ziehen. }\end{array}$ & $\begin{array}{l}\text { Länder- und sektorenübergreifend erar- } \\
\text { beiteter Radrundkurs (Maßnahme) }\end{array}$ & $\mathrm{x}$ & & \\
\hline Qualitätskriterien & Schwäche & D & A & I \\
\hline $\begin{array}{l}\text { Projekt-/ Maßnahmen, aus denen min- } \\
\text { destens zwei Interessengruppen einen } \\
\text { gemeinsamen Gewinn ziehen. }\end{array}$ & - & & & \\
\hline
\end{tabular}

\section{Strategie: Probleme und Lösungswille}

Zum einen existiert im Projektnetzwerk mit der Steuerungsgruppe ein Gremium, das zur Verhandlung und zur Kompromissfindung dient. Kompromisse werden vereinbart, Konfliktpunkte umgangen bzw. gelöst. Zum Beispiel wurden bei der Konzeption des Radrundkurses empfindliche Biotope besonders berücksichtigt 
und ihre Störung vermieden. Das Aushandeln solcher Kompromisse unterstützt die diskursive Qualität des Netzwerks.

Allerdings kritisieren einige Akteure, dass einzelne Vorschläge und Ideen nicht ausreichend beachtet werden und somit auch nicht in einem angemessenen Maß diskutiert werden. Dadurch entsteht ein gewisser Eindruck des „Nicht-MiteinanderRedens", was vor allem die Auswahl der umzusetzenden Maßnahmen zu Beginn des Projekts (Antragstellung) betrifft. Diese Wahrnehmung durch die Akteure hemmt wiederum die diskursive Qualität des Netzwerks.

Tabelle 11: Strategie: Probleme und Lösungswille in der Region Elbe-Altmark-Wendland $(D=$ diskursiv, $A=$ akteursorientiert, $I=$ integrativ)

\begin{tabular}{|l|l|l|l|l|}
\hline Qualitätskriterien & Stärke & D & A & I \\
\hline Intensive Dialogphasen & & & & \\
\hline Vereinbarte Kompromisse & $\begin{array}{l}\text { Steuerungsgruppe dient als Gremium zur Kompro- } \\
\text { missfindung } \\
\text { Konfliktpunkte werden umgangen, zum Beispiel: } \\
\text { Radrundkurs }\end{array}$ & $\mathrm{x}$ & & \\
\hline Qualitätskriterien & Stärke & D & A & I \\
\hline Intensive Dialogphasen & $\begin{array}{l}\text { Eindruck des ,Nicht-Miteinander-Redens“ bei der } \\
\text { Auswahl von Maßnahmen zu Beginn des Projekts }\end{array}$ & $\mathrm{x}$ & & \\
\hline Vereinbarte Kompromisse & - & & & \\
\hline
\end{tabular}

\section{Strategie: Transparen₹, Offenheit und Flexibilität}

Die Transparenz innerhalb des Projektnetzwerks wird dadurch sichergestellt, dass die in der Steuerungsgruppe getroffenen Entscheidungen an die betroffenen Akteure weitergegeben werden. Damit wird auch die Diskursivität im Netzwerk befördert. Allerdings sind die Entscheidungswege nicht immer eindeutig nachvollziehbar. Diese mangelnde Transparenz wird von den Akteuren auch hinsichtlich der Entscheidungen, die im Rahmen des E+E-Vorhabens für alle drei Modellregionen gelten, kritisiert. So wird nicht deutlich, ob die Entscheidung gefallen ist, weil es eine projektinterne Notwendigkeit ist, oder eine externe Vorgabe. Vor allem denjenigen, die nicht regelmäßig in der Steuerungsgruppe sitzen, ist nicht klar, wer die Entscheidungen fällt. Auch wer innerhalb des Projekts, welche Aufgaben übernimmt, wer in welcher Form wo eingebunden ist, ist nicht allen Beteiligten ausreichend transparent. Dies steht wiederum der diskursiven Qualität entgegen.

Zum Rückzug einzelner Akteure hat geführt, dass in der Projektantragsphase nicht zu 100\% offen gelegt wurde, welche Ziele mit dem Projekt verfolgt werden. Die Vielfalt an kleinen Maßnahmen führt zusätzlich dazu, dass es den fallbezogen eingebundenen Akteuren an Überblick mangelt. Mögliche Synergien scheinen so nicht ausreichend nutzbar. Einige Akteure vermuten auch, dass bei höherer 
Transparenz und Übersichtlichkeit ein höheres Potential an win-win-Situationen erreichbar wäre und die Integrationsfähigkeit erhöht würde.

Das Netzwerk wird von den Befragten im Wesentlichen als offen beschrieben, da die Möglichkeit besteht, jederzeit neue Akteure aufzunehmen. Diese grundsätzliche Offenheit steht für eine Akteursorientierung des Netzwerks. Andererseits gilt, wie bereits unter dem Merkmal „Breite Beteiligung“ beschrieben, der „Sack“ als „zu“, da neue Akteure keine neuen Maßnahmen mit einbringen können, wenn diese nicht kostenneutral eingebunden werden können. Dies wirke sich negativ auf die Motivation der Akteure und das Innenimage des Projekts aus und ist daher auch als hemmend für die Integration im Netzwerk einzuordnen.

Tabelle 12: Strategie: Transparen₹, Offenheit und Flexibilität in der Region Elbe-Altmark-Wendland $(D=$ diskursiv, $A=$ akteursorientiert, $I=$ integrativ)

\begin{tabular}{|c|c|c|c|c|}
\hline Qualitätskriterien & Stärke & $\mathbf{D}$ & $\mathbf{A}$ & $\mathbf{I}$ \\
\hline $\begin{array}{l}\text { Nachvollziehbarkeit von Abstimmungs- } \\
\text { und Entscheidungsprozessen. }\end{array}$ & $\begin{array}{l}\text { Mit der Steuerungsgruppe existiert ein } \\
\text { Gremium, das die Transparenz sicher- } \\
\text { stellen soll. }\end{array}$ & $\mathrm{x}$ & & \\
\hline $\begin{array}{l}\text { Fortwährende Zugangsmöglichkeit von } \\
\text { neuen Akteuren ins Netzwerk. }\end{array}$ & $\begin{array}{l}\text { Steter Versuch ein Unternehmensnetz- } \\
\text { werk aufzubauen. } \\
\text { Offenheit: Es können zu jedem Zeit- } \\
\text { punkt weiter Akteure beteiligt werden. }\end{array}$ & & $\mathrm{x}$ & \\
\hline $\begin{array}{l}\text { Inhaltliche und organisatorische Flexibi- } \\
\text { lität: Integration neuer Ideen, kreative } \\
\text { Lösungen für reg. Herausforderungen. }\end{array}$ & 1 & & & \\
\hline Qualitätskriterien & Schwäche & D & A & $\mathbf{I}$ \\
\hline $\begin{array}{l}\text { Nachvollziehbarkeit von Abstimmungs- } \\
\text { und Entscheidungsprozessen. }\end{array}$ & Entscheidungswege nicht eindeutig & $\mathrm{x}$ & & \\
\hline $\begin{array}{l}\text { Fortwährende Zugangsmöglichkeit von } \\
\text { neuen Akteuren ins Netzwerk. }\end{array}$ & - & & & \\
\hline \multirow{2}{*}{$\begin{array}{l}\text { Inhaltliche und organisatorische Flexibi- } \\
\text { lität: Integration neuer Ideen, kreative } \\
\text { Lösungen für reg. Herausforderungen. }\end{array}$} & $\begin{array}{l}\text { Umfang des Projekts minimiert die } \\
\text { Überschaubarkeit. }\end{array}$ & & & $x$ \\
\hline & $\begin{array}{l}\text { Akteure können nach Bewilligung des } \\
\text { Antrags keine neuen Maßnahmenideen } \\
\text { einbringen. }\end{array}$ & & & $\mathrm{x}$ \\
\hline
\end{tabular}

\section{Instrumente: Leitbild}

In einer Vorstudie der wissenschaftlichen Begleitung wurden ein touristisches Leitbild und naturschutzfachliche Ziele für das Hauptvorhaben formuliert. Eine Formulierung des Leitbilds während der eigentlichen Projektphase fand nicht statt. Für einen umfangreichen Prozess der Leitbildentwicklung ist die eigentliche Projektlaufzeit auch zu kurz. Durch das Vorziehen der Leitbildentwicklung wird Zeit für die Ausarbeitung und Umsetzung der Maßnahmen gewonnen. 
Das Leitbild ist in der Folge davon den Akteuren nicht in seinem vollen Umfang so präsent, als wenn es innerhalb des Projekts formuliert worden wäre. Dennoch wurde das Leitbild mit eigenen Begriffen adaptiert und mit eigenen Zielen untersetzt und die Maßnahmen entsprechend ausgerichtet. Die Formulierung des Leitbilds ,außerhalb“ des Netzwerks scheint daher für ein thematisch eng begrenztes Projekt, wie das E+E-Vorhaben kein Problem zu sein, wenn gewährleistet wird, dass das Leitbild entsprechend durch Multiplikatoren transportiert und von den Akteuren adaptiert werden kann.

Das in der Vorstudie formulierte Leitbild wird durch die in den Projektbausteinen formulierten Ziele weiter konkretisiert und spricht die drei Säulen (Ökologie, Ökonomie, Soziales) der Nachhaltigkeit an. Damit ist das Leitbild integrativ ausgerichtet. Besonders der Projektbaustein 2 „Entwicklung von Grenzerfahrungspunkten“ entfaltet eine Orientierungswirkung für die Akteure unterschiedlicher Bereiche, so dass auch hier die integrative Qualität befördert wird.

Trotz der feststellbaren Orientierungswirkung eines Projektbausteins, ist das gesamte Leitbild nicht durchgehend im Netzwerk bekannt, so dass Integrationspotentiale nicht vollständig ausgeschöpft wurden.

Tabelle 13: Instrument: Leitbild in der Region Elbe-Altmark-Wendland $(D=\operatorname{diskursiv,~} A=$ akteursorientiert, $I=$ integrativ)

\begin{tabular}{|l|l|c|c|c|}
\hline Qualitätskriterien & Stärke & D & A & I \\
\hline Vorhandensein eines Leitbildes. & $\begin{array}{l}\text { Das Leitbild ist in den einzelnen Pro- } \\
\text { jektbausteinen durch Zielformulierun- } \\
\text { gen konkretisiert. }\end{array}$ & x \\
\hline $\begin{array}{l}\text { Qualität des Leitbildes: Berücksichtigung } \\
\text { der drei Säulen d. Nachhaltigkeit. }\end{array}$ & $\begin{array}{l}\text { In den Zielen werden die Bereiche } \\
\text { Naturschutz, Tourismus, Bildung und } \\
\text { Zusammenarbeit angesprochen. }\end{array}$ & & $\mathrm{x}$ \\
\hline $\begin{array}{l}\text { Bekanntheit und Verankerung (Orientie- } \\
\text { rungswirkung!) in den Köpfen der } \\
\text { Netzwerkakteure. }\end{array}$ & $\begin{array}{l}\text { Vorwiegend sind die Ziele des Projekt- } \\
\text { bausteins 2 bekannt. }\end{array}$ & x \\
\hline Qualitätskriterien & Schwäche & D & A & I \\
\hline Vorhandensein eines Leitbildes. & - & & \\
\hline $\begin{array}{l}\text { Qualität des Leitbildes: Berücksichtigung } \\
\text { der drei Säulen d. Nachhaltigkeit. }\end{array}$ & - & & $\mathrm{x}$ \\
\hline $\begin{array}{l}\text { Bekanntheit und Verankerung (Orientie- } \\
\text { rungswirkung!) in den Köpfen der } \\
\text { Netzwerkakteure. }\end{array}$ & $\begin{array}{l}\text { Das Leitbild ist nicht durchgehend im } \\
\text { Netzwerk bekannt bzw. präsent. }\end{array}$ & & \\
\hline
\end{tabular}

\section{Instrumente: Prozessmanagement}

Der Projektträger, auch des Öfteren als Koordinierungsstelle bezeichnet, ist der zentrale Akteur für das Prozessmanagement. Er sorgt für den geregelten Ablauf und die Entwicklung des Projekts („treibt voran und hält die Fäden zusammen“). Ein Teil des Netzwerks pflegt intensive Kontakte und fördert auch über Projektveran- 
staltungen einen Austausch zur Information und Kooperation. Daneben unterstreicht besonders die positive Ausprägung der diskursiven Qualität, dass die Akteure die Kommunikation als ausreichend empfinden und dem Projektträger einen geregelten Ablauf bescheinigen. Der Projektträger, der zum einen als Koordinator, Organisator und Moderator aber auch als Übersetzer und Vermittler zwischen den unterschiedlichen Sektoren wahrgenommen wird, trägt zur integrativen Ausrichtung des Merkmals bei. Dies schlägt sich auch im Zufriedenheitsgrad der Akteure nieder (siehe hierzu Unterabschnitte von 3.2.2.1).

Bei manchen Akteuren entsteht allerdings der Eindruck des „Nicht-miteinanderredens". Auf einzelne Belange und Interessen verschiedener Akteure wurde nicht mit der gewünschten Aufmerksamkeit reagiert. Aber ein Versuch, die interne Kommunikation mit einem elektronischen Newsletter zu erhöhen, scheiterte, da der Newsletter von den Akteuren nicht ausreichend wahrgenommen wurde. Die hohe Zahl an seltenen Kontakten spricht gegen eine aktive Teilhabe dieser Akteure im Netzwerk und steht damit auch einem intensiven Austausch im Netzwerk entgegen.

Diese beiden Aspekte sowie organisatorische Probleme und inhaltliche Konflikte hemmen die diskursive Qualität. Die unzureichende Berücksichtigung der Belange einzelner Akteure steht dem integrativen Aspekt im Netzwerk entgegen, da die Einbindung unterschiedlicher Sichtweisen nicht gelungen scheint.

Zum Zeitpunkt der Interviews wurde auch die Verankerung des Projekts in der Öffentlichkeit als noch ausbaufähig beschrieben. Im weiteren Verlauf des Projekts wurde eine umfangreiche Pressearbeit betrieben, wie vielfache Pressemeldungen zeigten, die Einbindung der Öffentlichkeit wurde so zumindest für Teilgebiete der Projektregion intensiviert.

In Anbetracht der finanziellen und zeitlichen Ressourcen wird das Prozessmanagement der Koordinierungsstelle positiv herausgestellt. Für eine wesentliche Verbesserung und Reduzierung möglicher Defizite wäre ein Ausbau der verfügbaren Ressourcen notwendig. Darüber hinaus kann eine Intensivierung der Netzwerkarbeit nach Meinung einzelner Akteure nur über weitere Diskussionsrunden zu bestimmten Themen gelingen, reine Informationen über andere Maßnahmen innerhalb des Netzwerks reichen hier nicht aus. Die Bedeutung von derartigen „face-to-face"-Veranstaltungen zeigte sich auch bereits in der strukturellen Erhebung.

Tabelle 14: Instrument: Prozessmanagement in der Region Elbe-Altmark-Wendland ( $D=$ diskursiv, $A$ = akteursorientiert, $I=$ integrativ)

\begin{tabular}{|l|l|l|l|l|}
\hline Qualitätskriterien & Stärke & D & A & I \\
\hline $\begin{array}{l}\text { Vorhandensein einer (externen) Modera- } \\
\text { tion, die den Prozess steuernd begleitet. }\end{array}$ & $\begin{array}{l}\text { Projektträger fungiert als „Dolmetscher“ } \\
\text { zwischen Sektoren/ Akteuren. }\end{array}$ & & \\
\hline
\end{tabular}




\begin{tabular}{|l|l|l|l|l|}
\hline $\begin{array}{l}\text { Aktive Teilhabe der Akteure verschiede- } \\
\text { ner Sektoren. }\end{array}$ & $30 \%$ der Kontakte erfolgen häufig. & $\mathrm{x}$ & $\mathrm{x}$ & \\
\hline $\begin{array}{l}\text { Zufriedenheitsgrad der Akteure mit } \\
\text { Prozess. }\end{array}$ & $\begin{array}{l}\text { Großenteils erscheint die Kommunika- } \\
\text { tion ausreichend. }\end{array}$ & $\mathrm{x}$ & & \\
\hline $\begin{array}{l}\text { Präsenz des Netzwerks bzw. des Pro- } \\
\text { jekts in der Öffentlichkeit. }\end{array}$ & - & & $\mathrm{x}$ \\
\hline $\begin{array}{l}\text { Wahrnehmung aller Aufgaben des } \\
\text { Prozessmanagements durch Netzwerk- } \\
\text { manager. }\end{array}$ & $\begin{array}{l}\text { Projektträger als Koordinator, Modera- } \\
\text { tor und Organisator wahrgenommen. }\end{array}$ & & & \\
\hline
\end{tabular}

\begin{tabular}{|l|l|l|l|l|}
\hline Qualitätskriterien & Schwäche & D & A & I \\
\hline $\begin{array}{l}\text { Vorhandensein einer (externen) Modera- } \\
\text { tion, die den Prozess steuernd begleitet. }\end{array}$ & - & & & \\
\hline $\begin{array}{l}\text { Aktive Teilhabe der Akteure verschiede- } \\
\text { ner Sektoren. }\end{array}$ & $46 \%$ der Kontakte erfolgen selten. & $\mathrm{x}$ & & \\
\hline \multirow{2}{*}{$\begin{array}{l}\text { Zufriedenheitsgrad der Akteure mit } \\
\text { Prozess. }\end{array}$} & $\begin{array}{l}\text { Z. T. Eindruck des „Nicht-Miteinander- } \\
\text { Redens“. }\end{array}$ & $\mathrm{x}$ & & \\
\cline { 2 - 5 } & $\begin{array}{l}\text { Organisatorische Probleme und inhaltli- } \\
\text { che Konflikte. }\end{array}$ & $\mathrm{x}$ & & \\
\hline $\begin{array}{l}\text { Präsenz des Netzwerks bzw. des Pro- } \\
\text { jekts in der Öffentlichkeit. }\end{array}$ & $\begin{array}{l}\text { Zum Zeitpunkt der Erhebung wird das } \\
\text { Projekt als nicht in der Öffentlichkeit } \\
\text { präsent eingeordnet. }\end{array}$ & & $\mathrm{x}$ \\
\hline $\begin{array}{l}\text { Wahrnehmung aller Aufgaben des } \\
\text { Prozessmanagements durch Netzwerk- } \\
\text { manager. }\end{array}$ & $\begin{array}{l}\text { Einzelne Belange der Akteure nicht } \\
\text { ausreichend vom Projektträger berück- } \\
\text { sichtigt. }\end{array}$ & & $\mathrm{x}$ \\
\hline
\end{tabular}

\subsubsection{Maßnahmenperspektive}

Gemäß dem in Kapitel 2 beschriebenen Untersuchungsansatz erfolgt in diesem Abschnitt die Darstellung der Ergebnisse für das Erprobungs- und Entwicklungsvorhaben Erlebnis Grünes Band in der Untersuchungsregion Elbe-AltmarkWendland. Wie bereits in Kapitel 2 erwähnt, ist das E+E-Vorhaben im Durchführungszeitraum dieses Projektes noch nicht vollständig abgeschlossen gewesen. Um dennoch der Forschungshypothese, dass die Maßnahmen des E+E-Vorhabens auf regionale Nachhaltigkeit wirken, nachgehen zu können, wird angenommen, dass alle Maßnahmen, die im Projektantrag niedergeschrieben sind, auch so zur Umsetzung gelangen. ${ }^{96}$ Das E+E-Vorhaben „Erlebnis Grünes Band in der Mo-

96 Zumeist wird ein Projektantrag nicht eins zu eins umgesetzt, da sich im Fortgang eines Projektes Anpassungen ergeben. So wird auch hier die Möglichkeit von Änderungen in der Umsetzung von Maßnahmen nicht ignoriert. Nichtsdestotrotz ist die Struktur des Erprobungs- und Entwicklungsvorhaben mit seinen vier Projektbausteinen klar strukturiert, so dass einzelne abweichend vom Projektantrag durchgeführte Maßnahmen, die regionale Gesamtwirkung des E+E- 
dellregion Elbe-Altmark-Wendland, Grenzerfahrung im Vierländereck“ setzt sich aus vier Projektbausteinen zusammen, die wiederum verschiedene Einzelmaßnahmen umfassen: Projektbaustein 1 (Pb 1) ,Schutz, Pflege und Entwicklung von Lebensräumen am Grünen Band und ihre Einbindung in das touristische Erlebniskonzept“" umfasst sieben Einzelmaßnahmen (M 1.1 bis 1.7); Projektbaustein 2 (Pb 2) „Entwicklung von „Grenzerfahrungspunkten“ mit Einbindung in touristische Routen“ besteht aus dreizehn Einzelmaßnahmen (M 2.1 bis 2.13); Projektbaustein $3(\mathrm{~Pb} 3)$,Entwicklung und Umsetzung von Bildungsangeboten und Veranstaltungen" umfasst sieben Einzelmaßnahmen (M 3.1 bis 3.7); Projektbaustein 4 (Pb 4) „Marketing und Kommunikation“ umfasst verschiedene Einzelmaßnahmen, die dem Innen- und Außenmarketing zugeordnet werden können. Im Zusammenwirken der einzelnen Bausteine soll die Zusammenführung von Schutzund Nutzaspekten gelingen, die charakteristisch für das E+E-Vorhaben sein soll. Jeder dieser vier Projektbausteine umfasst verschiedene Einzelmaßnahmen $\left(M_{i}\right)$. Das Wirkungspotenzial dieses E+E-Vorhabens ergibt sich aus der Zusammenschau aller Maßnahmen. Um nun gemäß der Forschungshypothese zu einer Aussage zu kommen, werden Maßnahmen und Themenfelder der regionalen Nachhaltigkeit durch eine Matrix miteinander in Beziehung gesetzt.

\subsubsection{Maßnahmenbasismatrix}

Die Maßnahmenbasismatrix enthält in den Spalten die Projektbausteine inklusive der jeweils zugehörigen Maßnahmen. In den Zeilen sind die üblichen regionalen Nachhaltigkeitsthemenfelder ${ }^{97}$ abgetragen, eingruppiert in die drei üblichen übergeordneten Dimensionen ${ }^{98}$ Ökologie, Ökonomie und Soziales (siehe Abbildung 6). Die Lesart erfolgt von den Spalten zu den Zeilen: Eine Maßnahme X des Projektbausteins $Z$ kann direkt oder indirekt Wirkung entfalten auf die Themenfelder 1 bis 21. Dieser Zusammenhang wird hergestellt zum einen basierend auf Plausibilitätsüberlegungen und zum anderen basierend auf Literaturquellen. Abhängig vom Konkretisierungsgrad der geplanten Maßnahmen sind die Abschätzungen hinsichtlich des Wirkungspotenzials in unterschiedlicher Genauigkeit möglich. So wird in deskriptiver Form das Gesamtwirkungspotenzial des E+E-Vorhabens in seiner Verteilungswirkung über die einzelnen Themenfelder der regionalen Nachhaltigkeit sichtbar. Im Folgenden wird das Wirkungspotenzial für jeden Projektbaustein einzeln betrachtet:

Vorhabens nicht erheblich verändern. Aus diesem Grund ist das Heranziehen des Projektantrages als Grundlage, um der Forschungshypothese nachgehen zu können, unproblematisch.

97 Siehe zum Thema „Nachhaltige Regionalentwicklung als Evaluationsgegenstand“ auch Pollermann, K. (2007); Ness, B. et al. (2007); Graymore, M. et al. (2008). 
Wirkungspotenzial des Projektbausteins 1 (Pb 1) „Schutz, Pflege und Entwicklung von Lebensräumen am Grünen Band und ibre Einbindung in das touristische Erlebniskonzept ${ }^{\circ 99}$

Pb 1 wirkt auf das Themenfeld Ökosystem und Artenvielfalt: Alle sieben Maßnahmen (M 1.1 bis 1.7) tragen zur Entwicklung des Themenfeldes (TF) Ökosysteme und Artenvielfalt bei. ${ }^{100} \mathrm{Zu}$ nennen sind hier Renaturierungsmaßnahmen (M 1.1), die Lebensraum für unterschiedliche Arten, durch Schaffung von Schlaf- und Rastplätzen, begünstigen sollen. Positive Effekte werden erwartet für Kraniche und nordische Gänse, Limikolen (Brutplätze, Durchzug), See- und Fischadler, Schwarzstorch, Fledermäuse (Jagdhabitat) sowie Amphibien und Reptilien. Insbesondere die Amphibienfauna soll durch Anlage eines Kleingewässers positiv beeinflusst werden (M 1.7 „Anlage eines Kleingewässers bei Kietz im Landkreis Prignitz, Brandenburg'). Außerdem sollen Maßnahmen zur Entwicklung und Pflege von Heide- und Trockenlebensräumen (M 1.2 - 1.6) unterschiedliche Arten positiv beeinflussen (Kreuzotter, Schlingnatter, Ziegenmelker, Heidelerche, diverse Insektenarten, Schmetterlings- und Heuschreckenfauna, unterschiedliche Amphibienarten, Neuntöter und Wachtelkönig).

$\mathrm{Pb} 1$ wirkt auf das Themenfeld ökologische Landwirtschaft: Einige Maßnahmen sehen eine extensive Bewirtschaftung von Flächen vor sowie eine Schafbeweidung in Kooperation mit ortsansässigen Landwirten (M 1.1 - M 1.2). An einigen Stellen sind vorab Entkusselungsarbeiten durchzuführen (M 1.3). Diese Formen der Kulturlandschaftspflege kann als ökologische Nutzungsform unter dieses Themenfeld subsumiert werden. Von extensiv genutzten Flächen kann ein ganzes Bündel von Effekten $^{101}$ ausgehen, die auch andere Themenfelder der Maßnahmenbasismatrix tangieren. Allgemein werden extensiv genutzten Landschaftselementen folgende positive Eigenschaften zugeschrieben: Verminderung von Bodenerosion, Beeinflussung der Wasseraufnahmefähigkeit des Bodens sowie ästhetische Gründe. Somit kann durch diese Maßnahmen auch eine mögliche Wirkung auf die Themenfelder Boden und Wasser in der Dimension Ökologie und das Themenfeld Ästhetik ${ }^{102}$ der Landschaft in der Dimension Soziales gegeben sein.

Pb 1 wirkt auf das Themenfeld regionale Identität: Das mit den Landschaftspflegemaßnahmen verfolgte Ziel der Wiederherstellung und Erlebbarkeit des Grünen Bandes leistet einen Beitrag in diesem Themenfeld.

99 Siehe zur ökonomischen Bewertung von Umweltleistungen Hartje, Volkmar (2003); Günther, E. et al. (2008).

100 Siehe zu den Grundproblemen landschaftsökologischer Daten Leser, H. (1997), S. $278 \mathrm{ff}$.

101 Vgl. Nitsche, S.. et al. (1994).

102 Unter das Themenfeld Ästhetik der Landschaft werden auch Themen wie ,,(emotionaler) Eigenwert der Natur“ und „Naturethik“ subsumiert. Im Bezug auf die Perspektiven einer touristischen Inwertsetzung sind diese Themen mit von Bedeutung. Siehe dazu Krebs , A. (1996); Krause, R. et al. (2008). Zur Naturethik siehe u.a. Bundesministerium für Umwelt, Naturschutz und Reaktorsicherheit (2007), S. 15. 
$\mathrm{Pb} 1$ wirkt auf das Themenfeld regionale Wirtschaft: Einige Maßnahmen sehen Vertragsnaturschutz durch ortsansässige Landwirte explizit vor. Dieser soll stattfinden in Form von Schafbeweidung oder Ziegen im Hütebetrieb (M 1.1; M 1.2; M 1.4). Die vorgesehene Verwendung von Pflanzenmaterial aus dem Altmarkkreis Salzwedel ist ein Beispiel für die Nutzung von regionalen Potentialen (M 1.2). In wieweit dies geeignete Voraussetzungen sind, um einen regionalen Wirtschaftskreislauf zu initiieren, kann aus theoretischer Sicht nicht allgemein beantwortet werden. ${ }^{103}$

Pb 1 wirkt auf das Themenfeld Tourismus: Damit die Idee des E+E-Vorhabens, nämlich die Zusammenführung von Schutz- und Nutzaspekten, gelingen kann, leisten einige Maßnahmen einen Beitrag, zu einer touristischen Inwertsetzung. Die Errichtung eines Aussichtpunktes als Naturerfahrungspunkt (M 1.1) ist eine Möglichkeit, um die durch Landschaftspflegemaßnahmen im Themenfeld Ökosystem und Artenvielfalt erzielten Effekte, erfahrbar zu machen. Maßnahmen zur Befestigung des Mittelstreifens der Betonspurplatten des Kolonnenweges sind als notwendige Vorarbeiten zu sehen, um entsprechende Abschnitte am Grünen Band als Wander- und Radweg entwickeln zu können (M 1.2). Über Bedeutung von Flächen sowohl aus Perspektive des Naturschutzes als auch aus geschichtlicher Perspektive sollen Informationstafeln informieren (M 1.2 und M 1.3). Diese Maßnahmen sind ebenfalls als notwendige Vorarbeiten zu interpretieren um Schutzaspekte und Nutzaspekte zusammenzuführen. ${ }^{104}$

$\mathrm{Pb} 1$ wirkt auf die Themenfelder Familienfreundliche Strukturen und Integration: Durch die geplante Einbindung des Kinder- und Jugenderholungszentrums KIEZ am Arendsee in Pflegearbeiten am Grünen Band besteht die Möglichkeit, dass familienfreundlichen Strukturen regional Aufmerksamkeit geschenkt wird (M 1.2). Die barrierefreie Erschließung des Gebietes bei Konau-Popelau auf Grund der Nähe zur Ferienstätte für Menschen mit Behinderung in Gülstorf ist ein Aspekt von Integration, der im Rahmen einer nachhaltigen regionalen Entwicklung bedeutungsvoll ist und hier eine konkrete Entsprechung findet (M1.4).

Für den Projektbaustein 1 „Schutz, Pflege und Entwicklung von Lebensräumen am Grünen Band und ihre Einbindung in das touristische Erlebniskonzept" ist festzuhalten, dass direkte Wirkungen in allen drei Dimensionen der regionalen Nachhaltigkeit möglich sind. Insbesondere bei den Maßnahmen M 1.1 bis 1.3 besitzt jede Einzelmaßnahme gleichzeitig Wirkungspotenzial in allen drei Dimensionen (siehe Abbildung 6) Die Maßnahmen besitzen durch die mehrdimensionale Ansprache von Themenfeldern einen integrativen Charakter.

103 Zu Möglichkeiten und Grenzen von regionalen Wirtschaftskreisläufen siehe u.a. Petermann, C. (2001), S. 16.; Walz, R. et al. (2003-2004), S. 269; Kullmann, A. (2003).

104 Da während der Laufzeit dieses Projektes die Maßnahmenumsetzung im Rahmen des E+E-

Vorhabens noch nicht abgeschlossen war, kann nicht an Hand von Fallbeispielen argumentiert werden. Einen Überblick über Untersuchungen zu ökonomischen Effekten von Großschutzgebieten gibt Job, H. et al. (2005). Hier werden Methoden aufgezeigt und angewandt um ökonomische Effekte des Tourismus messbar zu machen. 


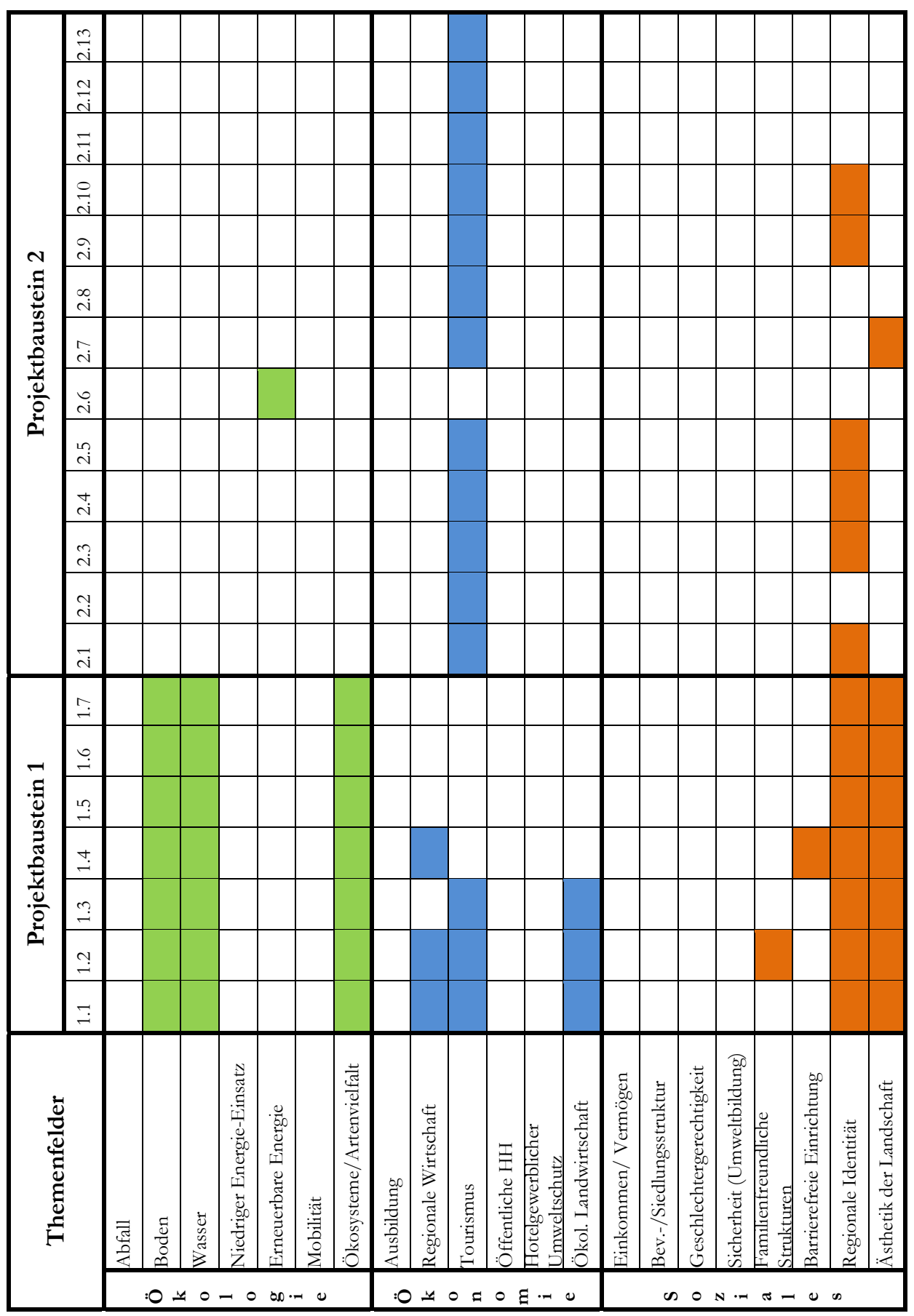




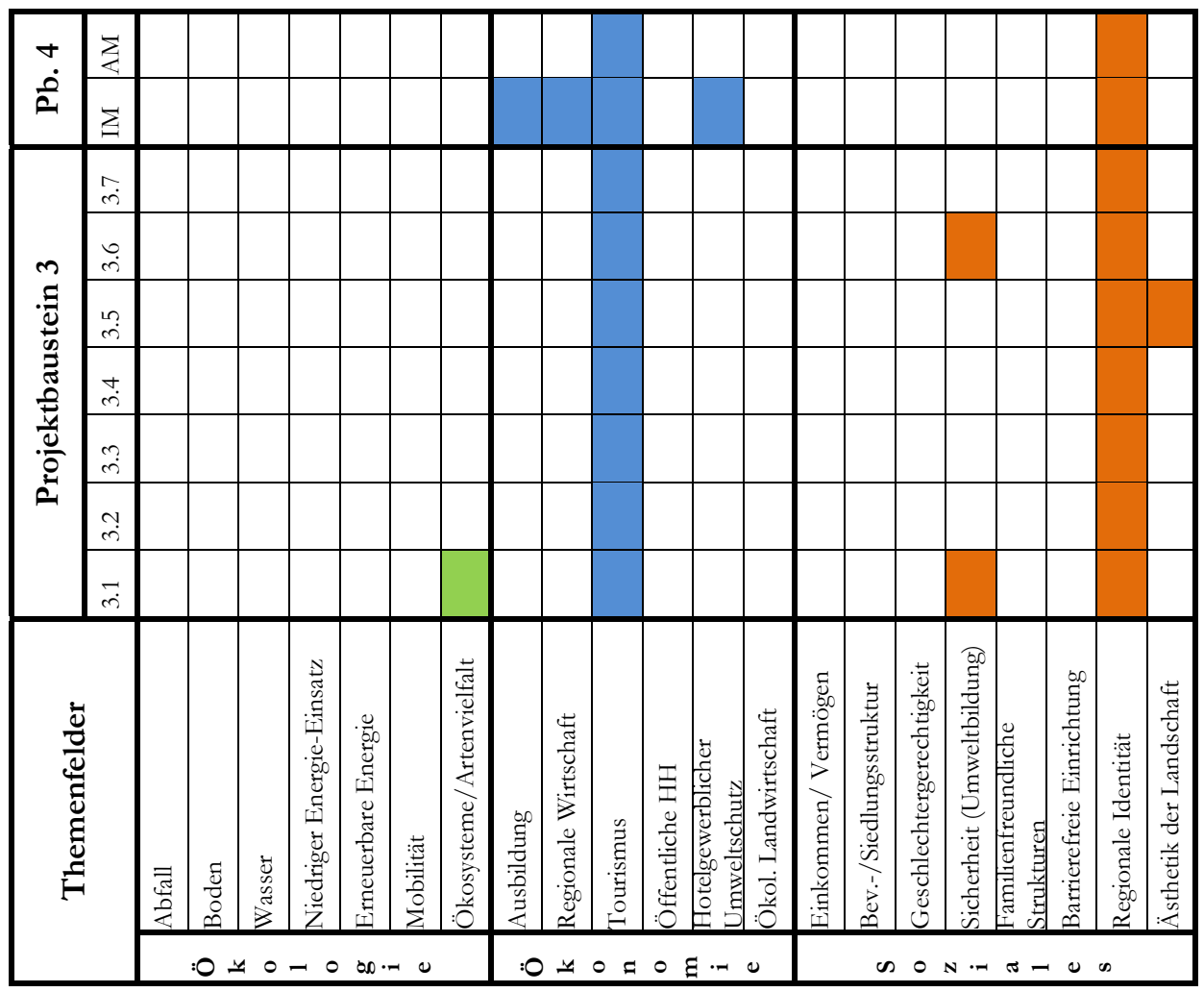

Abbildung 11: Maßnahmenbasismatrix für das E+E Vorhaben Elbe-Altmark-Wendland

Wirkungspotenzial des Projektbausteins 2 (Pb 2) „Entwicklung von Grenzerfahrungspunkten mit Einbindung in touristische Routen"

Pb 2 wirkt auf das Themenfeld Tourismus: Fast alle Einzelmaßnahmen dieses Projektbausteins haben Wirkungspotenzial auf das Themenfeld Tourismus. Die dreizehn Einzelmaßnahmen lassen sich grob folgendermaßen zu drei Maßnahmenschwerpunkten zusammenfassen. Maßnahmenschwerpunkt Lehrpfade und Rundwege, Maßnahmenschwerpunkt Grenzerfahrungspunkte und Maßnahmenschwerpunkt Fernradroute/Wassererlebnis. Im ersten Maßnahmenschwerpunkt geht es konkret darum, den bestehenden Naturlehrpfad „Bürgerholz“ (M 2.1) zu verlängern, einen grenzhistorischen Rundweg Konau, Popelau, Darchau (M 2.2) sowie eine Grenzerlebnisroute Schnackenburg, Stresow, Bömenzien, Gartow (M 2.3) auszubauen. Ferner sollen vorhandene natürliche und kulturhistorische Besonderheiten bei Rüterberg zu einem Rundweg zusammengeführt werden. Die Einzelmaßnahmen dienen der Qualifizierung und Professionalisierung bereits bestehender Infrastruk- 
tur. Die Maßnahmen sind als notwendige Vorarbeiten anzusehen, um diese Bereiche touristisch in Wert zu setzen. Neben den Maßnahmen zur Verbesserung vorhandener Infrastruktur stehen Überlegungen zur Vermittlung von naturkundlichen und historischen Informationen (z.B. Broschüren, Informationstafeln) im Mittelpunkt. Im zweiten Maßnahmenschwerpunkt geht es konkret um die ErschlieBung eines Grenzturmes (M 2.5), die Einrichtung einer Ausstellung im Dorf Unbesandten zum geschichtlichen Hintergrund des Dorfes sowie die naturkundlichen Besonderheiten im Umfeld (M 2.7) sowie den Bau eines Steges am Gewässer „Alte Jeetzel“ (M 2.8). Diese punktuellen Erschließungsarbeiten ermöglichen Nutzungen als Grenzerfahrungspunkte und damit die Einbindung in entsprechende touristische Konzepte. Auch sie sind als notwendige Vorarbeiten zur touristischen Inwertsetzung zu begreifen. Im dritten Maßnahmenschwerpunkt soll eine mehrtägige Fernradroute mit thematischen Schwerpunkten zur Natur-, Kultur- und Grenzgeschichte weiterentwickelt und umgesetzt werden (M 2.9) sowie die touristischen Nutzungsmöglichkeiten durch Bau von Anlegestellen am Grenzfluss Elbe (M 2.11) und am Gewässer Jeetzel (M 2.12) aufgewertet werden. Auch diese Maßnahmen können als Vorarbeiten begriffen werden, um entsprechende touristische Angebote (touristische Pauschalen) entwickeln zu können.

Pb 2 wirkt auf das Themenfeld regionale Identität: Die im Projektbaustein 2 geplanten Maßnahmen zur Entwicklung von Grenzerfahrungspunkten mit Einbindung in touristische Routen haben als gemeinsames Merkmal, dass sie grenzübergreifend ausgerichtet sind. Gab es bis zum Fall des Eisernen Vorhangs eine klar definierte Grenze und folglich auch klar definierte „,angrenzende“ Regionen und regionale Identitäten, so besteht die Chance die regionale Identität ${ }^{105}$ dahingehend (weiter) zu entwickeln, dass nicht mehr in diesseits und jenseits der Grenze differenziert wird. Sozialer Zusammenhalt in einer Region braucht häufig ein verbindendes Element. Der Projektbaustein 2 und die in ihm enthaltenen Maßnahmen bieten die Möglichkeit, dieses verbindende Element zu schaffen. ${ }^{106}$ Insbesondere die Maßnahmenschwerpunkte Rundwege und Grenzerfahrungspunkte bieten hierzu gute Voraussetzungen (M 2.1; M 2.3-M 2.5).

Pb 2 wirkt auf das Themenfeld Ästhetik der Landschaft: Im Rahmen der Ausgestaltung des Grenzerfahrungspunktes „Wischedorf“ (M 2.7) wurde eine Streuobstwiese angelegt, die den Obstanbau in der DDR thematisiert. ${ }^{107}$

$\mathrm{Pb} 2$ wirkt auf das Themenfeld erneuerbare Energien: Im Rahmen der Maßnahme „Grenzerfahrungs- und Naturerlebnispunkt Grenzturm Gandow“ (M 2.6) soll der Grenzturm mit einem Stromanschluss ausgestattet werden, um ihn noch besser als Veranstaltungsort nutzen zu können, insbesondere für Beamer-Präsentationen und Diavorführungen. Dazu soll eine Solarstromanlage auf dem Dach des Grenzturmes eingerichtet werden. Die Nutzung erneuerbarer Energien spielt im Rah-

\footnotetext{
105 Vgl. BUND (2008), S. 12.

106 Siehe hierzu auch die Ergebnisse der Netzwerkperspektive unter Kapitel 3.2.3.

107 Zum ästhetischen Eigenwert von Landschaften siehe Krebs, A. (1996), S. 4.
} 
men von nachhaltiger, regionaler Entwicklung eine wichtige Rolle. Diese Maßnahme ist unter diesem Aspekt positiv in ihrer Wirkung auf das Themenfeld zu bewerten

Für den Projektbaustein 2 „Entwicklung von Grenzerfahrungspunkten mit Einbindung in touristische Routen“ lässt sich klar feststellen, dass sein Hauptwirkungspotenzial im Themenfeld Tourismus liegt. In seltenen Fällen werden weitere Themenfelder der regionalen Nachhaltigkeit direkt angesprochen (M 2.6; M 2.7). Im Vergleich zum Projektbaustein 1 lässt sich daher sagen, dass die Maßnahmen im Projektbaustein 2 eine relativ geringere Wirkung besitzen im Hinblick auf die Integration von ökologischen, ökonomischen und sozialen Themen.

\section{Wirkungspotenzial des Projektbausteins $3(\mathrm{~Pb} 3)$ „Entwicklung von Bildungsangeboten und Veranstaltungen"}

$\mathrm{Pb} 3$ wirkt auf das Themenfeld Tourismus: Alle Maßnahmen in diesem Projektbaustein haben einen Bezug zur touristischen Inwertsetzung entsprechender Bereiche des Grünen Bandes. Geplant sind verschiedene Kategorien von Bildungsangeboten wodurch unterschiedliche Zielgruppen angesprochen werden sollen. So sind explizit Projektwochen für Kinder und Jugendliche (M 3.1) geplant. Ferner sollen die in Projektbaustein 3 gewonnenen Erkenntnisse in eine Materialiensammlung fließen, die Ideen, Vorschläge und Wissenswertes zum Thema Grünes Band enthält. Hieraus können sich interessierte Anbieter bedienen, wenn sie touristische Pauschalen entwickeln und diese Themen zielgruppenorientiert weiter entwickeln wollen (M 3.7). Weitere Kategorien von geplanten Bildungseinrichtungen und Veranstaltungen sind literarische Reiseangebote zum Grünen Band (M 3.3), halbbis eintägig geführte Touren am Grünen Band (M 3.2), sowie ein Künstlerworkshop zum Thema Grünes Band (M 3.5). Die Initiierung einer Geschichtswerkstatt (M 3.4) sowie die Einführung von Tagen der Grenzerfahrung mit EventCharakter (M 3.6) runden die Vielfalt der Kategorien ab. Da die Zielgruppen der Maßnahmen verschieden sind und auch die Möglichkeiten hinsichtlich der Vernetzung mit anderen Maßnahmen, die der touristischen Inwertsetzung dienen sollen (siehe entsprechende Maßnahmen aus den Projektbausteinen 1 und 2), sehr unterschiedlich sind, kann hier keine allgemeine Aussage zum Wirkungspotenzial gemacht werden.

$\mathrm{Pb} 3$ wirkt auf das Themenfeld regionale Identität: Die regionale Identität wird ebenfalls durch sämtliche Maßnahmen dieses Projektbausteines angesprochen. Bei allen zuvor unter „Wirkung auf das Themenfeld Tourismus“ angesprochenen Maßnahmen ist ein Zusammenwirken von Akteuren diesseits und jenseits der Grenze wünschenswert und zum Großteil auch erforderlich (insbesondere bei der Veranstaltungskategorie „Tage der Grenzerfahrung (M 3.6)“ und geführte Touren (M 3.2)). Dies gilt für die Aufarbeitung aller hier geplanter Themenbereiche im Zusammenhang mit der ehemaligen Grenze: Themenbereich Natur, Themenbe- 
reich Geschichte und Themenbereich Kultur und Kunst. Durch das Zusammenwirken von Akteuren diesseits und jenseits der ehemaligen Grenze, können unterschiedliche regionale Identitäten durch das Grüne Band ein verbindendes Element bekommen. ${ }^{108}$

$\mathrm{Pb} 3$ wirkt auf das Themenfeld Sicherheit (Umweltbildung): Sicherheit wird im Rahmen regionaler Nachhaltigkeit als ein wichtiges Themenfeld erachtet und steht deshalb auch in der Maßnahmenbasismatrix. Das Wirkungspotenzial dieses „Erprobungs- und Entwicklungsvorhabens“ kann auch in einer Sensibilisierung für Umweltthemen gesehen werden. Hierdurch kann indirekt ein Beitrag geleistet werden, sorgsamer mit der Umwelt umzugehen. Obgleich der direkte Einfluss einer entsprechenden Maßnahme auf das Ausmaß von Straftaten gegen die Umwelt empirisch ${ }^{109}$ nur schwer festgestellt werden kann, so ist nicht unplausibel, dass Naturbeobachtungen und Exkursionen am Grünen Band für Kinder und Jugendliche sowie Tage der Grenzerfahrung mit Events im Bereich Natur sowie naturkundlicher Führungen und Beobachtungen zur Sensibilisierung ${ }^{110}$ für entsprechende Themen beitragen (insbesondere M 3.1 und M 3.6) und so langfristig auch auf die Sicherheit positiv wirken.

Pb 3 wirkt auf das Themenfeld Ästhetik der Landschaft: Die Nachzeichnung des Grenzverlaufes durch künstlerisch bearbeitete Naturmaterialien ergänzt das natürlich vorhandene Landschaftsbild (M 3.5). Damit besteht ein Wirkungspotenzial im Hinblick auf die Ästhetik der Landschaft. Der Bereich des Grünen Bandes umfasst auf Grund der geschichtlichen Ereignisse auch emotionale Aspekte. Es ist davon auszugehen, dass diese emotionalen Aspekte durch diese Maßnahme ebenfalls nachgezeichnet und herausgestellt werden, wodurch sie deutlich und sichtbar mit der Ästhetik der Landschaft verknüpft werden. ${ }^{111}$

$\mathrm{Pb} 3$ wirkt auf das Themenfeld Ökosysteme und Artenvielfalt: Projektwochen für Kinder und Jugendliche (M 3.1), die inhaltlich durch Mitarbeit bei Naturschutzmaßnahmen am Grünen Band ausgestaltet sind, leisten einen Beitrag im Themenfeld Ökosystem und Artenvielfalt. Art und Umfang des Beitrages hängt von der Umsetzung der entsprechenden Maßnahme ab.

Für den Projektbaustein 3 ,Entwicklung und Umsetzung von Bildungsangeboten und Veranstaltungen“ lässt sich klar feststellen, dass sein Hauptwirkungspotenzial in den Themenfeldern Tourismus und regionale Identität gesehen werden kann. In seltenen Fällen werden weitere Themenfelder der regionalen Nachhaltig-

108 Vgl. BUND (2008), S. 12.

109 Grundvoraussetzung ist das Vorhandensein von entsprechendem Datenmaterial. Aber auch bei Anwendung von Methoden der Korrelationsstatistik ist die Ableitung von kausalen Bezügen, die allein auf entsprechenden Signifikanzniveaus beruhen, nicht möglich. Für die betrachtete Modellregion fehlt es aber bereits an entsprechendem Datenmaterial.

110 Vgl. Knolle, Friedhart (2004), S. 65, sowie Bittner A. (2003).

111 Diese Überlegungen sind sicherlich theoretisch. Dennoch wird der „emotionale Eigenwert“ der Natur und seine Beeinflussung wissenschaftlich diskutiert (vgl. hierzu insbesondere Krebs, A. (1996)). 
keit direkt angesprochen (M 3.1; M 3.5). Im Vergleich zum Projektbaustein 1 lässt sich daher sagen, dass die Maßnahmen im Projektbaustein 3 ein relativ geringeres Wirkungspotenzial besitzen im Hinblick auf die Integration von ökologischen, ökonomischen und sozialen Themen.

\section{Wirkungspotenzial des Projektbausteins 4 (Pb 4) „Marketing und Kommunikation“}

Mit Beginn des Erprobungs- und Entwicklungsvorhabens wird ein Marketingbeirat institutionalisiert, in dem neben dem Projektträger auch die Tourismusorganisationen der beteiligten Bundesländer vertreten sind. Das Gremium soll während der Laufzeit des E+E-Vorhabens regelmäßig zusammentreten und eine koordinierende Funktion übernehmen. Analog zu den Maßnahmen (M) der Projektbausteine 1 bis 3 lassen sich die Aktivitäten im Projektbaustein 4 in Innenmarketing (IM) und Außenmarketing (AM) unterscheiden. Maßnahmen, wie sie in den Projektbausteinen 1 bis 3 beschrieben wurden, sind zum einen nicht aus sich heraus wirksam noch können sie längerfristig bestehen bleiben, wenn niemand von ihren weiß. Daher bietet dieser Projektbaustein 4 für die Projektbausteine 1 bis 3 flankierende Maßnahmen.

$\mathrm{Pb} 4$ wirkt auf das Themenfeld Tourismus: $\mathrm{Zu}$ den Maßnahmen im Innenmarketing zählen die Etablierung von Kommunikationswegen zu den (potentiellen) Leistungserbringern. Hierzu dienen Mailing-Aktionen, Infobriefe, elektronische Newsletter sowie Workshops und Foren der Tourismusorganisationen ${ }^{112}$. Die im Rahmen des E+E-Vorhabens geplanten Maßnahmen können ausstrahlen und weitere Akteure motivieren, sich mit eigenen Maßnahmen in einem der Projektbausteine zu engagieren. Gleichzeitig soll aber auch aktiv versucht werden, weitere Leistungserbringer zu gewinnen. Zur Professionalisierung der Entwicklung von passenden touristischen Pauschalen wird über die Tourismusorganisation Viabono und die damit verbundenen Chancen und Möglichkeiten der Vermarktung informiert. Damit das Angebot von touristischen Pauschalen auch auf eine Nachfrage trifft, wird dieses nach außen entsprechend vermarktet. Neben der Nutzung bestehender Vermarktungswege der Marketingpartner sind verschiedene Einzelmaßnahmen geplant (Pressefahrt, Printprodukte, Messebesuche). Mittlerweile existiert für die Erlebnisregionen „Eichsfeld“, „Elbe-Altmark-Wendland“, „Harz“ und „Thüringer Wald und Schiefergebirge/Frankenwald“ ein einheitlicher Internetauftritt. ${ }^{113}$ Hier können die touristischen Pauschalen nach den Kriterien Besinnung, Genuss und Freiheit sortiert und über entsprechende Kontaktdaten, die zu jeder Pauschale angegeben sind, gebucht werden. Das touristische Produkt soll als Paket mit allen seinen Eigenschaften vermarktet werden. Hierzu zählen insbeson-

112 Hier kann exemplarisch die Internetplattform www.prignitzforum.de genannt werden, die im Rahmen eines BMBF-Projektes entwickelt wurde.

113 Siehe hierzu http://reisen.erlebnisgruenesband.de/HeaderTabs/erlebnisregionen/regionen.aspx (abgerufen am 30.03.2010). 
Die Untersuchungsregionen

dere auch die Informationen über Möglichkeiten der umweltfreundlichen Anreise und Mobilität vor Ort aber auch die Vermittlung der Naturschutzidee des Gesamtprojektes. ${ }^{114}$ Hierüber werden die Themenfelder Mobilität und Sicherheit (Umweltbildung) indirekt adressiert.

$\mathrm{Pb} 4$ wirkt auf das Themenfeld regionale Identität: Gelingt es im Rahmen des E+EVorhabens, insbesondere über die Maßnahmen dieses Projektbausteines 4, Leistungsträger aus den verschiedensten Bereichen entlang des Grünen Bandes zu gewinnen, sich an der Entwicklung von touristischen Angeboten zu beteiligen und ihr jeweiliges regionales Erfahrungswissen einzubringen, dann kann hierdurch ein Beitrag zur Entwicklung der regionalen Identität geleistet werden.

$\mathrm{Pb} 4$ wirkt auf das Themenfeld Ausbildung: Im Rahmen der Projektlaufzeit des $\mathrm{E}+\mathrm{E}-$ Vorhabens soll ein fünftägiger Fortbildungskurs für Gästeführer am Grünen Band entwickelt werden.

$\mathrm{Pb} 4$ wirkt auf das Themenfeld hotelgewerblicher Umweltschutz: Die Entwicklung der touristischen Angebote soll bestimmte (zum Teil noch zu konkretisierende) Kriterien erfüllen. Unter anderem spielt im Rahmen von regionaler Nachhaltigkeit auch der schonende Umgang mit Ressourcen eine Rolle. Das Hotelgewerbe kann hierzu natürlich einen Beitrag leisten und sich dafür zertifizieren lassen. Durch entsprechende Kommunikation dieser Anforderungen kann hier ein Beitrag geleistet werden, hotelgewerblichen Umweltschutz in der Region (weiter) zu verankern.

$\mathrm{Pb} 4$ wirkt auf das Themenfeld regionale Wirtschaft: Durch Kommunikation in die Region wird es möglich, regionale Akteure (kleine Betriebe z.B. Hotels, Gaststätten,...) über Möglichkeiten der Einbindung in Maßnahmen des E+E-Vorhabens $\mathrm{zu}$ informieren. Hierzu soll insbesondere die Internetplattform www.prignitzforum.de ${ }^{115}$ genutzt werden. Die theoretischen Voraussetzungen, Wirkungspotenzial in der regionalen Wirtschaftsstruktur zu entfalten, sind damit gegeben. ${ }^{116}$

Für den Projektbaustein 4 ,Marketing und Kommunikation“ lässt sich klar feststellen, dass sein Hauptwirkungspotenzial in den Themenfeldern Tourismus und regionale Identität gesehen werden kann. Aber auch das Wirkungspotenzial im Bereich Ausbildung ist hervorzuheben.

Insgesamt ist für das hier betrachtete Erprobungs- und Entwicklungsvorhaben „Erlebnis Grünes Band in der Modellregion Elbe-Altmark-Wendland“ festzuhalten, dass das von den Einzelmaßnahmen der Projektbausteine ausgehende Wirkungspotenzial auf die Themenfelder der regionalen Nachhaltigkeit eine gewisse Streuung besitzt. Dennoch lässt sich eine Konzentration von Einzelmaßnahmen für die Themenfelder „Ökosysteme und Artenvielfalt“ (8 Einzelmaßnahmen),

114 Siehe hierzu ebenfalls den Internetauftritt: http://reisen.erlebnisgruenesband.de (abgerufen am 30.03.2010).

115 Die Internetplattform www.prignitzforum.de wurde im Rahmen eines BMBF Projektes entwickelt.

116 Vgl. Petermann, C. (2001), S. 5f. 
„Tourismus“ (24 Einzelmaßnahmen), „regionale Identität“ (22 Einzelmaßnahmen) und „Ästhetik der Landschaft“ (9 Einzelmaßnahmen) feststellen. Hierüber sind alle drei Dimensionen (Ökologie, Ökonomie uns Soziales) bereits berücksichtigt. Dieses Ergebnis dokumentiert, dass es durch die entsprechende Ausgestaltung der Einzelmaßnahmen gelungen ist, Schutzaspekte und Nutzaspekte zusammen zu führen und damit der Zielsetzung des E+E-Vorhabens aus theoretischer Perspektive gerecht zu werden. Die vorstehende qualitative Auswertung bestätigt damit die Forschungshypothese, dass die Maßnahmen des E+E-Vorhabens auf regionale Nachhaltigkeit wirken. Die deskriptive Darstellung der Arten von Einzelmaßnahmen zeigt, wie diese auf regionale Nachhaltigkeit wirken bzw. wirken können.

\subsubsection{Nachbaltigkeitsspinne und regionale Verbaltensweisen}

Die Nachhaltigkeitsspinne dient dazu, wie unter Kapitel 2 beschrieben, den Prozess einer nachhaltigen regionalen Entwicklung zu organisieren. Inhaltlich bildet sie die Maßnahmenbasismatrix ab. Durch sie soll in deskriptiver Form ein Eindruck gewonnen werden, wie das E+E-Vorhaben auf regionale Nachhaltigkeit wirkt bzw. wirken kann. Für die hier betrachtete Untersuchungsregion zeigt die Nachhaltigkeitsspinne folgendes Bild (siehe Abbildung 12)

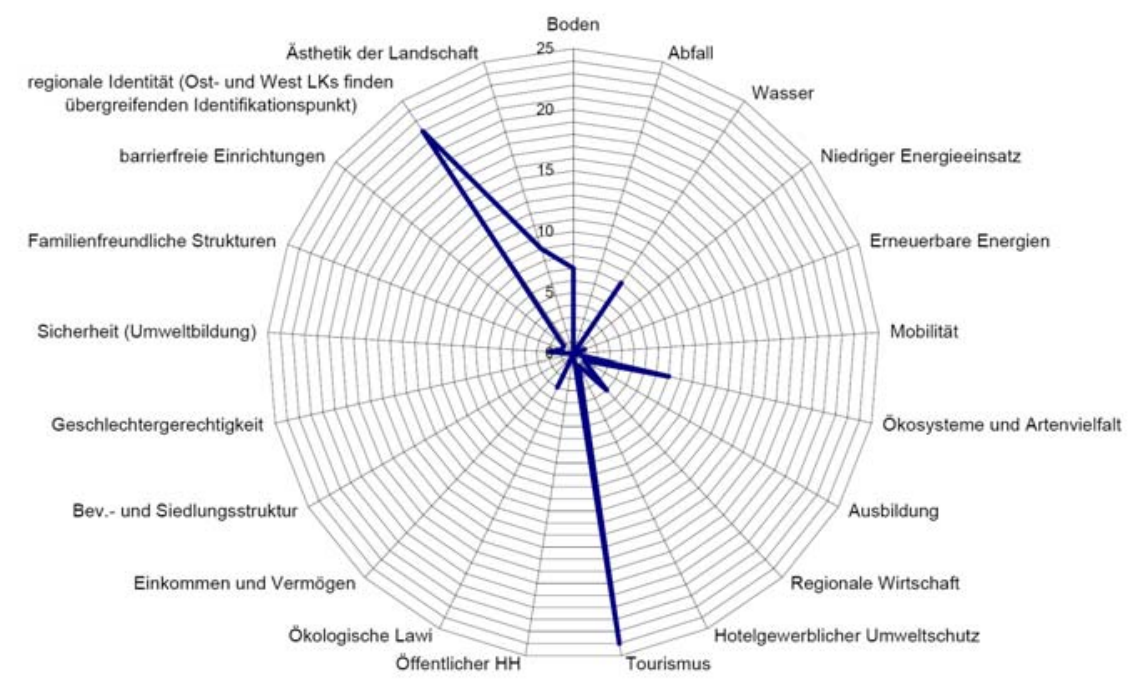

Abbildung 12: Nachbaltigkeitsspinne des E+E Projekts Elbe-Altmark-Wendland

Die Darstellungsform als Nachhaltigkeitsspinne (Kreisdiagramm) lässt sofort erkennen, in welchem Themenfeld der regionalen Nachhaltigkeit eine Maßnahmen- 
konzentration vorliegt. Je nach zu Grunde liegendem regionalem Nachhaltigkeitsverständnis wird deutlich, in welchen Themenfeldern das E+E-Vorhaben (bei entsprechender Ausgestaltung der Einzelmaßnahmen) einen Beitrag oder Mehrwert zur regionalen Nachhaltigkeit leisten kann. Die Maßnahmenkonzentration in den Themenfeldern „Ökosysteme und Artenvielfalt“, „Tourismus“, „regionale

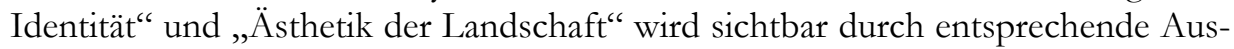
schläge im Kreisdiagramm. Die hier gewählte Dimension ist die der Maßnahmenanzahl.

Diese Form der deskriptiven Auswertung des E+E-Vorhabens auf regionale Nachhaltigkeit beschreibt eine statische Perspektive. Damit Nachhaltigkeitswirkungen festgestellt werden können, müssen im Vorfeld entsprechende Prozesse initiiert und durchgeführt werden. Dazu bedarf es bestimmter Verhaltensweisen, die eine Entwicklung und Ausgestaltung von regionalen Nachhaltigkeitsthemen ermöglichen. Die statische Perspektive ist deshalb um eine dynamische Perspektive, welche die prozessualen Eigenheiten in den Blick nimmt, zu ergänzen.

Gemäß den zehn Managementregeln der bundesnationalen Nachhaltigkeitsstrategie lassen sich auch für die regionale Ebene Verhaltensweisen identifizieren, die eine entsprechende Entwicklung in den Themenfeldern der regionalen Nachhaltigkeit begünstigen können.

Um die statische Perspektive (hier Wirkungspotenzial des E+E-Vorhabens in der Untersuchungsregion Elbe-Altmark-Wendland (siehe Basismatrix bzw. Nachhaltigkeitsspinne) mit der dynamischen Perspektive zu kombinieren, wurden Interviews, die mit regionalen Akteuren geführt wurden, unter diesem Aspekt ausgewertet. Die Tabelle gibt eine Übersicht über die regionalen Verhaltenweisen, die aus den Interviews herausgearbeitet wurden.

Tabelle 15: Synopse regionaler und nationaler Verbaltensweisen

\begin{tabular}{|c|c|}
\hline $\begin{array}{c}\text { Identifizierte regionale Managementregeln (Projektebene) } \\
\text { (aus Interviews mit regionalen Akteuren) }\end{array}$ & $\begin{array}{c}\text { Korrespondierende } \\
\text { Managementregeln } \\
\text { der nationalen } \\
\text { Nachhaltigkeitsstra- } \\
\text { tegie }^{118}\end{array}$ \\
\hline $\begin{array}{c}\text { 1. Die Region übernimmt eigenverantwortlich Vorsorge für } \\
\text { - ,saubere Energie“; } \\
\text { - Daseinsvorsorge in der Region; } \\
\text { - dauerhafte Maßnahmenfortführung. }\end{array}$ & $\mathrm{R} 1$ \\
\hline $\begin{array}{l}\text { 2. Erneuerbare Naturgüter (wie z.B. Ackerboden) dürfen auf Dauer nur } \\
\text { im Rahmen ihrer Fähigkeit zur Regeneration genutzt werden. Nicht } \\
\text { erneuerbare Naturgüter (wie z.B. mineralische Rohstoffe oder fossile }\end{array}$ & $\mathrm{R} 2$ \\
\hline
\end{tabular}

117 Die Nummerierung gibt die Stellung im Rahmen der 10 Regeln der nationalen Nachhaltigkeitsstrategie wieder.

118 Vgl. Die Bundesregierung (2008). 


\begin{tabular}{|c|c|}
\hline $\begin{array}{l}\text { Energieträger) dürfen auf Dauer nur in dem Umfang genutzt werden, } \\
\text { wie ihre Funktionen durch andere Materialien oder durch andere Ener- } \\
\text { gieträger ersetzt werden können }\end{array}$ & \\
\hline $\begin{array}{l}\text { 3. Die Region muss ihre gewachsenen Strukturen sowie Potenziale } \\
\text { kennen und ihr Handeln daran ausrichten. Das Handeln soll ferner dem } \\
\text { Grundsatz der Verhältnismäßigkeit genügen } \\
\text { Physisches Kapital (z.B. vorhandene Infrastruktur nutzen,...); } \\
\text { Immaterielles Kapital (z.B. regionale Kreativität und Ideenreichtum } \\
\text { nutzen,...). }\end{array}$ & $\mathrm{R} 3$ \\
\hline $\begin{array}{l}\text { 4. Der Strukturwandel in der Region soll wirtschaftlich erfolgreich } \\
\text { sowie ökologisch und sozial verträglich gestaltet werden. Zu diesem } \\
\text { Zweck sind die Themenfelder der regionalen Nachhaltigkeit so zu } \\
\text { integrieren, dass regionale wirtschaftliche Entwicklung, sozialer Zu- } \\
\text { sammenhalt und Umweltschutz Hand in Hand gehen. Dabei sind ins- } \\
\text { besondere die Themen } \\
\quad \text { - Naturschutz; } \\
\quad \text { - Regionale Entwicklung durch Tourismus; } \\
\quad \text { - Geschichte und Kultur; } \\
\text { integrativ zu betrachten. }\end{array}$ & R5 \\
\hline $\begin{array}{l}\text { 5. Die Bewahrung und Schaffung von historischem Wissen durch } \\
\text { Forschung und Dokumentation sowie die Weitergabe dieses Wissens } \\
\text { durch spezifische Bildungsmaßnahmen spielt eine entscheidende Rolle } \\
\text { für die regionale Identität. }\end{array}$ & R6 \\
\hline $\begin{array}{l}\text { 6. Um den sozialen Zusammenhalt zu stärken, sollen } \\
\text { - Armut und sozialer Ausgrenzung soweit wie möglich vorge- } \\
\text { beugt werden; } \\
\text { - Allen Bevölkerungsschichten Zugang zu angebotenen Bil- } \\
\text { dungsmaßnahmen (Kunst, Kultur, Geschichte) ermöglicht } \\
\text { werden; } \\
\text { - Alle am gesellschaftlichen und politischen Leben teilhaben. } \\
\text { Eine Möglichkeit, der Teilhabe kann darin bestehen, sich regional zu } \\
\text { engagieren (z.B. als Leistungserbringer im Rahmen des E+E-Vorhaben) }\end{array}$ & R9 \\
\hline $\begin{array}{l}\text { 7. Grünes Band - Ein Europäischer Gedanke. Der Gedanke Austausch } \\
\text { und Grenzüberwindung soll nicht nur intranational sondern auch inter- } \\
\text { national gedacht werden. }\end{array}$ & R10 \\
\hline
\end{tabular}

Tabelle 5 zeigt, dass in der Region Verhaltensweisen bereits etabliert sind, die eine günstige Entwicklung in Themenfeldern der regionalen Nachhaltigkeit unterstützen können. Betrachtet man die einzelnen, identifizierten Verhaltensweisen im Detail, so sind sie in unterschiedlichem Maße integrativ angelegt. Die regionale Managementregel Nr. 4 fokussiert simultan wirtschaftliche, ökologische und soziale Aspekte. Die Regel Nr. 5 hingegen adressiert einzeln das Themenfeld der regionalen Identität. Von Interesse ist in diesem Kontext, ob jenen Themenfeldern der regionalen Nachhaltigkeit, die durch die Maßnahmen des E+E-Vorhabens Erlebnis Grünes Band in der Untersuchungsregion Elbe-Altmark-Wendland angespro- 
chen werden, auch regionale Verhaltensweisen zugeordnet werden können. Hierüber gelingt die Verbindung von statischer und dynamischer Perspektive. Diese Verbindung gelingt für die vier Themenfelder mit der höchsten Konzentration von Einzelmaßnahmen: dem Themenfeld „Ökosysteme und Artenvielfalt“ können die regionalen Regeln 1, 2, 4, dem Themenfeld „Tourismus“ können die Regeln 1, 3, 4, 5, 6, 7, dem Themenfeld ,,regionale Identität“" können die Regeln 3, 4, 5, 6 und dem Themenfeld „Ästhetik der Landschaft“ die Regeln 3 und 4 zugeordnet werden. Somit bestehen zur positiven Gestaltung der Themenfelder, in denen das E+E-Vorhaben Wirkungspotenzial aufweist, theoretisch gute Ausgangsbedingungen.

\subsubsection{Nachhaltigkeitsinstitutionen}

Die in Tabelle 15 in Zeile 2 identifizierte regionale Managementregel benennt als einen Aspekt der eigenverantwortlichen Vorsorge die „dauerhafte Maßnahmenfortführung“. Um dem gerecht werden zu können, ist möglichst zu Beginn eines Förderprojektes nach Institutionen Ausschau zu halten, welche die im Rahmen des Förderzeitraumes initiierten Prozesse anschließend fortführen können.

Um diese Möglichkeiten zu prüfen, wurden in Landkreisen, die Teil der Modellregion Elbe-Altmark-Wendland des E+E-Vorhabens Erlebnis Grünes Band sind, Interviews mit Bürgermeistern geführt. Hauptziel der Erhebung ist die Identifizierung der unterschiedlichen Arten von regionalen Institutionen, die einen Bezug zu Themenfeldern der regionalen Nachhaltigkeit aufweisen. Grundlage für die Interviews ist ein standardisierter Fragenkatalog (siehe Anhang). In dieser Untersuchungsregion wurden in den Landkreisen Lüneburg, Altmarkkreis Salzwedel, Stendal und Prignitz Interviews geführt und folgende Arten von Nachhaltigkeitsinstitutionen identifiziert:

- Integrierte Stadtentwicklungskonzepte (ISEK): Auch wenn „Nachhaltigkeit" in diesem Zusammenhang nicht immer explizit erwähnt wird, so sind Charakteristika der ISEK eine vorausschauende Perspektive einzunehmen und damit die Aufgabe der Vorsorge zu adressieren. Dazu werden in unterschiedlichem Ausmaß und landkreisspezifisch verschiedene Themenfelder simultan und integrativ betrachtet. Die Verantwortlichkeiten sind in der Stadtverwaltung unterschiedlich verteilt.

- Verschiedene regionale Einzelinitiativen, für die sich keine allgemeinen Aussagen ableiten lassen (dies sind z.B. unterschiedliche Vereine ${ }^{119}$, engagierte Bürgermeister). Die Regionalinitiativen beziehen sich meistens nicht auf einen konkreten Ort sondern ein Gebiet, welches durch ganz unter-

119 Vgl. More-Hollerweger, E. (2008). 
schiedliche Kriterien abgegrenzt wird (naturräumlich, ökonomisch, kulturell,...) und beziehen Gemeinden von ganz unterschiedlicher Größe ein. ${ }^{120}$

- Regionaler Wachstumskern Perleberg-Wittenberge-Karstädt ${ }^{121}$ : Regionale Wachstumskerne (RWK) sind Elemente der Förderpolitik des Bundeslandes Brandenburg. In den strategischen Zielen des obigen RWK heißt es u.a., „1. Nachhaltige Stärkung der wirtschaftlichen Potenziale: 2. Zielgruppenorientierte Erweiterung der touristischen Angebotspalette zur Akquisition von Besuchern. 3. Schaffung einer ausgewogenen Bildungslandschaft..." 122

Zunächst ist festzustellen, dass regionale Institutionen existieren, denen eine inhaltliche Nähe zu den Themenfeldern der regionalen Nachhaltigkeit bescheinigt werden kann. Die zuvor genannten Institutionen weisen in unterschiedlicher Weise und Intensität eine Verbindung zu einzelnen oder mehreren Themenfeldern der regionalen Nachhaltigkeit auf. Wird also über die Fortführung von im Förderzeitraum initiierten Prozessen und Maßnahmen nachgedacht, so bieten sich hier verschiedene Anknüpfungspunkte um die regionale Managementregel der dauerhaften Fortführung auszugestalten und anzuwenden. Exemplarisch kann dies an der Institution „Regionaler Wachstumskern“ verdeutlicht werden. Die Maßnahmen des Projektbaustein 2 „Entwicklung von Grenzerfahrungspunkten mit Einbindung in touristische Routen" haben inhaltlich eine Nähe zum strategischen Ziel des RWK „Zielgruppenorientierte Erweiterung der touristischen Angebotspalette“. Ferner schließt das strategische Ziel „Schaffung einer ausgewogenen Bildungslandschaft" nicht die Möglichkeit aus, inhaltliche Bezüge zum Projektbaustein 3 ,Entwicklung und Umsetzung von Bildungsangeboten und Veranstaltungen“" herzustellen. Durch das Vorhandensein von entsprechenden regionalen Institutionen, die inhaltlich eine Nähe zu den Themenfeldern der regionalen Nachhaltigkeit haben, in denen die Maßnahmen des E+E-Vorhabens Wirkungspotenzial entfalten können, bestehen institutionell gute Ausgangsbedingungen um im Förderzeitraum entwickelte Maßnahmen und initiierte Prozesse langfristig fortführen zu können. ${ }^{123}$

Abschließend kann festgehalten werden, dass das Erprobungs- und Entwicklungsvorhaben „Erlebnis Grünes Band in der Modellregion Elbe-AltmarkWendland" über seine Maßnahmen Wirkungspotenzial in verschiedenen Themenfeldern entfalten kann, die im Rahmen einer nachhaltigen Regionalentwicklung wichtig sind. Dies sind insbesondere die Themenfelder „Ökosysteme und Arten-

120 Vgl. Segert, A. (2007), S.423ff. sowie allgemein zum Thema Regionalmanagement Mehl, D. et al. (2008).

121 Siehe dazu RWK Perleberg-Wittenberge-Karstädt (2006) sowie den Internetauftritt http://www.wachstumskern-prignitz.de (zuletzt aufgerufen am 24.03.2010).

122 Vgl. RWK Perleberg-Wittenberge-Karstädt (2006), S. 10.

123 Zur allgemeinen Diskussion der Rolle von Institutionen im Kontext einer nachhaltigen Entwicklung siehe Rennings, K. et al. (1999), S. $203 \mathrm{ff}$. 
vielfalt", „Tourismus“, „regionale Identität“ und „Ästhetik der Landschaft". Die Existenz von regionalen Managementregeln und regionalen Institutionen, die inhaltlich einen Bezug zu Nachhaltigkeitsthemen haben, bilden flankierend die Voraussetzungen, um die im Förderzeitraum umgesetzten Maßnahmen und angestoßenen Prozesse im Sinne der nachhaltigen regionalen Entwicklung erfolgreich umsetzen und nach Förderablauf fortsetzen zu können.

Da zum Zeitpunkt dieses Projektes viele Maßnahmen noch in der Umsetzungsphase sind, lässt sich die Forschungshypothese, dass die Maßnahmen des E+E-Vorhabens auf regionale Nachhaltigkeit wirken, aus instituionelltheoretischer Perspektive bestätigen.

\subsubsection{Beispielhafte Synthese zwischen den Perspektiven}

Damit Nachhaltigkeitswirkungen einer Maßnahme festgestellt werden können (statische Perspektive), müssen im Vorfeld entsprechende Prozesse initiiert und durchgeführt werden (dynamische Perspektive). Dazu bedarf es bestimmter Verhaltensweisen, die eine Entwicklung und Ausgestaltung der entsprechenden Maßnahme ermöglichen. Die statische Perspektive muss nach Möglichkeit um eine dynamische Perspektive, welche die prozessualen Eigenheiten in den Blick nimmt, ergänzt werden. Gemäß den zehn Managementregeln der nationalen Nachhaltigkeitsstrategie lassen sich auch für die regionale Ebene Verhaltensweisen identifizieren, die eine entsprechende Entwicklung in den Themenfeldern der regionalen Nachhaltigkeit begünstigen können (siehe Abbildung 6).

An der Maßnahme „Radrundkurs“ lässt sich die Dependenz der Netzwerkund Maßnahmenperspektive zeigen. Die Zusammenführung von Schutz- und Nutzaspekten ist beim E+E-Vorhaben Erlebnis Grünes Band handlungsleitend. Durch die Maßnahme wird ein länderübergreifendes Routenkonzept entwickelt.

Aus statischer Perspektive werden hier die Themenfelder Tourismus und regionale Identität angesprochen. Gemäß einer komparativ-statischen Analyse kann das Wirkungspotenzial gemäß einem Vergleich vor und nach Durchführung der Maßnahme festgestellt werden. Die Maßnahme Radrundkurs wäre demnach erfolgreich umgesetzt, wenn die Nutzaspekte (Auswirkungen der touristischen Nutzung des Radweges) und die Schutzaspekte (Schonung von bestimmten Bereichen des Grünen Bandes aus naturschutzfachlicher Sicht) in einem ausbalancierten Verhältnis zueinander stehen.

Die Entwicklung und Umsetzung der Maßnahmen wird maßgeblich durch das Netzwerk bestimmt. Dazu sind ,passende“ Verhaltensweisen im Netzwerk erforderlich. Für die hier betrachtete Modellregion wurden aus Interviews mit regionalen Akteuren Managementregeln im regionalen Kontext identifiziert, die allgemein bei der Maßnahmenumsetzung Bedeutung besitzen bzw. zur Anwendung kommen. Für das Fallbeispiel Radrundkurs waren die regionalen Regeln 3 und 4 maßgeblich. Regel 3 sagt aus, dass ,die Region ihre gewachsenen Strukturen sowie 
Potenziale kennt und ihr Handeln daran ausrichten [muss]. Das Handeln soll ferner dem Grundsatz der Verhältnismäßigkeit genügen (Physisches Kapital: z.B. vorhandene Infrastruktur nutzen, ... bzw. Immaterielles Kapital: z.B. regionale Kreativität und Ideenreichtum nutzen,...).“ ${ }^{124}$ Regel 4 ergänzt, dass „der Strukturwandel in der Region [...] wirtschaftlich erfolgreich sowie ökologisch und sozial verträglich gestaltet werden [soll]. Zu diesem Zweck sind die Themenfelder der regionalen Nachhaltigkeit so zu integrieren, dass regionale wirtschaftliche Entwicklung, sozialer Zusammenhalt und Umweltschutz Hand in Hand gehen. Dabei sind insbesondere die Themen Naturschutz, Regionale Entwicklung durch Tourismus sowie Geschichte und Kultur integrativ zu betrachten." 125

Durch die Anwendung dieser Verhaltensweisen im regionalen Netzwerk kann der notwendige Beitrag geleistet werden, um die Maßnahme Radrundkurs in ausbalancierter Weise (Zusammenführung von Schutz- und Nutzaspekten) zu realisieren. Tatsächlich zeigten die regionalen Workshops, dass auf jeden Fall implizit, bei Konflikten häufig aber auch explizit die Verhaltensregeln Anwendung in der Diskussion zwischen den Akteuren finden. Entsprechend wurde bei der Konzeption der Route des Radrundkurses im Netzwerk vorgegangen. Der Projektträger fungierte als Moderator zwischen den unterschiedlichen Schutz- und Nutzungsinteressen (Netzwerkmerkmal „Prozessmanagement“). Die klare Zielsetzung der Maßnahme ermöglichte den Akteuren eine Orientierung und erleichterte so die Verständigung zwischen den Akteuren (Netzwerkmerkmal „Leitbild“) und damit auch die Anwendung der identifizierten regionalen Verhaltensweisen. Beide Ausprägungen können als integrativ eingeordnet werden. Die Akteursorientierung zeigt sich vor allem im Netzwerkmerkmal „breite Beteiligung“, das sich vor allem dadurch auszeichnet, dass „unproblematisch grenzübergreifend“ (Aussage auf dem regionalen Auftaktworkshop) gearbeitet wurde. Zudem wurde bei der Konzeption der Route flexibel auf die unterschiedlichen Belange reagiert. So wurde beispielsweise der optimale Weg zwischen den verschiedenen zu verbindenden Grenzerfahrungspunkten gesucht und zudem darauf geachtet, naturschutzfachlich zu schützende Biotope keiner zusätzlichen Belastung durch den Rundkurs auszusetzen. Auch hieran zeigt sich die integrative Qualität des Netzwerks. Die Dependenz von dynamischer und statischer Perspektive wird hier offenkundig. 


\subsection{E+E Region Thüringer Wald \& Schiefergebir- ge/Frankenwald}

\subsubsection{Situation und Kontext}

Das Projektgebiet liegt in den Landkreisen Saale-Orla-Kreis (Thüringen), Hof (Bayern), Saalfeld-Rudolstadt (Thüringen), Sonneberg (Thüringen) und Kronach (Bayern) und reicht von Mödlareuth, dem ,geteilten Dorf“, bis nach Stockheim im Landkreis Kronach. Die Einwohnerdichten in den Landkreisen liegen zwischen 78 Einwohner $/ \mathrm{km}^{2}$ im Saale-Orla-Kreis und 141 Einwohner $/ \mathrm{km}^{2} \mathrm{im}$ Landkreis Sonneberg. Das Projektgebiet liegt zudem im Gebiet dreier Naturparke, des Naturparks Frankenwald auf bayerischer Seite und der Naturparke Thüringer Wald und Thüringer Schiefergebirge/Obere Saale auf thüringischer Seite.

Die Modellregion ist eine typische Mittelgebirgsregion mit einem hohen Anteil an Waldgebieten, Heideflächen, Feuchtgrünland sowie Sukzessionsflächen. Gerade die Sukzession auf vielen Flächen bedroht die Wahrnehmbarkeit des Grünen Bandes, weswegen nach Einschätzung des BN Bayern Landschaftspflegemaßnahmen zur Offenhaltung beispielsweise der Heideflächen im Grünen Band notwendig werden. ${ }^{126}$

Projektträger des Projekts ist der Regionalverbund Thüringer Wald e.V. der neben der Verbesserung der touristischen Situation auch die Erhaltung und Entwicklung von Natur und Landschaft in seiner Satzung verankert hat. In das Projekt als Projektpartner eingebunden sind die Naturparkverwaltungen, Landschaftspflegeverbände, Naturschutzverbände und Tourismusverbände, die mit dem Projektträger Kooperationen geschlossen haben. In einer Laufzeit von drei Jahren (März 2007 bis März 2010) wurden über einen inhaltlichen dreistufigen Aufbau Maßnahmen zum Naturschutz und zur Tourismusentwicklung umgesetzt. Hauptziele dabei sind der „Schutz und [die] nachhaltige Entwicklung des Grünen Bandes als naturräumlich in Deutschland einzigartigen Biotopverbund [zusammen] mit möglichst effizienten Methoden zur dauerhaften Absicherung der Pflegeergebnisse“ ${ }^{\text {127 }}$, sowie die „Entwicklung eines Tourismuskonzeptes (...)“128 das in engem Zusammenhang zum Grünen Band erarbeitet wird und auf die Naturschutzbelange Rücksicht nimmt. Außerdem soll durch das Projekt eine „Öffentliche Bewusstseinsstärkung bezüglich des historischen Werts der ehemaligen innerdeutschen Grenze und der damit zusammenhängenden historischen Ereignisse "129 erreicht werden.

\footnotetext{
126 BN Bund Naturschutz in Bayern e.V./BUND Bund für Umwelt und Naturschutz (2006), S. 63

127 Regionalverbund Thüringer Wald e.V. (2006), S. 8.

128 Vgl. ebenda.

129 Vgl. ebenda.
} 


\subsubsection{Netzwerkperspektive}

\subsubsection{Strukturelle Analyse des Projektnetziperks}

\section{Datenbasis}

Insgesamt wurden über zwei Runden 172 Akteure angeschrieben. Davon sendeten 43 Akteure den Fragebogen zurück. Das entspricht einem positiven Rücklauf von $25 \%$. Zehn Akteure äußerten sich zur Befragung, ohne den Fragebogen auszufüllen, was einem negativen Rücklauf von 7\% entspricht. Über die 43 Fragebögen sowie eine bestehende Akteursliste wurden insgesamt 200 Akteure erfasst (Grundgesamtheit). Für 120 der Akteure der Grundgesamtheit konnte eine Beziehung im Netzwerk nachgewiesen werden (Analyseeinheit). Im Folgenden sind kurz die Zusammensetzungen des positiven Rücklaufs, der Grundgesamtheit und der Analyseeinheit dargelegt.

Die 43 Akteure aus dem positiven Rücklauf verteilen sich vorrangig auf die Bereiche Tourismus (34,9\%), Soziales $(25,6 \%)$ und Naturschutz (14\%). Akteure der Bereiche Landwirtschaft und Wirtschaft sind jeweils mit 7,0\% vertreten. Vertreter der Regionalentwicklung konnten mit 9,3\% nachgewiesen werden, Politiker und Kommunalvertreter sind jeweils mit 2,3\% vorhanden. Vertreter der Wirtschaftsförderung konnten nicht nachgewiesen werden.

Die Akteure verteilen sich in Bezug auf die Institutionengruppen zu 34,9\% auf die Gruppe Vereine, zu 25,6\% auf die Gruppe Behörden. 30,2\% gehören zur Gruppe Privatunternehmen und 2,3\% zur Gruppe Politik. 9,3\% sind private Akteure.

Die akteursbezogenen Daten zeigen folgendes Bild: 50\% der Akteure besitzen einen Hochschulabschluss, 36,4\% eine Ausbildung und für 13,6\% konnten keine Angaben ermittelt werden. Zudem verteilen sich 4,7\% der Akteure in die Altersgruppe 20-29, 25,6\% in die Gruppe der 30-39-Jährigen, 27,9\% in die Altersgruppe 40-49. Der Altersgruppe 50-59 konnten 23,3\% der Akteure zugeordnet werden, und der Gruppe 60-69 Jahre 11,6\%. Für 7,0\% der Akteure konnten keine Angaben ermittelt werden. Ähnlich wie in der Untersuchungsregion Elbe-AltmarkWendland spricht auch in dieser Region das Projekt eher Akteure mittleren Alters mit gehobenem Bildungsabschluss an.

Die meisten der in der Grundgesamtheit erfassten 200 Akteure sind dem Fachbereich Tourismus (33,5\%) zuzuordnen. Die Bereiche Soziales und Naturschutz bilden mit 18\% bzw. 14\% die nächstgrößeren Gruppen. Für die Landnutzung konnten $6,0 \%$ und für die Kommunalverwaltung konnten 7,0\% an Akteuren nachgewiesen werden. Politische Vertreter sind zu 10,0\% vorhanden, Vertreter der Regionalentwicklung zu 4,5\% und Vertreter der Wirtschaftsförderung mit 1,0\%, 4,0\% der Akteure wurden dem Wirtschaftsbereich zugeordnet und 2,0\% 
wurden als „interessierte Bürger“ eingruppiert, da für sie keine fachliche Zugehörigkeit ermittelt werden konnte.

Den Hauptanteil mit 33,5\% nehmen in den Institutionengruppen die Privatunternehmen (Hotelbesitzer, etc.) ein. Immerhin 27,5\% der Akteure sind Vereinsmitglieder und 23,5\% Behördenvertreter. 9,5\% werden der Gruppe politische Vertreter zugeordnet und 6,0\% können als private Akteure keiner anderen Institutionengruppe zugeordnet werden.

Die „Ost-West-Verteilung“ ist nahezu ausgeglichen. 50\% der Akteure stammen aus den neuen Bundesländern, 48\% der Akteure sind den alten Bundesländern zuzuordnen. 0,5\% der Akteure wurden als Akteure der Bundesebene eingeordnet und 1,5\% der Akteure konnten nicht eindeutig zugeordnet werden.

In der Analyseeinheit befinden sich 120 Akteure, für die eindeutig Beziehungen nachgewiesen werden konnten. Davon sind 26\% Tourismusakteure, 19\% Naturschutzvertreter und 18\% sind dem Bereich Soziales zuzuordnen. Jeweils 8\% der Akteure sind Vertreter der Bereiche Politik bzw. Landnutzung. Dem Bereich Wirtschaft wurden 7\% der Akteure zugeordnet und 6\% der Akteure sind Kommunalvertreter. Akteure aus dem Bereich Regionalentwicklung sind mit 4\% vertreten und mit 1\% Akteure aus dem Bereich Wirtschaftsförderung, dazu kommen noch 3\% interessierte Bürger, die keinem andere Fachbereich zugeordnet werden konnten (siehe Abbildung 13). 

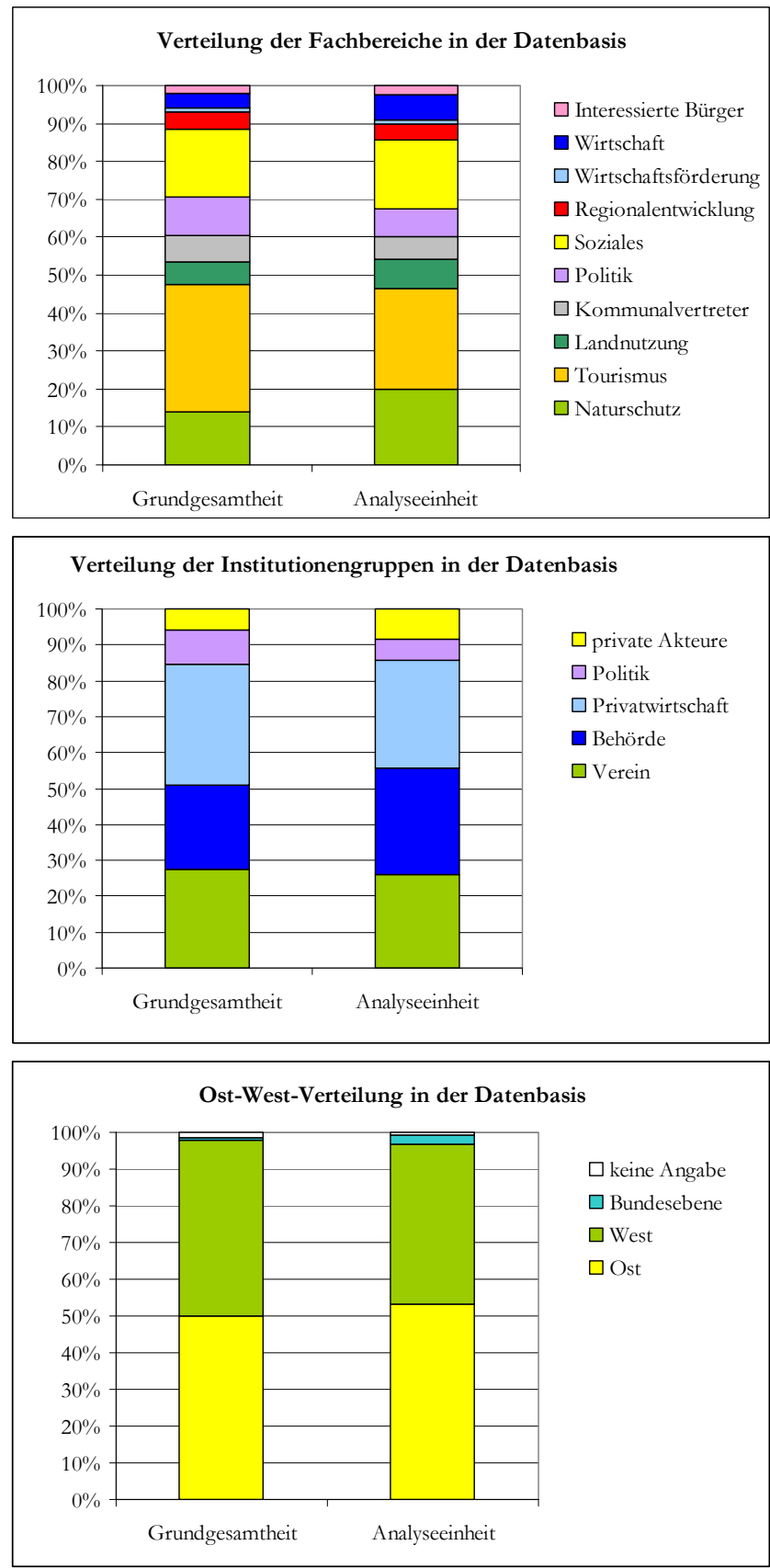

Abbildung 13: Verteilung der Fachbereiche, Institutionengruppen und Ost-West-Verteilung der Akteure der Grundgesamtheit und der Analyseeinheit im Netzwerk Thüringer W ald \& Schiefergebirge/ Frankenwald. 
Die Akteure verteilen sich in den Institutionengruppen mit 25,8\% auf die Gruppe „Vereine/Verbände“, mit 30,0\% auf die Gruppe Behörden und mit 30\% auf die Gruppe Private Unternehmen. 5,8\% der Akteure sind politische Akteure und 8,3\% der Akteure sind private Akteure.

Gegenüber der Grundgesamtheit verschiebt sich in der Analyseeinheit die „Ost-West-Verteilung“ leicht. So sind hier 53,3\% der Akteure aus den neuen Bundesländern und 43,3\% aus den alten Bundesländern. 0,8\% der Akteure sind auf der Bundesebene anzusiedeln und 2,5\% der Akteure konnten nicht eindeutig zugeordnet werden.

Wie auch in der Untersuchungsregion Elbe-Altmark-Wendland lässt sich der Schwerpunkt der Fachbereiche Naturschutz und Tourismus in der Verteilung der Akteure durch die Zielsetzung des Projekts erklären. So sind hier „Schutz und nachhaltige Entwicklung des Grünes Bandes als naturräumlich (...) einzigartigen Biotopverbunds (... “130. Hauptziel aus naturschutzfachlicher Sicht. Die „Entwicklung eines Tourismuskonzeptes (...)“131 ist aus touristischer Sicht zentrales Umsetzungsziel. Jedoch ist die Dominanz an Vertretern aus dem Tourismus auffällig. Da es sich um ein Projekt aus dem Bereich Naturschutzförderung handelt, wäre eigentlich eine Dominanz an Vertretern aus dem Naturschutzbereich zu erwarten, wie es sich auch in der Region Elbe-Altmark-Wendland darstellt. Der Grund hierfür ist vermutlich in der fachlichen Ausrichtung des Projektträgers zu suchen, der im Rahmen des Projekts für die regionale Entwicklung des Tourismus eintritt. Die dritte große Gruppe an Akteuren aus dem Fachbereich Soziales erklärt sich durch die große Zahl an im Projekt beteiligten Museen, die aufgrund des Umsetzungsziels „Öffentliche Bewusstseinsstärkung bezüglich des historischen

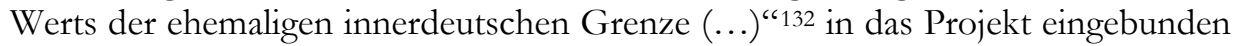
sind.

Die im Vergleich zur Untersuchungsregion Elbe-Altmark-Wendland hohe Zahl an privaten Unternehmern erklärt sich vor allem aus der Vielzahl an Hotelbetrieben und touristischen Anbietern, die sich in das Projekt einbringen. Dies spricht wiederum für einen intensiven Aufbau eines Unternehmensnetzwerkes in der Region. Zudem weist es darauf hin, dass hier entsprechende Voraussetzungen für die Etablierung der im Projekt entwickelten touristischen Produkte geschaffen wurden.

\section{Konstellation}

Die Vernetzung (siehe Abbildung 14) zeigt, dass vor allem bezogen auf die Sektoren das Netzwerk als offen bezeichnet werden kann. Innerhalb des Netzwerks zeigt sich ein Kern (rot umrandet) mit besonders intensiver Vernetzung. Die hier

130 Regionalverbund Thüringer Wald e.V., 2006, S. 8.

131 Ebenda.

132 Regionalverbund Thüringer Wald e.V., 2006, S. 8. 
zugehörigen Akteure sind aus unterschiedlichen Fachbereichen, lediglich die Landwirtschaft ist nicht in diesem Kern vertreten. Der abgrenzbare Kern innerhalb des Netzwerks ist besetzt von Vertretern aus unterschiedlichsten Bereichen, Tourismus, Naturschutz, Soziales, Regionalentwicklung, Politik und Wirtschaft und unterstreicht damit die sektorenbezogene Offenheit des Netzwerkes.

Die Dichte im Kern weist auf einen besonders hohen Austausch zwischen den Akteuren hin. Da es sich um ein Projektnetzwerk handelt, ist die Ausprägung eines verdichteten Kerns zu erwarten, ebenso das Vorhandensein von fallbezogen eingebundenen Akteuren. Ein derartiger Kern zeigt i.d.R. das höchste Interesse an der Umsetzung eines Projekts und ist von wesentlichen Macht-, Fach und Prozesspromotoren besetzt. Diese Promotoren in dem auch als „Inner Circle“ bezeichneten Kreis nehmen üblicherweise verschiedene Führungsaufgaben mit eingeschränkter Kompetenz wahr und dienen als Vorentscheiderzirkel ${ }^{133}$. Dadurch wird die Effektivität der Zusammenarbeit im Netzwerk deutlich erhöht. ${ }^{134}$ Ein Abgleich zwischen den im Projektantrag genannten Projektpartnern und den im Kern vorhandenen Akteuren ergab, dass diese zu 77\% übereinstimmen. Der Abgleich mit den qualitativen Interviews ergab zudem, dass diese Akteure ebenfalls als Ansprechpartner bzw. zentrale Promotoren im Netzwerk gelten. Daher kann davon ausgegangen werden, dass die Akteure im Kern auch in eingeschränktem Maße Führungsaufgaben übernehmen (siehe hierzu Netzwerkmerkmal „Multiplikatoren").

133 Vgl. Diller (2002), S. 103.

134 Vgl. ebenda. 


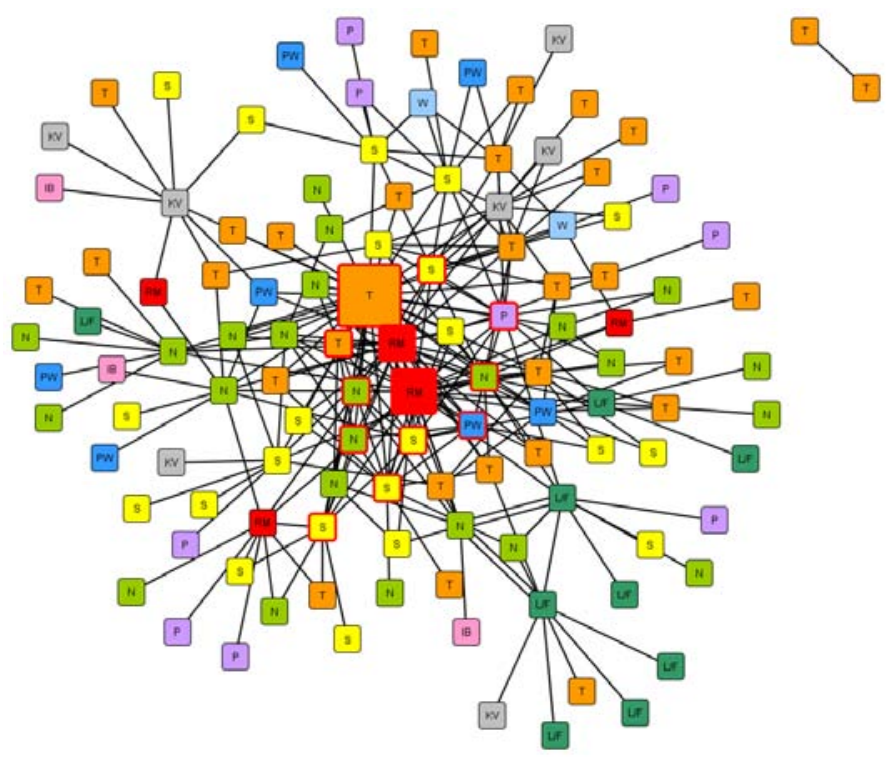

Abbildung 14: Graph des Netzwerks Thüringer Wald \& Schiefergebirge / Frankewald. $N=$ Naturschutz, $T=$ Tourismus, $P=$ Politik, $S=$ Soziales $/$ Kultur, $K V=$ Kommunalverwaltung, $P W=$ Privatwirtschaft, $W=$ Wirtschaftsförderung, $R M=$ Regionalentwicklung, $I B=$ Interessierte Bürger

Die Abbildung 14 zeigt auch die drei Akteure mit der größten Gradzentralität. Dabei ist ein Vertreter aus dem Tourismus mit 0,345 der Akteur mit der größten Zentralität. Erst danach schließt sich der Projektträger mit 0,235 bzw. 0,227 an.

Ähnliches gilt für die anderen Zentralitätsmaße. So zeigt der Tourismusvertreter für die nähebasierte Zentralität ebenfalls das höchste Maß (0,54). Der Projektträger, vertreten durch zwei Akteure, schließt sich mit 0,487 bzw. 0,483 an. Damit zeigt der Tourismusvertreter das höchste $\mathrm{Maß}$ an Kommunikationsaktivität und effizienz.

Zudem hat er mit einer Intermediationszentralität von 0,401 mit Abstand die größte Möglichkeit zur Kommunikationskontrolle. Hier schließt der Projektträger erst mit 0,181 bzw. 0,107, also einem deutlichen Abstand an.

Was sich bei der Zentralität der Akteure abzeichnet, spiegelt sich auch bei der Betrachtung des Prestiges wider. Der Tourismusvertreter ist mit einem indegree von 0,295 der Akteur, der mit Abstand am häufigsten um Rat gefragt wird. Zudem ist den Akteuren der Kontakt zu diesem Akteur besonders wichtig (indegree von 0,269), der Projektträger folgt mit einem indegree von 0,202. Auch wird dieser Tourismusvertreter als der Schlüsselakteur sowohl im Projekt (indegree von 0,338 ) als auch in der Region (indegree von 0,351) benannt. Nachfolgende Akteu- 
re schließen sich erst mit einem deutlichen Abstand an. Der nächste Akteur ist der Projektträger mit einem indegree von 0,221.

Das Netzwerk hat damit neben dem Projektträger einen eindeutigen Führungsakteur, vor allem auch, da nach dem Tourismusvertreter und dem Projektträger die Zentralitätsmaße und Prestigemaße deutlich abnehmen und die Abstände zwischen den Akteuren auch deutlich geringer werden.

Das Problem bei derartigen „Single-Leader-Networks“ ist die Gefahr der Überlastung des zentralen Akteurs und damit die Fehleranfälligkeit dieser Netzwerke. ${ }^{135} \mathrm{Ob}$ es sich bei dem Netzwerk, wie angezeigt, um ein wirkliches SingleLeader-Network handelt, oder ob es sich um ein Flexible-Task-Network handelt, und die beiden Promotoren darin die wesentliche Koordinierungsrolle übernehmen, kann aus den strukturellen Daten nicht abgelesen werden und muss mit den Daten aus den qualitativen Interviews überprüft werden (siehe Netzwerkmerkmal „Multiplikatoren").

\section{Netzwerkarbeit}

Das Projektnetzwerk hat sich für das Projekt knapp zur Hälfte (49\%) neu aufgebaut. Von den neuen Kontakten sind 27\% zudem länderübergreifend. Auch im Kern des Netzwerks wurden neue Verbindungen geknüpft, die nun als Brücken zwischen bestehenden Kontakten dienen. Auch dieses Netzwerk zeigt, dass das E+E-Vorhaben „Erlebnis Grünes Band“ zur länderübergreifenden Vernetzung regionaler Akteure beigetragen hat.

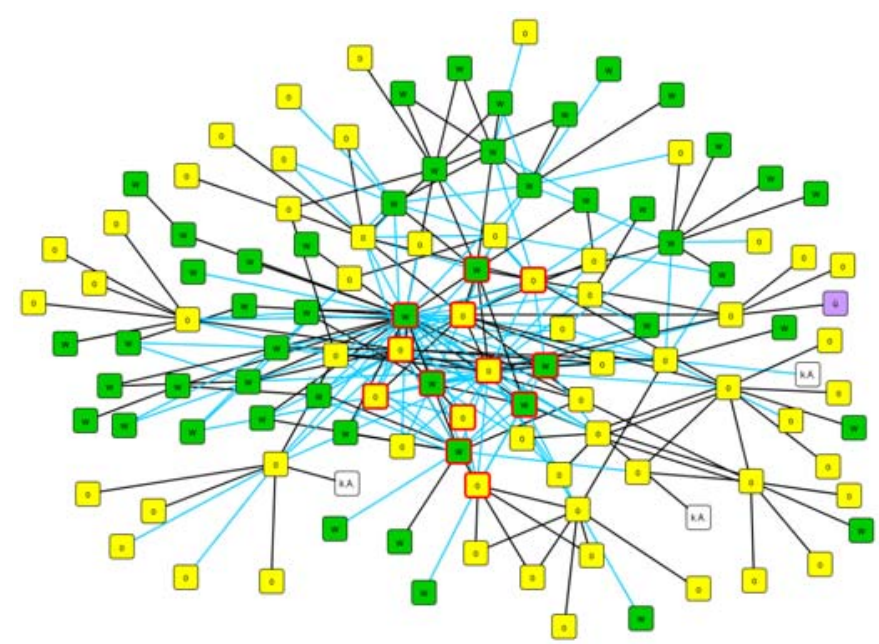

Abbildung 15: Ost-West-Vernetzung des Netzwerks Thüringer Wald \& Schiefergebirge / Frankenwald

135 Vgl. Diller (2002), S. 102 f. 
Damit zeigt sich aber auch, dass sich aus verschiedenen bestehenden ungerichteten Netzwerken ein neues, projektbedingt gerichtetes Netzwerk entwickelt hat, das nun vor der Herausforderung steht, in ein neues regionales Netzwerk überführt zu werden. Zudem wird hier die spezifische Stärke von Netzwerken deutlich, die in der ,innovative[n] Ausgestaltung von Entwicklungskorridoren“136 liegt. Die Bildung einer Vielzahl von neuen Kontakten innerhalb des Entwicklungskorridors „Erlebnis Grünes Band“ hat beispielsweise in Form von spezifischen Angebotspauschalen zu jenen innovativen Ausgestaltungen geführt, von denen Diller ${ }^{137}$ spricht.

Bei der Wahl der Kontaktarten zeigt sich eine relative Gleichverteilung. So machen Telefon und Mail 31\% der Kontakte aus, Projektveranstaltungen sind 34\% der Kontakte und Arbeitsbesprechungen 35\%. Hauptzweck der Kontaktaufnahme war der Austausch von Informationen und die Gestaltung von Kooperationen. Die Gründe dafür sind, wie bei der Region Elbe-Altmark-Wendland, in der Ausgestaltung des Förderprojekts zu suchen (siehe Unterabschnitte von 3.2.3.1).

Von den Kontakten sind 37\% häufig, ebenso viele Kontakte sind selten. Daran zeigen sich im Wesentlichen zwei Gruppen an Akteuren im Netzwerk: Der zuvor bereits beschriebene Kern hat häufig Kontakt zueinander, und die fall- oder maßnahmenbezogen eingebundenen Akteure haben eher selten Kontakt zu den Netzwerkpartnern.

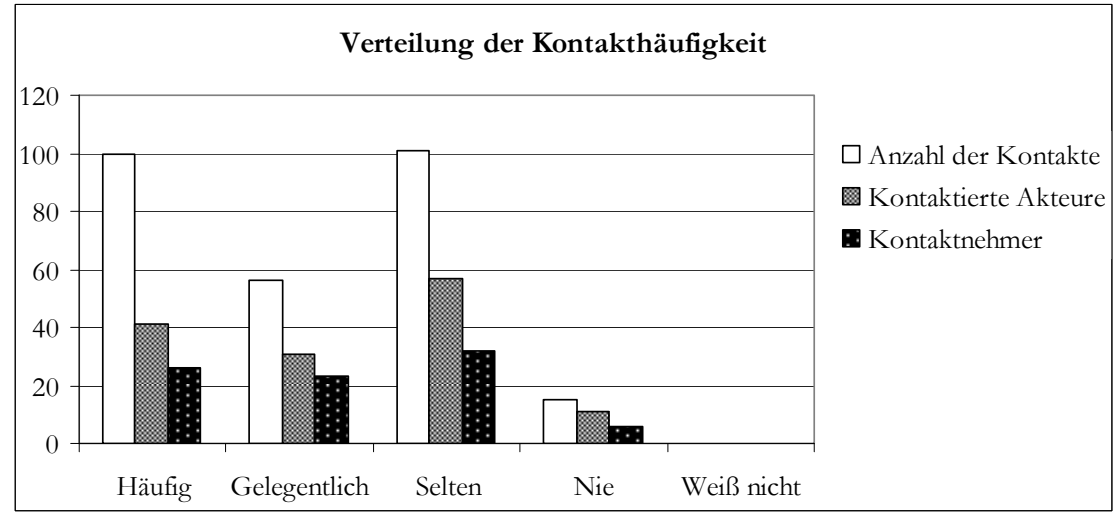

Abbildung 16: Verteilung der Kontakthäufigkeiten im Netzwerk Thüringer W ald \& Schiefergebirge / Frankenwald

136 Diller (2002), S. 202.

137 Vgl. ebenda. 


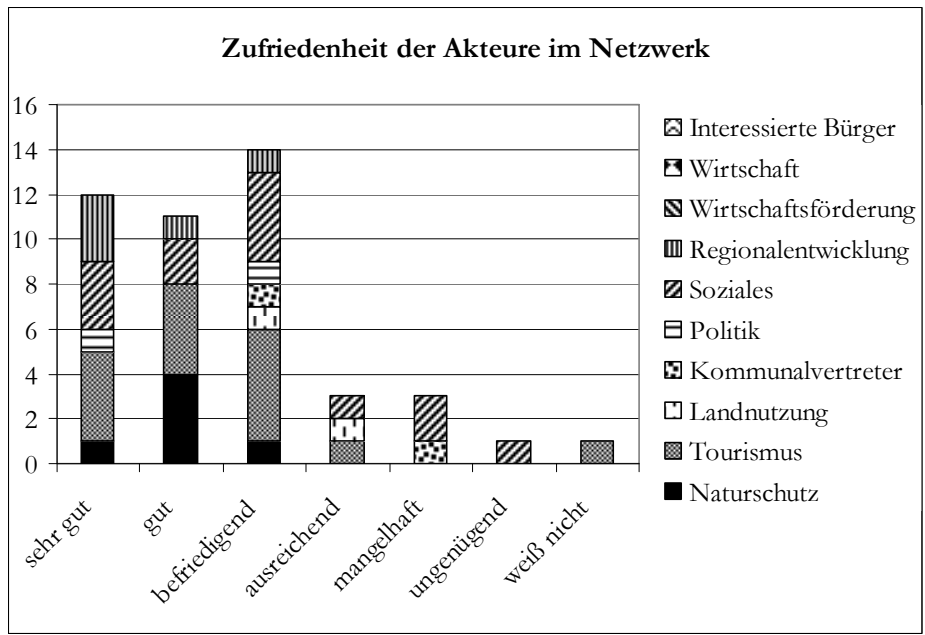

Abbildung 17: Zufriedenheit der Akteure im Netawerk. Thüringer W ald \& Schiefergebirge/Frankenwald

Aus dem positiven Rücklauf fühlen sich 53,5\% der Akteure sehr gut bzw. gut in das Netzwerk eingebunden, 32,5\% sind mit ihrer Einbindung zufrieden, 7\% der Akteure bewerten ihre Einbindung als ausreichend und ebenso viele als mangelhaft. Ein Akteur bewertet seine Einbindung sogar als ungenügend. Ähnlich wie im Netzwerk Elbe-Altmark-Wendland kann dies auch hier am jeweiligen Vernetzungsgrad der Akteure liegen. Besonders bei fallbezogen eingebundenen Akteuren ist anzunehmen, dass sie sich als nicht ausreichend im Netzwerk integriert betrachten. Genauso kann es an inhaltlichen Konflikten innerhalb des Netzwerks, die aufgrund des Interessenausgleichs zwischen unterschiedlichen Fachbereichen entstehen, liegen. Mögliche Quelle der Unzufriedenheit können aber auch die festgestellten organisatorischen Probleme sein.

\subsubsection{Analyse der Netawerkmerkmale}

Zur Analyse der Netzwerkmerkmale und ihrer strategischen Qualitäten werden, wie auch in den anderen Untersuchungsregionen die Erkenntnisse der strukturellen Netzwerkanalyse ebenso herangezogen, wie die Ergebnisse der durchgeführten regionalen Workshops und der Interviews. Mit Hilfe eines StärkenSchwächenprofils werden die Netzwerkmerkmale und die Ausprägungen ihrer strategischen Qualitäten charakterisiert. Jedoch konnte nicht zwingend für jedes Kriterium ein Hinweis auf seine Ausprägung nachgewiesen werden, in diesen Fällen bleibt das Profil leer. 


\section{Konstellation: Breite Beteiligung}

Die aktiven Akteure kommen aus unterschiedlichen Bereichen, z.B. touristische Leistungsträger, Vertreter von Museen, politische Akteure und Naturschützer, die auch über das Grüne Band hinaus daran interessiert sind, ihre Region weiterzuentwickeln. Die Einbindung der Akteure fand über ein Regionales Auftaktforum zur Motivation der Akteure sowie über die Organisationsstrukturen (vgl. Netzwerkmerkmal „Arbeiten auf verschiedenen Ebenen“) statt. Nach Aussagen der Akteure treten innerhalb des Netzwerks keine großen Lücken auf, es sind ,alle relevanten Akteure dabei" (Aussage eines Akteurs). Auch verschiedene regionale Unternehmen sind in das Projekt eingebunden, vor allem bei der Entwicklung regionaler touristischer Angebote. Da die Region als, ,ganz einfach wirtschaftsschwach“ (Aussage eines Akteurs) eingeordnet wird, sind keine anderen Wirtschaftsbereiche vorhanden, die eingebunden werden könnten (Aussage eines Akteurs). Diese Ausprägung des Netzwerkmerkmals, das Vorhandensein aller relevanten regionalen Akteure, kann als akteursorientiert eingeordnet werden.

Das anfängliche Interesse und die Motivation vieler Akteure verringerten sich jedoch im Projektverlauf, in den meisten Fällen war vermutlich der Aufwand zu groß für den zu erwartenden Nutzen für die Akteure. In der Folge verringerte sich ebenfalls die Zahl der beteiligten Akteure. Zudem wurde die Intensität der Beteiligung von Dienstleistern am Netzwerk von interviewten Akteuren als zu gering eingestuft. Die abnehmende Motivation und die ausbaufähige Intensität der Beteiligung stehen der Akteursorientierung im Netzwerk entgegen, da für eine Akteursorientierung eine passive Beteiligung der relevanten Akteure nicht ausreichend ist.

Tabelle 16: Konstellation: Breite Beteiligung in der Region Thüringer Wald \& Schiefergebirge/Frankenwald ( $D=$ diskursiv, $A=$ akteursorientiert, $I=$ integrativ)

\begin{tabular}{|c|c|c|c|c|}
\hline Qualitätskriterien & Stärke & D & $\mathbf{A}$ & $\mathbf{I}$ \\
\hline $\begin{array}{l}\text { Projektträger und in Projektstruktu- } \\
\text { ren/Gremien integrierte Personen }\end{array}$ & $\begin{array}{l}\text { Große Zahl an interessierten Akteuren } \\
\text { Regionales Auftaktforum zur Breiten } \\
\text { Beteiligung }\end{array}$ & & $\mathrm{x}$ & \\
\hline $\begin{array}{l}\text { Maßnahmenbezogene Partner des } \\
\text { Projekts, Fach- oder Machtposition } \\
\text { sowie Stakeholder in der Region }\end{array}$ & $\begin{array}{l}\text { Alle relevanten Akteure vorhanden } \\
\text { Alle Funktionsbereiche vertreten } \\
\text { Einbindung der regional vorhandenen } \\
\text { Wirtschaft }\end{array}$ & & $\mathrm{x}$ & \\
\hline Qualitätskriterien & Schwäche & $\mathrm{D}$ & $\mathbf{A}$ & I \\
\hline $\begin{array}{l}\text { Projektträger und in Projektstrukturen/ } \\
\text { Gremien integrierte Personen. }\end{array}$ & $\begin{array}{l}\text { Motivation der Beteiligten schwindet } \\
\text { im Verlauf des Projekts }\end{array}$ & & $\mathrm{x}$ & \\
\hline $\begin{array}{l}\text { Maßnahmenbezogene Partner des } \\
\text { Projekts repräsentieren relevante Sekto- } \\
\text { ren. }\end{array}$ & $\begin{array}{l}\text { Beteiligung von Dienstleistern zu } \\
\text { gering }\end{array}$ & & $\mathrm{x}$ & \\
\hline
\end{tabular}




\section{Konstellation: Multiplikatoren}

Die interviewten Akteure bestätigen im Wesentlichen die über die strukturelle Erhebung herausgefilterten Akteure als die wichtigsten Multiplikatoren, vor allem aufgrund ihrer Außenwirkung, aber auch als erste Ansprechpartner und Impulsgeber. Den beiden nach der strukturellen Netzwerkanalyse zentralen Akteuren wurde unabhängig der eigenen Sektorenzugehörigkeit der befragten Akteure ein hohes Prestige zugewiesen. Dies ist ein Indiz für die Bedeutung der beiden Motoren für die Integration unterschiedlicher Bereiche in das Netzwerk.

Neben diesen beiden werden für die Kommunikation nach innen zudem Akteure benannt, die sich auch im Kern des Netzwerks befinden. Diese treten als weitere Ansprechpartner in Erscheinung und daher wird ihnen ebenfalls eine Entscheidungskompetenz zugewiesen. Die Bedeutung dieser Multiplikatoren für das Projekt wird hoch eingeschätzt und als wesentlich für das Gelingen des Projekts eingeordnet („Die sind schon wichtig“). Daher kann man von einem tendenziellen Flexible-Task-Network sprechen, allerdings mit starken Tendenzen zu einem Single-Leader-Network mit Doppelspitze. Da neben dem Projektträger auch Akteure anderer relevanter Sektoren eine zentrale Position innerhalb des Netzwerks spielen und entsprechende Promotorenfunktionen einnehmen, kann die Ausprägung des Merkmals „Multiplikatoren“ als akteursorientiert eingestuft werden.

In der Region wurde die Beteiligung der politischen Promotoren, von einigen Ausnahmen abgesehen, als eher zurückhaltend eingestuft. Dies kann eine Akteursorientierung behindern, da besonders politische Promotoren aufgrund der ihnen zugewiesenen Funktion und ihrer Verbindungen in die unterschiedlichsten Bereiche zu einer Einbindung der relevanten Akteure beitragen können.

Tabelle 17: Konstellation: Multiplikatoren in der Region Thüringer W ald \& Schiefergebirge/Frankenwald $(D=$ diskursiv, $A=$ akteursorientiert, $I=$ integrativ)

\begin{tabular}{|l|l|c|c|c|}
\hline Qualitätskriterien & Stärke & D & A & I \\
\hline Motor an der Spitze & $\begin{array}{l}\text { Zwei zentrale Akteure mit entspre- } \\
\text { chendem regionalen Standing wirken } \\
\text { als Motoren an der Spitze }\end{array}$ & x \\
\hline $\begin{array}{l}\text { Integration regionaler Meinungsführer } \\
\text { und anerkannter Fachexperten aus den } \\
\text { relevanten Sektoren. }\end{array}$ & $\begin{array}{l}\text { Im Kern sind Akteure unterschiedli- } \\
\text { cher Funktionen vorhanden }\end{array}$ & $\mathrm{x}$ & \\
\hline Qualitätskriterien & Schwäche & D & A & $\mathbf{I}$ \\
\hline Motor an der Spitze. & - & & & x \\
\hline $\begin{array}{l}\text { Integration regionaler Meinungsführer } \\
\text { und anerkannter Fachexperten aus den } \\
\text { relevanten Sektoren. }\end{array}$ & $\begin{array}{l}\text { Potentielle politische Promotoren der } \\
\text { Region eher zurückhaltend }\end{array}$ & & \\
\hline
\end{tabular}




\section{Konstellation: Personelle Kontinuität}

Die kontinuierliche Bearbeitung des Projekts durch zwei Projektmanager während der Projektlaufzeit stellt sicher, dass vorhandenes Wissen, beispielsweise im Rahmen des Projekts gesammelte Erfahrungen, dauerhaft verfügbar ist. Unterschiedliche regional relevante Themen bekommen so durchgehend ihre Bedeutung beigemessen, daher kann diese Kontinuität als ,integrativ ausgerichtet“ eingeordnet werden. Gerade die Dauerhaftigkeit der Netzwerktätigkeit von Akteuren kann die Nachhaltigkeit befördern, da gewonnenes Wissen über die Region und erarbeitetes Vertrauen auch über die Projektlaufzeit hinaus weiter vorhanden ist.

Der zentrale Akteur des Projekts und des Netzwerks hat jedoch das Projekt vor Abschluss verlassen. Damit fehlt für das Netzwerk im Zusammenhang mit dem Grünen Band eine wesentliche Schlüsselfigur. Die befragten Akteure schätzen dies als sehr negativ für die Netzwerkarbeit ein. Mit der entstehenden Lücke kann besonders die Integration regional bedeutsamer Politikfelder und relevanter Akteure beeinträchtigt werden, da Akteure, die diese Lücken schließen müssen, nicht zwingend in gleichem Maße über regionales Wissen und Vertrauen verfügen.

Ähnliches gilt für Wechsel von Zuständigkeiten beim Projektträger zu Beginn der Projektumsetzung. Dadurch wird der Aufbau von Vertrauen und regionalem Wissen besonders bei einem sich aufbauenden Netzwerk wie dem der Region Thüringer Wald \& Schiefergebirge/Frankenwald beeinträchtigt und damit die Integrativität beeinträchtigt.

Tabelle 18: Konstellation: Personelle Kontinuität in der Region Thüringer $W$ ald \& Schiefergebirge/Frankenwald ( $D=$ diskursiv, $A=$ akteursorientiert, $I=$ integrativ)

\begin{tabular}{|c|c|c|c|c|}
\hline Qualitätskriterien & Stärke & $\mathbf{D}$ & $\mathbf{A}$ & I \\
\hline $\begin{array}{l}\text { Kontinuierliches Engagement der Mul- } \\
\text { tiplikatoren im Netzwerk/ für das Pro- } \\
\text { jekt }\end{array}$ & - & & & \\
\hline $\begin{array}{l}\text { Kontinuierliche Betreuung des Pro- } \\
\text { zessmanagement/ Netzwerkarbeit durch } \\
\text { geeignete Person. }\end{array}$ & $\begin{array}{l}\text { Kontinuierliche Bearbeitung des Pro- } \\
\text { jekts durch zwei Projektmanager }\end{array}$ & & & $\mathrm{x}$ \\
\hline Qualitätskriterien & Schwäche & $\mathbf{D}$ & $\mathbf{A}$ & I \\
\hline $\begin{array}{l}\text { Kontinuierliches Engagement der Mul- } \\
\text { tiplikatoren im Netzwerk/ für das Pro- } \\
\text { jekt }\end{array}$ & $\begin{array}{l}\text { Wegfall des zentralsten Akteurs zum } \\
\text { Ende des Projekts }\end{array}$ & & & x \\
\hline $\begin{array}{l}\text { Kontinuierliche Betreuung des Pro- } \\
\text { zessmanagement/ Netzwerkarbeit durch } \\
\text { geeignete Person. }\end{array}$ & $\begin{array}{l}\text { Zu Beginn viele Wechsel der Zuständig- } \\
\text { keiten beim Projektträger }\end{array}$ & & & x \\
\hline
\end{tabular}




\section{Strategie: Lernen}

Im Projekt fanden Workshops sowie Arbeitsbesprechungen statt, die zu einer sehr intensiven Vernetzung zwischen den Akteuren geführt haben. Dies hat wiederum die Kenntnisse über die eigene Region bei den Akteuren erhöht. So gab es zwischen den Projektpartnern regelmäßige Sitzungen mindestens einmal im halben Jahr und zudem regen Austausch per Mail und Telefon. Auch die unter dem Netzwerkmerkmal „Arbeiten auf verschiedenen Ebenen“ dargestellten Arbeitsgruppen und Workshops haben zu diesem Austausch geführt.

Die Akteure traten in diesen Workshops miteinander in einen Diskurs. Es wurde mehrfach bestätigt, dass der in den Workshops geführte intersektorale Austausch zu einem Verständnis für das jeweilige andere Fach geführt hat:

„Wir haben das regelmäßig gemacht und auf die Weise - denk' ich - auch das Verständnis für die jeweiligen Bedürfnisse des anderen gesteigert und auch dazu beitragen

können, dass aus dem jeweils anderen Disziplinbereich dann auch die Argumente verstanden werden" (Zitat aus einem Interview, Fachbereich Tourismus)

Damit ist eine wesentliche Anforderung der Nachhaltigkeit in Bezug auf Lernen erfüllt. Zudem fand innerhalb des E+E-Vorhabens ein Austausch zwischen den Modellregionen statt, der den Blick öffnet und die Möglichkeit bietet, die eigenen Erfahrungen auszutauschen. Die Ausprägung des Netzwerks ist daher als diskursiv einzuordnen.

Allerdings kritisierten einige Akteure, dass der Informationsfluss und damit auch der Austausch nicht gleichermaßen kontinuierlich zwischen allen Beteiligten aufrechterhalten wurden. Die oben beschriebenen Treffen zwischen den Akteuren fanden während der Projektlaufzeit nicht in regelmäßigen Abständen statt, was vor allem den Austausch mit den nicht stark eingebundenen (oder fallbezogen eingebundenen) Akteuren beeinträchtigt. Zudem werden keine Instrumente eingesetzt, die der Reflexion der Netzwerkarbeit dienen können. Die für die Nachhaltigkeit notwendige Teilhabe wird daher nicht durchgehend gewährleistet und wirkt sich negativ auf die strategische Qualität „diskursiv“ aus. 
Tabelle 19: Strategie: Lernen in der Region Thüringer Wald \& Schiefergebirge/Frankenwald $(D=$ diskursiv, $A=$ akteursorientiert, $I=$ integrativ)

\begin{tabular}{|c|c|c|c|c|}
\hline Qualitätskriterien & Stärke & $\mathbf{D}$ & $\mathbf{A}$ & $\mathbf{I}$ \\
\hline \multirow[b]{2}{*}{$\begin{array}{l}\text { direkter und regelmäßiger - sektoren- } \\
\text { übergreifender - Austausch zwischen } \\
\text { den Akteuren des Netzwerks }\end{array}$} & $37 \%$ der erfassten Kontakte: häufig & $\mathrm{x}$ & & \\
\hline & $\begin{array}{l}\text { Workshops als regionale Plattform zur } \\
\text { Entwicklung eines Verständnisses für } \\
\text { andere Disziplinen }\end{array}$ & $\mathrm{x}$ & & \\
\hline $\begin{array}{l}\text { Einsatz reflexiver Instrumente (Evalu- } \\
\text { ierungsmaßnahmen, Reflexionswork- } \\
\text { shops, Monitoring etc.) }\end{array}$ & 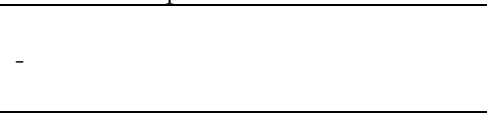 & & & \\
\hline $\begin{array}{l}\text { Erfahrungsaustausch mit Netzwerken } \\
\text { ähnlicher inhaltlicher Ausrichtung }\end{array}$ & $\begin{array}{l}\text { Austausch zwischen den Projektregio- } \\
\text { nen des E+E-Vorhabens (Sommer } \\
2009 \text { ) }\end{array}$ & $\mathrm{x}$ & & \\
\hline Qualitätskriterien & Schwäche & D & A & I \\
\hline \multirow{2}{*}{$\begin{array}{l}\text { direkter und regelmäßiger - sektoren- } \\
\text { übergreifender - Austausch zwischen } \\
\text { den Akteuren des Netzwerks }\end{array}$} & $37 \%$ der erfassten Kontakte: selten & $\mathrm{x}$ & & \\
\hline & $\begin{array}{l}\text { Lücken in der Kontinuität des Aus- } \\
\text { tauschs }\end{array}$ & $\mathrm{x}$ & & \\
\hline $\begin{array}{l}\text { Einsatz reflexiver Instrumente (Evalu- } \\
\text { ierungsmaßnahmen, Reflexionswork- } \\
\text { shops, Monitoring etc.) }\end{array}$ & $\begin{array}{l}\text { Kein Einsatz von Instrumenten zur } \\
\text { Reflexion der Netzwerkarbeit }\end{array}$ & $\mathrm{x}$ & & \\
\hline $\begin{array}{l}\text { Erfahrungsaustausch mit Netzwerken } \\
\text { ähnlicher inhaltlicher Ausrichtung }\end{array}$ & - & & & \\
\hline
\end{tabular}

\section{Strategie: Arbeiten auf verschiedenen Ebenen}

Die in Abbildung 18 schematisch dargestellte Struktur bildet die Organisation des Netzwerks im Projekt ab. Hinzugekommen sind im Laufe der Zeit weitere Arbeitsgruppen. Diese Arbeitsgruppen werden im Wesentlichen von interessierten und betroffenen Akteuren besetzt. Den einzelnen Akteuren ist dabei freigestellt, wo sie sich engagieren. ${ }^{138}$ Die Arbeitsgruppen werden als Diskussionsplattform begriffen, die zur Entscheidungsfindung einzelner Probleme benötigt werden. Da sie allen Interessierten offen stehen, können die Akteure je nach Interessenlage entscheiden, in welcher AG sie sich einbringen. So besteht die Möglichkeit, beispielsweise in Arbeitsgruppen, die sich mit Naturschutzfragen beschäftigen, auch Aspekte des Tourismus mit einzubringen und umgekehrt. Im Rahmen des Projekts waren die gleichen Akteure oftmals in mehreren Arbeitsgruppen aktiv, so dass hierüber der Austausch zwischen den Sektoren ermöglicht wurde.

138 „Also, es war je nach Aufgabenstellung und je nach Interessenlage ganz unterschiedlich, wer da alles mitgemacht hat" (Interviewte Akteurin). 


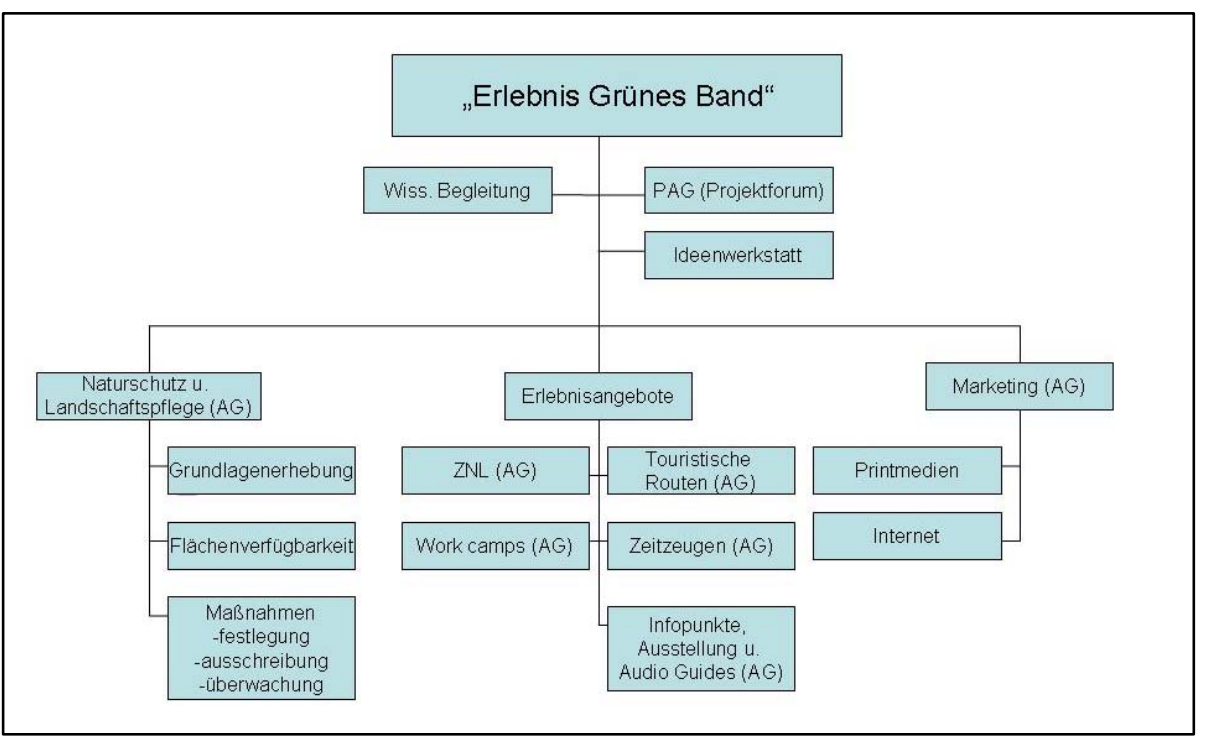

Abbildung 18: Organigramm des Netzwerks Tbüringer Wald \& Schiefergebirge / Frankenwald (Quelle: Regionalverbund Tbüringer Wald (2008, unveröff.), verändert)

Die Gruppe an Projektpartnern, die im Projekt die wesentlichen projektsteuernden Entscheidungen fällt, ist mit Vertretern unterschiedlicher Bereiche besetzt, so dass ein Interessenausgleich zwischen unterschiedlichen Sektoren stattfinden muss.

Aufgrund dieser Offenheit der Organisationsstrukturen und der Tatsache, dass die Arbeitsgruppen als Diskussionsplattform genutzt werden, um unterschiedliche Interessen auszugleichen, kann die Ausprägung des Netzwerks den Qualitäten „diskursiv“ und „,integrativ“ zugeordnet werden.

Das Projektgebiet wurde aufgrund seiner Größe und seines Zuschnitts in vier Bereiche eingeteilt und die Akteure diesen Bereichen zugeordnet. Innerhalb dieser Bereiche gab es zu Beginn auch jeweils einen zuständigen Akteur. Diese ursprünglich geplante „Zwischenebene“ zwischen Projektträger und Akteuren mit jeweiligen Regionalverantwortlichen, die die Projektregion aufgrund ihrer Größe weiter gliedern sollte und zudem die Einbindung der Akteure unterstützen sollte, geriet aber im Verlauf des Projekts eher ins Hintertreffen. Dies hat dazu beigetragen, dass Informationsflüsse nicht ausreichend ausgeprägt waren, oder zumindest einige Akteure es so empfanden. In der Projektpraxis haben sich Kleingruppen herauskristallisiert, die dann ausschließlich ihre Aufgabe, z.B. die Ausarbeitung einer speziellen touristischen Pauschale, bearbeiteten. Die Chance der Nachhaltigkeit als „Querschnittsaufgabe“ gerecht zu werden und damit die Integration unterschied- 
licher Bereiche weiter voranzutreiben, wurde damit nicht genutzt. Diese Ausprägung hemmt daher die integrative Qualität des Netzwerks.

Tabelle 20: Strategie: Arbeiten auf verschiedenen Ebenen in der Region Thüringer Wald \& Schiefergebirge/Frankenwald ( $D=$ diskursiv, $A=$ akteursorientiert, $I=$ integrativ)

\begin{tabular}{|l|l|c|c|c|}
\hline Qualitätskriterien & Stärke & D & A & I \\
\hline $\begin{array}{l}\text { Vorhandensein projekt- bzw. regi- } \\
\text { onsspezifischer Organisationstruk- } \\
\text { turen im Netzwerk, mit denen eine } \\
\text { Aufgabenteilung (Steuerung, Ent- } \\
\text { scheidung, Umsetzung, Diskussion, } \\
\text { Information) vorgenommen wird. }\end{array}$ & $\begin{array}{l}\text { Themenbezogene Aufteilung in } \\
\text { dungsbefugnis } \\
\text { AG-übergreifender, „Kern“ an } \\
\text { Projektpartnern als zentrales Ent- } \\
\text { scheidungs- und Steuerungsorgan }\end{array}$ & x & & \\
\hline Qualitätskriterien & Schwäche & D & A & I \\
\hline $\begin{array}{l}\text { Vorhandensein projekt- bzw. regi- } \\
\text { onsspezifischer Organisationsstruk- } \\
\text { turen im Netzwerk, mit denen eine } \\
\text { Aufgabenteilung (Steuerung, Ent- } \\
\text { scheidung, Umsetzung, Diskussion, } \\
\text { Information) vorgenommen wird. }\end{array}$ & $\begin{array}{l}\text { Ungenutztes Potential einer, ,Zwi- } \\
\text { schenebene“ }\end{array}$ & & & \\
\hline
\end{tabular}

\section{Strategie: Kooperation und win-win}

Das neu entstandene (Projekt-)Netzwerk wird von den interviewten Akteuren positiv beurteilt, es stellt einen „Gewinn für die Region“ (Zitat aus einem Interview) dar. Positiv ist vor allem die Möglichkeit, sich im Rahmen des Projekts kennen zu lernen und so neue Kooperationen zu schaffen und Synergieeffekte zu entdecken und zu nutzen. Dies betrifft sowohl länderübergreifende Kooperationen, beispielsweise in Form von Zusammenarbeiten zwischen zwei touristischen Leistungsträgern, aber auch die sektorenübergreifende Zusammenarbeit, vor allem zwischen Naturschutz und Tourismus.

Vor Beginn des Projekts war die länderübergreifende Kooperation zwischen den erfassten Akteuren nur bedingt vorhanden, mit dem Projekt hat sich dies deutlich verändert. Das Projekt hat dazu beigetragen, dass sich die Akteure beiderseits der ,imaginären Grenze“" (Aussage eines Akteurs) kennen lernten und die Möglichkeiten hatten, Synergien für weitere Projekte zu schaffen.

So sind verschiedene Organisationen aus denselben Bereichen beiderseits der Grenze vorhanden, die im Projekt jeweils eng zusammengearbeitet haben. Beispielsweise hat die intensive Zusammenarbeit zwischen den Naturparken Frankenwald und Thüringer Schiefergebirge/Obere Saale im Projekt dazu geführt, mit dem GeoPark „Schieferland“ ein weiteres länderübergreifendes gemeinsames Projekt zu verfolgen. Innerhalb derartiger Organisationen wird das Grüne Band nach Abschluss des Projekts in der jeweiligen sektoralen Ausrichtung auch weiter- 
hin eine Rolle spielen. Aber auch einzelne Akteure wie z.B. touristische Leistungsträger konnten im Rahmen des Projekts erste länderübergreifende Kooperationen ins Leben rufen.

Diese neu entstandenen Kooperationen zeigen, dass das Netzwerk während des Projekts funktionierte und wie sich der „Gewinn für die Region“ ausgestaltete. Eine Zuordnung der Ausprägungen zu den strategischen Qualitäten erscheint jedoch nicht sinnvoll, da es sich dabei eher um Folgen der Netzwerkarbeit handelt. Anhand der Schwächen kann hingegen dargestellt werden, wie Ausprägungen des Netzwerks die strategischen Qualitäten beeinflussen.

Allerdings ist nach Ablauf des Projekts die Gefahr groß, dass diese Kooperationen nicht weitergeführt werden. Dies liegt nicht nur an den finanziellen Herausforderungen, sondern auch daran, dass in der Region eine Organisation fehlt, die dauerhaft länderübergreifend agieren (Einschätzung eines Akteurs) und für Projekte relevante Akteure zusammenbringen kann. Dies steht der Akteursorientierung des Netzwerks entgegen.

Tabelle 21: Strategie: Kooperation und win-win in der Region Thüringer W ald \& Schiefergebirge/Frankenwald ( $D=$ diskursiv, $A=$ akteursorientiert, $I=$ integrativ)

\begin{tabular}{|l|l|c|c|c|}
\hline Qualitätskriterien & Stärke & D & A & I \\
\hline konkrete Projekt-/ Maßnahmenbei- & $\begin{array}{l}\text { Schaffung neuer Zusammenarbeiten } \\
\text { außiele, aus denen mindestens zwei } \\
\text { Interessengruppen einen gemeinsa- } \\
\text { men Gewinn ziehen }\end{array}$ & $\begin{array}{l}\text { derübergreifende Kooperation } \\
\text { GeoPark Schieferland }\end{array}$ & & \\
\hline Qualitätskriterien & Schwäche & & \\
\hline $\begin{array}{l}\text { konkrete Projekt-/ Maßnahmenbei- } \\
\text { spiele, aus denen mindestens zwei } \\
\text { Interessengruppen einen gemeinsa- } \\
\text { men Gewinn ziehen }\end{array}$ & $\begin{array}{l}\text { Fehlende länderübergreifend ausge- } \\
\text { richtete Organisation erschwert die }\end{array}$ & Zusammenarbeit & A & I \\
\hline
\end{tabular}

\section{Strategie: Probleme und Lösungswille}

Von den interviewten Akteuren wurde vor allem die harmonische und pragmatische Zusammenarbeit betont. Dies zeigt sich auch in der Beschreibung mit möglichen Konfliktpotentialen. Probleme innerhalb des Projekts entstanden in der Zusammenarbeit zwischen den unterschiedlichen Akteuren und den damit einhergehenden unterschiedlichen Interessen, die ausgeglichen werden müssen. Daraus entstehende Konflikte wurden in Diskussionen vorwiegend in den entsprechenden Arbeitsgruppen bzw. zwischen den Entscheidungsbefugten ausgetragen und es wurde versucht, Lösungen im Sinne der Projektziele zu finden. Ein Beispiel hierfür ist der Umgang mit für den Naturschutz sensiblen Bereichen im Projektgebiet. Um diese Bereiche zu schützen, wurden für die touristische Nutzung Alternativrouten erarbeitet, die in weniger sensible Bereiche führte. Damit wurden 
vor allem die im Sinne der Nachhaltigkeit notwendigen Verhandlungen offen geführt. Die Ausprägung ist daher als diskursiv für beide Kriterien einzuordnen.

Allerdings gibt es Probleme, die zwar die Netzwerk- und die Projektarbeit beeinflussen, jedoch nicht vom Netzwerk gelöst werden können. Vor allem ungeklärte Eigentumsverhältnisse von Flächen im Grünen Band erschweren die Maßnahmenumsetzung und wirken sich somit auch negativ auf die Zusammenarbeit im Netzwerk aus. Diese Schwäche kann jedoch nicht zu den strategischen Qualitäten in Bezug gesetzt werden, da es als eine die Netzwerkarbeit beeinflussende Rahmenbedingung zu sehen ist.

Tabelle 22: Strategie: Probleme und Lösungswille in der Region Tbüringer Wald \& Schiefergebirge/Frankenwald $(D=$ diskursiv, $A=$ akteursorientiert, $I=$ integrativ)

\begin{tabular}{|l|l|c|c|c|}
\hline Qualitätskriterien & Stärke & D & A & I \\
\hline Intensive Dialogphasen & $\begin{array}{l}\text { Offener Umgang mit Konflikten in- } \\
\text { nerhalb des Netzwerks in den jeweils } \\
\text { zuständigen Arbeitsgruppen }\end{array}$ & $\mathrm{x}$ & & \\
\hline Vereinbarte Kompromisse & $\begin{array}{l}\text { Lösungswille von möglichen Kon- } \\
\text { flikten im Sinne der Projektziele } \\
\text { vorhanden }\end{array}$ & $\mathrm{x}$ & & \\
\hline Qualitätskriterien & Schwäche & D & A & I \\
\hline Intensive Dialogphasen & - & & & \\
\hline Vereinbarte Kompromisse & - & & & \\
\hline
\end{tabular}

\section{Strategie: Transparenz, Offenheit und Flexibilität}

Insbesondere bei Entscheidungen wurden nicht immer alle Beweggründe vollständig berichtet, sondern den Akteuren in Form von Ergebnisprotokollen mitgeteilt, sofern sie für den jeweiligen Akteur von Bedeutung waren. Dies wurde von den Akteuren insbesondere unter dem Aspekt der „Effektivität des Netzwerks“ positiv beurteilt. Sie fühlten sich ausreichend an den Entscheidungen beteiligt, wobei sie die Information über die Entscheidung und die damit entstehende potentielle Möglichkeit der Einflussnahme auch als Beteiligung betrachteten. Das Projektnetzwerk wurde zudem als offen und flexibel eingeordnet. So konnten während des gesamten Projektverlaufs Akteure hinzukommen, was für eine Offenheit des Netzwerks spricht. Allerdings war dies im Rahmen des Projekts kaum der Fall, da das Potenzial an neuen Akteuren verhältnismäßig schnell erschöpft war („Die sind ja schon alle dabei“ (Aussage eines Akteurs)). Daher kann die Ausprägung des Merkmals als diskursiv und integrativ eingeordnet werden.

Ausnahmen bilden jene Akteure, die nur fallbezogen in das Projekt eingebunden waren. Diesen Akteuren erschienen einzelne Entscheidungen nicht nachvollziehbar, was mit dem unterschiedlich intensiven Informationsfluss im Projektver- 
lauf zusammenhängt. Kritisiert wurde zudem die mangelnde Transparenz von Entscheidungsprozessen von Organisationen außerhalb der Projektregion, die das Netzwerk jedoch maßgeblich beeinflussen. Hier wird der Verhandlungsprozess negativ beeinflusst, was sich auch auf die Transparenz gefällter Entscheidungen auswirkt. Daher steht diese Ausprägung der diskursiven Qualität entgegen.

Tabelle 23: Strategie: Transparenz, Offenheit und Flexibilität in der Region Thüringer $W$ ald \& Schiefergebirge/Frankenwald ( $D=$ diskursiv, $A=$ akteursorientiert, $I=$ integrativ)

\begin{tabular}{|c|c|c|c|c|}
\hline Qualitätskriterien & Stärke & $\mathbf{D}$ & $\mathbf{A}$ & I \\
\hline $\begin{array}{l}\text { Nachvollziehbarkeit von Abstim- } \\
\text { mungs- und Entscheidungsprozes- } \\
\text { sen }\end{array}$ & $\begin{array}{l}\text { Entscheidungen und Entschei- } \\
\text { dungsprozesse unter Berücksichti- } \\
\text { gung der Effektivität nachvollzieh- } \\
\text { bar dargestellt. }\end{array}$ & $\mathrm{x}$ & & \\
\hline $\begin{array}{l}\text { Fortwährende Zugangsmöglichkeit } \\
\text { von neuen Akteuren ins Netzwerk }\end{array}$ & Netzwerk offen für neue Akteure & & & $\mathrm{x}$ \\
\hline $\begin{array}{l}\text { Inhaltliche und organisatorische } \\
\text { Flexibilität: Integration neuer } \\
\text { Ideen, kreative Lösungen für regi- } \\
\text { onalspezifische Herausforderungen }\end{array}$ & - & & & \\
\hline Qualitätskriterien & Schwäche & $\mathbf{D}$ & $\mathbf{A}$ & I \\
\hline $\begin{array}{l}\text { Nachvollziehbarkeit von Abstim- } \\
\text { mungs- und Entscheidungsprozes- } \\
\text { sen }\end{array}$ & $\begin{array}{l}\text { selektiver Informationsfluss } \\
\text { Entscheidungsprozesse für fakulta- } \\
\text { tiv eingebundene Akteure nicht } \\
\text { immer nachvollziehbar }\end{array}$ & $\mathrm{x}$ & & \\
\hline $\begin{array}{l}\text { Fortwährende Zugangsmöglichkeit } \\
\text { von neuen Akteuren ins Netzwerk }\end{array}$ & - & & & \\
\hline $\begin{array}{l}\text { Inhaltliche und organisatorische } \\
\text { Flexibilität: Integration neuer } \\
\text { Ideen, kreative Lösungen für regi- } \\
\text { onalspezifische Herausforderungen }\end{array}$ & - & & & \\
\hline
\end{tabular}

\section{Instrumente: Leitbild}

Vor allem die Ziele im touristischen Bereich sind den Akteuren präsent und wurden auch entsprechend unterstützt und mitgetragen. Unter den Stichworten „Ruhe, Besinnung und Freiheit" wurden Pauschalen entwickelt und touristische Projekte konzipiert. Für den naturschutzfachlichen Bereich wurden ähnliche Zielformulierungen nicht von allen interviewten Akteuren benannt, einige stellten aber die Erhaltung und Entwicklung des Grünen Bandes und seines naturschutzfachlichen Wertes als Zielsetzung für dieses Projekt heraus. Das in der Vorstudie des $\mathrm{E}+\mathrm{E}-$ Vorhabens formulierte Leitbild ist den Akteuren kein Begriff, wurde aber 
weitestgehend von der deutlichen Zielformulierung „Naturschutz und Tourismus“ und dem regionalen touristischen Projektleitbild (siehe oben) ersetzt. Insofern trägt die Zielformulierung zum gemeinsamen Verständnis und der gemeinsamen Orientierung der Akteure bei. Die Ausprägung des Merkmals kann daher als integrativ eingeordnet werden.

Obwohl auch für den naturschutzfachlichen Bereich Zielformulierungen das Leitbild konkretisieren, sind diese bei den Akteuren weniger bekannt. Damit ist zwar der ökologische Bereich im Leitbild im Sinne der Nachhaltigkeit vertreten, jedoch bei den Akteuren nicht ausreichend verankert und kann daher auch nicht die entsprechende Orientierungswirkung entfalten. Diese Schwäche ist auch der strategischen Qualität integrativ zuzuordnen, da zwar das Politikfeld Naturschutz im Projekt bearbeitet wird, aber die „Basis gemeinsam geteilter Werte und Normen"139 bei den Akteuren nicht ausreichend gegeben ist.

Tabelle 24: Instrumente: Leitbild in der Region Thüringer W ald \& Schiefergebirge/Frankenwald $(D=$ diskursiv, $A=$ akteursorientiert, $I=$ integrativ)

\begin{tabular}{|l|l|l|l|l|}
\hline Qualitätskriterien & Stärke & D & A & I \\
\hline Vorhandensein eines Leitbildes & $\begin{array}{l}\text { Touristisches „Leitbild“ in Form } \\
\text { von Zielformulierungen vorhanden }\end{array}$ & $\begin{array}{l}\text { Leitbild der Vorstudie in Form } \\
\text { einer Zielformulierung im Projekt- } \\
\text { netzwerk verankert. }\end{array}$ & $\mathrm{x}$ \\
\hline $\begin{array}{l}\text { Qualität des Leitbildes hinsichtlich } \\
\text { der Berücksichtigung der drei Di- } \\
\text { mensionen der Nachhaltigkeit }\end{array}$ & $\begin{array}{l}\text { Zielformulierungen decken die } \\
\text { Bereiche Tourismus, Naturschutz } \\
\text { und Bildung ab }\end{array}$ & $\mathrm{x}$ \\
\hline $\begin{array}{l}\text { die Bekanntheit und Verankerung } \\
\text { (Orientierungswirkung!) in den } \\
\text { Köpfen der Netzwerkakteure }\end{array}$ & $\begin{array}{l}\text { Touristisches ,Leitbild“ in Form } \\
\text { von Zielformulierungen den Akteu- } \\
\text { ren bekannt }\end{array}$ & & $\mathrm{x}$ \\
\hline Qualitätskriterien & Schwäche & D & A & I \\
\hline Vorhandensein eines Leitbildes & - & & \\
\hline $\begin{array}{l}\text { Qualität des Leitbildes hinsichtlich } \\
\text { der Berücksichtigung der drei Di- } \\
\text { mensionen der Nachhaltigkeit }\end{array}$ & - & & & \\
\hline $\begin{array}{l}\text { die Bekanntheit und Verankerung } \\
\text { (Orientierungswirkung!) in den } \\
\text { Köpfen der Netzwerkakteure }\end{array}$ & $\begin{array}{l}\text { Naturschutzfachliches Leitbild } \\
\text { weniger präsent }\end{array}$ & $\mathrm{x}$ \\
\hline
\end{tabular}

139 Nohlen (2008), S. 229. 


\section{Instrumente: Prozessmanagement}

Die „Motoren an der Spitze“ sowie die Projektmanager teilten sich als Netzwerkmanager die Aufgabe des Prozessmanagements. Moderation, Verhandlung und Vermittlung zwischen Akteuren waren projektintern organisiert. Die Wahrnehmung dieser Aufgaben durch eindeutig benannte Netzwerkmanager ermöglichte einen Dialog zwischen den Akteuren und ist daher als diskursiv einzuordnen. Zudem wird das Prozessmanagement von einem recht aktiven Netzwerkkern betrieben, der untereinander einen regen Austausch pflegt und so den Netzwerkprozess maßgeblich beeinflusst.

Ein Indiz für die integrative Qualität des Netzwerks ist die Zufriedenheit der Akteure mit dem Prozess. Da im Netzwerk unterschiedliche Bereiche vertreten sind, und sich die Vertreter aus den meisten Bereichen zufrieden über den Prozess äußerten, kann von einer Akteursorientierung des Prozesses sowie von einer Integration der relevanten Politikfelder ausgegangen werden.

Die interviewten Akteure haben für die zukünftige Netzwerkarbeit ein hohes Interesse daran, das Netzwerk weiter zu erhalten und die angestoßenen Entwicklungen weiter voran zu treiben. Allerdings wird zum Teil auch erwartet, dass dieses Netzwerk von jemandem (von außerhalb) für sie erhalten wird. Diese passive Erwartungshaltung kann sich negativ auf die entstandenen länder- und sektorenübergreifenden Kooperationen auswirken. Denn nach Abschluss des Projekts stehen die Projektmanager, die maßgeblich auch die Aufgaben der Netzwerkmanager übernahmen, nicht mehr zur Verfügung.

Kritisiert wird zum Teil der nicht kontinuierliche Informationsfluss von den als „Köpfe des Projekts“ betrachteten Akteuren hin zu nur temporär eingebundenen Akteuren. $\mathrm{Zu}$ gewissen Stoßzeiten wurde die Information dieser Akteure etwas vernachlässigt. So hätte aus Sicht einiger Akteure hier die ursprüngliche Einteilung der Projektregion in Gebiete mit „Regionalverantwortlichen“ (vgl. Netzwerkmerkmal „Arbeiten auf verschiedenen Ebenen“) verwendet werden können, um einen kontinuierlichen Informationsfluss sicherzustellen. Diese Unzufriedenheit trug auch dazu bei, dass sie sich seltener aktiv am Netzwerk beteiligten, was wiederum einem intensiven gleichmäßigen Diskurs im Netzwerk entgegensteht. Zudem brachten sich Akteure, die vom Projekt betroffen sind, in der Folge nicht sehr intensiv in das Projekt ein, was wiederum nicht der Akteursorientierung entspricht. Dem Integrativen entgegen steht auch hier die kaum realisierte Nutzung der ursprünglich geplanten Zwischenebene sowie vor allem für die weitere Zukunft des Netzwerks die Beendigung der Arbeit der Netzwerkmanager mit Abschluss des Projekts.

Allerdings sind auch einige Akteure der Meinung, dass jeder Akteur hier eine gewisse Eigenverantwortung hat (,wir waren eigentlich auch aus eigenem Interesse immer über den Stand der Dinge informiert" (Aussage eines Akteurs)) und Informationen auf 
Nachfrage immer verfügbar waren. Dennoch fehlte zumeist die Zeit, um eine regelmäßige Weitergabe neuer Informationen an alle Interessierten sicherzustellen. Dies deutet auf ein wesentliches Problem im Prozessmanagement hin, das Zeitmanagement. Sowohl Projektträger als auch andere Akteure stellen den Zeitdruck, der sich im Projekt ergab, als eine große Herausforderung dar, unter dem bestimmte Dinge zu kurz kamen und dem nur standgehalten werden konnte, da einige Akteure mit hohem Einsatz das Projekt vorangetrieben haben. Gründe für den Zeitdruck sind der von außen vorgegebene Zeitplan - aufgrund der historischen Bedeutung des Themas musste ein Großteil der Projektaufgaben zum Jahr 2009 abgearbeitet sein - sowie der Tatsache, dass viel Zeit ,verloren ging“, da das Netzwerk und die Hauptverantwortlichen sich erst finden mussten. 
Tabelle 25: Instrument: Prozessmanagement in der Region Thüringer Wald \& Schiefergebirge/Frankenwald ( $D=$ diskursiv, $A=$ akteursorientiert, $I=$ integrativ)

\begin{tabular}{|c|c|c|c|c|}
\hline Qualitätskriterien & Stärke & D & $\mathbf{A}$ & I \\
\hline $\begin{array}{l}\text { das Vorhandensein einer (externen) } \\
\text { Moderation, die den Prozess steu- } \\
\text { ernd begleitet }\end{array}$ & $\begin{array}{l}\text { Wahrnehmung der internen Mode- } \\
\text { ration durch Netzwerkmanager }\end{array}$ & $\mathrm{x}$ & & \\
\hline $\begin{array}{l}\text { aktive Teilhabe der unterschiedli- } \\
\text { chen Akteursfunktionen am Netz- } \\
\text { werk (strukturelle Erhebung) }\end{array}$ & $\begin{array}{l}\text { Aktiver Kern, besetzt aus unter- } \\
\text { schiedlichen Funktionen }\end{array}$ & $\mathrm{x}$ & & \\
\hline $\begin{array}{l}\text { der Zufriedenheitsgrad der Akteure } \\
\text { mit dem Prozess }\end{array}$ & $\begin{array}{l}\text { Ca. } 80 \% \text { der Akteure mit dem } \\
\text { Prozess zufrieden }(\mathrm{N}=43)\end{array}$ & & & $\mathrm{x}$ \\
\hline $\begin{array}{l}\text { Präsenz des Netzwerks bzw. des } \\
\text { darin bearbeiteten Projekts in der } \\
\text { Öffentlichkeit }\end{array}$ & - & & & \\
\hline $\begin{array}{l}\text { Wahrnehmung aller Aufgaben des } \\
\text { Prozessmanagements durch Netz- } \\
\text { werkmanager }\end{array}$ & $\begin{array}{l}\text { Netzwerkmanager als Ansprech- } \\
\text { partner für die Akteure vorhanden }\end{array}$ & $\mathrm{x}$ & & \\
\hline Qualitätskriterien & Schwäche & D & $\mathbf{A}$ & I \\
\hline $\begin{array}{l}\text { das Vorhandensein einer (externen) } \\
\text { Moderation, die den Prozess steu- } \\
\text { ernd begleitet }\end{array}$ & - & & & \\
\hline $\begin{array}{l}\text { aktive Teilhabe der unterschiedli- } \\
\text { chen Akteursfunktionen am Netz- } \\
\text { werk (strukturelle Erhebung) }\end{array}$ & $\begin{array}{l}\text { Fallbezogen eingebundene Akteu- } \\
\text { re, die sich eher passiv verhalten }\end{array}$ & & $\mathrm{x}$ & \\
\hline \multirow[t]{2}{*}{$\begin{array}{l}\text { der Zufriedenheitsgrad der Akteure } \\
\text { mit dem Prozess }\end{array}$} & $\begin{array}{l}\text { Zeitweilige Unzufriedenheit mit } \\
\text { dem Informationsfluss zwischen } \\
\text { Netzwerkkern und fallbezogen } \\
\text { beteiligten Akteuren }\end{array}$ & $\mathrm{x}$ & & \\
\hline & $\begin{array}{l}\text { inhaltliche Konflikte zw. Interes- } \\
\text { sengruppen }\end{array}$ & $\mathrm{x}$ & & \\
\hline $\begin{array}{l}\text { Präsenz des Netzwerks bzw. des } \\
\text { darin bearbeiteten Projekts in der } \\
\text { Öffentlichkeit }\end{array}$ & - & & & \\
\hline $\begin{array}{l}\text { Wahrnehmung aller Aufgaben des } \\
\text { Prozessmanagements durch Netz- } \\
\text { werkmanager }\end{array}$ & $\begin{array}{l}\text { Nach Abschluss des Projekts keine } \\
\text { Netzwerkmanager mehr vorhanden } \\
\text { Ungenutztes Potential der „Zwi- } \\
\text { schenebene“ (vgl. Arbeiten auf } \\
\text { verschiedenen Ebenen) }\end{array}$ & $\mathrm{x}$ & & \\
\hline
\end{tabular}

\subsubsection{Maßnahmenperspektive}

In diesem Abschnitt erfolgt die Darstellung der Ergebnisse für das Erprobungsund Entwicklungsvorhaben Erlebnis Grünes Band in der Untersuchungsregion Thüringer Wald \& Schiefergebirge/Frankenwald (TSF). Wie bereits in Kapitel 2 
erwähnt, war das E+E-Vorhaben im Durchführungszeitraum dieses Projektes noch nicht abgeschlossen. Um dennoch der Forschungshypothese „Die Maßnahmen des E+E-Vorhabens wirken auf regionale Nachhaltigkeit" nachgehen zu können, wird die Annahme zugrunde gelegt, dass alle Maßnahmen, die im Projektantrag ${ }^{140}$ niedergeschrieben sind, auch so zur Umsetzung gelangen. ${ }^{141}$

Das E+E-Vorhaben „Erlebnis Grünes Band in der Modellregion Thüringer Wald \& Schiefergebirge/Frankenwald“ setzt sich aus mehreren Projektbausteinen zusammen, die wiederum verschiedene Einzelmaßnahmen umfassen:

- Projektbaustein 1 „Maßnahmen Naturschutz und Landschaftspflege“ umfasst zehn Einzelmaßnahmen (M 1.1 bis 1.10);

- Projektbaustein 2 „Entwicklung von Erlebnisangeboten“ besteht aus sechs Einzelmaßnahmen (M 2.1 bis 2.6);

- Projektbaustein 3 „Informationsangebot und Kommunikation vor Ort“: umfasst fünf Einzelmaßnahmen (M 3.1 bis 3.5);

- Projektbaustein 4 „Marketing und externe Kommunikation“ umfasst vier verschiedene Maßnahmenbereiche (M 4.1 bis 4.4).

Im Zusammenwirken der einzelnen Bausteine soll die Zusammenführung von Schutz- und Nutzaspekten gelingen, die charakteristisch für das E+E-Vorhaben sein soll. Die im Rahmen dieses E+E-Vorhabens geplanten Grundlagenerhebungen sind zur Verwirklichung der Projektbausteine eins bis vier notwendige Vorarbeiten. Das Wirkungspotenzial dieses Vorhabens ergibt sich aus der Zusammenschau aller Maßnahmen. Um in Bezug auf die Forschungshypothese zu einer Aussage zu kommen, werden Maßnahmen und Themenfelder der regionalen Nachhaltigkeit durch eine Matrix miteinander in Beziehung gesetzt (siehe nächsten Abschnitt).

\subsubsection{Maßnahmenbasismatrix}

Die Maßnahmenbasismatrix enthält in den Spalten die Projektbausteine inklusive der jeweils zugehörigen Maßnahmen. In den Zeilen sind die üblichen regionalen Nachhaltigkeitsthemenfelder ${ }^{142}$ abgetragen, eingruppiert in die drei üblichen übergeordneten Dimensionen Ökologie, Ökonomie und Soziales. Die Lesart erfolgt von den Spalten zu den Zeilen: Eine Maßnahme $\mathrm{M}_{\mathrm{i}}$ des Projektbausteins $\mathrm{Pb}_{\mathrm{i}}$ kann direkt oder indirekt Wirkung entfalten auf die Themenfelder 1 bis 21. Dieser Zu-

140 Vgl. Regionalverbund Thüringer Wald e.V. (2006)

141 Zumeist wird ein Projektantrag nicht eins zu eins umgesetzt, da sich im Fortgang eines Projektes Anpassungen ergeben. So wird auch hier die Möglichkeit von Änderungen in der Umsetzung von Maßnahmen nicht ignoriert. Nichtsdestotrotz ist die Struktur des Erprobungs- und Entwicklungsvorhabens mit seinen vier Projektbausteinen klar strukturiert, so dass einzelne abweichend vom Projektantrag durchgeführte Maßnahmen, die regionale Gesamtwirkung des E+EVorhabens nicht erheblich verändern. Aus diesem Grund ist das Heranziehen des Projektantrages als Grundlage, um der Forschungshypothese nachgehen zu können, unproblematisch.

142 Siehe zum Thema „Nachhaltige Regionalentwicklung als Evaluationsgegenstand“ auch Pollermann, K. (2007); Ness, B. et al. (2007); Graymore, M. et al. (2008). 
sammenhang wird hergestellt zum einen basierend auf Plausibilitätsüberlegungen und zum anderen basierend auf Literaturquellen. Abhängig vom Konkretisierungsgrad der geplanten Maßnahmen sind die Abschätzungen hinsichtlich des Wirkungspotenzials in unterschiedlicher Genauigkeit möglich. So wird in deskriptiver Form das Gesamtwirkungspotenzial des Vorhabens in seiner Verteilungswirkung über die einzelnen Themenfelder der regionalen Nachhaltigkeit sichtbar. Im Folgenden wird das Wirkungspotenzial für jeden Projektbaustein einzeln erläutert:

Wirkungspotenzial des Projektbausteins $1(\mathrm{~Pb}$ 1) „Maßnabmen Naturschutz, und Landschaftspflege" "143

$\mathrm{Pb} 1$ wirkt auf das Themenfeld Ökosystem und Artenvielfalt: Alle zehn Maßnahmen (M 1.1 bis 1.10) tragen zur Entwicklung des Themenfeldes (TF) Ökosysteme und Artenvielfalt bei. ${ }^{144}$ Die Einzelmaßnahmen verteilen sich auf zehn Testgebiete (TGs) die nach den Kriterien „Erhalt oder Wiederherstellung des Grünen Bandes aus naturschutzfachlicher Sicht“, „Wiederherstellung und Erlebbarkeit von typischen Landschaftsbildern“ (z.B. Wiesentäler), „Verbesserung der Lebensräume gefährdeter Arten“ (z.B. Wiesenbrütergebiete), „Möglichkeiten, Flächen in manchen Abschnitten vollständig aus der Nutzung zu nehmen“ (z.B. unterschiedlich fortgeschrittene Sukzessionsflächen) ausgesucht wurden. Je nach Testgebiet und seinen Charakteristika (geografische Besonderheiten, Artenzusammensetzung) erfolgt eine Anpassung der Landschaftspflegemaßnahmen. Die Landschaftspflegemaßnahmen können konkret zu vier Schwerpunkten zusammengefasst werden:

Schwerpunkt 1: „Erhaltung beziehungsweise teilweise Wiederherstellung des Grünlands mit entsprechender Artenzusammensetzung“ (TG 1 bis 4, TG 6). Pflegeziel hinsichtlich der Artenzusammensetzung sind u.a. der Schwarzstorch, Arnika, Bärwurz, Orchideen, Fledermäuse, der Falter Spanische Flagge, Kryptogamen, Schlingnatter, Uhu sowie Perückenflockenblume.

143 Siehe zur ökonomischen Bewertung von Umweltleistungen Hartje, Volkmar (2003); Günther, E. et al. (2008).

144 Siehe zu den Grundproblemen landschaftsökologischer Daten Leser, H. (1997), S. 278ff. 


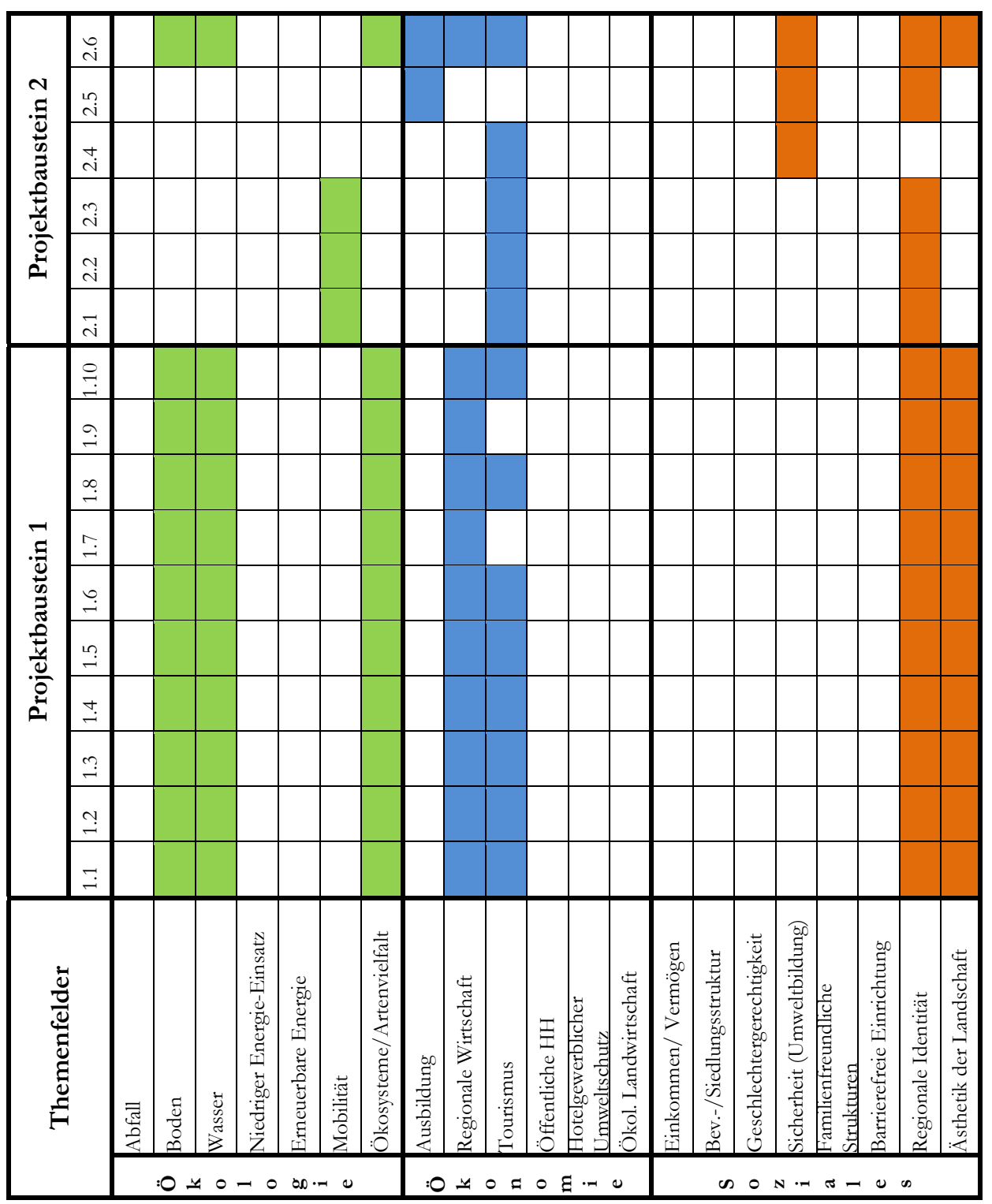




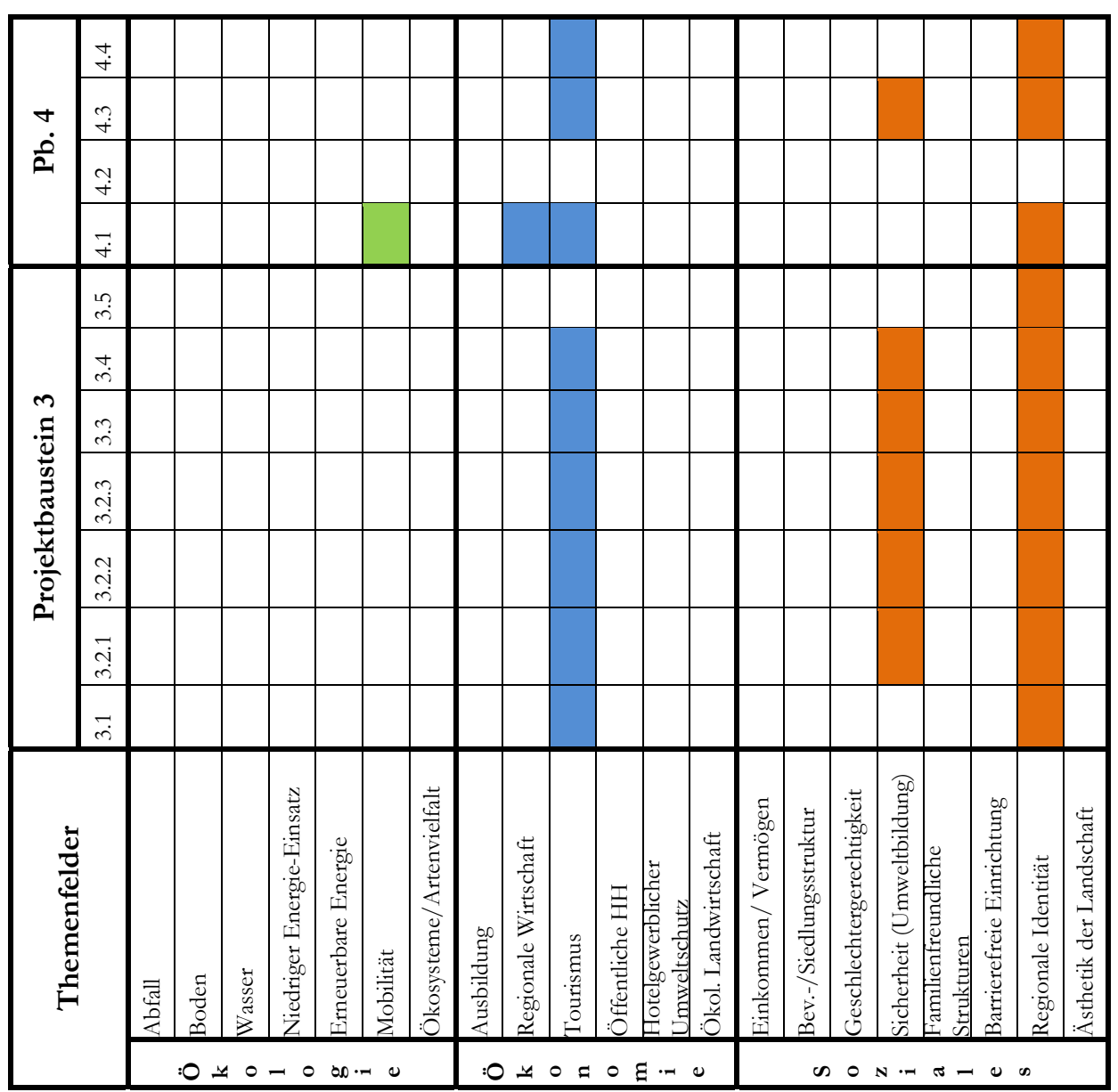

Abbildung 19: Maßnahmenbasismatrix für das E+E Projekt Thüringer W ald und Schiefergebirge/Frankenwald

Schwerpunkt 2: „Erhaltung von offenen Heideflächen“ (TG 6; TG 9). Pflegeziel hinsichtlich der Artenzusammensetzung sind u.a. der Schwarzstorch, verschiedene Eulen, diverse geschützte Farne und Flechten.

Schwerpunkt 3: „Entfernung standortuntypischer Gehölze und Förderung von Bergmischwald“ (TG 7 bis 8). Pflegeziel hinsichtlich der Artenzusammensetzung sind u.a Schwarzstorch, Uhu, Bärwurz, Orchideen, Arnika, Fischotter, Haselhuhn, diverse Fledermäuse.

Schwerpunkt 4: „Erhaltung und Entwicklung der Auenbereiche, offene Felsstandorte" (TG 10). Pflegeziel hinsichtlich der Artenzusammensetzung sind u.a Tagfalter und Vögel. Von den Landschaftspflegemaßnahmen können weitere 
Effekte ausgehen. So werden extensiv genutzten Landschaftselementen als positive Eigenschaften ${ }^{145}$ die Verminderung von Bodenerosion, Beeinflussung der Wasseraufnahmefähigkeit des Bodens sowie ästhetische Gründe zugeschrieben. Somit kann durch diese Maßnahmen auch eine mögliche Wirkung auf die Themenfelder Boden und Wasser in der Dimension Ökologie und das Themenfeld Ästhetik ${ }^{146}$ der Landschaft in der Dimension Soziales gegeben sein.

$\mathrm{Pb} 1$ wirkt auf Themenfeld Regionale Wirtschaft: Handlungsleitend bei den Landschaftspflegemaßnahmen in den Testgebieten (TG 1 bis 10) soll die Zusammenführung von Schutz- und Nutzaspekten sein. So sollen die bei den Landschaftspflegemaßnahmen anfallenden Produkte ökonomisch genutzt werden. Mischgut aus Entbuschungsmaßnahmen soll z.B. in Biogasanlagen verwertet werden unter Einbeziehung vorhandener Anlagen im erweiterten Projektgebiet. Älterer Birkenaufwuchs soll Anliegern als Kaminholz angeboten und extensive Beweidung mit Fleischvermarktung kombiniert werden.

Pb 1 wirkt auf Themenfeld Tourismus: Damit die Idee des E+E-Vorhabens - die Zusammenführung von Schutz- und Nutzaspekten - gelingen kann, leisten einige Maßnahmen einen Beitrag, damit eine touristische Inwertsetzung möglich ist. So sind die meisten Landschaftspflegemaßnahmen zum Erhalt beziehungsweise zur Wiederherstellung des Grünen Bandes aus naturschutzfachlicher Sicht notwendig. Die Wiederherstellung und Erlebbarkeit von typischen Landschaftsbildern ist aber auch notwendige Voraussetzung, um (naturnahen) Tourismus entwickeln zu können. Maßnahmen, wie zur Sicherung des Kolonnenweges sind diesbezüglich als notwendige Vorarbeiten zu interpretieren, da damit entsprechende Abschnitte am Grünen Band als Wander- und Radweg entwickelt werden können (TG 5). ${ }^{147}$

$\mathrm{Pb} 1$ wirkt auf Themenfeld regionale Identität: Die Landschaftspflegemaßnahmen haben die Wiederherstellung und Erlebbarkeit des Grünen Bandes zum Ziel. Damit leisten sie einen Beitrag in diesem Themenfeld.

Für den Projektbaustein 1 „Maßnahmen Naturschutz und Landschaftspflege“ ist festzuhalten, dass direkte Wirkungen in allen drei Dimensionen (Ökonomie, Ökologie und Soziales) der regionalen Nachhaltigkeit möglich sind. In diesem Projektbaustein besitzt jede Einzelmaßnahme gleichzeitig Wirkungspotenzial in

145 Vgl. Nitsche, S. et al. (1994).

146 Unter das Themenfeld Ästhetik der Landschaft werden auch Themen wie „(emotionaler) Eigenwert der Natur“ und „Naturethik“ subsumiert. Im Bezug auf die Perspektiven einer touristischen Inwertsetzung sind diese Themen mit von Bedeutung. Siehe dazu Krebs , A. (1996); Krause, R. et al. (2008). Zur Naturethik siehe u.a. Bundesministerium für Umwelt, Naturschutz und Reaktorsicherheit (2007), S. 15.

147 Da während der Laufzeit dieses Projektes die Maßnahmenumsetzung im Rahmen des E+EVorhabens noch nicht abgeschlossen war, kann nicht an Hand von Fallbeispielen argumentiert werden. Einen Überblick über Untersuchungen zu ökonomischen Effekten von Großschutzgebieten gibt Job, H. et al. (2005). Hier werden Methoden aufgezeigt und angewandt um ökonomische Effekte des Tourismus messbar zu machen. 
allen drei Dimensionen (siehe Abbildung 13). Die Maßnahmen besitzen durch die mehrdimensionale Ansprache von Themenfeldern einen integrativen Charakter.

\section{Wirkungspotenzial des Projektbausteins 2 (Pb 2) „Entwicklung von Erlebnisangeboten“}

Pb 2 wirkt auf das Themenfeld Tourismus: Im Projektbaustein 2 haben fast alle Einzelmaßnahmen Wirkungspotenzial auf das Themenfeld Tourismus. Die sechs Einzelmaßnahmen lassen sich grob zu drei Maßnahmenschwerpunkten zusammenfassen:

- „Wandern, Radfahren, Wasserwandern“ (M 2.1 bis M 2.4)

- „Geführte Touren“ (M 2.5) und

- ,Work-Camps“ (M 2.6).

Im ersten Maßnahmenschwerpunkt geht es konkret um die Entwicklung von touristischen Pauschalen. Hierzu wurden entlang des Grünen Bandes in der Modellregion vier Entwicklungsbereiche (M 2.1 bis M 2.4) definiert und unter ein Thema gestellt, welches die natürlichen Potenziale des Entwicklungsbereichs widerspiegelt: „Schwarzes Gold und Natur am Grünen Band“ (Entwicklungsbereich Sonneberg - Stockheim; M 2.1); „Auf den Spuren des blauen Goldes am Grünen Band“ (Entwicklungsbereich Probstzella - Ludwigsstatt - Lehesten; M 2.2); „Ein Fenster in die (Erd-)Geschichte am blauen Grünen Band“ (Entwicklungsbereich Blankenstein - Lichtenberg - Rudolfstein - Hirschberg; M 2.3) und „Das andere Grenzerlebnis“ (Entwicklungsbereich Wasserwandern auf der Saale im Bereich Blankenstein - Lichtenberg - Rudolfstein - Hirschberg; M 2.4). In den einzelnen Entwicklungsbereichen existieren verschiedene Perspektiven der touristischen Entwicklungsmöglichkeiten, deren Entwicklungspotenzial während der Laufzeit dieses Projektes noch nicht abschließend erhoben und ausgewertet werden konnte. ${ }^{148} \mathrm{Um}$ die jeweiligen regionalen Potenziale der Entwicklungsbereiche erhalten und herausstellen zu können, leisten die entsprechenden Einzelmaßnahmen einen Beitrag zur Professionalisierung der vorhandenen Infrastruktur. Für die Entwicklungsbereiche M 2.1 bis M 2.3 sollen ca. $200 \mathrm{~km}$ Wanderwege und $150 \mathrm{~km} \mathrm{Rad}$ wege mit Markierungszeichen und Beschilderungen versehen werden. Ferner soll für das Erlebnisangebot Wasserwandern die notwendige Infrastruktur etabliert werden (Ein- und Ausstiegsstellen sowie Rast- und Biwakplätze (M 2.4). In allen Entwicklungsbereichen sollen Erläuterungstafeln auf die naturschutzfachlichen und historischen Besonderheiten hinweisen. Neben der Erarbeitung von verschiedenen Informationsmaterialien (Flyer, Karten, Broschüren) ist die Installation eines GPS-gestützten Orientierungssystems vorgesehen. Diese Maßnahmen

148 Wie bereits in Kapitel 2 beschrieben lief dieses Projekt parallel zum E+E-Vorhaben. Die Maßnahmen (hier konkret die touristischen Pauschalen) befanden sich noch in der Entwicklung beziehungsweise Umsetzungsphase und konnten daher nicht als Fallbeispiele dienen. Bis zur Abschlusstagung dieses Projektes im Februar 2010 wurde eine touristische Pauschale gebucht (nach mündlicher Aussage eines Teilnehmers der Abschlusstagung). 
sind notwendige Vorarbeiten, damit die regionalen Potenziale touristisch in Wert gesetzt werden können. Im zweiten Maßnahmenschwerpunkt soll ein Weiterbildungsmodul „Grünes Band“ für zertifizierte Natur- und Landschaftsführer entstehen. Die im Rahmen des Weiterbildungsmoduls erworbenen Kenntnisse sollen in die Durchführung von geführten Touren am Grünen Band eingehen (M 2.5). Die entsprechende Aus- und Weiterbildung von Natur- und Landschaftsführern ist ebenfalls als Beitrag zu sehen, um die touristische Nutzung von Teilen des Grünen Bandes zu professionalisieren. Der dritte Maßnahmenschwerpunkt ist die Durchführung von Work-Camps (M 2.6). Durch die Verbindung von Naturerleben, ökologischer Bildung sowie erlebnispädagogischen Elementen soll ein interessantes Erlebnisangebot entstehen. Die maximal zehntägigen Camps verfolgen simultan multiple Ziele. Neben der touristischen Inwertsetzung von Bereichen des Grünen Bandes (durch Bewerbung der Work-Camps) soll gleichzeitig eine ökologische Inwertsetzung (u.a. durch landschaftspflegerische und artenschützerische Beiträge an konkreten Punkten), eine ökonomische Inwertsetzung von Bereichen des Grünen Bandes (z.B. durch Gewinnung von Heizmaterial), eine soziale Inwertsetzung des Grünen Bandes (z.B. durch Einbeziehung von Grundeigentümern und der Öffentlichkeit sowie von Schulen und Jugendlichen aus der Region) und eine kulturhistorische Inwertsetzung von geeigneten Bereichen des Grünen Bandes (z.B. durch Wiederherstellung und nachhaltige Nutzung von Nieder- und Mittelwaldstrukturen) erfolgen. In Folge dieser integrativ ausgestalteten Maßnahmen können auch positive Effekte in den Themenfeldern Boden, Wasser sowie Ökosysteme und Artenvielfalt und auch im Themenfeld Ästhetik der Landschaft (insbesondere durch dabei durchzuführende Pflegemaßnahmen) erreicht werden. Das im Rahmen der Work-Camps erworbene Wissen hinsichtlich der historischen und naturschutzfachlichen Besonderheiten spricht ebenfalls das Themenfeld Ausbildung an.

Pb 2 wirkt auf das Themenfeld Mobilität: Bei der Ausgestaltung der Entwicklungsbereiche M 2.1 bis M 2.4 soll der öffentliche Nahverkehr stärker eingebunden und genutzt werden. Dies kann neben einem positiven Effekt auf die Reduktion von Treibhausgasemissionen auch zu positiven Effekten für strukturschwache und bevölkerungsarme Bereiche unter Mobilitätsaspekten führen. ${ }^{149}$

Pb 2 wirkt auf das Themenfeld Ausbildung: Im Rahmen dieses E+E-Vorhabens soll ein Aus- und Weiterbildungsmodul „Grünes Band“ für zertifizierte Naturund Landschaftsführer entwickelt und erprobt werden (M 2.5). Das Curriculum soll u.a. folgende Themengebiete beinhalten: Beherrschung und Umgang mit der im Projektgebiet vorgesehenen Informations-Infrastruktur, Kenntnisse der touris-

149 Im Projektantrag ist der Punkt „Einbindung des öffentlichen Nahverkehrs“ nicht weiter ausgeführt. Zu denken wäre hier an einen Ausbau und eine Verstetigung der Erreichbarkeit von bevölkerungsarmen Regionen am Grünen Band mit öffentlichen Verkehrsmitteln. Hieraus könnte unter Mobilitätsaspekten ein Nutzen für die dort lebenden Menschen entstehen. 
tischen Infrastruktur im Projektgebiet und der naturschutzfachlichen Besonderheiten sowie Entwicklung von modellgebietsspezifischen Exkursionsangeboten.

$\mathrm{Pb} 2$ wirkt auf das Themenfeld Sicherheit (Umweltbildung): Sicherheit wird im Rahmen einer nachhaltigen regionalen Entwicklung als ein wichtiges Themenfeld erachtet und steht deshalb auch in der Maßnahmenbasismatrix (siehe Abbildung 13). Das Wirkungspotenzial dieses „Erprobungs- und Entwicklungsvorhabens" besteht auch in einer Sensibilisierung für Umweltthemen. Diese kann indirekt einen Beitrag leisten, sorgsamer mit der Umwelt umzugehen. Obgleich der direkte Einfluss einer entsprechenden Maßnahme auf die Höhe von Straftaten gegen die Umwelt empirisch nur schwer nachweisbar ist, ${ }^{150}$ so ist die Annahme nicht unplausibel, dass Naturbeobachtungen und Exkursionen am Grünen Band sowie Tage der Grenzerfahrung mit Events im Bereich Natur sowie naturkundliche Führungen und Beobachtungen zur Sensibilisierung für entsprechende Themen beitragen (insbesondere M 2.4 und M 2.6). ${ }^{151}$

$\mathrm{Pb} 2$ wirkt auf das Themenfeld regionale Identität: Die im Projektbaustein 2 geplanten Maßnahmen zur Entwicklung von Erlebnisangeboten haben als gemeinsames Merkmal, dass sie grenzübergreifend ausgerichtet sind. Vor dem Fall des Eisernen Vorhangs gab es eine klar definierte Grenze und folglich auch klar definierte ,angrenzende“ Regionen und „regionale“ Identitäten. Heute besteht die Chance die regionale Identität ${ }^{152}$ dahingehend zu entwickeln, dass nicht mehr in diesseits und jenseits der Grenze differenziert wird. Der Projektbaustein 2 und die in ihm enthaltenen Maßnahmen bieten die Möglichkeit ein verbindendes Element zu schaffen und dadurch einen Beitrag zur Entwicklung des regionalen Zusammenhaltes zu leisten.

Für den Projektbaustein 2 „Entwicklung von Erlebnisangeboten“ lässt sich klar feststellen, dass sein Hauptwirkungspotenzial im Themenfeld Tourismus liegt. Durch den Maßnahmenbereich Work-Camps werden weitere Themenfelder regionaler Nachhaltigkeit direkt angesprochen (M 2.6). Im Vergleich zum Projektbaustein 1 lässt sich sagen, dass die Maßnahmen im Projektbaustein 2 eine relativ geringere Ausgestaltung besitzen im Hinblick auf die Integration von ökologischen, ökonomischen und sozialen Themen.

150 Grundvoraussetzung ist das Vorhandensein von entsprechendem Datenmaterial. Aber auch bei Anwendung von Methoden der Korrelationsstatistik ist die Ableitung von kausalen Bezügen, die allein auf entsprechenden Signifikanzniveaus beruht, nicht möglich. Für die betrachtete Modellregion fehlt es aber bereits an entsprechendem Datenmaterial.

151 Vgl. Knolle, Friedhart (2004), S. 65, sowie Bittner A. (2003).

152 Vgl. BUND (2008), S. 12. 
Wirkungspotenzial des Projektbausteins $3(\mathrm{~Pb} 3)$ „Informationsangebot und Kommunikation vor Ort"

$\mathrm{Pb} 3$ wirkt auf das Themenfeld Tourismus: Die meisten Maßnahmen in diesem Projektbaustein haben einen Bezug zur touristischen Inwertsetzung entsprechender Bereiche des Grünen Bandes. Geplant sind verschiedene Formen der Informationsvermittlung. Um konkrete Stationen bei Führungen nacherlebbar zu machen, sollen u.a. Tondokumente verwendet werden, die in Zusammenarbeit mit Zeitzeugen und Experten erstellt werden sollen (M 3.1). Ferner ist geplant jeweils eine Informationsstelle „Grünes Band“ in Form einer Ausstellung im Grenzmuseum Mödlareuth (M 3.2.1) und im Wasserschloss Mitwitz (M 3.2.3) einzurichten. Die Ausstellungen sollen gleichsam die historisch-politisch bedeutsamen Aspekte des Grünen Bandes sowie die naturschutzfachlichen Aspekte herausarbeiten. Beide Ausstellungsorte liegen an entgegen gesetzten Punkten der Modellregion. Daher soll zusätzlich eine Wanderausstellung mit gleichem inhaltlichem Fokus für das Projektgebiet eingerichtet werden (M 3.2.2). Daneben sollen auch ca. fünf Infopunkte „Grünes Band“ in Einrichtungen mit Personal (Museen, Gastronomie) in der Modellregion entstehen. Diese Infopunkte sollen in die touristischen Angebote (siehe Projektbaustein 2) bestmöglich integriert werden (M 3.3). Die Bereitstellung von Informationen über mobile Endgeräte mit Benutzerlenkung (M 3.4) dient der Professionalisierung der touristischen Pauschalen im Bereich der Routen- und Wanderkonzepte (siehe Projektbaustein 2). Die unterschiedlichen Formen der Informationsvermittlung sollen verschiedene Zielgruppen ansprechen.

$\mathrm{Pb} 3$ wirkt auf das Themenfeld regionale Identität: Die regionale Identität wird ebenfalls durch sämtliche Maßnahmen dieses Projektbausteines angesprochen. Bei allen zuvor unter „Wirkung auf das Themenfeld Tourismus“ angesprochenen Maßnahmen ist ein Zusammenwirken von Akteuren diesseits und jenseits der ehemaligen Grenze wünschenswert und zum Großteil auch erforderlich. Dies gilt für die Aufarbeitung aller geplanter Themen im Zusammenhang mit der ehemaligen Grenze wie im Themenbereich Natur und im Themenbereich Geschichte. Durch das Zusammenwirken von Akteuren diesseits und jenseits der ehemaligen Grenze, können unterschiedliche regionale Identitäten durch das Grüne Band ein verbindendes Element bekommen. ${ }^{153}$ Wie diese Zusammenarbeit im Rahmen des Projekts aussah, zeigen die Kapitel 3.3.3.1 ff.

$\mathrm{Pb} 3$ wirkt auf das Themenfeld Sicherheit (Umweltbildung): Durch Sensibilisierung für Umweltthemen kann ein Beitrag geleistet werden, sorgsamer mit der Umwelt umzugehen. Einen direkten Zusammenhang zwischen einer entsprechenden Maßnahme und der Höhe von Straftaten gegen die Umwelt empirisch ${ }^{154}$ nachzu-

153 Vgl. BUND (2008), S. 12.

154 Grundvoraussetzung ist das Vorhandensein von entsprechendem Datenmaterial. Aber auch bei Anwendung von Methoden der Korrelationsstatistik ist die Ableitung von kausalen Bezügen, die 
weisen ist schwierig. Dennoch ist die Annahme nicht unplausibel, dass die Informationsangebote, die im Rahmen des Projektbaustein 3 angeboten werden, zur Sensibilisierung ${ }^{155}$ für entsprechende Themen beitragen können.

Für den Projektbaustein 3 „Informationsangebot und Kommunikation vor Ort“ lässt sich klar feststellen, dass sein Hauptwirkungspotenzial in den Themenfeldern Tourismus, regionale Identität und Sicherheit (Umweltbildung) liegt. Im Vergleich zum Projektbaustein 1 lässt sich sagen, dass die Maßnahmen im Projektbaustein 3 eine relativ geringere Wirkung besitzen im Hinblick auf die Integration von ökologischen, ökonomischen und sozialen Themen.

\section{Wirkungspotenqial des Projektbausteins 4 (Pb 4) „Marketing und externe Kommunikation“}

Maßnahmen, wie sie in den Projektbausteinen 1 bis 3 enthalten sind, sind nicht aus sich heraus wirksam und können nicht längerfristig bestehen bleiben, wenn niemand von ihnen weiß. Daher bietet dieser Projektbaustein 4 für die Projektbausteine 1 bis 3 flankierend konzentrierte Maßnahmen

$\mathrm{Pb} 4$ wirkt auf das Themenfeld Tourismus: Zu den Maßnahmen im Innenmarketing zählen die Etablierung von Kommunikationswegen zu den (potentiellen) Leistungserbringern ( $\mathrm{M}$ 4.1). Hierzu dienen regionale Informationsveranstaltungen und Workshops. Zur Professionalisierung der Entwicklung von passenden touristischen Pauschalen informiert die Tourismusorganisation Viabono über die damit verbundenen Chancen und Möglichkeiten der Vermarktung. Damit das Angebot von touristischen Pauschalen auch auf eine Nachfrage trifft, wird dieses nach außen entsprechend vermarktet (M 4.3 und M 4.4). Neben der Nutzung bestehender Vermarktungswege der Marketingpartner sind verschiedene Einzelmaßnahmen geplant (Druckerzeugnisse, Internetinformationen, CD-ROM). Dabei wird Wert auf einen (nach außen) möglichst einheitlichen Vermarktungsauftritt entlang des Grünen Bandes gelegt. Mittlerweile existiert für die Erlebnisregionen „Eichsfeld“, „Elbe-Altmark-Wendland“, „Harz“ und „Thüringer Wald \& Schiefergebirge/Frankenwald“ ein einheitlicher Internetauftritt. ${ }^{156}$ Hier können die touristischen Pauschalen nach den Kriterien Besinnung, Genuss und Freiheit sortiert und über die entsprechenden Kontaktdaten jede Pauschale gebucht werden. Das touristische Produkt soll als Paket mit allen seinen Eigenschaften vermarktet werden. Zu den Eigenschaften zählen insbesondere auch die Informationen über Möglichkeiten der umweltfreundlichen Anreise und Mobilität vor Ort aber auch

allein auf entsprechenden Signifikanzniveaus beruht, nicht möglich. Für die betrachtete Modellregion fehlt es aber bereits an entsprechendem Datenmaterial.

155 Vgl. Knolle, Friedhart (2004), S. 65, sowie Bittner A. (2003).

156 Siehe hierzu:

http://reisen.erlebnisgruenesband.de/HeaderTabs/erlebnisregionen/regionen.aspx (abgerufen am 30.03.2010). 
die Vermittlung der Naturschutzidee des Gesamtprojektes. ${ }^{157}$ Hierüber werden die Themenfelder Mobilität und Sicherheit (Umweltbildung) indirekt adressiert.

$\mathrm{Pb} 4$ wirkt auf das Themenfeld regionale Identität: Gelingt es im Rahmen des E+EVorhabens, insbesondere über die Ansprache durch Maßnahmen des Projektbausteins 4, Leistungsträger aus den verschiedensten Bereichen entlang des Grünen Bandes zu gewinnen, sich an der Entwicklung von touristischen Angeboten zu beteiligen und ihr jeweiliges regionales Erfahrungswissen einzubringen, dann entsteht dadurch auch ein Beitrag zur Entwicklung der regionalen Identität.

$\mathrm{Pb} 4$ wirkt auf das Themenfeld regionale Wirtschaft: Durch Kommunikation in die Region wird es möglich, regionale Akteure (kleine Betriebe, Hotels, Gaststätten etc.) über Möglichkeiten der Einbindung in Maßnahmen des E+E-Vorhabens zu informieren. Die theoretischen Voraussetzungen, Wirkungspotenzial in der regionalen Wirtschaftsstruktur zu entfalten, sind damit gegeben. ${ }^{158}$

Für den Projektbaustein 4 „Marketing und externe Kommunikation“ lässt sich klar feststellen, dass sein Hauptwirkungspotenzial in den Themenfeldern Tourismus und regionale Identität gesehen werden kann. Aber auch das Wirkungspotenzial im Themenfeld Mobilität und Sicherheit (Umweltbildung) ist hervorzuheben.

Insgesamt ist für das Erprobungs- und Entwicklungsvorhaben „Erlebnis Grünes Band in der Modellregion Thüringer Wald \& Schiefergebirge/Frankenwald" festzuhalten, dass das von den Einzelmaßnahmen der Projektbausteine ausgehende Wirkungspotenzial auf die Themenfelder der regionalen Nachhaltigkeit eine Streuung aufweist. Jedoch lässt sich eine Konzentration von Einzelmaßnahmen für die Themenfelder „Ökosysteme und Artenvielfalt“ (11 Einzelmaßnahmen), „Regionale Wirtschaft“ (12 Einzelmaßnahmen), „Tourismus“ (22 Einzelmaßnahmen), ,regionale Identität“ (25 Einzelmaßnahmen) und „Ästhetik der Landschaft“ (11 Einzelmaßnahmen) feststellen (siehe Abbildung 20). Hierüber sind alle drei Dimensionen (Ökologie, Ökonomie uns Soziales) bereits berücksichtigt. Dieses Ergebnis dokumentiert, dass es durch die entsprechende Ausgestaltung der Einzelmaßnahmen gelingt, Schutz- und Nutzaspekte zusammen zu führen und damit der Zielsetzung des E+E-Vorhabens aus theoretischer Perspektive gerecht zu werden. Gemäß der vorstehenden qualitativen Auswertung kann die Forschungshypothese, dass die Maßnahmen des E+E-Vorhabens auf regionale Nachhaltigkeit wirken, bestätigt werden.

\subsubsection{Nachbaltigkeitsspinne und regionale Verhaltensweisen}

Die Nachhaltigkeitsspinne dient dazu (siehe Kapitel 2) den Prozess einer nachhaltigen regionalen Entwicklung zu organisieren. Durch sie soll in deskriptiver Form ein Eindruck gewonnen werden, wie das E+E-Vorhaben auf regionale Nachhal-

157 Siehe hierzu ebenfalls den Internetauftritt: http://reisen.erlebnisgruenesband.de (abgerufen am 30.03.2010).

158 Vgl. Petermann, C. (2001), S. 5f. 
tigkeit wirkt bzw. wirken kann. Für die hier betrachtete Untersuchungsregion ist die Nachhaltigkeitsspinne in Abbildung 20 dargestellt. Die Ausschläge zeigen die Zahl der Maßnahmen im jeweiligen Themenfeld.

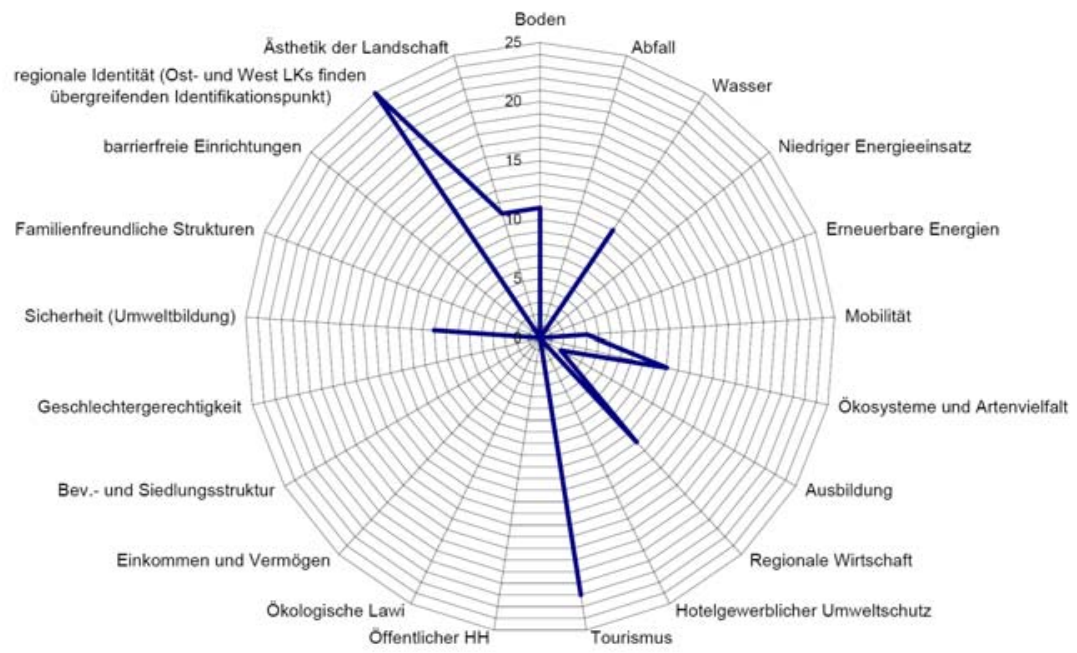

Abbildung 20: Nachbaltigkeitsspinne der Modellregion Thüringer W ald \& Schiefergebirge/Frankenwald

Die Maßnahmenkonzentration in den Themenfeldern „Ökosysteme und Artenvielfalt“, „Regionale Wirtschaft“, „Tourismus“, „regionale Identität“ und „Ästhetik der Landschaft" wird sichtbar durch entsprechende Ausschläge im Kreisdiagramm.

Diese Form der deskriptiven Auswertung des E+E-Vorhabens auf regionale Nachbaltigkeit stellt eine statische Perspektive dar. Damit Nachbaltigkeitswirkungen festgestellt werden können, bedarf es bestimmter Verhaltensweisen, die eine Entwicklung und Ausgestaltung von regionalen Nachbaltigkeitsthemen ermöglichen. Die statische Perspektive muss möglichst um eine dynamische Perspektive ergänzt werden. Um die statische Perspektive (hier Wirkungspotenzial des E+E-Vorhabens in der Untersuchungsregion Thüringer W ald \& Schiefergebirge/Frankenwald, siehe Basismatrix und Nachbaltigkeitsspinne) mit der dynamischen Perspektive zu kombinieren, wurden Interviews mit regionalen Akteuren geführt und ausgewertet. Tabelle 26 gibt eine Übersicht über die regionalen Verbaltenweisen, die aus den Interviews herausgearbeitet wurden. 
Tabelle 26: Synopse regionale und nationale Verbaltensweisen

\begin{tabular}{|c|c|}
\hline $\begin{array}{l}\text { Identifizierte regionale Managementregeln (Projektebene) } \\
\text { (aus Interviews mit regionalen Akteuren) }\end{array}$ & $\begin{array}{l}\text { Korrespondierende } \\
\text { Managementre- } \\
\text { geln } 159 \\
\text { der nationalen } \\
\text { Nachhaltigkeitsstra- } \\
\text { tegie }\end{array}$ \\
\hline $\begin{array}{l}\text { 1. Die Region übernimmt eigenverantwortlich Vorsorge für: } \\
\text { - ,saubere Energie“; } \\
\text { - Daseinsvorsorge in der Region, } \\
\text { - } \text { dauerhafte Maßnahmenfortführung. }\end{array}$ & R1 \\
\hline $\begin{array}{l}\text { 2. Die Region muss ihre gewachsenen Strukturen sowie Potenziale } \\
\text { kennen und ihr Handeln daran ausrichten. Das Handeln soll ferner dem } \\
\text { Grundsatz der Verhältnismäßigkeit genügen in Bezug auf: } \\
\quad \text { - physisches Kapital (z.B. vorhandene Infrastruktur nutzen,...), } \\
\quad \text { - immaterielles Kapital (z.B. regionale Kreativität und Ideenreich- } \\
\quad \text { tum nutzen,...). }\end{array}$ & R3 \\
\hline $\begin{array}{l}\text { 3. Der Strukturwandel in der Region soll wirtschaftlich erfolgreich sowie } \\
\text { ökologisch und sozial verträglich gestaltet werden. Zu diesem Zweck } \\
\text { sind die Themenfelder der regionalen Nachhaltigkeit so zu integrieren, } \\
\text { dass regionale wirtschaftliche Entwicklung, sozialer Zusammenhalt und } \\
\text { Umweltschutz Hand in Hand gehen. Dabei sind insbesondere folgende } \\
\text { Themen integrativ zu betrachten: } \\
\quad \text { - Naturschutz, } \\
\text { - regionale Entwicklung durch Tourismus, } \\
\text { - Geschichte und Kultur. }\end{array}$ & R5 \\
\hline $\begin{array}{l}\text { 4. Die Bewahrung und Schaffung von historischem Wissen durch For- } \\
\text { schung und Dokumentation sowie die Weitergabe dieses Wissens durch } \\
\text { spezifische Bildungsmaßnahmen spielt eine entscheidende Rolle für die } \\
\text { regionale Identität. }\end{array}$ & R6 \\
\hline $\begin{array}{l}\text { 5. Um den sozialen Zusammenhalt zu stärken, sollen } \\
-\quad \text { Armut und sozialer Ausgrenzung soweit wie möglich vorgebeugt } \\
\text { werden. } \\
\text { - allen Bevölkerungsschichten Zugang zu angebotenen Bildungs- } \\
\text { maßnahmen (Kunst, Kultur, Geschichte) ermöglicht werden. } \\
\text { - alle am gesellschaftlichen und politischen Leben teilhaben. } \\
\text { Eine Möglichkeit, der Teilhabe kann darin bestehen sich regional zu } \\
\text { engagieren (z.B. als Leistungserbringer im Rahmen des E+E-Vorhabens) }\end{array}$ & R9 \\
\hline
\end{tabular}

Für die regionale Ebene lassen sich Verhaltensweisen identifizieren, die eine entsprechende Entwicklung in den Themenfeldern der regionalen Nachhaltigkeit

159 Die Nummerierung gibt die Stellung im Rahmen der 10 Regeln der nationalen Nachhaltigkeitsstrategie wieder.

160 Vgl. Die Bundesregierung (2008). 
begünstigen können. Vorbild sind hier die zehn Managementregeln der Nachhaltigkeitsstrategie des Bundes. In Tabelle 26 sind regionale und nationale Regeln einander zugeordnet.

Die Tabelle zeigt, dass die befragten Akteure Hinweise für Verhaltensweisen liefern, die den Prozess einer nachhaltigen Regionalentwicklung günstig beeinflussen können. Allen identifizierten regionalen Verhaltensweisen kann eine korrespondierende Managementregel aus der bundesnationalen Nachhaltigkeitsstrategie zugeordnet werden. Die identifizierten regionalen Managementregeln 1 bis 5 weisen jeweils einen unterschiedlichen Grad an integrativem Denken auf. So fokussiert die Regel unter 3. alle drei Dimensionen einer nachhaltigen regionalen Entwicklung (Wirtschaft, Ökologie, Soziales) wohingegen die Regel unter 4. eher spezifisch auf die „regionale Identität“ abstellt, die im Rahmen einer nachhaltigen regionalen Entwicklung als wichtig erachtet wird. Um die statische Perspektive nun mit der dynamischen zu verbinden, ist zu prüfen, ob den Themenfeldern der regionalen Nachhaltigkeit, die durch die Maßnahmen des E+E-Vorhabens mit beeinflusst werden, korrespondierende Verhaltensweisen gegenüberstehen. Dies ist hier für die fünf Themenfelder mit der höchsten Konzentration von Einzelmaßnahmen zu bejahen. Dem Themenfeld „Ökosysteme und Artenvielfalt“ und dem Themenfeld „Regionale Wirtschaft" können jeweils die beiden Regeln 1 und 3, dem Themenfeld „Tourismus" können die Regeln 1, 2, 3, 4, 5, dem Themenfeld „regionale Identität“" können die Regeln 2, 3, 4, 5 und dem Themenfeld „Ästhetik der Landschaft" die Regeln 2 und 3 zugeordnet werden.

Durch das Vorhandensein von entsprechenden regionalen Managementregeln, die Verhaltensweisen für die Themenfelder formulieren, in denen die Maßnahmen des E+E-Vorhabens Wirkungspotenzial entfalten, bestehen theoretisch gute Ausgangsbedingungen, diese Themenfelder nachhaltig zu gestalten.

\subsubsection{Nachhaltigkeitsinstitutionen}

Die in Tabelle 26 identifizierte regionale Managementregel 1 benennt als einen Aspekt der eigenverantwortlichen Vorsorge die „dauerhafte Maßnahmenfortführung“". Daher ist möglichst zu Beginn eines Förderprojektes nach Institutionen Ausschau zu halten, welche die im Rahmen des Förderzeitraumes initiierten Prozesse anschließend fortführen können.

Zur Prüfung dieser Möglichkeiten wurden in Landkreisen, die Teil der Modellregion Thüringer Wald \& Schiefergebirge/Frankenwald des E+E-Vorhabens Erlebnis Grünes Band sind, Interviews mit Bürgermeistern geführt. Hierzu wurde ein standardisierter Fragenkatalog (siehe Anhang) benutzt. In dieser Untersuchungsregion wurden in den Landkreisen Saalfeld-Rudolstadt, Sonneberg, Hildburghausen, Schmalkalden-Meiningen und Gotha erhoben. Dabei wurden folgende Arten von Nachhaltigkeitsinstitutionen identifiziert:

- Integrierte Stadtentwicklungskonzepte (ISEK); siehe zur genaueren Beschreibung Kap 3.2.2.3. 
- Verschiedene regionale Einzelinitiativen, für die sich keine allgemeinen Aussagen ableiten lassen; siehe zur genaueren Beschreibung Kap 3.2.2.3.

- Lokales Agenda-21-Büro. In der Stadt Gotha wurde ein lokales Agenda-21Büro eingerichtet, um auf kommunaler Ebene gemäß Kapitel 28 der Agenda 21 „Initiativen der Kommunen zur Unterstützung der Agenda 21“ einen Beitrag zur nachhaltigen Entwicklung zu leisten. Das Agenda-Büro ist im Stadtplanungsamt der Stadtverwaltung Gotha angesiedelt. Koordiniert durch einen Agenda-Beirat entwickeln engagierte Bürgerinnen und Bürger sowie Verbände und Firmen Maßnahmen und Projekte zur Gestaltung eines kommunalen Nachhaltigkeitsprozesses. Das Engagement erfolgt ehrenamtlich in vier Arbeitsgruppen (Bildung \& Soziales, Energie \& Wirtschaft, Kinder \& Jugend sowie Stadtentwicklung \& Naturschutz). Das Agenda-Büro leitet die Ideen und Projekte aus der ehrenamtlichen Tätigkeit direkt an die Stadtverwaltung weiter.

Die zuvor genannten Institutionen haben in unterschiedlicher Weise und Intensität eine Verbindung zu einzelnen oder mehreren Themenfeldern der regionalen Nachhaltigkeit. Sofern über die Fortführung von im Förderzeitraum initiierten Prozessen und Maßnahmen nachgedacht wird, bieten sich hier verschiedene Anknüpfungspunkte. Exemplarisch ${ }^{161}$ kann dies an der Institution „Lokales-Agenda21-Büro der Stadt Gotha“ verdeutlicht werden. Obschon diese Nachhaltigkeitsinstitution einen stadtbezogenen Fokus besitzt, bestehen inhaltliche Bezüge zwischen den Arbeitsgruppen des Agenda-Büros und den Projektbausteinen des E+E-Vorhabens. Die Maßnahmen der Projektbausteine 2 „Entwicklung von Erlebnisangeboten“ und 3 „Informationsangebot und Kommunikation vor Ort“ zeigen inhaltlich eine Nähe zur Arbeitsgruppe Bildung und Soziales. Die Fortführung von erlebnispädagogischen Maßnahmen wie den Work-Camps (Pb2, M 2.6) kann inhaltliche Schnittmengen entwickeln mit den Themen der Arbeitsgruppe Kinder und Jugend. Ferner könnte die Arbeitsgruppe „Stadtentwicklung und Naturschutz“ inhaltliche Bezüge zum Projektbaustein 4 ,Marketing und externe Kommunikation" herstellen.

Durch das Vorhandensein von entsprechenden regionalen Institutionen, die inhaltlich eine Nähe zu den Themenfeldern der regionalen Nachhaltigkeit haben, in denen die Maßnahmen des E+E-Vorhabens Wirkungspotenzial entfalten können, bestehen theoretisch gute Ausgangsbedingungen, um im Förderzeitraum entwickelte Maßnahmen und initiierte Prozesse langfristig fortführen zu kön-

161 Obgleich die Stadt Gotha geografisch nicht in direkter Nachbarschaft zum Grünen Band liegt, so kann das Beispiel „Lokales-Agenda-21-Büro der Stadt Gotha“ dennoch verdeutlichen, worauf es bei der Identifikation von Nachhaltigkeitsinstitutionen ankommt. Vorstellbar wären zum Beispiel Partnerschaften mit städtischen Bildungseinrichtungen, die entsprechende erlebnispädagogische Angebote am Grünen Band nutzen möchten. 
nen. ${ }^{162}$ Eine möglichst frühzeitige Kontaktaufnahme zu den identifizierten regionalen Nachhaltigkeitsinstitutionen, um Möglichkeiten der Weiterführung von Projekten auszuloten, erhöht die Wahrscheinlichkeit, dass diese nicht mit Ende des Förderzeitraumes ebenfalls enden.

Abschließend kann im Rahmen des Kapitel 3.3.3 festgehalten werden, dass das Erprobungs- und Entwicklungsvorhaben „Erlebnis Grünes Band in der Modellregion Thüringer Wald \& Schiefergebirge/Frankenwald“ über seine Maßnahmen Wirkungspotenzial in verschiedenen Themenfeldern, die im Rahmen einer nachhaltigen Regionalentwicklung für wichtig erachtet werden, entfalten kann. Dies sind insbesondere die Themenfelder „Ökosysteme und Artenvielfalt“, „Regionale Wirtschaft", „Tourismus“, „Regionale Identität" und „Ästhetik der Landschaft“. Die identifizierten regionalen Managementregeln und regionalen Institutionen, die inhaltlich einen Bezug zu Nachhaltigkeitsthemen haben, bilden flankierend die Voraussetzungen, um die im Förderzeitraum umgesetzten Maßnahmen und angestoßenen Prozesse im Sinne der nachhaltigen regionalen Entwicklung erfolgreich umsetzen und nach Ablauf der Förderung fortsetzen zu können.

Da zum Zeitpunkt dieser Untersuchung viele Maßnahmen noch nicht umgesetzt bzw. in der Umsetzungsphase sind, kann die Forschungshypothese, dass die Maßnahmen auch dieses E+E-Vorhabens auf regionale Nachhaltigkeit wirken, aus einer theoretischen Perspektive bestätigt werden.

\subsubsection{Beispielhaft Synthese der Perspektiven}

Die Entwicklung und Umsetzung der Projektmaßnahmen werden maßgeblich durch die Verhaltensweisen der Akteure im Netzwerk bestimmt. Damit die Maßnahmen folglich im Sinne der Nachhaltigkeit umgesetzt werden, müssen die Akteure im Netzwerk entsprechende zur Nachhaltigkeit passende Verhaltensweisen an den Tag legen.

Wie die Verhaltensweisen der Akteure im Netzwerk wirken, kann an zwei Beispielen veranschaulicht werden. Im Rahmen des Projekts sollte eine Form des Tourismus verfolgt werden, die mit dem Stichwort „Ruhe“ überschrieben wurde und naturschutzrelevante Aspekte bei der Entwicklung berücksichtigte. Also „,...) keine actionreichen Dinge, sondern es soll, ja, mit dem Grünen Band irgendwo und der Natur im Einklang stehen." (Zitat aus einem Interview). Hierzu lässt sich als Verhaltensweise die regionale Regel 4 (siehe Abschnitt 3.3.3.2) zuordnen, die auf eine integrative Betrachtung unterschiedlicher Themenfelder abzielt und in der Folge die Berücksichtigung selbiger bei der Maßnahmengestaltung mit sich bringt.

Entsprechend wurde bei der Entwicklung touristischer Pauschalen auf den Naturschutz eingegangen und damit dieses Themenfeld integrativ betrachtet. So

162 Zur allgemeinen Diskussion der Rolle von Institutionen im Kontext einer nachhaltigen Entwicklung siehe Rennings, K. et al. (1999), S. $203 \mathrm{ff}$. 
wurde bei der Auswahl von Wanderrouten auf für den Naturschutz sensible Bereiche Rücksicht genommen und im Zweifelsfalle Alternativrouten entwickelt. So genannte „motorisierte touristische Erkundungsfahrten auf dem Kolonnenweg““ wurden im Rahmen von Diskussionen in verschiedenen Workshops von den Akteuren im Netzwerk als „nicht im Sinne des Projekts“ eingestuft und daher nicht weiter verfolgt bzw. beworben. Daran zeigt sich die Funktionsweise der Netzwerkmerkmale „Probleme und Lösungswille“ und „Lernen“. So wurden zum einen im Diskurs zwischen den Akteuren Kompromisse erarbeitet und zum anderen haben die Akteure ein Verständnis für die Belange der jeweils anderen Fachrichtungen entwickelt. Beides unterstreicht die strategische Qualität „diskursiv“ im Netzwerk. 


\subsection{Naturschutzgroßprojekt Schaalsee-Landschaft}

\subsubsection{Situation und Kontext}

Das 1992 gestartete Naturschutzgroßprojekt „Schaalsee Landschaft“ ist die nördlichste der vier Untersuchungsregionen. Sie liegt im schleswig-holsteinischen Kreis Herzogtum Lauenburg (Naturpark Lauenburgische Seen) sowie in den mecklenburgischen Landkreisen Nordwestmecklenburg und Ludwigslust (Biosphärenreservat Schaalsee).

Das Naturschutzgroßprojekt befindet sich seit Beginn der Förderung in der Trägerschaft eines Zweckverbandes Schaalsee-Landschaft (ZSL) mit Sitz in Ratzeburg, Schleswig-Holstein. Mitglieder des Verbandes sind der Kreis Herzogtum Lauenburg (Schleswig-Holstein), der Landkreis Nordwestmecklenburg (Mecklenburg-Vorpommern) sowie der Landkreis Ludwigslust (MecklenburgVorpommern) sowie die Umweltstiftung WWF Deutschland. Die erste Fördertranche erstreckte sich vom 20. November 1992 bis 31. Dezember 2003, die zweite Fördertranche erstreckte sich vom 11. Februar 2005 bis 31. Dezember 2009. Die zweite Tranche wurde im Kontext der Optimierung der Förderrichtlinie unter veränderten Rahmenbedingungen durchgeführt, so dass das Projekt mit einer Moderationsphase begonnen wurde. Das Projekt „Schaalsee-Landschaft" gehört innerhalb der vom Bundesministerium für Umwelt, Naturschutz und Reaktorsicherheit geförderten Naturschutzgroßprojekte mit einer Fläche von 32.867 ha und einem Investitionsvolumen von rund 26 Millionen Euro zu den fünf größten Projekten dieser Art in Deutschland. ${ }^{163}$

\subsubsection{Netzwerkperspektive}

Bei der Netzwerkanalyse ist zu beachten, dass das Projektnetzwerk die aktivste Phase bereits durchschritten hat und nach 17 Jahren ein, im Vergleich zu den Netzwerken in den drei anderen Untersuchungsregionen, etabliertes Netzwerk vorliegt. Das Förderprojekt steht zum Untersuchungszeitpunkt kurz vor Ende der zweiten Fördertranche (Ende 2009) und beschäftigt sich vorwiegend mit Feinheiten des PEPL und verwaltenden Tätigkeiten. Die regionalen Erfahrungen bezüglich der Verankerung des Projekts in die Region und Verknüpfung mit anderen sektoralen Interessen sind aufgrund des langen Projektzeitraums entsprechend umfangreich und werden im Folgenden erfasst und analysiert.

163 http://schaalsee.herzogtum-lauenburg.de/(Zugriff 19.03.2010) 


\subsubsection{Strukturelle Analyse des Projektnetzoverks}

\section{Datenbasis}

Ausgangsbasis für die strukturelle Netzwerkanalyse ist die Projektbegleitende Arbeitsgruppe (PAG) mit 73 Vertretern der beteiligten Behörden, Ämter und Verbände auf Bundes-, Länder- und Landkreis-, sowie Kommunalebene.

In der ersten Befragungsrunde gaben 36 Akteure, d.h. 49\%, Rückmeldung und in der zweiten Befragungsrunde gaben 15 Akteure, d.h. 38\%, Rückmeldung. Daraus ergibt sich bezogen auf alle 112 Befragte eine Rücklaufquote von $46 \%$. Die Hälfte sendete den Fragebogen ausgefüllt zurück (25 Akteure), während die andere Hälfte anderweitig telefonische oder schriftliche Rückmeldung gab. ${ }^{164}$ Dementsprechend stützt sich die strukturelle Netzwerkanalyse auf 25 Fragebögen.

Diese 25 Akteure charakterisieren sich wie folgt: $36 \%$ stammen aus dem Kreis Herzogtum Lauenburg, $48 \%$ aus den beiden ostdeutschen Kreisen, $12 \%$ vertreten den Projektträger und 8\% die Bundesebene. Fachlich gehören 40\% dem Naturschutz an, 32\% der Landnutzung, 16\% der Politik und jeweils 4\% der Regionalentwicklung, Kommunalverwaltung und dem Wassersektor. Soziales, Tourismus, Wirtschaftsförderung und die Privatwirtschaft fehlen. Institutionell vertreten sie zu 56\% Behörden, mit 32\% Vereine und 16\% sind politische Vertreter. $85 \%$ sind zwischen 40 und 60 Jahre alt, $84 \%$ der Akteure sind männlich und $75 \%$ besitzen einen Hochschulabschluss. 64\% der im Rücklauf erfassten Akteure haben seit mehr als 10 Jahren die derzeit besetzte berufliche Position inne, so dass sie über entsprechende Erfahrungen und Kenntnisse in diesem Kontext verfügen. Dies ist insofern relevant, als sich - wie die Analyse zeigt - ein berufsbezogenes Netzwerk gebildet hat.

Die Grundgesamtheit des Netzwerks umfasst 125 Akteure. Die Analyseeinheit, auf der sich die Netzwerkanalyse stützt, umfasst 84 Akteure, denn für 33\% der 125 erfassten Akteure konnten auf Basis der vorliegenden Daten keine Beziehungen zum Projektnetzwerk nachgewiesen werden. Da jedoch nur ein Netzwerkausschnitt erfasst wurde, ist nicht belegt, ob die Akteure tatsächlich nicht bzw. nur schwach in das Projektnetzwerk eingebunden sind. Strukturell spiegelt sich in den erfassten Daten der Fördertatbestand in der prozentualen Teilhabe der Fachbereiche wider (siehe Abbildung 21). In der Grundgesamtheit ist der Naturschutz mit $29 \%$ vertreten, die Landnutzung mit $27 \%$, politische Interessenvertreter mit

\footnotetext{
16425 Befragte (22\%) haben sich mit einer begründeten Absage rückgemeldet. Davon gaben gut zwei Drittel an, nicht oder nicht ausreichend in das Projekt eingebunden zu sein, um den Fragebogen sinnvoll ausfüllen zu können. Vier Akteure leiteten den Fragebogen an Akteure weiter, von denen sie sich vertreten sahen. Dies waren drei unterschiedliche Akteure bzw. Institutionen, die sich bei der weiteren Analyse als dem Netzwerkkern zugehörig erwiesen. Zwei Akteure lehnten die Beantwortung aus Datenschutzgründen ab, während jeweils ein Akteur erst seit kurzer Zeit in der Region tätig ist bzw. kein Interesse an dem Forschungsvorhaben hatte.
} 
$10 \%$, Akteure der Regionalentwicklung und Tourismus mit jeweils $8 \%$, der Wassersektor mit $5 \%$, Fischerei und Jagd mit $6 \%$, die Raumplanung mit $4 \%$, Soziales mit $3 \%$, Kommunalvertretung mit $1 \%$, interessierter Bürger mit $2 \%$, Regionalentwicklung mit $1 \%$. Die Wirtschaftsförderung fehlt. Die Analyseeinheit ist ähnlich aufgestellt, in der Naturschutz mit 27\% vertreten ist, Landnutzung mit 24\%, Politik mit $18 \%$, Tourismus mit $11 \%$, Regionalentwicklung und der Wassersektor mit jeweils $7 \%$, Soziales mit $2 \%$, Planung und Kommunalvertretung mit jeweils $1 \%$. Das Vorhandensein der z. T. schwach vertretenen Fachbereiche in der Grundgesamtheit kann jedoch zugleich als Indiz für eine zwar ebenso schwache, aber immerhin bestehende übersektorale Vernetzung des Projekts mit regionalen Initiativen gewertet werden.

Die Interessenvertretung ist überwiegend organisierter Art. Die Grundgesamtheit wird mit $42 \%$ von Behörden dominiert, gefolgt von Vereinen mit $34 \%$, Politik mit $10 \%$, der Privatwirtschaft mit $9 \%$ und 5\% privaten Akteuren. Dies ist im Kontext des konzeptionellen Charakters von Naturschutzgroßprojekten zu sehen, die zum Ziel haben, einen Pflege- und Entwicklungsplan (= Naturschutzfachplan) $\mathrm{zu}$ erstellen und umzusetzen. Naturschutzgroßprojekte sind- bezogen auf die Planumsetzung - nicht auf breite bürger- bzw. zivilgesellschaftliche Beteiligung angewiesen. Diese erfordert die Zusammenarbeit mit betroffenen Grundeigentümern, die bspw. in Bauernverbänden organisiert sind, und insbesondere mit entscheidungszuständigen Stellen der Verwaltung. Das Auswertungsergebnis der Frage nach den Gründen für das Engagement (Frage 10, siehe Anhang) stützt dies, da sich die Akteure überwiegend im beruflichen Kontext für das Naturschutzgroßprojekt (68\%) engagieren. In der Analyseeinheit sind Behörden mit 37 $\%$ die stärkste Gruppe, gefolgt von Vereinen (30 \%). Politische Vertreter sind mit $18 \%$ vertreten, die Privatwirtschaft mit $11 \%$ und private Akteure mit $5 \%$.

Der größere Flächenanteil des Projektgebietes liegt in den ostdeutschen Kreisen. Das spiegelt sich so auch in der räumlichen Verteilung der Akteure wider. In der Grundgesamtheit stammen $55 \%$ der Akteure aus den beiden ostdeutschen Kreisen, $38 \%$ aus dem Kreis Herzogtum Lauenburg. Den Projektträger als länderübergreifende Institution vertreten $4 \%$, Bund/Land sind mit $2 \%$ vertreten. Der Überhang ostdeutscher Vertreter verstärkt sich in der Analyseeinheit mit 63 $\%$. Akteure des westdeutschen Kreises sind mit $30 \%$ vertreten, der Projektträger mit $6 \%$, Bund/Land mit $1 \%$. Vertreter der Landes- bzw. Bundesebene fehlen in der Analyseeinheit. Dies lässt im Vergleich zu deren Präsenz in der Region Eichsfeld-Werratal eine abnehmende Präsenz dieser Ebene im Laufe der Projektumsetzung vermuten. 

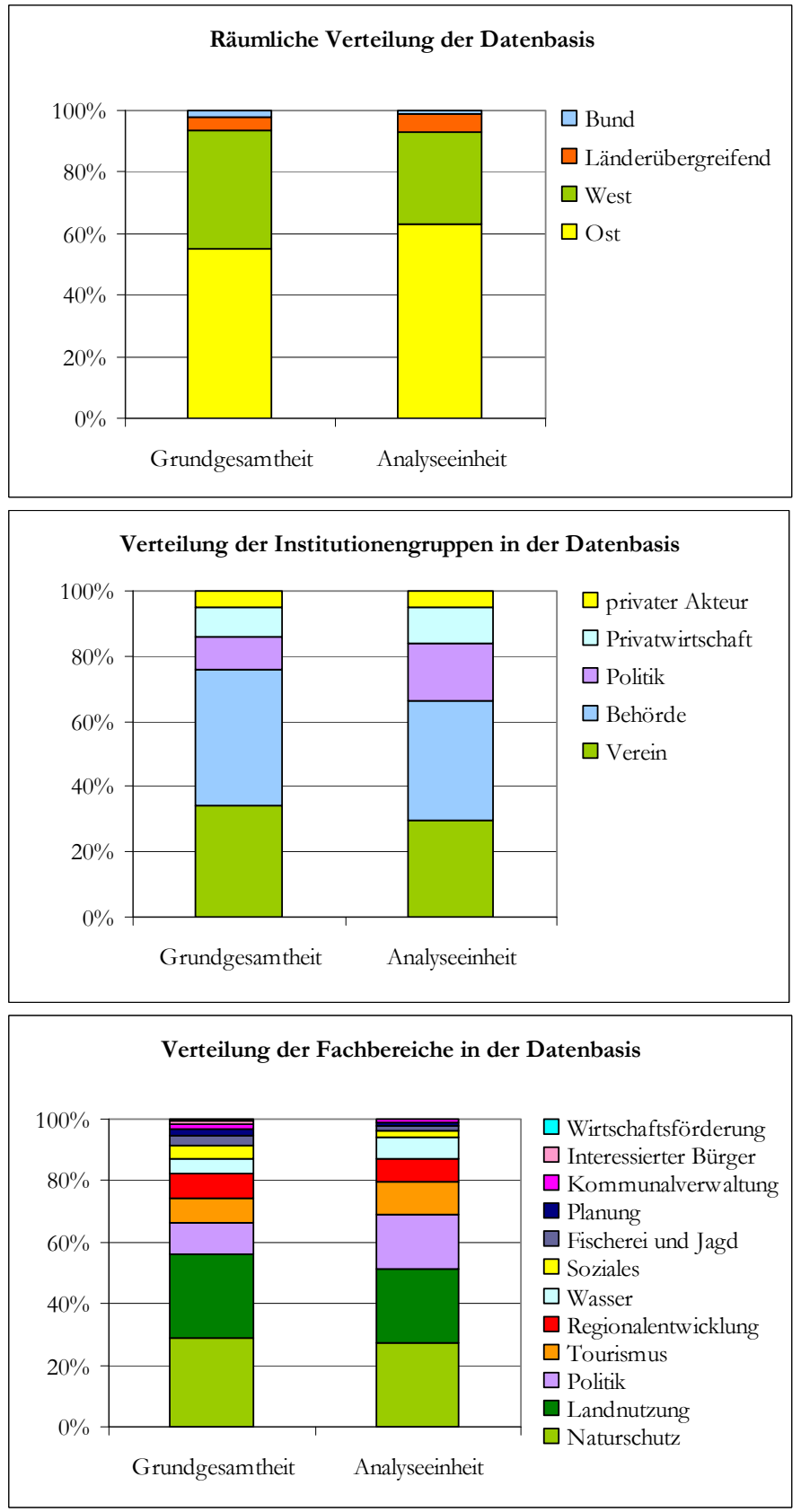

Abbildung 21: NGP Schaalsee-Landschaft - Verteilung der Fachbereiche, Institutionengruppen und räumliche Verteilung im Netzwerk. 


\section{Konstellation}

Die Gradzentralität zeigt, dass sich die sieben Akteure mit der höchsten Netzwerkaktivität auf sechs Stufen verteilen und dem Naturschutzbereich angehören (Zentralitätsindizies 0.333 bis 0.17 ), von denen sich jedoch zwei deutlich an die Spitze setzen - beide gehören dem Projektträger an. Die nähebasierte Zentralität im Netzwerk zeigt, dass sich eine Gruppe von zehn Akteuren, die relativ nahe beieinander liegen (0.332 bis 0.3), eine vorteilhafte Position durch den Zugriff auf effektive, schnelle und unabhängige Kontakte im Netzwerk erschlossen hat. Sie setzt sich aus sechs Naturschutz-, einem Landnutzung-, einem politischen Vertreter sowie einem Vertreter des Wassersektors zusammen. Besonders hervorgehobene „Maklerpositionen“" haben zwei Naturschutzvertreter als zentrale Akteure inne (0.100 und 0.093), wovon einer den Projektträger vertritt. Zusammen erweisen sich diese drei Akteure als wichtige fachübergreifende Anker im Netzwerk. Mit bereits deutlichem Abstand folgen zwei Vertreter der Politik und ein Naturschutzvertreter (siehe Abbildung 22).

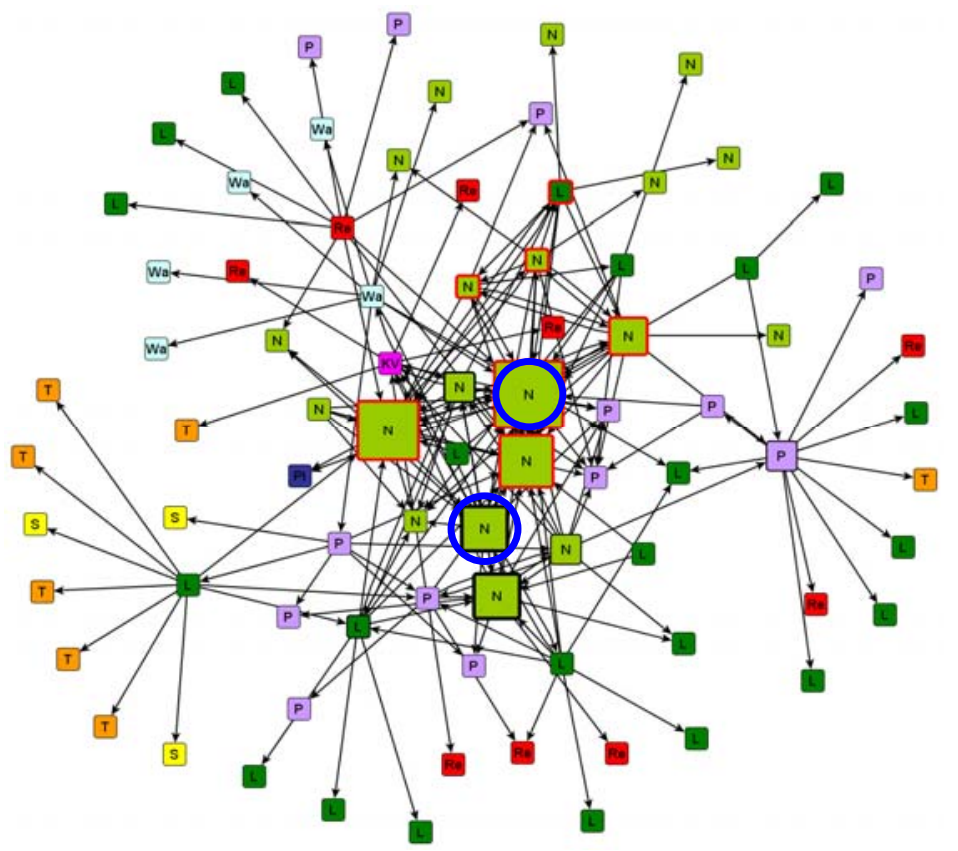

Abbildung 22: NGP Schaalsee-Landschaft - Graph des Projektnetzwerks ( $=$ Landnutzung, $N=$ Naturschutr, $S=$ Soziales, $P=$ Politik, $R_{e}=$ Regionalentwicklung, $K V=$ Kommunalverwaltung, $T=$ Tourismus, $W a=W$ asser, $\mathrm{P}=$ Planung, $\mathrm{O}=$ Makler, $\square=$ Kern 
Bezüglich der länderübergreifenden Zusammenarbeit im Netzwerk zeigt sich, dass die zentrale Spitze (Gradzentralität) durch den Zweckverband Schaalsee-Landschaft grundsätzlich länderübergreifend angelegt ist. Eine länderübergreifende Zusammenarbeit lässt sich aus dem Netzwerkgraphen anhand der Durchmischung der Akteure erkennen. Die länderübergreifenden Impulse werden jedoch durch weitere Analyseergebnisse relativiert (siehe Abbildung 23).

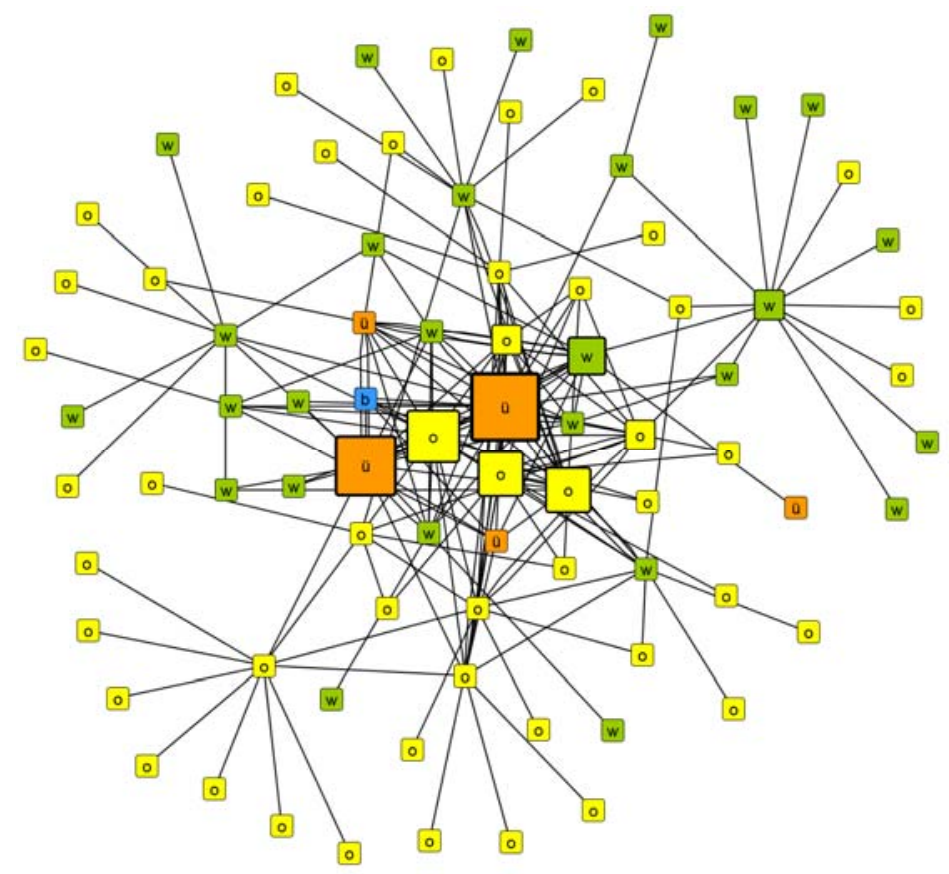

Abbildung 23: NGP Schaalsee-Landschaft - Länderïbergreifende Vernetzung im Projektnetzwerk (W= ,West, $O=$,Ost, $\ddot{u}=$ übergreifend, $B=$ Bund)

Bei der Analyse der ,indegree centrality "(Fragen 6 bis 8,) fällt zunächst die ansteigende Zahl der unverbundenen Akteure auf, je unmittelbarer nach Prestige gefragt wird, woraus eine Konzentration des Prestiges im Netzwerk auf eine relativ kleine Gruppe ablesbar ist. Ein Fünftel der genannten Beziehungen werden als nicht wichtig betrachtet, so dass 17 Akteure unverbunden sind. 25 Akteure bzw. Beziehungen gehen bei der Betrachtung von Abhängigkeiten verloren, da sie nicht für das Einholen von projektbezogenen Ratschlägen genutzt werden. 48 Akteure bzw. Beziehungen fallen schlussendlich aus dem Netzwerk heraus, wenn mit der Frage nach Schlüsselakteuren das offensichtliche Prestige angesprochen wird. Auf Basis der den erfassten Beziehungen zugewiesenen Wichtigkeit treten die drei Naturschutzvertreter $(0.239,0.194,0.149)$ als zentral hervor, die zuvor bereits die drei höchsten Gradzentralitätsindizies erlangt haben - zwei von ihnen vertreten den 
Projektträger. Bereits mit großem Abstand folgt ein weiterer Naturschutzvertreter (0.09), dann gemeinsam ein Naturschutz- und Politikvertreter (0.075). Bezogen auf die Frage nach dem eingeholten Rat erweisen sich die drei Naturschutzvertreter abermals als zentral $(0.237,0.186,0.153)$. Erst mit Abstand (0.102) folgt eine Vierergruppe von zwei Naturschutzvertretern und jeweils einem Politik- und Kommunalvertreter. Die unmittelbare Frage nach dem offensichtlichen Prestige bestätigt die drei bereits erfassten Naturschutzvertreter als Schlüsselakteure, wobei einer von ihnen besonderes Prestige gewonnen hat (0.371) und die beiden anderen erst mit Abstand (0.2) folgen. Ein Politiker (0.171) und eine Gruppe von einem Naturschutzvertreter sowie zwei politische Vertreter (0.114) schließen sich mit Abstand daran an.

Schon jetzt zeigt sich, dass sich der Fördertatbestand unmittelbar auf die Struktur des Netzwerks des Naturschutzgroßprojekts Schaalsee-Landschaft ausgewirkt hat. Es hat sich ein relativ großes Netzwerk gebildet, das durch eine Vielfalt schwach vertretener Fachbereiche am Netzwerkrand und eine starke sektorale Orientierung (Naturschutz und Landnutzung) insgesamt und insbesondere im fachlich spezialisierten Kern geprägt ist. Ein integrativer Charakter ist zwar ansatzweise erkennbar, aber die Vielfalt von Einzelakteuren unterschiedlicher Fachbereiche rückt rein quantitativ und bei Betrachtung der Wichtigkeit, der Abhängigkeiten und der Wertschätzung von Akteuren im Netzwerk in den Hintergrund.

\section{Netzwerkarbeit}

Die Wirkung des Naturschutzgroßprojekts auf die Vernetzung in der Region erscheint insofern schwach, als dass die Mehrheit der genannten Kontakte (72\%) bereits vor dem Naturschutzgroßprojekt bestand, während $28 \%$ der Kontakte durch das NGP geknüpft wurden. Nichtsdestotrotz ist die Bedeutung der neuen Kontakte nicht zu unterschätzen, denn es lässt sich eine Intensivierung und Ausweitung des regionalen Netzwerks - mit Fokus auf den Naturschutz - ablesen. Darüber hinaus zeigt sich, dass Vertreter des Zweckverbands SchaalseeLandschaft am häufigsten durch das Projekt kennen gelernt wurden (35\% der neuen Kontakte). Diese Kontakte können nicht als länderübergreifend eingeordnet werden, da der Verband eine länderübergreifende Institution ist. Im Rücklauf zeichnet sich ab, dass das Projekt nicht von länderübergreifender Zusammenarbeit geprägt und dass projektbezogen eine stärkere Vernetzung in den Landkreisen zu vermuten ist. 


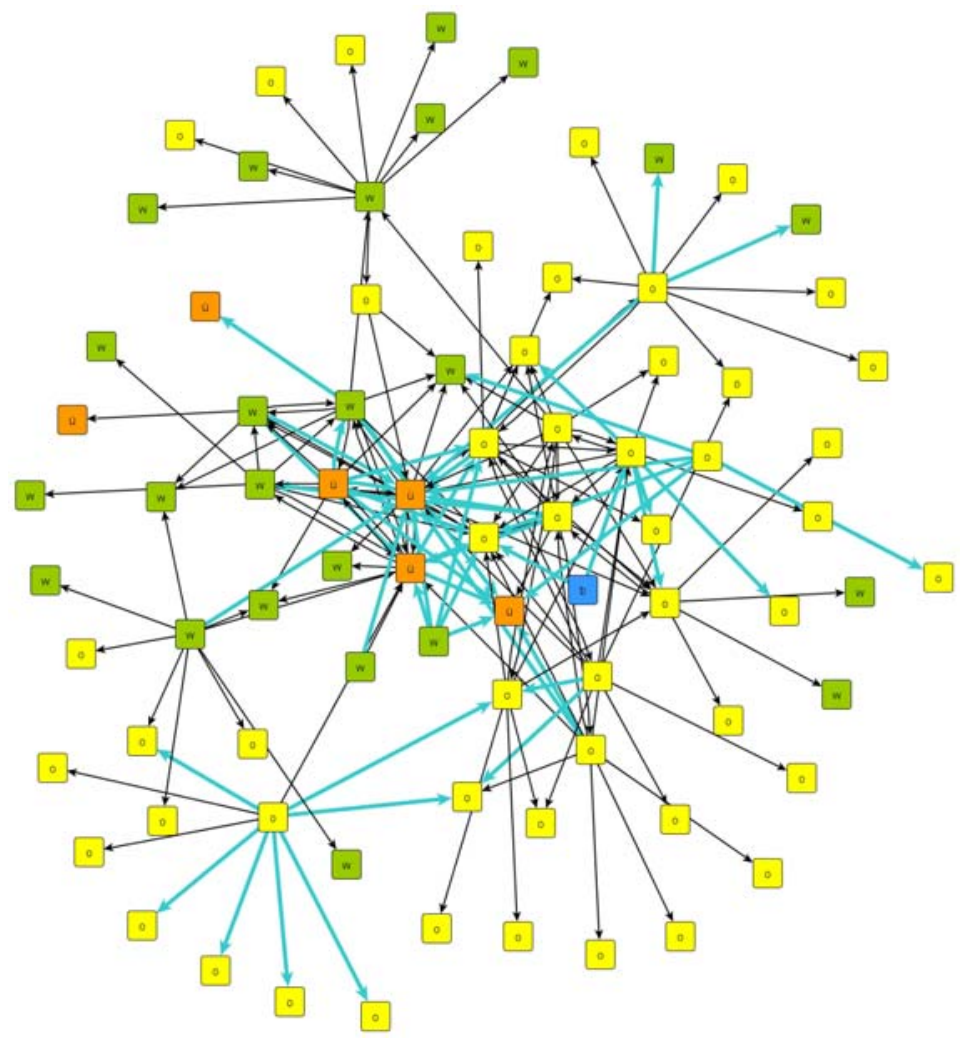

Abbildung 24: NGP Schaalsee-Landschaft - Alte (schwarz) und neue (blau) Kontakte im Netrwerk

Die Netzwerkarbeit wird maßgeblich durch deren Intensität bestimmt. Rein quantitativ erhielt der „häufige Kontakt“ die meisten Nennungen (34\%). Diese konzentrieren sich jedoch auf 31 Akteure, d.h. $37 \%$ des analysierten Netzwerks. Die Netzwerkarbeit wird des Weiteren durch die seltenen oder nie genutzten Kontakte charakterisiert, die zusammen $36 \%$ der erfassten Kontakte und $21 \%$ der erfassten Akteure betreffen. Betrachtet man die Fachbereichszugehörigkeit der häufig kontaktierten Akteure wird eine Fokussierung der Kontaktintensität auf den Naturschutzsektor deutlich (45\% der häufigen Kontakte; Politik und Landnutzung jeweils $19 \%$ ). 


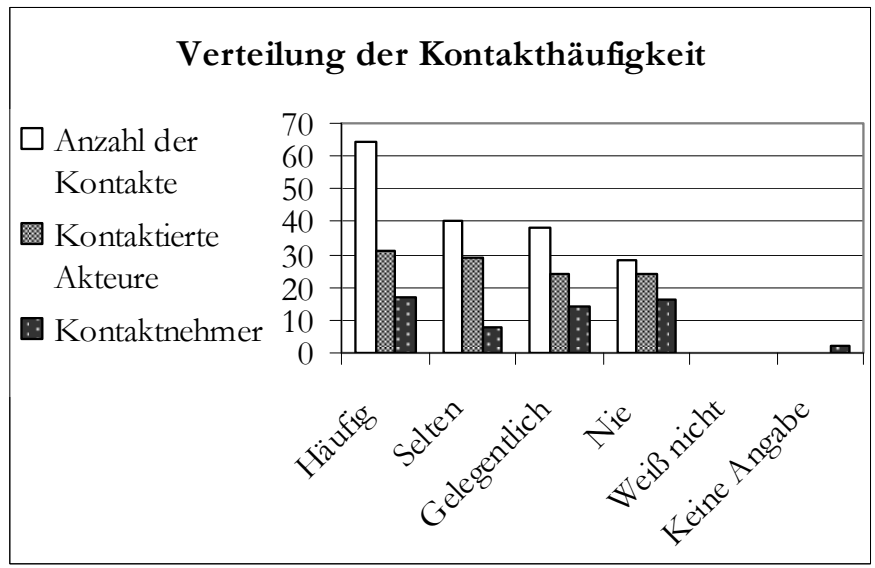

Abbildung 25: NGP Schaalsee-Landschaft - Verteilung der Kontakthäufigkeit

Im Netzwerk sticht keine favorisierte Kontaktart hervor. 52\% der Akteure, die geantwortet haben, nutzen alle Kontaktarten. Zusätzlich haben sie bestimmte Kontaktarten ausgewählt, so dass gemeinsam besuchten NGPProjektveranstaltungen oder Arbeitsbesprechungen ebenso wie den gängigen Kommunikationsmedien (Telefon, E-Mail) die gleiche Bedeutung zuzuweisen ist. Daraus ergibt sich ein vielfältiges Kommunikationsnetz im aktiven Netzwerkausschnitt. Betrachtet man die Vertretung der Fachbereiche in dieser Kategorie, zeigt sich ein weiteres Mal die Dominanz des Fachbereiches Naturschutz, gefolgt von der Landnutzung und Politik.

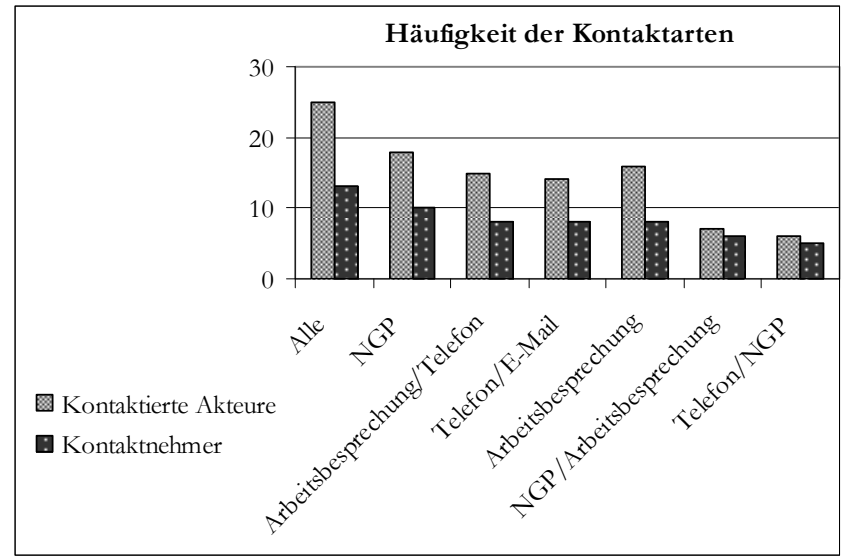

Abbildung 26: NGP Schaalsee-Landschaft - Häufigkeit der Kontaktarten 
Die Frage nach den Kontaktzwecken im Netzwerk zeigt, dass der Austausch von Ressourcen keine Rolle spielt. Dies erklärt sich angesichts der Förderkulisse, die die Fördermittel nur für klar abgegrenzte Zwecke bereitstellt. Hingegen kristallisiert sich die Kooperation zur Realisierung bestimmter Ziele sowie des Informationsaustausches als häufigster Grund der Netzwerkaktivität heraus.| Daraus lassen sich Bemühungen der Abstimmung zwischen dem NGP und anderen Initiativen ablesen.

Um erste Informationen bezüglich der Zufriedenheit der Akteure mit dem Netzwerk im Allgemeinen zu gewinnen, wurde nach Defiziten gefragt. Das am häufigsten benannte Defizit im Netzwerk ist die Kombination aus inhaltlichen und persönlichen Konflikten (29 \%). Die inhaltlichen Konflikte können als fachbzw. interessenbezogene Zieldivergenzen eingeordnet werden, die sich in der Zusammenarbeit unterschiedlichster Fachbereiche an einem naturschutzfachlichen Großprojekt ergeben. Unterschiedlichste Interessen und fachliche Meinungen werden in die Planung und Umsetzung des Naturschutzgroßprojektes einbezogen, die, kaum überraschend, nicht immer harmonisiert werden konnten. Persönliche Konflikte können stets bei intensiver Zusammenarbeit zwischen mehreren Personen entstehen und werden nur sekundär von fachlichen Divergenzen geprägt, wobei diese auch auf die Persönlichkeitsebene projiziert werden können. Grundsätzlich ist anzunehmen, dass im erhobenen Netzwerk die Netzwerkarbeit so intensiv ist, dass die Akteure, die geantwortet haben, diese Form von Konflikten wahrnehmen.

$40 \%$ der Akteure, die geantwortet haben (Naturschutz, Landnutzung, Politik, Wassersektor) bewerten ihre Einbindung in das Netzwerk mit sehr gut bis gut. Gleichwohl bewerten $24 \%$ der Akteure (Naturschutz, Landnutzung, Sportfischerei, Kommunalverwaltung) ihre Einbindung mit befriedigend bis ausreichend und 16\% mit mangelhaft bis ungenügend (Naturschutz, Politik). Eine bessere Einbindung der beiden dominanten Fachbereiche lässt sich folglich nicht bestätigen. 


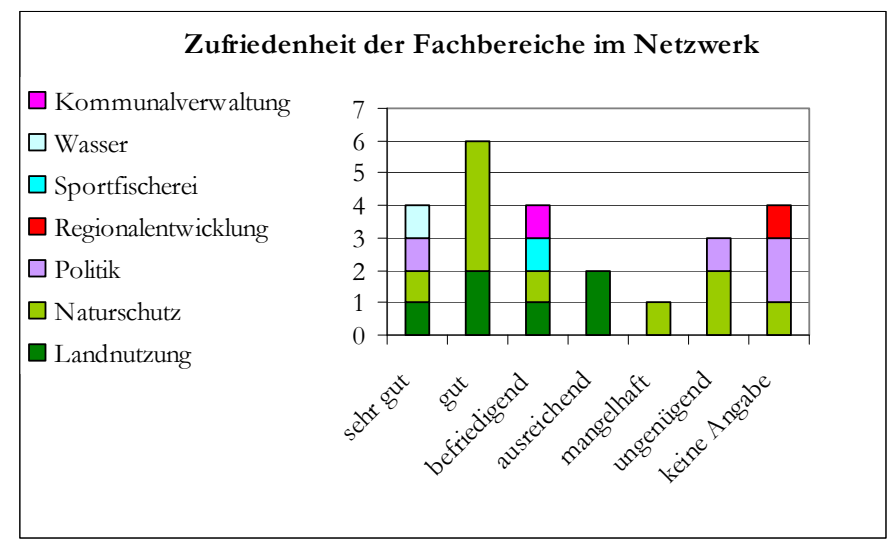

Abbildung 27: NGP Schaalsee-Landschaft-Zufriedenheit der Akteure mit Einbindung in Projektnetzwerk

Die erhobenen strukturellen Daten belegen für einen Ausschnitt des Netzwerks häufige Netzwerkaktivität, die sich zudem auf die Sektoren Naturschutz und Landnutzung konzentriert. Diese Erkenntnis ist vor dem Hintergrund des Befragungszeitpunkts und -kontexts einzuordnen. Es wurde spezifisch nach projektbezogener Aktivität gefragt, und dass diese im Jahr 2008 (Auslauf der Fördertranche 2009 und weitgehende Umsetzung des PEPL) bereits ein niedriges und fachlich konzentriertes Niveau erreicht hat, leitet sich aus der Tatsache ab, dass die Verhandlungen zum PEPL in der PAG und Region längst abgeschlossen sind. Zum Befragungszeitpunkt wurden Feinheiten des PEPL zwischen den formal Zuständigen verhandelt, so dass die fachliche Konzentration eine logische Folge ist. Folgende strukturelle Merkmale prägen das Netzwerk des Naturschutzgroßprojektes Schaalsee-Landschaft, die sich überwiegend durch die formelle Struktur des Fördertitels, zum anderen jedoch durch die regionsspezifische Netzwerkdynamik erklären.

Projektträger als zentraler Netzwerkmotor: Der Projektträger des NGP besitzt eine besonders vorteilhafte Position aufgrund seiner durch das Design des Fördertitels vorgegebenen Multifunktionalität. Diese spiegelt sich in dessen Zentralität wider. Im Moderationsbericht 2005 steht:

„Der Zweckverband hat einerseits als Eigentümer und Käufer bzw. Verpächter von Flächen, sowie als Verhandlungsführer bzgl. Flächenkauf und Flächentausch und andererseits als Vertreter des Naturschutzes und drittens als Mittler zwischen den Interessen der Länder, Kreise und Kommunen sowie der Bebörden und Ämter, eine wesentliche Schlüsselstellung in diesem Projek.t. "(Redlefsen 2006, S. 34)

Diese Schlüsselstellung zeigt sich in der Konstellation des Netzwerks, die von einer deutlichen Orientierung hin zum Projektträger geprägt ist. 
Spezialisiertes, starkes Kernnetzwerk: Das Projektnetzwerk ist insgesamt aufgrund dessen Fokussierung auf die Umsetzung des PEPL fachlich spezialisiert. Diese Spezialisierung wird durch ein starkes Kernnetzwerk von Akteuren des Naturschutzes betont.

Professionell und administrativ: Das Projektnetzwerk ist als professionell zu charakterisieren, da die große Mehrheit der Akteure über einen beruflichen Kontext insbesondere zuständige Behörden - mit dem Projekt verbunden sind.

Dialogorientiertes Netzwerk: Förderpolitisches Ziel des Netzwerkes ist es, den PEPL umzusetzen und die aus der Umsetzung resultierenden Nutzungskonflikte zu lösen. Die Kontaktzwecke bestätigen dieses Ziel, da sie eine Ausrichtung auf Information und Kooperation vorweisen.

Regional eingebettet: Der erhobene Ausschnitt des Projektnetzwerks zeigt, dass trotz der fachlichen Fokussierung eine Vielzahl weiterer Akteursfunktionen aus der Region in dem Projektnetzwerk vertreten ist, so dass sich eine Vernetzung mit vorhandenen regionalen Netzwerken zeigt. Dies wird darüber hinaus dadurch belegt, dass das Projektnetzwerk zu fast zwei Dritteln auf bestehende regionale Kontakte aufbauen konnte.

Potenzielle Multiplikatoren sind spezialisierte Fachpromotoren: Mindestens drei potenzielle Naturschutzvertreter wurden als Multiplikatoren des Projekts identifiziert. Ob sie diese Funktion aktiv ausfüllen, muss durch qualitative Erkenntnisse geklärt werden. Die Rolle der identifizierten Makler könnte, trotz ihrer schwachen Präsenz im Netzwerk, ebenso multiplikativ im Sinne regionaler Verankerung sein.

\subsubsection{Analyse der Netzwerkmerkmale und ihrer strategischen Qualität(en)}

Im folgenden Abschnitt werden die Ausprägungen der Netzwerkmerkmale auf Basis der strukturellen Netzwerkanalyse, der Interviewtranskripte sowie der Ergebnisprotokolle der Regionalen Workshops (RW1 und RW2) beschrieben. Dazu wird ein Stärken-Schwächenprofil genutzt, in das zugleich die Merkmalsausprägungen hinsichtlich der strategischen Qualität des Netzwerks eingeordnet werden.

\section{Konstellation: Breite Beteiligung}

Die Einrichtung eines länderübergreifenden Zweckverbandes ist positiv bezüglich der Projektverankerung vor Ort zu bewerten, da damit formal der politische Rückhalt gesichert ist. ${ }^{165}$ Durch die Beteiligung der Landkreise/Kreistage ist eine breite Interessenvertretung und damit auch Akteursorientierung hergestellt. Die Beteiligung eines Naturschutzverbandes als Mitglied des Zweckverbands ist als

\footnotetext{
165, ,...] Es ist sicherlich auch deshalb gut angekommen, weil es auch im politischen Raum gut verankert war. Sofort mit diesen demokratischen Strukturen eines ZV, manchmal etwas holprig, manchmal schwierig für die Akteure damit klar zu kommen. Aber es dient letztlich dazu, diese politische Verankerung zu finden, was andere Projekte eben nicht so baben, dadurch in dem Raum auch wieder kritisch beäugt werden, das muss man vielleicht auch dazu sagen" [...] (Zitat aus einem Interview: Naturschutz).
} 
integrativ einzuordnen, da die Vereinigung der politischen und naturschutzfachlichen Interessen unter einem institutionellen Dach dazu beitragen kann, dass sich ein gemeinsames Ziel- und Werteverständnis entwickelt. Das Netzwerk ist insofern akteursorientiert, als dass eine Vielfalt von Fachbehörden vertreten ist und das Umsetzungsverständnis des Trägers netzwerkorientiert angelegt ist. Auf ein Netzwerk ist das Projekt jedoch auch aufgrund des Freiwilligkeitsprinzips angewiesen. Als akteursorientiert erweist sich darüber hinaus die projektorientierte Vernetzung des NGP mit Vertretern der regionalen Wirtschaft, z.B. Landwirten oder Vereinen, die sich in unterschiedlichsten Bereichen betätigen (s. Tabelle 37 und Tabelle 38).

Die Ausprägung der Qualitätskriterien zeigen neben den Stärken auch Schwächen. So weist das Netzwerk eine starke sektorale Fokussierung insgesamt und im Kern auf, die die integrative Qualität schwächt. In diskursiver Hinsicht erweist sich die Beschränkung der Beteiligung ebenso negativ. Ein Interviewpartner (Politik und Regionalentwicklung) stellt den Vergleich zu der LEADER Initiative in Landkreis Nordwestmecklenburg an:

„,[...] als das Ergebnis nachber fertig war, das Rahmenkonzept (LEADER Schaalsee Region) erstellt wurde, das war wirklich ein Gemeinschaftswerk von Nord bis Süd, sag ich mal, hier im Biosphärenreservat. Das ist leider so im Rahmen des Großschutzprojektes nicht gewesen [...]". (Zitat aus einem Interview: Politik)

Ohne die beiden von Grund auf verschiedenen Förderinitiativen über einen Kamm scheren zu wollen, zeigt sich, welche mobilisierende und in Folge integrierende Wirkung Akteursorientierung haben kann. Dies wird bestätigt durch die Wahrnehmung der (formalen) Projektstruktur als Expertenkreis durch außen stehende Akteure. Zugleich kritisierte ein interviewter Experte und Mitglied der PAG (Forstwirtschaft), dass maßnahmenbezogen regionales Expertenwissen nicht ausreichend integriert werde: ,[...] das Ziel stellen wir ja überhaupt nicht in Frage, [...], aber der Weg dabin... der Weg dabin, den müsst ihr uns Fachleuten überlassen [...] ". Daraus ist zu schließen, dass der regionale sowie maßnahmenbezogene Diskurs nicht ausreichend ermöglicht wurde bzw. die Verteilung von Verantwortung auf den Projektebenen hätte besser gestaltet werden können (siehe Tabelle 27). 
Tabelle 27: NGP Schaalsee-Landschaft - Konstellation: Breite Beteiligung $(D=$ diskursiv, $A=$ akteursorientiert, $I=$ integrativ)

\begin{tabular}{|c|c|c|c|c|}
\hline Qualitätskriterien & Stärke & D & A & I \\
\hline \multirow{3}{*}{$\begin{array}{l}\text { Projektträger und in Projektstruktu- } \\
\text { ren/Gremien integrierte Personen. }\end{array}$} & $\begin{array}{l}\text { Länderübergreifende Trägerstruktur mit } \\
\text { (formaler) politischer Verankerung: } \\
\text { Zweckverband. }\end{array}$ & & $\mathrm{X}$ & \\
\hline & $\begin{array}{l}\text { Naturschutzverband in Trägerstruktur } \\
\text { ergänzt öffentliche/politische Interes- } \\
\text { senvertretung. }\end{array}$ & & & $\mathrm{X}$ \\
\hline & $\begin{array}{l}\text { Projekt wird von Netzwerkgedanke } \\
\text { getragen. }\end{array}$ & & $\mathrm{X}$ & \\
\hline & $\begin{array}{l}\text { Breite Beteiligung zuständiger Fachbe- } \\
\text { hörden (PAG) }\end{array}$ & & $\mathrm{X}$ & \\
\hline $\begin{array}{l}\text { Maßnahmenbezogene Partner des Pro- } \\
\text { jekts repräsentieren relevante Sektoren. }\end{array}$ & $\begin{array}{l}\text { Projektorientierte Vernetzung mit regio- } \\
\text { naler Wirtschaft: Landwirte, Vereine. }\end{array}$ & & $\mathrm{X}$ & \\
\hline Qualitätskriterien & Schwäche & $\mathbf{D}$ & $\mathbf{A}$ & I \\
\hline \multirow{2}{*}{$\begin{array}{l}\text { Projektträger und in Projektstruktu- } \\
\text { ren/Gremien integrierte Personen. }\end{array}$} & $\begin{array}{l}\text { Starke sektorale Prägung im gesamten } \\
\text { Netzwerk und dessen Kern }\end{array}$ & & $\mathrm{X}$ & \\
\hline & $\begin{array}{l}\text { Beschränkung der Beteiligung der Fach- } \\
\text { behörden auf landschaftsbezogene Abtei- } \\
\text { lungen }\end{array}$ & & $\mathrm{X}$ & \\
\hline \multirow{2}{*}{$\begin{array}{l}\text { Maßnahmenbezogene Partner des Pro- } \\
\text { jekts aus den relevanten Sektoren. }\end{array}$} & \begin{tabular}{|l|} 
NGP nicht als regionales Gemein- \\
schaftsprojekt wahrgenommen
\end{tabular} & & $\mathrm{X}$ & \\
\hline & $\begin{array}{l}\text { Einbeziehung regionalen Expertenwis- } \\
\text { sens teilweise als nicht ausreichend emp- } \\
\text { funden. }\end{array}$ & $x$ & & \\
\hline
\end{tabular}

\section{Konstellation: Multiplikatoren}

Das Projektmanagement des Projektträgers wird im Netzwerk sektorübergreifend als zentraler Prozess- und Netzwerkmotor wahrgenommen. Dies erklärt sich insbesondere durch dessen Multifunktionalität (s. S. 157). Der Netzwerkmotor bestehend aus dem Projektmanagement und dem Naturschutzvertreter als Mitglied des Zweckverbandes - ist einseitig sektoral aufgestellt, was sich auf die Wahrnehmung des NGP auswirkt und die Integrativität hemmt. Als Motoren an der Spitze des Netzwerks agierten zu Projektbeginn die Landräte. Aufgrund der grundsätzlich integrativen Position der politischen Vertreter, hat deren Einsatz dem Projekt regionalen Rückhalt und Dynamik verliehen. Deren Rückzug stellt nun eine Schwäche der Integrativität dar. Die in logischer Konsequenz sektorale 
Wahrnehmung des Projekts schwächt ein weiteres Mal dessen integrative Ausrichtung. Eine große integrative Schwäche des Projekts ist darin zu sehen, dass diejenigen Akteure, denen innerhalb des Netzwerks eine zentrale Stellung zugesprochen wird, nicht immer die positive Promotorenfunktion übernehmen, wie sie es könnten. Durch Passivität oder Abgrenzung bleiben Synergiemöglichkeiten ungenutzt. Hier sei auf den regionalspezifischen Kontext verwiesen: Die Überlappung mehrerer Schutzgebiete (Biosphärenreservat, Naturpark) mit dem Naturschutzgroßprojekt hat Abstimmungen erfordert, die nur in Teilen synergetisch gestaltet wurden (siehe Tabelle 28).

Tabelle 28: NGP Schaalsee-Landschaft - Konstellation: Multiplikatoren $(D=$ diskursiv, $A=$ akteursorientiert, $I$ = integrativ)

\begin{tabular}{|c|c|c|c|c|}
\hline Qualitätskriterien & Stärke & $\mathbf{D}$ & A & $\mathbf{I}$ \\
\hline \multirow{3}{*}{ Motor an der Spitze. } & $\begin{array}{l}\text { Einsatz politischer Repräsentanten für } \\
\text { das NGP bei Projektstart. }\end{array}$ & & & $\mathrm{X}$ \\
\hline & $\begin{array}{l}\text { Heute: Fachpromotor i.S. charismatischer } \\
\text { Persönlichkeit identifiziert: Naturschutz. }\end{array}$ & & $\mathrm{X}$ & \\
\hline & $\begin{array}{l}\text { Projektträger ist Prozessmotor: Multi- } \\
\text { funktionalität. } \\
\end{array}$ & & $\mathrm{X}$ & \\
\hline $\begin{array}{l}\text { Integration regionaler Meinungsführer } \\
\text { und anerkannter Fachexperten aus den } \\
\text { relevanten Sektoren. }\end{array}$ & - & & & \\
\hline Qualitätskriterium & Schwäche & $\mathbf{D}$ & $\mathbf{A}$ & $\bar{I}$ \\
\hline \multirow{3}{*}{ Motor an der Spitze. } & $\begin{array}{l}\text { Projektträger und zwei Naturschutzver- } \\
\text { treter erweisen sich als Netzwerkspitze. }\end{array}$ & & & $\mathrm{X}$ \\
\hline & $\begin{array}{l}\text { Politische Promotoren haben sich zu- } \\
\text { rückgezogen bzw. haben gewechselt. } \\
\text { Politisches Zugpferd fehlt. }\end{array}$ & & & $\mathrm{X}$ \\
\hline & $\begin{array}{l}\text { Sektorale Wahrnehmung des NGP wird } \\
\text { durch Einzelkämpfer des Naturschutzes } \\
\text { verstärkt. }\end{array}$ & & & $\mathrm{X}$ \\
\hline $\begin{array}{l}\text { Integration regionaler Meinungsführer } \\
\text { und anerkannter Fachexperten aus den } \\
\text { relevanten Sektoren. }\end{array}$ & - & & & \\
\hline $\begin{array}{l}\text { Integration regionaler Meinungsführer } \\
\text { und anerkannter Fachexperten aus den } \\
\text { relevanten Sektoren. }\end{array}$ & $\begin{array}{l}\text { Zentrale Akteure füllen ihre Schlüsselpo- } \\
\text { sition nicht im positiven Sinne aus (Pro- } \\
\text { motorenfunktion), sondern verhalten sich } \\
\text { passiv. }\end{array}$ & & & $\mathrm{X}$ \\
\hline
\end{tabular}




\section{Konstellation: Personelle Kontinuität}

Als positiv bezüglich der integrativen Ausrichtung erweist sich die personell kontinuierliche und kompetente Bearbeitung des Projekts im Projektmanagement, da dies den Zusammenhalt des Netzwerks aufgrund des aufgebauten Vertrauens, der gemeinsamen Erfahrungen, der daraus gewonnenen Kenntnisse und ggf. geteilter Perspektiven bedingt.

Schwächen des Projektmanagements ergeben sich aufgrund der Diskrepanz zwischen Kapazitäten und Aufgabenspektrum, da nicht alle Aufgaben in der Weise erfüllt werden können, wie es notwendig wäre (Moderation, Verhandlung, Öffentlichkeitsarbeit). Diese Schwäche wird als diskursiv eingeordnet, da sie den Dialog und das Kommunikationsklima im Netzwerk belastet und Unzufriedenheit entstehen lässt (siehe Tabelle 29).

Tabelle 29: NGP Schaalsee-Landschaft - Konstellation: Personelle Kontinuität ( $D=$ diskursiv, $A=$ akteursorientiert, $I=$ integrativ)

\begin{tabular}{|l|l|l|l|l|}
\hline Qualitätskriterium & Stärke & D & A & I \\
\hline $\begin{array}{l}\text { Kontinuierliches Engagement der Multi- } \\
\text { plikatoren im Netzwerk/ für das Projekt }\end{array}$ & - & & \\
\hline $\begin{array}{l}\text { Kontinuierliche Betreuung des Prozess- } \\
\text { managements/ Netzwerkarbeit durch } \\
\text { geeignete Person. }\end{array}$ & $\begin{array}{l}\text { Projektträger begleitet Projekt seit Beginn } \\
\text { (Vertrauen und Netzwerkstabilität auf- } \\
\text { grund Persönlichkeits- und Fachkompe- } \\
\text { tenz bestätigt) }\end{array}$ & X & X \\
\hline Qualitätskriterium & Schwäche & D & A & I \\
\hline $\begin{array}{l}\text { Kontinuierliche Betreuung des Prozess- } \\
\text { managements/ Netzwerkarbeit durch } \\
\text { geeignete Person. }\end{array}$ & $\begin{array}{l}\text { Aufgabenvielfalt durch Projektressourcen } \\
\text { nicht kontinuierlich wahrnehmbar. }\end{array}$ & X & & \\
\hline
\end{tabular}

\section{Strategie: Lernen}

Bezüglich der diskursiven Qualität des Netzwerks können zwei Stärken festgestellt werden: Zum einen fordern die Akteure im Netzwerk den direkten und intensiven Austausch - insbesondere im Konfliktfall - ein. Eine intensive Phase der Konfliktbearbeitung hat dabei Früchte getragen: „Es geht jetzt nicht darum, dass man erstmal die harten Positionen sich an den Kopf wirft, sondern man weiß, welche Position der andere hat. Inzwischen sieht das so aus, dass man zumindest Verständnis gegenseitig aufbringt, für die jeweilige Situation, man 
kennt gegenseitig die Möglichkeiten. Von daher ist also deutlich einfacher geworden, dass heißt nicht, dass die Umsetzung deutlich einfacher geworden ist, aber eben die Gesprächskultur ist positiv beeinflusst worden. Ganz eindeutig. "(Zitat aus einem Interview: Naturschutz)

Zum anderen findet ein fachlicher Erfahrungsaustausch zwischen den Projektträgern von aktuell laufenden Naturschutzgroßprojekten statt. Beide Ausprägungen zeigen, dass Diskurse und Verhandlungen auch auf unterschiedlichen Ebenen im Netzwerk stattfinden.

Der Austausch im Netzwerk konzentriert sich zum Befragungszeitpunkt jedoch nur auf einen kleinen sektoral geprägten Teil des Netzwerks, so dass der Diskurs nicht übersektoral und damit auch nicht akteursorientiert stattfindet. Die fehlende Reflexion auf regionaler Ebene sowie die Fokussierung des Erfahrungsaustauschs auf der Förderebene auf Fach- und Abwicklungsfragen ist ein Defizit des Diskurses und erschwert das Lernen darin (siehe Tabelle 30).

Tabelle 30: NGP Schaalsee-Landschaft - Strategie: Lernen $(D=$ diskursiv, $A=$ akteursorientiert, $I=$ integrativ)

\begin{tabular}{|c|c|c|c|c|}
\hline Qualitätskriterium & Stärke & $\mathbf{D}$ & A & I \\
\hline $\begin{array}{l}\text { Direkter und regelmäßiger - sektoren- } \\
\text { übergreifender - Austausch zwischen den } \\
\text { Akteuren }\end{array}$ & $\begin{array}{l}\text { Intensiver Austausch (zur Konfliktbear- } \\
\text { beitung) wurde eingefordert und stärkte } \\
\text { Verhandlungsfähigkeit. }\end{array}$ & $\mathrm{x}$ & & \\
\hline $\begin{array}{l}\text { Einsatz reflexiver Instrumente (Evaluie- } \\
\text { rungsmaßnahmen, Reflexionsworkshops, } \\
\text { Monitoring etc.) }\end{array}$ & $\begin{array}{l}\text { Externe Moderation zu Beginn der zwei- } \\
\text { ten Fördertranche }\end{array}$ & $\mathrm{X}$ & & \\
\hline $\begin{array}{l}\text { Erfahrungsaustausch mit Netzwerken } \\
\text { ähnlicher inhaltlicher Ausrichtung }\end{array}$ & $\begin{array}{l}\text { Austausch auf Förderebene zwischen den } \\
\text { Projektträgern: Naturschutzfachlicher } \\
\text { Dialog. }\end{array}$ & $x$ & & \\
\hline Qualitätskriterium & Schwäche & $\mathbf{D}$ & A & I \\
\hline $\begin{array}{l}\text { Direkter und regelmäßiger - sektoren- } \\
\text { übergreifender - Austausch zwischen den } \\
\text { Akteuren }\end{array}$ & $\begin{array}{l}37 \% \text { der erfassten Kontakte werden } \\
\text { selten oder nie genutzt }\end{array}$ & $\mathrm{X}$ & & \\
\hline $\begin{array}{l}\text { Einsatz reflexiver Instrumente (Evaluie- } \\
\text { rungsmaßnahmen, Reflexionsworkshops, } \\
\text { Monitoring etc.) }\end{array}$ & $\begin{array}{l}\text { Keine Reflexion des Umsetzungsprozes- } \\
\text { ses während erster Förderphase. }\end{array}$ & $\mathrm{x}$ & & \\
\hline $\begin{array}{l}\text { Erfahrungsaustausch mit Netzwerken } \\
\text { ähnlicher inhaltlicher Ausrichtung }\end{array}$ & $\begin{array}{l}\text { Austausch auf Projektträger-Ebene auf } \\
\text { naturschutzfachliche Fragen beschränkt. }\end{array}$ & $x$ & & \\
\hline
\end{tabular}




\section{Strategie: Transparenz, Offenheit, Flexibilität}

Die klare Verhandlungslinie - ein Resultat der strikten Fokussierung auf Naturschutzziele auf Grundlage des Zuwendungsbescheids - ist als diskursive Stärke des Netzwerks einzuordnen, da dies die Verhandlung zwischen den Akteuren im Netzwerk erleichtert:

„[...] Das ist ein Projekt von bundesweiter Bedeutung, mit EU-Sachen, also ich kann es nicht den Spekulationen der jeweiligen Akzeptanzfindung oder zur Ak.zeptanzfindung überlassen, ich muss da schon eine klare Linie fahren. Habe aber auch festgestellt, wenn man diese klare Linie vertritt, dass man auch Anerkennung findet, auch in den Gemeinden, dass sie sagen, na ja gut, da hat er kein Spielraum, sehen wir das mal ein, und man trifft sich im Leben auch häufiger und da würde ich sagen, haben wir auf Gemeindeebene eine Menge erreicht" (Zitat aus einem Interview: Naturschutz).

Es zeigt sich, dass durch einen transparenten und offenen Umgang Akzeptanz für sektorale Förderziele geschaffen wird. Die diskursive Qualität wird durch notwendigerweise vertrauliche Verhandlungen über Flächenverkäufe insofern geschwächt, als dass die Veränderung der Eigentumsstruktur nicht zu jedem Zeitpunkt öffentlich dargestellt werden kann. Dies ist eine Schwäche, die nicht unmittelbar geändert werden kann, aber flankierende Maßnahmen können die strategische Ausrichtung verbessern. Die langwierige und folglich ermüdende Verhandlung über Kooperationsspielräume erschwert den Diskurs, da das übersektorale Verständnis unter dem Aufwand und den Reibungsverlusten leidet. Dies schwächt die Akzeptanz von Einschränkungen der eigenen Interessen zugunsten z.B. des Naturschutzes. Die kapazitätsbedingte Beschränkung der Beteiligung steht der Akteursorientierung entgegen, da die Zugangsmöglichkeiten für Interessierte und potenziell relevante Akteure im Sinne der Nachhaltigkeit beschränkt war. Die generell wahrgenommene Beschränkung des regionalen, kreativen Anknüpfungsspielraumes durch den Zuwendungsbescheid hat es dem Netzwerk erschwert, regionalspezifisch integrative Lösungen für Interessenausgleiche zu finden (siehe Tabelle 31). 
Tabelle 31: NGP Schaalsee-Landschaft - Strategie: Transparenæ, Offenheit, Flexibilität $(D=$ diskursiv, $A=$ akteursorientiert, $I=$ integrativ)

\begin{tabular}{|c|c|c|c|c|}
\hline Qualitätskriterium & Stärke & D & A & I \\
\hline $\begin{array}{l}\text { Nachvollziehbarkeit von Abstimmungs- } \\
\text { und Entscheidungsprozessen }\end{array}$ & $\begin{array}{l}\text { Klare Verhandlungslinie des NGP erfährt } \\
\text { Wertschätzung in der Region. }\end{array}$ & $\mathrm{X}$ & & \\
\hline $\begin{array}{l}\text { Fortwährende Zugangsmöglichkeit von } \\
\text { neuen Akteuren ins Netzwerk }\end{array}$ & - & & & \\
\hline $\begin{array}{l}\text { Inhaltliche und organisatorische Flexibi- } \\
\text { lität: Integration neuer Ideen, kreative } \\
\text { Lösungen für reg. Herausforderungen }\end{array}$ & - & & & \\
\hline Qualitätskriterium & Schwäche & D & A & I \\
\hline \multirow{2}{*}{$\begin{array}{l}\text { Nachvollziehbarkeit von Abstimmungs- } \\
\text { und Entscheidungsprozessen }\end{array}$} & $\begin{array}{l}\text { Wahrnehmung des Projekts als verdeck- } \\
\text { ter Prozess (Flächenverkauf). }\end{array}$ & $\mathrm{X}$ & & \\
\hline & $\begin{array}{l}\text { Abstimmung von Spielräumen für Ko- } \\
\text { operation zwischen NGP und anderen } \\
\text { Landnutzergruppen langwierig. }\end{array}$ & $\mathrm{X}$ & & \\
\hline $\begin{array}{l}\text { Fortwährende Zugangsmöglichkeit von } \\
\text { neuen Akteuren ins Netzwerk }\end{array}$ & $\begin{array}{l}\text { Breite Beteiligungsmöglichkeiten auf- } \\
\text { grund Ressourcen und Zielsetzung nicht } \\
\text { geschaffen. }\end{array}$ & & $\mathrm{x}$ & \\
\hline $\begin{array}{l}\text { Inhaltliche und organisatorische Flexibi- } \\
\text { lität: Integration neuer Ideen, kreative } \\
\text { Lösungen für reg. Herausforderungen }\end{array}$ & $\begin{array}{l}\text { Inhaltliche Flexibilität aufgrund Zuwen- } \\
\text { dungsbescheids gering. }\end{array}$ & & & $\mathrm{X}$ \\
\hline
\end{tabular}

\section{Strategie: Kooperation und win-win}

Gelungene Synergieprojekte lassen annehmen, dass sich die Beteiligten insbesondere auch durch die Projektplanung und -umsetzung ein gemeinsames, integratives Zielverständnis geschaffen haben. Eine (maßnahmen-)spezifischere Einordnung in die strategischen Qualitäten ist jedoch nicht möglich, da es sich bei den Projekten um Effekte einer erfolgreichen übersektoralen Zusammenarbeit von Akteuren handelt, die wiederum durch andere Eigenschaften im Netzwerk ermöglicht wurden. Eher die Schwächen zeigen auf, wie Kooperation und win-win die strategische Qualität des Netzwerks beeinflussen.

Dauerhafte Nutzungskonflikte zwischen Naturschutz und z.B. Tourismus oder Landeigentümern stehen der Integration entgegen, da sich diese in divergie- 
renden Ziel- und Wertevorstellungen manifestieren. Auch die Mitnahme positiver Sekundäreffekte (z.B. intakte Landschaft $=$ weicher Standortfaktor) ohne Gegenleistung verhindert die Ausprägung eines integrativen Entwicklungsverständnisses (siehe Tabelle 32).

Tabelle 32: NGP Schaalsee-Landschaft - Strategie: Kooperation und win-win $(D=$ diskursiv, $A=$ akteursorientiert, $I=$ integrativ)

\begin{tabular}{|l|l|c|c|c|}
\hline Qualitätskriterium & Stärke & D & A & I \\
\hline $\begin{array}{l}\text { Projekt-/ Maßnahmen, aus denen min- } \\
\text { destens zwei Interessengruppen einen } \\
\text { gemeinsamen Gewinn ziehen. }\end{array}$ & $\begin{array}{l}\text { Vereinbarung unterschiedlicher Rauman- } \\
\text { sprüche und Schaffung von Synergieef- } \\
\text { fekten zwischen Naturschutz in beispiel- } \\
\text { hafter Weise sichtbar (s. Tabelle 37, } \\
\text { Tabelle 38) }\end{array}$ & & X \\
\hline Qualitätskriterium & Schwäche & D & A & I \\
\hline \multirow{2}{*}{$\begin{array}{l}\text { Projekt-/ Maßnahmen, aus denen min- } \\
\text { destens zwei Interessengruppen einen } \\
\text { gemeinsamen Gewinn ziehen. }\end{array}$} & $\begin{array}{l}\text { win-win-Potenziale aufgrund strikter } \\
\text { Nutzungsrestriktionen für z.B. Landei- } \\
\text { gentümer/ Gemeinden/ Tourismus z.T. } \\
\text { auch verhindert. }\end{array}$ & $\begin{array}{l}\text { Kooperation mit regionalen Institutionen } \\
\text { des Tourismus/ der Wirtschaftsförde- } \\
\text { rung nicht gegeben, wobei Produktquali- } \\
\text { tät von diesen jedoch ,mitgenommen“. }\end{array}$ & X & X \\
\hline
\end{tabular}

\section{Strategie: Arbeiten auf verschiedenen Ebenen}

Der Zweckverband mit seinen Organen wird als geeignete Verhandlungsarena bestätigt, da sie die komplexen Fragestellungen der Projektumsetzung in handhabbare Aufgabenpakete herunter bricht und den Diskurs sinnvoll, d.h. zielorientiert gestaltet.

Trotz der positiven Einschätzung der Projektorganisationsstrukturen zeigen sich die negativen Konsequenzen der selektiven Beteiligung an den inhaltlichen Konflikten, die nicht ausgehandelt, über einen langen Zeitraum „mitgetragen“ und in der strukturellen Erhebung als häufigstes Defizit genannt wurden. Die Organisationsstrukturen fangen folglich den Abstimmungs- und Verhandlungsbedarf nicht umfassend auf, so dass eine diskursive Schwäche relativ zu Aufgabe feststellbar ist. Die (wahrgenommene) Geschlossenheit verstärkt dies und schwächt die Akteursorientierung durch den Ausschluss relevanter Akteure (siehe Tabelle 33). 
Tabelle 33: NGP Schaalsee-Landschaft - Strategie: Arbeiten auf verschiedenen Ebenen $(D=$ diskursiv, $A=$ akteursorientiert, $I=$ integrativ)

\begin{tabular}{|c|c|c|c|c|}
\hline Qualitätskriterium & Stärke & D & A & I \\
\hline $\begin{array}{l}\text { Projekt- bzw. regionsspezifische Organi- } \\
\text { sationsstrukturen im Netzwerk zur Auf- } \\
\text { gabenteilung (Steuerung, Entscheidung, } \\
\text { Umsetzung, Diskussion, Information) }\end{array}$ & $\begin{array}{l}\text { ZSL/ PAG als geeignete Struktur bestä- } \\
\text { tigt: fallbezogene AG, breites fachliches } \\
\text { Beratungsspektrum }\end{array}$ & $x$ & & \\
\hline Qualitätskriterium & Schwäche & D & $\mathbf{A}$ & I \\
\hline \multirow{2}{*}{$\begin{array}{l}\text { Projekt- bzw. regionsspezifische Organi- } \\
\text { sationsstrukturen im Netzwerk zur Auf- } \\
\text { gabenteilung (Steuerung, Entscheidung, } \\
\text { Umsetzung, Diskussion, Information) }\end{array}$} & $\begin{array}{l}\text { Inhaltliche Konflikte = häufigstes Defi- } \\
\text { zit: Diskurs wurde nicht ausreichend } \\
\text { organisiert }\end{array}$ & $\mathrm{X}$ & & \\
\hline & $\begin{array}{l}\text { Wahrnehmung der PAG als Experten- } \\
\text { kreis; keine offene regionale Diskussi- } \\
\text { onsplattform; Mitwirkungsmöglichkeiten } \\
\text { Interessierter und anderer Sektoren } \\
\text { begrenzt. }\end{array}$ & & $\mathrm{x}$ & \\
\hline
\end{tabular}

\section{Instrumente: Leitbilder, Visionen, Ziele}

Die gemeinsame Orientierung scheint grundsätzlich möglich, da ein projektbezogenes Leitbild und regionale Zukunftsszenarien als Gemeinschaftswerke der PAG erarbeitet wurden. Die Rückmeldung der Interviewpartner legt jedoch nahe, dass sie weder für die PAG noch für andere Akteure handlungsweisend sind. Der Beitrag des Leitbilds bzw. der Szenarien zur Ausrichtung des Netzwerks an Nachhaltigkeit ist insofern als gering einzuschätzen, als dass inhaltliche Konflikte die am häufigsten genannten Defizite sind und somit die integrative Ausrichtung des Netzwerks hemmen. Sie scheinen auch durch die Erarbeitung des Leitbildes und der Szenarien nicht ausgeräumt worden zu sein und die Vernachlässigung des Qualitätskriteriums „Berücksichtigung der drei Dimensionen der Nachhaltigkeit“ legt den Grund nahe. Das Leitbild sowie die Szenarien weisen trotz der Benennung der Bedeutung umweltschonender Bewirtschaftung und Nutzung einen starken Natur(schutz)fokus auf, so dass eine Ansprache der relevanten Akteure der regionalen Nachhaltigkeit nur sehr eingeschränkt einzulösen ist, so dass auch hier eine Schwäche der Integrativität festzustellen ist (siehe Tabelle 34). 
Tabelle 34: NGP Schaalsee-Landschaft - Instrumente: Leitbilder, Visionen, Ziele (D = diskursiv, $A$ = akteursorientiert, $I=$ integrativ)

\begin{tabular}{|c|c|c|c|c|}
\hline Qualitätskriterium & Stärke & $\mathbf{D}$ & A & I \\
\hline Vorhandensein eines Leitbildes. & $\begin{array}{l}\text { Projektbezogenes Leitbild vorhanden. } \\
\text { Zukunftsszenarien entwickelt. }\end{array}$ & $\mathrm{X}$ & & \\
\hline $\begin{array}{l}\text { Qualität des Leitbildes: Berücksichtigung } \\
\text { der drei Säulen d. Nachhaltigkeit. }\end{array}$ & $\begin{array}{l}\text { Projektleitbild und Zukunftsszenarien } \\
\text { stellen Bezug zwischen Naturschutz und } \\
\text { umweltschonender Bewirtschaft und } \\
\text { Nutzung her. }\end{array}$ & & & $\mathrm{x}$ \\
\hline $\begin{array}{l}\text { Bekanntheit und Verankerung (Orientie- } \\
\text { rungswirkung!) in den Köpfen der Netz- } \\
\text { werkakteure }\end{array}$ & $\begin{array}{l}\text { Leitbilder des NGP sind Gemeinschafts- } \\
\text { werk der PAG. }\end{array}$ & & & $\mathrm{X}$ \\
\hline Qualitätskriterium & Schwäche & $\mathbf{D}$ & A & I \\
\hline Vorhandensein eines Leitbildes. & - & & & $\mathrm{x}$ \\
\hline $\begin{array}{l}\text { Qualität des Leitbildes: Berücksichtigung } \\
\text { der drei Säulen d. Nachhaltigkeit. }\end{array}$ & $\begin{array}{l}\text { Inhaltliche und persönliche Konflikte = } \\
\text { häufigstes Defizit; Naturschutzfokus des } \\
\text { Projektleitbildes. }\end{array}$ & & & $\mathrm{x}$ \\
\hline \multirow{2}{*}{$\begin{array}{l}\text { Bekanntheit und Verankerung (Orientie- } \\
\text { rungswirkung!) in den Köpfen der Netz- } \\
\text { werkakteure }\end{array}$} & $\begin{array}{l}\text { Handlungsorientierte Wirkung - auch für } \\
\text { PAG - beschränkt. }\end{array}$ & & & $\mathrm{X}$ \\
\hline & $\begin{array}{l}\text { Leitbilder ohne Breitenwirkung - kein } \\
\text { regionales Gemeinschaftswerk. }\end{array}$ & & & $\mathrm{X}$ \\
\hline
\end{tabular}

\section{Instrumente: Prozessmanagement}

Die diskursive Ausrichtung des Netzwerks wird aufgrund der moderierenden Tätigkeit des Projektmanagements bestätigt. Die Moderation strukturiert die Verhandlungen und Diskurse im Netzwerk und verschafft ihnen einen Raum zur Aushandlung der regionalen Nachhaltigkeit im Projektkontext. Die bestätigte Präsenz des Projekts in der Öffentlichkeit stärkt ebenso die diskursive Qualität, da die Region über das Projekt und dessen Ziele informiert wird und sich die nur interessierten oder betroffenen Akteure darüber austauschen können, wie die Umsetzung gestaltet werden kann.

Einige Schwächen des Prozessmanagements beeinflussen die strategische Ausrichtung des Netzwerks negativ. So verhindert die beschränkte Beteiligung die Initiierung eines regionalen Dialogs über die Einbettung oder Verknüpfung des NGP mit Initiativen der regionalen Entwicklung. Die Akteursorientierung bleibt also auch bedingt durch die fehlenden Ressourcen schwach, da die Einbindung der relevanten Akteure der zur Gestaltung regionaler Nachhaltigkeit wichtigen Sektoren über die Verwaltungsebene hinaus, lückenhaft bleibt. Die Zufriedenheit 
der Akteure mit der Moderationsfähigkeit des Projektträgers steht den ungelösten inhaltlichen Konflikten gegenüber, die zeigen, dass die Kapazitäten nicht ausreichten, um alle relevanten Akteure und Konflikte in die Moderation aufzunehmen. Ein Interviewpartner aus dem Naturschutz unterstreicht die Bedeutung entsprechender Ressourcen und einer externen Moderation:

„[...] wäre es besser gewesen, wenn man diese Dinge von vornherein sachlicher vermittelt bätte und diese, ja, zunächst unversöhnlichen Standpunkte dort wirklich in Rube bätte ausdiskutieren können, und das wäre eben letztendlich nur sinnvoll unter Mithilfe einer Moderation gewesen $[\ldots]^{6}$. (Zitat aus einem Interview: Naturschutz)

Eine integrative Schwäche zeigt sich in dem Erklärungsbedarf von Naturschutzmaßnahmen. Das Verständnis für diese ist nicht durch Aufklärung geschaffen, so dass die Entwicklung eines integrativen Verständnisses erschwert wird. Während zwar die Präsenz des NGP in den regionalen Medien bestätigt wird, weist ein Interviewpartner aus dem Bereich der Forstwirtschaft auf ein großes Informations- bzw. Aufklärungsdefizit hin:

„,[...] aber das wäre tatsächlich wichtig gewesen, ich sage mal, dass man diese Leistungen und so weiter nach außen noch ein bisschen mehr kommuniziert hätte. Da hat der ZV genau das gleiche Problem wie wir alle hier, dass die Mittel teilweise auch knapp sind für Öffentlichkeitsarbeit [...]".(Zitat aus einem Interview: Forstwirtschaft)

Damit bestätigt er zugleich den Optimierungspunkt der Förderrichtlinie bezüglich der projektbegleitenden Informationsmaßnahmen (siehe Abschnitt 3.1.2). Die Dynamik des Prozessmanagements berührt jede strategische Qualität, und prägt schließlich die integrative Ausrichtung, die sich hier zusammenfassend nur schwach zeigt (siehe Tabelle 35). 
Tabelle 35: NGP Schaalsee-Landschaft - Instrumente: Prozessmanagement $(D=\operatorname{diskursiv,~} A=$ akteursorientiert, $I=$ integrativ)

\begin{tabular}{|c|c|c|c|c|}
\hline Qualitätskriterium & Stärke & D & $\mathbf{A}$ & I \\
\hline $\begin{array}{l}\text { Vorhandensein einer (externen) Modera- } \\
\text { tion, die den Prozess steuernd begleitet. }\end{array}$ & $\begin{array}{l}\text { Wertschätzung des Projektträgers auf- } \\
\text { grund Verhandlungsführung und ausglei- } \\
\text { chender Moderationsfunktion. }\end{array}$ & $\mathrm{X}$ & & \\
\hline $\begin{array}{l}\text { Aktive Teilhabe der Akteure verschiede- } \\
\text { ner Sektoren }\end{array}$ & - & & & \\
\hline $\begin{array}{l}\text { Zufriedenheitsgrad der Akteure mit } \\
\text { Prozess }\end{array}$ & - & & & \\
\hline $\begin{array}{l}\text { Präsenz des Netzwerks bzw. des Projekts } \\
\text { in der Öffentlichkeit }\end{array}$ & $\begin{array}{l}\text { Präsenz des Projekts in der Öffentlich- } \\
\text { keit/ in den regional-lokalen Medien } \\
\text { bestätigt. }\end{array}$ & $\mathrm{X}$ & & \\
\hline $\begin{array}{l}\text { Wahrnehmung aller Aufgaben des Pro- } \\
\text { zessmanagements durch Netzwerkmana- } \\
\text { ger }\end{array}$ & - & & & \\
\hline Qualitätskriterium & Schwäche & $\mathbf{D}$ & $\mathbf{A}$ & I \\
\hline $\begin{array}{l}\text { Vorhandensein einer (externen) Modera- } \\
\text { tion, die den Prozess steuernd begleitet. }\end{array}$ & $\begin{array}{l}\text { Organisation breiter Beteiligungsprozesse } \\
\text { aufgrund Ressourcenzuweisung/ Zielset- } \\
\text { zung nicht erfolgt. Eingrenzung der } \\
\text { Beteiligung zugunsten der effektiven } \\
\text { Planumsetzung. }\end{array}$ & & $x$ & \\
\hline $\begin{array}{l}\text { Aktive Teilhabe der Akteure verschiede- } \\
\text { ner Sektoren }\end{array}$ & $\begin{array}{l}\text { Großer Anteil passiver Akteure im } \\
\text { Netzwerk. }\end{array}$ & & $\mathrm{X}$ & \\
\hline $\begin{array}{l}\text { Zufriedenheitsgrad der Akteure mit } \\
\text { Prozess }\end{array}$ & $\begin{array}{l}\text { Erklärungsbedarf bzgl. Naturschutzmaß- } \\
\text { nahmen nicht immer gedeckt. }\end{array}$ & $\mathrm{X}$ & & \\
\hline $\begin{array}{l}\text { Präsenz des Netzwerks bzw. des Projekts } \\
\text { in der Öffentlichkeit }\end{array}$ & - & & & \\
\hline $\begin{array}{l}\text { Wahrnehmung aller Aufgaben des Pro- } \\
\text { zessmanagements durch Netzwerkmana- } \\
\text { ger }\end{array}$ & $\begin{array}{l}\text { Dynamik des Prozessmotors geschwächt } \\
\text { aufgrund Ressourcenmangel (Personal, } \\
\text { etc.) }\end{array}$ & $\mathrm{X}$ & & \\
\hline
\end{tabular}

\subsubsection{Ergänzende Situationsanalyse}

\section{Länderübergreifende Kooperation}

Die Diskussion mit den Akteuren über den Beitrag des NGP zur länderübergreifenden Kooperation hat relativ kohärente Einschätzungen ergeben. Die Erwartung einer länderübergreifenden „Klammer“ konnte nicht erfüllt werden, da das NGP überwiegend auf administrativen Ebenen agiert und sich an formalen Zu- 
ständigkeitsgrenzen orientiert ${ }^{166}$. Die Beteiligung zweier Länder führte dazu, dass es bei der Abwicklung wie zwei Projekte gehandhabt wurde. Lediglich bei grenzüberschreitenden Wirkungen von Naturschutzmaßnahmen werden gemeinsame Arbeitsgruppen eingerichtet. Eine Stärkung der länderübergreifenden Zusammenarbeit in dem Projektgebiet wurde stärker von anderen Initiativen der Regionalentwicklung gefördert. Trotzdem wird auch heute das NGP als ein wichtiger Akteur für länderübergreifende Zusammenarbeit wahrgenommen ${ }^{167}$.

Grundsätzlich herrscht Einigkeit bezüglich funktionierender Kooperationsstrukturen „im Kleinen“, d.h. Gemeinden oder Anbieter touristischer Infrastrukturen arbeiten über Ländergrenzen hinweg zusammen, um sich als Region zu profilieren:

„[...] So, es gibt aber eine Strategie dazu, nämlich, dass uns diese Grenze überhaupt nicht interessiert, diese Landesgrenze. In unserer Arbeit. So, das beißt, wir arbeiten mit Mölln und Ratzeburg, dem Tourismusverband in Schleswig-Holstein ganz eng zusammen und sagen, die Sachen, wo wir Regionalentwicklung organisieren können, weil der Gast hier in der Region nimmt sowieso nicht wahr, wo die Grenze ist [...]" (Zitat aus einem Interview: Naturschutz und Regionalentwicklung).

Im „Großen“ allerdings funktioniert die Kooperation weniger gut und wird auch perspektivisch kritisch eingeschätzt:

„[...] Und ich weiß, denn es sind ja oftmals Versuche gestartet worden hier und da doch etwas gemeinsam zu machen, aber es ist lange Zeit nie realisiert worden. Also ich glaube schon, dass hier beide Seiten irgendwo ein gemeinsames Ziel haben, nur der eine läuft rechts, der andere läuft links des Schaalsees und man versucht da irgendwo zusammen zu kommen. Das ist natürlich schade. Das stimmt schon. Kann man glaube ich nur verändern, wenn man die Bundesländer zusammenschmeißt und einen Nordstaat bildet [...] (Zitat aus einem Interview: Politik).

Während also viele Akteure in der Region bereits beispielhaft länderübergreifend arbeiten, wird den höheren Ebenen eine Verantwortung für die Erleichterung dieser im Kontext des NGP und im Allgemeinen zugesprochen:

„[...] Der Wille müsste da sein in beiden Ländern, ganz. oben, in der Politik, dass man hier auf Dauer etwas gemeinsam macht und das grenzüberschreitende Organisationsstrukturen geschaffen werden, ja. Wo dann diese rechtlichen Dinge zumindest mal außer Kraft gesetzt werden bzw. zumindest so harmonisiert werden, dass sie tragbar sind [...]" (Zitat aus einem Interview: Forstwirtschaft).

166 „[...] Also, ich glaube, dass die, diese Situation ausgerechnet, was hier jetzt das Großschutaprojekt anbelangt, diese Situation, nicht die Effekte gebracht hat, die man sich nach außen hin mit grenzübergreifend vorstellt. Was wir geschafft haben, ist, diesseits und jenseits der Grenze Flächen so zu arrondieren, dass das Naturschutziziel besser umgesetzt wurde, aber dass nach außen bin darstellen und vermarketen ist aus verschiedenen Gründen nicht erfolgt" [...]. (Zitat aus einem Interview: Naturschutz)

167, ,...] der ZV ist nicht nur allein für's grenzüberschreitende Verständnis und diese Dinge da gewesen, auch nicht, sondern das sind mehrere, das sind viele Akteure. Das sind das BR, der Naturpark und andere Projekte, die da gelaufen sind, durchaus von Privaten oder auch Verbänden. Da würde ich den ZV schon als wichtigen Akteur einstufen, aber nicht allein eben [... "(Zitat aus einem Interview: Forstwirtschaft). 
Zusammenfassend kann festgehalten werden, dass die Gestaltung länderübergreifender Zusammenarbeit schwieriger wird, je mehr Projekte tatsächlich gemeinsamer Umsetzung bedürfen. Um also durch ein Naturschutzgroßprojekt auch einen Impuls zur länderübergreifenden Arbeit zu geben, bedarf es erleichternder Rahmenbedingungen, um nicht an bürokratischen Hürden zu scheitern.

\section{Verknüpfung von Förderprojekten}

Von den Akteuren wurden eine Reihe von Förderprogrammen und Fördermittelgebern mit besonderer Bedeutung für Naturschutz und Regionalentwicklung genannt (siehe Tabelle 36).

Tabelle 36: NGP Schaalsee-Landschaft - Übersicht der regionalen Förderkulisse

\begin{tabular}{|l|l|}
\hline Fördermittelgeber & Finanzinstrument \\
\hline $\begin{array}{l}\text { EU, Umsetzung und Ko-Finanzierung durch die Fachmi- } \\
\text { nisterien der Bundesländer Schleswig-Holstein und Meck- } \\
\text { lenburg-Vorpommern }\end{array}$ & $\begin{array}{l}\text { Landwirtschaftsfonds der EU } \\
\text { (ELER), hier auch LEADER }\end{array}$ \\
\hline $\begin{array}{l}\text { EU, Umsetzung und Ko-Finanzierung durch jeweils zu- } \\
\text { ständige Fachministerien der Bundesländer Schleswig- } \\
\text { Holstein und Mecklenburg-Vorpommern }\end{array}$ & $\begin{array}{l}\text { Fonds für regionale Entwicklung der } \\
\text { EU (EFRE) }\end{array}$ \\
\hline $\begin{array}{l}\text { EU, Umsetzung und Ko-Finanzierung durch das zuständi- } \\
\text { ge Fachministerium des Bundeslandes Mecklenburg- } \\
\text { Vorpommern }\end{array}$ & Europäischer Sozialfonds (ESF) \\
\hline EU, genannt für M-V (Ostseeraum) & Interreg III B \\
\hline
\end{tabular}

Alle regionalen Akteure sind sich einig, dass aktuell nur eine unzureichende Verknüpfung der Themenfelder und Förderprogramme von Naturschutz und Regionalentwicklung erfolgt. Die Gründe für die unzureichende Vernetzung können insbesondere auf die klassische Ausrichtung der Förderprogramme zurückgeführt werden sowie auf das Fehlen einer Institution, die sich dieser Aufgabe integrativ annimmt.

\subsubsection{Zusammenfassung}

Auf Basis aller vorliegenden Erkenntnisse können zuvor getroffene Aussagen spezifiziert und ergänzt sowie Schlüsse hinsichtlich des Einflusses des Projektnetzwerks auf den Beitrag des betrachteten Naturschutzgroßprojekts auf die regionale Nachhaltigkeit gezogen werden.

Der Projektträger kristallisiert sich in allen Daten und Analyseeinheiten als zentraler „Knoten im Netz“ heraus. Die Aufgaben- und Rollenvielfalt des Projekt- 
trägers wird von ihm selbst sowie den beteiligten Akteuren als geeignet wahrgenommen, da sie der Umsetzung des PEPL dient. Das Prozessmanagement des Trägers wurde grundsätzlich positiv bewertet: Dessen Moderationsfähigkeit wurde als wichtigste Kompetenz in den Interviews herausgearbeitet, um Vertrauen als Fundament in das interessenreiche Netzwerk zu bringen. Demgegenüber erwies sich jedoch die für diese Aufgabe zu begrenzte Ressourcenausstattung angesichts dieser Aufgabenvielfalt als vielfaches Hemmnis für den Beitrag des NGP zur regionalen Nachhaltigkeit. Dieser Beitrag zeichnet sich aus Netzwerkperspektive dadurch aus, dass mittels einer integrativen, akteursorientierten und diskursiven Netzwerkstrategie Akzeptanz für die sektoralen Förderziele geschaffen wird. Diese Akzeptanz zu schaffen, hängt davon ab, ob es gelingt, die Förderziele zu erklären und mit anderen Interessen zu verknüpfen. Beides ist im Förderkontext als Kommunikationsaufgabe zu verstehen, wodurch die Bedeutung der diskursiven Qualität des Netzwerks hervortritt. Diese erfüllen zu können, setzt voraus, dass über entsprechende harte (finanzielle, personelle, sachbezogene) und weiche (Wissen) Ressourcen verfügt werden kann. Die Fallstudie zeigt jedoch, dass die erfolgreiche Bewältigung der „Kommunikationsaufgabe Naturschutz“ im vorgegebenen Förderkontext mit dem oben formulierten Anspruch kaum realisierbar scheint. Eine dynamische und integrative Prozessgestaltung mit dem Anspruch die Förderziele gegenüber den relevanten Akteuren zu erklären und über den regionalen Mehrwert und Synergiemöglichkeiten aufzuklären wurde nicht in einer, der regionalen Nachhaltigkeit angemessenen Weise realisiert. Dies erklärt sich anhand der unzureichenden Bedeutung, die der kommunikativen Facette des Fördertitels in den Förderrichtlinien zugewiesen wird. Die offensichtlichen Folgen sind unbefriedigter Erklärungsbedarf von Naturschutzmaßnahmen aus Sicht von Forstrevieren sowie Grundeigentümern oder Gemeinden, und daraus resultierende Ablehnung der Maßnahmen bzw. Verzögerungen in der PEPL-Umsetzung. Subjektiv empfundene Willkür aufgrund des Aufklärungsdefizits wirkt sich deutlich negativ auf die Wahrnehmung des NGP und in Folge der Naturschutzmaßnahmen aus, obwohl eine grundsätzliche Befürwortung des Naturschutzes gegeben ist. Auch die regionale Verankerung über win-win-Projekte und deren wirksame Darstellung in der Öffentlichkeit wird als vielversprechende Strategie der gesellschaftlichen Akzeptanzschaffung für klassische Naturschutzmaßnahmen kaum genutzt. Auch der Leitbildprozess bzw. die Leitbilder als integrative Instrumente des Prozessmanagements haben wenig zur Aufklärung bzw. zur Entwicklung eines gemeinsamen Verständnisses von den Zielen und regionalen Chancen des NGP beigetragen. Die Schwäche der Leitbilder korreliert mit der Ausprägung der Breiten Beteiligung, die angesichts der Ressourcenausstattung sowie Zielsetzung (Planumsetzung) in selektiver Weise durchgeführt wurde. Die Verbandsstruktur ist zwar im Sinne der Arbeit auf verschiedenen Ebenen grundsätzlich als geeignet zu bewerten (z.B. PAG als Koordinierungs- bzw. Konfliktregelungsorgan, fallbezogene Arbeitsgruppen, Verbandsversammlung als Entscheidungsgremium und der Projekt- 
träger als Mittler zwischen diesen Organen und Moderator auf allen Ebenen). Aber die selektive Beteiligung und Fokussierung auf zuständige Behörden und ausschließlich direkt Betroffene hatte deutliche negative Konsequenzen. Zum einen mag der konfliktreiche Projektstart aus ihr resultiert sein, zum anderen erschwerte sie die regionale Verankerung des Projekts über die Ebene der Behörden hinaus. Beispielhaft konnte aufgezeigt werden, welche Synergiepotenziale zwischen Naturschutz und sanfter Nutzung bestehen. Diese hätten jedoch stärker genutzt werden können, um die Impulsfunktion des NGP im Sinne regionaler Nachhaltigkeit voll zu entfalten. Die aufgeführten Fallbeispiele (Tabelle 37, Tabelle 38) decken das ,stille Potenzial“ beispielhaft auf. Eine gezielte Kooperation mit Tourismusverbänden oder der Wirtschaft wurde nicht geknüpft, so dass kein gemeinsames Entwicklungsverständnis geschaffen wurde. Darüber hinaus sollte die erfasste beispielhafte, aktive Einforderung von Beteiligungs- bzw. Kooperationsmöglichkeiten mit dem NGP durch eine Gemeinde in der SchaalseeLandschaft als Schlüssel für die gesellschaftliche Akzeptanz von Naturschutzmaßnahmen verstanden und entsprechend im Netzwerk aufgewertet werden.

Die klare Verhandlungslinie des NGP wird wertgeschätzt, da es so als transparenter Verhandlungspartner wahrgenommen wird. Die stringente, und damit auch unflexible Strategie der Flächensicherung vor Aushandlung von Nutzungskorridoren sorgt jedoch für zum Teil starke Konfrontationen zwischen den Schutzinteressen einerseits und den Nutzungsinteressen der direkt Betroffenen (Forstwirtschaft, Landwirtschaft, Grundeigentümer) sowie indirekt Betroffener (Anwohner, Touristiker). Auch hier erweist sich der Ressourcenmangel als wesentliches Hemmnis, da die Aushandlung der Anknüpfungspunkte/Entwicklungskorridore zeit-, personal- und ressourcenintensiv wäre. Trotz formell begrenzter Kooperationsmöglichkeiten, zeigt das „stille Potenzial“, dass im Rahmen eines NGP der Einklang von Schutz und Nutzung der Natur mit entsprechend flankierenden Maßnahmen der Öffentlichkeitsarbeit und Projektkooperationen möglich ist.

Ein kaum beeinflussbares Netzwerkmerkmal sind die Multiplikatoren. An dieser Stelle sei dazu lediglich festgehalten, dass der politische Rückhalt für solche sektoralen Förderungen wie die des BMU, die stets mit Einschränkungen anderer Interessen einher gehen, von zentraler Bedeutung ist, um eine Integration mit den regional relevanten Politikbereichen und damit auch eine regionale Verankerung realisieren zu können.

Die Optimierung der Förderrichtlinie durch die Einrichtung einer Moderationsphase ist ein wesentlicher Meilenstein im Sinne der integrativen, diskursiven und akteursorientierten Ausrichtung der Projektnetzwerke. Das Netzwerk in dieser Untersuchungsregion profitierte erst zu Beginn der zweiten Fördertranche von diesem Instrument. Deren Sinn und Mehrwert wurde jedoch in der Region bestätigt. Die Möglichkeit eine externe Moderation einzusetzen, scheint über die gesamte Projektlaufzeit sinnvoll, da sich verändernde Rahmenbedingungen und 
gesellschaftliche Interessenlagen ggf. eine Neuverhandlung nach einigen Jahren auslösen.

Insgesamt ist festzuhalten, dass sich das NGP als ein langfristiges regionales Lernprojekt darstellt. Der Aufbau und die Pflege von verhandlungsfähigen und konsensfähigen Netzwerkstrukturen, die auf einem Vertrauensverhältnis begründen, bedürfen ausreichend bemessener Ressourcen und Kompetenzen. Die Ergebnisse der Netzwerkanalyse haben Anknüpfungsmöglichkeiten für die Optimierung der Rahmenbedingungen und die Umsetzung des regionalen Lernprojekts „Naturschutzgroßprojekt“ im Sinne der regionalen Nachhaltigkeit aufgezeigt.

\subsubsection{Maßnahmenperspektive}

\subsubsection{Maßnabmenbasismatrix}

\section{Grundlegendes Vorgehen}

Wie Kapitel 2 beschreibt, geschieht die Herstellung der kausalen Wirkungszusammenhänge von Projektmaßnahmen des Naturschutzgroßprojekts und regionaler Nachhaltigkeit über die so genannten Entwicklungszielbereiche (EZB). Die EZB orientieren sich dabei am „PEPL II“, konkret an der Zielplanung auf den Eigentumsflächen im Projektkerngebiet. Davon ausgehend lassen sich drei wesentliche EZB ableiten: „Waldflächen“, „Grünlandflächen“ und „Moorflächen“. Diese EZB sind dabei als Summe der jeweiligen Einzelziele eines Ökosystems zu verstehen (Tabelle aller Einzelziele siehe Anhang). Waldflächen besitzen in der Untersuchungsregion NGP Schaalsee-Landschaft den Hauptanteil von rund $50 \%$ der Entwicklungsziele, gefolgt von „Grünlandflächen“ mit ca. $32 \%$ und „Moorflächen“ mit ca. $10 \%$. Die restlichen $8 \%$ beinhalten Kleinstrukturen, Wasserflächen und Ackernutzung. Daran anknüpfend werden im Folgenden kausale Wirkungszusammenhänge zwischen den EZB und den Handlungsfeldern der gewählten Nachhaltigkeitsdefinition hergestellt. Dies geschieht zum einen auf Basis bestehender Erkenntnisse in Form von Fachliteratur, zum anderen über ausgewählte Fallbeispiele aus der Untersuchungsregion NGP Schaalsee-Landschaft, die die Erkenntnisse aus der Literatur unterstützen bzw. erweitern. Die entsprechenden Wirkungszusammenhänge lassen sich anschließend in eine Maßnahmenbasismatrix übertragen und anwenderfreundlich in Form der Nachhaltigkeitsspinne präsentieren. 


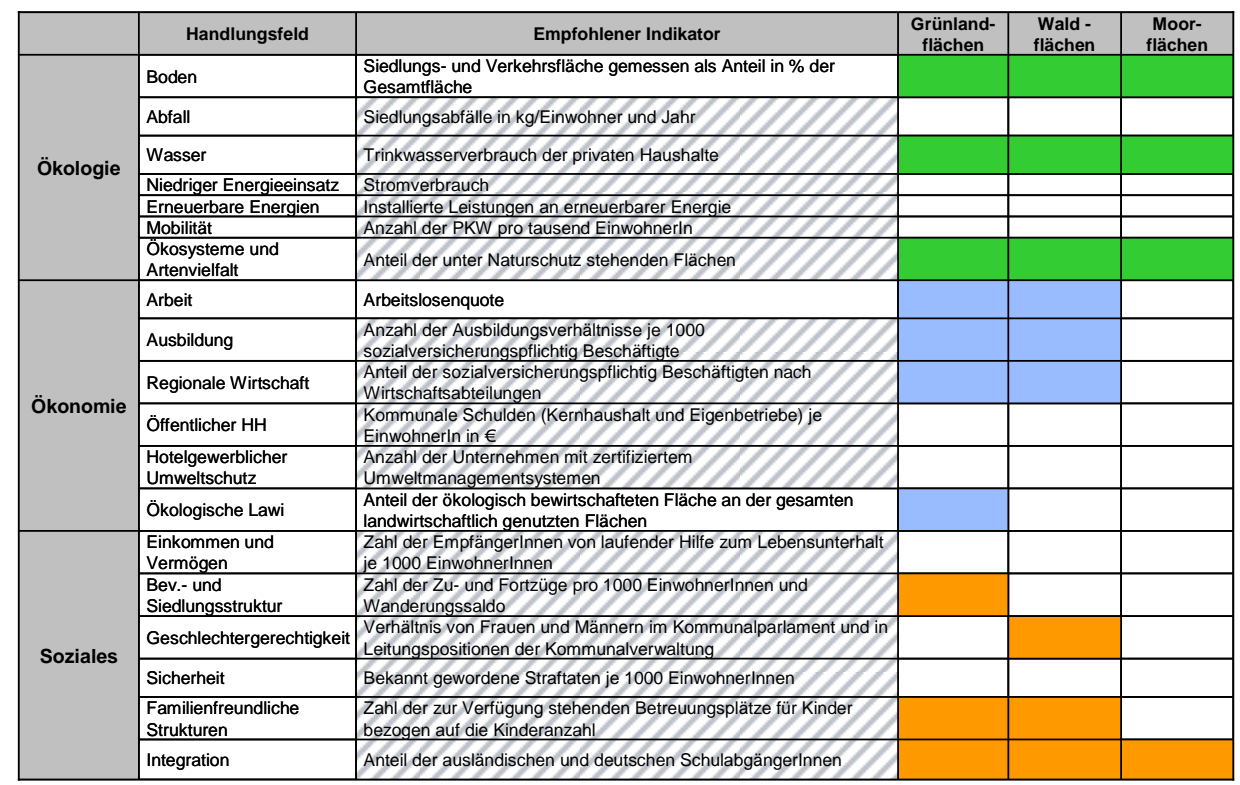

Abbildung 28: NGP Schaalsee-Landschaft-Maßnahmenbasismatrix

Im Folgenden gilt es die Ergebnisse der Wirkungszusammenhänge, die in Abbildung 28 zusammengefasst sind, zu erläutern. Dabei repräsentieren die farblich markierten Felder jeweils den möglichen Kausalzusammenhang zwischen dem jeweiligen Flächentyp und dem entsprechenden Handlungsfeld. Die empfohlenen Indikatoren finden zum größten Teil keine Anwendung (schraffiert), sind aber zur Orientierung mit aufgeführt. Die fehlende Anwendung begründet sich durch das Fehlen einer entsprechenden Datenerhebung bzw. Datenbasis für die jeweiligen Indikatoren in der Untersuchungsregion.

\section{Wirkung des Maßnahmenindikators „Waldfläche“}

Auf ca. $50 \%$ der 4500 ha großen Fläche, die der Zweckverband SchaalseeLandschaft erwarb, dominiert der Entwicklungszielbereich „Wald“. Darin finden sich vor allem Ziele, die eine Umstrukturierung von Waldgebieten, Neuwaldbildung sowie Rückführung von Waldgebieten aus der Nutzung betreffen. Bei der Betrachtung der Wirkungskette wird der Maßnahmenindikator „Wald“ bewusst sehr allgemein gewählt, da es schwierig ist, differenzierte Nachhaltigkeitswirkungen der zusammengefassten Zielbereiche des eben sehr spezifischen PEPL nachzuweisen.

Bei der Betrachtung des Handlungsfeldes Boden bildet der prozentuale Anteil der Siedlungs- und Verkehrsfläche an der Gesamtfläche den Nachhaltigkeitsindi- 
kator. In diesem Zusammenhang kann von einer grundsätzlichen Konkurrenzsituation zwischen der Waldfläche und Siedlungs- und Verkehrsfläche ausgegangen werden. Schon Plausibilitätsüberlegungen verdeutlichen, dass Konflikte zwischen den beiden Flächennutzungsformen existieren, wie beispielsweise der Zielkonflikt von Straßenbau und Freiflächen bzw. Freiraum. In der folgenden Ausführung entspricht „Freiraum“ der Waldfläche, da sie als spezielle Form von Freiraum zu sehen ist.

Eine der primären Zielsetzung von Naturschutzgroßprojekten ist die Schaffung eines Naturschutzgebietes, dessen Hauptmerkmale Großflächigkeit und Komplexität ist. ${ }^{168}$ Dies steht deutlich im Widerspruch zur Nutzung der entsprechenden Fläche zu Siedlungs- und Verkehrszwecken. Polyzentrische Verdichtungsräume, die sich aus dem Zusammenwachsen bzw. Annähern von Städten und Gemeinden im näheren und weiteren Umland entwickeln, führen zu einer Zerstückelung bzw. Zerschneidung des Freiraums, die irreversible Schäden im Naturhaushalt und Landschaftsbild bewirken kann. ${ }^{169}$ Vor allem die Verkehrsinfrastruktur stellt hierbei eine besondere Problematik dar; Straßen und Eisenbahnstrecken stellen Barrieren dar, die zusammenhängende Erlebbarkeit und die Zugänglichkeit von Freiräumen verringern und Biotope zerstören. Aber nicht nur Verkehrsfläche besitzt diese Wirkung, auch disperse Siedlungsflächen wirken sich in Form von Gewerbe- und Industriegebieten, Deponien, Abbaugebieten und Berghalden negativ auf großflächige Freiräume aus. ${ }^{170}$ Hinzu kommt der Effekt, dass Verkehrswachstum und Siedlungs- und Verkehrsfläche sich noch gegenseitig verstärken. Disperse und autoorientierte Siedlungsstrukturen führen zu einer Ausweitung der Verkehrsfläche, da die entsprechenden Wohngebiete erreichbar sein müssen. Verbleibende zusammenhängende Freiflächen werden dadurch zerschnitten. ${ }^{171}$ Das macht deutlich, dass die Bewahrung von Waldfläche als Teil des Freiraums und des Bodens massiv durch Siedlungs- und Verkehrsfläche beeinträchtigt werden. Der Zukauf von Flächen durch den ZSL kann die Ausdehnung der Siedlungs- und Verkehrsfläche in der Region zumindest punktuell verlangsamen.

Das Handlungsfeld „Ökosystem und Artenvielfalt" hingegen steht in einem positiven Zusammenhang mit dem Maßnahmenindikator Wald. Den Indikator für dieses Handlungsfeld bildet der Anteil der unter Naturschutz stehenden Flächen an der Gesamtfläche einer zu betrachtenden Fläche. Laut Umweltbericht der strategischen Umweltprüfung im Rahmen der ex-ante Evaluierung des operationellen Programms für den EFRE 2007-2013 des Freistaates Thüringen kann davon ausgegangen werden, dass sich eine positive quantitative Entwicklung von Wald- und Naturschutzflächen auch positiv auf Artenvielfalt und bedrohte Tierarten aus-

\footnotetext{
168 Vgl. BfN (2008).

169 Vgl. TMLNU (2006), S.11.

170 Vgl. Gailing (2005), S. 42.

171 Vgl. Gailing (2005), S. 44.
} 
wirkt. Des Weiteren beschreibt diese Umweltprüfung den Bedarf an besonders großen, zusammenhängenden Flächen in Form von Naturschutzgebieten, Biosphärenreservaten und Nationalparken, um Artenvielfalt nachhaltig (dauerhaft) zu sichern. Die Ausweisung von Schutzgebieten mit Waldflächenanteilen ist deshalb als ein wichtiges Instrument zur Erhöhung und Bewahrung von Artenvielfalt zu sehen. ${ }^{172}$

Betrachtet man das Handlungsfeld Arbeit mit dem Indikator „Arbeitslosenquote“, so ist zunächst von einem positiven Zusammenhang auszugehen. Besonders deutlich wird dies bei Eigentumswechsel von „Familienbesitz“ in staatliches Eigentum. Die in Familienbetrieben bestehende Arbeitselastizität kann bewirken, dass Mehrarbeit nicht unbedingt mit Mehrbeschäftigung einhergeht. Tritt hingegen Mehrarbeit in staatlichen Wäldern auf, besteht die Möglichkeit diese mit zusätzlichen Beschäftigten zu bewältigen. Dies liegt in der Hauptsache an dem gröBeren Umfang von Möglichkeiten, die der Staat im Gegensatz zu privaten Kleinbetrieben besitzt, flexibel auf Mehrarbeit zu reagieren. Des Weiteren verstärkt sich der positive Effekt durch den Zusammenschluss von Kleinflächen zur Großfläche. Dies liegt vor allem daran, dass Waldbesitzvereinigungen großflächigere waldverbessernde Maßnahmen ermöglichen. ${ }^{173}$

Betracht man das Handlungsfeld Geschlechtergerechtigkeit, so erscheint es zunächst schwierig, einen Zusammenhang zwischen diesem Handlungsfeld und dem Maßnahmenindikator Wald herzustellen. Zunächst ist festzuhalten, dass die Forstverwaltung noch immer eine Männerdomäne darstellt und traditionell auch in Jagd- und Forstvereinigungen sowie in der Holzindustrie der Frauenanteil gering ist ${ }^{174}$. Dennoch lässt sich eine positive Wirkung des Maßnahmenindikators Wald auf die Geschlechtergerechtigkeit erkennen. Um diese Hypothese zu untermauern, müssen neben den traditionellen waldwirtschaftlichen Kernthemen die neuen Themenfelder betrachtet werden, die im Zusammenhang mit Wald stehen. Vor allem der Bereich der Umwelt- und Waldpädagogik sowie der Öffentlichkeitsarbeit werden von Frauen favorisiert und vorwiegend ausgeübt. 175 Aufgrund des gesellschaftlichen Wandels im Hinblick auf die Ansprüche an Wälder steht heutzutage immer weniger die reine Holzproduktion im Mittelpunkt des allgemeinen Interesses. Vielmehr rücken Themen in den Vordergrund, die sich mit Umwelt-, Freizeit- und Erholungsleistungen des Waldes befassen, also Themenfelder, in denen vorwiegend Frauen tätig sind. ${ }^{176}$ Damit besteht ein positiver Zusammenhang zwischen dem Maßnahmenindikator Wald und der Geschlechtergerechtigkeit.

\footnotetext{
172 Vgl. TAURUS (2006). S.8.

173 Vgl. Tamme (2001), S.7 f.

174 Vgl. Katz (2004), S. 84.

175 Vgl. Späth (2000).

176 Vgl. Katz (2004), S.89, Vgl. Wonneberg (2004), S. 19.
} 
Bezüglich der Wirkung auf das Handlungsfeld „familienfreundliche Strukturen“ ist es schwierig eine direkte kausale Beziehung herzustellen. Dennoch ist von einer indirekten positiven Wirkung des Maßnahmenindikators Wald auf dieses Handlungsfeld auszugehen, wenn man den Wirkungszusammenhang von familienfreundlichen Strukturen und Geschlechtergerechtigkeit betrachtet. Die Verbesserung der Vereinbarkeit von Familie und Beruf für Frauen und Männer ist derzeit das zentrale gleichstellungspolitische Anliegen: Ohne eine Aufhebung der geschlechtsspezifischen Verantwortlichkeiten in Familie und Beruf und ohne das Bereitstellen der hierfür erforderlichen Rahmenbedingungen, wie familienfreundliches Arbeitsleben, Kinderbetreuung und Pflege ist Gleichstellung nicht durchsetzbar. ${ }^{177}$ Das macht die positive Beziehung dieser beiden Handlungsfelder deutlich.

\section{Wirkung des Maßnahmenindikators „Grünlandflächen“"}

Auf ca. $33 \%$ der 4500 ha ${ }^{178}$, die im Rahmen des Naturschutzgroßprojekts erworben wurden, stellt der Entwicklungszielbereich „Grünland“ den Förderschwerpunkt dar. Unter diesem Zielbereich finden sich vor allem Ziele, die eine Umwandlung von Acker in Grünland, Extensivierung von intensiv genutztem Grünland, Erhalt von Feuchtgrünland, etc. betreffen.

Betrachtet man zunächst die Wirkung des exogenen Einflusses des Maßnahmenindikators auf das Handlungsfeld „Boden“ so lässt sich schnell eine ähnliche Beziehung wie bei Waldflächen erkennen. Auch Grünland steht in Konkurrenz zu Siedlungsflächen, so dass ein negativer Zusammenhang besteht. Laut der Studie „Energie aus dem Grünland - eine nachhaltige Entwicklung?“ des Forschungszentrums Karlsruhe wird trotz sinkender Flächeninanspruchnahme für Siedlungsund Verkehrsfläche die Umwandlung von Grünland in diese Flächenformen anhalten und damit Verluste von Kulturlandschaft andauern. ${ }^{179}$ Vor allem diese Flächennutzungsform steht in großer Konkurrenz zu Grünlandflächen. Laut Verordnung der Europäischen Union (EG) Nr. 1782/2003 verpflichten sich die Mitgliedsländer zum Erhalt von Grünland. In Deutschland erfolgt diese Umsetzung über die Festlegung, dass ausgehend vom Referenzjahr 2003 die Abnahme der Dauergrünlandflächen maximal $5 \%$ betragen darf. Dem entgegen steht die stetige Verdrängung des Grünlandes durch die zu anderen landwirtschaftlichen Zwecken genutzten Flächen. Ein erheblicher Anteil des Grünlandumbruchs findet zu Gunsten von Mais für die Bioenergieproduktion statt. Den entscheidenden Anteil haben daran mitunter Mecklenburg-Vorpommern, Niedersachsen und SchleswigHolstein, also gerade auch die Bundesländer, die Flächenanteile im NGP Schaal-

\footnotetext{
177 Vgl. BMFSFJ (2008).

178 Vgl. Zweckverband Schaalsee-Landschaft (2006).

179 Vgl. Rösch et al. (2007).
} 
see-Landschaft haben. ${ }^{180}$ Somit wird auch hier der negative Zusammenhang von intensiv genutzter Landwirtschaftsfläche und Grünlandflächen deutlich.

Betrachtet man hingegen das Handlungsfeld „Ökosysteme und Artenvielfalt“, ist von einem stark positiven Zusammenhang auszugehen. Auf Dauergrünland mit extensiver Futternutzung befinden sich ca. 1000 unterschiedliche Pflanzenarten, von denen 300-500 zu den gefährdeten Pflanzenarten gehören. Damit ergibt sich, dass etwa ein Viertel aller Pflanzenarten, die in Deutschland vorkommen, auf Dauergrünland zu finden sind. ${ }^{181}$ Somit tragen der Erhalt und die Entwicklung von Grünlandflächen eindeutig zu einer positiven Entwicklung von Ökosystemen und Artenvielfalt bei.

Bei Betrachtung des Handlungsfeldes „Arbeit“ ergibt sich eine positive Wirkung des Maßnahmenindikators Grünland. Grünland, welches extensiv genutzt wird, stellt eine wesentliche Grundlage für die Vieh- bzw. Milchwirtschaft dar, die in den Landkreisen der Untersuchungsregion eine bedeutende Rolle spielen (vgl. Abbildung 22). ${ }^{182}$

\footnotetext{
180 Vgl. BfN (2008), S. 6 f.

181 Vgl. Bayrisches Staatsministerium für Landwirtschaft und Forsten (2003), S. 6 f.

182 Vgl. Mecklenburg-Vorpommern Ministerium für Landwirtschaft, Umwelt und Verbraucherschutz (2008).
} 


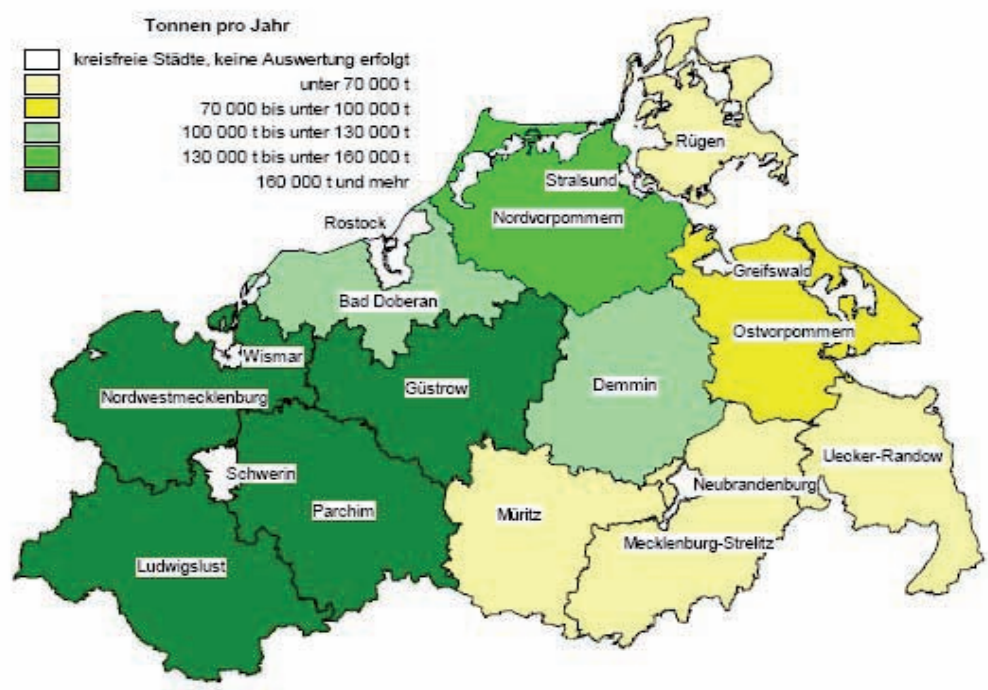

Abbildung 29: Milcherzengung in MV im Jahr 2007 in den Landkreisen, Statistisches Landesamt MV (2008)

Mutterkuhhaltung mit einer geringen Besatzstärke ist tierfreundlich und naturverträglich und eine extensive Bewirtschaftungsform von Grünland. Sie trägt zur Offenhaltung des Grünlandes bei und leistet somit einen Beitrag zum Erhalt einer reich strukturierten Kulturlandschaft mit hohem Erholungswert. Darüber hinaus hat sie eine besondere Schutzfunktion für die Lebensgrundlagen von vielen seltenen Pflanzen und Tierarten. Nicht zuletzt entschärft sie bei extensiver Bewirtschaftung die Überschusssituation auf den Agrarmärkten und kann in strukturschwachen Regionen Arbeitsplätze und in Verbindung mit Ausgleichszahlungen ein gewisses Einkommen sichern. ${ }^{183}$

Auch das Handlungsfeld „Ökologische Landwirtschaft" weist einen positiven Zusammenhang zu dem Maßnahmenindikator Grünland auf. Nach Angaben der Zentralen Markt- und Preisberichtstelle (ZMP) nahm der ökologische Landbau im Jahr 2007 auf Dauergrünland um $5 \%$ zu und erstreckte sich damit auf ca. 450.000 ha in Deutschland. ${ }^{184}$

183 Vgl. Mecklenburg-Vorpommern Ministerium für Landwirtschaft, Umwelt und Verbraucherschutz (2008).

184 Vgl. ZMP (2008). 


\section{Wirkung des Maßnahmenindikators „Moorfläche“}

Der dritte Entwicklungszielbereich „Moorflächen“ bildet den dritten Schwerpunkt der erworbenen Fläche des PEPL II. Knapp 11 \% der durch den ZSL erworbenen 4500 ha wurden mit dem Ziel erworben, Maßnahmen rund um den Bereich Moor durchzuführen. Der Maßnahmenindikator „Moorflächen“ beinhaltet Ziele wie den Erhalt von Moorflächen, Entwicklung von Moorflächen zu feuchten Moorwäldern sowie Entwicklung von Hochmoorkern- und Niedermoorflächen.

In Bezug auf das Handlungsfeld Boden stehen Moorflächen deutlich im Widerspruch zur Nutzung entsprechender Flächen zu Siedlungs- und Verkehrszwecken. Jedoch ist dieser Zusammenhang nur noch in sehr schwacher Form zu sehen, da es sich heutzutage kaum noch wirtschaftlich lohnt Moore zu entwässern, um daraus Siedlungsfläche zu gewinnen. Des Weiteren stehen Moorflächen inzwischen größtenteils unter Naturschutz, was eine Nutzung als Siedlungs- oder Verkehrsfläche wesentlich erschwert. ${ }^{185}$ Im Rahmen des Handlungsfeldes Boden ist auch auf die Konkurrenz von Moorflächen zu land- und forstwirtschaftlich genutzten Flächen hinzuweisen. Aufgrund der stark mineralhaltigen Böden von Moorflächen erschien es jahrelang sehr reizvoll diese Flächen zu entwässern, dies gilt auch für die Schaffung von landwirtschaftlich genutzten Flächen. Aber auch diese Konkurrenzsituation ist heutzutage in Deutschland weitgehend entschärft.

Bezüglich des Handlungsfeldes Ökosysteme und Artenvielfalt kann von einer direkt positiven Wirkung ausgegangen werden. Durch Schutz der entsprechenden Moorflächen kann die Artenvielfalt auf diesen Flächen gesichert werden. Moorflächen haben allgemein eine sehr ausgeprägte Artenvielfalt, mit einer erheblichen Anzahl von „Überlebensspezialisten“. Vieler dieser Arten befinden sich auf der „Roten Liste“, so dass der Erhalt sowie die Erweiterung dieser Flächen eine stark positive Wirkung auf das Handlungsfeld ergeben.

Die Wirkung auf das Handlungsfeld Arbeit des Maßnahmenindikators Moorflächen kann als marginal positiv gesehen werden. Allgemein ist davon auszugehen, dass zur Instandhaltung von Feuchtgebieten, entsprechend auch Mooren, eine gewisse Anzahl von Arbeitskräften notwendig ist. Darüber hinaus können indirekt Arbeitsplätze in Bereich Umweltpädagogik und Umweltschutz entstehen. Dies liegt in der Notwendigkeit der öffentlichen Sensibilisierung für die Notwendigkeit des Erhalts von Mooren.

Im Kontext der Wirkungsanalyse ist dabei die Eigentumsbildung während der Projektlaufzeit von besonderem Interesse, da sie verdeutlicht, wie sich der seit 1994 kontinuierliche Flächenankauf und die damit verbundenen Nachhaltigkeitseffekte bis 2008 entwickelten (vgl. Abbildung 30).

185 Vgl. Joosten (2006), S.38. 


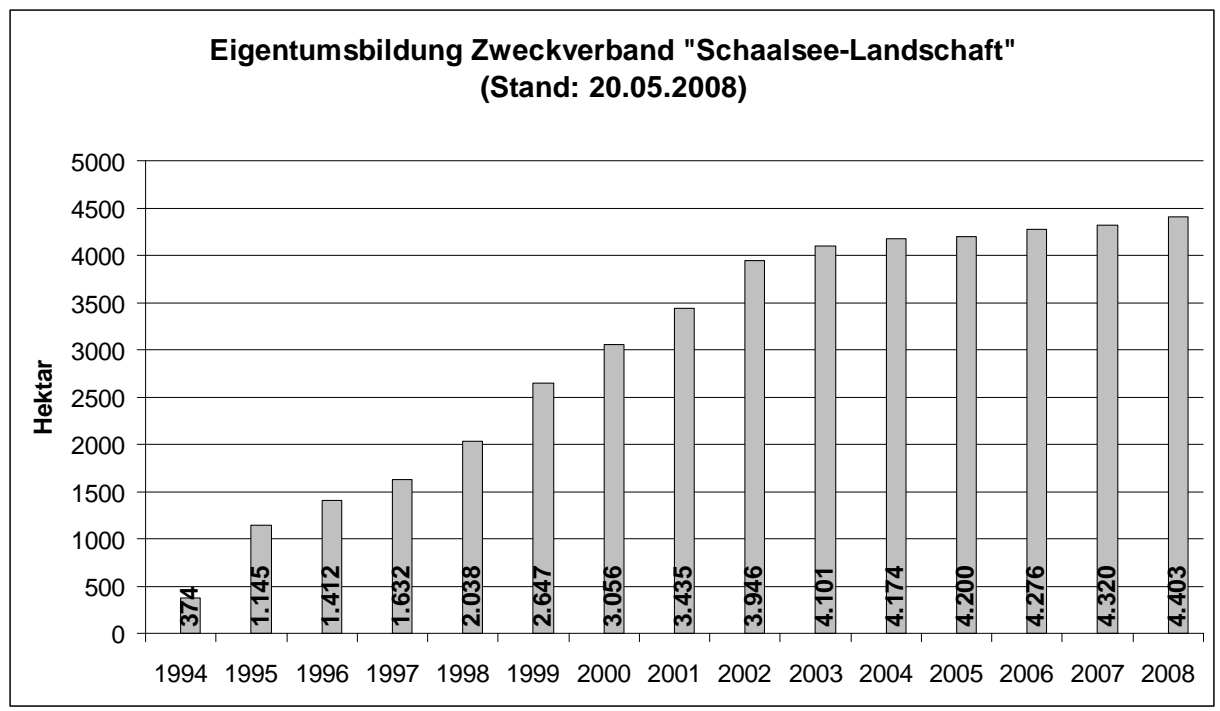

Abbildung 30: NGP Schaalsee-Landschaft - Absolute Eigentumsbildung des ZSL 1994 - 2008; Quelle: ZSL (2008)

\section{Wirkungszusammenhänge aus Fallbeispielen}

Zusätzlich zu der Literaturauswertung werden die ermittelten Wirkungszusammenhänge mit Fallbeispielen untermauert. Um den Regionalbezug herzustellen, bedurfte es einer Diskussion mit den relevanten Akteuren. Dies geschah zum einen im Rahmen der beiden Workshops zum anderen durch Telefoninterviews. In der Diskussion mit den Workshopteilnehmern ließen sich die wesentlichen Sekundäreffekte der Maßnahmen des Naturschutzgroßprojekts SchaalseeLandschaft bzw. regionaler Projekte, die aufgrund der Maßnahmen des NGP ermöglicht wurden, erfassen. Konzentrierte sich der vorangegangene Abschnitt auf die Primäreffekte, die aus den Maßnahmen des PEPL II resultieren, so sind die regionalen Fallbeispiele als Sekundäreffekte zu verstehen, die den regionalen Bezug herstellen und partiell die Wirkungsbereiche erweitern. Diese Sekundäreffekte resultieren aus der Umsetzung von Naturschutzmaßnahmen oder werden durch deren Leistungen ermöglicht und zeigen in der Praxis, wie neben der reinen Wirkung auf die ökologische Säule auch ökonomische und soziale Aspekte berührt werden.

Im Nachgang zu der exemplarischen Benennung einzelner Beispiele durch kleinere Akteursgruppen während der regionalen Workshops, fand eine umfassend Erhebung statt. Dazu stellte der ZSL eine Liste aller Pächter der sich in Eigentum des Projektträges befindenden Flächen zur Verfügung. Diese Pächter 
verpflichten sich im Rahmen des Naturschutzgroßprojekts zur Umsetzung von Projektmaßnahmen und können die gepachteten Flächen im begrenzten Rahmen für vereinbarte $Z$ wecke nutzen. Anknüpfend an diese Tatsache fand eine Befragung zu diesen Nutzungen auf den Flächen des Projektträgers statt. Die Befragung führte zu dem Ergebnis, dass derartige Nutzungen nur im geringen Rahmen erfolgen. Die durchgeführten Tätigkeiten konzentrieren sich im Wesentlichen auf Viehhaltung auf den Grünlandflächen. Landwirte nutzen zur Maßnahmenerfüllung (Grünlandoffenhaltung) Vieh jeglicher Form (Mutterkühe, Schafe, Galloways). Die positiven externen Effekte einer Offenhaltung des Grünlandes durch Viehhaltung nutzen die Landwirte dann jeweils unterschiedlich. Die Bandbreite geht dabei von Mutterkuhhaltung über extensive Beweidung durch Hochlandrinder bis hin zu Herstellung und Vermarktung von Bio-Fleischprodukten. Ein weiterer Schwerpunkt von Nutzungen bezieht sich im Wesentlichen auf soziale Projekte, die darauf abzielen, Synergien zwischen der Ökologie und dem Sozialem herzustellen. Häufig koppeln sich diese Projekte an Umweltbildungsmaßnahmen, die auf die Entwicklung eines Umweltbewussteins abzielen und somit auch einen Beitrag zur Verankerung des Naturschutzgroßprojekts in der Region leisten.

Tabelle 37 und Tabelle 38 fassen die Fallbeispiele mit ihren jeweiligen Sekundäreffekten auf die Nachhaltigkeitssäulen zusammen. 
Tabelle 37: NGP Schaalsee-Landschaft - Mögliche Nachbaltigkeitswirkungen der Sekundäreffekte der Projektmaßnabmen Teil I

\begin{tabular}{|c|c|c|c|}
\hline 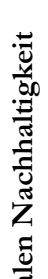 & 竎 & 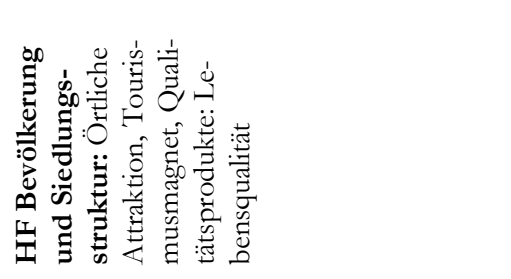 & 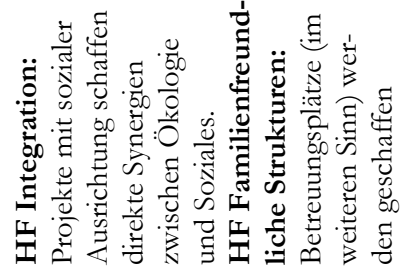 \\
\hline 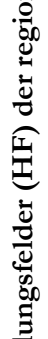 & $\begin{array}{c}.00 \\
0 \\
0 \\
0 \\
: 0 \\
: 0\end{array}$ & 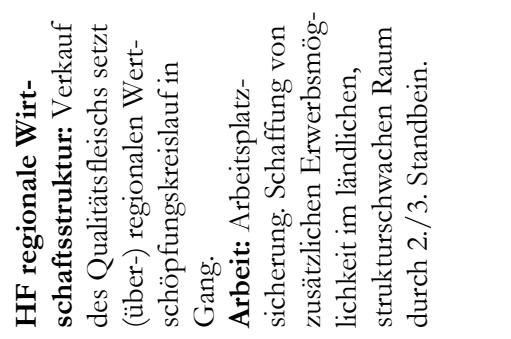 & 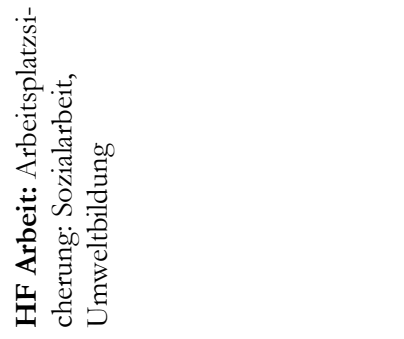 \\
\hline 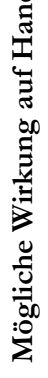 & $\begin{array}{l}\text { 엉 } \\
0 \\
0 \\
: 0\end{array}$ & 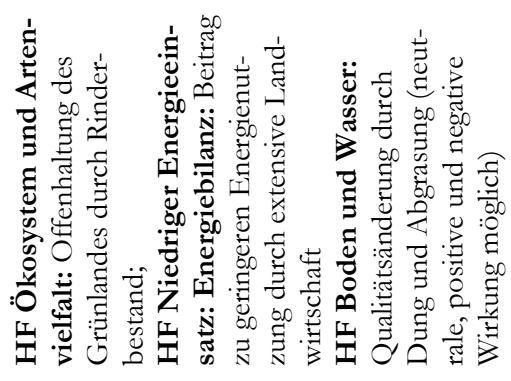 & 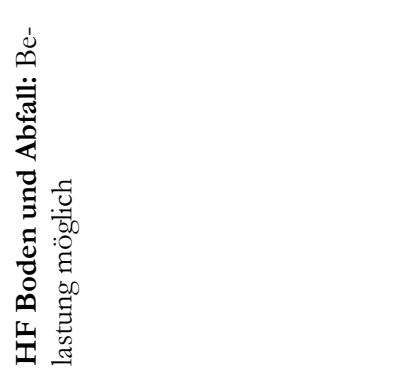 \\
\hline 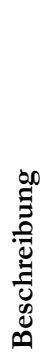 & & 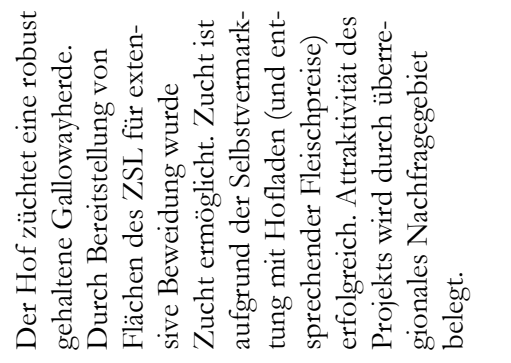 & 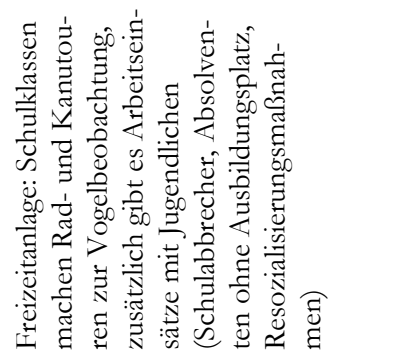 \\
\hline 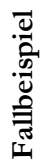 & & 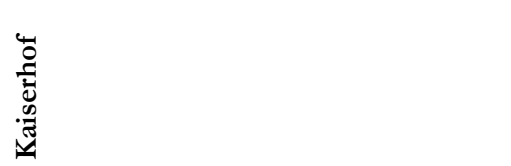 & 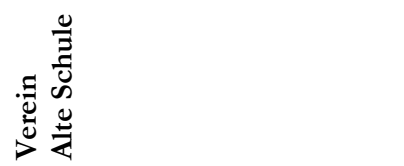 \\
\hline
\end{tabular}


Tabelle 38: NGP Schaalsee-Landschaft: Mögliche Nachbaltigkeitswirkungen der Sekundäreffekte der Projektmaßnahmen Teil II

\begin{tabular}{|c|c|c|c|}
\hline 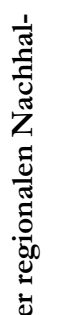 & $\begin{array}{l}\frac{0}{\pi} \\
\frac{\pi}{N} \\
\stackrel{D}{0}\end{array}$ & 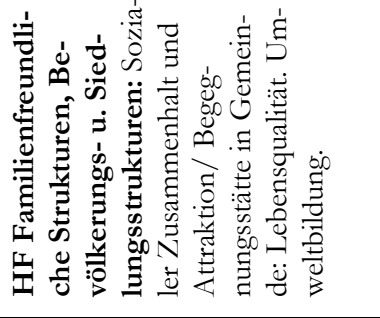 & 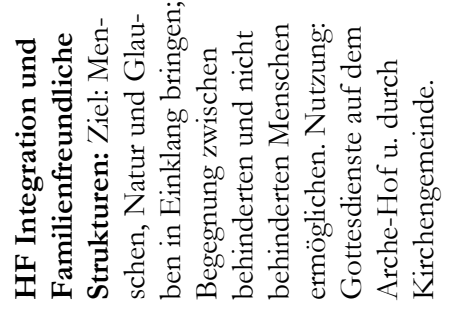 \\
\hline 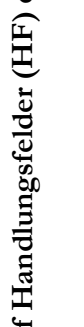 & 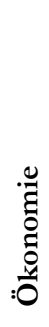 & 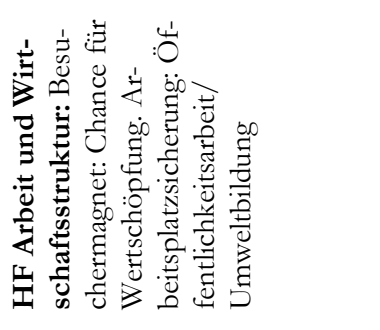 & 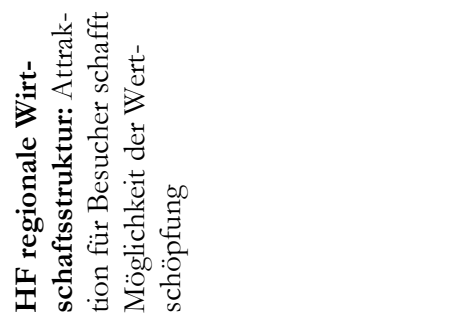 \\
\hline 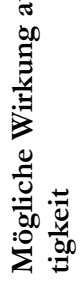 & $\begin{array}{l}\frac{0}{0} \\
\frac{0}{0} \\
0 \\
0 \\
0\end{array}$ & & 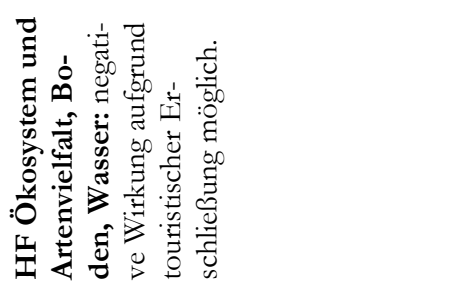 \\
\hline $\begin{array}{l}\infty \\
\vdots \\
0 \\
0 \\
0 \\
0 \\
0 \\
0 \\
0 \\
0 \\
0\end{array}$ & & 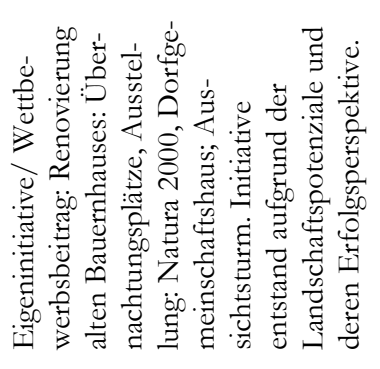 & 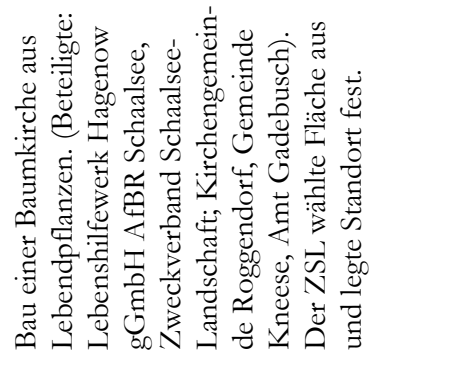 \\
\hline 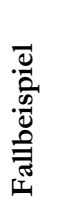 & & 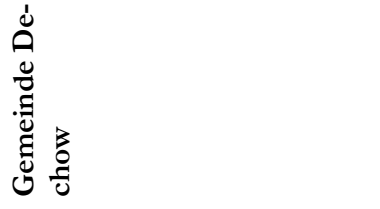 & 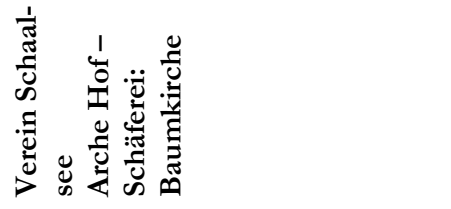 \\
\hline
\end{tabular}




\section{Zusammenfassung der Ergebnisse}

Basierend auf den Ergebnissen der Literaturrecherche und der Fallbeispiele lassen sich Rückschlüsse auf Nachhaltigkeitswirkungen ableiten. Diese besitzen jedoch in erster Linie keinen wertenden, sondern dokumentarischen Charakter

Dazu bedarf es zunächst der Übertragung der Ergebnisse in die Maßnahmenbasismatrix und in die Nachhaltigkeitsspinne, um so einen Überblick über die Wirkungsfelder der einzelnen Maßnahmenindikatoren zu erhalten. Ebenso wie die Maßnahmenbasismatrix bildet die Basisspinne die grundlegenden Wirkungszusammenhänge von Projektmaßnahmen und den jeweiligen Maßnahmenindikatoren ab. In Bezug auf die Möglichkeiten der Nutzung der Nachhaltigkeitsspinne besteht natürlich eine Vielzahl von Variationsmöglichkeiten. So können beispielsweise Grundzusammenhänge im Hinblick auf Investitionsvolumina, Anzahl der Maßnahmen oder Flächenanteile ausgestaltet werden (vgl. Kapitel 4).

Im vorliegenden Fall des NGP Schaalsee-Landschaft lässt sich eindeutig erkennen, dass die Projektmaßnahmen zunächst ausschließlich auf die ökologische Säule der Nachhaltigkeit abzielen (vgl. EZB Anhang). Diese Wirkungszusammenhänge und die damit verfolgten Ziel stehen nicht im Fokus der Analyse, da der positive Beitrag des NGP auf die ökologische Dimension der Nachhaltigkeit außer Frage steht und vielmehr die Effekte des Projekts auf die beiden anderen Dimensionen der Nachhaltigkeit zu erfassen sind, um Akzeptanzprobleme in den Regionen zu mindern.

Im Hinblick auf die positiven Effekte der Projektmaßnahmen auf die ökonomische und die soziale Säule kristallisieren sich drei grundlegenden Typen heraus. Der erste Typus positiver Wirkungen ist die extensive Ackerbewirtschaftung, die ökonomische Effekte aus der Bewirtschaftung der geschützten Flächen zieht. Hierbei findet bewusst keine Berücksichtigung von Verdrängungseffekten der konventionellen Landwirtschaft statt, die unter Umständen einen höheren Ertrag und damit einen höheren ökonomischen Effekt erreichen könnte. Vielmehr ist die extensive Bewirtschaftung als Kompromisslösung auf Naturschutzflächen zu interpretieren, die im Kontext eines naturschutzorientierten Förderprojekts eine Form der Bewirtschaftung zulässt, dem Naturschutz sogar zuträglich sein kann.

Der zweite Typus, der positive Effekte auf Ökonomie und Soziales bewirkt, ist die Viehwirtschaft auf den verpachteten Flächen des Projektträgers. Wie aus den Fallbeispielen und den Interviews ersichtlich wurde, erzielen einzelne Akteure durch die Umsetzung der in den Pachtverträgen festgelegten Auflagen, wie die Grünlandoffenhaltung, die offensichtlichsten ökonomischen Zusatzeffekte von Naturschutzmaßnahmen. Die zur Verfügung gestellten Flächen dienen den Viehwirten als Weidefläche und damit als Futtergrundlage. Im Falle eines Hofs konnten sich dadurch sogar ein Vertrieb von Qualitätsfleisch, ein Hofladen sowie ein Hofcafe etablieren.

Der dritte Typus von Nachhaltigkeitseffekten, der im NGP SchaalseeLandschaft existent ist, besitzt seinen Wirkungsschwerpunkt auf der sozialen Säu- 
le. Vor allem Initiativen mit dem Schwerpunkt Umweltbildung und -erziehung aber auch Bürgerinitiativen koppeln sich an die Maßnahmenindikatoren. In diesem Zusammenhang erscheint eine Differenzierung der Maßnahmenindikatoren wenig sinnvoll, vielmehr ist von einer umfassenden Wirkung aller Flächenformen auf einzelne soziale Handlungsfelder auszugehen.

Zusammenfassend lässt sich ableiten, dass neben den Wirkungen von Projektmaßnahmen auf die ökologische Säule auch ökonomische und soziale Wirkungen möglich sind. Jedoch beschränken sich diese auf eher marginale Größe, was sie als Nebenprodukte des Naturschutzgroßprojekts charakterisiert. Dies begründet sich primär durch zwei Hauptaspekte. Zum einen ist das NGP SchaalseeLandschaft ein Naturschutzfördertitel, d.h. es existierte kein Nachhaltigkeitsmanagement im Rahmen der Projektumsetzung. Die dennoch entstandenen Nachhaltigkeitswirkungen entwickelten sich durch das besondere Engagement des ZSL, der durch die Projektmaßnahmen regionale Akteure einbinden konnte und somit die Entstehung der beschriebenen Effekte förderte. Es lässt sich daher vorsichtig folgern, dass ein gezieltes, explizites Nachhaltigkeitsmanagement noch größere Nachhaltigkeitseffekte generiert hätte.

Zum anderen führt die NGP-Förderrichtlinie zu einer starken Fokussierung auf den Naturschutz, die sich aus dem Förderziel wie auch dem Ressortprinzip ergibt. Dies bewirkt aber, dass die Projektmaßnahmenplanung sowie -umsetzung nur Naturschutzziele verfolgt, da jede Form von Regionalförderung unzulässig wäre. Somit lassen sich keine ökonomischen oder sozialen Maßnahmen durchführen. Da die direkte Umsetzung eines nachhaltigen Leitbildes der Projektausrichtung aufgrund der besagten Richtliniengrenzen momentan nicht möglich ist, kann dies nur durch eine indirekte Ausrichtung geschehen. Als Konsequenz daraus können Naturschutzmaßnahmen so ausgestaltet sein, dass sie gezielt positive Effekte im Bereich des Sozialen und der Ökonomie bewirken. Ausgehend von den genannten Fallbeispielen sollte beispielsweise eine bewusste Ausgestaltung und Ankopplung von Projektmaßnahmen mit regionalen Akteuren der Ökonomie und des Sozialen geschehen, um gezielt Nachhaltigkeitseffekte zu generieren und damit die Akzeptanz für den Naturschutz zu steigern.

Abschließend sei auch auf die grundlegende Zielausrichtung des Naturschutzgroßprojekts und seine Positionierung in der Untersuchungsregion hingewiesen. Wie geschildert bewirkt das NGP nur marginale Effekte im ökonomischen und sozialem Bereich, was im Wesentlichen mit der naturschutzorientierten Zielausrichtung und entsprechenden rechtlichen Vorgaben zusammenhängt. Wenn man davon ausgeht, dass sich die Richtlinie im Hinblick auf die Integration von Nachhaltigkeitsaspekten nicht ändert, ist eine andere Perspektive denkbar. Durch den Beitrag in der ökologischen Säule kann das NGP als ökologischer „Spezialist“ in Kombination mit anderen Förderprojekten in der Untersuchungsregion seinen Beitrag zu nachhaltigen Entwicklung leisten. Mit dem Blick auf Folgeprojekte ist daher zu klären, welche Ausrichtung Naturschutzgroßprojekte aufweisen sollen: 
Nachhaltigkeitsgeneralist mit eine möglichst breiten Wirkung in allen Nachhaltigkeitsfeldern oder ein Nachhaltigkeitsspezialist mit einer weiterhin gezielten ökologischen Ausrichtung in Kombination mit anderen Förderprojekten.

Um diese Entscheidung zu vereinfachen, bietet die Nachhaltigkeitspinne den entscheidenden Vorteil, dass sie die Wirkungsbereiche des Projektes verdeutlicht und eine Hilfestellung zu Entscheidungen der beiden Ausrichtungstypen liefert.

\subsubsection{Nachbaltigkeitsspinne und regionale Verbaltensweisen}

Zur Abbildung der eben genannten Wirkungen des NGP Schaalsee-Landschaft können nun die in der Maßnahmenbasismatrix (siehe Abbildung 28) abgebildeten Wirkungszusammenhänge in die Darstellungsform der Nachhaltigkeitsspinne (siehe Abbildung 31) übertragen werden.

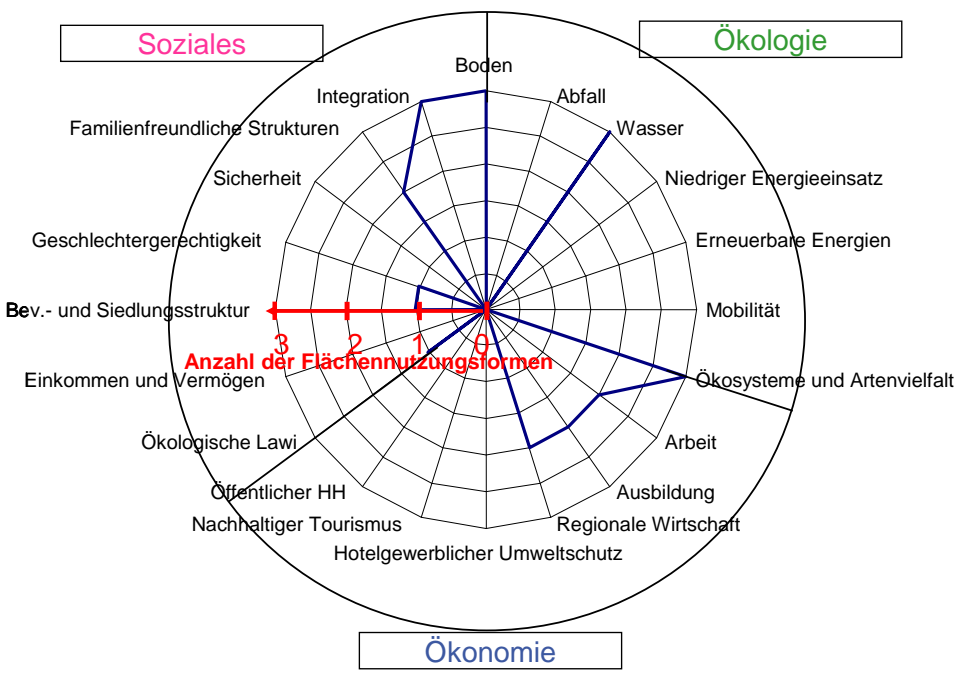

Abbildung 31: NGP Schaalsee-Landschaft - Nachbaltigkeitsspinne

Diese Darstellung der Basisspinne verdeutlicht die Streuung der Wirkungen über die einzelnen Handlungsfelder hinweg, in Bezug auf die jeweiligen Flächennutzungsformen (max. 3: Waldfläche, Grünlandfläche und Moorfläche). Dabei bilden die drei Flächennutzungsformen die Skala, welche abbildet, ob die geplanten Projektmaßnahmen der jeweiligen Flächennutzungsformen eine Wirkung entfalten können oder nicht. Es wird deutlich, dass das NGP Schaalsee-Landschaft seine größten Wirkungen im ökologischen Sektor entfaltet, mit einigen Wirkungen im ökonomischen und sozialen Sektor. 
Anknüpfend an die Abbildung der Maßnahmenwirkungen ließen sich Verhaltensweisen für Nachhaltigkeit erkennen. Dies geschah auf Basis der geführten leitfadengestützen Interviews der Netzwerkanalyse im Hinblick auf Verhaltens- und Handlungsweisen der regionalen Akteure bei der Umsetzung von Projektmaßnahmen. Dieses Vorgehen orientiert sich an den Verhaltensweisen, die die Nationale Nachhaltigkeitsstrategie für eine nachhaltige Entwicklung vorgibt. Tabelle 39 beschreibt die regionalen Verhaltensweisen und ordnet sie den nationalen Regeln (vgl. Kapitel 2) zu.

Tabelle 39: NGP Schaalsee-Landschaft - Regionale Verbaltensweisen für Nachbaltigkeit

\begin{tabular}{|l|c|}
\hline $\begin{array}{l}\text { Identifizierte regionale Managementregeln (Projektebene) } \\
\text { (aus Interviews mit regionalen Akteuren) }\end{array}$ & $\begin{array}{l}\text { Korrespondierende } \\
\text { Managementregeln } \\
\text { der nationalen } \\
\text { Nachhaltigkeitsstra- } \\
\text { tegie186 }\end{array}$ \\
\hline $\begin{array}{l}\text { Die Region übernimmt eigenverantwortlich Vorsorge für: } \\
\quad \text { - landschaftliche Entwicklung, } \\
\quad-\quad \text { Integration. }\end{array}$ & R 1 \\
\hline $\begin{array}{l}\text { Erneuerbare Naturgüter (wie z.B. Kulturlandschaft) dürfen auf Dauer } \\
\text { nur im Rahmen ihrer Fähigkeit zur Regeneration genutzt werden. Dies } \\
\text { gilt im Hinblick auf: } \\
\quad-\quad \text { Tourismus, } \\
\quad-\quad \text { Landwirtschaft. }\end{array}$ \\
\hline $\begin{array}{l}\text { Die Region muss ihre gewachsenen Strukturen sowie Potenziale kennen } \\
\text { und ihr Handeln daran ausrichten. Das Handeln soll ferner dem Grund- } \\
\text { satz der Verhältnismäßigkeit genügen in Bezug auf: } \\
\quad-\quad \text { physisches Kapital (z.B. vorhandene Infrastruktur,...), } \\
\quad-\quad \text { immaterielles Kapital (z.B. Kreativität, Ideen, Know-how, histo- } \\
\quad \text { risches Wissen), }\end{array}$ \\
$\quad$ natürliche Systeme.
\end{tabular}

186 Die Nummerierung gibt die Stellung im Rahmen der 10 Regeln der nationalen Nachhaltigkeitsstrategie wider. 


\begin{tabular}{|c|c|}
\hline $\begin{array}{l}\text { Der Strukturwandel in der Region soll wirtschaftlich erfolgreich sowie } \\
\text { ökologisch und sozial verträglich gestaltet werden. Zu diesem Zweck } \\
\text { sind die Themenfelder der regionalen Nachhaltigkeit so zu integrieren, } \\
\text { dass regionale wirtschaftliche Entwicklung, sozialer Zusammenhalt und } \\
\text { Umweltschutz Hand in Hand gehen. Dabei sind insbesondere folgende } \\
\text { Themen integrativ zu betrachten: } \\
\quad \text { - Sicherstellung der Erreichung der gestellten Naturschutzziele } \\
\text { unter Berücksichtigung von sozialen und ökonomischen As- } \\
\text { pekten, } \\
\text { - Integration von externen Experten, } \\
\text { - länderübergreifende Kooperation. } \\
\text { Kooperation in Handlungsräumen statt in Verwaltungsräumen: } \\
-\quad \text { Bottom-up Vernetzung an Projekten bzw. Themen orientiert, } \\
\text { nicht an administrativen Grenzen. }\end{array}$ & R 5 \\
\hline $\begin{array}{l}\text { Schaffung von Wissen durch Austausch zwischen regionalen Interes- } \\
\text { sengruppen sowie Möglichkeiten der Umweltbildung: } \\
\text { - } \quad \text { i.S. einer „gemeinsamen Sprache“ zwischen den unterschiedli- } \\
\text { chen Akteuren; } \\
\text { - } \quad \text { i.S. eines Verständnisses für unterschiedliche Schutzmaßnah- } \\
\text { men; } \\
\text { - durch Integration von Expertenwissen („Offenlegung“, „Inven- } \\
\text { - tur“ von Wissen in der Region); } \\
\text { - international; } \\
\text { - durch regionale Kooperation zwischen Akteuren. }\end{array}$ & R 6 \\
\hline $\begin{array}{l}\text { Sozialer Ausgrenzung vorbeugen, indem: } \\
\quad \text { - } \text { allen Bevölkerungsschichten Chancen eröffnet werden, am wirt- } \\
\text { schaftlichen Erfolg teilzuhaben; } \\
\quad \text { - alle am gesellschaftlichen und politischen Leben teilhaben. }\end{array}$ & R 9 \\
\hline $\begin{array}{l}\text { Lernen von anderen internationalen Beispielen: } \\
\quad \text { - } \text { statisch: Vergleich von Daten } \\
\quad-\quad \text { dynamisch: Strategieanalyse }\end{array}$ & R 10 \\
\hline
\end{tabular}

Betrachtet man die zusammengefassten Verhaltensregeln, so lässt sich eine breite Streuung über annähernd alle Felder des nationalen Pendants erkennen. Dies lässt den Schluss zu, dass die regionalen Akteure bereits ein implizit ausgeprägtes Nachhaltigkeitsbewusstsein besitzen, welches sie im Kontext der Maßnahmenumsetzung steuert. Die erfassten Verhaltensweisen geben damit einen Überblick über implizit nachhaltiges Handeln, welches in Zukunft zu konkretisieren bzw. zu bewussten Regeln für die Region auszugestalten ist.

\subsubsection{Nachbaltigkeitsinstitutionen}

Wie beschrieben, befindet sich die Untersuchungsregion in den Landkreisen Herzogtum Lauenburg (Schleswig-Holstein) Nordwestmecklenburg (MecklenburgVorpommern) und Ludwigslust (Mecklenburg-Vorpommern). Im Hinblick auf 
eine dauerhafte Etablierung des Naturschutzgroßprojekts während der Förderung und nach der Förderung wurden in den genannten Landkreisen potentielle Nachhaltigkeitsinstitutionen erhoben. Dazu erfolgten Interviews mit den Bürgermeistern der größten an die Untersuchungsregion angrenzenden Städte, mit dem Ziel zu erfahren, welche bereits existierenden Nachhaltigkeitsinstitutionen eine potentielle Andockmöglichkeit für einzelne Projektbausteine des Naturschutzgroßprojekts Schaalsee-Landschaft bieten. Exemplarisch fand die Erhebung in den drei Städten Grevesmühlen, Ratzeburg und Ludwigslust statt, die jeweils in den einzelnen Landkreisen der Untersuchungsregion liegen.

Quintessenz der Untersuchung ist, dass Grevesmühlen und Ratzeburg Nachhaltigkeit nicht als direktes Ziel aber über die jeweiligen „Integrierten Stadtentwicklungskonzepte“ (ISEK) indirekt verfolgt. Die Auswertung der Interviews ergab, dass in den besagten Städten Nachhaltigkeit schon immer eine wesentliche Rolle spielte, man es jedoch nicht für notwendig erachtete, dieses ,integrierte“ Vorgehen explizit als Nachhaltigkeit zu bezeichnen. Allein die Stadt Ludwigslust setzt neben dem ISEK auf einen Lokalen Agenda 21 Prozess, der gezielt einen zusätzlichen Mehrwert in der bewussten Ausgestaltung regionaler Nachhaltigkeit sieht.

Tabelle 40: NGP Schaalsee-Landschaft: - Überblick über bestehende Nachbaltigkeitsinstitutionen in den Landkreisen

\begin{tabular}{|l|l|l|l|l|}
\hline Stadt & Bürgermeister & $\begin{array}{l}\text { Stadt- } \\
\text { /Regionalentwicklun } \\
\text { gskonzept (ISEK) }\end{array}$ & $\begin{array}{l}\text { Wachstums- } \\
\text { kern }\end{array}$ & $\begin{array}{l}\text { Lokale } \\
\text { Agenda }\end{array}$ \\
\hline Grevesmühlen & & & & \\
\hline Ratzeburg & & & & \\
\hline Ludwigslust & & & \\
\hline
\end{tabular}

Damit besteht für den ZSL die Möglichkeit mit den entsprechenden Institutionen in Dialog zu treten, um über Abstimmung und Integration Projektbausteine dauerhaft in der Region zu verankern. Aus den Interviews lässt sich ableiten, dass alle Institutionen ein hohes Interesse am Naturschutz aufweisen, so dass es durchaus denkbar ist, einzelne Projektmaßnahmen fortzuführen bzw. weiterzuentwickeln.

In Bezug auf das Nachhaltigkeitsmanagement sollte bereits vorab eine Erhebung relevanter Nachhaltigkeitsinstitutionen geschehen. Die hier vorgestellten Beispiele zeigen, dass die Notwendigkeit besteht, über die Projektgrenzen hinweg in Kooperation mit Partnern wie beispielsweise größere Nachbarstädte eine langfristige und selbsttragende Projektstruktur zu entwickeln. Dies gilt nicht nur für die Projektmaßnahmen, sondern ebenso für die Sicherung der Projektnetzwerke nach dem Auslaufen der Förderphase. 


\subsubsection{Beispielhafte Synthese zwischen den Perspektiven}

Die Ausweisung des Kerngebietes im NGP Schaalsee Landschaft I und II weicht vom ersten Entwurf des Pflege- und Entwicklungsplanes ab, da - angestoßen durch die Kritik der betroffenen Forstämter und Bauernverbände - Verhandlungen über mehrere Ebenen hinweg sowie zwischen unterschiedlichen Akteuren eine Anpassung der Gebietsausweisung bewirkten. Verschiedene Netzwerkmerkmale lassen diese Anpassung erklären (s. Tabelle 41).

Tabelle 41: Fallbeispiele für die Wirkung des Netzwerks auf Maßnabmen

\begin{tabular}{|c|c|c|}
\hline Netzwerkmerkmal & Ausprägung & $\begin{array}{l}\text { Strategische } \\
\text { Qualität }\end{array}$ \\
\hline $\begin{array}{l}\text { Arbeiten auf ver- } \\
\text { schiedenen Ebenen }\end{array}$ & $\begin{array}{l}\text { Der Verhandlungsprozess verknüpfte drei Ebenen des } \\
\text { Projektnetzwerks: die Region, den ZSL und das BfN. } \\
\text { Über diese Verknüpfung konnte das naturschutzfach- } \\
\text { liche Expertenwissen der überregionalen und förder- } \\
\text { mittelgebenden Ebene um das regionale Wissen er- } \\
\text { gänzt werden und somit eine Optimierung des Fach- } \\
\text { plans herbeigeführt werden. }\end{array}$ & Integrativ \\
\hline $\begin{array}{l}\text { Transparenz, Offen- } \\
\text { heit und Flexibilität }\end{array}$ & $\begin{array}{l}\text { Die Offenheit und inhaltliche Flexibilität während des } \\
\text { PEPL-Erarbeitungsprozesses wurde von der Region } \\
\text { eingefordert und die verhandlungsoffene Positionie- } \\
\text { rung der anderen Ebenen erwies sich als Schlüssel für } \\
\text { einen Konsenses. }\end{array}$ & $\begin{array}{l}\text { Diskursiv und } \\
\text { akteursorientiert }\end{array}$ \\
\hline $\begin{array}{l}\text { Lernen und Qualifi- } \\
\text { zierung }\end{array}$ & $\begin{array}{l}\text { Es zeigt sich ein Lernprozess, in dem fachliche Ent- } \\
\text { scheidungen im Sinne der Optimierung revidiert } \\
\text { wurden. Sektorales Wissen wurde um weitere Perspek- } \\
\text { tiven ergänzt, d.h. die Naturschutzperspektive hat } \\
\text { durch den Austausch mit den Interessen der Forst- } \\
\text { und Landwirtschaft die Notwendigkeit zur Plananpas- } \\
\text { sung erkannt. }\end{array}$ & $\begin{array}{l}\text { Diskursiv und } \\
\text { integrativ }\end{array}$ \\
\hline $\begin{array}{l}\text { Kompetentes Pro- } \\
\text { zessmanagement }\end{array}$ & $\begin{array}{l}\text { Nur die intensive Begleitung des Dialogs zwischen } \\
\text { den Konfliktparteien konnte eine Einigung herbeifüh- } \\
\text { ren. Der ZSL spielte dabei eine zentrale Rolle. Einer- } \\
\text { seits Sprachrohr für den Fördermittelgeber (Vermitt- } \\
\text { lung der naturschutzfachlichen Ziele in die Region), } \\
\text { andererseits Sprachrohr für die Region (Vermittlung } \\
\text { der regionalen Erwartung, Kritik und Konflikte an } \\
\text { den Bund) oblag es ihm, die unterschiedlichen An- } \\
\text { sprüche zu moderieren und Lösungskorridore heraus- } \\
\text { zufiltern. }\end{array}$ & $\begin{array}{l}\text { Diskursiv, Ak- } \\
\text { teursorientiert, } \\
\text { integrativ }\end{array}$ \\
\hline
\end{tabular}

Durch den regionalen Druck bzw. die Abhängigkeit der Umsetzungsmöglichkeit der naturschutzfachlichen Maßnahmen von deren regionaler Akzeptanz (Freiwilligkeitsprinzip) wurde eine Mitentscheidung von der unteren Ebene eingefordert und dies in Folge auch ermöglicht. Das führte im Ergebnis dazu, dass von dem Ziel, alle Waldflächen aus der Nutzung zu nehmen und zugleich große zusam- 
menhängende Waldkomplexe als Kernzone auszuweisen, Abstand genommen und ebenso wertvolle Ackerflächen aus der ursprünglichen Kernzone herausgenommen und weiterhin zur (extensiven) Nutzung zur Verfügung gestellt wurden. Diese Anpassung der ursprünglichen Kerngebietsplanung kann als ein Aushandlungsprozess regionaler Nachhaltigkeit verstanden werden, da ein von Experten formuliertes und ausschließlich naturschutzfachliches Ziel um weitere Perspektiven ergänzt werden musste, um auf regional-lokaler Ebene realisiert werden zu können. Bei der Vereinbarkeit der unterschiedlichen Raumansprüche konnte man sich annähern - auch wenn noch heute kleinräumige Nutzungskonflikte bestehen.

Aus den Interviewtranskripten ließen sich wiederum Managementregeln ableiten, die ihre Wirkung im Kontext des angeführten Fallbeispiels entfalten (Tabelle 42).

Tabelle 42: Korrespondierende regionale und nationale Managementregeln

\begin{tabular}{|l|l|}
\hline Managementregel national & Managementregel Projektebene \\
\hline $\begin{array}{l}\text { Jede Generation muss ihre Aufgaben selbst } \\
\text { lösen und Vorsorge treffen. }\end{array}$ & $\begin{array}{l}\text { Region übernimmt Eigenverantwortung für an sie } \\
\text { gestellte Aufgaben: Natur-/ Ressourcenschutz }\end{array}$ \\
\cline { 2 - 2 } & $\begin{array}{l}\text { Regional bedeutsame Projekte werden regional } \\
\text { verankert: regionales Wissen - regionale Interessen }\end{array}$ \\
\hline $\begin{array}{l}\text { Strukturwandel soll wirtschaftlich erfolgreich } \\
\text { sowie ökologisch und sozial verträglich gestal- } \\
\text { tet werden. Zu diesem Zweck sind die Politik- } \\
\text { felder zu integrieren. }\end{array}$ & $\begin{array}{l}\text { Der Landnutzungswandel soll wirtschaftlich erfolg- } \\
\text { reich sowie ökologisch und sozial verträglich gestal- } \\
\text { tet werden. Zu diesem Zweck sind die betroffenen } \\
\text { Politikfelder zu integrieren. }\end{array}$ \\
\hline
\end{tabular}

Daran anknüpfend lässt sich verdeutlichen, dass die Netzwerkarbeit die Maßnahmenumsetzung in der Modellregion Schaalsee Landschaft maßgeblich beeinflusst. Die in Tabelle 42 beispielhaft zusammengefassten regionalen Verhaltensregeln für Nachhaltigkeit bilden dabei die entsprechende Brücke von statischer Maßnahmenperspektive zur dynamischen Netzwerkperspektive. Die regionale Ausgestaltung verdeutlicht dabei beispielhaft die Synthese beider Perspektiven. Die genannten regionalen Verhaltensregeln für Nachhaltigkeit lassen sich exemplarisch am Fallbeispiel des „Kaiserhofs“ (siehe Tabelle 27) konkretisieren.

Die Bereitstellung der Fläche durch den ZSL an den Pächter zeichnet sich durch mehrere Faktoren als besonders nachhaltig aus: Das Beispiel veranschaulicht, wie die Region eigenverantwortlich die gestellten Aufgaben, in dem Fall die Umsetzung der Projektmaßnahmen des NGP Schaalsee-Landschaft, umsetzt. Dabei wird die Projektmaßnahme „Offenhaltung des Grünlandes“ durch einen regionalen Akteur in Verbindung mit ökonomischen und sozialen Effekten (vgl. Tabelle 27: Kaiserhof) bewältigt. Auch fließt in die Umsetzung gleichzeitig regionales Wissen hinein, d.h. die Generierung ökonomischer Effekte, in dem Fall resultierend aus der Gallowayzucht, orientiert sich idealer Weise an den regionalen 
Rahmenbedingungen. Gleichzeitig lässt sich ein wirtschaftlich sinnvoller Landnutzungswandel erkennen, der ökologisch und sozial verträglich erscheint.

Damit wird die Brücke zwischen der Netzwerkperspektive und der Maßnahmenperspektive geschlagen, denn nur durch eine entsprechend nachhaltige Netzwerkarbeit innerhalb der Untersuchungsregion, die letztendlich in den regionalen Verhaltensregeln für Nachhaltigkeit mündet, lassen sich entsprechend nachhaltige Maßnahmen umsetzen. 


\subsection{Naturschutzgroßprojekt Grünes Band Eichsfeld- Werratal}

\subsubsection{Situation und Kontext}

Die Antragsphase (2003 bis 2009) des Naturschutzgroßprojekts Grünes Band Eichsfeld-Werratal wurde am 20. August 2009 erfolgreich durch die Übergabe des Zuwendungsbescheids abgeschlossen. Antragsteller und Projektträger der dreijährigen Förderphase I ist die Heinz Sielmann-Stiftung.

Das Projekt wird als Meilenstein der dreißigjährigen Geschichte des Fördertitels des Bundes beschrieben, da sich erstmals drei Bundesländer gemeinsam daran beteiligen: Niedersachsen, Thüringen und Hessen (BfN 2010).

Das Projektgebiet erstreckt sich über sechs Landkreise: Osterode am Harz (Niedersachsen), Nordhausen (Thüringen), Göttingen (Niedersachsen), Eichsfeld (Thüringen), Werra-Meißner-Kreis (Hessen), Unstrut-Hainich-Kreis (Thüringen). Naturräumlich ist es den Einheiten Weser-Leine-Bergland im Westen, Thüringer Becken und Randplatten im Osten und Osthessisches Bergland im Südosten zuzuordnen. Das Projektgebiet hat eine Flächengröße von rund 31.300 ha und verteilt sich mit etwa 59\% (ca. 18.500 ha) auf Thüringen, mit etwa 24\% (ca. 7.560 ha) auf Niedersachsen und mit etwa 17\% (ca. 5.270 ha) auf Hessen (vgl. HeinzSielmann Stiftung 2007, Kap. 2, S. 1). Das Gebiet zeichnet sich durch die vorhandenen Naturparke auf hessischer sowie thüringischer Seite aus: Naturpark Meißner-Kaufunger Wald und Naturpark Eichsfeld-Hainich-Werratal. Die Naturparke, die sowohl dem Schutz und Erhalt der Kulturlandschaften als auch der Erholung, dem natur- und umweltverträglichen Tourismus und einer dauerhaft natur- und umweltverträglichen Landnutzung dienen (vgl. BfN 2010), können wichtige Partner des Naturschutzgroßprojekts im Kontext regionaler Nachhaltigkeit werden, da sie bereits über 20 bzw. fast 40 Jahre Erfahrung im Bereich Naturschutz und Regionalentwicklung verfügen. Neben den Naturparken ist die regionale Förderkulisse zu beachten. So werden alle Landkreise derzeit durch die EU (Leader-Ansatz in den Landkreisen Göttingen, Eichsfeld, Werra-Meißner Kreis und Nordhausen), über die Gemeinschaftsaufgabe Regionale Wirtschaftsförderung (Unstrut-Hainich Kreis) oder das Land (Landkreis Osterode) im Bereich der Regionalentwicklung durch ein Regionalmanagement gefördert. 


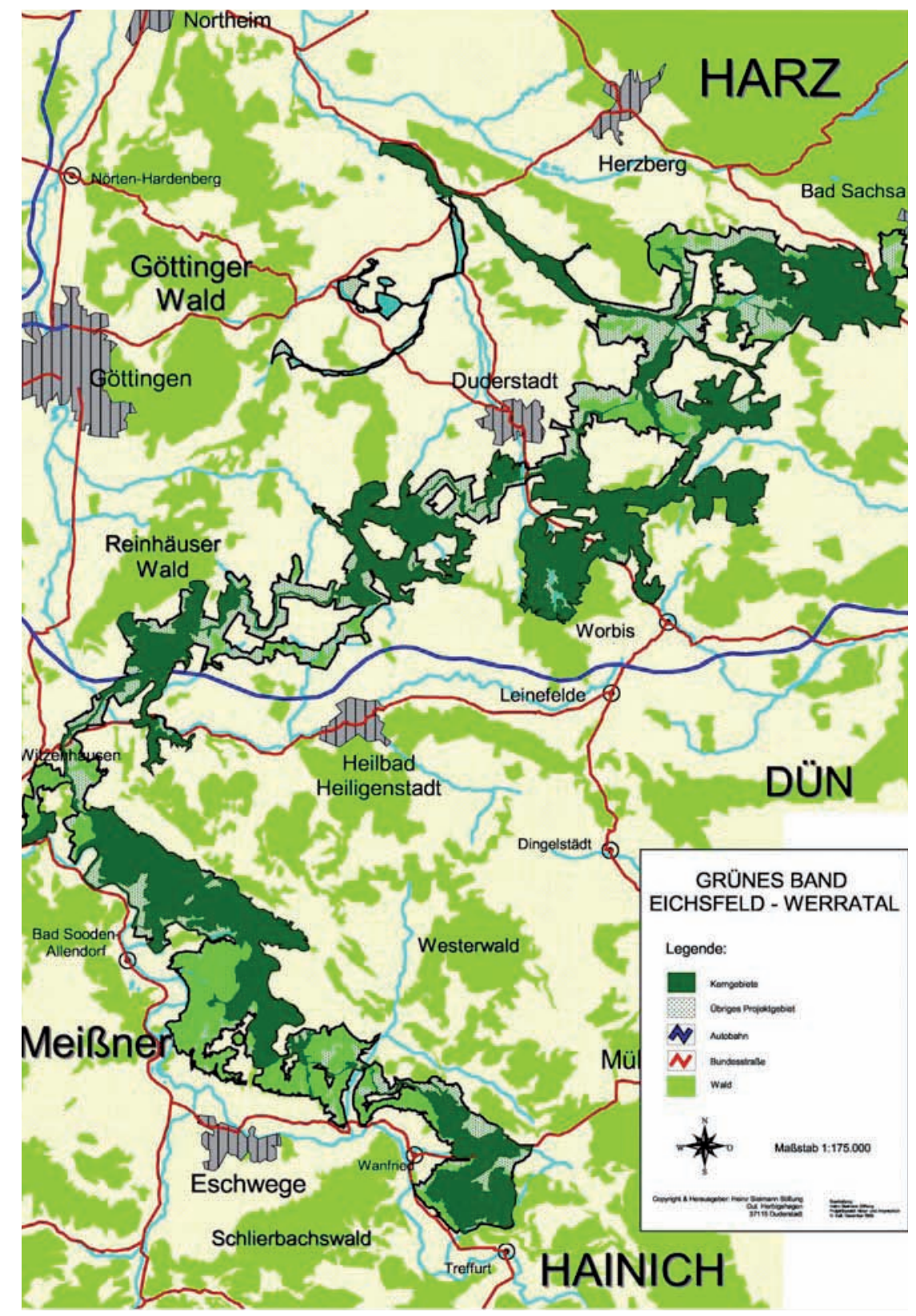

Abbildung 32: NGP Grünes Band Eichsfeld-Werratal: Großräumige Lage des Projektgebietes (Quelle: Heinz. Sielmann Stiftung, 2007) 
Laut Projektantrag werden im Rahmen des NGP Grünes Band Eichsfeld-Werratal überregional bedeutsame Naturschutzziele verfolgt, nämlich der

„Erhalt und die Entwicklung der naturnahen, großräumigen und weitgehend unzerschnittenen Waldkomplexe; die Entwicklung einer überregional bedeutsamen Biotopverbundstruktur sowie die Pflege der historisch gewachsenen Kulturlandschaft" (Heinz Sielmann Stiftung 2007, Kap. 1, S. 2)

Der diesen Zielen zugrunde liegende integrative Projektansatz wird im Antrag deutlich. Der Schutz der Natur wird als Chance für eine nachhaltige Regionalentwicklung benannt und das Projekt als „ökonomisch Erfolg versprechende Perspektive für den (Er-)Lebensraum Eichsfeld-Werratal'“ (Heinz Sielmann Stiftung 2007, Kap. 16, S. 1ff) beschrieben. Das Bewusstsein für die Bedeutung der Netzwerkarbeit im Kontext des Naturschutzgroßprojektes wird bestätigt:

„Für den Erfolg des Naturschutzgroßprojekts Grünes Band Eichsfeld-Werratal ist es von zentraler Bedeutung, die regionalen Akteure auf thüringischer, niedersächsischer und hessischer Seite schon sehr früh über die Ziele und Maßnahmen zu informieren und sie in den Entwicklungsprozess einzubeziehen. Verständigung und Vertrauensbildung benötigen Zeit. Diesen Kommunikationsprozess, der beispielsweise in der Region Eichsfeld bereits seit Jabren erfolgreich praktiziert wird, gilt es auf einer verbesserten Wissensbasis in den nächsten Jahren überregional fortzuführen" (Heinz Sielmann Stiftung, 2007, Kap. 1, S. 2).

Für die gestartete Förderphase I stellt der Bund 758.473 Euro zur Verfügung. Weitere 162.195 Euro kommen von den Bundesländern Thüringen, Niedersachsen und Hessen. Die Heinz-Sielmann-Stiftung beteiligt sich mit 90.630 Euro an den Kosten der ersten drei Jahre (BfN 2010).

\subsubsection{Inhaltliche und methodische Anpassung der disziplinären Forschung}

Das NGP Grünes Band Eichsfeld-Werratal weicht von den anderen Untersuchungsregionen ab, da das Projekt erst im Sommer 2009 bewilligt und gestartet wurde. Die Untersuchungen haben folglich vor der Projektumsetzung begonnen, so dass eine Anpassung des Vorgehens beider Forschungsperspektiven notwendig war. Eine beispielhafte Synthese zwischen den beiden Forschungsperspektiven konnte nicht ausgearbeitet werden, da dazu die Fallbeispiele, d.h. umgesetzte Projektmaßnahmen, als Datengrundlage fehlten. Trotz dieser Lücke zeigt sich, dass beide Perspektiven durch die Betrachtung der Projektvorbereitungsphase relevante Erkenntnisse für die Erarbeitung der Perspektiven (s. Kapitel 4) hervorbringen konnten.

\subsubsection{Netzwerkanalyse}

Die Netzwerkanalyse in der Untersuchungsregion NGP Grünes Band EichsfeldWerratal wurde aufgrund oben genannter Gründe vor der eigentlichen Projektumsetzung durchgeführt. Ziel dabei ist, die Effekte der Antragstellung und Projektvorbereitung auf die regionale Vernetzung zu erfassen. Bereits während der An- 
tragsphase findet ein projektbezogener Austausch zwischen Akteuren und Institutionen unterschiedlicher Sektoren in der Region statt, es werden Ziele und Erwartungen an die Förderung geknüpft und diskutiert. Das Projekt baut auf die alten und während der Antragsphase neu entstandenen Kontaktstrukturen sowie die entstandene Erwartungshaltungen in der Region auf. Je nach Divergenz der Interessen in der Region und der Divergenz zwischen diesen und den Spielräumen der Förderrichtlinien wirken sie sich positiv oder negativ auf die weitere Motivation, Akzeptanz und Unterstützung des Projekts aus. Aus diesem Grunde wurden die Erwartungen an die Förderung erhoben und das regionale Erfahrungswissen zur Netzwerkarbeit im Kontext von Naturschutz und Landnutzung sowie Regionalentwicklung erfasst, um Schlüsse hinsichtlich der Umsetzungsstrategie des Naturschutzgroßprojektes auszuarbeiten.

\subsubsection{Nachbaltigkeitsforschung}

Aufgrund der Tatsache, dass seit August 2009 die Moderationsphase sowie Erarbeitung des PEPL unter der Trägerschaft der Heinz-Sielmann-Stiftung vorbereitet werden, lag während des Untersuchungszeitraums der PEPL für das Naturschutzgroßprojekt noch nicht vor. Dies bedeutet wiederum eine fehlende Grundlage für eine Dokumentation von Projektmaßnahmen im Hinblick auf ihre Nachhaltigkeitswirkungen. Das führt zu der Einsicht, dass keine sinnvolle Bewertung bei fehlenden Projektmaßnahmen durchführbar ist. Daher lag der Fokus der Nachhaltigkeitsforschung im Aufzeigen von Anwendungspotenzialen des Nachhaltigkeitsmanagement während der Moderationsphase.

\subsubsection{Netzwerkperspektive}

\subsubsection{Strukturelle Netrwerkanalyse}

\section{Datenbasis}

Ausgangsbasis für die strukturelle Netzwerkanalyse ist eine Liste von Akteuren, die bereits in der Antragsphase als Projektpartner des Antragstellers bzw. Trägers involviert waren und als Ausschnitt der zukünftigen projektbegleitenden Arbeitsgruppe (PAG) zu werten ist. Die Gruppe besteht aus 86 Personen aus beteiligten Behörden, Ämtern und Verbänden auf Bundes-, Länder- und Landkreisebene, sowie der Städte und Gemeinden.

Die Befragung erzielte einen Gesamtrücklauf von $39 \%$. Während $34 \%$ (29 Akteure) der Ausgangsbasis den Fragebogen zurücksandten, gaben 24 \% (21 Ak- 
teure) negative Rückmeldungen. ${ }^{187} 83$ Akteure wurden neu genannt, von denen 11 $\%$ (9 Akteure) den Fragebogen ausgefüllt zurücksandten und $7 \%$ (6 Akteure) negative Rückmeldungen gaben. Die strukturelle Netzwerkanalyse stützt sich folglich auf 38 Fragebögen.

Diese 38 Akteure charakterisieren sich wie folgt: $87 \%$ der Akteure sind zwischen 40 und 60 Jahre alt, $92 \%$ sind männlich und $53 \%$ sind seit mehreren Jahren und $37 \%$ seit mehr als 10 Jahren in der beruflichen Position tätig, die sie zum Befragungszeitpunkt besetzen. Von allen Akteuren, die geantwortet haben, verfügen $84 \%$ über einen Hochschulabschluss, $8 \%$ über einen Doktortitel und $11 \%$ sind als staatliche Geprüfte in verschiedenen Bereichen tätig.

Die Frage nach den Gründen für das Engagement im Netzwerk zeigt, dass sich die überwiegende Mehrheit der Akteure (92\%) aufgrund ihrer beruflichen Tätigkeit im Netzwerk engagieren.

Die Grundgesamtheit (169 Akteure) und Analyseeinheit (139 Akteure) weisen deutliche Ähnlichkeiten bei der Verteilung der Fachbereiche, Interessenvertretung und räumlichen Verteilung auf (siehe Abbildung 33). Das betrachtete Netzwerk kann daher als repräsentativ eingeschätzt werden, ohne jedoch den Anspruch einer vollständigen Netzwerkerfassung erheben zu können.

187 Hauptgrund ist keine Beteiligung am Projekt; in zwei Fällen wurde der Fragebogen weitergeleitet, eine Ablehnung aufgrund des Datenschutzes, eine Ablehnung als Akteur des politischen Raumes (Bundesebene). 

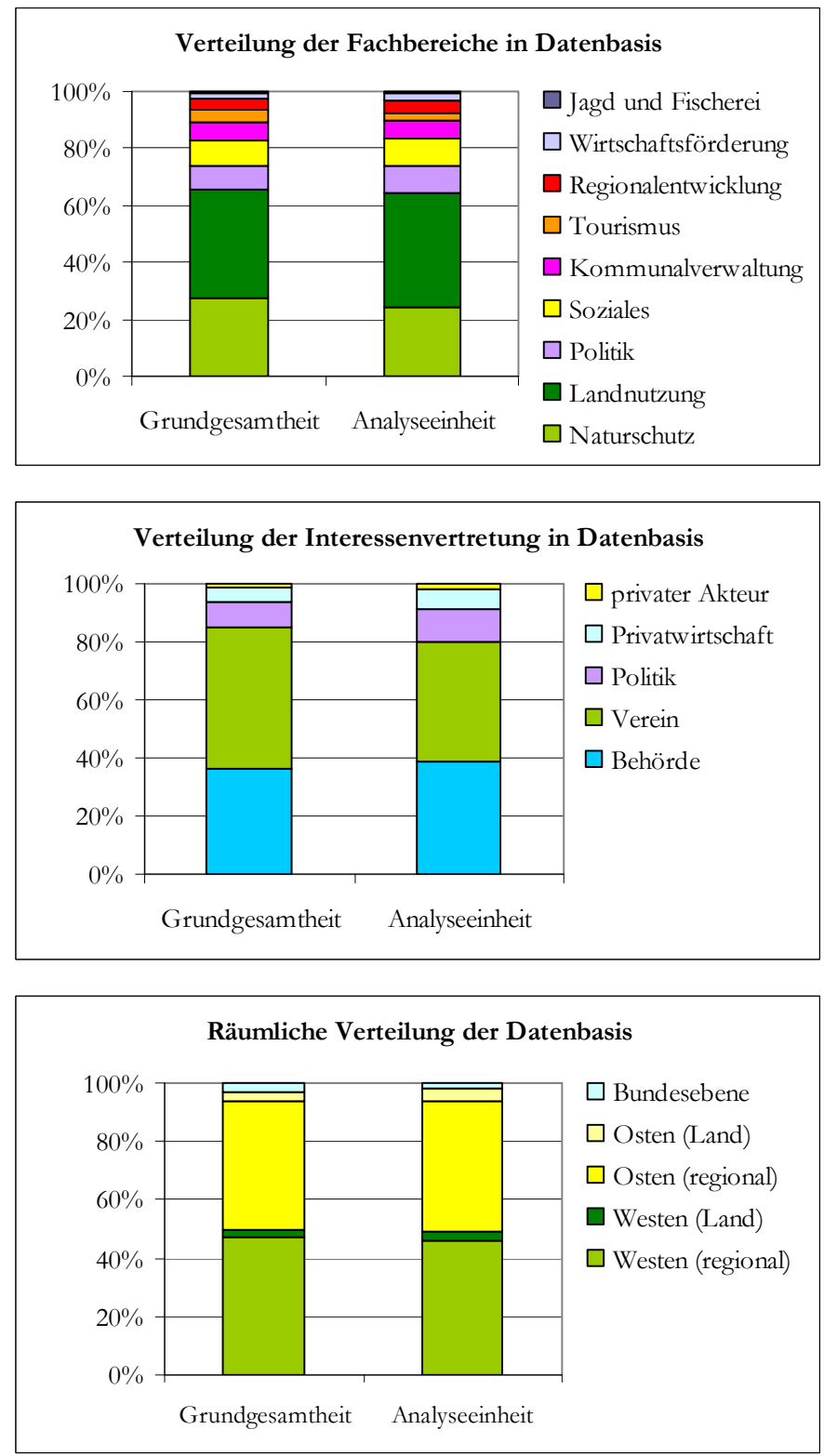

Abbildung 33: NGP Grünes Band Eichsfeld-Werratal - Verteilung der Fachbereiche, Institutionengruppe und räumliche Verteilung im Netzwerk. 
Die Dominanz der Bereiche Naturschutz und Landnutzung zeigt sich durchgängig stark mit über zwei Dritteln (64 - 68\%). Die Präsenz der weiteren relevanten Fachbereiche von zusammen 32 - $36 \%{ }^{188}$ zeigt, dass das Projekt bereits von mulitsektoralem Interesse ist. Die Interessenvertretung findet mit deutlicher Mehrheit organisiert und öffentlich statt. Private Einzelakteure und Vertreter der Privatwirtschaft spielen zur Zeit der Befragung kaum eine Rolle.

\section{Konstellation}

Die Gradzentralität (degree) innerhalb der Analyseeinheit lässt einen Naturschutzvertreter, konkret den Antragsteller bzw. Projektmanager, als zentralen Akteur hervortreten (0.216). Dies ist gleichzusetzen mit der stärksten Aktivität im Netzwerk, die sich durch seine Rolle als Antragsteller erklären lässt. Drei weiteren Akteuren können zentrale, d.h. vorteilhafte Positionen aufgrund der Kontaktzahl zugesprochen werden, nämlich einem Vertreter des Sozialen ${ }^{189}$, des Naturschutzes ${ }^{190}$ und des Tourismus ${ }^{191}$. Dies ist insofern bemerkenswert, als dass sich - bezogen auf die Gradzentralität - eine Vielfalt von Sektoren bereits im Netzwerkkern zeigt. Die Kommunikationseffizienz ist auf einen sektoralen und ebenenübergreifenden Kern konzentriert, da die vier nähebasiert zentralen Akteure (closeness) den Naturschutz vertreten $(0.255$ bis 0.228$)$. Darunter finden sich ein behördlicher Naturschutzvertreter ${ }^{192}$, zwei eines regionalen Naturschutzvereins ${ }^{193}$ sowie ein Vertreter des Bundesnaturschutzes, der sich auch bei der Gradzentralität auf Rang fünf fand. Der Projektmanager positioniert sich im Anschluss an diese vier Akteure (0.218), jedoch in unmittelbarer Nähe zu weiteren Akteuren aus dem Bereich Regionalentwicklung und Landnutzung. Zentrale Maklerpositionen (betweenness) nehmen drei Akteure ein, die auch bei den anderen Zentralitäten hohe Werte erzielt haben, so der Naturschutzvertreter (0.07), der Projektmanager (0.06) und der Vertreter des Bundesnaturschutzes (0.02).

Betrachtet man in Ergänzung die Akteursverflechtung innerhalb des Netzwerks (cohesiveness) wird ein Kern von acht Akteuren identifiziert, indem die regional aktiven Akteure in den Fokus treten, während der Bundesvertreter nicht dazugehört. Der Kern ist multisektoral aufgestellt: zwei zentrale Naturschutzvertreter, u.a. der Projektmanager, zwei Politiker (ost und west), der zentrale Touris-

188 Grundgesamtheit: Naturschutz $27 \%$, Landnutzung $38 \%$, Politik $8 \%$, Soziales $9 \%$, Kommunalverwaltung 7\%, Tourismus 4\%, Regionalentwicklung $4 \%$, Wirtschaftsförderung $2 \%$, Jagd und Fischerei $1 \%$; Analyseeinheit: Naturschutz $24 \%$, Landnutzung $40 \%$, Politik $10 \%$, Soziales $9 \%$, Kommunalverwaltung $6 \%$, Tourismus $2 \%$, Regionalentwicklung $5 \%$, Wirtschaftsförderung $2 \%$, Jagd und Fischerei $1 \%$

189 west/Behörde, 0.151

190 ost/ Verein, 0.137

191 ost/ Verein, 0.122

192 ost/ Verein, 0.255

193 ost/ Verein, 0.232, 0.228 
musvertreter (ost), sowie zwei Vertreter des Sozialen (west und ost) und einer der Regionalentwicklung (ost). Alle besetzen Führungspositionen in regional bedeutenden Institutionen der genannten Fachbereiche (siehe Abbildung 34).

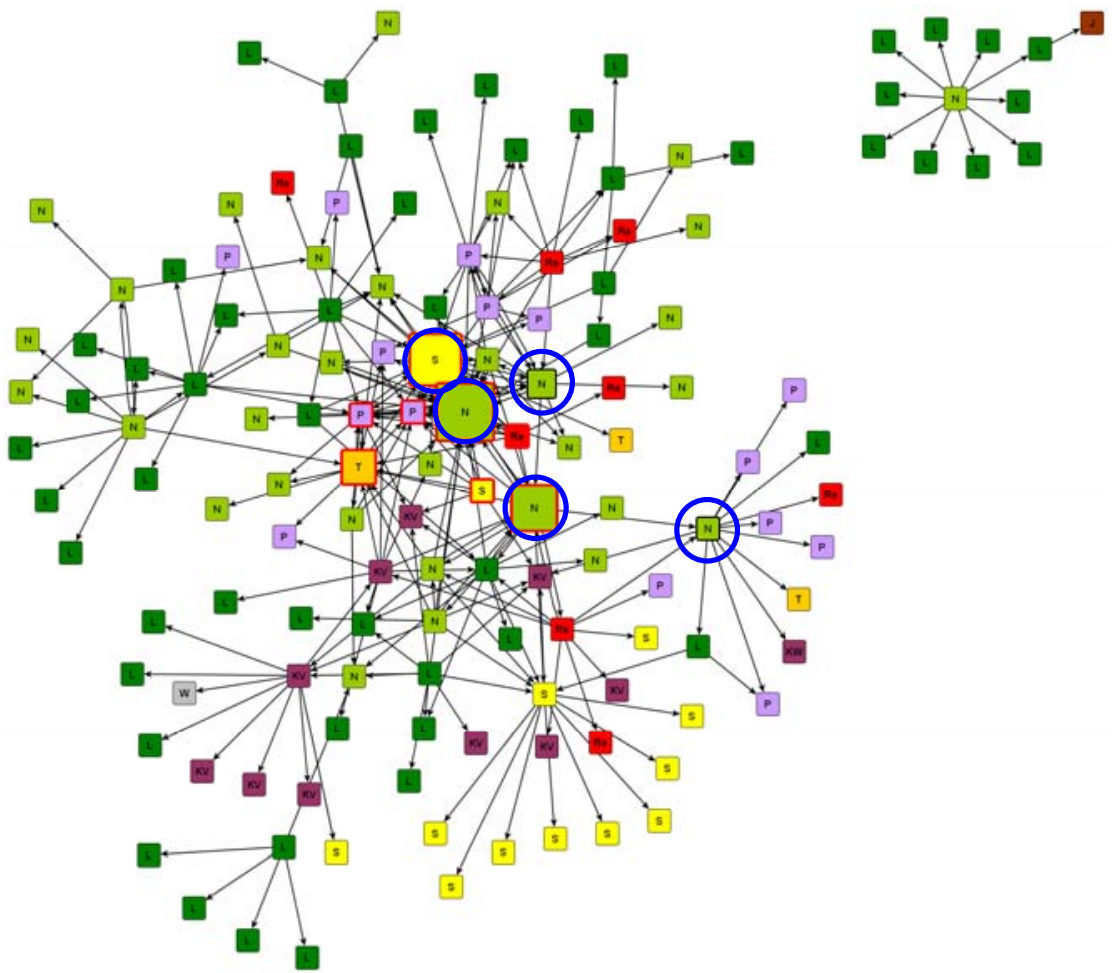

Abbildung 34: NGP Grünes Band Eichsfeld-Werratal-Graph des Projektnetzwerks (L= Landnutzung, $N=$ Naturschutr, $S=$ Soziales, $P=$ Politik, $R=$ Regionalentwicklung, $K V=$ Kommunalverwaltung, $T=$ Tourismus, $W=$ Wirtschaftsförderung, $\mathbf{O}=$ Makler, $\square=$ Kern)

Die Fragen zur Erfassung des Prestiges im Netzwerk zeigen dessen Konzentration auf einen Netzwerkausschnitt, da über die Wichtigkeit (allgemeine Wertschätzung), den Rat (Abhängigkeit) und Schlüsselakteure (offensichtliches Prestige) die Zahl der nicht gewählten Akteure generell hoch ist und in der genannten Reihenfolge deutlich zunimmt $(77,99,153)$. Die Frage nach der Wichtigkeit, die die Befragten den genannten Akteuren zuweisen, zeigt einen deutlichen Zentralitätsvorsprung des Projektmanagers, der sich bereits als besonders zentral erwiesen hat (0.164). Mit Abstand folgen gemeinsam der zweite zentrale Naturschutzvertre- 
ter $^{194}$ und der Vertreter des Sozialen ${ }^{195}$. Auf der nachfolgenden Zentralitätsstufe stehen der Vertreter der Politik (w) und des Tourismus ${ }^{196}$.

Die Frage, von welchem Akteur projektbezogener Rat eingeholt wird, zeigt ebenso ein deutliches Zentralitätsgewicht des Projektmanagers (0.164). Bereits mit Abstand folgt wieder der Vertreter des Sozialen ${ }^{219}$ (0.096) und des Tourismus 220 (0.069). Es folgt eine Gruppe bestehend aus einem Politiker (w), einem Naturschutzvertreter der Region und einem des Bundes (0.055).

Das offensichtliche Prestige, d.h. die Anerkennung als Schlüsselakteur, ist auf einen kleinen Teil des Netzwerks konzentriert. Fünf Akteure, die auch dem Kern angehören, darunter jeweils ein Politiker der kommunalen Ebene (w) und Landkreisebene (o), zwei Naturschutzvertreter (Projektmanager und o/B) und der Tourismusvertreter (o/V) belegen die Spitzenposition (0.1). Auch unter den nachfolgenden Schlüsselakteuren fehlen die Vertreter der Naturschutzvereine, die sich zuvor unter den zehn zentralen Akteuren positionieren konnten.

Anhand der Ergebnisse der Zentralitäts- und Prestigeanalysen können poten iielle Multiplikatoren im Netzwerk identifiziert werden. Ein besonderes multiplikatives Potenzial ist dem stets auf den vordersten Plätzen positionierten Projektmanager zuzuweisen. Er nimmt bei jeder prestigebezogenen Fragestellung die zentralste Position im Netzwerk ein und rangiert bei den drei Zentralitätsmaßen auf den obersten Rängen. Dies leitet sich aus der Rolle der antragstellenden Institution in dieser Phase logisch ab, die der Projektmanager vertritt. Des Weiteren ist die multisektorale Zusammensetzung der Prestige-Inhaber, die zuvor auch als zentrale Akteure im Netzwerk identifiziert wurden, positiv hinsichtlich der Chance das Projekt übersektoral, d.h. integrativ in der Region zu verankern sowie den notwendigen (politischen) Rückhalt zu entwickeln, einzuordnen.

In den weiteren Analysen wird sich zeigen, ob der in struktureller Hinsicht erkennbare multisektorale innere Kreis (vgl. Diller 2002) mit aktiven, führenden Promotoren besetzt ist, die steuernde und koordinierende Funktionen erfüllen. Aufgrund des starken Gefälles der degree-Indizies wäre eher ein SingleLeadership-Netzwerk zu vermuten. Während der Antragsphase ist dies logisch zu erklären aufgrund der Promotorenfunktion des Antragstellers. Da jedoch die Gefälle der beiden anderen Zentralitätsindizes wesentlich flacher verlaufen, scheint es Potenzial für ein Flexible-Task-Netzwerk zu geben. Hierzu wäre die strategische Vernetzung des Antragstellers mit regionalen Partnern der Schlüssel.

Bezüglich der länderübergreifenden Zusammenarbeit im Netzwerk lässt sich zum Befragungszeitpunkt und auf Grundlage der Datenbasis feststellen, dass die oben identifizierten zentralen Akteure (Projektmanager, Naturschutz- und Tourismusvertreter, Vertreter des Sozialen) zum Befragungszeitpunkt und in diesem

\footnotetext{
194 ost/ Behörde

195 west/ Behörde, 0.082

196 ost/ Verein, 0.064
} 
konkreten Projektkontext länderübergreifende Ankerfunktionen erfüllen. Eine Durchmischung der Akteure ist noch nicht feststellbar wie Abbildung 35 deutlich zeigt. Mit dem Start des Projekts ist hier eine Veränderung zu erwarten.

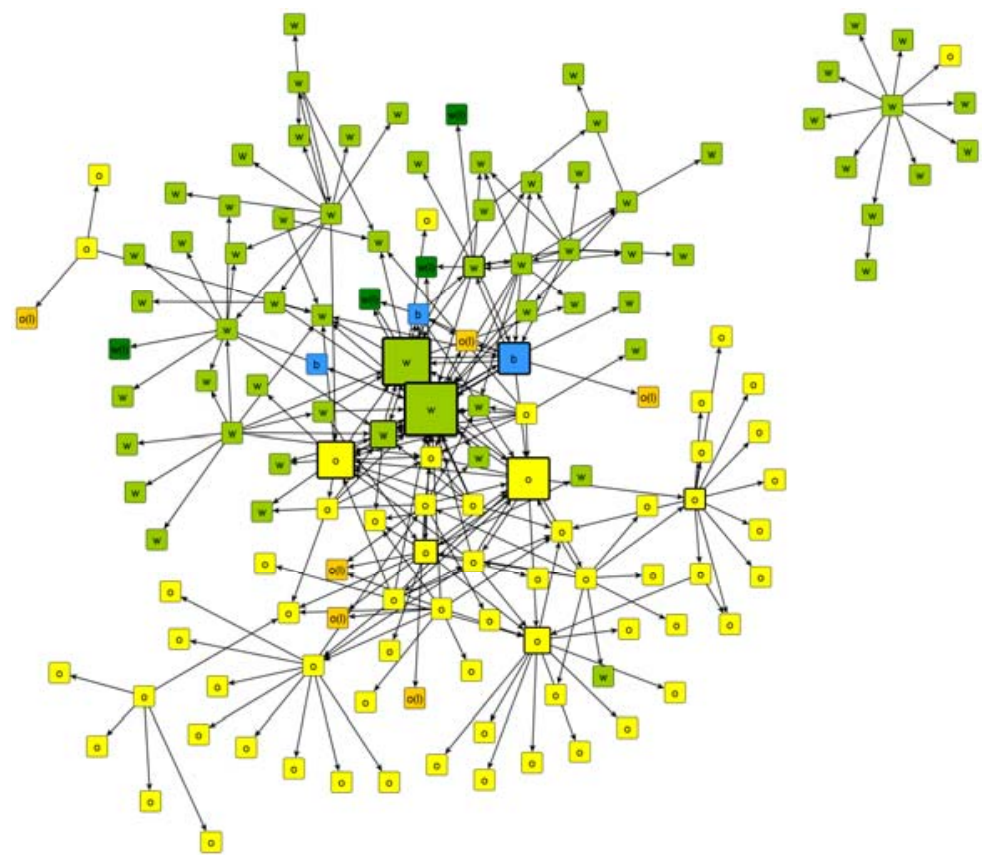

Abbildung 35: NGP Grünes Band Eichsfeld-Werratal: Länderübergreifende Vernetzung im Projektnetzwerk $(w=W$ est, $o=O s t, o(l)=$ Landesebene ost, $w(l)=$ Landesebene west, $b=$ Bund $)$

\section{Netzwerkarbeit}

Die rein quantitative Betrachtung des Verhältnisses von alten und neuen Kontakten im Netzwerk (47\% zu 53 \%), zeigt, dass bereits diese Projektvorphase einen Beitrag zur Akteursverflechtung unterschiedlicher Fachbereiche in der Region geleistet hat. Betrachtet man zudem die räumliche Verteilung der neuen Kontakte, so zeigt sich, dass grundsätzlich eine stärkere Wirkung auf die Vernetzung auf westdeutscher oder ostdeutscher Seite entstanden ist. 

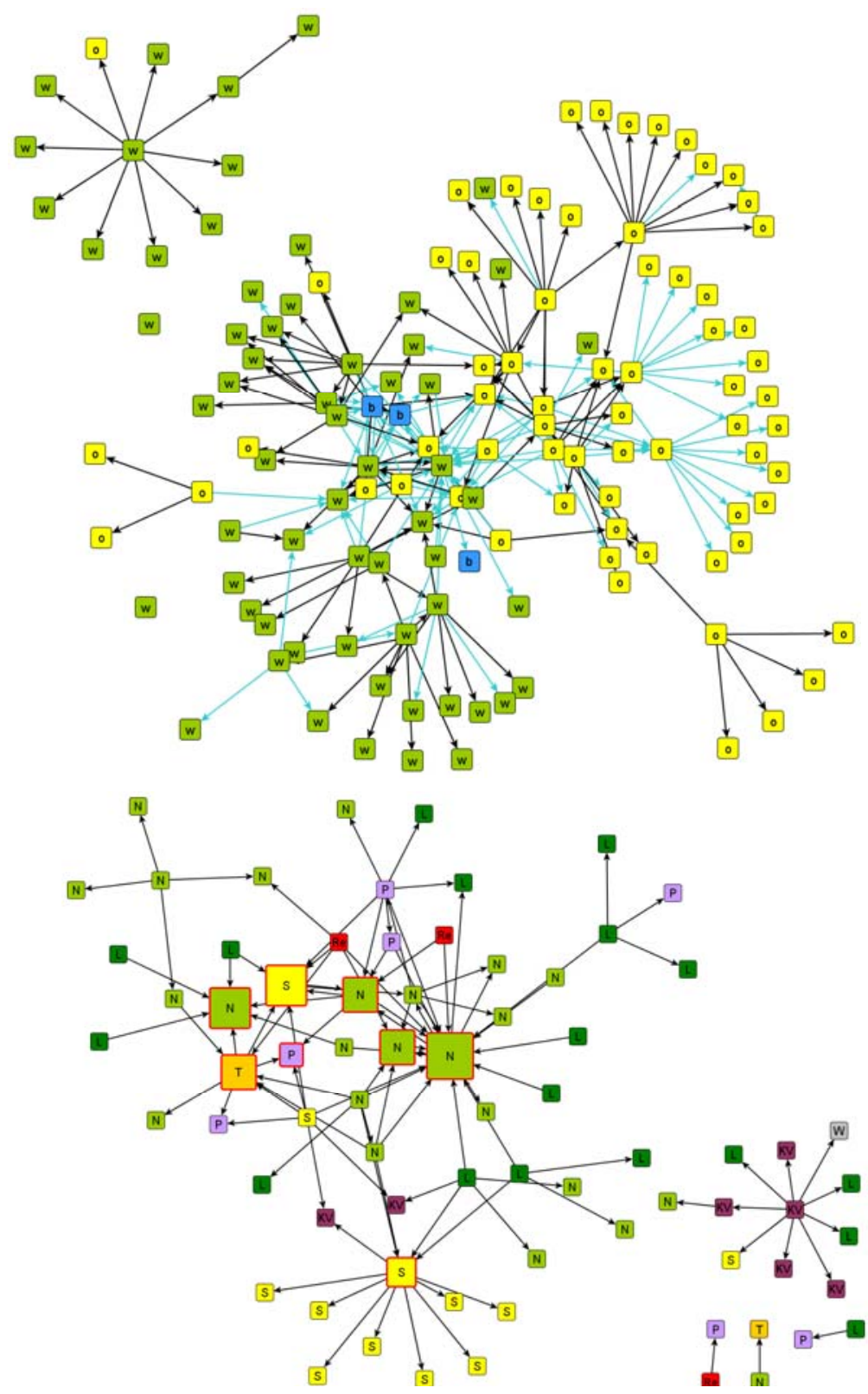

Abbildung 36: NGP Grünes Band Eichsfeld-Werratal - Oben: Neue Kontakte (blau) im Gesamtnetzwerk. $(w=W e s t, o=O s t, b=$ Bund); Unten: Selektion aller neuen Kontakte im Netzwerk. $\bar{L}=$ Landnutzung, $N=$ Naturschutz, $S=$ Soziales, $P=$ Politik, $R=$ Regionalentwicklung, $K V=$ Kommunalverwaltung, $T=$ Tourismus, $W=$ Wirtschaftsförderung) 
Die Kontaktintensität, d.h. gelegentliche und häufige Kontakte, konzentriert sich auf weniger als ein Drittel (50) der Akteure im erfassten Netzwerk. Trotz dieser Konzentration, zeigt sich im Sinne der breiten Beteiligung ein positives Bild, da die 50 Akteure eine Mischung der relevanten Fachbereiche aufweisen.

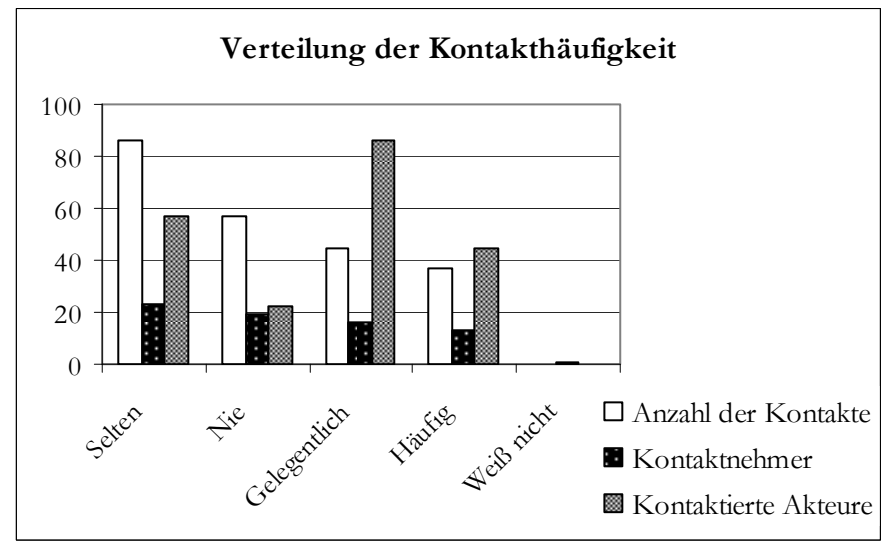

Abbildung 37: NGP Grünes Band Eichsfeld-Werratal - Verteilung der Kontakthäufigkeit

Im Kontext des frühen Projektstadiums (Antragsphase) erklärt sich, weshalb der Informationsaustausch der häufigste Kontaktzweck ist (34\% der erfassten Kontakte). Die Kooperation für gemeinsame Ziele (17\%) sowie die Kombination aus diesem Zweck und dem Informationszweck (14\%) schließen sich an. Die Akteure tauschen sich über Inhalte und Ziele der Förderung aus, informieren sich über eigene Betroffenheit und Chancen. Das Projektnetzwerk hat folglich eine diskursive, informatorische Ausrichtung - Ressourcenaustausch spielt im Prinzip noch keine Rolle. Die Kontaktarten bestätigen die informatorische, diskursive Ausrichtung und Fokussierung auf das NGP, da die meisten Kontakte über Telefon oder E-Mail gepflegt werden und an zweiter Stelle Projektveranstaltungen rangieren. 


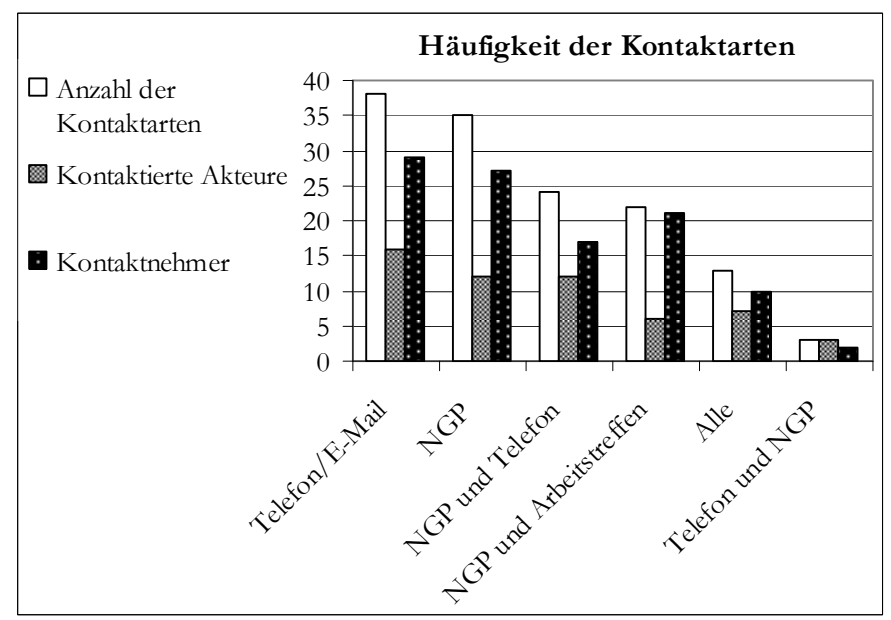

Abbildung 38: NGP Grünes Band Eichsfeld-Werratal - Häufigkeit der Kontaktarten

Es zeigt sich, dass zum Befragungszeitpunkt insbesondere inhaltliche Probleme im Netzwerk wahrgenommen werden. Auch dies lässt sich durch das frühe Projektstadium erklären, da das Projekt unterschiedliche Interessengruppen betrifft, deren Interessen jedoch erst intensiv während der ersten Projektphase (Moderationsphase und Erarbeitung des PEPL) bearbeitet werden können. Der Bedarf des Interessenausgleichs ist folglich bereits vor dem eigentlichen Projektstart sichtbar. Die organisatorischen Lücken können durch die Projektstrukturen, die während des Antragverfahrens auf einem niedrigen bzw. informellen Level gehalten werden, erklärt werden.

\section{Zusammenfassung der strukturellen Ausprägungen}

Das Netzwerk des NGP Grünes Band Eichsfeld-Werratal weist einige deutliche Ausprägungen auf, die sich zum einen durch das Projektstadium (Antragsphase) sowie die Ausrichtung des Förderprogramms erklären lassen.

Projektträger ist zentraler Netzwerkmotor: Der Projektträger des NGP besitzt eine herausgehobene Position, da er zur Zeit der Befragung die aktivste Rolle aufgrund der angestrebten Projektträgerschaft spielt. ${ }^{197}$

197 Auch zukünftig wird sich seine Position kaum weniger zentral ausprägen, da die Multifunktionalität des Projektträgers diese eher zusätzlich verstärkt. Im Moderationsbericht (2005), S. 34 des ZV Schaalsee Landschaft steht: „Der Zweckverband hat einerseits als Eigentümer und Käufer bzw. Verpächter von Flächen, sowie als Verhandlungsführer bzgl. Flächenkauf und Flächentausch und andererseits als Vertreter des Naturschutzes und drittens als Mittler zwischen den 
Multisektorales Kernnetzwerk: Die Vielfalt der vertretenen Fachbereiche ist gekennzeichnet von einer deutlichen Dominanz des Naturschutzes und der Landnutzung. Trotzdem ist die Struktur im Sinne der Definition des Netzwerkmerkmals „Breite Beteiligung“ positiv zu bewerten, da sich ein multisektoraler Kern gebildet hat. Die jeweils zehn Akteure mit den höchsten Zentralitäten und dem höchsten Prestige vertreten den Naturschutz, Soziales/Bildung/Kultur, Politik, und Tourismus. Sie können als Keimzellen einer übersektoralen Integration des NGP wirken.

Diskursiv und informatorisch: Da zum Zeitpunkt der Befragung das Projekt noch nicht bewilligt oder mit dessen Umsetzung begonnen wurde, liegt der Schwerpunkt der Aktivität im Netzwerk darin, inhaltliche Fragen und Probleme zu diskutieren, Informationen zu erhalten, weiterzugeben und gemeinsame Ziele abzustecken.

Regional eingebettet: Je zentraler die Akteure, desto stärker sind sie regional bzw. lokal verankert. Es zeigt sich, dass die potenziellen Zugpferde des Projekts zentrale Positionen unterschiedlicher Fachbereiche - Naturschutz, Politik, Tourismus und Soziales - in der Region einnehmen. Die starke Beteiligung regional tätiger Vereine zeigt die Bedeutung, die dem Projekt in der Region zugewiesen wird (Ehrenämter).

Die Orientierung des Netzwerks auf wenige Einzelakteure ist durch das frühe Projektstadium zu erklären. Die an der Beantragung und Bewerbung des Projekts in der Region beteiligten Akteure sind die aktivsten und entsprechend präsent im Netzwerk. Der Projektmanager ist der Akteur mit dem höchsten Prestige. Dies leitet sich aus seiner Rolle als Antragsteller ab und bringt ihm eine Koordinationsfunktion im Netzwerk ein (vgl. Jansen/Wald in Benz et. al. 2007). Die verschiedenen Zentralitäten verteilen sich auf eine kleine Gruppe von Akteuren, der auch der Projektmanager angehört. Die Gruppe setzt sich aus regional bedeutenden Akteuren verschiedener Fachbereiche zusammen. Bezüglich des Beitrages zur regionalen Nachhaltigkeit, aber auch der allgemeinen Projektumsetzung, wird es eine Herausforderung sein, die Tendenz der Fokussierung auf den Projektmanager und die Gefahr seiner Überlastung und in Konsequenz der Demotivierung des Netzwerks (vgl. Diller 2002) entgegenzuwirken, indem die anderen zentralen, multisektoralen Akteure Führungsaufgaben übernehmen. Darüber hinaus könnte bspw. den Naturschutzvereinen der Region, die sich vorwiegend auf ehrenamtliche Tätigkeiten stützen, eine wichtige Rolle zukommen, da sie einen Beitrag zur Verteilung der Arbeit im Netzwerk leisten können.

Interessen der Länder, Kreise und Kommunen sowie der Behörden und Ämter, eine wesentliche Schlüsselstellung in diesem Projekt“". 


\subsubsection{Qualitative Analyse}

\section{Erwartungen an das Naturschutzgroßprojekt}

Die Interviews mit den regionalen Akteuren deckten eine Vielfalt von Erwartungen an die Wirkung des NGP auf, die im Folgenden beispielhaft mit Zitaten belegt werden:

Das Oberziel Naturschutz ist akzeptiert, jedoch werden darüber hinaus entwicklungsbezogene Erwartungen an die Förderung geknüpft:

„Dass es sicherlich in erster Linie um Naturschutz geht, steht außer Frage. Aber man darf wohl nicht vergessen, dass in diesen Bereichen auch Menschen wohnen. Und da ist es natürlich wichtig, dass man bei den Projekten auch immer im Auge hat, "wie könnte man eventuell dort auch eine Wertschöpfungskette aufbauen?", dass also die, auch die Menschen, die dort wohnen, wissen oder die Gewissheit haben, also, das Naturschutzprojekt bringt uns auch ein bisschen was wieder zurück, dass wir auch hier in der, in diesem ländlichen Raum davon ein bisschen davon profitieren und dass so eine Entwicklung nicht über die Köpfe von den Menschen binweg geht. "(Zitat aus einem Interview: Kommunalverwaltung).

Als grundsätzliche Anforderung an Förderungen im ländlichen Raum werden folglich Entwicklungschancen geknüpft. Die notwendige Beteiligung der Akteure vor Ort wird angesprochen, worin die Befürchtung eines „Käseglockenschutzes“ mitschwingt. Es sei darauf zu achten,

„dass es für die unterschiedlichen Interessen die entsprechenden Wege, Wegeverbindungen, Leitsysteme gibt, und auch Besucherleitsysteme, dass es irgendwo - wie gesagt - dann letztendlich auch ein bisschen verknüpft wird, und dass trotzdem aber der Landwirt keine Angst haben muss, dass er vielleicht wegen einem Naturschutzprojekt jetzt - sagen wir mal - 30 Kilometer Umweg fahren muss, weil er vielleicht seine Felder im, in der Nachbarschaft nicht mehr erreicht; also nur mal so ein praktisches Beispiel. Irgendwo muss man da dran denken, dass eine Erreichbarkeit bestehen bleibt, dass man Verknüpfungen hat und dass man da nicht irgendwas trennt oder abschottet". (Zitat aus einem Interview: Kommunalverwaltung)

In Regionen mit Naturschutzgroßprojekten ist auch der Lebensraum der Bevölkerung zu bedenken, um weder für die dort Wirtschaftenden, noch für die Erholungssuchenden an Attraktivität zu verlieren. Eine flankierende Begleitplanung mit dem Ziel der intelligenten Verknüpfung der landschaftsbezogenen Interessen wird als notwendig erachtet.

Dem zukünftigen Netzwerk des NGP wird das Potenzial einer Impulsfunktion zugesprochen:

„Und da könnte wiederum das Netzwerk die Partner zusammenfübren, über ein gemeinsames Thema, das Grüne Band, eine neue Produktentwicklung erzielen“. (Zitat aus einem Interview: Soziales)

Die besondere Qualität des Fördergebietes an der ehemaligen innerdeutschen Grenze wurde mehrfach unterstrichen und dessen Potenzial für die Außendarstel- 
lung der (geförderten) Region und des NGP klar formuliert. Auch der Tourismus sieht Chancen:

„Ist ja auch wirklich etwas Bemerkenswertes, dass sozusagen entlang einer ehemaligen Grenze oder eines ehemaligen Todesstreifens neues Leben entstehen kann. Das ist medienwirksam, auf jeden Fall. Also insofern ist es für uns dann auch nutzbar". (Zitat aus einem Interview: Tourismus).

Mit dem NGP kann sich die Region Eichsfeld-Werratal eine verbindende Klammer schaffen, die zum Aufbau gemeinsamer, regionaler touristischer Wertschöpfungsketten genutzt werden kann. Eine Stärkung der bereits vorhandenen Regionalmarken durch eine regionalspezifische Marke „Grünes Band“ stufen die Akteure auch während der Diskussion auf dem Regionalen Workshop (06/2009) als Entwicklungschance ein. Die gebietsübergreifende Zusammenarbeit wird in diesem Zusammenhang als Schlüssel für die Ausschöpfung der Potenziale des NGP genannt, jedoch auch als zentrale Herausforderung wahrgenommen. Dabei gelte es, auf Bestehendes aufzubauen:

„Also, Teilregionen sollte man auf jeden Fall als Partner ins Boot holen, unabhängig davon, wie die übergeordnete Struktur aussieht und wie stark sie ist. Das ist schon mal ganz. klar. Zumal die Teilregionen auch wirklich starke Marken sind, die möglicherweise ... Also, manchmal hat man es so, dass die, die Untermarken stärker sind als die Dachmarke". (Zitat aus einem Interview: Tourismus)

Die aufgeführten, beispielhaften Aussagen belegen, dass aus regionaler Sicht mit der Förderinitiative des Bundes mehr verbunden wird, als die primären Förderziele. An den Aufbau neuer Netzwerkstrukturen und der Schaffung einer thematischen Klammer für die Region Eichsfeld-Werratal werden neue Entwicklungschancen geknüpft. Als möglicher Stolperstein für die Umsetzung und Akzeptanz können sich jedoch Erwartungshaltungen erweisen, die von den Zielen und Förderrichtlinien der Naturschutzgroßprojekte stark abweichen:

„,...] Aber ich glaube, das ist einn ganz großes Problem, dass also viele da meinen,

"oh, super, Millionen und da können wir alles mit machen"'. (Zitat aus einem Interview: Regionalentwicklung).

Transparente Förderrichtlinien mit entsprechender Kommunikation durch den Projektträger und Fördermittelgeber erweisen sich auch hier als wichtige Voraussetzung für die Zielsetzung „Projektumsetzung“ und „Nachhaltigkeit“.

\section{Erfahrungswissen: Naturschutz \& Regionalentwicklung}

Während der Interviews wurde anhand beispielhafter Initiativen in dem Projektgebiet sowie allgemeiner Erfahrungen bezüglich der Zusammenarbeit von Naturschutz und Landnutzung oder Tourismus die Bedeutung der Netzwerkmerkmale hinterfragt. Mit Hilfe der regionalspezifischen Erfahrungen wurden sie untermauert oder konkretisiert. 
Vertrauensnetzwerke werden als Schlüssel für erfolgreiche regionale Kooperationen und Projekte beschrieben. Über Jahre gewachsene, persönliche Netzwerke im beruflichen Kontext geben der Netzwerkarbeit das notwendige Vertrauen und gegenseitige Wissen, um einen konstruktiven Umgang miteinander auch übersektoral realisieren zu können. ${ }^{198}$ Einzelakteuren oder Institutionen, die über einen langen Zeitraum als Ansprechpartner oder Berater in der Region zur Verfügung stehen und über regionalspezifische Orts-, Netzwerk- oder Geschichtskenntnisse verfügen, wird eine Rolle als „Anker“ regionaler Entwicklung zugesprochen. Insbesondere im Kontext regionaler Förderprojekte, die personelle Fluktuation begünstigen, seien die in der Region fachlich profilierten Einzelakteure bzw. Institutionen für die Verstetigung sehr wichtig ${ }^{199}$. In der Region Eichsfeld-Werratal können bereits erste Erträge des Vertrauensaufbaus erkannt werden, denn beispielhaft wurde Dialogbereitschaft trotz harter Konflikte zwischen Naturschutz und Landwirtschaft bestätigt. Dies belegt die Relevanz des Netzwerkmerkmals „personelle Kontinuität".

Die Bedeutung von Multiplikatoren in regionalen Projektnetzwerken wurde insofern konkretisiert als mehrfach die Bedeutung der politischen Promotoren benannt wurde. So seien Projekte mit regionalem Maßstab in besonderer Weise auf den politischen Rückhalt und deren Motorenfunktion angewiesen, da sie sonst aufgrund des hohen Arbeits- und Abstimmungsaufwandes kaum Durchsetzungschancen haben. ${ }^{200}$

Als notwendiges Merkmal der Netzwerkarbeit im Kontext der „Kommunikationsaufgabe Naturschutz“ wird der unmittelbare Ansatz bei der „Betroffenheit“ bzw. den Akteuren, die man für das Ziel „naturschutzgerechte Landschaftsnutzung" gewinnen möchte, gesehen:

„Das ist immer, so ein Rezept zum Erfolg. Man muss wirklich vor Ort gucken, „wen betrifft es", um die Menschen mitzunehmen. [...] Also, auf die Dörfer zu gehen, ist ganz. wichtig. Also, ich kann jetat nicht immer sagen, "wir machen hier einen Obstbaumschnittkurs und treffen uns hier in der Volkshochschule in Göttingen", sondern wir gehen dann in irgend eine alte Schule in einem kleinen Dorf und schneiden dann nachmittags auch die Bäume der Gemeinde dann da oder am Feldweg von der Feldmarkinteressenschaft. Und dann kommen die Leute auch". (Zitat aus einem Interview: Naturschutz und Landnutzung)

198 „Und das, was ich jetzt in den, in den, ja, mebr oder weniger vier Jabren, wo ich das jetzt bier mache, festgestellt hab, ist, dass, also, dieses, dieses persönliche Netzwerk und das persönliche Kennen und auch Wissen, was man von einem erwarten kann, aber auch, was nicht, dass das quasi immer der Schlüssel ist". (Zitat aus einem Interview: Regionalentwicklung)

199 s. Protokoll des ersten Regionalen Workshops in der Untersuchungsregion NGP Grünes Band Eichsfeld-Werratal am 12.06.2009, Gut Herbigshagen - Duderstadt, S. 7

200 „Wichtig ist natürlich auch, dass man Multiplikatoren in der Region - also, dazu zäblen natürlich die gewählten Vertreter, also für mich sind da schon Bürgermeister sehr wichtig-, dass man die auf seiner Seite bat, die natürlich dann in den Gremien und im Kontakt mit den Bürgern auch immer wieder erklären, was dieses Projekt für Ziele hat. Also, das trägt sicherlich zur Integration bei“. (Zitat aus einem Interview: Soziales) 
Aktive Beteiligung der relevanten Akteure bedeutet dementsprechend auch die (sekundären) Zielgruppen einzubinden. Das Beispiel zeigt, dass landschaftspflegerische Bildungsangebote ein Weg zur Schaffung eines allgemein naturschutzbewussten Handelns in der Region sein können.

Regionale Identität wird als Motivationsfaktor für Entwicklungsinitiativen beschrieben. Das Engagement der Akteure wird in direktem Zusammenhang mit der Verbundenheit mit der Region, mit der Wertschätzung der Lebensqualität und dem Bedürfnis der positiven Gestaltung der Entwicklung gesetzt. Die Untersuchungskulisse „Grünes Band“ birgt eine besondere historische Prägung, die zu berücksichtigen ist.

Regionale (Gemeinschafts-)Aktivitäten abseits der Förderkanäle werden als zunehmend wichtiger eingeschätzt, um eine dauerhafte und zukunftsfähige Entwicklung anstoßen und verstetigen zu können. Die Abhängigkeit von oder Orientierung an Fördermöglichkeiten hemmt aus Erfahrung das regionale kreative Potenzial und letztlich auch die regionale Eigenständigkeit, auf die es ankommt. Das Netzwerkmerkmal Probleme und Lösungswille steht hiermit in Zusammenhang und wird um die Qualität der Eigenständigkeit ergänzt. Ist diese nicht ausreichend ausgeprägt, zeigen sich negative Wirkungen auf die allgemeine Projekt- und Maßnahmenplanung in der Region. Auch die Netzwerkarbeit leidet unter der Orientierung an Förderfristen:

„Denn ich glaube, es gibt unzäblige von Projekten, die halbwegs gelaufen sind, solange die Förderungen geflossen sind, und schnell zusammengebrochen sind, wenn die Förderungen nicht mehr geflossen sind". (Zitat aus einem Interview: Tourismus)

Negative Erfahrungen wurden in der Vergangenheit aufgrund unzureichender Transparenz im Kontext von Naturschutzmaßnahmen gesammelt. Beispielhaft kann das „Rebhuhnprojekt“ der Abteilung Naturschutzbiologie der Universität Göttingen und der Biologischen Schutzgemeinschaft Göttingen e.V. genannt werden. Ein von den regionalen Bauernverbänden und der Jägerschaft akzeptiertes und unterstütztes Projekt löst Diskussionen und z. T. Konfrontationen durch befürchtete „strategische Probleme“ als Folge der Projektumsetzung durch erhöhte Auflagen bei Stall- oder Hallenbauten oder gar der Bewirtschaftung von Flächen aus. ${ }^{201}$ Eine höhere Transparenz von Naturschutzprojekten und ihren Zielen sowie den Konsequenzen für Wirtschaftende in der Landschaft könnte dazu beitragen, dass die Akzeptanz auch bei der praktischen Maßnahmenumsetzung gesichert bleibt. Der Eindruck, mit der Unterstützung von Naturschutzprojekten unbewusst eine Kettenreaktion bezüglich weiterer gesetzlicher Auflagen auszulösen, die Nachteile für das eigene Wirtschaften mit sich bringt, belastet die Integration der Schutz- und Nutzungsinteressen.

Das Bewusstsein für die Relevanz funktionierender Netzwerke für regionale Projekte und die Entwicklung im Allgemeinen ist bei den Interviewpartnern aus-

201 S. Informationsblatt Landvolk Göttingen (November 2008) 
geprägt. Einig sind sie sich jedoch, dass die vorhandenen Kapazitäten mit dem Mehraufwand, den Abstimmungen und Kooperationen mit sich bringen, nicht im Gleichgewicht stehen. Personal- und damit Zeitressourcen sind knappe Güter und angesichts der großen Projektregion des NGP Eichsfeld-Werratal wird in Frage gestellt, wie eine regionale Netzwerkstruktur aufzubauen ist, damit der Mehraufwand tragbar bleibt und der Gewinn daraus dessen Einsatz rechtfertigt. Transparente Förderstrukturen und „lückenlose“ Informationsflüsse werden als wichtige Bedingungen genannt, um diese Herausforderung zu meistern.

Die länderübergreifende Zusammenarbeit stellt im Kontext der Förderung am Grünen Band eine zentrale Herausforderung dar. Scheinbar einfache, praktische Projekte stoßen schnell an Grenzen:

„Also, gerade im Bereich des Werra-Burgen-Steigs stellen wir das fest, dass eben, was die Förderung betrifft, es da doch eklatante Unterschiede zwischen Thüringen und Hessen gibt, was eben auch schwierig auf einen Nenner zu bringen ist, wenn es darum geht, Sachen möglichst zeitgleich zu takten oder, ja, auch die Beschilderung an Bundesstraßen. Da werden in Thüringen die Gesetze wesentlich freier ausgelegt - obwobl sie eigentlich Bundesgesetze sind - als bei uns. Das ist sehr schwierig, dann hier vor Ort zu erklären, warum es dafür eben kein Schild gibt". (Zitat aus einem Interview: Tourismus).

Die unterschiedlichen gesetzlichen Rahmenbedingungen in den Bundesländern erweisen sich als Hürden in der gemeinsamen Projektpraxis. Eine Wertschätzung und gewisse Einforderung der Unterstützung durch die übergeordneten Ebenen gerade bei länderübergreifenden Initiativen auf regionaler Ebene wurde deutlich:

„Aber da gibt es ja das gute Beispiel, jetzt aktuell, die Neukonzeption unseres Grenzlandmuseums, wo sich die Länder Niedersachsen, Thüringen zusammen mit dem Bund beteiligen. Und auch hier wollen wir gar nicht, oder als, als Verantwortliche vor Ort wollen wir gar nicht - und schätzen das auch - beispielsweise den fachlichen Rat und diese fachliche Kompetenz, die bei den Ländern vorhanden ist. Das heißt, ich mach es jetzt gar nicht mal an Geld fest, sondern dass man einfach auch die fachliche Kompetenz der Länder natürlich unbedingt braucht und auch, ja, Wege aufgezeigt bekommt, wie man vielleicht dann auch die Ziele erreichen kann. " (Zitat aus einem Interview: Soziales).

Die aufgeführten Erkenntnisse aus den Interviews belegen beispielhaft die Bedeutung der Netzwerkmerkmale aus regionalspezifischer Perspektive. Jedoch zeigen sich Übereinstimmungen mit den Untersuchungsergebnissen in der Region Schaalsee-Landschaft, so dass sich konkrete Ansatzpunkte für die Perspektiven bzw. Empfehlungen aus Sicht der Netzwerkforschung ableiten lassen.

\subsubsection{Ansatzpunkte für Empfehlungen}

Die Untersuchungen in den NGP-Regionen Schaalsee-Landschaft und Grünes Band Eichsfeld-Werratal haben die Annahme bestätigt, dass der Beitrag von Naturschutzgroßprojekten zur regionalen Nachhaltigkeit in erster Linie von der Verknüpfung des sektoralen Projekts mit anderen regionalen Initiativen oder Sektoren abhängig ist. Die ausschließliche Beteiligung von Akteuren aus den betroffenen 
Kernsektoren (Naturschutz und Landnutzung) während der PEPL-Erarbeitung im Sinne der effizienten Planerstellung und -umsetzung kann wie gezeigt mehrere negative Auswirkungen bzw. Missverständnisse zur Folge haben:

- Wahrnehmung des NGP als Expertenprojekt ohne Mitwirkungsmöglichkeiten weiterer Interessierter oder indirekt Betroffener (Gemeinden),

- Wahrnehmung des NGP als Projekt der Verwaltung bzw. des Naturschutzes, nicht der Region;

- ungenutzte Synergiepotenziale,

- negative Belastung des Images des Naturschutzes.

Um diese negativen Auswirkungen zu vermeiden, die der regionalen Nachhaltigkeit aber auch langfristig den Naturschutzinteressen entgegen stehen, sollte die Definition der „relevanten Akteure“ nicht zu eng gefasst werden, wenn das Projekt einen Beitrag zur regionalen Nachhaltigkeit leisten soll. Hier wird es in der Untersuchungsregion Grünes Band Eichsfeld-Werratal insbesondere auf die Bewahrung der multisektoralen Zusammensetzung des Netzwerks im Kern und insgesamt ankommen.

Grundsätzlich zielen die Empfehlungen ausschließlich auf die Netzwerkarbeit ab, die als Schlüssel zum Beitrag der NGP zur Nachhaltigkeit verstanden wird. Deren Grundlage bildet die Verteilung von Verantwortungen zwischen Projektträger und Projektregion. Das Ziel, ein NGP als einen Beitrag zur regionalen Nachhaltigkeit umzusetzen, hängt von der bewussten Zielformulierung durch die Projektregion und nicht von den Projektträgern allein ab. Der Projektträger spielt zwar die zentrale Rolle als ein Motor des Netzwerks. Um aber die Offenheit und integrative Ausrichtung des Projekts zu sichern, und die Anknüpfung bzw. Nutzung der landschaftsbezogenen Potenziale, die ein NGP schaffen kann, zu realisieren, müssen jedoch viele Akteure in der Region das NGP initiieren und tragen:

„Was natürlich nicht geschehen darf - und das würde den Projektträger überfordern -, man darf nicht den Projektmanager dafür verantwortlich machen, wenn plötzlich keine touristische Entwicklung da ist oder keine Regionalentwicklung im weitesten Sinne. Das ist nicht in seiner Verantwortung. In seiner Verantwortung liegt natürlich primär, liegen die Naturschutziziele und liegt im weitesten Sinne noch der Aufbau des Netzoverkes. Und dieses Netzwerk, hat aber eine eigene Verantwortung für die Realisierung über den Naturschutz, binaus gehender Zielsetzungen. Also, das ist eigentlich selbstverständlich. Aber man, heute hat man ja oft damit zu tun, dass dann gesagt wird, "na, ibr bekommt die Förderung; also seht doch mal $\approx u$ ". Das ist ein völlig falsch verstandenes Agieren im Netziperk". (Zitat aus einem Interview: Soziales)

Wenn ein NGP einen Beitrag zur regionalen Nachhaltigkeit leisten soll, müssen folglich zwei Handlungsebenen, die sich aus den Rahmenbedingungen der Förderung ergeben, bewusst wahrgenommen und „aktiviert" werden. Auf den Ebenen kommen unterschiedliche Akteurs- und Verantwortungskonstellationen zum Tragen, die sich verschiedenen Aufgaben widmen (siehe Abbildung 39). 


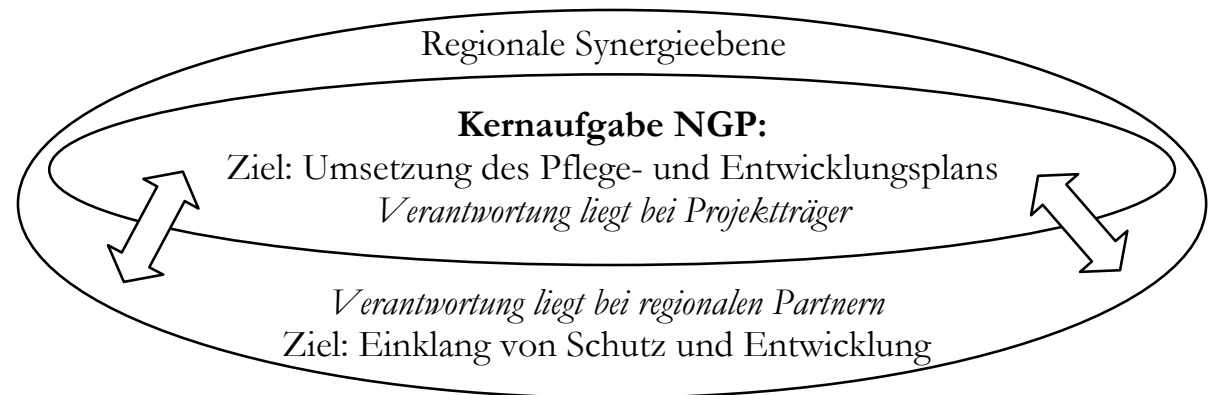

Abbildung 39: Ebenen der Projektarbeit von Naturschutzgroßprojekten im Kontext regionaler Nachbaltigkeit

Empfehlungen für die Ebene der Kernaufgabe zielen insbesondere auf die Verhandlungsführung zwischen den unmittelbar Betroffenen und Zuständigen ab, wobei dem Projektträger sowie der externen Moderation die Hauptverantwortung zukommt.

Auf die regionale Synergieebene zielen die Empfehlungen für den Aufbau geeigneter Kommunikations- und Organisationsstrukturen in der Projektregion. Ziel sollte sein, im multisektoralen Dialog zu klären, welche Entwicklungschancen die NGP-Region den Teilregionen bieten kann. Auf Basis der Untersuchung können mindestens zwei Handlungsfelder abgesteckt werden, nämlich „Naturschutz und Tourismus“ und „Naturschutz und Wirtschaft“. Diese Ansatzpunkte werden in Kapitel 4 durch die Empfehlungen entsprechend ausgeführt.

\subsubsection{Maßnahmenperspektive}

\subsubsection{Maßnahmenbasismatrix}

\section{Anwendungspotenzial}

In der Entwicklung des PEPL während der dreijährigen Moderationsphase liegt eine besondere Chance für die Region, da sie die Planung und Integration des PEPL in die Region im Sinne von regionaler Nachhaltigkeit vornehmen kann.

An diesen Gestaltungsprozess kann sich nun die Nachhaltigkeitsausrichtung von Projektmaßnahmen andocken. Die im Rahmen des Kapitel 2 genannte Organisation von regionaler Nachhaltigkeit kann dabei direkt in die Planung des NGP Eichsfeld-Werratal einfließen. Dazu besteht die Möglichkeit, aus den Erfahrungen der Schaalseeregion zu lernen. Aufgrund der gleichen Projektausrichtung, also 
dem Erwerb von Flächeneigentum durch den Projektträger, können exemplarisch die Fallbeispiele und Literaturnachweise herangezogen werden. Bei der Planung von Maßnahmen auf den unterschiedlichen Flächentypen (Grünlandfläche, Waldflächen, Wasserflächen, etc.) lassen sich damit bereits Sekundäreffekte mit einkalkulieren, die die jeweiligen Flächentypen bewirken. Damit besteht die Möglichkeit die entwickelten Instrumente der Nachhaltigkeitsdokumentation anzuwenden.

Dazu müssen die regionalen Akteure, die in der Projektverantwortung stehen, zunächst das regionale Nachhaltigkeitsverständnis eruieren. Dazu liefert die Moderationsphase die ideale Möglichkeit der Implementation der generierten Ergebnisse dieser Arbeit. An den Handlungsfeldern der regionalen Nachhaltigkeit orientierend können mit den relevanten Akteuren der Region Eichsfeld-Werratal die regionalen Ausprägungen von Nachhaltigkeit diskutiert und damit das regionale Nachhaltigkeitsverständnis in Erfahrung gebracht werden. Diese sollten sich aus den Zielsetzungen einzelner Akteursgruppen ableiten. Es gilt also zu klären, welche Zielsetzung die Landwirte bisher auf den potenziellen Projektflächen verfolgen, was die Naturschutzverbände planen und welche sozialen Belange die regionale Bevölkerung besitzt. Damit würde die spezialisierte, d.h. sektorale Ausrichtung des Naturschutzgroßprojekts, um eine generalistische, d.h. integrative Perspektive ergänzt. Nur wenn diese Zielsetzungen aller drei Säulen der Nachhaltigkeit in den PEPL Eingang finden, erscheint eine nachhaltige Ausrichtung des NGP Grünes Band Eichsfeld-Werratal möglich. Das bedeutet, dass im Rahmen der förderrechtlichen Möglichkeiten, der Projektträger zusammen mit den regionalen Partnern und den übrigen relevanten Akteuren Sekundäreffekte auf die ökonomische und soziale Säule generieren kann.

Betrachtet man dazu nochmals die zweite mögliche Perspektive, in der das NGP Grünes Band Eichsfeld-Werratal einen Beitrag zur regionalen Nachhaltigkeit leisten kann, so lassen sich unterschiedliche Schlussfolgerungen für die Maßnahmenplanung ableiten. Im Gegensatz zur generalistisch-nachhaltigen Ausrichtung des Naturschutzgroßprojekts verfolgt die spezialisierte Ausrichtung eine Konzentration auf die Zielsetzung in der ökologischen Säule der regionalen Nachhaltigkeit. Bei dieser Ausrichtung bildet auch hier die Moderationsphase eine ideale Plattform, um in den Dialog mit Vertretern anderer Förderprojekte zu treten. Dieser Dialog müsste sicherstellen, dass bei reinen Naturschutzförderprojekten Förderprojekte wie LEADER oder die regionale Wirtschaftsförderung die anderen Säulen der regionalen Nachhaltigkeit bedienen. Dieses Vorgehen kommt der regionalen Förderung eines regionalen Nachhaltigkeitsmanagement nach.

Unabhängig von der gewählten Perspektive bietet das vorliegende Dokumentations-Tool den Vorteil, dass es implizite Zielvorstellungen in Form der Nachhaltigkeitsspinne transparent darstellt. Diese wiederum ist als Basis für den Dialog in der Moderationsphase zur Erreichung einer möglichst hohen Nachhaltigkeitsausprägung zu sehen. Denn nur das Bewusstsein über die Gesamtheit aller Ziele der jeweiligen Akteursgruppen von Nachhaltigkeit kann Basis für Ausgestaltung des 
Naturschutzgroßprojekts Grünes Band Eichsfeld-Werratal im Sinne regionaler Nachhaltigkeit sein.

\subsubsection{Nachbaltigkeitsspinne und regionale Verhaltensweisen}

Zur Abbildung möglicher Wirkungen des NGP Grünes Band Eichsfeld-Werratal können die jeweiligen Zielsetzung und Ausgestaltungen in die Maßnahmenbasismatrix übertragen werden. Daran anknüpfend besteht die Notwendigkeit die Wirkung der entsprechenden Flächenform zuzuordnen. Zum großen Teil bietet das NGP Schaalsee-Landschaft schon die grundlegenden Wirkungszusammenhänge von Flächenformen und den Handlungsfeldern, wie Abbildung 40 verdeutlicht.

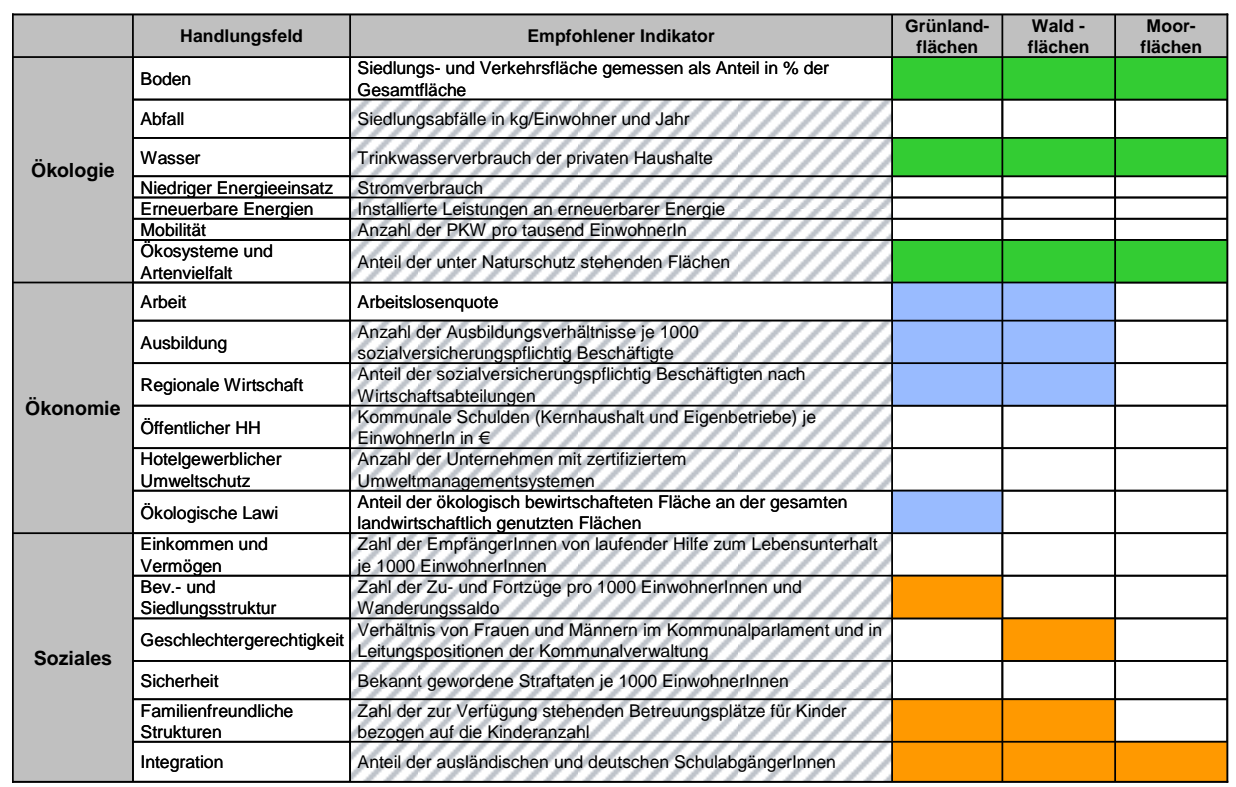

Abbildung 40: NGP Schaalsee-Landschaft-Maßnahmenbasismatrix

Im Gegensatz zur Schaalseeregion besteht hier die bereits diskutierte Chance der Konkretisierung der Handlungsfelder von Nachhaltigkeit im Verlauf der Moderationsphase.

Im Unterschied zur fehlenden Projektumsetzung besteht bereits ein Netzwerk in der Untersuchungsregion NGP Grünes Band Eichsfeld-Werratal. Dies begründet sich in der Tatsache, dass im Vorfeld ein Netzwerk von relevanten Akteuren notwendig war, um die Förderung in die Region zu holen. Diese Vernetzung wurde durch die Netzwerkanalyse belegt. Durch die Interviews könnten sich auch für diese Region Verhaltensweisen bzw. -grundsätze ableiten lassen, die mit denen der Managementregelen für Nachhaltigkeit korrespondieren (siehe Tabelle 43). 
Tabelle 43: Region Eichsfeld-Werratal - Regionale Verhaltensweisen für Nachhaltigkeit

\begin{tabular}{|l|l|}
\hline $\begin{array}{l}\text { Identifizierte regionale Managementregeln (Projekt- } \\
\text { ebene) } \\
\text { (aus Interviews mit regionalen Akteuren) }\end{array}$ & $\begin{array}{l}\text { Korrespondierende } \\
\text { Managementregeln der nationalen } \\
\text { Nachhaltigkeitsstrategie }{ }^{202}\end{array}$ \\
\hline $\begin{array}{l}\text { Die Region übernimmt eigenverantwortlich Vorsorge für } \\
\text { regionale Entwicklung und fokussiert sich auf Projekte } \\
\text { mit regionalspezifischem Bezug. }\end{array}$ & R 1 \\
\hline $\begin{array}{l}\text { Die Region muss ihre gewachsenen Strukturen in aktuelles } \\
\text { und zukünftiges Handeln integrieren und Geschichtsbe- } \\
\text { wusstsein bewahren. }\end{array}$ & R 3 \\
\hline $\begin{array}{l}\text { Der Strukturwandel in der ländlichen Region soll wirt- } \\
\text { schaftlich erfolgreich sowie ökologisch und sozial verträg- } \\
\text { lich gestaltet werden. Naturschutzziele sind in den Kon- } \\
\text { text der sozialen und ökonomischen Dimension der } \\
\text { Nachhaltigkeit einzubetten, indem die Region als Lebens- } \\
\text { raum der Bevölkerung betrachtet wird. Kooperation in } \\
\text { Handlungsräumen statt in Verwaltungsräumen. }\end{array}$ & R 5 \\
\hline $\begin{array}{l}\text { Allen an der Mitgestaltung regionaler Entwicklung interes- } \\
\text { sierten Bürgern ist die Möglichkeit dazu zu geben. }\end{array}$ & R 9 \\
\hline Lernen durch interregionalen Austausch. & R 10 \\
\hline
\end{tabular}

Folglich zeigt sich auch in der Untersuchungsregion NGP Grünes Band Eichsfeld-Werratal ein Nachhaltigkeitsbewusstsein, das positive Effekte auf die Entwicklung des PEPL erwarten lässt. Mit Blick auf die Region Schaalsee-Landschaft lässt sich vieles auch in der Region Eichsfeld-Werratal wieder finden.

Dennoch konnte zumindest in der vorliegenden Reihe von Interviews kein Hinweis für regionale Verhaltensregeln gefunden werden, die den Regeln 2 und 6 der nationalen Nachhaltigkeitsstrategie entsprechen. Dies führt zu dem Schluss, dass der Umgang von erneuerbaren Gütern im Hinblick auf Landwirtschaft und Tourismus noch nicht geklärt ist (Regel 2). Dies bestätigt die im vorangegangen Abschnitt erläuterte Notwendigkeit, Akteure aus allen Säulen der Nachhaltigkeit in die Moderationsphase einzubinden, um auf der Basis der Nachhaltigkeitsdiskussion die Akzeptanz für den Naturschutz zu stärken. Dies erscheint notwendig, um bestehende Zielkonflikte im Rahmen der Nachhaltigkeit zu lösen und damit einen Beitrag zur Integration des Projekts in die Region zu leisten. Regel 6 knüpft hierzu an eine gemeinsame Sprache zwischen den unterschiedlichen Akteuren an, die sich aus den geführten Interviews noch nicht ableiten ließ. Hier besteht die Notwendigkeit, durch Austausch Wissen über den anderen Akteur zu schaffen, das im Idealfall als Regionswissen allen Akteuren zur Verfügung steht und zur Kooperation zwischen den Akteursgruppen führen kann.

202 Die Nummerierung gibt die Stellung im Rahmen der 10 Regeln der nationalen Nachhaltigkeitsstrategie wider. 
Dennoch ist darauf hinzuweisen, dass es sich bei den eruierten Verhaltensweisen um Verhaltensweisen des vorläufigen Projektnetzwerkes handelt, das einer gewissen Dynamik unterliegt und sich über die Zeit hinweg weiter entwickelt. Die bewusste Integration in die Moderationsphase führt unter Umständen zu einer erheblichen Beschleunigung dieses Entwicklungsprozesses und damit zu einer möglicherweise beschleunigten Nachhaltigkeitsentwicklung in der Region.

\subsubsection{Nachbaltigkeitsinstitutionen}

Wie beschrieben, befindet sich die Untersuchungsregion in den Landkreisen Osterode am Harz, Nordhausen, Göttingen, Eichsfeld, Werra-Meißner-Kreis und Unstrut-Hainich-Kreis. Im Hinblick auf eine dauerhafte Etablierung des Naturschutzgroßprojekts während der Projektumsetzung und nach der Förderperiode wurde in den benannten Landkreisen eine Erhebung potentieller Nachhaltigkeitsinstitutionen durchgeführt. Dazu erfolgten Interviews mit den Bürgermeistern der größten an die Modellregion angrenzenden Städte, mit dem Ziel zu erfahren, welche bereits existierenden Nachhaltigkeitsinstitutionen eine potentielle Andockmöglichkeit für einzelne Projektbausteine des NGP Grünes Band EichsfeldWerratal bieten. Exemplarisch fand die Erhebung in den Städten Osterode, Bleicherode, Witzenhausen und Heiligenstadt statt, die jeweils in den einzelnen Landkreisen der Modellregion liegen.

Tabelle 44: NGP Grünes Band Eichsfeld-Werratal - Beispiele von Nachhaltigkeitsinstitutionen in den Landkreisen

\begin{tabular}{|l|l|l|l|l|}
\hline Stadt & $\begin{array}{l}\text { Bürgermeis- } \\
\text { ter }\end{array}$ & $\begin{array}{l}\text { Stadt- } \\
\text { /Regionalent } \\
\text { wicklungs- } \\
\text { konzept } \\
\text { (ISEK) }\end{array}$ & $\begin{array}{l}\text { Wachstums- } \\
\text { kern }\end{array}$ & $\begin{array}{l}\text { Lokale Agen- } \\
\text { da }\end{array}$ \\
\hline Osterode & & & & \\
\hline Bleicherode & & & & \\
\hline Witzenhausen & & & & \\
\hline Heiligenstadt & & & & \\
\hline
\end{tabular}

Im Gegensatz zur Schaalseeregion sind die größeren Städte, die an die Untersuchungsregion angrenzen viel mehr auf die Bürgermeister als treibende Nachhaltigkeitsinstitution ausgerichtete (siehe Tabelle 44). Dies erlaubt die Schlussfolgerung diese Repräsentanten der jeweiligen Städte zumindest in der Anfangsphase der Moderationsphase mit einzubinden, um damit einen Beitrag zur langfristigen Sicherung des NGP Grünes Band Eichsfeld-Werratal zu leisten. Durch den Dialog mit den jeweiligen Bürgermeistern eröffnet sich die Perspektive, die Projektmaßnahmen so zu gestalten, dass die betroffenen Landkreise bereits zu Beginn äußern können, was für sie langfristig von Interesse ist. 


\section{Perspektiven und Empfehlungen}

\subsection{Einführung}

Aufbauend auf den Analyseergebnissen in den vier Regionen stellt dieses Kapitel Empfehlungen zur Netzwerkarbeit und eine Perspektive zur Nachhaltigkeitsdokumentation vor. Letztere dient als Darstellungsform für Nachhaltigkeitserfolge, die Fördermittelempfängern eine frühzeitige Ausrichtung auf Synergieeffekte in der Region über den Naturschutz hinaus und damit eine gesteigerte Akzeptanz des Naturschutzes ermöglicht.

Zu Beginn werden Empfehlungen zur Netzwerkarbeit dargestellt, aus denen Folgerungen für die zukünftige Netzwerkarbeit in Naturschutzgroßprojekten und E + E-Vorhaben gewonnen werden können. Die Empfehlungen gliedern sich nach den einzelnen Netzwerkmerkmalen. Grundlage sind die jeweils festgestellten positiven wie negativen Ausprägungen in den Netzwerken (siehe Kapitel 3), aus denen sich Problemlagen der Netzwerkarbeit ableiten lassen. Wo erforderlich werden die Problemlagen und Empfehlungen auf die beiden betrachteten Fördertitel weiter fokussiert. Zudem werden mögliche Werkzeuge vorgestellt, welche die Netzwerkarbeit unterstützen und dafür sorgen, dass durch diese umfassendere Effekte für die regionale Nachhaltigkeit insgesamt entstehen. In Abschnitt 4.3 werden die Perspektive zur Nachhaltigkeitsdokumentation, die so genannte „Nachhaltigkeitsspinne" und ihre unterschiedlichen Anwendungsmöglichkeiten dargestellt. Diese kann sowohl für die Offenlegung der durch das Projekt angesprochenen Themen- 
felder der Nachhaltigkeit, der Nachhaltigkeitspostulate als auch der Institutionen für Nachhaltigkeit verwendet werden.

\subsection{Empfehlungen zur Netzwerkarbeit}

Die Empfehlungen zur Netzwerkarbeit werden anhand der Netzwerkmerkmale in den drei Kategorien „Konstellation“, „Strategie“ und „Instrumente“ dargestellt. Werkzeuge zur Netzwerkarbeit als Vorschläge zur Realisierung einzelner Empfehlungen werden daran anschließend kurz vorgestellt. Die Überschneidungen zwischen den Werkzeugen sind vielfältig und sie sprechen oftmals mehrere Netzwerkmerkmale an. Diese Überschneidungen sind an den entsprechenden Stellen durch Querverweise kenntlich gemacht. Zudem stellt Tabelle 45 die angesprochenen Merkmale noch einmal in einer Übersicht dar. Die Perspektiven der Umsetzung zeigen auf, wer welche Bausteine dieser Werkzeuge zur Netzwerkarbeit realisieren muss, damit sie im Sinne der Nachhaltigkeit wirken können.

\subsubsection{Konstellation: Breite Beteiligung}

\section{Problemlagen}

Breite Beteiligung in Netzwerken erweist sich bei allen Untersuchungsregionen als wesentliche Einflussgröße für die Akzeptanz von Förderzielen sowie für die Motivation regionaler Akteure einen eigenen Beitrag für diese zu leisten. Es zeigt sich, dass eine sektoral ausgerichtete, selektive Beteiligung eine regionale Verankerung von Naturschutzförderprojekten oder die regionale Sensibilisierung für Naturschutzziele sowie die Verknüpfungen mit weiteren Sektoren (Tourismus, Regionalentwicklung) erschwert. Die Dauer der Förderung ist ein wichtiger Einflussfaktor der Beteiligungsmöglichkeit.

\section{Empfehlungen}

Partizipation alleine führt nicht automatisch zu mehr Nachhaltigkeit203, jedoch kann über eine breitere Beteiligung der Anforderung an Netzwerke Rechnung getragen werden, einen Diskussionsprozess zu gestalten und sich integrativ auszurichten. Breite Beteiligung muss sich an den Kriterien der Effizienz und Effektivität messen lassen. D.h. der investierte Mehraufwand darf nicht auf Kosten der primären Förderziele geleistet und regionale Strukturen nicht überlastet werden. Wenn eine integrative Ausrichtung der Projektnetzwerke durch die Integration der relevanten Akteure unterschiedlicher Fachbereiche gewünscht wird, ergeben sich Chancen und Risiken bei der Umsetzung. Als Chance ist die gesteigerte Akzeptanz

203 Vgl. Oels (2007). 
für Naturschutzziele durch Einbindung, Abstimmung und Information zu sehen, sowie die Schaffung von Synergien in regionalspezifischer Ausprägung. Als Risiko ist der erhöhte Aufwand für den Projektträger (Personal, Zeit, Kompetenzen, Ressourcen) zu nennen.

Mit Hilfe breiter Beteiligung kann ein Verhandlungsraum geschaffen werden, in dem projektrelevante Themen der regionalspezifischen Nachhaltigkeit diskutiert und, im Rahmen des Projekts, entsprechend gestalten werden. Im jeweiligen Projektkontext sollte daher mittels regionalem Screening geklärt werden, welche Akteure die relevanten sind, um sie aktiv einzubinden. Allerdings ist dabei das Verhältnis aktiver Projektsteuerung und akteursbezogener Eigeninitiative zu beachten: Der relevante Akteurskreis ist variabel und muss auf ein handhabbares Maß begrenzt werden. Dennoch sollte das Netzwerk für die Einbindung weiterer Akteure, die durch ihr Engagement und Interesse Relevanz gewinnen können, offen sein (vgl. hierzu Abschnitt 4.2.4).

Um die Beteiligung im Projektverlauf zielführend umzusetzen, ist es entscheidend, Verantwortungen im Planungs- und Umsetzungsprozess zu klären, denn der Träger von Förderprojekten kann die breite Beteiligung mit dem Ziel der Verankerung des Projekts und die Aushandlung von Synergien nicht allein realisieren, sondern braucht dazu regionale Partner. Aus den Ergebnissen der Netzwerkanalysen erweist sich ein „offener Projektstart“ als positiv im Sinne der Akzeptanzgewinnung und Motivation. Während der Projekte kann eine ausreichende und zielführende Beteiligung durch Workshops, Arbeitsgruppen oder Steuerungsgruppen (siehe Abschnitt 4.2.6) gestaltet werden. Der Fördermittelempfänger muss sich folglich bereits in der Antragsphase mit der Organisation, der Auswahl und dem Umfang dieser Beteiligungswerkzeuge auseinandersetzen und sollte klären, welche bestehenden Organisationen einzubinden sind.

Fördertitelfokus NGP: In der Moderationsphase sollte der Aufgabe „übersektorale Verknüpfung“ Zeit und vor allem personelle Ressourcen zugesprochen werden. Dies unterstützt zugleich das primäre Interesse des Fördermittelgebers, Akzeptanz durch Aufklärung und durch die Ableitung eines konkreten Nutzens für andere Interessengruppen zu schaffen. Ideen und konkrete Projekte zur Gestaltung dieses Nutzens sollten in der Moderationsphase erarbeitet werden.

Fördertitelfokus E+E-Vorhaben: Aufgrund der kurzen Projektlaufzeit von durchschnittlich drei Jahren bedarf die breite Beteiligung einer straffen Organisation. Das Beispiel aus der Region Thüringer Wald \& Schiefergebirge/Frankenwald hat gezeigt, dass dennoch eine breit angelegte Beteiligung aller Interessierten möglich und sinnvoll ist. 


\section{Werkzenge zur Netzwerkarbeit}

Regionalforum: Als ein geeignetes Werkzeug für eine breite Beteiligung bietet sich das Regionalforum an. Damit lässt sich breite Beteiligung initiieren und verstetigen, um die relevanten, aber auch (potenziell) interessierten Akteure über dessen Ziele, Inhalte und Vorgehensweisen zu informieren. Bei räumlich ausgedehnten, länderübergreifenden und neuen Regionszuschnitten sind sie besonders hilfreich, um alle relevanten Akteure anzusprechen ${ }^{204}$. Je nach Projektlaufzeit und Arbeitsphasen sind sie halb- oder einjährig und öffentlich durchzuführen, um über Stand und Entwicklung des Projekts zu informieren und mit den Beteiligten und Interessierten zu diskutieren. Dies trägt zu einer Erhöhung der Transparenz bei und kann sich positiv auf die Akzeptanz des Projekts auswirken. Ein solches Regionalforum kann als ein den Projektprozess strukturierendes Element seitens des Fördermittelgebers vom Projektträger eingefordert werden. Allerdings entstehen durch dieses Beteiligungsinstrument Kosten, die bei der Projektplanung entsprechend zu berücksichtigen sind. So muss beispielsweise überprüft werden, welchen Spielraum die einzelnen Richtlinien für die Finanzierung derartiger Veranstaltungen bieten, oder ob über andere unterstützende Finanzierungsmöglichkeiten nachgedacht werden muss.

\subsubsection{Konstellation: Multiplikatoren}

\section{Problemlagen}

Regionale Multiplikatoren sind für die Übernahme von projektspezifischen Förderzielen als regionale Ziele entscheidend. Je sektoraler ein Förderprojekt ausgerichtet ist, desto wichtiger ist eine Unterstützung durch Multiplikatoren aus anderen Sektoren. Der politische Rückhalt, der in besonderer Weise zur Integration von Interessen erforderlich ist, nimmt mit der Projektdimension (Dauer, Fördermittelumfang, regionale Bedeutung) zu.

\section{Empfehlungen}

Die Gewinnung regionaler Schlüsselakteure ist eine personen- und netzwerkspezifische Aufgabe, die kaum durch äußere Maßnahmen initiiert werden kann. Grundsätzlich müssen sich regionale Akteure in zentralen (Führungs-, Entscheidungs-, Steuerungs-)Positionen darüber bewusst sein, dass sie potentiell eine Multiplika-

204 Dabei kann man sich an den in verschiedenen Planungsprozessen üblichen Bürgerversammlungen und anderen Partizipationsformen, wie dem runden Tisch orientieren. Fürst et al. (2008). 
torposition innerhalb des Projekts einnehmen können. Ihnen fällt die Aufgabe zu, im Rahmen des Projektprozesses sicherzustellen, dass das Projekt während seiner Laufzeit (und auch darüber hinaus) an regional vorhandene Strukturen, wie Planungsgrundlagen, Entwicklungskonzepte oder andere Projekte anknüpft oder mit diesen abgestimmt wird, um Synergien zu erzeugen und Ressourcen effizient zu nutzen.

\section{Werkenenge zur Netzwerkarbeit}

Zur Einbindung von Multiplikatoren sei auf Abschnitt 4.2.6 verwiesen, denn die regionale Steuerungsgruppe - zusammengesetzt aus regionalen Schlüsselakteuren aller relevanten Fachbereiche - dient diesem Zweck.

\subsubsection{Strategie: Lernen}

\section{Problemlagen}

Die Lernfähigkeit der Netzwerke wird in den untersuchten Netzwerken durch mehrere Faktoren behindert. Zum einen fehlt häufig eine Reflexion des Umsetzungsprozesses, die eine Optimierung von Verhandlungsweisen, Beteiligungsstrukturen und Entscheidungsfindungen ermöglichen kann. Darüber hinaus schränkt die Fokussierung der Netzwerkaktivität auf kleinere Ausschnitte der Netzwerke in allen Regionen das regionale Lernen ein.

\section{Empfehlungen}

Lernen im Kontext der Förderprojekte bezieht sich neben der Generierung von fachlichem und fachübergreifendem Wissen auch auf Wissen über die Herausforderungen der Netzwerkarbeit, der Förderspielräume sowie auf Wissen über die vorhandenen regionalen Potentiale. Besonders im Hinblick auf das Leitbild Nachhaltigkeit ist vielfältiges Wissen erforderlich, um die Akteure in die Lage zu versetzen, das Leitbild entsprechend der regionalen Voraussetzungen zu konkretisieren. So konnte aus den Netzwerkanalysen neben der Bedeutung des intraregionalen Austauschs auch der interregionale Austausch als eine sehr wertgeschätzte, jedoch nicht ausreichend ausgeschöpfte Chance herausgefiltert werden. Der stetige Austausch über regionale Erfahrungen mit Fragen zur Förderung kann die Umsetzung erleichtern und dazu beitragen, Probleme schneller zu überwinden.

Da der länderübergreifende Charakter eine Besonderheit am Grünen Band ist, ist ein regelmäßiger Austausch zwischen allen Förderprojekten mit thematisch ähnlichem Bezug entlang des Grünen Bandes zu empfehlen. Dieser sollte frühzeitig sichergestellt und durch die Fördermittelgeber organisiert werden. 
Fördertitelfokus NGP: Der Austausch zwischen Projektträgern der NGP findet bereits auf regelmäßigen Tagungen unter Federführung des BfN statt. Diese Tagungen beschäftigen sich mit Fragen der Naturschutzfachplanungen. Eine Ausweitung des Themenspektrums sollte im Sinne der regionalen Verankerung und des positiven Beitrags der NGP zur regionalen Nachhaltigkeit erfolgen. Der Austausch zu Erfahrungen mit der Moderations- und Umsetzungsphase sowie der Schaffung von Verknüpfungen mit anderen Sektoren, kann das Projektmanagement erleichtern.

Fördertitelfokus E+E-Vorbaben: Die Bedeutung des Austauschs zwischen den Modellregionen wurde von den Akteuren verschiedentlich hervorgehoben. Hier kommt die Besonderheit des untersuchten E+E-Vorhabens mit drei Modellregionen zum Tragen, während andere E+E-Vorhaben meist nur eine Gebietskörperschaft umfassen. Die Unterstützung eines intensiveren Austauschs im Rahmen von E+E-Vorhaben kann jedoch auf Ebene der fünf Förderschwerpunkte der $\mathrm{E}+\mathrm{E}-$ Vorhaben sinnvoll sein.

\section{Werkzenge zur Netzwerkarbeit}

Darstellung von guten und schlechten Beispielen der Netzwerkarbeit. Die Darstellung von guten Beispielen der Netzwerkarbeit sowie ein offener Umgang mit gescheiterten Maßnahmen und Projekten können einen Beitrag zum Lernen auch bezüglich der Herausforderungen der Netzwerkarbeit leisten. Diese Beispiele können intra- aber ebenso auch interregional z.B. auf Tagungen der Projektträger präsentiert und konstruktiv reflektiert werden.

Netzwerktreffen: Regelmäßige Netzwerktreffen erhalten den Dialog aufrecht, verstetigen Kontakte und fördern Vertrauen. Während der Projektlaufzeit können solche Netzwerktreffen zum gezielten Austausch über das Projekt und eventuelle Anknüpfungspunkte mit anderen Projekten dienen. Nach Abschluss des jeweiligen Förderprojekts sollte aber auch gewährleistet sein, dass diese Treffen weiter stattfinden, um die erreichten Lerneffekte weiter zu fördern. Diese können zum Beispiel in Form von Arbeitsgruppen ausgestaltet werden, oder aber, einfacher gehalten, als eine Form des „Stammtisches“. Diese lose Form von Treffen ermöglicht eine hohe Flexibilität bezüglich ihrer Zusammensetzung und inhaltlichen Ausrichtung, birgt aber darin auch die Gefahr, dass sie als unnütz und belastend seitens der Akteure wahrgenommen wird. Wichtig ist daher bei derartigen Treffen, dass sie immer eine gewisse Zielorientierung beinhalten und am Ende ein Ergebnis (z.B. in Form kleiner Arbeitsaufträge) aufweisen können. Zudem muss es bei solchen Treffen einen oder mehrere sich verantwortlich Fühlende geben, die die Organisation dieser Treffen in die Hand nehmen. In besonders großräumigen Regionen könnten über ein Rotationsverfahren und der regelmäßigen Weitergabe der Verantwortung die Überlastung einzelner Akteure verringert werden. 
Moderierter Abschlussworkshop: Ein großer moderierter Abschlussworkshop des Projekts mit einem veröffentlichten Protokoll kann der Region als Wegweiser für zukünftige Projekte dienen, indem auf gesammelten Erfahrungen aufgebaut werden kann.

\subsubsection{Strategie: Transparenz, Offenheit, Flexibilität}

\section{Problemlagen}

Festzustellen ist, dass Akteure in den Netzwerken mit Erwartungen an das Projekt herangingen, die auf Grundlage der Förderrichtlinie nicht realisierbar sind. In Folge war es zeitaufwendig, Gestaltungsspielräume abzustecken. Je höher die Fördersumme, desto eher werden Erwartungen geweckt, die über die Förderziele hinaus reichen. Enttäuschte regionale Erwartungen aufgrund begrenzter und unflexibler Förderspielräume bergen die Gefahr des Akzeptanz- oder Motivationsverlusts gegenüber der Förderung. Mangelnde Transparenz einiger Entscheidungsprozesse in E+E sowie NGP Regionen verstärken diese Gefahr zusätzlich.

Die Herstellung echter Offenheit des Netzwerks, die die Einbindung neuer Akteure zu jeder Zeit in das Netzwerk erlaubt, ist eine zentrale Herausforderung und notwendig, um die Projektdynamik und regionale Verankerung zu fördern.

\section{Empfehlungen}

Die netzwerkinterne Kommunikation bedarf einer Regelmäßigkeit und Dauerhaftigkeit, die zum Beispiel über regelmäßige Veranstaltungen erreicht werden kann. Transparenz und Offenheit zu schaffen, liegt in der Verantwortung des Projektträgers, jedoch in gleicher Weise auch beim Fördermittelgeber. Dabei ist wichtig, den regionalen Akteuren aufzuzeigen, ob und wie sie mit ihren (regionalen) Zielvorstellungen und Ideen an die Förderung anknüpfen können. Je komplexer ein Projekt konzipiert ist, desto schwieriger ist es, dieses transparent und nachvollziehbar darzustellen. Genau dies ist jedoch vom Projektträger zu leisten, um aus Nachhaltigkeitsperspektive eine erfolgreiche Umsetzung des Projekts realisieren zu können. Der Fördermittelgeber und Projektträger können gemeinsam durch frühzeitige Informationsveranstaltungen über die Grenzen und Möglichkeiten der Förderung sowie regionale Anknüpfungsmöglichkeiten vor Ort aufklären, um so regionale Akteure in die Lage zu versetzen, andere Förderprojekte entsprechend einzubinden und das Projekt mit regionalen Initiativen zu flankieren. Eine aktive Aufklärungsarbeit in Verbindung mit einer zielgruppenspezifischen Öffentlichkeitsarbeit, die berücksichtigt, dass die Sprache der Fachexperten nicht der Sprache der Öffentlichkeit entspricht, ist ein Schlüsselfaktor für die regionale Verankerung und positive Wahrnehmung des Naturschutzes. 
Die Offenheit des Projektnetzwerks muss der Projektträger aktiv gestalten. Die passive Haltung nach dem Prinzip ,jeder kann bei uns mitmachen“, reicht nicht aus, um Mitstreiter zu gewinnen. Im Sinne der Effizienz ist zudem auf die Holschuld der beteiligten Akteure zu verweisen. Sie müssen auch eigeninitiativ beim Projektträger Beteiligungsmöglichkeiten und Informationen einfordern.

\section{Werkzenge zur Netzwerkarbeit}

Regionalforum und Auftaktworkshop: Das regelmäßige Regionalforum (s. 4.2.1) kann zu einer angemessenen Transparenz im Projekt beitragen. Der regionale Auftaktworkshop in der Untersuchungsregion Thüringer Wald \& Schiefergebirge/Frankenwald hat sich dabei als probates Mittel erwiesen, um Offenheit und Transparenz gleich zum Projektstart herzustellen.

Internetplattform: Moderne Medien eignen sich bei professionellem Einsatz zur effektiven und kontinuierlichen Gestaltung von Information und Kommunikation in Projektprozessen. Es gäbe hier die Möglichkeiten,

a) eine Internetplattform zur öffentlichen Bereitstellung von prozessbegleitenden Informationen zu nutzen, und/oder

b) die Plattform zusätzlich als Forum zum Austausch zwischen den Projektbeteiligten zu verwenden.

Option a) stellt eine Informationsplattform für den interessierten Akteur dar. Benötigte Informationen stehen bereit und sind jederzeit zugänglich. Der Vorteil liegt in der zeitlichen Unabhängigkeit der Informationsbereitstellung und in der Dauerhaftigkeit der Informationenverfügbarkeit. Ggf. kann eine solche „one-way“ Plattform durch einen geschützten Bereich ergänzt werden, um sensiblere Informationen den Projektpartnern zur Verfügung zu stellen, ohne sie für die breite Öffentlichkeit zu öffnen. Der Fördermittelgeber kann auf die Gestaltung einer Internetplattform hinwirken, indem er fordert, dass sie bereits zu Beginn des Projekts zur Verfügung steht.

Option b) geht über die einseitige Informationsstreuung hinaus, da die Plattform den Austausch zwischen Interessierten ermöglichen soll und ist wesentlich aufwändiger in der Betreuung.

Die untersuchten Projektregionen zeigen, dass eine regionalspezifische Entscheidung sinnvoll ist, denn die Akteure wissen, welche Informations- oder Kommunikationsinstrumente genutzt werden. 


\subsubsection{Strategie: Kooperation und win-win}

\section{Problemlagen}

Sektorale Projekte wie NGP können mit „Trittbrettfahrerverhalten“ konfrontiert werden. So können regionale Institutionen mit den Leistungen des Naturschutzgroßprojekts wie der Herstellung und Erhaltung einer intakten Landschaft werben und dadurch mehr Besucher oder Investoren anlocken, ohne dass sie aber im Gegenzug einen angemessenen Beitrag zur Umweltbildung, Aufklärung oder Öffentlichkeitsarbeit für das Projekt leisten.

\section{Empfehlungen}

Hier muss vor allem der Fördermittelempfänger darauf achten, Möglichkeiten für derartiges Verhalten rechtzeitig zu erkennen und die entsprechenden Institutionen als Partner in das Netzwerk einzubinden, um Kooperationen vereinbaren zu können. Diese können insbesondere im Bereich der Öffentlichkeitsarbeit erfolgen.

\subsubsection{Strategie: Arbeiten auf verschiedenen Ebenen}

\section{Problemlagen}

In allen vier Untersuchungsregionen zeigt sich die Herausforderung, in einem neuen Regionsgebilde, das kreis- und darüber hinaus länderübergreifend aus Teilen bereits verfasster Regionen besteht, eine leistungsfähige Arbeitsstruktur auf verschiedenen Ebenen (Projektebene und regionale Ebene, aber auch Umsetzungs-, Diskussions- und Entscheidungsebene) aufzubauen. Aufgrund der Gebietsgrößen verstärkt sich die Herausforderung, die Projekte „über die Fläche hinweg“ zu verankern. In den Projektregionen haben sich dafür unterschiedliche Strukturen gebildet, wie in den entsprechenden Abschnitten (vgl. Abschnitt 3.2.2.2, Abschnitt 3.3.2.2, Abschnitt 3.4.2.2) dargestellt ist.

\section{Empfehlungen}

Projektträger, die über wenige Erfahrungen mit Projekten von regionaler Bedeutung verfügen, können bereits mit der Schaffung eines Bewusstseins für die organisatorische Herausforderung in der Phase der Beantragung unterstützt werden. So kann dem Mehrebenenproblem bereits im Antrag eine Lösung zugeführt werden. 


\section{Werkzenge zur Netziwerkarbeit}

Thematische Arbeitsgruppen: Thematische Arbeitsgruppen bieten sich bei der betrachteten Projektgebietsgröße an, um die inhaltliche Komplexität sowie die Akteursvielfalt in einen organisatorisch handhabbaren Rahmen zu fügen. Dies kann nur mit Hilfe regionaler Eigeninitiative gelingen, denn regionale Akteure müssen sich für die Organisation und Arbeit dieser thematisch ausgerichteten Gruppen einsetzen. Die thematischen Arbeitsgruppen sind als Ergänzung bzw. Vertiefung und Verstetigung der Arbeit der PAG oder Steuerungsgruppe zu verstehen, die nicht immer den Raum für übersektorale Verknüpfungen und flankierende Projektentwicklung aufweisen. Die Verantwortung für die regionale Vernetzung liegt in hohem Maße bei den Arbeitsgruppen, da sie vom Projektträger allein nicht zu bewältigen ist.

Steuerungsgruppe: Übergreifend über alle Arbeitsgruppen ist eine sektoren- und je nach Projektregion auch länderübergreifende Steuerungsgruppe mit den entsprechenden Entscheidungsbefugnissen einzurichten. Durch sie wird ein Ausgleich unterschiedlicher Interessen im Sinne der Nachhaltigkeit auf Projektebene sichergestellt. Im NGP Grünes Band Eichsfeld-Werratal wäre der „Regionale Projektbeirat" in dieser Funktion zu sehen.

„Regionaler Nachhaltigkeitsbeirat": Die Sicherstellung des Interessenausgleichs und einer konsistenten Entwicklung im Sinne der Nachhaltigkeit auf regionaler Ebene steht vor der Herausforderung des mit jedem neuen Förderprojekt neu entstehenden Regionszuschnitts. Selten decken unterschiedliche Projekte das gleiche Gebiet ab, wodurch eine konsistent regionale Entwicklung erschwert wird. Nichtsdestotrotz ist eine projektbezogene Zusammenarbeit in unterschiedlichen räumlichen Kontexten sinnvoll bzw. notwendig (Beispiel Grünes Band). Die Landkreise sind jedoch für eine konsistente Steuerung verantwortlich, um die Zersplitterung der Entwicklung zu vermeiden. Um diese Steuerung zu unterstützen, könnte ein „Nachhaltigkeitsbeirat“ auf Landkreisebene benannt werden. Aus allen relevanten Fachbereichen würden Schlüsselakteure zur Verfügung stehen, die sich dann als Beraterinnen und Berater in den Förderprojekten des jeweiligen Landkreises einbringen.

Sie beraten bei der Auswahl und begleiten die regionale Verankerung der Förderprojekte kurz- und langfristig. Die Beraterinnen und Berater nehmen einen fachlichen und übergeordneten Blick auf das Kreisgebiet ein und ermöglichen aus der Nachhaltigkeitsperspektive ${ }^{205}$ eine strategische Ausrichtung der Projekte und

205 Beispiele hierzu auf Landesebene: http://www.nachhaltigkeitsbeirat.brandenburg.de; http://www.nachhaltigkeitsbeirat-thueringen.de (Zugriff: 15.01.2010) 
des Kreises. Zudem können sie den Fördermittelempfänger bei der Erstellung der Nachhaltigkeitsspinne unterstützen.

Der „Nachhaltigkeitsbeirat“ kann wie folgt eingebunden werden: Eine (aktive bzw. passive) Vorschlagsliste benennt jene regionalen Akteure unterschiedlicher Sektoren, die als potentielle Berater zur Verfügung stehen. Der Projektträger benennt im Einvernehmen mit den Landkreisen jeweils die Vertreter der Sektoren Ökonomie, Soziales und Ökologie für die Beratung in dem jeweils zentralen Projektgremium (Steuerungsgruppe oder Regionaler Projektbeirat). Wenn nun ein Ausschnitt eines Landkreises im Projektgebiet liegt, werden die regionalen Experten aus den Landkreisen in das jeweilige Projektgremium eingebunden und so die strategische Orientierung des Projekts sichergestellt. So erscheint es möglich, dass dauerhaft Synergien zwischen unterschiedlichen Förderstrukturen erzeugt werden und die unterschiedlichen Förderprojekte ihren Beitrag zur regionalen Nachhaltigkeit leisten können.

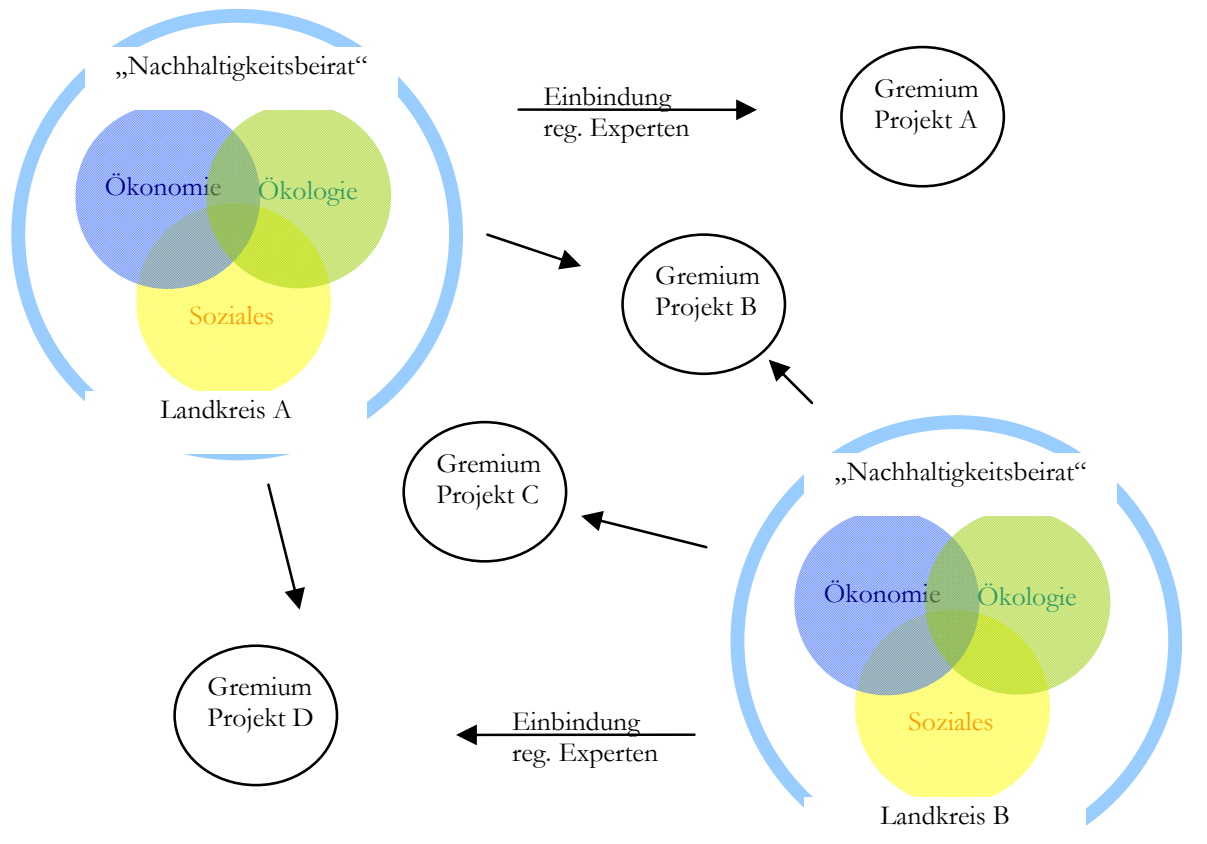

Abbildung 41: Der „Nachbaltigkeitsbeirat“ und seine Einbindung in die unterschiedlichen Förderprojekte 


\subsubsection{Instrumente: Leitbild, Visionen und Ziele}

\section{Problemlage}

In den untersuchten Regionen zeigen sich ganz unterschiedliche Qualitäten und Wirkungen von Leitbildern, wobei in den E+E-Regionen, in denen die Leitbilder bekannt sind, sie als wichtig für die gemeinsame Orientierung der Akteure aus unterschiedlichen Funktionsbereichen eingeschätzt werden.

Im NGP Schaalsee Landschaft hat das Projektleitbild lediglich für die an der Pflege- und Entwicklungsplanung beteiligten Akteure eine Bedeutung, jedoch entfaltet es auch hier über die Jahre der Laufzeit eine recht geringe handlungsleitende Wirkung. Eine formale Integration der Naturschutzziele bzw. des NGP in Entwicklungsprogramme wurde vorgenommen, ohne jedoch in der Projektpraxis zu integrativen Verknüpfungen geführt zu haben.

Die betrachteten Fördertitel erstrecken sich über eine relativ kurze (2-3 Jahre) oder sehr lange (mehr als 10 Jahre) Laufzeit. Zum einen ist kaum Zeit für einen intensiven Leitbildprozess vorhanden, zum anderen stehen generell sehr wenige Ressourcen für derartige integrative Prozesse zur Verfügung.

\section{Empfeblungen}

Das Leitbild sollte entsprechend der drei Säulen der Nachhaltigkeit dreidimensional ausgerichtet sein und Anknüpfungspunkte zu den Handlungsfeldern der Dimensionen aufzeigen. Hier kann auch das Instrument der Nachhaltigkeitsspinne Verwendung finden.

Die Leitbilder sollten an regionalen Leitbildern der Regionalentwicklung andocken. Die Zusammenhänge sollten klar herausgestellt werden. Im besten Falle konkretisiert das Projekt-Leitbild das regionale (Nachhaltigkeits-)Leitbild in den Förderzielen.

Fördertitelfokus NGP: Das Leitbild von Naturschutzgroßprojekten ist auf naturschutzfachliche Aspekte ausgerichtet. Dieses Leitbild kann regionale Leitbilder in Handlungsfeldern, wie „Natur und Landschaft", weiter konkretisieren. Diese Konkretisierung ist bereits in der Antragsphase, spätestens in der Moderationsphase zu bedenken.

Fördertitelfokus E+E: Ähnlich, wie in den NGP, haben Leitbilder in E+EVorhaben ihren Schwerpunkt im naturschutzfachlichen Bereich. Jedoch ist der Spielraum größer, als es bei Naturschutzgroßprojekten der Fall ist. So können Projekte, die „naturschutzgerechte Regionalentwicklung anstoßen“206, durchgeführt werden. Deswegen und wegen der geringen Laufzeit ist ein klarer Bezug zu regional vorhandenen Leitbildern notwendig. In der Voruntersuchung, die in je-

206 http://www.bfn.de/0202_eue.html (letzter Zugriff 03.05.2010) 
dem E+E-Vorhaben optional durchgeführt werden kann, sollte geprüft werden, in welchen Bereichen das Projekt an regionale Leitbilder anschließt.

\subsubsection{Instrumente: Prozessmanagement}

\section{Problemlagen}

Bezüglich des Prozessmanagements ist die Aufgabenvielfalt anzuerkennen, die ein Projektträger zu bewältigen hat. Kurz gefasst steht er vor der Herausforderung, die Förderziele mit entsprechenden Maßnahmen zu erreichen, das Projekt richtlinienkonform inhaltlich und administrativ abzuwickeln, gleichzeitig jedoch auch regionale, gelegentlich divergierende Interessen in die Maßnahmen zu integrieren und als eigenständiger regionaler Akteur seine Position wahrzunehmen.

\section{Empfehlungen}

Eine Möglichkeit, der Aufgabenvielfalt zu begegnen, kann die stärkere Differenzierung der Aufgabenschwerpunkte in den verschiedenen Projektphasen sein. Über eine angemessene Ressourcenzuweisung sollte der Stellenwert und die Bedeutung einzelner Aufgaben, z, B. der Öffentlichkeitsarbeit, bereits durch die Förderrichtlinien geregelt werden.

Außerdem erscheint es notwendig, den Projektträger durch die Vermittlung von Wissen über die Herausforderungen und Möglichkeiten in der Netzwerkarbeit zu unterstützen und zu qualifizieren.

Fördertitelfokus NGP: Die Untersuchungsregion Schaalsee-Landschaft hat gezeigt, dass es für die Umsetzung des NGP als langfristiges regionales Lernprojekt bzgl. der Gestaltung der Nachhaltigkeit, sinnvoll wäre, die externe Moderation über die zwei- oder dreijährige erste Förderphase hinaus bedarfsbezogen einsetzen zu können. Konfliktbearbeitungen sind auch nach der Erstellung des Pflege- und Entwicklungsplans notwendig, da sich Rahmenbedingung oder Akteurskonstellationen verändern können. Die bereits in Abschnitt 4.2.1 und Abschnitt 4.2.3 empfohlenen Regionalforen oder Abschlussworkshops bedürften ebenfalls einer externen Moderation, da der Projektträger zu positioniert im Netzwerk ist, um ohne eigene Agenda derartige Foren zu moderieren. Die Öffentlichkeitsarbeit im Rahmen des NGP ist ein wichtiges Element zur Akzeptanzförderung, so dass eine angemessene Mittelausstattung zu gewährleisten ist. 


\section{Werkzenge zur Netzwerkarbeit}

Netzperkleitfaden: Der Fördermittelgeber kann den Projektträger im Prozessmanagement durch die Erstellung eines Leitfadens zur Netzwerkarbeit unterstützen, in dem bspw. andere Projekte desselben Fördertitels ihre Erfahrungen darstellen und Hinweise geben, worauf zu achten ist. Inhalte dieses Leitfadens wären unter anderem:

- die unterschiedlichen Möglichkeiten zur Gestaltung der Organisationsstruktur des Projektnetzwerks inklusive ihrer Vor- und Nachteile

- die Gestaltung der Öffentlichkeitsarbeit, um Erfahrungen, bspw. zum notwendigen Aufwand, auszutauschen,

- welche Themen die Auftakt- bzw. Abschlussveranstaltungen beinhalten kann

- wie etwaige Reflexionsphasen zur Netzwerkarbeit gestaltet und in den Projektprozess sinnvoll eingebunden werden können.

Diese Liste an Themen sollte je nach Fördertitel entsprechend ausgestaltet werden.

\subsubsection{Perspektiven der Umsetzung}

Der Erfolg der vorgestellten Werkzeuge hängt stark davon ab, dass jede Ebene ihrer jeweiligen Verantwortung nachkommt. Wie die Wahrnehmung der jeweiligen Verantwortung aussehen könnte, ist in der nachfolgenden Tabelle 45 dargestellt. Zudem zeigt sie in der Übersicht, welches Netzwerkmerkmal vom jeweiligen Instrument bedient wird und welcher strategischen Qualität das Instrument zuzuordnen ist. Die meisten Instrumente sind der strategischen Qualität „diskursiv“ zuzuordnen. In der Anwendung besteht die Perspektive, dass die strategische Qualität „,integrativ“ sekundär bedient wird. 
Tabelle 45: Werkezenge für Netzwerkarbeit: Zuständigkeiten, bediente Merkmale und strategische Qualitäten

\begin{tabular}{|c|c|c|c|c|c|c|}
\hline 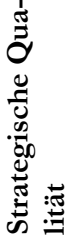 & 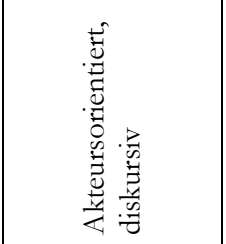 & 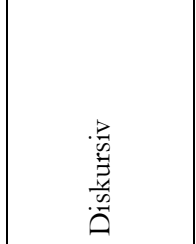 & 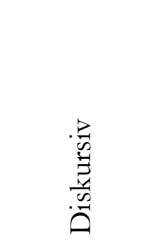 & & 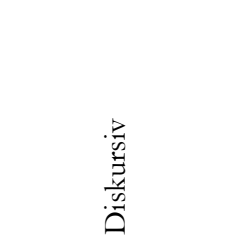 & 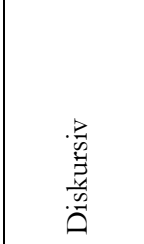 \\
\hline 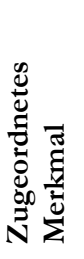 & 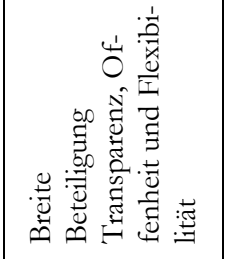 & 氖 & 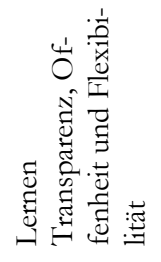 & 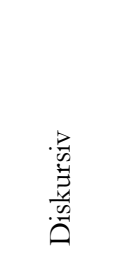 & 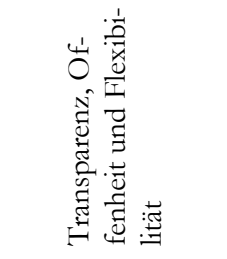 & 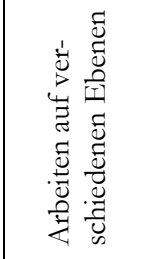 \\
\hline 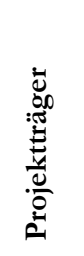 & 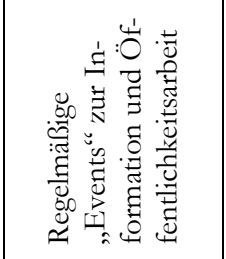 & & 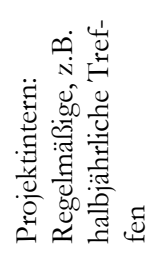 & 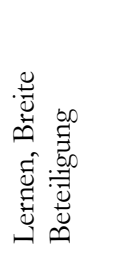 & 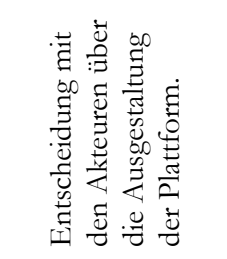 & 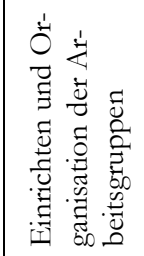 \\
\hline 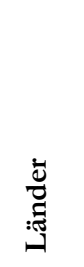 & 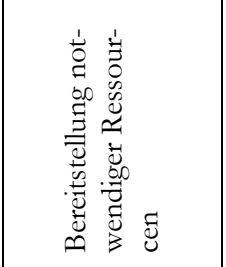 & 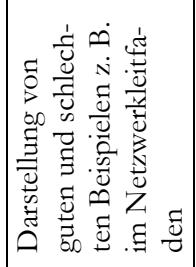 & 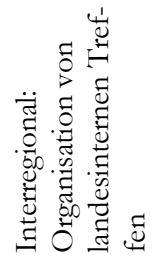 & 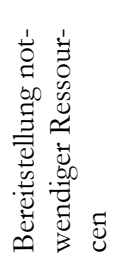 & 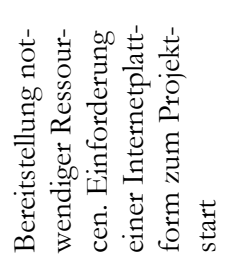 & 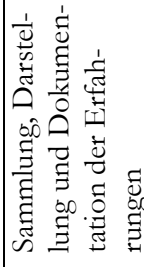 \\
\hline$\underset{\mathscr{n}}{\vec{E}}$ & 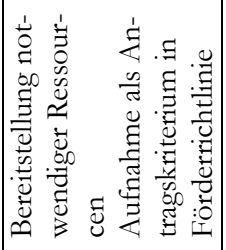 & 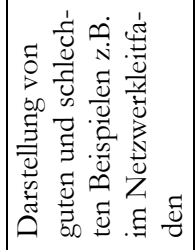 & 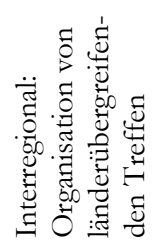 & 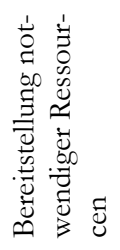 & 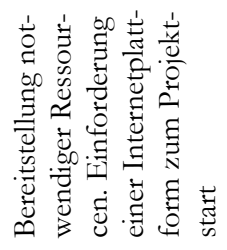 & \\
\hline 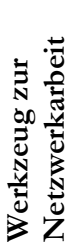 & 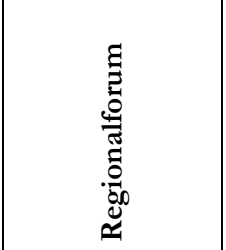 & 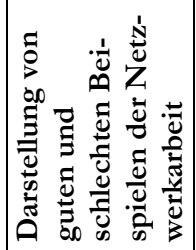 & 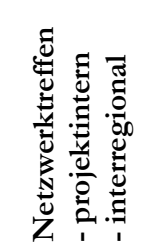 & 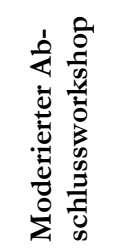 & 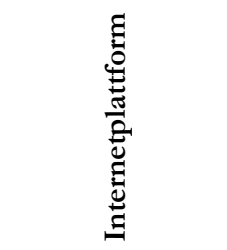 & 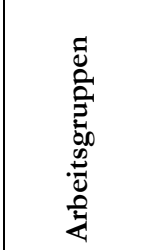 \\
\hline
\end{tabular}




\begin{tabular}{|c|c|c|c|c|}
\hline 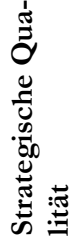 & 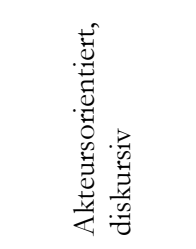 & \multirow{5}{*}{ 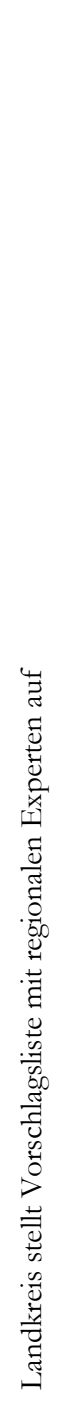 } & 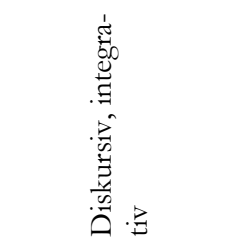 & 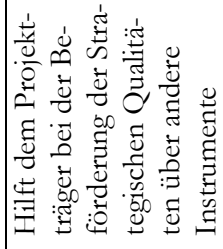 \\
\hline 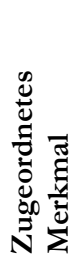 & 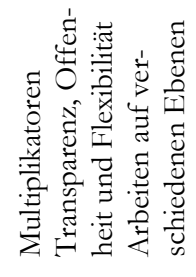 & & 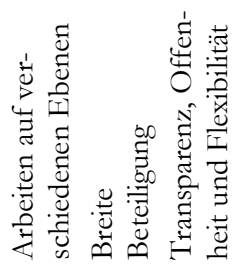 & 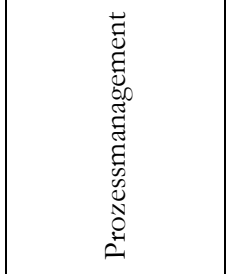 \\
\hline 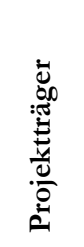 & \multirow[t]{3}{*}{ 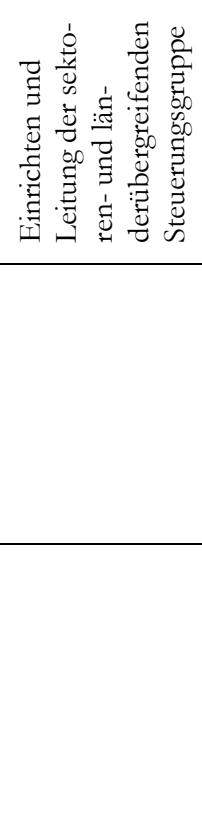 } & & \multirow[b]{2}{*}{ 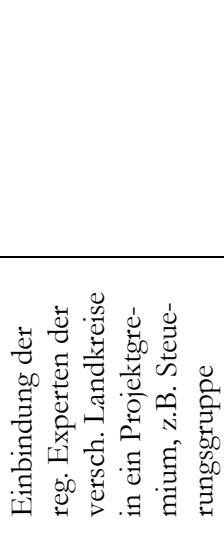 } & 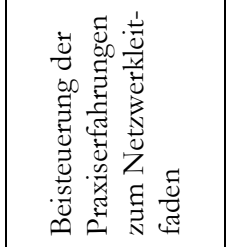 \\
\hline 离 & & & & 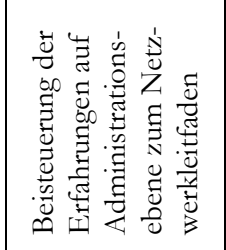 \\
\hline$\underset{\tilde{D}}{\widetilde{D}}$ & & & & 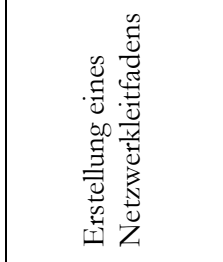 \\
\hline 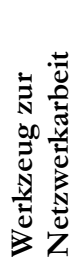 & 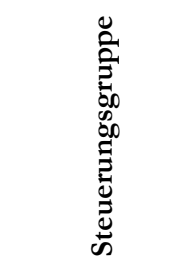 & & 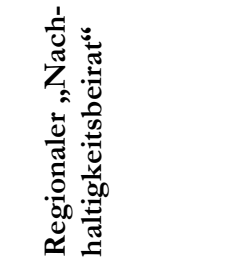 & 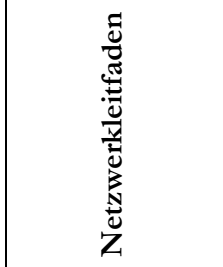 \\
\hline
\end{tabular}




\subsection{Regionale Nachhaltigkeit organisieren}

Die Auswirkungen von regionalen Aktivitäten und Maßnahmen auf Nachhaltigkeit zu kennen, ist Voraussetzung dafür, regionale nachhaltige Entwicklung gestalten zu können. Die Gestaltung von regionaler Nachhaltigkeit erfordert die Integration verschiedener Perspektiven (statisch und dynamisch) durch ein Managementkonzept. ${ }^{207}$ Das Managementkonzept der bundesnationalen Nachhaltigkeitsstrategie mit seinen drei Komponenten Managementregeln, Nachhaltigkeitsthementableau und begleitender Dokumentation ist Vorbild für ein Managementkonzept von Nachhaltigkeitsprozessen auf regionaler Ebene. Wie in der Einführung kurz dargelegt, wird der Begriff der Nachhaltigkeit in verschiedenen Gebrauchskategorien verwendet, die jeweils einen unterschiedlichen Grad an inhaltlicher Bestimmtheit aufweisen.

Der in Kapitel 2 beschriebene Untersuchungsansatz der Maßnahmenperspektive beschreibt die Nutzung eines Mehrkomponenten-Managementkonzeptes für die Ebene der Regionen. In Kapitel 3 wurden die dafür notwendigen Schritte für die vier im Rahmen dieses Projektes betrachteten Modellregionen durchgeführt. Diese Erörterungen sind Grundlage für die Ableitung von Perspektiven und Empfehlungen zur Nutzung eines Nachhaltigkeitsmanagementkonzeptes im Kontext von Naturschutzfördertiteln. Die folgenden vier Thesen formulieren Anhaltspunkte für Anforderungen an das Managementkonzept, durch welches der Beitrag von Naturschutzfördertiteln zur nachhaltigen Regionalentwicklung sichtbar gemacht und das Wirkungspotenzial der Fördertitel für eine nachhaltige regionale Entwicklung effektiv genutzt werden kann:

1. Nachhaltige Entwicklung kann nicht organisiert werden ohne regionale Ausdifferenzierung des Nachhaltigkeitsbegriffes (z.B. in Form eines regionalen Nachhaltigkeitsthementableaus).

2. Nachhaltige Entwicklung kann nicht organisiert werden ohne bestimmte Ausprägungsformen von regionalen Verhaltensweisen.

3. Nachhaltige Entwicklung kann nicht organisiert werden ohne das Vorhandensein von regionalen Nachhaltigkeitsinstitutionen.

4. Nachhaltige Entwicklung kann nicht organisiert werden ohne begleitende Dokumentation.

Ausgehend von diesen vier Thesen umfassen auch die Empfehlungen vier Schritte, die die Übersetzung der Projektergebnisse in die Anwendung vornehmen.

1. Entwicklung eines regionalen Nachhaltigkeitsthementableaus sowie Überführung in eine Maßnahmenbasismatrix und Nachhaltigkeitsspinne

207 Vgl. Die Bundesregierung (2008) 
Nachhaltigkeit auf regionaler Ebene wird häufig durch so genannte bottom-upProzesse inhaltlich ausgestaltet. Dies führt dazu, dass sich auf regionaler Ebene eine Vielzahl von unterschiedlichen Interpretationen des Nachhaltigkeitsbegriffes herausbildet. Daher ist das Wirkungspotenzial von Fördertiteln immer nur vor dem Hintergrund des Nachhaltigkeitsverständnisses der jeweiligen (Modell)Region zu betrachten.

\section{Identifizieren von regionalen Managementregeln}

Managementregeln repräsentieren die notwendigen Verhaltensweisen für eine erfolgreiche nachhaltige Entwicklung in einer Region. Ferner stellen sie im gewählten Untersuchungsansatz die Brücke von der statischen Maßnahmenperspektive zur dynamischen Netzwerkperspektive dar. Denn nur entsprechend nachhaltige Verhaltensweisen innerhalb der Projektnetzwerkstruktur führen auch zu nachhaltigen Projektmaßnahmen und deren Umsetzung. Netzwerkanalysen können hierzu als Instrument zur Ermittlung des jeweiligen regionalen Standards dienen, so dass auch bereits im Vorfeld der Projektbewilligung ersichtlich wird, wie weit regionale Verhaltensweisen nachhaltig ausgeprägt sind bzw. der Anpassung bedürfen. Das Gleiche gilt für die Nacherhebung, die eine Aussage über die Entwicklung regionaler Nachhaltigkeitsmanagementregeln zulässt und somit ein Indiz über eine nachhaltige Umsetzung der Projektmaßnahmen liefert.

\section{Identifizieren von regionalen Nachhaltigkeitsinstitutionen}

Neben der Konzentration auf die reine Projektumsetzung besteht die Notwendigkeit einer langfristigen Verankerung der Projekte in den jeweiligen Modellregionen. E+E-Projekte und Naturschutzgroßprojekte besitzen in der Regel nur einen begrenzten Förderzeitraum, was dazu führen kann, dass nach dem Auslaufen der Förderung die erreichten Ziele wieder auf die Ausgangssituation zurückfallen und auch die erarbeiteten Netzwerkstrukturen nicht erhalten werden können. Um dies zu verhindern, erscheint es sinnvoll, Anschlussmöglichkeiten für die jeweiligen Projektformen am Grünen Band zu finden. Um selbst tragende sowie nachhaltiglangfristige Strukturen für beide Projektformen zu etablieren, sind die bereits bestehenden Nachhaltigkeitsstrukturen jeder Region in die Projektplanung zu integrieren. In diesem Zusammenhang hat sich gezeigt, dass oftmals bereits etablierte Nachhaltigkeitsinstitutionen existieren, zumeist jedoch unter einem anderen Leitbild als dem der regionalen Nachhaltigkeit. Dies hängt grundsätzlich damit zusammen, dass in den Regionen Nachhaltigkeit schon immer eine wesentliche Rolle spielte, jedoch selten in einer so expliziten Form, wie im Rahmen des vorliegenden Projektes. Damit lässt sich die durchgeführte Erhebung regionaler Nachhaltigkeitsinstitutionen wiederum als Konkretisierung von impliziten Tatbeständen charakterisieren. Es ist notwendig, die entsprechenden Förderprojekte in Hinblick auf diese Institutionen auszurichten, um selbst tragende und nachhaltiglangfristige Strukturen zu nutzen. 
4. Nachhaltige Entwicklung begleitend dokumentieren

Zusätzlich besteht die Notwendigkeit einer entsprechenden Dokumentation der vollzogenen Entwicklungsschritte. Das ist wichtig, damit der Fördermittelgeber einen Nachweis für die Entwicklung in den einzelnen Punkten erhält und damit gegebenenfalls eine nachhaltige Ausrichtung der Projekte gezielt steuern kann. Außerdem liefert eine begleitende Dokumentation der Region transparent Hinweise, in welchem Entwicklungsstadium regionaler Nachhaltigkeit sie sich befindet und an welchen Stellen sie unter Umständen ihr Vorgehen anpassen muss. Damit kann die Nachhaltigkeitsdokumentation, unabhängig von ihrer Darstellungsform, als Erfolgskontrolle für eine nachhaltige Entwicklung interpretiert werden. Dies gilt gleichermaßen für Projektmaßnahmen und Netzwerkarbeit aber vor allem auch für das Zusammenspiel beider Komponenten.

Zur Durchführung der vier Schritte kann eine Arbeitshilfe nützlich sein, die einfach und ohne großen Zeitaufwand nutzbar ist. Hier sind verschiedene Möglichkeiten denkbar, vom einfachen Arbeitsblatt bis hin zu einem programmierten Software-Tool. Die Grundidee bleibt jedoch dieselbe: Unterstützung bei der Durchführung der vier obigen Schritte, damit regionale Nachhaltigkeit beim Vollzug von Fördertiteln gleichzeitig mit gestaltet werden kann. Exemplarisch lässt sich dies an der Arbeitshilfe „Arbeitsblatt“ veranschaulichen (siehe Abbildung 42).

Abbildung 42: Arbeitshilfe für die Maßnabmenebene

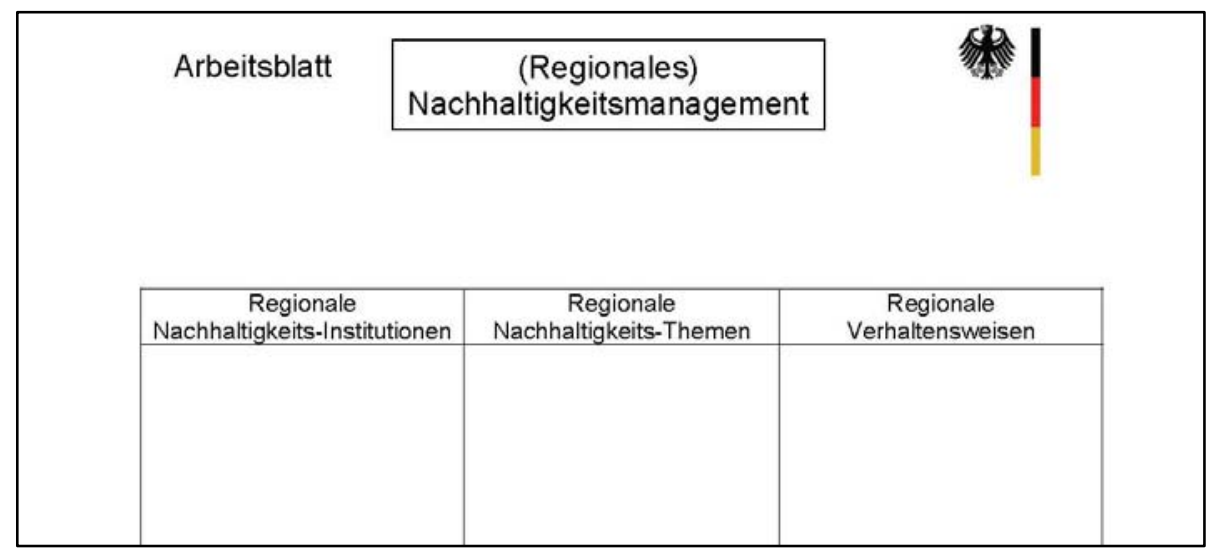

Das Arbeitsblatt besteht aus drei Spalten. Die mittlere Spalte umfasst das regionale Nachhaltigkeitsverständnis. Hier sollen die Regionalen Nachhaltigkeits-Themen, die den jeweiligen regionalen Nachhaltigkeitsbegriff ausdifferenzieren, dokumentiert werden. Um einen Eindruck vom Wirkungspotenzial des betrachteten Fördertitels vor dem Hintergrund der jeweiligen thematischen Ausdifferenzierung zu bekommen, ist die mittlere Spalte des Arbeitsblattes zu einer so genannten Maß- 
nahmenbasismatrix zu erweitern. Dazu werden die Themenfelder der regionalen Nachhaltigkeit (mittlere Spalte des Arbeitsblattes) in Beziehung gesetzt zu den Maßnahmen, die das jeweilige E+E-Vorhaben und NGP ausmachen. Wie in Kapitel 2 beschrieben muss das Wirkungspotenzial der Maßnahmen im Hinblick auf die Themenfelder abgeschätzt werden. Das Gesamtwirkungspotenzial des E+EVorhabens oder des Naturschutzgroßprojekts in seiner Verteilungswirkung über die Themenfelder des jeweiligen, regionalen Nachhaltigkeitsverständnisses wird sichtbar (Schritt 1).

Im Fortgang soll eine Verbindung von nachhaltigkeitsrelevanten Themenfeldern mit Verhaltensweisen erfolgen, die eine entsprechend günstige Entwicklung im jeweiligen Themenfeld herbeiführen können. Diese Verhaltensweisen werden als regionale Managementregeln bezeichnet und können einen „Soll-Zustand“ definieren. Die themenfeldspezifische Zuordnung von regionalen Managementregeln schlägt eine Brücke zwischen der Betrachtung regionaler Nachhaltigkeit in statischer Perspektive und dynamischer Perspektive. Diese Brücke ist in zweierlei Hinsicht zu nutzen: Zum einen erhält jedes Themenfeld eine regionale Managementregel, die deutlich macht, wie sich die Akteure im jeweiligen Projektnetzwerk verhalten sollen. Zum anderen kann diese Zuordnung auch zur Ergründung des Ist-Zustandes verwendet werden. Immer dann, wenn themenfeldspezifisch bereits Verhaltensweisen praktiziert werden, werden diese an entsprechender Stelle vermerkt, wodurch Lücken bei den Verhaltensweisen sichtbar werden (Schritt 2).

$\mathrm{Da}$ in diesem Projekt Fördertitel und ihr Wirkungspotenzial auf nachhaltige, regionale Entwicklung untersucht werden, muss der Aspekt der Befristung von Förderzeiträumen ebenfalls Berücksichtigung finden. Der Beitrag von E+EVorhaben und NGP zur Organisation von regionaler Nachhaltigkeit wäre als relativ geringer einzuschätzen, wenn die im Förderzeitraum initiierten Prozesse und Maßnahmen zeitlich ausschließlich an den Förderzeitraum gebunden wären. Das Ziel der Fördertitel, eine Impulsfunktion auszuüben, kann im Sinne einer nachhaltigen regionalen Entwicklung nur dann als erreicht gelten, wenn die im Förderzeitraum initiierten Prozesse und umgesetzten Maßnahmen auch über den Förderzeitraum hinaus wirksam sind und somit Förderzeitraum und Wirkungspotenzial entkoppelt werden. Dazu sind Institutionen zu identifizieren, die zur Fortführung der Prozesse und Maßnahmen in Frage kommen. Diese müssen jeweils einen inhaltlichen Bezug aufweisen zu den Themenfeldern der regionalen Nachhaltigkeit, in denen die E+E-Vorhaben sowie die Naturschutzgroßprojekte Wirkungspotenzial entfalten. Durch den Abgleich korrespondierender Institutionen zu entsprechenden Themenfeldern kann bei frühzeitiger Durchführung ein Handlungsbedarf identifiziert werden, um die Wahrscheinlichkeit der Fortführung von Prozessen und Maßnahmen über den Förderzeitraum hinaus zu erhöhen (Schritt 3). Durch die wiederholte Anwendung der Schritte 1 bis 3 kann der Weg einer nachhaltigen Regionalentwicklung dokumentiert und damit transparent und nachvollziehbar gemacht werden (Schritt 4). In der Zusammenschau der Schritte 1 bis 
4 entsteht ein Managementkonzept, welches es ermöglicht, eine nachhaltige regionale Entwicklung effektiv mit zu gestalten bei der Durchführung von Naturschutzfördertiteln (siehe Abbildung 43).

\begin{tabular}{|c|c|c|}
\hline Arbeitsblatt & $\begin{array}{c}\text { (Regionales) } \\
\text { Nachhaltigkeitsmanagement }\end{array}$ & \\
\hline \multicolumn{3}{|c|}{ Beispiel NGP Schaalseelandschaft } \\
\hline \begin{tabular}{|c|} 
Regionale \\
Nachhaltigkeits-Institutionen
\end{tabular} & \begin{tabular}{|c} 
Regionale \\
Nachhaltigkeits-Themen
\end{tabular} & $\begin{array}{c}\text { Regionale } \\
\text { Verhaltensweisen }\end{array}$ \\
\hline $\begin{array}{l}\text { Lokale Agenda } 21 \\
\text { Ludwigslust: } \\
\text { Arbeitskreis Natur-, } \\
\text { Landschafts-, } \\
\text { Artenschutz/Wasser- und } \\
\text { Bodenschutz }\end{array}$ & $\begin{array}{l}\text { - Boden } \\
\text { - Wasser } \\
\text { - Ökosysteme und } \\
\text { Artenvielfalt }\end{array}$ & $\begin{array}{l}\text { Erneuerbare Naturgüter } \\
\text { (wie z.B. Kulturlandschaf) } \\
\text { dürfen auf Dauer nur im } \\
\text { Rahmen ihrer Fähigkeit } \\
\text { zur Regeneration genutzt } \\
\text { werden. Dies gilt im } \\
\text { Hinblick auf: } \\
\text {-Tourismus } \\
\text {-Landwirtschaft } \\
\text {-... }\end{array}$ \\
\hline
\end{tabular}

Abbildung 43: Beispiel für die Anwendung des Arbeitsblattes

\subsubsection{Perspektiven für den Fördermittelgeber}

Die Ergänzung der nachhaltigkeitsrelevanten Themenfelder um die regionalen Verhaltensweisen sowie potenziellen Fortführungsinstitutionen ermöglicht in strukturierter Form, notwendige Bedingungen für nachhaltige regionale Entwicklung explizit darzustellen. Der Fördermittelgeber könnte entsprechende Dokumentationen bereits bei der Beantragung von Förderungen als Anlage an den Förderantrag in seinen Richtlinien vorsehen. Die erfolgten Angaben können wichtige Hinweise geben, um die Förderungen spezifischer auszurichten. Das möglichst frühzeitige Erkennen von fehlenden Handlungsweisen und nicht vorhandenen Institutionen zur Fortführung der Förderung hilft die Verbindung von statischer und dynamischer Perspektive sowie die Verstetigung von Prozessen zu gewährleisten. Eine möglichst umfassende Kenntnis hierüber kann auf Seite des Fördermittelgebers dazu beitragen, die Förderpolitik noch effektiver zu gestalten. Folgende Schritte kann der Fördermittelgeber dazu initiieren:

1. Aufnahme einer entsprechenden Arbeitshilfe in die Förderrichtlinien als obligatorischen Schritt zur Beantragung von Fördergeldern. 
2. Verbreitung von Informationen zur Nutzung der Arbeitshilfe durch frühzeitige Diskussion mit Antragstellern und anderen, die im Prozess der Bewilligung von Fördergeldern integriert sind. Dabei ist zu beachten, dass folgende Inhalte zu vermitteln sind:

- Thematischer Kontext: die drei Dimensionen Ökonomie, Ökologie und Soziales sollen integrativ gedacht werden und nachhaltige regionale Entwicklung ist entsprechend zu organisieren.

- Aufbau der Arbeitshilfe (regionale Nachhaltigkeitsthemen, regionale Verhaltensweisen sowie Fortführungsinstitutionen identifizieren) und Anwendung auf den jeweiligen regionalen Kontext.

- Akzeptanzförderung in Bezug auf das Instrument Arbeitshilfe durch Beispiele. Außerdem sollte der Fördermittelgeber verdeutlichen, warum ihm an der Verstetigung der Maßnahmen liegt und welche Motive er mit regionaler Nachhaltigkeit verfolgt.

3. Bereitstellung der Arbeitshilfe über das Internet.

\subsubsection{Perspektiven für den Fördermittelempfänger}

Auch der Fördermittelempfänger kann das Instrument der Arbeitshilfe nutzen. Auf Grund der einfachen Handhabbarkeit können sowohl im Vorfeld als auch während des Förderzeitraumes leicht Informationen, die die Effektivität der Förderung beeinflussen, gewonnen und aufbereitet werden. Unabhängig davon, ob der Fördermittelempfänger regionale Nachhaltigkeit direkt organisieren möchte oder nicht, kann er sich mit Hilfe der Nachhaltigkeitsspinne Klarheit darüber verschaffen, inwieweit wichtige Voraussetzungen dafür bereits existieren bzw. erst geschaffen werden müssen. ${ }^{208}$ So verdeutlicht die Arbeitshilfe, wo die Brücke zwischen statischer Perspektive (nachhaltigkeitsrelevante Themenfelder) und dynamischer Perspektive (themenfeldspezifische Verhaltensweisen) noch nicht tragfähig ist. Sie regt aber auch dazu an, schon vor Beginn bzw. während des Förderzeitraumes darüber nachzudenken, wie angestoßene Prozesse verstetigt werden können und erhöht damit auch Bewilligungschancen. Da sowohl die Identifikation und Zuordnung von Nachhaltigkeitspostulaten als auch die Erhebung des regionalen Institutionengefüges leicht und ohne großen Ressourcen-Einsatz möglich sind, liegen die potentiellen Schwellen der Anwendung eher niedrig. Auch wenn nicht alle Zuordnungen in idealtypischer Weise erfolgen, was in der Praxis häufig der Fall sein dürfte, schmälert dies den Vorteil nicht, der durch die strukturierte Analyse entsteht. Der Fördermittelempfänger verschafft sich selbst gegenüber Stärken und Schwächen im Bezug auf wichtige Punkte der nachhaltigen, regionalen Entwicklung Transparenz. Er kann darüber hinaus ein Monitoring-System im Projektverlauf entwickeln, indem er die Arbeitshilfe in regelmäßigen Abständen

208 Dies gilt unabhängig von bereits bestehenden Regularien einzelner Förderrichtlinien. 
für einen Erhebungs- und Dokumentationsprozess aktualisiert und diskutiert. Sowohl innerhalb der Region als auch gegenüber dem Fördermittelgeber kann er die so ermittelten Daten als Kommunikationsinstrument nutzen.

Sofern die Arbeitshilfe als Instrument durch den Fördermittelgeber als Schritt bei der Antragserstellung vorgeschrieben ist, kann der potentielle Fördermittelempfänger das Instrument auch in folgender Weise für sich nutzen:

1. Informieren über die Schritte der Fördermittelbeantragung und Herunterladen der Arbeitshilfe (z.B. als Arbeitsblatt oder Software)

2. Lesen der die Arbeitshilfe begleitenden Dokumentation (Schritt der Antragserstellung)

3. Bestandsaufnahme: Welche Themenfelder prägen die Nachhaltigkeitsdiskussion im potentiellen Fördergebiet? Diese Themenfelder sind den drei Dimensionen Ökologie, Ökonomie und Soziales zuzuordnen.

4. Erstellung der Maßnahmenbasismatrix: Auf welche Themenfelder der drei Dimensionen wirkt die geplante Förderung aller Voraussicht nach? Dazu sind Plausibilitätsüberlegungen anzustellen.

5. Zuordnung von regionalen Verhaltensweisen: Welche Handlungsweisen werden im potentiellen Fördergebiet praktiziert, die eine günstige Entwicklung in den jeweiligen Themenfeldern unterstützen? Gibt es entsprechende Handlungsweisen, dann sind diese entsprechend hinter dem jeweiligen Themenfeld zu vermerken.

6. Zuordnung von potentiellen Fortführungsinstitutionen: Welche Institutionen existieren im potentiellen und erweiterten Fördergebiet, die geeignet sind, die durch die Förderung angestoßenen Prozesse nach Ablauf des Förderzeitraumes fortzuführen? Gibt es entsprechende Institutionen, dann ist darauf hinter dem jeweiligen Themenfeld hinzuweisen.

7. Beifügen der Dokumentation zum Förderantrag. Sie bietet einen ersten Anhaltspunkt, in welchem Ausmaß die beantragte Förderung auf regionale Nachhaltigkeit wirkt. 



\section{Fazit und Ausblick}

Wie wirken Naturschutzgroßprojekte und das E+E Vorhaben „Erlebnis Grünes Band“ auf regionale Nachhaltigkeit und welchen Beitrag leisten die Projektnetzwerke zu dieser Wirkung? Diese Frage galt es im Rahmen des interdisziplinären Forschungsvorhabens „Grünes Band - Modellregion für Nachhaltigkeit“ zu beantworten.

Eine qualitative Maßnahmenanalyse in Verbindung mit einer umfassenden Netzwerkanalyse mit dem gemeinsamen Forschungsbezug „Nachhaltigkeit“ wurde als geeigneter Ansatz herausgearbeitet. Die Verknüpfung der beiden disziplinären Perspektiven als zentrale Herausforderung des Forschungsvorhabens gelang im Sinne eines Brückenschlags. Dieser Brückenschlag kann in Form eines Flussdiagramms visualisiert werden und besteht aus den fünf Schritten (1) Erhebung von Netzwerkmerkmalen, (2) Zusammenfassung dieser in strategische Qualitäten, (3) Zusammenführung dieser mit den regionalen Verhaltensweisen für Nachhaltigkeit ${ }^{209}$, (4) Zuordnung selbiger zu den regionalen Handlungsfeldern der Nachhaltigkeit, die sich (5) anhand geeigneter Indikatoren messen lassen.

Die Erhebung und Analyse der Projektnetzwerke in den vier Untersuchungsregionen war dabei eine wesentliche Grundlage des Brückenschlags. Basierend auf

209 Regionale Verhaltensweisen für Nachhaltigkeit setzen sich aus den Managementregeln und Postulaten zusammenfassen. 
einschlägiger Literatur der Netzwerkforschung im Kontext regionaler Entwicklung und Nachhaltigkeit wurden Merkmale von Netzwerken herausgearbeitet, die den Untersuchungsgegenstand „Netzwerk“ operationalisieren. Die Netzwerkerhebungen und -analysen orientierten sich an diesen und erfassten die unterschiedlichen Ausprägungen der Merkmale in den Projektnetzwerken. Die Verknüpfung der Netzwerkmerkmale mit regionaler Nachhaltigkeit erfolgt über die in der Nationalen Nachhaltigkeitsstrategie des Bundes formulierten Anforderungen an den gesellschaftlichen Prozess, der notwendig ist, um das Leitbild Nachhaltigkeit umzusetzen. Demnach muss Netzwerkarbeit diskursiv, akteursorientiert und integrativ sein. Diese Zusammenführung von Netzwerkmerkmalen und den so genannten „strategischen Qualitäten“ ist ein qualitativer Analyseansatz, mit dem sich der Zusammenhang von Netzwerken und Nachhaltigkeit untersuchen lässt.

Als grundsätzliche Erkenntnis der Netzwerkanalyse ist festzuhalten, dass Netzwerke eine zentrale Rolle bei der Verwirklichung von Nachhaltigkeitszielen im Kontext von Naturschutzförderung spielen. Die Ergebnisse der Netzwerkanalyse belegen, dass der Beitrag der einzelnen, geförderten Projektmaßnahmen sowie der Förderprojekte insgesamt zur regionalen Nachhaltigkeit unmittelbar sowie mittelbar von dem begleitenden Prozess der Maßnahmenplanung und -umsetzung durch die Projektnetzwerke abhängig ist. Die Fallstudien der Projektnetzwerke in den vier Untersuchungsregionen zeigen, dass positive Merkmalsausprägungen stets einen positiven Einfluss auf die Ausrichtung der Netzwerke als Ganzes im Sinne der regionalen Nachhaltigkeit haben. Hingegen hemmen negative Merkmalsausprägungen die Netzwerkarbeit oftmals stärker und in mehrfacher Hinsicht als positive Ausprägungen die Arbeit unterstützen. Die negativen Merkmalsausprägungen können beispielsweise in Ablehnung der sektoralen Förderziele, manifestierte Interessenkonflikte in der Region, nicht ausgeschöpfte Synergiepotenziale oder Unzufriedenheit und Passivität von Akteuren in den Netzwerken resultieren. Aus diesem Grund sollte Netzwerkarbeit deutlich stärker in die Konzeption der Fördertitel einbezogen werden.

Die betrachteten Naturschutzfördertitel wiederum haben grundsätzlich einen positiven Einfluss im Sinne der Intensivierung und Ausweitung der Netzwerkarbeit. Die Aushandlung gemeinsamer Ziele und Interessen sowie Konfliktbearbeitung wurde angestoßen und somit auch der gesellschaftliche Prozess zur Übersetzung der Nachhaltigkeit in ein regionales Handlungskonzept initiiert. Dennoch können auch prozesshemmende Defizite identifiziert werden. So erfordert die Organisation der Projekte, d.h. insbesondere das Management der notwendigen intensiven Interaktion, aber auch die regionale Verankerung und Ausrichtung der Netzwerke auf Nachhaltigkeit entsprechende Ressourcen und das notwendige Wissen. Diese beiden Voraussetzungen sind in den Untersuchungsregionen nicht immer ausreichend erfüllt. Die Ableitungen, die sich daraus aus Netzwerkperspektive formulieren lassen, fokussieren sich daher auf diejenigen Netzwerkmerkmale, die der Organisation der Netzwerkarbeit und Generierung von Wissen dienen: 
Arbeiten auf verschiedenen Ebenen, Prozessmanagement und Lernen. Über die Beachtung dieser Netzwerkmerkmale kann in besonderem Maße steuernd auf die Entwicklung des Netzwerks eingewirkt werden.

Die in den Perspektiven dargelegten Werkzeuge der Netzwerkarbeit zur Optimierung der Netzwerkarbeit im Kontext der betrachteten Naturschutzfördertitel, knüpfen an den erfassten zentralen Herausforderungen dieser Netzwerkarbeit an: Akzeptanz schaffen, indem regionaler Mehrwert aufgezeigt wird; zur Mitwirkung motivieren, indem regionale Anknüpfungspunkte herausgestellt werden; Mitwirkung organisieren, indem leistungsfähige Arbeitsstrukturen in der Fläche das Mehrebenenproblem lösen; Netzwerkarbeit verstetigen, um Perspektiven über den Förderzeitraum hinaus zu schaffen.

Wie in den Perspektiven dargestellt, werden konkrete Optimierungsmöglichkeiten mit Hilfe von Werkzeugen der Netzwerkarbeit beschrieben. Die Verdeutlichung der Zusammenhänge zwischen regionaler Entwicklung und den Förderprojekten, z.B. durch die Integration der Projektleitbilder in die regionalen Leitbilder, ist zentral, um abgestimmte und konsistente regionale Entwicklung voranzutreiben. An Nachhaltigkeit ausgerichtete Netzwerke müssen zudem lernfähig sein, um Verhaltensweisen, Beteiligungsstrukturen, Entscheidungsfindungen und die gesamte Projektumsetzung stetig zu optimieren. Eine zielgruppengerechte Kommunikation der Förderziele auf den verschiedenen Netzwerkebenen sollte verstärkt werden, um das notwendige Wissen zu schaffen, auf das dann auch Akzeptanz sektoraler Ziele aufbauen kann.

Während die Netzwerkstrukturen die Grundlage bilden, können die Projektmaßnahmen einen entscheidenden Beitrag zur regionalen Nachhaltigkeit leisten und bedurften daher ebenso einer Analyse. Wie erwähnt, war die ursprüngliche Überlegung die Nachhaltigkeitswirkung von Projektmaßnahmen über ein gewähltes Indikatorset zu operationalisieren aufgrund fehlender Datensätze nicht umsetzbar. Dies ist auch bedingt durch die Abgrenzung der Untersuchungsregionen, die nicht mit Landkreisgrenzen übereinstimmt und damit die Anwendung von Daten des statistischen Landesamtes nicht möglich ist. Aber auch das fehlende regionsspezifische Nachhaltigkeitsmonitoring erschwert den Aufbau einer Datenbasis. Resultierend aus diesen Tatbeständen bedurfte es einer Anpassung des Vorgehens seitens der Maßnahmenperspektive. Zum einen soll dieses weiterhin den Brückenschlag zur Netzwerkanalyse aufrechterhalten, zum anderen die Dokumentation möglicher Nachhaltigkeitswirkungen der Projekte in den Untersuchungsregionen sicherstellen, um die vorgefundenen Defizite strukturell zu beheben. Der Brückenschlag erfolgt über einen weiteren Baustein der Nationalen Nachhaltigkeitsstrategie, nämlich die Nachhaltigkeitspostulate und Managementregeln für Nachhaltigkeit. Während Managementregeln allgemeingültigen und integrativen Charakter haben, sind die Nachhaltigkeitspostulate als spezifische und nichtintegrative Verhaltensregeln in den jeweiligen Handlungsfeldern regionaler Nachhaltigkeit zu verstehen. Die durch die Netzwerkerhebung geschaffene Datenbasis 
ermöglichte in den Untersuchungsregionen geltende Regeln und Postulate dieser Art zu erfassen. Diese Erhebung wird ergänzt durch eine Erhebung der regionalen Institutionen von Nachhaltigkeit in den Untersuchungsregionen. Diese wird im Sinne eines Brückenbausteins als Instrument des Nachhaltigkeitsmanagements in die Entwicklung einer Nachhaltigkeitsspinne integriert. Aufgrund der fehlenden Möglichkeit der unmittelbaren Operationalisierung orientiert sich die Nachhaltigkeitspinne an den Handlungsfeldern und ermöglicht damit der Region durch eine sehr weite und flexible Zuordnung der Projektmaßnahmen eine mittelbare Nachhaltigkeitsdokumentation.

NGP und E+E-Vorhaben bedürfen in ihren Wirkungszusammenhängen einer differenzierten Betrachtung. Fokussieren sich Naturschutzgroßprojekte in der Regel auf eine reine Naturschutzförderung mit dem Ziel großflächige Verbundsysteme zu schaffen, bietet der Fördertitel E+E-Vorhaben auch die Möglichkeit, punktuell Projekte zur naturschutzorientierten Regionalentwicklung zu entwickeln. Diese unterschiedlichen Eigenschaften der Förderkulissen führten zu unterschiedlichen Skalen bei der Dokumentation der Nachhaltigkeitswirkungen. Die analysierten E+E-Projekte verwenden die Anzahl der Projektmaßnahmen als Skalierung, die Naturschutzgroßprojekte hingegen die unterschiedlichen Flächenformen. Dazu erfolgte zunächst die Übertragung der jeweiligen Projektmaßnahmen in eine Maßnahmenbasismatrix, welche die Grundlage für die Nachhaltigkeitsspinne liefert. Mit ihrer Hilfe ist eine differenzierte Darstellung der Wirkungen aller Projektmaßnahmen möglich, welche primär zur Unterstützung der Entscheidungsprozesse in der Maßnahmenplanung sowie deren Umsetzungen dienen soll.

Der Schwerpunkt der Dokumentation liegt auf der Verdeutlichung impliziter Zusammenhänge. Den Adressaten der Nachhaltigkeitsspinne soll ein Instrument an die Hand gegeben werden, welches einen Abgleich von „Ist“ und „Soll“ im Kontext von Nachhaltigkeit ermöglicht. Daran knüpfte sich ferner der Gedanke an, ein Instrument zu entwickeln, das sich zudem mit möglichst geringem Aufwand implementieren lässt. Die Brücke zu den Netzwerken besteht dabei in der Zuordnung von Nachhaltigkeitspostulaten, welche innerhalb der Netzwerke Anwendung finden (sollen).

Neben der reinen Brückenfunktion und grundlegenden Nachhaltigkeitsdokumentation entwickelten sich im Gespräch mit dem BfN, BUND BN Bayern sowie dem Alpenforschungsinstitut weiterführende Fragestellungen, die an das Grundkonzept der Nachhaltigkeitspinne anknüpfen. Vor allem die Frage nach der Fortführung von Strukturen nach dem Förderzeitraum stand im Raum, d.h. was passiert mit den Projektbausteinen und den Projektnetzwerken in den Regionen, wenn keine Fördermittel mehr fließen? Diese Fragstellung aufgreifend wurden bestehende Nachhaltigkeitsinstitutionen am Grünen Band selbst und in angrenzenden Regionen eruiert. Die dazu durchgeführte Erhebung erfasst regional vorhandene Nachhaltigkeitsinstitutionen. Die Grundidee liegt hierbei in der Überle- 
gung, naturschutzorientierte Projekte so zu planen und auszugestalten, dass im Idealfall nach dem Auslaufen der Förderphase die Projektbausteine interessant genug sind, dass vorhandene Nachhaltigkeitsinstitutionen sie weiterführen. Das entspricht dem grundlegenden Gedanken der Nachhaltigkeit. Neben der nachhaltigen Ausrichtung von Projektbausteinen gilt dies ebenso für die Weiterführung von durch die Projekte initiierten Netzwerkstrukturen, die es auf gleiche Art und Weise auf eine Zeit nach der Förderung vorzubereiten gilt.

Im Rahmen der Untersuchung ließen sich drei wesentliche Adressaten für Empfehlungen identifizieren: die Region, die Länder sowie der Bund. Der Bund bzw. der Fördermittelgeber kann durch ergänzende Förderkriterien oder gezielte Ressourcenverteilung die geförderten Regionen auf die Bedeutung der Netzwerkarbeit im Kontext von Naturschutzförderung die aufmerksam machen und entsprechend Anreize schaffen, dass sie sich im Sinne der regionalen Nachhaltigkeit organisieren. Länder und Regionen selbst stehen ebenfalls in der Verantwortung, ihren Beitrag zur Ausrichtung von Förderprojekten des Naturschutzes an regionaler Nachhaltigkeit auszurichten. Die Umsetzung der konkreten Empfehlungen obliegt jedoch der Region, die dabei auch einen besonderen Anteil an Eigeninitiative vorweisen muss. Mit dem Blick auf die Nachhaltigkeitsdokumentation spielen auch wiederum der Bund und die Region die entscheidenden Rollen. Dabei besteht die Überlegung, die Nachhaltigkeitsspinne bereits in den Förderantrag der Region zu implementieren. Bei Festlegung eines nachhaltigen Leitbildes für die Projektgestaltung durch den Bund könnte dies zu einer nachhaltigen Ausrichtung der Projektmaßnahmen führen.

Zusammenfassend lässt sich festhalten, dass das Forschungsvorhaben „Grünes Band - Modellregion für Nachhaltigkeit" verschiedene Instrumente und Werkzeuge zur bewussten Ausrichtung und Umsetzung der Förderung im Sinne der regionalen Nachhaltigkeit aufzeigt. Deren Nutzung und Ausgestaltung liegt nun im Handlungsbereich der Zielgruppen der Forschung, d.h. dem Fördermittelgeber (BMU/BfN) und den geförderten Regionen. 



\section{Literatur}

Adrian, L. (2003): Regionale Netzwerke als Handlungskonzept. Erfolg versprechender Weg einer innovationsorientierten Regionalentwicklung. Herausgegeben von Deutsches Institut für Urbanistik difu. Berlin: difu.

agenda-transfer (2003): Gemeinsam empfohlene Indikatoren zur kommunalen Nachhaltigkeit, zugegriffen am 22.10.2008, in: http:/ /databases.euccd.de/files/documents/00000205_Gemeinsame_Indikatoren_03.pdf

Bayrisches Staatsministerium für Landwirtschaft und Forsten (2003), Grünlandwirtschaft in Bayern, München.

Benz, A.; Fürst, D.; Kilper, H.; Rehfeld, D. (1999): Regionalisierung. Theorie, Praxix, Perspektiven. Leske \& Buderich, Opladen

Benz, A.; Meincke, A. (9.5.2007): Regionen Aktiv - Land gestaltet Zukunft. Begleitforschung 2004 bis 2006. Endbericht der Module 3 und 4. Regionalwissenschaftliche Theorieansätze. Analyse der Governance Strukturen. Hagen.

BfN (1993): Richtlinien zur Förderung der Errichtung und Sicherung schutzwürdiger Teile von Natur und Landschaft mit gesamtstaatlich repräsentativer Bedeutung einschließlich der Förderung von Gewässerrandstreifen (Förderrichtlinie für Naturschutzgroßprojekte) vom 28. Juni 1993

BfN (2003): Eckpunkte zur Optimierung des Förderinstrumentariums für Naturschutzgroßprojekte im Zeitraum vom 01.09.2003 bis 31.12.2007 
BfN (2007): Eckpunkte zur Optimierung des Förderinstrumentariums für Naturschutzgroßprojekte im Zeitraum 01.01.2008 bis 31.12.2012

BfN (2008): Where have all the flowers gone? Grünland im Umbruch, Hintergrundpapier und Empfehlung des BfN, Bonn.

BfN (2009): Erprobungs- und Entwicklungsvorhaben.

http://www.bfn.de/0202_eue.html (Stand 17.12.2009)

BfN (2010): Naturschutzgroßprojekte, http:/ / wmw.bfn.de/0203_grossprojek.te.html (Zugriff: 01.04.2010)

BfN (2010): Naturparke, http:/ / wnw.bfn.de/0308_np.html (Zugriff 26.03.2010)

BfN (2010): Naturschutzgroßprojekt Grünes Band Eichsfeld - Werratal startet in in erste Phase,

bttp:/ / wnw.bfn.de/ 6.html?.cHash $=d 1$ e06b409devtx_ttnews\%5Btt_news $\% 5 D=1436$ (Zugriff 26.03.2010)

BfU Beratungsbüro für Umwelt- und Unternehmensentwicklung (2005): Projekt BeNN. Wissenschaftliche Begleitforschung, Moderation und Coaching regionaler Netzwerke auf dem Gebiet neuer Nutzungsstrategien. Abschlussbericht im Auftrag des Umweltbundesamtes (Projekt KONI). Unter Mitarbeit von Katrin Gillwald, Waltraud Höppner, Jörg Longmuss, Jurij Poelchau, Monika Stauber, Matthias Teller, Berlin.

Bittner, A. (2003) Außerschulische Umweltbildung in der Evaluation. Wirkungen kurzzeitpädagogischer Maßnahmen auf Umwelt- und Naturschutzinteressen von Schülerinnen und Schülern der Sekundarstufe I.- Didaktik in Forschung und Praxis, Bd. 5. - Hamburg (Verlag Dr. Kovac).

Bizer, K. (2000): Die soziale Dimension der Nachhaltigkeit, in: Zeitschrift für angewandte Umweltforschung (ZAU), Jg. 13, H. 3/4, S. 469-482.

Bizer, K.; Sternberg, R. (2001): Grundprobleme von Indikatorensystemen für Regionale Nachhaltigkeit, in: Zeitschrift für Raumforschung und Raumordnung, H. 5/6, S. 381-391.

BmFSFJ (2009): Gleichberechtigung in allen Lebensphasen, in: http:/ / wnw.bmfsfj.de/ bmfsfj/generator/BMFSFJ/gleichstellung, did=76920. html, zugegriffen am 05.01.2009.

BN-Bund Naturschutz in Bayern e.V./BUND-Bund für Umwelt und Naturschutz (2006): E+E-Vorhaben Vorstudie „Erlebnis Grünes Band“ (Abschlussbericht). Im Auftrag des Bundesamtes für Naturschutz. Nürnberg. Unveröffentlicht.

Böcher, M.; Krott, M.; Tränkner, S. (2007): Regionen Aktiv - Land gestaltet Zukunft. Begleitforschung 2004 - 2006. Endbericht des Moduls 5. Erfolgsfaktoen und Rahmenbedingungen. Online verfügbar unter http://www.regionenaktiv.de/Downloads/B2_Modul5_Endbericht1106200 7.pdf, zuletzt aktualisiert am 11.06.2007. 
Bohnsack, R. (2003): Rekonstruktive Sozialforschung: Einführung in qualitative Methoden, 5. Aufl., Opladen: Leske + Budrich, 2003

BUND (2008): Das Grüne Band - Vom „Eisernen Vorhang“ zum Biotopverbund.

Bundesministerium für Umwelt, Naturschutz und Reaktorsicherheit (1987):

Richtlinien zur Förderung von Erprobungs- und Entwicklungsvorhaben im Bereich Naturschutz und Landschaftspflege. http://www.bfn.de/0202_richtlinien.html (Zugriff am 31.03.2010)

Bundesministerium für Umwelt, Naturschutz und Reaktorsicherheit (2007): Nationale Strategie zur biologischen Vielfalt.

Die Bundesregierung (2008): Fortschrittsbericht 2008 zur Nationalen Nachhaltigkeitsstrategie - Für ein nachhaltiges Deutschland. Berlin.

Bundesregierung (o.J.): Perspektiven für Deutschland - Unsere Strategie für eine nachhaltige Entwicklung.

Diefenbacher, H.; Dümig, D. ; Teichert, V.; Wilhelmy, S. (2001): Die Analyse regionaler Nachhaltigkeit im Vergleich verschiedener Bewertungsmethoden, Forschungsbericht FZKA-BWPLUS.

Diller, C. (2002): Zwischen Netzwerk und Institution. Eine Bilanz regionaler Kooperationen in Deutschland. Opladen: Leske + Budrich.

Döring, T.; Heiland, S.; Tischer, M. (2004): Kommunale Nachhaltigkeitsindikatorensysteme in Deutschland - Zum aktuellen Stand von Entwicklung und Anwendung, in: Vierteljahreshefte zur Wirtschaftsforschung, 73 (2004), DIW Berlin.

Franke, K.; Wald, A. (2006): Möglichkeiten der Triangulation quantitativer und qualitativer Methoden in der Netzwerkanalyse. In: Hollstein, B.; Straus, F. (Hrsg.): Qualitative Netzwerkanalyse. Konzepte, Methoden, Anwendungen. VS Verlag für Sozialwissenschaften. Wiesbaden

Fürst, D.; Scholles, F. (Hg.) (2008): Handbuch Theorien und Methoden der Raum- und Umweltplanung / Dietrich Fürst ; Frank Scholles (Hg.). Mit Beitr. von Dietrich Fürst ... 3. Aufl. Dortmund: Rohn.

Gailing, L. (2005): Regionalparks - Grundlagen und Instrumente der Freiraumpolitik in Verdichtungsräumen. Dortmunder Beiträge zur Raumplanung Nr. 121. Dortmund.

Graymore, M.; Sipe, N.; Rickson, R. (2008): Regional sustainability: How useful are current tools of sustainability assessment at the regional scale?, in: Ecological Economics, 67, S. 362-372. 
Greif, M. (2000): Von der lokalen zur regionalen Nachhaltigkeit. Die Ausweitung lokaler Agenda 21-Prozesse auf die regionale Ebene am Beispiel der Region „Oldenburg und Umland“. [Electronic ed.]. Oldenburg: BIS - Bibliotheksund Informationssystem der Universität Oldenburg; Bibliotheks- und Informationssystem der Univ. [Host]. (Studien zur Soziologie- und Politikwissenschaft).

Günther, E.; Kaulich, S. (2008): Umweltleistung und ihre Messung, in: WISU, 11, S. 1504-1505.

Hahne, U. (2002): Lokale Agenda 21 als Basis nachhaltiger Regionalentwicklung Dilemmata eines neuen Politiktypus. In: Geographische Revue, Jg. 4, H. 2, S. 21-33. Online verfügbar unter http://www.geographischerevue.de/archiv/gr2-02.pdf.

Hartje, V. (2003): Monetäre Bewertung einer nachhaltigen Entwicklung der Stromlandschaft Elbe, Forschungsvorhaben gefördert durch das Bundesministerium für Bildung und Forschung (BMBF) im Rahmen des Programms Elbe-Ökologie, Endbericht.

Heinl, W.(2005): Integrative Nachhaltigkeit. Erweitertes Orientierungswissen für nachhaltige Entwicklung am Beispiel der Lokalen Agenda 21. Dissertation. Betreut von Holger Magel. München. TU München, Lehrstuhl für Bodenordnung und Landentwicklung.

Heinz Sielmann Stiftung (2007): Projektantrag zur Errichtung und Sicherung schutzwürdiger Teile von Natur und Landschaft mit gesamtstaatlich repräsentativer Bedeutung Naturschutzgroßprojekt Grünes Band Eichsfeld-Werratal

Hertwig, F., Priebe, R. (o.J): Umweltschonende Grünlandnutzung mit Mutterkühen, in: bttp:/ / wmw.mluv.brandenburg.de/cms/media.php/2331/flrindj.pdf, zugegriffen am 06.01.2009.

Jansen, D. (2006): Einführung in die Netzwerkanalyse. Grundlange, Methoden, Forschungsbeispiele. (1. Aufl. 1999) VS Verlag für Sozialwissenschaften, Wiesbaden.

Job, H.; Bernhard Harrer; Daniel Metzler; David Hajizadeh-Alamdary (2005):

Ökonomische Effekte von Großschutzgebieten, Untersuchung der Bedeutung von Großschutzgebieten für den Tourismus und die wirtschaftliche Entwicklung der Region, in BfN (Hrsg.), BfN-Skripten 135.

Joosten, H.; Augustin, J. (2006): Peatland restoration and climate: on possible fluxes of gases and money. In: Bambalov, N.N. (ed.): Peat in solution of energy, agriculture and ecology problems. Proceedings of the International Conference Minsk, May 29 - June 2, 2006. Tonpik, Minsk, 412 - 417.

Joosten, H. (2005): Moorschutz in Europa-Restauration und Klimawandel, Europäisches Symposium „Moore in der Regionalentwicklung“ 
Katz, C. (2004): Waldnutzung und Waldschutz im Wandel - Neue Chancen für mehr Geschlechtergerechtigkeit?, in: BfN, Hayn, Doris (Bearb.) (2004), Gender Mainstreaming im Naturschutz, Bonn-Bad Godesberg.

Kaufmann, R.; Cleveland, C. (1995): Measuring sustainability: needed - an interdisciplinary approach to an interdisciplinary concept, in: Ecological Economics, 15, S. 109-112.

Klump, R. (2006): Wirtschaftspolitik - Instrumente, Ziele und Institutionen.

Knolle, F. (2004): Öffentlichkeitsarbeit und Umweltbildung im Nationalpark Harz - Das Thema „Gender“ in der alltäglichen Naturschutzarbeit, in BfN (Hrsg.), Gender Mainstreaming im Naturschutz.

Krause, R.; Meyer, R.; Marggraf, R. (2008): Kann die Umweltökonomie den intrinsischen Wert der Natur berücksichtigen?, in: Zeitschrift für Umweltpolitik \& Umweltrecht 31(3), 2008, 293-313.

Krebs, A. (1996): Ich würde gern mitunter aus dem Haus tretend ein paar Bäume sehen, Philosophische Überlegungen zum Eigenwert der Natur, in: Nutzinger, H. (Hrsg.) Naturschutz - Ethik - Ökonomie, S. 31-38, Metropolis, Marburg.

Kuckartz, U. (2007): Einführung in die computergestützte Analyse qualitativer Daten, 2. aktualisierte und erweiterte Auflage, VS Verlag für Sozialwissenschaften/ GWV Fachverlage GmbH, Wiesbaden, 2007

Kullmann, A. (2003): Status Quo der Regionalvermarktung in den deutschen Biosphärenreservaten, in: Deutsches MAB-Nationalkomitee (Hg.), Voller Leben, S. 225-233.

Leser, H. (1997): Landschaftsökologie, 4.A., UTB.

Lindloff, K. (2003): Kooperation erfolgreich gestalten. Erfolgsfaktoren kooperativer Prozesse in der Regionalentwicklung. Dortmund: Dortmunder Vertrieb für Bau- und Planungsliteratur (Kommunikation im Planungsprozeß, 5).

Maier, J.; Obermaier, F. (2000): Regionalmanagement in der Praxis - Erfahrungen aus Deutschland und Europa - Chancen für Bayern; Bayrisches Staatsministerium für Landesentwicklung und Umweltfragen - München 2000

Mayer, H. (2006): Interview und schriftliche Befragung: Entwicklung, Durchführung und Auswertung, 3. überarb. Aufl., München 2006

Mecklenburg-Vorpommern Ministerium für Landwirtschaft, Umwelt und Verbraucherschutz (2008): Trends zur Entwicklung der Milchproduktion und der Molkereiwirtschaft in Mecklenburg-Vorpommern unter Beachtung der Diskussionen zum health check, in: http://www.rinderzuchtmv.de/fileadmin/pdf/managementseminar/2_Dr-Gerhard-Rudolphi.pdf, zugegriffen am 22.12.2008. 
Mehl, D.; Bollmohr, A.; Bittl, R.; Reimann, T. (2008): Regionalmanagement in drei kooperierenden Amtsbereichen, Ein innovatives Pilotvorhaben integrierter ländlicher Entwicklung zur Umsetzung von GAKG und ELERVerordnungen in Mecklenburg-Vorpommern, in RuR 1, S. 76-83.

Meister, H. (2002): Politik, Wirtschaft, Gesellschaft: Partner oder Konkurrenten in der nachhaltigen Regionalentwicklung. In: Molitor, Reimar; Nischwitz, Guido (Hg.): Kommunikation für eine nachhaltige Entwicklung in der Region. Tagungs- und Forschungsprojekt KOMREG. Berlin: IÖW (Schriftenreihe des IÖW, 160/02), S. 51-59.

Molitor, R.; Nischwitz, G. (Hg.) (2002): Kommunikation für eine nachhaltige Entwicklung in der Region. Tagungs- und Forschungsprojekt KOMREG. Berlin: IÖW (Schriftenreihe des IÖW, 160/02).

More-Hollerweger, E. (2008): Der Rückzug von Markt und Staat im ländlichen Raum, Zur Rolle von Vereinen für die Dienstleistungen der Daseinsvorsorge, in: Ökologisches Wirtschaften, 1. S. 35-38.

Ness, B.; Urbel-Piirsalu, E.; Anderberg, S.; Olsson, L. (2007): Categorising tools for sustainability assessment, Survey, in: Ecological Economics, 60, S. 498508.

Nitsche, S.; Nitsche, L. (1994): Extensive Grünlandnutzung, Neumann Verlag.

Nohlen, D.; Grotz F. (Hg.) (2008): Kleines Lexikon der Politik. 4. Aufl. Bonn: Bundeszentrale für Politische Bildung $>$ (Schriftenreihe der Bundeszentrale für Politische Bildung).

Oels, A. (2007): Nachhaltigkeit, Partizipation und Macht - oder: Warum Partizipation nicht unbedingt zu Nachhaltigkeit führt. In: Jonuschat, H. et al. (2007): Partizipation und Nachhaltigkeit. Vom Leitbild zur Umsetzung. Oekom Verlag, München

Pappi, F.; van Koolwijk, J.; Albrecht, G. (1987): Methoden der Netzwerkanalyse. München: Oldenbourg (Techniken der empirischen Sozialforschung, : ein Lehrbuch in 8 Bänden / hrsg. von Jürgen van Koolwijk ... Bearb. von G. Albrecht; Bd. 1).

Payer, H. (2008): Die Kunst des Netzwerkens. In: Bauer-Wolf, Stefan; Payer, Harald; Scheer, Günter (Hg.): Erfolgreich durch Netzwerkkompetenz. Handbuch für Regionalentwicklung. Wien [u.a.]: Springer, S. 23-46.

Payer, H. (November 2002): Wieviel Organisation braucht ein Netzwerk. Entwicklung und Steuerung von Organisationsnetzwerken mit Fallstudien aus der Cluster- und Regionalentwicklung. Klagenfurt. Universität Klagenfurt, Fakultät für Kulturwissenschaften.

Petermann, C. (2001): Naturschutz als Impulsgeber für sozioökonomische Entwicklungen, Ergebnisse des Kolloquiums Instrumente und institutionelle Rahmenbedingungen für kooperative Naturschutzprojekte, FuE-Vorhaben (FKZ 89881 004). 
Pollermann, K. (2007): Prozessintegrierte Evaluationen zur Nachhaltigen Regionalentwicklung, in: disP 169, 2, S. 68-79.

Regionalverbund Thüringer Wald e.V. (2006): Das „Grüne Band“ (inter)aktiv erleben. Erprobungs- und Entwicklungsvorhaben „Erlebnis Grünes Band“. Projektbeschreibung. Oberhof

Rennings, K.; Koschel, H.; Brockmann, K.; Kühn, I. (1999): A regulatory framework for a policy of sustainability: lessons from the neo-liberal school, in: Ecological Economics, 28, S. 197-212.

Riemenschneider, R. (2002): Akteure einer nachhaltigen Regionalentwicklung: Neue Aufstellung - gleiches Spiel. In: Molitor, Reimar; Nischwitz, Guido (Hg.): Kommunikation für eine nachhaltige Entwicklung in der Region. Tagungs- und Forschungsprojekt KOMREG. Berlin: IÖW (Schriftenreihe des IÖW, 160/02), S. 121-125.

Rösch, C.; Raab, K.; Sharka, J.; Stelzer, V.. (2007): Energie aus dem Grünland eine nachhaltige Entwicklung?, Karlsruhe.

RWK Perleberg-Wittenberge-Karstädt (2006): Standortentwicklungskonzeptition des Regionalen Wachstumskerns, In:

http:/ /daten2.verwaltungsportal.de/dateien/seitengenerator/sek_2006.pdf, abgerufen am 23.03.2010.

Sauerborn, K.; Dehmel, C.; Fink, B.; Zagst, L.; Wagner, T. (2006): Umweltbericht der strategischen Umweltprüfung im Rahmen der ex-ante Evaluierung des operationellen Programms für EFRE 2007-2013 des Freistaates Thüringen, Trier.

Schägner, J. (2008): Der Wert des Waldes in einer ökonomisierten Welt, die monetäre Bewertung unserer Wälder, in: Ökologisches Wirtschaften, 1/2008, S. 24-26.

Schneider, W. (2009): Grundlagen der Garfinkel - RC - Habermas - Luhmann. 3. Aufl. Wiesbaden: VS Verl. für Sozialwissenschaften (Grundlagen der soziologischen Theorie, Bd. 2).

Segert, A.; Zierke, I. (2007): Regionalinitiativen - Entwicklungsressource strukturschwacher ländlicher Räume, in: RuR, 5, S. 421-434.

Selle, K. (2000): Freiräume, Siedlung, Kooperationen. Forschungsergebnisse, Hinweise für die Praxis, Folgerungen. Unter Mitarbeit von Sibille Hüchtker, Brigitte Scholz und Heidi Sinning et al. Dortmund: Dortmunder Vertrieb für Bau- und Planungsliteratur (Arbeits- und Organisationsformen für eine nachhaltige Entwicklung, 1).

Späth, R. (2000): German Forest Administrations and Female Foresters - Some Aspekts and Fields for Investigation and Research, in: Furuberg M.; Hylen G.; Strupstad L.M.; Kinderas K.; Follo, G. (eds.), Proceedings, Symposium on „Women and Forestry“ in Lillehammer, 12.-15. August 1999, Oslo 
Spitzer, H. (1995): Einführung in die räumliche Planung. 26 Tabellen. Stuttgart: Ulmer (UTB für Wissenschaft Grosse Reihe, 8106).

Stahl, T.; Schreiber, R. (2003): Regionale Netzwerke als Innovationsquelle. Das Konzept der "Lernenden Region" in Europa. Frankfurt/Main: Campus-Verl. (Campus-Forschung, 868).

Tamme, O. (2001): Beschäftigungseffekte der forstlichen Förderungen, in: Ländlicher Raum, Online-Fachzeitschrift des Bundesministeriums für Land- und Forstwirtschaft, Umwelt und Wasserwirtschaft Jahrgang 2001.

Thüringer Ministerium für Landwirtschaft, Naturschutz und Umwelt (2006): Landeswaldprogramm des Freistaates Thüringen. Gotha.

Trägerverbund Burg Lenzen e.V. (2006): Projektbeschreibung für das E+EVorhaben Erlebnis Grünes Band in der Modellregion Elbe - Altmark- Wendland, Grenzerfahrungen im Vierländereck.

Tröger-Weiss, G. (1998): Regionalmanagement: Ein neues Instrument der Landes- und Regionalplanung; Schriften zur Raumordnung und Landesplanung Band 2; Selbstverlag Fachgebiet Raumordnung und Landesplanung; Universität Augsburg - 1998

Wald, A.; Jansen D. (2007): Netzwerke. In: Benz, Arthur (Hg.): Handbuch Governance. Theoretische Grundlagen und empirische Anwendungsfelder. 1. Aufl. Wiesbaden: VS Verl. für Sozialwiss. , S. 93-105.

Walz, R.; Nathani, C.; Schleich, J.; Schwermer, S.(2003-2004): Wie nachhaltig sind Kreislaufwirtschaftsstrategien? - Ergebnisse von Fallbeispielen aus den Bereichen Recycling und umweltfreundliche Produktstrategien-, in: Zeitschrift für angewandte Umweltforschung (ZAU), Jg. 15/16, H. 2, S. 256-272.

Wiechmann, T. (2004): Das Modellvorhaben „Regionen der Zukunft“. Erfahrungen und Schlussfolgerungen für eine nachhaltige Regionalentwicklung in Deutschland. Dresden: Leibniz-Inst. für Ökologische Raumentwicklung (IÖR-Schriften, 45).

Wonneberg, E. (2004): Arbeit rund um Wald im Geschlechterblick, in: WaldArbeitspapier, Nr. 7.

Zahl, B. (2004): Soziale und regionale Netzwerkanalysen - Theoretische Grundlagen und ausgewählte Fallstudien, Arbeitspapier 1, Institut für Tourismus- und Bäderforschung in Nordeuropa GmbH, N.I.T. 2004

Zeck, H. (1998): Auswertung von Erfahrungen zur Kooperation in Regionen. In: Mayer-Ries, Jörg Friedrich (Hg.): Kooperation in der Region. Ein Ansatz für nachhaltige Entwicklung; [Dokumentation einer Tagung der Evangelischen Akademie Loccum in Kooperation mit der Akademie für Raumforschung und Landesplanung Hannover ... vom 26. bis 28. November 1997]. 1. Aufl. Rehburg-Loccum: Evang. Akad. Loccum Protokollstelle (Loccumer Protokolle, 1997,71). 
ZMP (2008), UK: Deutlich größere Bio-Fläche, in: http://www.zmp.de/agrarmarkt/branchen/oekomarkt/2008_09_01_Bio_U k.asp?id $=/$ suchfunktion. aspsearch $=$ Dauergr $\%$ FCnland $\&$ mode $=$ allwords, zugegriffen am 19.01.2009

Zweckverband Schaalsee-Landschaft (2006): Pflege- und Entwicklungsplan „Schaalsee-Landschaft II“ 

Anhang 

Anhang 1: Übersicht der EZB im Rahmen des PEPL Schaalsee-Landschaft II

\begin{tabular}{|c|c|c|}
\hline Liste der Entwicklungsziele auf Flächen des ZSL & EZB & $\begin{array}{l}\text { Anteil an Flä- } \\
\text { che des ZSL }\end{array}$ \\
\hline $\begin{array}{l}\text { A1 Extensive Ackernutzung } \\
\text { A2 Berücksichtigung Naturschutz bei Ackernutzung }\end{array}$ & 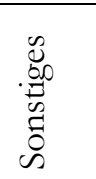 & $0,2 \%$ \\
\hline $\begin{array}{l}\text { W1a Buchenwald ohne Nutzung im Prozeß- } \\
\text { schutzwald } \\
\text { W1b Buchenwald ohne Nutzung im Wirtschaftswald } \\
\text { W1c Naturwaldparzellen (Forschung) } \\
\text { W2a naturnaher Wirtschaftswald (reine Laubwälder) } \\
\text { W2b Waldrandentwicklung } \\
\text { W3a Umwandlung Nadelgehölze zum Laubwald } \\
\text { WWirtschaftswald) } \\
\text { W3b Umwandlung Nadelgehölze zum Laubwald, } \\
\text { danach Nullwirtschaft } \\
\text { W4 Waldneubildung } \\
\text { B1a Bruchwald ohne Nutzung im Prozeßschutzwald } \\
\text { B1b Bruchwald ohne Nutzung im Wirtschaftswald } \\
\text { B2a Entwicklung Bruchwald } \\
\text { B2b Entwicklung Bruchwald aus Fichtenforst } \\
\text { B3 Gebiete für Feuchtwaldentwicklung } \\
\text { B4 Sukzession Feuchtbrachen Niederwaldnutzung }\end{array}$ & 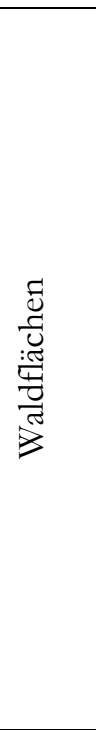 & $50,9 \%$ \\
\hline $\begin{array}{l}\text { S1a Große Seen von jeder Nutzung freihalten } \\
\text { S1b Kleine Seen von jeder Nutzung freihalten } \\
\text { S1c Ufer als Prozessschutz } \\
\text { S2a Große Seen extensiv nutzen } \\
\text { S2b Kleine Seen extensiv nutzen } \\
\text { S2c Ufer extensiv nutzen } \\
\text { S3a Seen entwickeln } \\
\text { S3b Ufer entwickeln } \\
\text { S4 Berücksichtigung Naturschutz auf Seen } \\
\text { F1a Erhalt Fließgewässer } \\
\text { F1b Erhalt Bachschlucht } \\
\text { F1c Erhalt und Entwicklung Flusslandschaft } \\
\text { F2a Entwicklung Fließgewässer } \\
\text { F3 Anhebung Grundwasserstand in Niederungen }\end{array}$ & 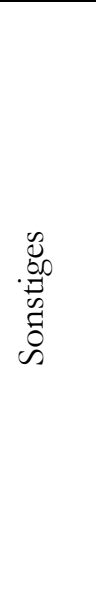 & $3,5 \%$ \\
\hline
\end{tabular}


G1a Erhalt wertvoller Feuchtgrünlandbestände durch Pflege

G1b Offenhalten von Feuchtgrünland, Streuwiesennutzung

G1c Erhalt und Entwicklung von Feuchtgrünland durch Pflege

G2 Entwicklung Feuchtgrünland aus intensivem

Grünland

G3a Erhalt Trocken/Magerstandorte

G3b Erhalt und Entwicklung Tro-

cken/Magerstandorte

G4a Erhalt strukturreiche Weidelandschaften

G4b Erhalt und Entwicklung Weidelandschaften

G4c Entwicklung Weidelandschaften aus Intensivgrünland

G5a Acker umwandeln in Grünland

G5b Intensives Grünland extensivieren

K1 Erhalt Klein- / Sonderstrukturen

K2 Entwicklung Hecken/Knicks, Gehölzstrukturen

K3 Entwicklung Kleingewässer

K4 Ackerrandstreifen

M1a Erhalt offene Hochmoorkernfläche

M1b Erhalt regionaltypische Moore

M1c Erhalt Moorflächen in Form nasser Torfstiche

M1d Erhalt Moorflächen in Form nasser Moorwälder

M2a Entwicklung Moorflächen zu feuchtem Moorwald

M2b Entwicklung Moorflächen aus Fichtenpflanzungen

M2c Entwicklung offener Moorflächen mit Pfeifengras

N1 Entwicklung nährstoffarme Niedermoorflächen

N2 Entwicklung nährstoffreiche Niedermoorflächen 
Wie wichtig sind die folgenden Ziele für nachhaltige Regionalentwicklung in den nächsten fünf Jahren?

nicht wichtig

Intakte Umwelt

Intakte Gesellschaft

Intakte Wirtschaft

Intakte Verwaltung

Langfristigkeit
$\mathrm{O} \quad \mathrm{O}$

$\mathrm{O} \quad \mathrm{O}$

$\mathrm{O} \quad \mathrm{O}$

$\mathrm{O} \quad \mathrm{O}$

$\mathrm{O} \quad \mathrm{O}$

sehr wichtig

Sonstiges

Wie relevant sind die folgenden Themen für regionale Nachhaltigkeit?

nicht relevant sehr relevant

$\begin{array}{lllll}\mathrm{O} & \mathrm{O} & \mathrm{O} & \mathrm{O} & \mathrm{O} \\ \mathrm{O} & \mathrm{O} & \mathrm{O} & \mathrm{O} & \mathrm{O} \\ \mathrm{O} & \mathrm{O} & \mathrm{O} & \mathrm{O} & \mathrm{O} \\ \mathrm{O} & \mathrm{O} & \mathrm{O} & \mathrm{O} & \mathrm{O}\end{array}$

O O O O O

O O O O O

$\begin{array}{lllll} & \mathrm{O} & \mathrm{O} & \mathrm{O} & \mathrm{O}\end{array}$

$\begin{array}{lllll}\mathrm{O} & \mathrm{O} & \mathrm{O} & \mathrm{O}\end{array}$

$\begin{array}{lllll}\mathrm{O} & \mathrm{O} & \mathrm{O} & \mathrm{O} & \mathrm{O}\end{array}$

$\begin{array}{lllll}\mathrm{O} & \mathrm{O} & \mathrm{O} & \mathrm{O} & \mathrm{O}\end{array}$

$\begin{array}{lllll}\mathrm{O} & \mathrm{O} & \mathrm{O} & \mathrm{O} & \mathrm{O}\end{array}$

$\begin{array}{lllll}\mathrm{O} & \mathrm{O} & \mathrm{O} & \mathrm{O} & \mathrm{O}\end{array}$

$\begin{array}{lllll}\mathrm{O} & \mathrm{O} & \mathrm{O} & \mathrm{O}\end{array}$

$\begin{array}{lllll}\mathrm{O} & \mathrm{O} & \mathrm{O} & \mathrm{O} & \mathrm{O}\end{array}$

$\begin{array}{lllll}\mathrm{O} & \mathrm{O} & \mathrm{O} & \mathrm{O} & \mathrm{O}\end{array}$

O $\quad \mathrm{O} \quad \mathrm{O} \quad \mathrm{O} \quad \mathrm{O}$

O $\quad \mathrm{O} \quad \mathrm{O} \quad \mathrm{O} \quad \mathrm{O}$

E-Partizipation 
Wer sind sichtbare Akteure / Institutionen („Leuchttürme“) in den jeweiligen Themenfeldern? Welches Maß an Verantwortlichkeit ist ihnen eigen?

Ak.teur/Institution

Verantwortlichkeit (boch, gering)

Politik

Gesellschaft

Umweltschutz

Regionale Wirtschaft

Soziale Gerechtigkeit

Generationengerechtigkeit

Bildung

Basis-Demokratie

Zukunftsfähigkeit

Klimaschutz

Gesundheit

Geschlechtergerechtigkeit

Ausweisung von Baugebieten

Demographie

Kooperation mit Kammern

Wettbewerb mit Nachbarkommunen/-regionen

Möglichkeiten von E-Partizipation

Wie wirken die Akteure/Institutionen auf die jeweiligen Themenfelder Ihrer Region? Welche Ressourcen und Befugnisse besitzen sie?

Akteur/Institution

Wirkung/Ressourcen/Befugnisse

Politik

Gesellschaft

Umweltschutz

Regionale Wirtschaft

Soziale Gerechtigkeit

Generationengerechtigkeit

Bildung

Basis-Demokratie

Zukunftsfähigkeit

Klimaschutz

Gesundheit

Geschlechtergerechtigkeit

Ausweisung von Baugebieten

Demographie 
Anhang 2: Interviewleitfaden der Institutionenanalyse von regionaler Nachhaltigkeit

\section{Institutionen der Nachhaltigkeit in den Regionen}

\section{TEIL 1: Einführung}

Wird in Ihrer Region Nachhaltigkeit bedacht? (Beispiele?)

Fordert die Region Nachhaltigkeit ein? (Beispiele?)

Warum fordert die Region Nachhaltigkeit ein, bzw. nicht?

Halten Sie ein Nachhaltigkeitsmanagement in der Region für sinnvoll? (Beispiele?)

Existiert in der Region ein Nachhaltigkeitsmanagement, Regionalmanagement bzw. Fördertitelmanagement?

Sehen Sie Bedarf eines „Regionalmanagements“, bzw. „Fördertitelmanagements“ in der Region?

Gibt es Institutionen, die sich in der Region um Nachhaltigkeit kümmern?

\section{TEIL 2: Leitfadeninterview}

Haben Sie ein Nachhaltigkeitskonzept?

Benutzen Sie eine Definition von Nachhaltigkeit?

$\mathrm{O} \mathrm{Ja}$

O Nein

\section{Wie lautet diese?}

Was ist Nachhaltigkeit für Sie?

O ein Leitbild

O eine Zukunftsstrategie

O ein Politischer Mehrwert

O ein Wirtschaftlicher Mehrwert

O ein Gesellschaftlicher Mehrwert

$\mathrm{O}$ eine Floskel

O Sonstiges 
Kooperation mit Kammern

Wettbewerb mit Nachbarkommunen/-regionen

Möglichkeiten von E-Partizipation

Welches Themenfeld sollte in Ihrer Region besonders gestärkt werden und warum?

Akteur/Institution

Begründung für die Stärkung

Politik

Gesellschaft

Umweltschutz

Regionale Wirtschaft

Soziale Gerechtigkeit

Generationengerechtigkeit

Bildung

Basis-Demokratie

Zukunftsfähigkeit

Klimaschutz

Gesundheit

Geschlechtergerechtigkeit

Ausweisung von Baugebieten

Demographie

Kooperation mit Kammern

Wettbewerb mit Nachbarkommunen/-regionen

Möglichkeiten von E-Partizipation

Wem oder was gegenüber fühlen Sie sich im Sinne von Nachhaltigkeit verpflichtet?

$$
\text { keine Verpflichtung bohe Verpflichtung }
$$

Gesellschaft

$$
\begin{array}{lllll}
\mathrm{O} & \mathrm{O} & \mathrm{O} & \mathrm{O} & \mathrm{O}
\end{array}
$$

Gesetz

$\mathrm{O} \quad \mathrm{O}$

$\mathrm{O}$

$\mathrm{O} \quad \mathrm{O}$

Moral

$\mathrm{O} \quad \mathrm{O}$

$\mathrm{O}$

$\mathrm{O} \quad \mathrm{O}$

Religion

O O

$\mathrm{O}$

$\mathrm{O} \mathrm{O}$

Anspruchsgruppen

$\mathrm{O} \quad \mathrm{O}$

$\mathrm{O}$

O O

Aktionäre

O O

$\mathrm{O}$

O O

Natur

$\mathrm{O} \quad \mathrm{O}$

$\mathrm{O}$

$\mathrm{O} \quad \mathrm{O}$

Kommende

O O

$\mathrm{O}$

$\mathrm{O}$

O 
In welchem zeitlichen Rahmen wirkt Nachhaltigkeit?

O Kurzfristig

O Mittelfristig

O Langfristig

In welchem zeitlichen Rahmen lässt sich Nachhaltigkeit messen?

O Immer

O Kurzfristig

O Mittelfristig

O Langfristig

O Gar nicht

Welche Indikatorensätze sind Ihnen bekannt?

Welche Priorität hat Nachhaltigkeit in Ihrer Region?

O Hohe Priorität

O Mittlere Priorität

O Niedrige Priorität

Haben Sie in Ihrer Region Empfehlungen für Verhaltensweisen im Sinne der Nachhaltigkeit?

Für wie wichtig halten Sie bei Projekten mit dem Bund und anderen Regionen ein gemeinsames Verständnis von Nachhaltigkeit?

O Wichtig

O Nicht so wichtig

O Unwichtig

Sollte eine verbindliche Definition von Nachhaltigkeit aufgestellt werden und wenn ja, von welchem Akteur/Institution?

TEIL 3: Beziehung vom „Grünen Band“ und regionaler Nachhaltigkeit

Welche Bedeutung ordnen Sie dem „Grünen Band“ im Kontext der regionalen Nachhaltigkeit zu? 
Anhang 3: Fragebogen der strukturellen Netzwerkanalyse

Name

Funktion/Vertreter von...

\section{Frage 1}

Bitte nennen Sie aus Ihrer Sicht die 10 wichtigsten Akteure aus der Region für die Initiierung, Planung und Umsetzung des Projekts.

\begin{tabular}{|l|l|l|}
\hline Name & Vorname & Institution/Vertreter von... \\
\hline & & \\
\hline & & \\
\hline & & \\
\hline & & \\
\hline & & \\
\hline & & \\
\hline & & \\
\hline & & \\
\hline
\end{tabular}

\section{Frage 2}

Haben Sie den Akteur durch das Projekt kennen gelernt?

\begin{tabular}{|l|l|l|}
\hline Name & Ja & Nein \\
\hline & & \\
\hline & & \\
\hline & & \\
\hline & & \\
\hline & & \\
\hline & & \\
\hline & & \\
\hline & & \\
\hline
\end{tabular}




\section{Frage 3}

Wie häufig hatten Sie im Jahr 2007 im Rahmen des Projekts mit dem Akteur zu tun?

\begin{tabular}{|l|l|l|l|l|l|}
\hline Name & $\begin{array}{l}\text { Selten } \\
(\max .3 x)\end{array}$ & $\begin{array}{l}\text { Gelegentlich } \\
(\text { max. 6x) }\end{array}$ & $\begin{array}{l}\text { Häufig } \\
\text { (mind. 7x) }\end{array}$ & Nie & Weiß nicht \\
\hline & & & & & \\
\hline & & & & & \\
\hline & & & & & \\
\hline & & & & & \\
\hline & & & & & \\
\hline & & & & & \\
\hline & & & & & \\
\hline & & & & & \\
\hline & & & & & \\
\hline & & & & & \\
\hline
\end{tabular}

\section{Frage 4}

Welcher Art waren diese Kontakt?

\begin{tabular}{|l|l|l|l|}
\hline Name & Telefon/ E-Mail & Projektveranstaltung o.ä. & $\begin{array}{l}\text { Arbeits- } \\
\text { besprechung o.ä. }\end{array}$ \\
\hline & & & \\
\hline & & & \\
\hline & & & \\
\hline & & & \\
\hline & & & \\
\hline & & & \\
\hline & & & \\
\hline & & & \\
\hline & & & \\
\hline
\end{tabular}




\section{Frage 5}

Der primäre Zweck des Kontakts zu Akteur Nr. war ...

\begin{tabular}{|c|c|c|c|c|}
\hline Name & $\begin{array}{l}\text { Austausch } \\
\text { von Informa- } \\
\text { tionen }\end{array}$ & $\begin{array}{l}\text { Ressourcen- } \\
\text { austausch }\end{array}$ & $\begin{array}{l}\text { Kooperation zur Reali- } \\
\text { sierung bestimmter } \\
\text { Ziele }\end{array}$ & $\begin{array}{l}\text { Weiß } \\
\text { nicht }\end{array}$ \\
\hline & & & & \\
\hline & & & & \\
\hline & & & & \\
\hline & & & & \\
\hline & & & & \\
\hline & & & & \\
\hline & & & & \\
\hline & & & & \\
\hline & & & & \\
\hline & & & & \\
\hline
\end{tabular}

\section{Frage 6}

Wessen Rat oder Unterstützung ist für Sie im Rahmen des Projekts wichtig? Bitte kereuzen Sie in der Tabelle den entsprechenden Akteur/ die entsprechenden Akteure an.

\begin{tabular}{|l|l|}
\hline Name & \\
\hline & \\
\hline & \\
\hline & \\
\hline & \\
\hline & \\
\hline & \\
\hline & \\
\hline & \\
\hline
\end{tabular}




\section{Frage 7}

Wie wichtig ist der Kontakt zum Akteur für Sie? Ist er...

\begin{tabular}{|c|c|c|c|c|c|}
\hline Name & Unwichtig & $\begin{array}{l}\text { Weniger } \\
\text { wichtig }\end{array}$ & wichtig & $\begin{array}{l}\text { Sehr } \\
\text { wichtig }\end{array}$ & $\begin{array}{l}\text { Weiß } \\
\text { nicht }\end{array}$ \\
\hline & & & & & \\
\hline & & & & & \\
\hline & & & & & \\
\hline & & & & & \\
\hline & & & & & \\
\hline & & & & & \\
\hline & & & & & \\
\hline & & & & & \\
\hline & & & & & \\
\hline & & & & & \\
\hline
\end{tabular}

\section{Frage 8}

Welche der 10 von Ihnen genannten Personen nehmen aus Ihrer Sicht eine Schlüsselposition im Projekt oder in der Region ein? (Mehrfachnennungen möglich)

\begin{tabular}{|l|l|l|}
\hline Name & $\begin{array}{l}\text { Im } \\
\text { Projekt }\end{array}$ & $\begin{array}{l}\text { In der } \\
\text { Region }\end{array}$ \\
\hline & & \\
\hline & & \\
\hline & & \\
\hline & & \\
\hline & & \\
\hline & & \\
\hline & & \\
\hline & & \\
\hline & & \\
\hline
\end{tabular}




\section{Frage 9}

Sehen Sie Defizite oder Lücken in Ihrer Kommunikation mit anderen Akteuren in dem Projekt?

\begin{tabular}{|l|l|}
\hline Inhaltliche Konflikte zwischen Interessengruppen & \\
\hline Persönliche Konflikte zwischen Akteuren & \\
\hline $\begin{array}{l}\text { Organisatorische Probleme, die die Kommunika- } \\
\text { tion stören }\end{array}$ & \\
\hline Es gibt keine & \\
\hline Weiß nicht & \\
\hline
\end{tabular}

Andere Defizite oder Lücken:

\section{Nun einige Fragen zu Ihrem persönlichen Engagement}

\section{Frage 10}

Aus welchen Gründen engagieren Sie sich

für das Projekt? (Bitte nur einen Grund angeben)

\begin{tabular}{|l|l|}
\hline Berufliches Interesse & \\
\hline Ehrenamt & \\
\hline Privates Interesse & \\
\hline
\end{tabular}

\section{Frage 11}

Wie hat sich Ihr Engagement für das Projekt ergeben?

\begin{tabular}{|l|l|}
\hline Eigeninitiative & \\
\hline Angesprochen aufgrund der & \\
Funktion/ Position & \\
\hline $\begin{array}{l}\text { Angesprochen aufgrund Res- } \\
\text { sourcen (Geld, Informationen) }\end{array}$ & \\
\hline $\begin{array}{l}\text { Angesprochen aufgrund persön- } \\
\text { licher Kontakte }\end{array}$ & \\
\hline
\end{tabular}




\section{Frage 12}

Wie gut fühlen Sie sich in das Akteursnetzwerk des Projekts eingebunden?

\begin{tabular}{|l|l|}
\hline Sehr gut & \\
\hline Gut & \\
\hline Befriedigend & \\
\hline Ausreichend & \\
\hline Mangelhaft & \\
\hline Ungenügend & \\
\hline Weiß nicht & \\
\hline
\end{tabular}

\section{Zum Schluss benötigen wir noch einige Angaben zu Ihrer Person}

\section{Daten 1}

In welchem Jahr sind Sie geboren?

\section{Daten 2}

Wo wohnen Sie?

\section{Daten 3a}

Welchen Beruf üben Sie aus und welchen Ausbildungsabschluss haben Sie?

Beruf:

Ausbildungsabschluss:

\section{Daten 3b}

Seit wann sind Sie in Ihrer jetzigen Position?

\begin{tabular}{|l|l|}
\hline Seit ein paar Monaten & \\
\hline Mindestens seit einem Jahr & \\
\hline Seit mehreren Jahren & \\
\hline Seit mehr als zehn Jahren & \\
\hline Weiß nicht & \\
\hline
\end{tabular}

\section{Daten 4a}

In welchen Vereinen, Organisationen, Parteien sind Sie Mitglied?

\section{Daten 4b}

Sind Sie in diesem Verein bzw. dieser Organisation/Partei ehrenamtlich aktiv?<smiles>C1CCC1</smiles>

\section{Nein}

\section{Daten 4c}

Haben Sie dort ein bestimmtes Amt inne?

\section{Daten 4d}

Seit wann üben Sie diese ehrenamtliche Tätigkeit aus?

\begin{tabular}{|l|l|}
\hline Seit ein paar Monaten & \\
\hline Mindestens seit einem Jahr & \\
\hline Seit mehreren Jahren & \\
\hline Seit mehr als zehn Jahren & \\
\hline Weiß nicht & \\
\hline
\end{tabular}




\section{Anhang 4: Interviewleitfaden zur Netzwerkanalyse}

1. Mit welchen Maßnahmen sind Sie in das Projekt eingebunden? Wer außer Ihnen noch? Können Sie mir die Maßnahme kurz erläutern? Warum beteiligen Sie sich im Projekt?

2. Wie funktioniert die Organisationsstruktur? Welche „Gremien“ gibt es? Ist die Organisationsstruktur des Projekts geeignet? Wo besteht Änderungsbedarf?

3. Wie werden die Akteure in den laufenden Prozess eingebunden? Haben Sie neue Akteure der Region kennengelernt?

4. Sind Ihrer Meinung nach alle relevanten Akteure in das Projekt integriert? Wie ist die Wirtschaft in das Projekt integriert?

5. Wie gestaltet sich die Arbeit innerhalb des Projekts/ der Maßnahme? Wo gibt es in der Zusammenarbeit mit den unterschiedlichen Akteuren Schwierigkeiten? Wie sieht dies aus und was ist der Grund dafür?

a. Wie funktioniert der Austausch mit anderen Beteiligten im Projekt/ in der Maßnahme?

b. Wie funktioniert der Informationsfluss innerhalb des Projekts/ in der Maßnahme? Wie werden Sie über den Stand der Dinge und die erzielten Erfolge im Projekt informiert?

c. Wie werden zentrale Entscheidungen im Projekt / in der Maßnahme gefällt? Sind sie transparent und nachvollziehbar?

6. Welche Bedeutung hat die personelle Kontinuität für das Projekt?

7. Wie schätzen Sie die Beteiligung und Motivation der Akteure ein im Projekt/ in der Maßnahme?

8. Gibt es im Rahmen des Projekts ein gemeinsames Leitbild? Welche Bedeutung hat es für Sie (in der Maßnahme)?

9. Wer gibt dem Projekt Rückhalt in der Region? Sind dies die gleichen Personen, wie für andere Projekte der Region?

10. Wer ist für das Projekt besonders wichtig? Wen würden Sie als Multiplikator bezeichnen? Warum?

11. Welche Rolle spielt das Projekt in der Öffentlichkeit? 
12. Welche Probleme sind durch das Projekt / die Maßnahme in der Region entstanden und wie wird damit umgegangen?

13. Welchen „Gewinn“ können Sie aus dem Netzwerk bereits ziehen? Haben sich bereits weitere Projekte aufgrund des Netzwerks entwickelt?

14. Wie soll es nach Abschluss des Projekts weitergehen? Wie werden die entwickelten Maßnahmen weitergeführt?

15. Gibt es für die Kommunikation bestimmte Instrumente, für deren Realisierung/ Anwendung Sie die Unterstützung durch den Fördermittelgeber benötigen?

16. Wie gestaltet sich die länderübergreifende Kooperation? Hat das Projekt dazu beigetragen?

17. Worin besteht bzgl. der länderübergreifenden Kooperation Handlungsbedarf? Wo funktioniert es bereits gut? Welche Faktoren wirken fördernd bzw. hemmend?

18. Was ist Ihnen bei der Arbeit im Projekt/ in der Maßnahme wichtig? Worauf kommt es Ihnen an? 
und und Länder engagieren sich seit der deutDschen Wiedervereinigung für die Bewahrung des ökologischen Potenzials am "Grünen Band", der ehemaligen innerdeutschen Grenze. Im Rahmen von Erprobungs- und Entwicklungsvorhaben sowie Naturschutzgroßprojekten setzt sich das Bundesministerium für Umwelt, Naturschutz und Reaktorsichrheit (BMU), für den Erhalt, die Pflege und Entwicklung großräumiger Landschaftsräume am Grünen Band ein. Die Verknüpfung der Naturschutzziele mit regionaler Nachhaltigkeit gewinnt für deren dauerhafte Verankerung vor Ort zunehmend an Bedeutung.

Die bislang unzureichend erfassten Wirkungen der Naturschutzförderung auf die Entwicklung der Regionen waren Anlass für das BMBF-Forschungsvorhaben „Grünes Band - Modellregion für Nachhaltigkeit" (2007-2010). Regionalwissenschaftler der Hochschule für angewandte Wissenschaft und Kunst (HAWK) untersuchten im interdisziplinären Verbund mit Wirtschaftswissenschaftlern der Universität Göttingen am Beispiel von vier Regionen die Wirkung der Bundesförderung auf die regionale Nachhaltigkeit. Der vorliegende Bericht präsentiert die disziplinären und interdisziplinären Ergebnisse sowie die jeweiligen und gemeinsam erarbeiteten Handlungsempfehlungen für Fördermittelgeber und -empfänger.

$\frac{3}{8}$

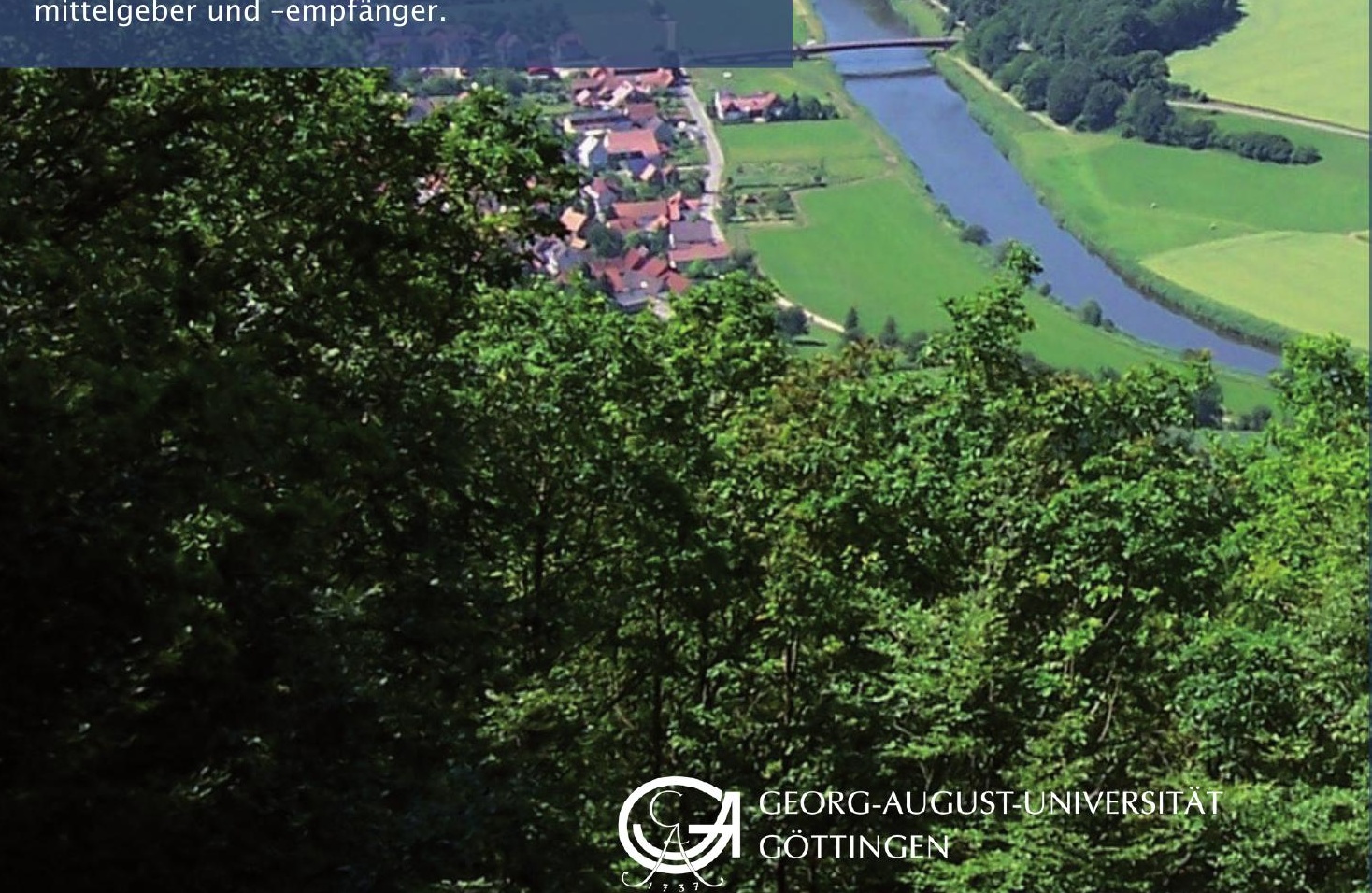

